\title{
Lasers in the Conservation of Artworks XI
}





\section{Lasers}

\section{in the Conservation}

of Artworks XI

\section{Proceedings of the International Conference LACONA XI,}

Kraków, Poland, 20-23 September 2016

\section{Editors:}

Piotr TARgowski,

Nicolaus Copernicus University in Toruń, Poland

MaŁgorzata Walczak,

The Jan Matejko Academy of Fine Arts in Kraków, Poland

Paraskevi Pouli,

Institute of Electronic Structure \& Laser, Foundation

for Research and Technology - Hellas (FORTH), Heraklion,

Crete, Greece

\section{Organisers:}

The Jan Matejko Academy of Fine Arts in Kraków

Nicolaus Copernicus University in Toruń

The Interacademic Institute of Conservation and Restoration of Art, Warszawa/Kraków

The National Museum in Kraków 
$11^{\text {th }}$ Conference on Lasers in the Conservation of Artworks is co-financed by the Minister of Science and Higher Education of Poland (Project No. 581/P-DUN/2016) from the resources allocated for the activities disseminating knowledge.

\section{How to cite this document:}

Authors, title, in: Lasers in the Conservation of Artworks XI, Proceedings of the International Conference LACONA XI, Kraków, Poland, 20-23 September 2016, P. Targowski, M. Walczak, P. Pouli (Eds.), NCU Press, Toruń 2017, DOI: $10.12775 / 3875-4$

On the cover:

The vault of the lantern in the Sigismund's Chapel of the Wawel Cathedral, Kraków, Poland, during laser cleaning. Photo: Jan Marczak

(C) Copyright by Nicolaus Copernicus University Press

Toruń 2017

ISBN 978-83-231-3875-4

\section{NICOLAUS COPERNICUS UNIVERSITY PRESS}

Editorial office: ul. Gagarina 5, 87-100 Toruń

tel. 566114295 , fax 566114705

e-mail: wydawnictwo@umk.pl

Distribution: ul. Mickiewicza 2/4, 87-100 Toruń

tel./fax 566114238

e-mail: books@umk.pl

www.wydawnictwoumk.pl

Printed by: The printing house of Nicolaus Copernicus University Press 


\section{Contents}

$\begin{array}{lr}\text { Foreword } & 9\end{array}$

Permanent Scientific Committee $\quad 13$

$\begin{array}{ll}\text { Local Organising Committee } & 14\end{array}$

Final Endeavors of "Monument Man"

[John F. Asmus, Vadim A. Parfenov, Jessica P. Elford]

\section{DEVELOPMENTS IN LASER REMOVAL OF UNWANTED SUBSTANCES ON CULTURALLY-IMPORTANT OBJECTS AND MONUMENTS}

Wavelength-dependent absorption and scattering effects on laser cleaning of a corroded iron alloy European scale armor

[Marlene April Yandrisevits, Pablo Londero, Federico Carò, Adriana Rizzo, Caterina Cappuccini]

From Earth to Outer Space: Laser cleaning semiprecious quartz and a novel application for meteoritic metal

[Rebecca A. Kaczkowski, Bartosz A. Dajnowski, Edward P. Vicenzi]

Laser yellowing effect: study of the nanophases created by laser irradiation of synthetic black crusts using transmission electron microscopy (TEM) and electron paramagnetic resonance (EPR) spectroscopy

[Marie Godet, Véronique Vergès-Belmin, Christine Andraud, Mandana Saheb, Judith Monnier, Eric Leroy, Julie Bourgon, Laurent Binet]

Laser Cleaning of a First Phase Navajo Wool Chief's Blanket by Nd:YAG

[Pablo Londero, Ludovica Corda, Daniele Ciofini, Christine Giuntini, Marco Leona]

Towards the understanding of the two wavelength laser cleaning in avoiding yellowing on stonework: a micro-Raman and LIBS study [Athanasia Papanikolaou, Panayiotis Siozos, Aggelos Philippidis, Kristalia Melessanaki, Paraskevi Pouli]

The potential of OCT for assessing laser assisted removal of deposits from ceramic tiles

[Magdalena Iwanicka, Jędrzej Musiela, Jadwiga W. Łukaszewicz, Henryk Stoksik,

Marcin Sylwestrzak] 
The Hanford Nuclear Plant: Radiation Ablation Meets History, Cultural Heritage, and Nuclear Decontamination

[John F. Asmus, Vadim A. Parfenov, Jessica P. Elford]

\section{COHERENT LIGHT-BASED METHODS FOR IMAGING, DOCUMENTATION, EXAMINATION AND ANALYSIS OF OBJECTS AND STRUCTURES}

Surface micro-profilometry for the assessment of the effects of traditional and innovative cleaning treatments of silver

[Claudia Daffara, Nicola Gaburro, Giacomo Marchioro, Alessandro Romeo, Giulia Basilissi, Andrea Cagnini, Monica Galeotti]

Nonlinear optical imaging techniques (NLO) for painting investigation [Alice Dal Fovo, Raffaella Fontana, Jana Striova, Enrico Pampaloni, Marco Barucci, Marco Raffaelli, Raffaella Mercatelli, Luca Pezzati, Riccardo Cicchi]

A moderate microsampling in Laser Ablation Inductively Coupled Plasma Mass Spectrometry analysis of cultural heritage objects: a review [Barbara Wagner, Olga Syta, Marek Sawicki]

A preliminary study of contemporary binders by Time Resolved Laser Induced Fluorescence (TR-LIF) spectroscopy: characterization of the painting Nascita Della Forma by Nato Frascà [Martina Romani, Marco Marinelli, Alessandra Pasqualucci, Gianluca Verona-Rinati]

LIBS, optical and multivariate analyses of selected $17^{\text {th }}$-century oil paintings from the Museum of King Jan III's palace at Wilanów [Agnieszka Pawlak, Wojciech Skrzeczanowski, Krzysztof Czyż]

Elemental analysis of Chinese red seal inks on xuan paper by ArF laserinduced plume fluorescence [Bruno Yue Cai, Vincent Motto-Ros, Vincent Detalle, Judy T.S. Lum, Kelvin S. Y. Leung, Nai-Ho Cheung]

\section{CASE STUDIES}

Using the new G.C. Laser Cleaning System for cleaning and surface preparation for re-gilding of a large outdoor bronze monument of Alexander Hamilton

[Andrzej Dajnowski, Bartosz A. Dajnowski] 
Experiences at the Academy of Fine Arts of Brera in Milan, Italy: the application of laser-technology on three case studies of the historical heritage

[Elisa Isella, Donatella Bonelli, Silvia Cerea, Francesca Mancini, Veronica Ruppen, Alessandra Botteon, Antonio Sansonetti]

Decontamination of biocidal loaded wooden artworks by means of laser and plasma processing

[Birgit Angelika Schmidt, Simone Pentzien, Andrea Conradi, Jörg Krüger,

Constanze Roth, Oliver Beier, Annett Hartmann, Bernd Grünler]

Laser-based techniques for a multidisciplinary action aimed at the restitutive restoration of S. Costanzo church in Ronciglione (Italy)

[Valeria Spizzichino, Luisa Caneve, Massimiliano Ciaffi, Roberta Fantoni, Massimo Francucci, Massimiliano Guarneri, Antonio Palucci, Gaetano Terranova, Franca Persia, Angelo Tati, Maria Fernanda Falcon Martinez, Chiara Giuffrida, Francesca Scirpa, Laura Bartoli, Alessandro Zanini]

Archaeometric investigations of medieval stained glass panels from Grodziec in Poland

[Dariusz Wilk, Marta Kamińska, Małgorzata Walczak, Ewa Bulska]

Easel paintings on canvas and panel: application of Nd:YAG laser at 355 $\mathrm{nm}, 1064 \mathrm{~nm}$ and UV, IR and visible light for the development of new methodologies in conservation

[Joakim Striber, Vanja Jovanović, Maja Jovanović]

White, yellow and green pigments on Polish artists' palettes in the period 1838-1938

[Mirosław Wachowiak, Grzegorz Trykowski, Iwona Żmuda-Trzebiatowska]

Spectral imaging of Dutch gilt leather for improved conservation strategies [Roger M. Groves, Vassilis M. Papadakis, Martine Posthuma de Boer, Tigran Mkhoyan, Bianca van Velzen, Kate Seymour] 



\section{Foreword}

The eleventh conference on Lasers in the Conservation of Artworks has been the latest of the LACONA conference series, initiated more than 20 years ago - in 1995 - by Costas Fotakis and held in FORTH in Heraklion, Greece. It was followed by the LACONA II meeting in Liverpool, UK in 1997, LACONA III in Florence, Italy in 1999, LACONA IV in Paris, France in 2001, and then Osnabrück, Germany in 2003. The LACONA conference was held in Vienna, Austria in 2005, followed by Madrid, Spain in 2007, Sibiu, Romania in 2009 and London, UK in 2011. The most recent LACONA X conference was planned in 2013 in Egypt; however, due to political disturbances it was transferred to Sharjah, UAE and held in 2014. The present edition was held in Kraków, Poland, hosted by the Jan Matejko Academy of Fine Arts. It was co-organized by Nicolaus Copernicus University in Toruń, The Interacademic Institute of Conservation and Restoration of Art in Warszawa and Kraków, and, last but not least, by the National Museum in Kraków.

The early editions of LACONA were devoted mostly to the application of lasers to cleaning of artworks, which, at the time, was an emerging topic. Today, we prefer to talk about "removal of unwanted layers and coatings" to emphasize that this application now goes far beyond simple "cleaning" and has become - for many classes of objects a mature procedure, widely accepted within the conservation/restoration community. Nevertheless, its effective and safe application to specific objects remains a subject of extensive study. Also, continual progress in the development of lasers, especially by utilizing the ultra-short pulses provided by compact and less expensive sources, opens new areas of application by placing a broader set of tools in the hands of the conservator/ restorer. Another significant feature of recent LACONA conferences is the much broader range of subjects presented, since the application of lasers in the conservation of artworks covers much more than simply "laser cleaning”. Appropriate restoration procedures are always preceded by examination and laser techniques - or, more broadly speaking, techniques utilizing coherent light - such as LIBS, Raman spectroscopy, optical coherence tomography and nonlinear or two-photon laser microscopy, feature regularly as significant subjects at LACONA.

A specific aim for the LACONA series has always been to bring together conservation scientists active in the field of developing methods and instruments with practising restorers and conservators who are the most important recipients of these new developments. Without the professional knowledge and experience of the latter it would not be possible to implement any of the results obtained in laboratory research in conservation practice. Therefore, LACONA has always been a unique forum for the exchange of ideas between scientists and practitioners. All these areas of interests are reflected in this year's conference programme, where the two largest sessions are devoted to fundamental research on the removal of unwanted 
substances with the aid of lasers and to case studies, in which reports of the use of lasers in restoration studios were presented. These two groups of contributions were complemented by coherent light-based methods for imaging, documentation and examination of objects and structures as well as laser-based spectroscopic techniques for the analysis of culturally-significant objects and the monitoring of restoration treatments.

During this, the latest, LACONA conference, 45 oral communications and 14 posters were presented. For the first time the LACONA conference was preceded by a oneday workshop for about 50 participants, dedicated to laser cleaning techniques for various cultural heritage objects. During hands-on sessions, the participants had an opportunity to work with four different specialized lasers to gain experience and a better understanding of the use of lasers in conservation. This session could not have been possible without a kind support from Dr. Alessandro Zanini from El.En., Ms. Małgorzata Musiela from Restauro Sp. z o.o. and Mr. Bartosz Dajnowski from GC Laser Systems Inc.

This volume comprises 20 peer-review research articles submitted by participants after the conference. They are organised in three chapters covering laser ablation, coherent light-related analytical techniques and case studies. Additionally, the first chapter contains two key-note articles, describing lesser-known aspects of the history of use of light and lasers in the context of preservation of cultural heritage.

Finally, as a chief editor I would like to express my gratitude to the co-editors of this volume, all referees, the Permanent Scientific Committee of LACONA Conferences and, last but not least, to the Local Organising Committee. Very special thanks go to His Magnificence, the Rector of the Jan Matejko Academy of Fine Arts in Kraków, Professor Stanisław Tabisz, and the Deans of the Department of Restoration and Conservation of Artworks. Without their enthusiastic support this event would not have been possible.

\section{Piotr Targowski \\ LACONA XI Chair \\ Kraków, Toruń - September 2016}




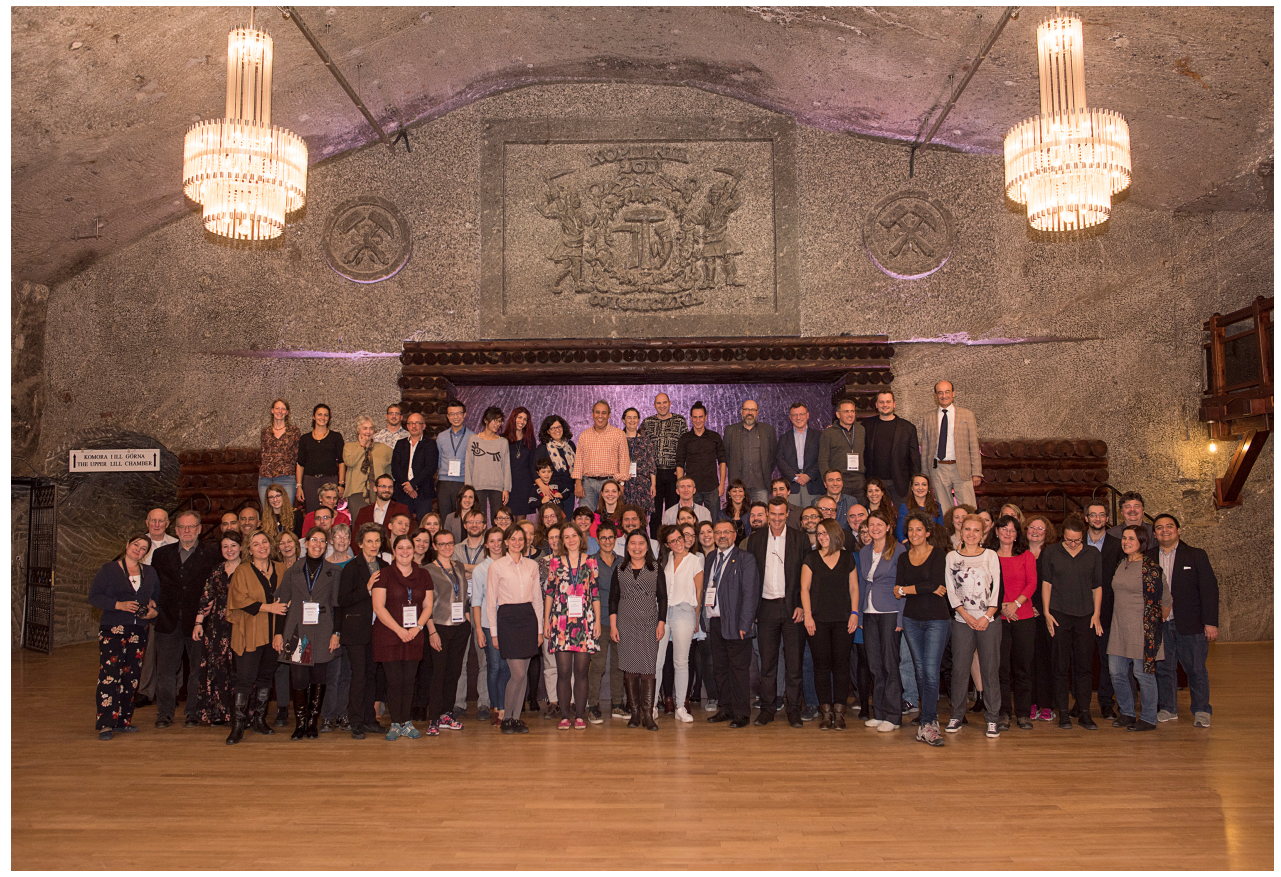

Lacona XI participants during the Conference Dinner in the "Wieliczka" Salt Mine, September 22nd, 2016. 



\section{Permanent Scientific Committee}

Margaret Abraham, Sr Project Lead, New Space Programs, NASA, The Aerospace Corp, El Segundo, CA, USA

John F. Asmus, Center for Advanced Nanotechnology, Department of Physics, University of California, San Diego (UCSD), La Jolla, CA, USA

Marta Castillejo, Instituto de Química Física Rocasolano, Consejo Superior de Investigaciones Científicas (CSIC), Madrid, Spain

Martin Cooper, Lynton Lasers Ltd., Holmes Chapel, Cheshire, United Kingdom

Vincent Detalle, Centre de recherche et de restauration des musées de France (C2RMF), Palais du Louvre, Paris, France

Abdelrazek Elnaggar, Conservation Department, Faculty of Archaeology, Fayoum University, Fayoum, Egypt

Costas Fotakis, Institute of Electronic Structure \& Laser (ISL) Foundation for Research and Technology - Hellas (FORTH), Heraklion, Crete, Greece

Wolfgang Kautek, Institut für Physikalische Chemie, Universität Wien, Austria

Austin Nevin, Institute for Photonics and Nanotechnologies - Consiglio Nazionale delle Ricerche, Politecnico di Milano, Milano, Italy

Johann Nimmrichter, Abteilung für Restaurierung und Konservierung, Bundesdenkmalamt, Wien, Austria
Vadim A. Parfenov, Department of Quantum Electronics and Opto-Electronic Devices, St. Petersburg State Electrotechnical University, Saint-Petersburg, The Russian Federation

Paraskevi Pouli, Institute of Electronic Structure \& Laser, Foundation for Research and Technology - Hellas (FORTH), Heraklion, Crete, Greece

Roxana Radvan, Centre for Restoration by Optoelectronic Techniques - CERTO, National Institute for Research and Development in Optoelectronics - INOE, Bucuresti - Magurele, Romania

Matija Strlič, UCL Institute for Sustainable Heritage, University College London, London, United Kingdom

Renzo Salimbeni, Istituto di Fisica Applicata „Nello Carrara” - Consiglio Nazionale delle Ricerche, Firenze, Italy

David Saunders, Honorary Research Fellow, Department of Conservation and Scientific Research, British Museum, London, United Kingdom

Manfred Schreiner, Institut für Naturwissenschaften und Technologie in der Kunst (INTK), Akademie der bildenden Künste, Wien, Austria

Salvatore Siano, Istituto di Fisica Applicata „Nello Carrara”- Consiglio Nazionale delle Ricerche, Firenze, Italy

Piotr Targowski, Institute of Physics, Nicolaus Copernicus University, Toruń, Poland 
Véronique Vergès-Belmin, Laboratoire de recherche des monuments historiques, Champs sur Marne, France

\section{Local Organising Committee}

Piotr Targowski, Chair of the Lacona IX Conference, Nicolaus Copernicus University, Toruń

Małgorzata Walczak, Secretary of the Organising Committee, Jan Matejko Academy of Fine Arts, Kraków

Andrzej Koss, Interacademic Institute of Conservation, Warszawa

Edyta Bernady, Jan Matejko Academy of Fine Arts, Kraków

Maria Goryl, Jan Matejko Academy of Fine Arts, Kraków
Kenneth Watkins, The University of Liverpool, Department of Mechanical Engineering, Liverpool, United Kingdom

Marta Kamińska, Jan Matejko Academy of Fine Arts, Kraków

Zofia Krzemińska, Jan Matejko Academy of Fine Arts, Kraków

Barbara Łydżba-Kopczyńska, The National Museum in Kraków

Michał Płotek, Jan Matejko Academy of Fine Arts, Kraków

Anna Sobesto, The National Museum in Kraków

Marcin Sylwestrzak, Nicolaus Copernicus University, Toruń 
Lasers in the Conservation of Artworks XI,

Proceedings of LACONA XI, P. Targowski et al. (Eds.),

NCU Press, Toruń 2017, DOI: 10.12775/3875-4.01

\title{
Final Endeavors of "Monument Man"
}

\author{
John F. Asmus ${ }^{1 \star}$, Vadim A. Parfenov ${ }^{2}$, Jessica P. Elford ${ }^{1}$ \\ 1 Department of Physics and Center for Advanced Nanotechnology, University of California, \\ San Diego, 9500 Gilman Dr., La Jolla, CA, 92093-0319, USA \\ 2 Department of Quantum Electronics and Opto-Electronic Devices, St. Petersburg State \\ Electrotechnical University, Popova str., 5, 197101, St. Petersburg, Russian Federation \\ * Corresponding author: jfasmus@ucsd.edu
}

\begin{abstract}
In 1973, as a Visiting Scholar at UCSD/IGPP (University of California, San Diego/ Institute for Geophysics and Planetary Physics, Scripps Institution of Oceanography), Mr. George L. Stout (1897-1978; co-author of the bible of art conservation: "Painting Materials, A Short Encyclopedia"; Director of the Isabella Stewart Gardner Museum; and co-founding Presidents of the International Institute of Conservation of Historic and Artistic Works as well as the later AIC) performed the first-ever experiments on laser divestment of embrittled varnishes from paintings. In that early year for laser technology, the only readily-available puled laser was an original Hughes ruby device (a copy Theodore Maiman's first laser) operating in the free-running mode. In spite of the very significant limitation imposed by the use of this primitive laser, he found that the varnish could be just crazed at the optical interaction threshold. Then, he found that the weakened varnish residue was easily removed with a moistened cotton swab. In the final years of his life (1975-1978), Mr. Stout participated in the founding of the UCSD-affiliated Balboa Art Conservation Center and the UCSD Center for Art/Science Studies (CASS). Through his affiliation with UNESCO, he initiated projects at CASS to employ laser divestment in saving the Egyptian temples at Philae. By 1978, his idea of utilizing digital computer image processing (borrowed from the SIO lunar space and moon-rock programs) had been pursued at CASS.
\end{abstract}

Keywords: Laser, Icon, Varnish, Gioconda, Leonardo, Philae

\section{Background}

The Science Advisor to President Richard M. Nixon (Dr. Edward E. David, Jr.) arranged for the leading USA laser scientists to assemble during the summer of 1971 to formulate a National Laser Research Plan. The most profound of the numerous recommendations that emerged from that policy study ("JASON 1971") led to a substantial expansion of the laser program at the Lawrence Livermore
National Laboratory (LLNL) and the establishment of the National Ignition Facility (NIF) with what was then named the Nova Laser, as its centerpiece, for the attainment of thermonuclear Inertial Confinement Fusion (ICF). A footnote in the Final Report Executive Summary cites recent advances in pulsed ruby laser holography that enable in-situ creation of 3D diffraction-limited images of meter-scale subjects: far beyond the state-of the-art centimeter-scale objects 
of traditional laboratory holography. This technology had been employed to diagnose the performances of rocket-propulsion plasma jets and space-satellite microwave antennas. The JASON Committee noted that pulsed holography had the potential to record high-resolution 3D images of crumbling artistic treasures. Shortly thereafter, at the occasion of being inducted into the Club of Rome, JASON member Professor Walter Munk was able to negotiate an agreement with the Italian Petroleum Institute (ENI) to fund a holographic feasibility experiment to record for posterity archival 3D images, in-situ, of endangered Venetian sculptural treasures.

In the aftermath of the Venetian Holography project numerous museums (e.g. The Gardner Museum in Boston and the Smithsonian Institution in Washington) and universities (e.g. Harvard University and Massachusetts Institute of Technology in Cambridge) extended invitations for lectures on the holography project and subsequent discovery of laser divestment and cleaning in art conservation. Subsequently, it was learned that these invitations came in response to private disclosures by an interested prominent art conservator. The Venetian Laser Holographic and Divestment exploratory investigations were being promoted by George L. Stout, Director of the Isabella Stewart Gardner Museum, co-author of Painting Materials, a short encyclopedia (the bible of art conservation), co-founder and founding President of both the International Institute for conservation (IIC) and the American Institute for Conservation AIC), and recipient of both a Bronze Star (army) and the Congressional Gold Medal. Furthermore, he arranged (with Clements Robertson, Director, Nelson Art Gallery) for the laser work to be presented at the inaugural conference of the AIC in Kansas City (1973). The following three sections summarize the investigations that Stout insisted would transform art conservation practice.

\section{Holographic replication and diagnostics}

The ENI-sponsored (US \$7,000) holographic conservation study took place in the winter of 1971-1972 and employed a 2J/pulse ruby laser oscillator and single-stage ruby amplifier yielding a coherence length of $10 \mathrm{~m}$. The holographic arrangement is shown in San Gregorio (Venice) together with its developer (Dr. Ralph Wuerker) in Fig. 1.

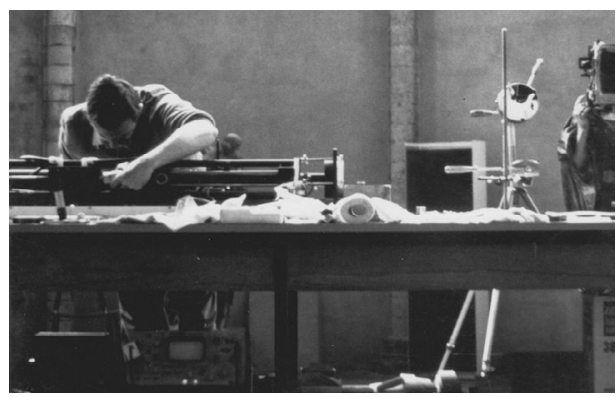

Fig. 1. Ralph Wuerker at S. Gregorio producing in-situ holograms of the Donatello John the Baptist carving.

During the three-month project, more than 50 transmission type archival holograms were produced of large $(1-2 \mathrm{~m})$ stone statues, woodcarvings, and paintings by Donatello, Nino Pisano, Caravaggio, and other artists. The holograms were recorded on 12- and $25-\mathrm{cm}$ glass plates and placed on display at the Academia Museum in Venice with HeNe laser illumination. A photograph of the reconstruction of one of the first in a series of Venetian holographic interferograms (the coherent interference pattern from super- 
imposed holographic images) is reproduced in Fig. 2. In this example of holographic NDT (Non-Destructive Testing) the painted wooden leg of the Donatello work was

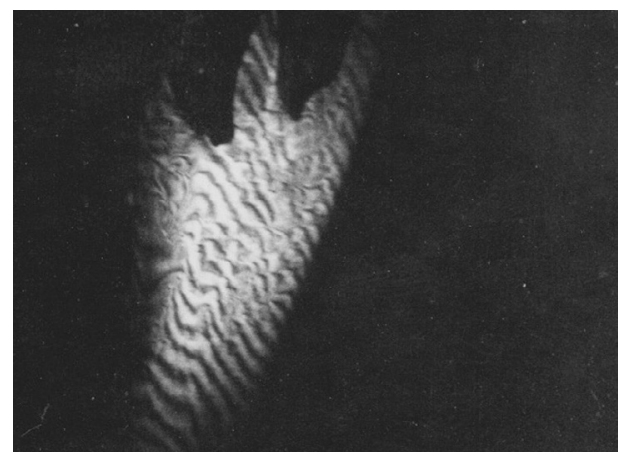

Fig. 2. Double-exposure hologram of the leg of the Donatello painted wood carving revealing hidden cracks.

subjected to a shift in humidity during the interval between a double-pulse exposure. The anomalous interference fringes reveal the locations of paint-layer detachments and subsurface cracks. Such interferograms may be employed to guide art conservators in repairing such defects [1].

\section{Laser stone divestment in Venice}

In the field of art conservation surface divestment frequently poses an array of vexing problems in the conservation, preservation, and presentation of artworks [2]. It is not uncommon to find that an encrustation, over paint, corrosion layer, soil, or biological growth to be removed is more durable (both chemically and physically) than its submerged artistic remnant. In traditional art-conservation practice the various mechanical and chemical surface treatments often attack the overburden and substrate with comparable vigor. Customarily, it is a matter of obser- vation, skill, and timing that leads to maximum divestment with minimum damage or alteration to the fabric of the artifact itself. The problem of ancillary damage to artwork surfaces during cleaning is exacerbated by collateral health hazards to workers. Protective equipment to shield conservators from vapors, chemicals, and dust can impede the observation and control needed for precise and optimum treatment. Consequently, there has been a prolonged interest in discovering a new divestment technology that is free of the limitations of conventional chemical, mechanical, and abrasive methods. One such candidate is radiation-induced divestment employing non-toxic and environmentally friendly photons.

Toward the end of the Venetian holographic project in February 1972 G. Musumeci (conservator) was shown illustrations revealing impressive impulses delivered to surfaces through laser ablation (Figs. 3 and 4). She returned to San Gregorio from a meeting of the Soprintendenza alle Gallerie e alle Opere d'Arte del Veneto with the suggestion that stone cleaning be attempted by concentrating the ruby laser beam on a specimen of the black encrustation ("stone cancer") that was continuing to consume the city's marble monuments. She explained to the hologra-

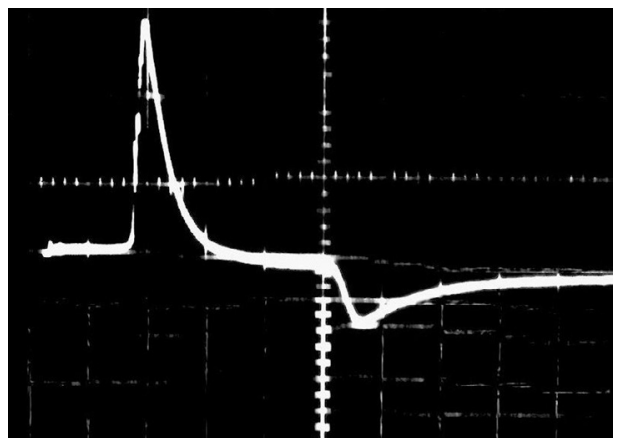

Fig. 3. Laser-induced stress wave and its reflection in a metal wafer. 


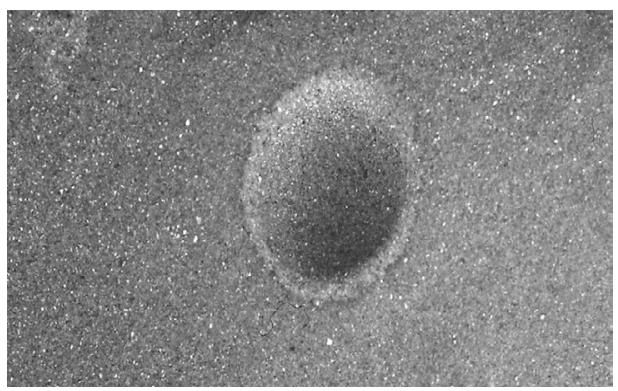

Fig. 4. Hypervelocity impact crater in a steel plate from a laser-propelled wafer.

phy team that outdoor sculptures in Venice were being "eaten" by the oxides of sulphur present in the local air pollution. In essence the air pollution destroys stone sculpture by converting solid marble (calcium carbonate) into granular gypsum (calcium sulphate). Conservation measures (cleaning, protection, and consolidation) were being impeded and avoided due to the propensity of the then current cleaning methods (abrasive, chemical, and mechanical) to damage friable stones. Proposed conservation projects failed to be granted official approval because available cleaning methods yielded an unacceptable patina with a "frosted" appearance. It was suggested that A. Schawlow's famous "laser eraser" technology [2] might be adapted to non-destructive and self-limiting stone conservation through the selective removal of black encrustations from crumbling marble sculpture without altering or damaging the weak surviving marble.

\section{Initial laser projects}

By March 1972, the holographic work had been completed. It was calculated that the $2 \mathrm{~J}$ free-running pulses (non Q-switched with pulse length determined by pump duration) should be applied to $4 \mathrm{~mm}$ spots and that the
1J Q-switched pulses should be applied to 1 $\mathrm{cm}$ spots. During a two-day interval about fifteen Venetian monument stone specimens were test cleaned and parametric variations in laser flux and fluence were performed. The results were assessed by Superintendent F. Valcanover and Prof. L. Lazzarini for the Veneto and Mr. K. Hempel of the Victoria and Albert Museum for the Venice in Peril Fund. The three were in accord that every result was superior to conventional surface divestment in aesthetic patina and avoidance of surface damage [3]. Consequently, a series of laser-divestment major stone-conservation projects followed, beginning with the Cremona Cathedral (Fig. 5).

The 3D archival holographic recording proceeded with the assembly of images of Greek and Roman antiquities at the Getty/ Malibu Museum (Fig. 6).

\section{George Stout at UCSD (1974-1977)}

In September 1973, two advisors to the World War II staff of General Eisenhower met for the first time in a hospital room in San Diego, California. George Stout, retired Director of the Gardner Museum of Boston, had led the Monuments Men (played by motion picture

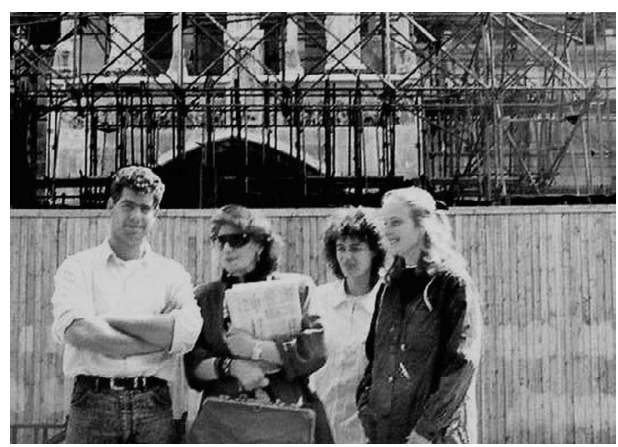

Fig. 5. Cremona Cathedral laser restoration team at the project site (1990). 


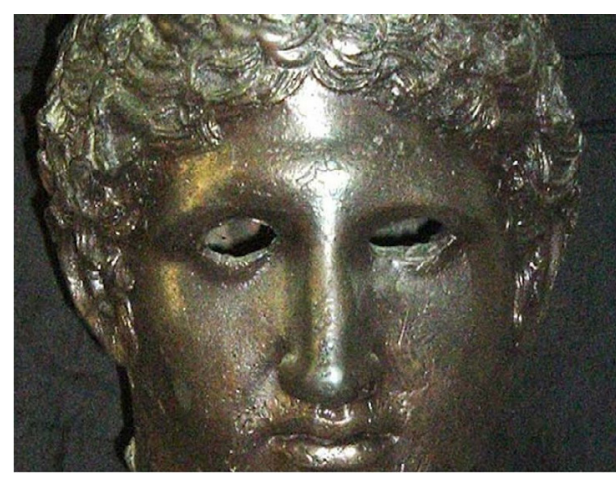

Fig. 6. Getty bronze of a young Greek that was holographically reproduced in 1974 for the opening of the Museum.

actor George Clooney) in recovering World War II looted artworks. Professor Walter Munk, (recovering from a skiing accident) was an Associate Director of the Scripps Institution of Oceanography/Institute for Geophysics and Planetary Physics (SIO/ IGPP) and had led the team that forecast the sea conditions for the WWII Normandy invasion sites and selected an optimum date for the Allied landings. At their hospital meeting, the pair set in motion events that led to the establishment of the Balboa Art
Conservation Center as well as the UCSD Center for Art/Science Studies (CASS).

Initially (1972-1974), laser cleaning tests and post-cleaning diagnostics at UCSD focused on a wide variety of antique stone specimens from monuments and buildings of Venice and London. This was a consequence of the major role in the laser evaluation program that was assumed by Sir Ashley Clark and Kenneth Hempel. However, George Stout's entire career had centered on paintings. His consulting tasks at The Timken concerned paintings, and he had just completed the second edition of his 1948 monograph: The Care of Pictures. Thus, it is understandable that he was eager to explore laser-ablation technology for improved conservation of paintings, rather than objects of stone, metal, ceramic, glass, or wood.

The CASS scientists and graduate students were uniformly skeptical as to the likelihood of Stout having any success in selectively removing a somewhat transparent varnish layer from the polychromatic paint layer of a painting with a single wavelength longpulse ruby laser (Fig. 7). To the surprise of all, he discovered that by setting the laser flux at

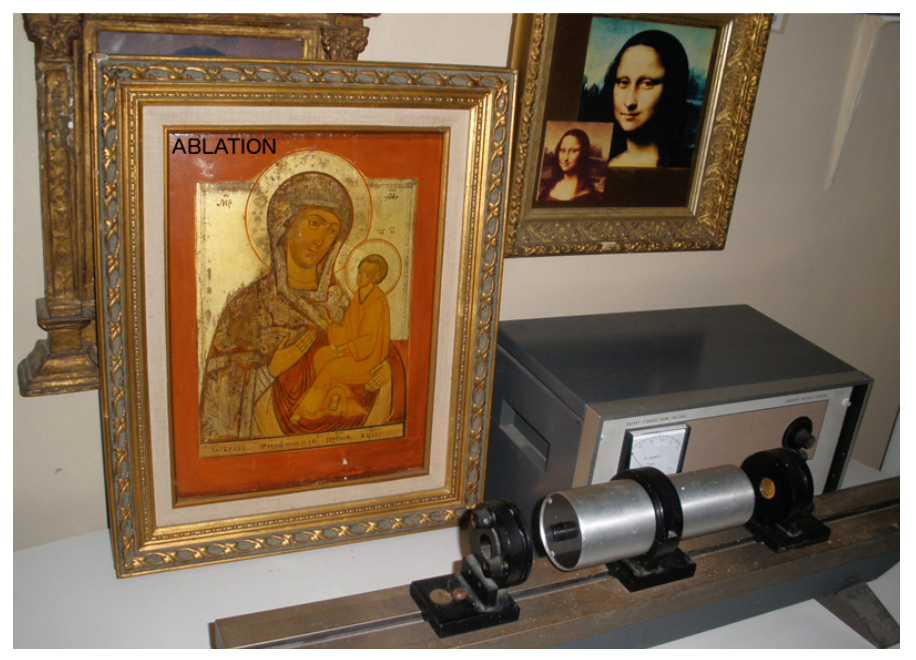

Fig. 7. 1962 Hughes

Company ruby laser copy of Theodore Maiman's original laser used by George Stout in the first radiation-divestment cleaning of a painting (Serbian Icon shown). 


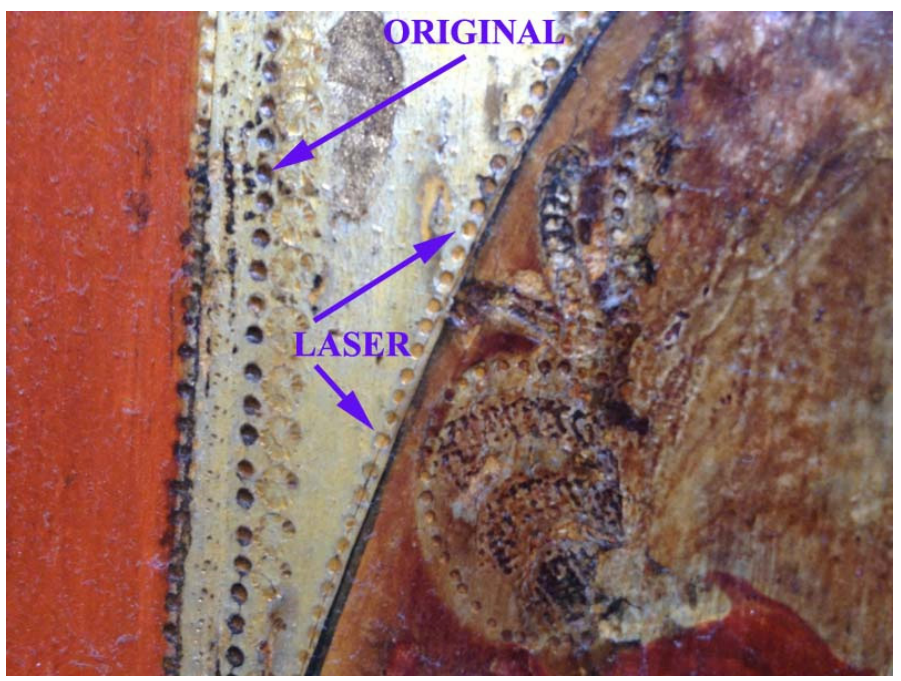

Fig. 8. Detail of a Timken Gallery Serbian icon revealing Stout's success in the laser-aided removal of darkened varnish residues from dimples in the gold gilding. the interaction threshold, the varnish would be crazed just enough so that the degraded residue could be easily removed with a moistened cotton swab. However, patience was still required as several cycles of irradiation followed by swabbing was required to completely remove the varnish. Figure 8 shows what Stout had accomplished early in the process of cleaning one of the Serbian icons (Lady of Tikhvin) of the Timken Museum collection.

The introduction and commercial availability of newer high-performance systems such as excimer, erbium, picosecond, and femtosecond lasers has amplified the utility a much broader range of applications. Reliable and long-lived laser systems operating on the harmonics of the Nd:YAG wavelength have also opened new opportunities for the laser divestment of difficult materials such as papers and paints. The improved access to alternative wavelengths and shorter pulse lengths enhances the likelihood of avoiding thermal damage to fragile materials and the production of unwanted chemical ablation byproducts. Finally, it has become practical to employ multiple wavelengths, simultaneously, in order to lessen unfortunate outcomes such as the yellowing of certain marbles during laser divestment.

\section{Laser removal of the Isis Temple of Philae painted stripes}

Upon returning to UCSD from a UNESCO meeting on the fate of Isis Temple of Philae under the encroaching waters of Lake Nasser, Stout divulged that one of the problems facing the conservation teams in Egypt was the removal of painted white alignment stripes from some of the temple stones. He calculated that the laser radiation flux "window" for successful self-limiting divestment was $142-164 \mathrm{~J} / \mathrm{cm}^{2}$ at the $10.6 \mathrm{um}$ wavelength of carbon dioxide lasers. It was proposed that a TEA laser divestment test would be performed on an Isis stone specimen supplied by UNESCO. First, the spot size for irradiation was adjusted for a flux of $50 \mathrm{~J} / \mathrm{cm}^{2}$. Only a single pulse was required to completely remove the white paint. A microscopic examination 
failed to reveal any sign of alteration (color or morphological) to the sandstone. A second portion of the paint stripe was irradiated at $100 \mathrm{~J} / \mathrm{cm}^{2}$. The results were indistinguishable from the earlier result. These observations deviate from the theoretical prediction. However, this is to be expected as the composition, consolidation, and homogeneity of both the paint and the stone are likely to be different from the handbook parameter values used in the calculations. The post-test condition of the Isis stone is displayed in Fig. 9.

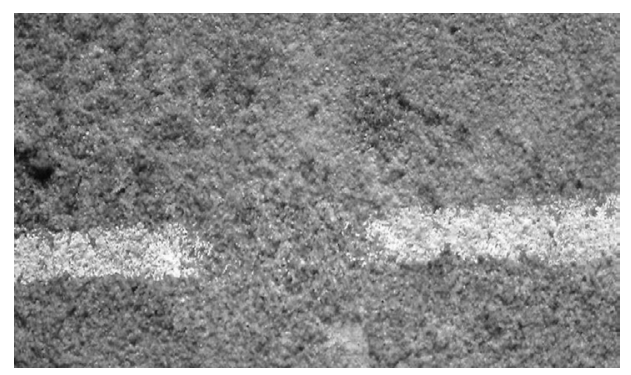

Fig. 9. A sandstone test block from the Isis Temple of Philae showing the region where the paintedwhite stripe was removed by $\mathrm{CO}_{2}$ TEA laser self-limiting ablation $\left(50-100 \mathrm{~J} / \mathrm{cm}^{2}\right)$.

Plans were formulated, after the successful laboratory paint removal tests, for the laser treatment of the reassembled Isis Temple of Philae on the island of Agilkia. As schedules and costs were being determined, the onsite team made an unforeseen discovery. They found that environmental exposure (temperature excursions, sunshine, wind, and blowing sand) of the temple was leading to the gradual disappearance of the white stripes. Consequently, it was decided to let Nature take its course and just remain patient and await the eventual disappearance of the paint without resorting to laser ablation. The years following the Isis white stripe investigation have witnessed numerous examples of laser ablation of encrustations from dark substrates (e.g. [4] and [5]). Increased understanding, newer laser types, and improved models have all contributed to broader venues of success in such circumstances.

\section{Digital enhancement of the "Mona Lisas"}

When Stout first arrived at UCSD/SIO/IGPP in 1974 the major research activities there were concerned with the analysis of Moon Rocks and Lunar Imaging from lunar orbiting satellites. Upon touring the computer imaging laboratory at the Satellite Remote Sensing Facility (SRSF) he reflected immediately: "Couldn't we do computer image restoration of paintings?". During that era, the Voyager probes were intercepting Saturn, Jupiter, and the Jovian moons so that no time was available on the image-processing computers. Other visitors in those years, such as Prof. Carlo Pedretti, Prof. James Arnold, Mr. Walter Cronkite, Lord Kenneth Clark, and Mr. Thomas Hoving, offered similar suggestions, independently. However, it was not until 1985 that the IBM Corporation offered unlimited access to their Palo Alto Supercomputer before significant progress was possible in digital-computer image restoration to recover the original Mona Lisa from beneath the fog of the brown varnish layers, the web of cleavages, and the earlier restorations [6]. Figure 10 illustrates one of the results of that effort in removing the craquelure through FFT and Bi-scatter digital filtering.

Through the ages, prodigious energies have been absorbed into controversies about the execution date (or dates) of the portrait. The artistic, historical, and philosophical concerns that hinge on this are too numerous, convoluted, and erudite to be summarized adequately here. Suffice it to say that on 


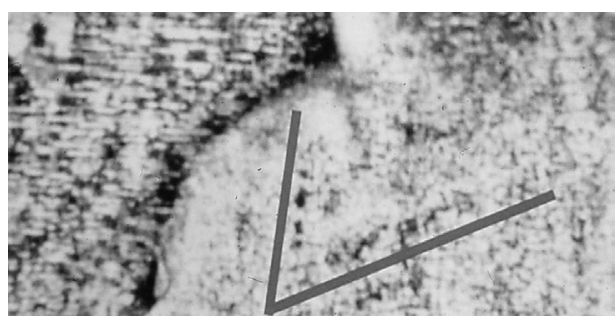

Fig. 10. Mona Lisa detail identifying varnish cleavages and craquelure (left side) by means of regional digital enhancement. The same numerical processing revealed vee-shaped pentimenti, indicating that Leonardo originally intended that the female figure wore a necklace (reproduced with permission from Mona Lisa Foundation).

largely stylistic grounds, Pedretti dates it to 1513-1516. The position taken by Lord Clark on historical evidence points to 1503 [7].

Also curious, is Leonardo's reason for producing duplicate versions of three of his other significant works. These are his Virgin of the Rocks, the Virgin and Child, and the Virgin and Child with St. Anne. Through the ages this recognition has triggered speculation that there may have been a second Mona Lisa by Leonardo, as well. A number of paintings have been advanced as the possible second Mona Lisa, only to be discarded after failing historical and/or scientific scrutiny.

The art connoisseur Hugh Blaker acquired a Mona Lisa painting in 1913 and placed it on display at his Isleworth studio near London. The apparent youth of the lady compared to the figure in the Louvre Mona Lisa as well as a completely different background clearly established that this painting was not simply a copy of the portrait in the Louvre. A number of art experts asserted that the painting, identified then as the Isleworth Mona Lisa, was of such a high quality that it had to be by the hand of Leonardo da Vinci. In a NY Times column, Paul G. Konody was unrestrained in his enthusiasm for the quality of the piece as well as its proportions and the arrangement of its elements [8].

By 1989, the Isleworth Mona Lisa had been in the hands of the Pulitzer Estate and an arrangement was made for a cursory scientific examination to determine whether the visual features of the painting were consistent with Leonardo's style and technique. This initial study was necessarily of limited scope as the artwork could not be touched or removed from its storage vault. Consequently, the analyses were performed on photographs taken at the storage facility in Lausanne. Upon digitizing these photographs and inspecting proportions and alignments it became evident immediately that the Isleworth painting was not a copy of the Louvre Mona Lisa that we had been studying and analyzing for the previous ten years $[5,6]$. On the other hand, it was clearly demonstrated that the artist's strategy in aligning elements in the composition followed identical rules. In the subsequent 27 years, the Isleworth painting has passed virtually every comparative scientific test available in art conservation science with respect to the Jaconde portrait in the Louvre Museum. These tests include pigment analyses, multispectral and hyperspectral imaging, 3D imaging, isotopic measurements, geometrical analyses pertaining to the Vertruvian proportion and golden ratio, radiocarbon dating, infrared scanning, and digital-image age regression (employing FBI programs pertaining to facial recognition.) In addition, the Isleworth Mona Lisa has been visually inspected by a number of notable Leonardo experts [9].

\section{Conclusion}

When the first manuscript describing the laser project in Venice was submitted to 
a journal for publication (1972), it was rejected without the benefit of a peer review with the editor's dismissal: "Cleaning with lasers is too hypothetical to be taken seriously." In contrast, George Stout's entire career marked him as a true visionary and his very early advocacy of laser divestment, holographic and digital imaging, and radiation ablation of coatings on paintings and dark substrates revealed his creative personality. Stout's participation in the initial research projects at CASS dictated the vectors for their evolution and fruition. Subsequent to Stout's passing in 1978, all of these art conservation opportunities have matured and found widespread application.
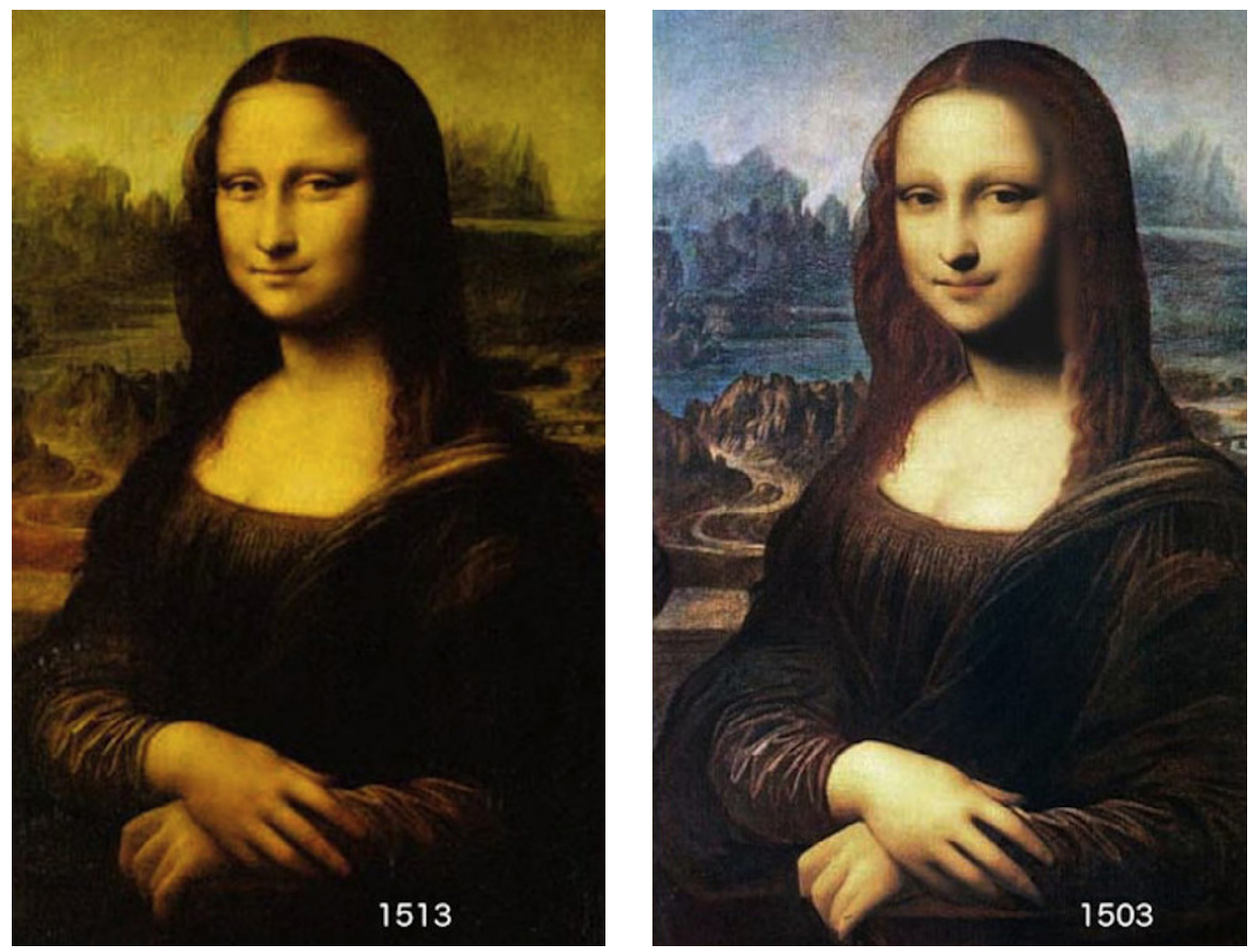

Fig. 11. The Mona Lisa in the Louvre Museum (L) and the Isleworth (Earlier) version (R) embedded in the digitally-corrected background of the Louvre painting (reproduced with permission from Mona Lisa Foundation). 


\section{References}

[1] J.F. Asmus, W.H. Munk, and R.F. Wuerker, "Lasers and Holography in Art Preservation and Restoration", IEEE Northeast Electronics Research and Engineering Meeting, Boston, 5-9, November 1972.

[2] E.H. Gombrich, The Story of Art, Phaidon, London 1967.

[3] J.F. Asmus, M. Seracini, and M.J. Zetler, "Surface Morphology of Laser-Cleaned Stone", Lithoclastia, 1 (1976) 23-46.

[4] O. Madden, M. Abraham, S. Scheerer, and L. Werden, "The Effects of Laser Radiation on Adhesives, Consolidants, and Varnishes", in: Lasers in the Conservation of Artworks, Lacona V Proceedings, Osnabrück, Germany, Sept. 15-18, 2003, K. Dickmann, C. Fotakis, J.F. Asmus (Eds.), Springer 2005, pp. 249-254.

[5] S. Chlouveraki, P. Pouli, K. Melessanaki, K. Zervaki, and M. Yiannakaki, "Laser Cleaning Studies of Hard Insoluble Aluminosilicate Crusts on Minoan
(LM IIIC) Pottery", in: Lasers in the Conservation of Artworks, Lacona V Proceedings, Osnabrück, Germany, Sept. 15-18, 2003, K. Dickmann, C. Fotakis, J.F. Asmus (Eds.),. Springer 2005, pp. 143-155.

[6] J.F. Asmus and N.F. Katz, "Digital image processing applied to problems of art and architecture," in: Applications of Digital Image Processing XI, Proc. SPIE, 974 (1988) 278-282.

[7] C. Pedretti, Leonardo: A Study in Chronology and Style, University of California Press, Berkeley 1973.

[8] P.G. Konody, "Another Mona Lisa found in London", The New York Times, February 15, 1914, http:// query.nytimes.com $/ \mathrm{mem} /$ archive-free/pdf?res $=9 \mathrm{~A} 0$ 7E2DD1E39E633A25756C1A9649C946596D6CF, (accessed 25.06.2017).

[9] The Mona Lisa Foundation, Leonardo da Vincis Earlier Mona Lisa, The Mona Lisa Foundation, Zurich 2015.

[10] K. Clark, Leonardo da Vinci, Cambridge University Press, Cambridge 1952. 


\section{Developments in laser removal of unwanted substances on culturally-important objects and monuments}





\title{
Wavelength-dependent absorption and scattering effects on laser cleaning of a corroded iron alloy European scale armor
}

\author{
Marlene April Yandrisevits ${ }^{1,2 \star}$, Pablo Londero ${ }^{3}$, Federico Carò ${ }^{1}$, \\ Adriana Rizzo ${ }^{1}$, Caterina Cappuccini ${ }^{1}$ \\ 1 The Metropolitan Museum of Art, 1000 Fifth Avenue, New York, NY 10028, USA \\ 2 Smithsonian National Air and Space Museum, Steven F. Udvar-Hazy Center, 14390 Air and Space \\ Museum Parkway, MRC 326, Chantilly, VA 20151, USA \\ 3 Institute for the Preservation of Cultural Heritage, Yale University, 300 Heffernan Road, Bldg. 800, \\ West Haven, CT 06516, USA \\ * Corresponding author: marlene.yandrisevits@metmuseum.org
}

\begin{abstract}
This research seeks to optimize Nd:YAG laser cleaning parameters for the removal of corrosion products on historic iron alloy surfaces. This article presents the treatment of a $19^{\text {th }}$-century, European scale armor jazeran in the collection of the Arms and Armor Department at The Metropolitan Museum of Art. Laser cleaning was coupled with traditional mechanical cleaning techniques to increase the time efficiency of treatment while best preserving the composite material construction of the artifact and the aesthetic expectations of treatment. Laser cleaning using an $8 \mathrm{~ns}$, Q-switched, Nd:YAG laser at $532 \mathrm{~nm}$ was found more effective at corrosion removal and less damaging to the underlying metal surface than laser cleaning at $1064 \mathrm{~nm}$. Wavelength-dependent absorption and scattering effects on the laser cleaning of the corroded iron alloy surfaces were investigated. The composition, morphology, and stratigraphy of the corrosion layers were characterized and the effects of laser cleaning of these corrosion layers at $1064 \mathrm{~nm}$ and $532 \mathrm{~nm}$ were examined using stereomicroscopy, scanning electron microscopy - energy dispersive spectroscopy and back-scattered and secondary electron imaging, Raman spectroscopy, Fourier transform - infrared spectroscopy, and thermally assisted hydrolysis and methylation - gas chromatography - mass spectrometry. It was found that the success of the $532 \mathrm{~nm}$ laser cleaning is consistent with the green laser's more resonant energy absorption and decreased scattering length as compared to $1064 \mathrm{~nm}$. These data were used to optimize the optical parameters of the laser energy interaction with the corrosion products to develop more effective and safer laser cleaning profiles for the removal of ferrous corrosion layers on the historic iron alloy surface.
\end{abstract}

Keywords: laser cleaning, iron, wavelength, absorption, scattering, armor

\section{Introduction}

The reduction of thick corrosion layers from historic iron artifacts has traditionally been accomplished through mechanical or chemical techniques. Mechanical methods typically employ an abrasive compound or sharp tool to separate corrosion layers from the underlying metal through physical forces. Chemical means often utilize $\mathrm{pH}$, reducing/ 
oxidizing agents, chelators, or electrical charge to solubilize or convert corrosion products on the metal surface. For some iron surfaces, however, traditional corrosion removal approaches may prove non-ideal. Mechanical and/or chemical techniques may lack adequate precision or control, may jeopardize adjacent alloys and/or associated non-metallic materials, might create an undesired surface appearance, or may be prohibitively time-consuming for effective treatment. As alternative treatment methods are explored, laser cleaning has become increasingly popular as a conservation tool for reducing corrosion layers from historic metals. In laser cleaning, the laser energy parameters can be tailored to remove metal oxides and other undesired surface material without damaging the underlying metal alloy.

For some metal surfaces, laser cleaning can prove more time efficient in reducing corrosion layers and more precise and controllable in protecting underlying and adjacent historic materials than traditional treatment methods, thereby increasing the efficacy of treatment and promoting the overall preservation of the artifact. After laser cleaning, the metal surface might still be treated using traditional finishing methods to achieve the desired surface aesthetic and to promote corrosion resistance.

\subsection{Background}

Laser cleaning as a tool for the removal of corrosion layers from metal surfaces has been investigated widely [1-4]. While much research has focused on copper alloys [5-13] and gilt surfaces [6,14-17], equally significant work has sought to characterize the cleaning of iron alloys by lasers [18-32].

Studies on the laser cleaning of corroded metal alloys often characterize the effects of variances in laser wavelength and/or pulse duration on corrosion removal and metal substrate preservation $[1,3,21,24,25,27$, 28]. Considering the diversity of corrosion compositions and laser parameters, it is unsurprising that these studies do not present complete agreement on optimized factors in the laser cleaning of iron. Some studies cite improved iron corrosion removal utilizing a $532 \mathrm{~nm}$ wavelength [25], while others promote $1064 \mathrm{~nm}[21,24]$, and still others find no distinguishing results [28]. It is generally agreed that a shorter pulse duration reduces thermal effects and lessens the risk of micromelting [1, 24, 33]. Hypotheses toward laser parameter optimization posit the critical roles of laser fluence $[3,21,27$, 28], materials absorptivity [1, 3, 23, 24, 27], and thermal conductivity $[1,34]$ in the safe and effective removal of corrosion layers.

Other typical studies compare the efficacy of laser cleaning against conventional abrasive methods for corrosion removal [21, 25, 27-29]. General conclusions suggest that laser cleaning better preserves surface topography [27, 29]; however, laser cleaning introduces the risks of micromelting and/ or redeposition of ablated material [10, $20,24,26,27]$. Many studies conclude that optimized iron corrosion removal combines laser cleaning and traditional mechanical techniques, citing a limit to the effectiveness of lasers in removing very thick corrosion layers or particular corrosion species $[18,21$, $23,26,28]$.

This study investigates the effect of wavelength variance on corrosion removal and underlying metal preservation in the laser cleaning of a historic iron alloy, with particular consideration given to wavelength-dependent absorption and scattering effects. The impact of laser cleaning used in combination with traditional mechanical 
techniques on the time efficiency of treatment is also evaluated.

\subsection{Case Study}

In this study, laser cleaning is coupled with traditional mechanical cleaning techniques to reduce thick corrosion layers on the iron alloy surfaces of a late $19^{\text {th }}$-century, European scale armor jazeran (Gift of Ordinance Dept., U.S. Army, through Chief Engineering Dept., 1919; 19.49.16) in the collection of the Arms and Armor Department at The Metropolitan Museum of Art in New York (Fig. 1).

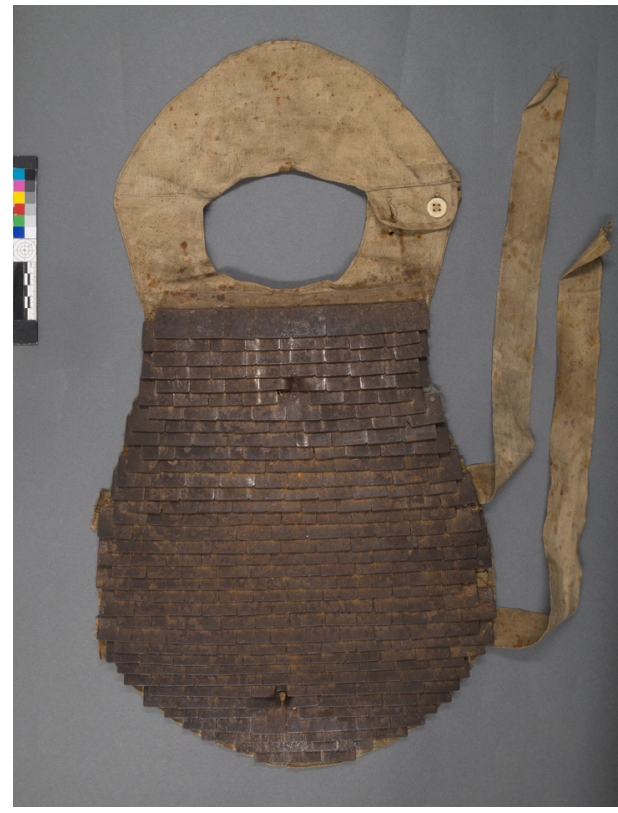

Fig. 1. The $19^{\text {th }}$-century, European scale armor (19.49.16) in the collection of the Arms and Armor Department of The Metropolitan Museum of Art which was the subject of this case study in laser cleaning of a corroded historic iron alloy surface.

The artifact belongs to a class of body defense worn to protect the breast and upper abdomen and is a variant on European scale armor typified by earlier brigandines [35].
The armor was gifted to The Met in 1919 by the Ordinance Department of the United States Army, in which The Met's founding Arms and Armor curator Bashford Dean served during the First World War [36]. Dean received his Army commission to research historic armors to design contemporary helmets and body defense for US armed forces; the jazeran of this case study featured in Dean's military experiments on ballistic resistance [37].

The armor consists of small, flat iron alloy rectangular plates which are riveted to both sides of textile strips subsequently sewn in overlapping horizontal rows across a bib-shaped textile support with textile back straps and bone collar buttons. The alloy composing the metal scales is observationally identified as "low carbon steel" [38]. No metallurgical testing was undertaken to characterize the composition of the alloy, as destructive testing was deemed too invasive; however, qualitative energy dispersive - $\mathrm{x}$-ray fluorescence spectroscopy confirms the ferrous nature of the metal and the lack of any surface enrichment or plating layers.

The surface of the armor's metal scales displays thick, non-homogenous corrosion layers (Fig. 2). The corrosion cross-section generally consists of localized surface spots

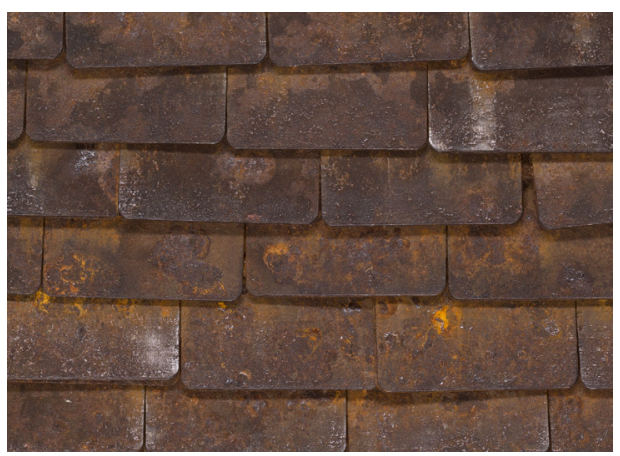

Fig. 2. Thick, non-homogenous corrosion layers on the surface of the armor's metal scales. 
of bright orange, friable corrosion; underlying compact, dark corrosion; and a lowest overall layer of red corrosion. A translucent film overlies the corrosion layers locally. The metal surface underneath the corrosion layers was darkly pitted in some areas, but brightly intact in other areas. It was decided for treatment that all corrosion layers would be reduced to reveal the aged, but clean, dark pitting patina of the underlying metal.

Comingling of the metal and textile elements within the object's construction complicated treatment. Often in the treatment of composite artifacts, components of different material types may be separated or masked to reconcile incompatibilities based on compositional sensitivities. In the case of this armor, with metal scales riveted on both sides of fabric supports, two material types with significantly different treatment vulnerabilities are permanently affixed and in intimate contact (Fig. 3).

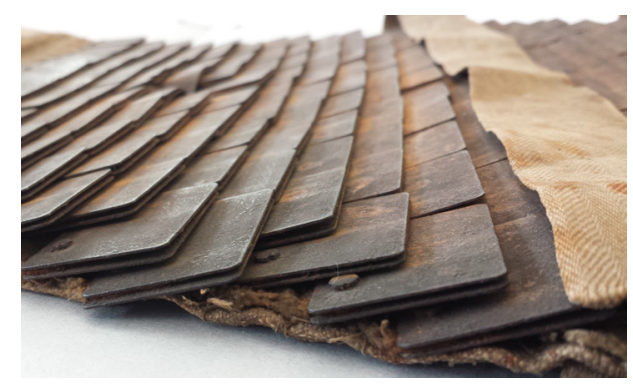

Fig 3. The armor's metal scales as riveted in rows to both sides of textile strips sewn onto a textile support.

With the pale fabric in such close proximity, corrosion reduction on ferrous scales by traditional mechanical techniques proceeded slowly and with limited lubrication to avoid saturation and possible staining of the textile. Mechanically reducing the extensive, thick corrosion layers using this restrained methodology proved time-consuming, and alternate options were pursued. Through analytical investigation and empirical testing, the surface corrosion was characterized and a treatment protocol was optimized combining laser cleaning and mechanical cleaning for the efficient and safe reduction of corrosion from the iron alloy armor scales.

\section{Methods of Analysis and Testing}

The composition, morphology, and stratigraphy of the corrosion layers were characterized and the effects of laser cleaning of these corrosion layers at $1064 \mathrm{~nm}$ and $532 \mathrm{~nm}$ were examined using stereomicroscopy, scanning electron microscopy - energy dispersive spectroscopy (SEM-EDS) and back-scattered and secondary electron (SEM-BSE and SEM-SE) imaging, Raman spectroscopy (Raman), Fourier transform - infrared spectroscopy (FTIR), and thermally assisted hydrolysis and methylation - gas chromatography - mass spectrometry (THM-GC-MS). The metal alloy composition was characterized using energy dispersive $\mathrm{x}$-ray fluorescence spectroscopy (ED-XRF).

\subsection{Stereomicroscopy}

Initial visual assessment of surface corrosion was performed using a Zeiss Discovery V12 stereomicroscope. The surface was illuminated from the side using a Scott white LED source. Low magnification visible images were taken using a Canon EOS-6D attached to the Discovery microscope, highly magnified visible images of the single plate were taken using a Keyence VHX-500 digital microscope, and highly magnified images of scrapings were taken using an Infinity-2 camera attached to a Bruker Senterra Raman microscope. 
2.2. Scanning Electron Microscopy - Energy

Dispersive Spectroscopy/ Backscattered

Electron Imaging/Secondary Electron

Imaging

The scale surface was analyzed by SEM-EDS and imaged with SEM-BSE/SE to investigate the composition and morphology of various corrosion products and surface features. SEM-EDS data was taken using a Zeiss Sigma HD VP electron microscope, equipped with an Oxford Instrument $\mathrm{X}$-MaxN 80 SDD detector. Samples were imaged and analyzed both in low and high vacuum, at a working distance of $8.5 \mathrm{~mm}$ and with a $20 \mathrm{kV}$ source.

\subsection{Raman Spectroscopy}

Scraping samples of each surface layer were analyzed using Raman spectroscopy to characterize corrosion species present. Raman spectroscopy was performed using a Bruker Senterra operating at $10 \mathrm{~mW}$ of power with an excitation wavelength of 785 $\mathrm{nm}$. The spectra were recorded at a resolution of $3-5 \mathrm{~cm}^{-1}$. Integration time and number of acquisitions varied depending on the signal and fluorescence levels of different samples, between 10-60 s and 2-12 acquisitions. The data was compared to an internal Raman reference spectral database which agglomerated data from various sources including RRUFF, the Tate Pigment Library, University College London, and self-made reference libraries.

\subsection{Fourier Transform -}

\section{Infrared micro-Spectroscopy}

Scraping samples of the translucent overlying film were crushed in a diamond compression cell (Spectra Tech) and analyzed by Fourier transform infrared micro-spectroscopy
(micro-FTIR) in transmission mode to characterize the composition. A Hyperion 3000 Microscope interfaced to a Tensor 27 spectrometer (Bruker Optics) equipped with a 15x FTIR objective and a liquid nitrogen-cooled, mercury cadmium telluride (MCT) detector was used. The FTIR spectra were acquired as a sum of 32 scans in the range 4000 to $600 \mathrm{~cm}^{-1}$ and $4 \mathrm{~cm}^{-1}$ resolution. An in-house made reference spectral library was used for data comparison.

\subsection{Thermally-assisted Hydrolysis and Methylation - Gas Chromatography - Mass Spectrometry}

A scraping sample of the translucent overlying film was analyzed by thermally assisted hydrolysis and methylation-gas chromatography/mass spectrometry (THM-GC/ MS) to clarify its organic composition. The sample for analysis measured $64 \mu \mathrm{g}$. The sample was accurately weighed on an Ultramicrobalance UMX2 (Mettler Toledo) in the pyrolysis cup (Eco-cup, Frontier lab) and treated with $3 \mu \mathrm{L}$ of (tetramethyl) ammonium hydroxide (TMAH) $25 \%$ in methanol. THM was performed at $550^{\circ} \mathrm{C}$ in the vertical micro-furnace of the double-shot 2020iD pyrolyzer (Frontier lab), equipped with the Auto-Shot sampler AS-1020E (Frontier lab). The micro-furnace is interfaced to the gas chromatograph Agilent 6890 coupled with the Agilent 5973 Network Mass Selective Detector. The analysis was carried out in split mode 20/1. A J\&W DB-5MS capillary column $(30 \mathrm{~m} \times 0.25 \mathrm{~mm} \times 0.25 \mu \mathrm{m})$ was used for the chromatographic separation. The inlet was kept at $320^{\circ} \mathrm{C}$ and the MS transfer line at $320^{\circ} \mathrm{C}$. Helium was used as the carrier gas, constant flow $1.5 \mathrm{~mL} / \mathrm{min}$. The GC oven temperature program was: $40^{\circ} \mathrm{C}$ for $1 \mathrm{~min}$ ramped to $320^{\circ} \mathrm{C}$ at $10^{\circ} \mathrm{C} / \mathrm{min}$, followed by 
$15 \mathrm{~min}$ isothermal period. Acquisition was performed in SCAN mode (m/z 35-600) with temperatures at MS Source $230^{\circ} \mathrm{C}$ and at Quadrupole $150^{\circ} \mathrm{C}$.

\subsection{Energy Dispersive X-Ray Fluorescence Spectroscopy}

Qualitative ED-XRF analysis was performed to characterize the metal alloy composition and to detect any surface enrichment or plating layers. No samples were taken; the analysis was performed with the instrument in contact with areas of uncorroded metal surface on scale undersides. A Bruker Tracer III-SD portable spectrometer with a rhodium anode tube and no filter run in air at $40 \mathrm{kV}$ and $9.60 \mathrm{~mA}$ for $60 \mathrm{~s}$ live time irradiation was used. Spectral data was collected and analyzed using S1 PXRF software.

\subsection{Laser Cleaning}

Laser cleaning tests were performed using a Quanta Systems Thunderart Nd:YAG Q-switched laser at $1064 \mathrm{~nm}$ and $532 \mathrm{~nm}$ outputs with a pulse duration of $8 \mathrm{~ns}$, a Gaussian beam spot of $10 \mathrm{~mm}$ at its fixed focus, and a repetition rate of $2 \mathrm{~Hz}$.

\section{Characterization of Surface Layers}

To optimize the laser cleaning protocol, it was important to understand the species and stratigraphy of corrosion present.

A discrete scale (measuring $30 \times 26 \times$ $2 \mathrm{~mm}$ ) which had previously detached from the armor was used for surface characterization and cleaning tests. Corrosion layers on the scale's surface were observed under stereomicroscopy. The scale was then placed inside the SEM chamber and its surface imaged through BSE and SE, as well as analyzed by EDS. Next, scrapings of corrosion layers were taken for analysis by Raman spectroscopy and SEM-EDS. Finally, the translucent film was sampled for investigation by FTIR and THM-GC-MS.

In order to determine the basic corrosion stratigraphy, observations under stereomicroscopy were combined with SEM imaging and point-based EDS analysis. It was deemed too destructive to take cross-sectional samples; however the gaps and local variations in the surface composition proved sufficient to establish the layered structure. The proposed multi-layered surface corrosion stratigraphy is shown in (Fig. 4).

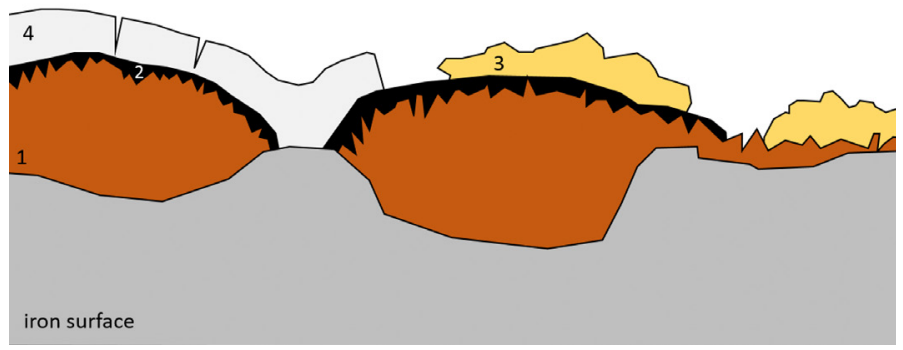

Fig. 4. Diagram showing the proposed multilayered surface corrosion stratigraphy: 1) matte, red layer consisting primarily of lepidocrocite; 2) compact, dark layer of magnetite; 3) fluffy, bright orange layer consisting predominantly of goethite; and 4) translucent film composed of waxes/oils and iron oxalates. Scale is arbitrary. 
Stereomicroscopy revealed the scale's iron alloy surface to be covered by layers of non-uniform, irregular corrosion products of varying color and texture (Fig. 5). Four visually distinct layers were observed in basic stratigraphy, from the metal scale upward: (1) a matte, red layer covering most of the iron alloy surface, over which (2) a compact, dark layer was often present, further topped locally by (3) a fluffy, bright orange layer; in some areas, (4) a brittle, translucent film of variable color was present on top. The layered structure varied locally, with surface corrosion measuring 500 microns at its thickest. Layers (1) and (2) are the most widely distributed across the armor's surface area, while layers (3) and (4) are more localized to areas of overlapping contact between scale layers.

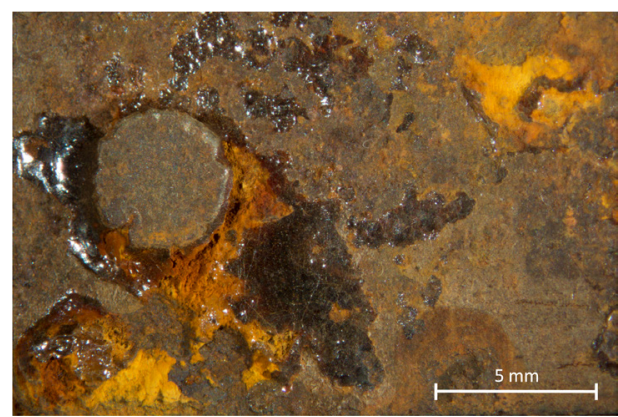

Fig. 5. Stereomicrograph showing the varying color and texture of corrosion products on the metal surface.

To clarify the stratigraphy and composition of the corrosion layers, $\sim 400 \times 500 \mathrm{~mm}$ areas representative of each of the distinct surface layers of the scale were imaged by SEM-BSE-SE. Point-based SEM-EDS analysis was then applied to the typical morphological structure of the different iron oxides and oxyhydroxides observed in each layer to inform characterization of the corrosion species. The matte, red layer (1) exhibits predominantly platy crystals of lepidocrocite ranging in size from $1-5 \mu \mathrm{m}$, stacked and/or interlocked to form a dense structure on top of the iron alloy surface (Fig. 6). The dark layer (2) consists of a compact and continuous magnetite crust, in intimate connection with the underlying lepidocrocite of layer (1) (Fig. 7). The bright orange layer (3) appears as fine, $2-3 \mathrm{~mm}$ wide, aggregates of acicular goethite, occurring in discrete localities on

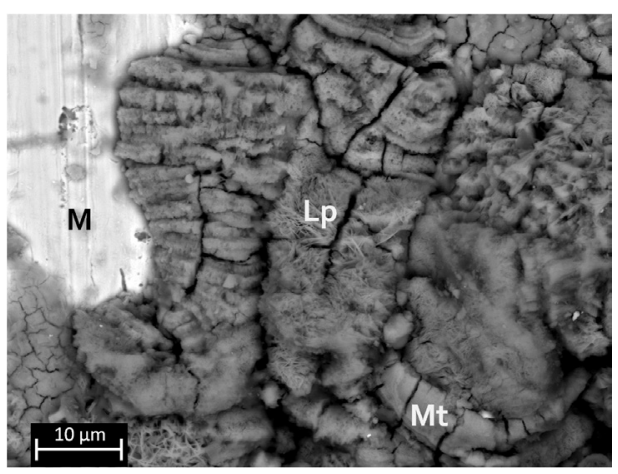

Fig. 6. SEM-BSE image of platey lepidocrocite (Lp) crystals stacked and interlocked to form a dense structure on top of the metal (M) surface. Magnetite (Mt) is also visible in this image.

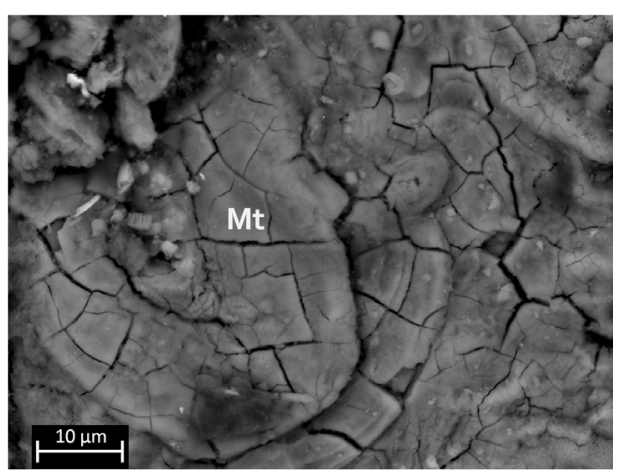

Fig. 7. SEM-BSE image of compact and continuous magnetite (Mt) crust.

top of and mixed with lesser amounts of lepidocrocite plates measuring up to $5 \mu \mathrm{m}$ in length (Fig. 8). 


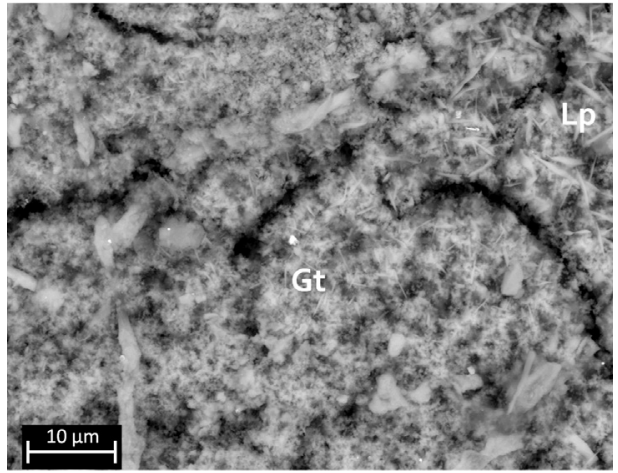

Fig. 8. SEM-BSE image of fine aggregates of acicular goethite (Gt) and mixed with lesser amounts of lepidocrocite (Lp) plates.

Examined by SEM, the translucent layer (4) presents as a continuous film, up to 20 $\mu \mathrm{m}$ thick, covering the other corrosion layers (Fig. 9). Under normal light, the color of

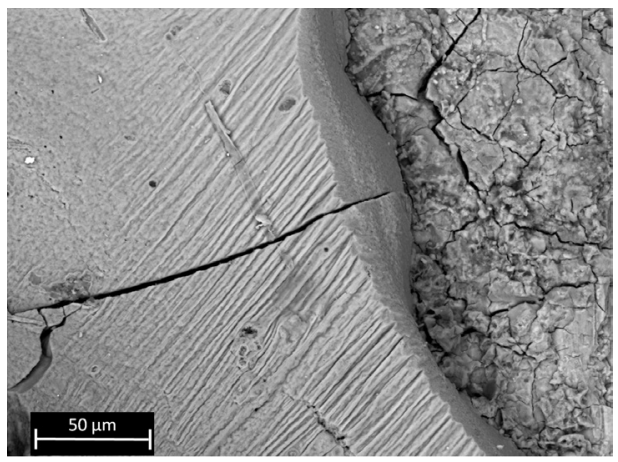

Fig. 9. SEM-BSE image of layer (4) continuous film over the corrosion crust.

this film varies from black to bright yellow, dependent on the color of the underlying iron oxide or oxyhydroxide. SEM-SE observation of the translucent material at low $\mathrm{kV}$ revealed a thin layer of sub-micron, rod-like particles measuring a few nanometers in diameter (Fig. 10). Further characterization of the material by FTIR identified iron oxalates, goethite corrosion, and an organic component of lipids and/or waxes (Fig. 11).

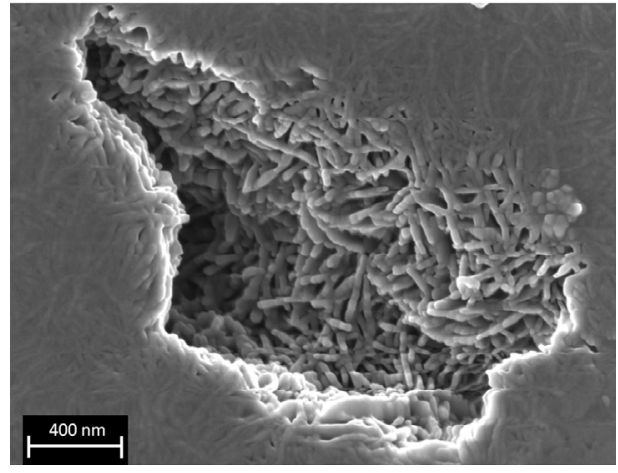

Fig. 10. SEM-SE image of layer (4) showing sub-micron, rod-like iron oxalates.

Iron oxalate formation may be associated with degradation of surface organic materials or possibly with metabolic corrosion processes of bacillus bacterial colonies found in areas on the scale's surface [39-46]. THMGC-MS confirmed the presence of wax and lipids, with fatty acids possibly from multiple sources (oils, fat, wax, soil).

To verify and complement the SEM-EDS/ BSE/SE characterization of the surface corrosion, scraping samples of each layer (1), (2), and (3) were collected for further investigation by Raman spectroscopy. It is noted that under certain Raman measurement conditions it is possible to induce some peak broadening in various phases of iron oxides and iron oxyhydroxides, and that magnetite in particular can experience a phase transformation to hematite [47-50]. However, good quality Raman spectra were not obtained in this study at lower power levels, therefore higher power measurement settings were employed. The Raman spectrum of layer (1) exhibits the primarily singular presence of lepidocrocite, indicated by peaks at $250 \mathrm{~cm}^{-1}$, $381 \mathrm{~cm}^{-1}$, and $533 \mathrm{~cm}^{-1}$; consistent with SEM findings, no other corrosion species were detected in layer (1) by Raman (Fig. 12). The Raman spectrum of layer (2) featured large 


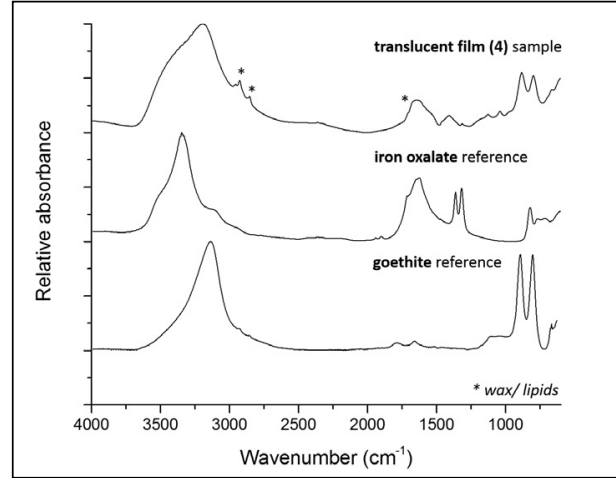

Fig. 11. FTIR spectra comparison of layer (4) with reference spectra of iron oxalate (main peaks around $3340,1648,1380,1317 \mathrm{~cm}^{-1}$ ) and goethite (diagnostic peaks at 800 and $889 \mathrm{~cm}^{1}$ ) and absorption bands of waxes and lipids $\left(^{*}\right)$.

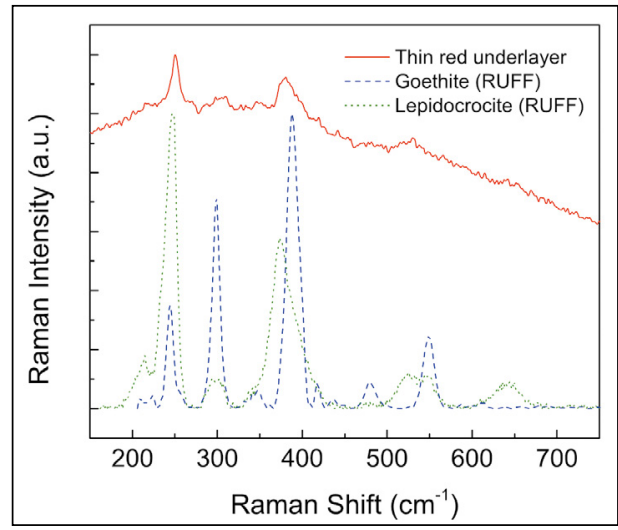

Fig. 12. Raman spectra comparison of layer (1) exhibiting the primarily singular presence of lepidocrocite with peaks at $250 \mathrm{~cm}^{-1}, 381 \mathrm{~cm}^{-1}$, and $533 \mathrm{~cm}^{-1}$.

amounts of fluorescence which inhibited the identification of corrosion species content (Fig. 13). This fluorescence is attributed to significant amounts of fluorescent organic matter contaminants, such as fibers, from the scale's surface; the lack of distinctive peaks could also result from the low Raman activity of magnetite, indicated by SEM as the layer's primary component [51]. The

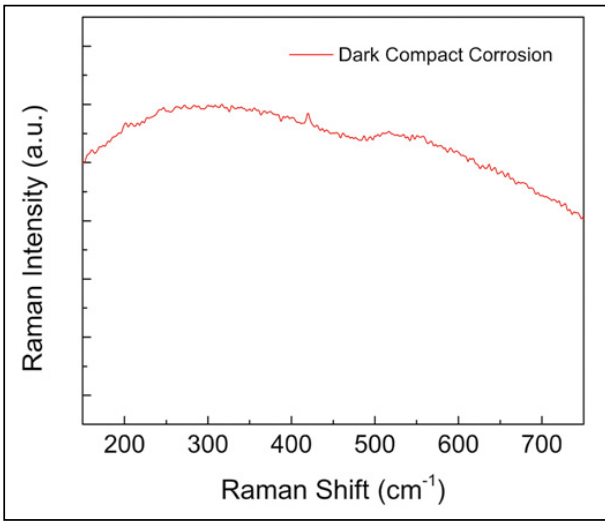

Fig. 13. Raman spectra comparison of layer (2) featuring large amounts of fluorescence, which inhibited the identification of corrosion species content.

Raman spectrum of layer (3) shows the presence of goethite, indicated by peaks at $300 \mathrm{~cm}^{-1}, 386 \mathrm{~cm}^{-1}$, and $549 \mathrm{~cm}^{-1}$ and consistent with SEM findings (Fig. 14). No other substances are evident in the Raman spectrum of layer (3), including the lepidocrocite observed in the SEM scans; this is likely due to the lesser amounts of lepidocrocite observed under SEM combined with

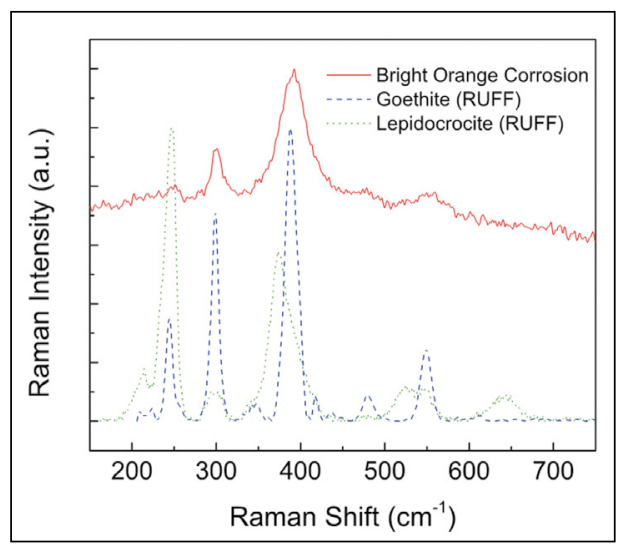

Fig. 14. Raman spectra comparison of layer (3) showing the presence of goethite with peaks at $300 \mathrm{~cm}^{-1}$, $386 \mathrm{~cm}^{-1}$, and $549 \mathrm{~cm}^{-1}$. 
the similar Raman activity levels of goethite and lepidocrocite [52].

Based on these analyses, a simplified stratigraphy of corrosion products on the surface of the metal scales can be summarized, with local variations and a maximum thickness of $500 \mu \mathrm{m}$, as follows: a lowest layer (1) of matte, red lepidocrocite platy crystals ranging in size from $1-5 \mu \mathrm{m}$ which stack and interlock in a dense structure covering most of the metal surface, over which a layer (2) of compact, dark magnetite approximately $1 \mu \mathrm{m}$ thick is often present, further topped locally by a layer (3) consisting of bright orange goethite in fine acicular aggregates $2-3 \mu \mathrm{m}$ wide, mixed with less amounts of lepidocrocite plates measuring $5 \mu \mathrm{m}$ long. In some areas, a translucent layer (4) is present on top as a continuous film, up to $20 \mu \mathrm{m}$ thick and containing sub-micron, rod-like iron oxalates possibly associated with bacillus bacterial colonies in an oily-waxy substance.

Additional information was gained from the SEM-EDS/BSE/SE analysis of the scale surface and from the SEM-EDS analysis of the isolated layer scraping samples. Besides the corrosion products formed on the scale's surface, several foreign particles were identified. Among the most recurrent particles were silicates and other inorganic materials consistent with soiling contamination, with quartz and feldspar grains recognized. Organic material consisting primarily of textile fibers were also found embedded within the surface layers and may originate from the armor's textile support or incidental contamination.

\section{Laser Cleaning Tests}

Following characterization of the corrosion layers, laser cleaning tests were performed to establish the damage threshold of the metal substrate and to evaluate the efficacy of various laser parameters in reducing the surface corrosion.

An Nd:YAG Q-switched laser was used at $1064 \mathrm{~nm}$ and $532 \mathrm{~nm}$ outputs with a pulse duration of $8 \mathrm{~ns}$ and a repetition rate of $2 \mathrm{~Hz}$. Fifty pulses were chosen as the experimental pulse number based on the variable thickness and composition of the surface corrosion layers which require a controlled and gradual reduction of corrosion layers. Mineral spirits was applied to the surface on a rolled cotton swab before laser cleaning to increase laser-induced corrosion removal and reduce the metal substrate's initial reactivity $[24,25$, $28,53]$. In areas of thicker corrosion, mineral spirits on a cotton swab was applied multiple times and also served as method of mild mechanical removal for material loosened during laser application.

The macroscopic damage threshold of the armor's iron alloy scale was empirically determined by applying laser pulses of the above parameters at each wavelength to a small, uncorroded region of the scale and gradually increasing the fluence until a very slight yellowing was visually observed after 50 pulses, indicating micromelting of the metal surface [2]. Effective fluences for corrosion removal were empirically found for each wavelength, constricted below the established macroscopic damage threshold and with diminishing results observed after 50 pulses. Cleaning tests were performed below the established damage thresholds and within the effective corrosion removal fluences, resulting in $175 \mathrm{~mJ} / \mathrm{cm}^{2}$ at 1064 $\mathrm{nm}$ and $90 \mathrm{~mJ} / \mathrm{cm}^{2}$ at $532 \mathrm{~nm}$. The tests were performed on two visually similar regions approximately $1 \mathrm{~cm}^{2}$ that possessed representative distributions of corrosion products. 
Comparative tests between laser cleaning with the $1064 \mathrm{~nm}$ infrared laser and the 532 $\mathrm{nm}$ green laser showed that a lower energy fluence could be employed using the $532 \mathrm{~nm}$ laser to produce more efficient iron corrosion reduction and significantly less risk of alteration to the underlying metal surface than could be achieved at the higher energy fluence required using the $1064 \mathrm{~nm}$ laser.

SEM - BSE images show magnifications of the metal scale surface after corrosion reduction utilizing laser cleaning at 1064 $\mathrm{nm}$ and at $532 \mathrm{~nm}$. Neither laser cleaning protocol completely removed all corrosion on the metal surface. It was not feasible in this study to take samples to compare the depth of surface layers before and after cleaning in order to obtain a more quantitative comparison of the amount of corrosion removal by each laser wavelength. However, the $100 \times$ magnification images show that a greater portion of corrosion was reduced by the $532 \mathrm{~nm}$ wavelength than by the 1064 $\mathrm{nm}$ wavelength, as evidenced in the greater revelation of the underlying metallic surface (Fig. 15-16). Images at higher magnification

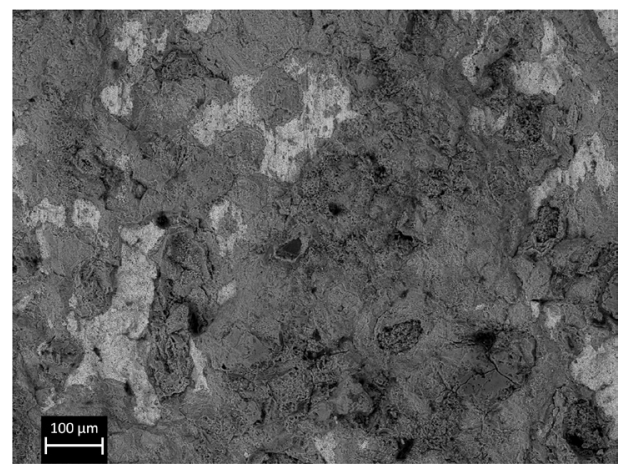

Fig. 15. SEM-BSE image $(100 \times)$ of surface of corroded metal armor scale after laser cleaning with $1064 \mathrm{~nm}$ at $0.175 \mathrm{~J} / \mathrm{cm}^{2}$ ( $8 \mathrm{~ns}, 2 \mathrm{~Hz}, 50$ pulses). Significant amount of corrosion remains on the surface, with some clean metal visible in local areas.

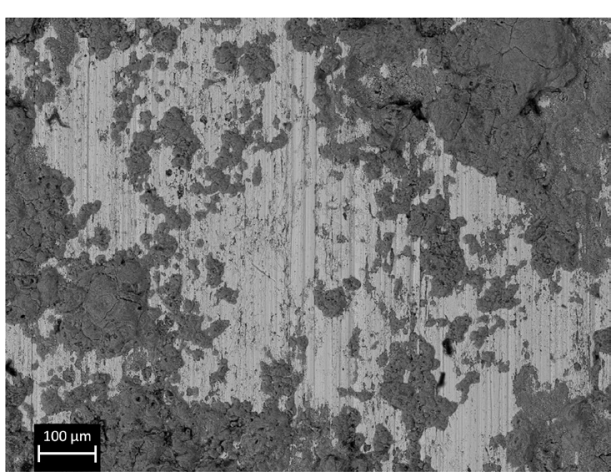

Fig. 16. SEM-BSE image $(100 \times)$ of surface of corroded metal armor scale after laser cleaning with $532 \mathrm{~cm}$ at $0.090 \mathrm{~J} / \mathrm{cm}^{2}$ (8 ns, $2 \mathrm{~Hz}, 50$ pulses). Some corrosion remains on the surface; however, significant areas of clean metal are also visible.

$(3100 \times)$ were taken to provide greater detail of the metallic surface as revealed by cleaning at each laser wavelength. The surface irradiated with $1064 \mathrm{~nm}$ laser pulses shows clear signs of significant micromelting, including bubbling, deformation, and metallic nanospheres (Fig. 17). The surface irradiated with $532 \mathrm{~nm}$ pulses, on the other hand, exhibits almost no signs of micromelting, except

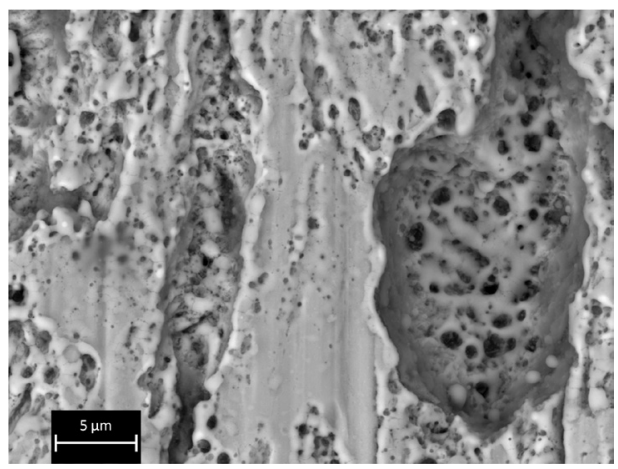

Fig. 17. SEM-BSE image (3100x) of surface of corroded metal armor scale after laser cleaning with $1064 \mathrm{~nm}$ at $0.175 \mathrm{~J} / \mathrm{cm}^{2}$ ( $8 \mathrm{~ns}, 2 \mathrm{~Hz}, 50$ pulses). The surface shows clear signs of significant micromelting, including bubbling, deformation, and metallic nanospheres. 
perhaps for a slight softening of some edges in pitted regions and several small, bright nanospheres (Fig. 18). These data suggest that the lower cleaning fluence at the $532 \mathrm{~nm}$ wavelength is both more effective in ferrous corrosion reduction and less damaging to the underlying iron alloy.

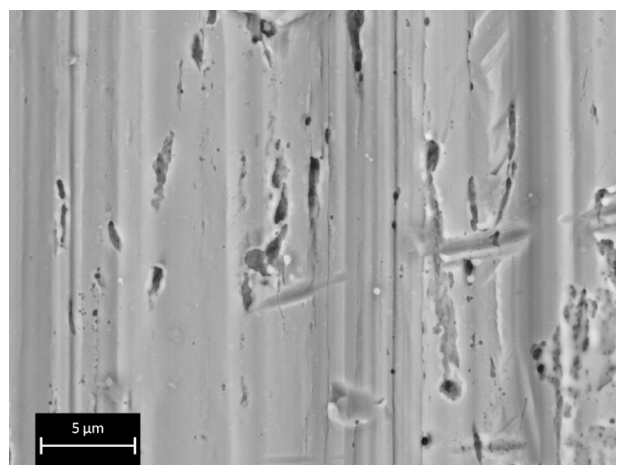

Fig. 18. SEM-BSE image $(3100 \times)$ of surface of corroded metal armor scale after laser cleaning with $532 \mathrm{~cm}$ at $0.090 \mathrm{~J} / \mathrm{cm}^{2}$ ( $8 \mathrm{~ns}, 2 \mathrm{~Hz}, 50$ pulses $)$. The surface exhibits almost no signs of micromelting, except perhaps for a slight softening of some edges in pitted regions and several small, bright nanospheres.

It is worth noting that after laser cleaning, a dark residue remained on portions of the surface. While conclusive characterization of this dark material was not pursued in this study, the observation is consistent with other investigations into the laser cleaning of iron surfaces in which the formation of hematite and/or magnetite induced by thermal effects and plasma generation from laser ablation processes results in a darkening of the cleaned metal surface $[4,19$, 24]. The thermal transformations amongst iron oxides and iron oxyhydroxides (in particular amongst goethite, magnetite, and hematite) are thoroughly researched by allied sciences [54-66] and for the cultural heritage community may indicate distinctions amongst laser cleaning parameters, such as pulse duration and thermal effects $[24,52]$.

\section{Discussion}

The improvement of the $532 \mathrm{~nm}$ treatment over the $1064 \mathrm{~nm}$ treatment can be attributed to more efficient and better localized energy transfer due to the optical scattering and absorption properties of each wavelength and of the iron oxide corrosion population as characterized.

Optical absorption by all detected iron oxide species increases dramatically at wavelengths less than $550 \mathrm{~nm}$ [67]. Absorption profiles extending from weak absorption in the near-IR to strong absorption in the near-UV is characteristic of ferric oxides and oxyhydroxides due to crystal field transitions [67]. This indicates that shorter wavelengths are closer to resonance for these electronic transitions and consequently should induce enhanced ablation from single- and multi-photon processes such as electronic-vibrational coupling and plasma generation, respectively [68]. This study further hypothesizes that, with increased optical scattering and absorption of the $532 \mathrm{~nm}$ wavelength by the iron oxides, more energy from the incident laser beam is absorbed by the upper corrosion layers and therefore less laser energy impinges on the underlying metal when cleaning at green wavelengths. Contrastingly, as less of the $1064 \mathrm{~nm}$ wavelength is absorbed by the corrosion layers, more energy is transmitted to the metal substrate, increasing the risk of damage and melting of the underlying metal during laser cleaning in the infrared.

In addition to strong absorption, shorter wavelengths experience increased scattering within iron corrosion layers and highly scat- 
tering media generate light intensity within the material greater than the incident intensity $[28,69]$. The scattering phenomenon in a laser-cleaned sample (in which the incident beam is much wider than the distance between scattering events) can be modeled analogous to the diffusion equation with light propagation approximated as a one-dimensional case with exact solutions $[70,71]$. The magnitude and distribution of light intensity in the sample have a complex relationship with several parameters including the spatial distribution of scattering events, the degree of scattering and absorption, and the thickness of the material [72]. The measurements needed to characterize these parameters were beyond the scope of this experiment; therefore, it was not possible to estimate the degree to which laser cleaning is dependent on wavelength-tunable scattering in the case of this study. However, in one-dimensional cases of light propagation in the diffusion equation, intensity increases due to scattering effects of 2-3x the incident light are not unusual [72]. This study posits that decreased scattering length at $532 \mathrm{~nm}$ as compared to $1064 \mathrm{~nm}$ limits the penetration depth of the laser energy and increases the effective fluence of each laser pulse nearer to the point of surface incidence, thereby allowing for a more localized impact of laser cleaning on the surface corrosion layers and a minimized load on the underlying metal substrate.

\section{Conclusions and further research}

In summary, the use of the $532 \mathrm{~nm}$ wavelength at a lower fluence produced more effective corrosion reduction and less alteration of the underlying iron surface than the use of the $1064 \mathrm{~nm}$ wavelength at a higher fluence. This result is attributed to increased retention of the incident laser energy within the corrosion layers and decreased penetration to the underlying metal surface due to increased optical absorption of shorter wavelengths by the iron oxide species and to increased and localized energy intensity within the corrosion layers due to decreased scattering length of shorter wavelengths.

Ultimately, in the practical treatment of the armor scales, laser cleaning was combined with traditional mechanical cleaning to produce optimal results. The incorporation of laser cleaning in the treatment protocol increased the overall time efficiency of the corrosion reduction by a factor of four (Figs. 19-20). Initial laser cleaning of the corroded

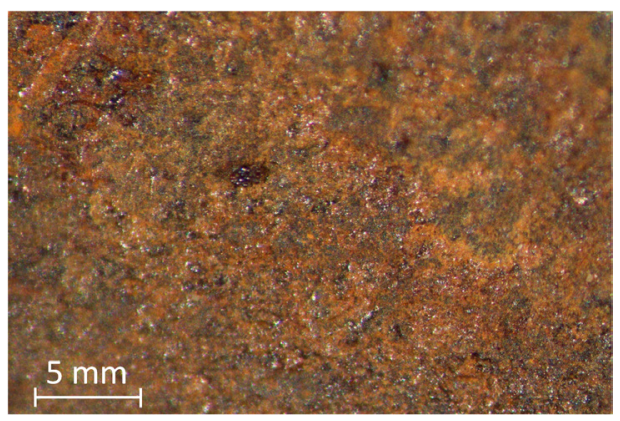

Fig. 19. Stereomicrograph of corroded surface of armor's metal scale before cleaning.

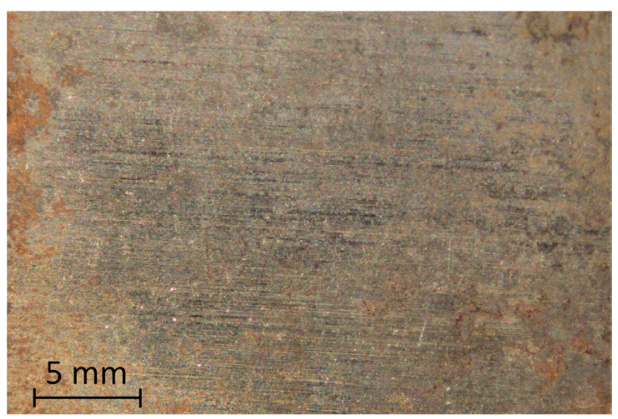

Fig. 20. Stereomicrograph of corroded surface of armor's metal scale after laser cleaning $(532 \mathrm{~nm}$ wavelength, $8 \mathrm{~ns}$ pulse duration, $2 \mathrm{~Hz}$ frequency, and $0.090 \mathrm{~J} / \mathrm{cm}^{2}$ fluence) and before mechanical cleaning. 
surface followed by mechanical cleaning performed with a scalpel blade allowed an increase in time-efficiency of treating the large metal surface area while protecting the adjacent sensitive textile and maintaining a finished surface appearance familiar to traditional techniques and established aesthetic expectations.

The research presented here is ongoing, as we strive to clarify and deepen our understanding of the effects of wavelength variances on the reduction of corrosion on iron surfaces. Relevant future studies would seek to model the heat-effected zone within the stratigraphy of the corrosion crust and the underlying metal and to identify any relevant wavelength-dependent components of thermal diffusion [12, 73-78]. Pulse duration and frequency could be introduced as variable factors within the study and ablation rates at different laser parameter combinations could be quantified $[79,80]$. Finally, investigative studies could be initiated into the potential for evaluating thermal transformations amongst iron oxides and oxyhydroxides under variable oxidizing/ reducing environments as an evaluative tool for the characterization and monitoring of the laser cleaning of iron alloys.

\section{Acknowledgements}

The authors thank The Metropolitan Museum of Art, the Smithsonian National Air and Space Museum, and Yale University for generous support of this research study. Special thanks are extended to The Met's Department of Scientific Research and Marco Leona, to The Met's Arms and Armor Department, Pierre Terjanian, and Edward Hunter, to The Met's Objects Conservation Department, Lawrence Becker, Lisa Pilosi, Carolyn Riccardelli, and Wendy Walker, and to the Smithsonian National Air and Space Museum's Conservation Unit and Malcolm Collum. We gratefully acknowledge Quanta System for providing the Thunder Art Laser to the Metropolitan Museum of Art. Further acknowledgements are offered to the Andrew W. Mellon Foundation, the National Endowment for the Humanities, and the Winterthur/University of Delaware Program in Art Conservation.

\section{References}

[1] M. Pires, C. Curran, W. Perrie, K. Watkins, "Laser cleaning: influence of laser beam characteristics", in: Lasers in the Conservation of Artworks (Proceedings of the International Conference LACONA VII), Madrid, Spain, 17-21 September 2007, M. Castillejo, P. Moreno, M. Oujja, R. Radvan, J. Ruiz (Eds.), CRC Press, Boca Raton 2008, pp. 329-333.

[2] A. Siatou, D. Charalambous, V. Argyropoulos, P. Pouli, "A comprehensive study for the laser cleaning of corrosion layers due to environmental pollution for metal objects of cultural value: preliminary studies on artificially corroded coupons", Laser Chemistry, (2006), Article ID 85324, DOI: 10.1155/2006/85324 (accessed: 17.06.2017).

[3] P. Mottner, G. Wiedemann, G. Haber, W. Conrad, A. Gervais, "Laser cleaning of metal surface - laboratory investigation", in: Lasers in the Conservation of Artworks (LACONA V Proceedings), Osnabrück, Germany, September 15-18, 2003, K. Dickmann, C. Fotakis, J. Asmus (Eds.), Springer, Berlin 2005, pp. 79-86.

[4] C. Cottam, D. Emmony, "TEA-CO2 laser surface processing of corroded metals", Corrosion Science 41 (1999) 1529-1538, DOI: 10.1016/S0010938X(98)00202-9.

[5] M. De Giorgi, E. D’Anna, A. Della Patria, H. De Rosa, A. Lorusso, P. Orfanou, A. Perrone, "Laser cleaning of copper-based coins", in: Lasers in the Conservation of Artworks IX (Proceedings of the International Conference on LACONA IX), London, United Kingdom, 07-10 September 2011, D. Saunders, M. Strlič, C. Korenberg, N. Luxford, K. Birkhölzer (Eds.), Archetype Publications, London 2013, pp. 192-193.

[6] H. Garbacz, E. Fortuna-Zaleśna, J. Marczak, M. Strzelec, A. Koss, A. Zatorska, "Evaluation of laser cleaning of old copper roofing and gilding using tun- 
able length laser pulses", in: Lasers in the Conservation of Artworks IX (Proceedings of the International Conference on LACONA IX), London, United Kingdom, 07-10 September 2011, D. Saunders, M. Strlič, C. Korenberg, N. Luxford, K. Birkhölzer (Eds.), Archetype Publications, London 2013, pp. 204-206.

[7] A. Dajnowski, A. Lins, "The practical use of lasers in removing deteriorated Incralac coatings from large bronze monuments", in: Lasers in the Conservation of Artworks VIII (Proceedings of the International Conference on LACONA VIII), Sibiu, Romania, 21-25 September 2009, R. Radvan, J. Asmus, M. Castillejo, P. Pouli, A. Nevin (Eds.), CRC Press, London 2011, pp. 47-52.

[8] A. Dajnowski, "Laser as a cleaning tool for the treatment of large-scale bronze monuments", in: Lasers in the Conservation of Artworks (Proceedings of the International Conference LACONA VII), Madrid, Spain, 17-21 September 2007, M. Castillejo, P. Moreno, M. Oujja, R. Radvan, J. Ruiz (Eds.), CRC Press, Boca Raton 2008, pp. 303-308.

[9] M. Froidevaux, P. Platt, M. Cooper, K. Watkins, "Laser interactions with copper, copper alloys and their corrosion products used in outdoor sculpture in the United Kingdom", in: Lasers in the Conservation of Artworks (Proceedings of the International Conference LACONA VII), Madrid, Spain, 17-21 September 2007, M. Castillejo, P. Moreno, M. Oujja, R. Radvan, J. Ruiz (Eds.), CRC Press, Boca Raton 2008, pp. 277-284.

[10] C. Korenberg, A. Baldwin, "Investigating the laser cleaning of archaeological copper alloys using different laser systems", in: Lasers in the Conservation of Artworks (Proceedings of the International Conference LACONA VII), Madrid, Spain, 17-21 September 2007, M. Castillejo, P. Moreno, M. Oujja, R. Radvan, J. Ruiz (Eds.), CRC Press, Boca Raton 2008, pp. 285-290.

[11] S. Agnoletti, A. Brini, L. Nicolai, "Current work in laser cleaning of the Porta del Paradiso", in: Lasers in the Conservation of Artworks (LACONA VI Proceedings), Vienna, Austria, 21-25 September 2005, J. Nimmrichter, W. Kautek, M. Schreiner (Eds.), Springer, Berlin 2007, pp. 29-36.

[12] S. Siano, R. Salimbeni, "The Gate of Paradise: physical optimization of the laser cleaning approach",
Studies in Conservation, 46 (2001) 269-281, DOI: 10.1179/sic.2001.46.4.269.

[13] R. Pini, S. Siano, R. Salimbeni, M. Pasquinucci, M. Miccio, "Tests of laser cleaning on archaeological metal artefacts", Journal of Cultural Heritage, 1 (2000) S129-S137, DOI: S1296-2074(00)00139-4/FLA .

[14] P. Fitzsimons, K. Watkins, A. El-Naggar, Z. Allen, "Removal of bronze gilding layers using a 20ps fibre laser", in: Lasers in the Conservation of Artworks IX (Proceedings of the International Conference on LACONA IX), London, United Kingdom, 07-10 September 2011, D. Saunders, M. Strlič, C. Korenberg, N. Luxford, K. Birkhölzer (Eds.), Archetype Publications, London 2013, pp. 201-203.

[15] M. Kono, K. Baldwin, A. Wain, M. Sawicki, I. Malkiel, A. Rode, "High repetition rate laser restoration and monitoring of large area gilded surfaces", in: Lasers in the Conservation of Artworks IX (Proceedings of the International Conference on LACONA IX), London, United Kingdom, 07-10 September 2011, D. Saunders, M. Strlič, C. Korenberg, N. Luxford, K. Birkhölzer (Eds.), Archetype Publications, London 2013, pp. 45-51.

[16] M. Barrera, C. Escudero, D. Ynsa, A. Climent-Font, "Application of ion beam analysis (IBA) techniques for the assessment of laser cleaning on gilded copper", in: Lasers in the Conservation of Artworks (Proceedings of the International Conference LACONA VII), Madrid, Spain, 17-21 September 2007, M. Castillejo, P. Moreno, M. Oujja, R. Radvan, J. Ruiz (Eds.), CRC Press, Boca Raton 2008, pp. 323-328.

[17] M. Panzer, G. Wiedemann, M. Meier, W. Conrad, A. Kempe, T. Hutsch, "Laser cleaning of gildings", in: Lasers in the Conservation of Artworks (LACONA VI Proceedings), Vienna, Austria, 21-25 September 2005, J. Nimmrichter, W. Kautek, M. Schreiner (Eds.), Springer, Berlin 2007, pp. 21-28.

[18] A. Dajnowski, B. Dajnowski, “A case study in the removal of paint and corrosion from cast iron using a Nd:YAG laser", in: Lasers in the Conservation of Artworks IX (Proceedings of the International Conference on LACONA IX), London, United Kingdom, 07-10 September 2011, D. Saunders, M. Strlič, C. Korenberg, N. Luxford, K. Birkhölzer (Eds.), Archetype Publications, London 2013, pp. 40-44. 
[19] J. Resende, A. Hipólito, M. Pires, "Modification of iron corrosion after pulsed laser irradiation", in: Lasers in the Conservation of Artworks IX (Proceedings of the International Conference on LACONA IX), London, United Kingdom, 07-10 September 2011, D. Saunders, M. Strlič, C. Korenberg, N. Luxford, K. Birkhölzer (Eds.), Archetype Publications, London 2013, pp. 176-178.

[20] I. Khalfallah, M. Rahoma, J. Abboud, K. Benyounis, "Microstructure and corrosion behavior of austenitic stainless steel treated with laser", Optics \& Laser Technology, 43 (2011) 806-813, DOI: 10.1016/j. optlastec.2010.11.006 .

[21] C. Korenberg, A. Baldwin, "Laser cleaning of iron: surface appearance and re-corrosion of model systems", in: Lasers in the Conservation of Artworks VIII (Proceedings of the International Conference on LACONA VIII), Sibiu, Romania, 21-25 September 2009, R. Radvan, J. Asmus, M. Castillejo, P. Pouli, A. Nevin (Eds.), CRC Press, London 2011, pp. 123-128.

[22] K. Alabeedi, J. Abboud, K. Benyounis, "Microstructure and erosion resistance enhancement of nodular cast iron by laser melting", Wear, 266 (2009) 925-933, DOI: 10.1016/j.wear.2008.12.015 .

[23] J. Chamón, J. Barrio, E. Catalán, M. Arroyo, A. Pardo, "Nd:YAG laser cleaning of heavily corroded archaeological iron objects and evaluation of its effects", in: Lasers in the Conservation of Artworks (Proceedings of the International Conference LACONA VII), Madrid, Spain, 17-21 September 2007, M. Castillejo, P. Moreno, M. Oujja, R. Radvan, J. Ruiz (Eds.), CRC Press, Boca Raton 2008, pp. 297-302.

[24] C. Korenberg, A. Baldwin, "Investigating and optimising the laser cleaning of corroded iron", in: Lasers in the Conservation of Artworks (Proceedings of the International Conference LACONA VII), Madrid, Spain, 17-21 September 2007, M. Castillejo, P. Moreno, M. Oujja, R. Radvan, J. Ruiz (Eds.), CRC Press, Boca Raton 2008, pp. 291-296.

[25] Y. Koh, J. Powell, A. Kaplan, J. Carlevi, "Laser cleaning of corroded steel surfaces: a comparison with mechanical cleaning methods", in: Lasers in the Conservation of Artworks (LACONA VI Proceedings), Vienna, Austria, 21-25 September 2005, J. Nim- mrichter, W. Kautek, M. Schreiner (Eds.), Springer, Berlin 2007, pp. 13-20.

[26] Y. Koh, J. Powell, A. Kaplan, "The removal of layers of corrosion from steel surfaces: a comparison of laser methods and mechanical techniques", Journal of Laser Applications 19 (2007) 99-106, DOI: 10.2351/1.2567378.

[27] Y. Koh, I. Sárady, "Cleaning of corroded iron artifacts using pulsed TEA $\mathrm{CO}_{2}$ and Nd:YAG-lasers", Journal of Cultural Heritage, 4, (2003) 129-133, DOI: 10.1016/S1296-2074(02)01140-8.

[28] K. Dickmann, J. Hildenhagen, J. Studer, E. Müsch, "Archaeological ironwork: removal of corrosion layers by Nd:YAG-laser", in: Lasers in the Conservation of Artworks (LACONA V Proceedings), Osnabrück, Germany, September 15-18, 2003, K. Dickmann, C. Fotakis, J. Asmus (Eds.), Springer, Berlin 2005, pp. 71-77.

[29] Y. Koh, I. Sárady, "Surface cleaning of iron artefacts by lasers", in: Lasers in the Conservation of Artworks (LACONA V Proceedings), Osnabrück, Germany, September 15-18, 2003, K. Dickmann, C. Fotakis, J. Asmus (Eds.), Springer, Berlin 2005, pp. 95-100.

[30] J. Asmus, "President Kennedy in Dallas: Dealey Plaza memorialized", Optical Metrology for Arts and Multimedia, 6618 (2007) 139-144, DOI: 10.1117/12.724292.

[31] J. Asmus, "Photonic restoration of marine artifacts and vessels of New Spain", in: Lasers in the Conservation of Artworks (Proceedings of the International Conference LACONA VII), Madrid, Spain, 17-21 September 2007, M. Castillejo, P. Moreno, M. Oujja, R. Radvan, J. Ruiz (Eds.), CRC Press, Boca Raton 2008, pp. 1-8.

[32] J. Brannon, J. Asmus, "Citric acid augmented flashlamp cleaning of corroded steel surfaces", Applications of Surface Science, 9 (1981) 14-21, DOI: 10.1016/0378-5963(81)90022-2 .

[33] C. Fotakis, D. Anglos, V. Zafiropulos, S. Georgiou, V. Tornari, Lasers in the Preservation of Cultural Heritage, Taylor and Francis, New York 2006.

[34] T. Burmester, M. Meier, H. Haferkamp, S. Barcikowski, J. Bunte, A. Ostendorf, "Femtosecond laser cleaning of metallic cultural heritage and antique 
artworks", in: Lasers in the Conservation of Artworks (LACONA V Proceedings), Osnabrück, Germany, September 15-18, 2003, K. Dickmann, C. Fotakis, J. Asmus (Eds.), Springer, Berlin 2005, pp. 61-69.

[35] S. Grancsay, "A Renaissance brigandine", The Metropolitan Museum of Art Bulletin, 37 (1942) 132-136, DOI: $10.2307 / 3256979$.

[36] The Metropolitan Museum of Art Collections Records 19.49.16, http://www.metmuseum.org/art/ collection $/$ search $/ 33499 ?=\&$ imgNo=0 $\&$ tabName $=$ re lated-objects (accessed 03.10.2016).

[37] B. Dean, Helmets and Body Armor in Modern Warfare, Yale University Press, New Haven 1920.

[38] B. Dean, Helmets and Body Armor in Modern Warfare, Yale University Press, New Haven 1920, pp. 59.

[39] H. Ashassi-Sorkhabi, M. Moradi-Haghighi, G. Zarrini, R. Javaherdashti, "Corrosion behavior of carbon steel in the presence of two novel iron-oxidizing bacteria isolated from sewage treatment plants", Biodegradation, 23 (2012) 69-79, DOI: 10.1007/ s10532-011-9487-8.

[40] L. Herrera, H. Videla, "Role of iron-reducing bacteria in corrosion and protection of carbon steel", International Biodeterioration \& Biodegradation, 63 (2009) 891-895, DOI: 10.1016/j.ibiod.2009.06.003.

[41] R. Jack, D. Ringelberg, D. White, "Differential corrosion rates of carbon steel by combinations of Bacillus sp., Hafnia alvei, and Desulfovibrio gigas established by phospholipid analysis of electrode biofilm", Corrosion Science, 33 (1992) 1843-1853, DOI: 10.1016/0010-938X(92)90188-9.

[42] B. Little, P. Wagner, "Microbiologically Influenced Corrosion", in: Peabody's Control of Pipeline Corrosion: $2^{\text {nd }}$ Edition, A. Peabody, R. Bianchetti (Eds.), NACE International, The Corrosion Society, Houston, Texas 2001, pp. 273-284.

[43] A. Rajasekar, B. Anandkumar, S. Maruthamuthu, Y. Ting, P. Rahman, "Characterization of corrosive bacterial consortia isolated from petroleum-product-transporting pipelines", Applied Microbiology and Biotechnology, 85 (2010) 1175-1188, DOI: $10.1007 / \mathrm{s} 00253-009-2289-9$.

[44] A. Rajasekar, Y. Ting, "Role of inorganic and organic medium in the corrosion behavior of Bacillus megaterium and Pseudomonas sp. in stainless steel SS 304", Industrial \& Engineering Chemistry Research, 50 (2011) 12534-12541, DOI: 10.1021/ie200602a.

[45] D. Xu, Y. Li, F. Song, T. Gu, "Laboratory investigation of microbiologically influenced corrosion of C1018 carbon steel by nitrate reducing bacterium Bacillus licheniformis", Corrosion Science, 77 (2013) 385-390, DOI: 10.1016/j.corsci.2013.07.044.

[46] K. Zarasvand, V. Rai, "Microorganisms: induction and inhibition of corrosion in metals", International Biodeterioration \& Biodegradation, 87 (2014) 66-74, DOI: 10.1016/j.ibiod.2013.10.023.

[47] D. de Faria, S. Venancio Silva, M. de Oliveira, "Raman microspectroscopy of some iron oxides and oxyhydroxides", Journal of Raman Spectroscopy, 28 (1997) 873-878, DOI: 10.1002/(SICI)10974555(199711)28:11<873::AID-JRS177>3.0.CO;2-B.

[48] O. Shebanova, P. Lazor, "Raman study of magnetite $\left(\mathrm{Fe}_{3} \mathrm{O}_{4}\right)$ : laser-induced thermal effects and oxidation", Journal of Raman Spectroscopy, 34 (2003) 845-852, DOI: $10.1002 /$ jrs. 1056 .

[49] O. Shebanova, P. Lazor, "Raman spectroscopic study of magnetite $\left(\mathrm{FeFe}_{2} \mathrm{O}_{4}\right)$ : a new assignment for the vibrational spectrum", Journal of Solid State Chemistry, 174 (2003) 424-430, DOI: 10.1016/ S0022-4596(03)00294-9.

[50] T. Glotch, M. Kraft, “Thermal transformations of akaganéite and lepidocrocite to hematite: assessment of possible precursors to Martian crystalline hematite", Physics and Chemistry of Minerals, 35 (2008) 569-581, DOI: 10.1007/s00269-008-0249-z .

[51] M. Hanesch, "Raman spectroscopy of iron oxides and (oxy)hydroxides at low laser power and possible applications in environmental magnetic studies", Geophysical Journal International, 177 (2009) 941-948, DOI: 10.1111/j.1365-246X.2009.04122.x.

[52] S. Oh, D. Cook, H. Townsend, "Characterization of iron oxides commonly formed as corrosion products on steel", Hyperfine Interactions, 112 (1998) 59-66.

[53] A. Sarzyński, K. Jach, J. Marczak, "Comparison of wet and dry laser cleaning of artworks", in: Lasers in the Conservation of Artworks (LACONA VI Proceedings), Vienna, Austria, 21-25 September 
2005, J. Nimmrichter, W. Kautek, M. Schreiner (Eds.), Springer, Berlin 2007, pp. 161-167.

[54] V. An, E. Ivchenko, C. de Izarra, "Laser induced oxidation processes in iron, copper and nickel nanopowders", Material Letter, 62 (2008) 2211-2214, DOI: 10.1016/j.matlet.2007.11.049.

[55] Y. Cudennec, A. Lecerf, "Topotactic transformations of goethite and lepidocrocite into hematite and maghemite", Solid State Sciences, 7 (2005) 520-529, DOI: 10.1016/j.solidstatesciences.2005.02.002.

[56] L. Davidson, S. Shaw, L. Benning, "The kinetics and mechanisms of schwertmannite transformation to goethite and hematite under alkaline conditions", American Mineralogist, 93 (2008) 1326-1337, DOI: 10.2138/am.2008.2761.

[57] D. de Faria, F. Lopes, "Heated goethite and natural hematite: can Raman spectroscopy be used to differentiate them?", Vibrational Spectroscopy, 45 (2007) 117-121, DOI: 10.1016/j.vibspec.2007.07.003.

[58] L. Diamandescu, D. Mihàilà-Tàràbàşanu, M. Feder, "On the solid phase transformation goethite - hematite”, Material Letters, 17 (1993) 309-311, DOI: 10.1016/0167-577X(93)90019-T.

[59] H. Fan, B. Song, Q. Li, "Thermal behavior of goethite during transformation to hematite", Materials Chemistry and Physics, 98 (2006) 148-153, DOI: 10.1016/j.matchemphys.2005.09.005.

[60] S. Gialanella, F. Girardi, G. Ischia, I. Lonardelli, M. Mattarelli, M. Montagna "On the goethite to hematite phase transformation", Journal of Thermal Analysis and Calorimetry, 102 (2010) 867-873, DOI: 10.1007/s10973-010-0756-2.

[61] G. González, A. Sagarzazu, R. Villalba, "Study of the mechano-chemical transformation of goethite to hematite by TEM and XRD", Materials Research Bulletin, 35 (2000) 2295-2308, DOI: 10.1016/S00255408(00)00434-7.

[62] F. Jia, K. Ramirez-Muñiz, S. Song, “Mechanism of the formation of micropores in the thermal decomposition of goethite to hematite", Surface and Interface Analysis, 47 (2015) 535-539, DOI: 10.1002/ sia. 5744 .

[63] L. Löffler, W. Mader, "Anisotropic x-ray peak broadening and twin formation in hematite derived from natural and synthetic goethite", Journal of the
European Ceramic Society, 26 (2006) 131-139, DOI: 10.1016/j.jeurceramsoc.2004.09.032.

[64] L. Pérez-Maqueda, J. Criado, C. Real, J. Šubrt, J. Boháček, "The use of constant rate thermal analysis (CRTA) for controlling the texture of hematite obtained from the thermal decomposition of goethite", Journal of Materials Chemistry, 9 (1999) 1839-1845, DOI: 10.1039/a901098j.

[65] K. Przepiera, A. Przepiera, "Kinetics of thermal transformations of precipitated magnetite and goethite", Journal of Thermal Analysis and Calorimetry, 65 (2001) 497-503, DOI: 10.1023/A:1012441421955.

[66] D. Walter, G. Buxbaum, W. Laqua, "The mechanism of the thermal transformation from goethite to hematite", Journal of Thermal Analysis and Calorimetry, 63 (2001) 733-748, DOI: 10.1023/A:1010187921227.

[67] R. Morris, H. Lauer, C. Lawson, E. Gibson, G. Nace, C. Stewart, "Spectral and other physicochemical properties of submicron powders of hematite $\left(\alpha-\mathrm{Fe}_{2} \mathrm{O}_{3}\right)$, maghemite $\left(\gamma-\mathrm{Fe}_{2} \mathrm{O}_{3}\right)$, magnetite $\left(\mathrm{Fe}_{3} \mathrm{O}_{4}\right)$, goethite $(\alpha-\mathrm{FeOOH})$, and lepidocrocite $(\gamma$-FeOOH)", Journal of Geophysical Research, 90 (1985) 3126-3144, DOI: 10.1029/JB090iB04p03126.

[68] R. Haglund, "Microscopic and mesoscopic aspects of laser-induced desorption and ablation", Applied Surface Science, 96-98 (1996) 1-13, DOI: 10.1016/0169-4332(95)00371-1.

[69] R. Cornell, U. Schwertmann, The Iron Oxides: Structure, Properties, Reactions, Occurrences and Uses: $2^{\text {nd }}$ Edition, WILEY-VCH Verlag GmbH \& Co. KGaA, Weinheim 2003, pp. 147-152.

[70] A. Ishimaru, "Diffusion of a pulse in densely distributed scatterers", Journal of the Optical Society of America, 68 (1978) 1045-1050, DOI: 10.1364/ JOSA.68.001045.

[71] S. Prahl, "Light transport in tissue", in: Doctoral thesis, University of Texas at Austin, United States 1988, http://omlc.org/ prahl/pubs/pdf/prahl88.pdf (accessed 09.10.2016).

[72] M. Van Gemert, S. Jacques, H. Sterenborg, W. Star, "Skin Optics", IEEE Transactions on Biomedical Engineering, 36 (1989) 1146-1154, DOI: 10.1109/10.42108. 
[73] A. Hassan, M. El-Nicklawy, M. El-Adawi, E. Nasr, A. Hemida, O. Abd El-Ghaffar, "Heating effects induced by a pulsed laser in a semi-infinite target in view of the theory of linear systems", Optics \& Laser Technology, 28 (1996) 337-343, DOI: 10.1016/0030-3992(95)00083-6.

[74] S. Kogou, A. Selimis, P. Pouli, S. Georgiou, C. Fotakis, "The role of the substrate in the laser cleaning process: a study on the laser assisted removal of polymeric consolidation materials from various substrates", in: Lasers in the Conservation of Artworks VIII (Proceedings of the International Conference on LACONA VIII), Sibiu, Romania, 21-25 September 2009, R. Radvan, J. Asmus, M. Castillejo, P. Pouli, A. Nevin (Eds.), CRC Press, London 2011, pp. 23-28.

[75] E. Saavedra, A. López, M. Fiorucci, J. Lamas, A. Ramil, T. Rivas, "Laser cleaning of granitic stones: modeling the heat-affected zone by means of a finite elements method", in: Lasers in the Conservation of Artworks IX (Proceedings of the International Conference on LACONA IX), London, United Kingdom, 07-10 September 2011, D. Saunders, M. Strlič, C. Korenberg, N. Luxford, K. Birkhölzer (Eds.), Archetype Publications, London 2013, pp. 243-245.

[76] E. Saavedra, A. Ramil, A. López, J. Álvarez, "Laser hardening of XC42 steel: numerical analysis of quenched area", Materials Science Forum, 636-637
(2010) 1165-1171, DOI: 10.4028/www.scientific.net/ MSF.636-637.1165.

[77] B. Taarnskov, P. Pouli, J. Bredal-Jørgensen, "Laser cleaning studies for the removal of tarnishing from silver and gilt silver threads in silk textiles", in: Lasers in the Conservation of Artworks VIII (Proceedings of the International Conference on LACONA VIII), Sibiu, Romania, 21-25 September 2009, R. Radvan, J. Asmus, M. Castillejo, P. Pouli, A. Nevin (Eds.), CRC Press, London 2011, pp. 67-73.

[78] N. Vasantgadkar, U. Bhandarkar, S. Joshi, "A finite element model to predict the ablation depth in pulsed laser ablation", Thin Solid Films, 519 (2010) 1421-1430, DOI: 10.1016/j.tsf.2010.09.016.

[79] S. Siano, M. Giamello, L. Bartoli, A. Mencaglia, V. Parfenov, R. Salimbeni, "Phenomenological characterisation of stone cleaning by different laser pulse duration and wavelength", in: Lasers in the Conservation of Artworks (LACONA VI Proceedings), Vienna, Austria, 21-25 September 2005, J. Nimmrichter, W. Kautek, M. Schreiner (Eds.), Springer, Berlin 2007, pp. 87-96.

[80] W. Svendsen, J. Schou, B. Thestrup, O. Ellegaard, "Ablation from metals induced by visible and UV laser irradiation”, Applied Surface Science, 96-98 (1996) 518-521, DOI: 10.1016/0169-4332(95)00506-4. 



\title{
From Earth to Outer Space: Laser cleaning semiprecious quartz and a novel application for meteoritic metal
}

\author{
Rebecca A. Kaczkowski ${ }^{1 \star}$, Bartosz A. Dajnowski ${ }^{2}$, Edward P. Vicenzi ${ }^{1}$ \\ 1 Museum Conservation Institute, Smithsonian Institution, 4210 Silver Hill Road, MRC 534, \\ Suitland, MD 20746, USA \\ 2 Conservation of Sculpture and Objects Studio, Inc., 900 S. Des Plaines Ave, Forest Park, \\ IL 60130, USA \\ * Corresponding author: KaczkowskiR@si.edu
}

\begin{abstract}
Varying the pulse duration, pulse frequency, and fluence (energy density) of conservation lasers can result in successful cleaning of mineralogical materials such as quartz crystals coated with iron oxide films and corroded iron meteorites. Semiprecious quartz is valued by specimen collectors for its euhedral crystal habit and is desirable for commercial applications given its uniform piezo-electric properties. Infrared laser pulses may provide a rapid means for removing metal oxide films from these rough crystals. Extraterrestrial minerals within meteorites provide scientists with valuable information about the origin of our solar system, as well as geologic events in the Earth's deep history. Iron meteorite samples are typically mechanically sectioned and polished to reveal crystalline structures displayed in reflected light microscopy. However, the surfaces of these iron-rich samples can easily oxidize when exposed to moisture and other environmental contaminants. Wet polishing methods, inappropriate handling, and atmospheric exposure can therefore result in surface corrosion in the form of iron oxy-hydroxide-hydrates (e.g. goethite and lepidocrocite). Further hydroxyl and chlorine uptake leads to formation of akaganéite $\left(\mathrm{Fe}^{3+} \mathrm{O}(\mathrm{OH}, \mathrm{Cl})\right)$. The use of $1064 \mathrm{~nm}$ laser pulses at various durations and fluences, in both atmospheric conditions and aided by an argon purge, is explored in this study as a means to remove the corrosion products from an iron meteorite. This method avoids the removal of a significant mass of specimen material involved with grinding and polishing, without the introduction of additional exposure to water. Effects of laser treatment were monitored using scanning electron microscopy and energy dispersive x-ray spectrometry (SEM-EDS). The SEM-EDS "postmortem" evaluation of corrosion removal from iron meteorites establishes the nature of the specimen surface chemistry and morphology following laser cleaning.
\end{abstract}

Keywords: high frequency laser cleaning, minerals, meteorite, natural history conservation, quartz, FeNi alloy

\section{Introduction}

At its core, natural science conservation is dedicated to preserving the scientific utility of the specimens within museum collections. Retaining physical evidence in a specimen allows scientists to weigh theory against observations. Specimens collected for research undergo various preparation techniques to enhance their scientific utility 
for a specific purpose, but each preparation technique can deleteriously impact that same usefulness to answer other, currently unanticipated hypotheses. Thus, collection staff have the challenging task to retain as much chemical, molecular, and physical integrity in the specimens held in museum collections as possible; this includes modifications induced by the initial preparation methodology, subsequent conservation treatment, storage, exhibition, and even analyses. For conservation treatment methodology to be adopted in a natural science context, it must be efficient, effective, and instigate the least amount of chemical or physical change in a material. For these reasons, a study to evaluate the feasibility of incorporating laser cleaning techniques, which can be markedly more efficient and non-destructive than traditional techniques, into the conservation treatment of mineralogical specimens was developed.

This study draws on previous work related to laser cleaning of iron alloys and quartz-containing matrices with a high-frequency system $[1,2]$; there are few published natural history-related investigations into the use of laser cleaning as a conservation tool to remove museum-applied coatings and efflorescence or as a preparation tool to enhance the utility of the specimen such as removal of matrix from fossilized bone [3-7]. In other studies involving iron alloys and quartz-containing stone matrices, laser cleaning at $1064 \mathrm{~nm}$ proved an effective tool to reduce corrosion and weathering products, respectively. The cleaning of iron alloys has been shown to be achievable at low fluences and even at slightly higher fluences with the addition of moisture or ethanol, while the formation of oxides and damage to the alloy can also easily occur [8-10]. Changing these laser parameters ultimately influences the relationships between the possible photomechanical, photochemical, and photothermal effects of the ablation process on the surface of the material. For example, shorter pulses can have more peak energy than longer pulses, and therefore can generate greater photoacoustic shock. Whereas longer pulses tend to generate less photoacoustic shock while having more plasma formation and more potential for photothermal effects [11]. The first part of this study focuses on the interaction of the laser beam with a relatively simple matrix to remove a thin coating formed in a geological setting: specifically, quartz $\left(\mathrm{SiO}_{2}\right)$ provides an excellent substrate to monitor the efficacy of the laser in removing an iron-oxide film. Post-cleaning evaluation of these laser cleaned surfaces using SEM-EDS was deemed a necessary step prior to approaching more chemically complex materials, such as corrosion from an iron meteorite, in the second part of this study.

\subsection{Iron Meteorites in Collections}

A meteorite is a fragment of extraterrestrial material that enters Earth's atmosphere and impacts our planet's surface. Most specimens represent material from the early solar system and reveal a host of processes related to planetary formation. Meteorites range from stony (chondrites and achondrites), to stony-iron, to iron [12]. Some of the greatest preservation challenges persist in the iron meteorite group, and an example metal corrosion issue is the focus of this study. The iron group is composed of predominantly iron-nickel alloys in two primary cubic metallic minerals: kamacite $(\alpha-(\mathrm{Fe}, \mathrm{Ni}) ; 5-12$ wt $\% \mathrm{Ni})$ and taenite $(\gamma-(\mathrm{Ni}, \mathrm{Fe}) ; 20-65 \% \mathrm{wt} \%$ 
$\mathrm{Ni})$. While iron and nickel comprise the most abundant elements in iron meteorites, phosphorous, sulphur, carbon, and silicon account for the remaining fraction of their chemical make-up.

Chlorine is a terrestrial contaminant derived from aqueous and atmospheric sources that threatens the stability of all iron meteorites buried in soil or water-saturated sediment $[12,13]$. The primary chlorine-bearing iron corrosion product formed is akaganéite, an iron III oxyhydroxide [14-16]. Akaganéite $(\beta-\mathrm{FeO}(\mathrm{OH}))$ is poorly ordered, and its small crystal sizes have a high capacity to take on moisture readily in ambient conditions $[15,17]$. The resulting corrosion products have a molar volume of approximately three times greater than the primary meteorite minerals, causing devastating losses to the specimen by way of cracks and exfoliation if left unchecked $[12,13,17]$. Chlorine ions are highly mobile in akaganéite, and the mineral can decompose in situ to intergrowths of goethite $(\gamma-\mathrm{FeO}(\mathrm{OH}))$ and maghemite $\left(\gamma-\mathrm{Fe}_{2} \mathrm{O}_{3}\right)$ or nickeliferous maghemite $[13,14,17,18]$.

The typical means for specimen preparation of iron meteorites includes diamond sawing followed by polishing; some samples can then be prepared as polished thick sections embedded in resin for transmitted light examination. Removing corrosion products often involves similar cutting and grinding methods. Because metallurgical changes can occur in iron meteorites at a few hundred degrees Celsius, so the use of a lubricant during cutting is used to mitigate frictional heating [12]. Cutting and polishing can add additional moisture to the system and can serve to exacerbate corrosion in chlorine-contaminated specimens. Additionally, polishing irreversibly removes bulk material from the specimen, compounding mass lost to corrosion. Although this removal of material from the specimen is minor, this can represent significant loss of material for a polished thin section.

Similar to the conservation of other iron-rich artifacts, e.g. archaeological objects, the removal or reduction of chlorine ions from the bulk of the material is necessary to mitigate the potential for future corrosion in moderate storage environments that do not rely on actively monitored hypoxic conditions $[13,19]$. The soluble nature of some chlorine species in iron and the knowledge of chlorine's role in corrosion has led to the development of desalination treatments; however, this process is difficult to monitor to an end point and is not fully effective in the complete removal of the chlorine contaminant. Experimental hydrogen plasma treatments have also been explored as a means both for iron species reduction (at low temperatures, $<150^{\circ} \mathrm{C}$ ) to facilitate corrosion removal and for chlorine extraction (at high temperatures, $>300-400^{\circ} \mathrm{C}$ ) [17-19]. The primary drawback of the process is that high temperatures in excess of $400-700^{\circ} \mathrm{C}$ are required for directly extracting chlorine contamination from the bulk of the iron meteorite, while temperatures as low as $250^{\circ} \mathrm{C}$ can result in micro-fracturing of the crystal structure. Micro-fracturing facilitates continued corrosion and subsequent loss of scientific integrity of the specimen $[17,20]$. Low temperature plasma cleaning does not impart metallurgical changes in the metal substrate, however chlorine is not directly extracted through this method. Follow-up mechanical and aqueous processes are required, demanding interventive and time-consuming treatment [18]. 


\section{Materials and Methods}

\subsection{Sample Materials and Preparation}

The first sample laser cleaned in this study was quartz mined from the Ouachita Mountains of Arkansas (Fig. 1).

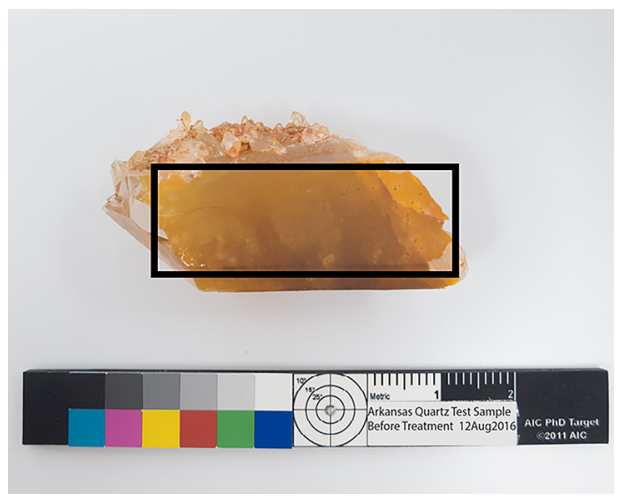

Fig. 1. Growth face of Arkansas quartz used for test cleaning, prior to treatment; region of interest is outlined in black rectangle.

The specimen was extracted by hand and was encapsulated in iron-rich clay; the clay was removed via rinsing with water. The quartz exhibited a thin iron oxide film/ deposit on the interface between the surface of the quartz and the surrounding matrix material. A thin film estimated to be less than $10 \mu \mathrm{m}$ thick determined from Hirox 3-D digital microscopy, the iron oxide coating was well bound to the surface of the crystal. Mechanical removal was, therefore, nearly impossible given the 3-D nature of the crystal growth surfaces and inefficient without marring the surfaces. Cleaning procedures used by mineral collectors include the mechanical reduction of all matrix material followed by immersion in an acidic solution with gradual heating to just below the boiling point for at least 8 hours, followed by a gradual cooling period to room temperature, and a final rinse with pure water to remove acidic residues.
Rapid temperature changes are avoided to prevent thermal shock and cracking of the crystal. A common recipe among quartz miners in Arkansas for preparing this acid bath is to use approximately $0.11 \mathrm{~kg}(0.25$ lbs.) of oxalic acid powder for every 3.8 liters (1.0 gallon) of water. Due to the hazardous fumes produced in the process, these acidic solutions are often cooked outdoors in containers over a heat source such as fire. Alternatively, rock collectors often use pressure cookers to heat the acid bath. For the purposes of this study, a large growth face of a single quartz crystal measuring roughly $3.0 \mathrm{~cm} \times 1.0 \mathrm{~cm}$ was selected due to the relative uniformity of the iron oxide film, in addition to ample surface area available for test cleaning. In an effort to gauge cleaning efficacy of traditional acid cleaning versus laser cleaning, an approximate $1.0 \mathrm{~cm} \times 0.5$ $\mathrm{cm}$ portion of the sample was removed by a water-cooled, low-speed diamond saw for acid cleaning. The remainder of the test face of the sample was masked into a grid-like pattern for laser cleaning tests. The masking material consisted of aluminum foil for aqueous cleaning and heavy white cardstock for cleaning in atmosphere.

The second sample utilized in this study was a $24.67 \mathrm{~g}$ cut, unpolished piece of Canyon Diablo (USNM 676), a well characterized iron meteorite (Fig. 2).

Canyon Diablo is the meteorite responsible for the geologically young ( 49 ka) impact crater in the southeastern United States, called Barringer Meteor Crater [21]. The sample was selected for its mineral phase chemistry and its presentation of typical corrosion found on similar irons stored in zip-top polyethylene bags within powder-coated stainless steel museum storage cases. Designated an iron meteorite class IAB-MG, Canyon Diablo is a coarse-grained 


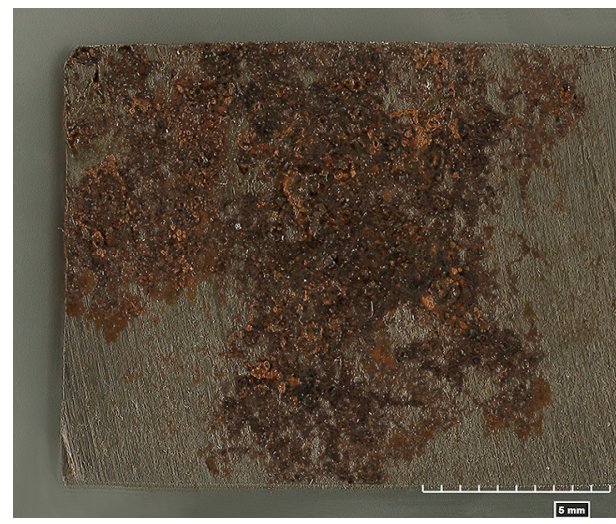

Fig. 2. Photomicrograph of iron meteorite sample (USNM 676), sawed and corroded surface used for laser test cleaning, prior to treatment; whole scale represents $5 \mathrm{~mm}$.

octahedrite. Kamacite ( $\alpha-(\mathrm{Fe}, \mathrm{Ni}) ; 5-12 \mathrm{wt} \%$ $\mathrm{Ni})$ and taenite $(\gamma-(\mathrm{Ni}, \mathrm{Fe}) ; 20-65 \% \mathrm{wt} \% \mathrm{Ni})$ comprise the majority of the volume, with minor schreibersite $\left((\mathrm{Fe}, \mathrm{Ni})_{3} \mathrm{P}\right)$ confirmed by SEM-EDS imaging and analysis. In addition to primary minerals kamacite, taenite, and schreibersite, chlorine was detected in significant concentration in association with the corroded surface and secondary mineralization (Fig. 3, Tab. 1).

Table 1. Semi-Quantitative Elemental Analysis of Meteorite Corrosion

\begin{tabular}{lcc}
\hline Element & Weight (\%) & Atomic (\%) \\
\hline Iron $(\mathrm{Fe})$ & 48.6 & 20.8 \\
Oxygen $(\mathrm{O})$ & 34.6 & 51.7 \\
Carbon $(\mathrm{C})$ & 12.7 & 25.3 \\
Chlorine $(\mathrm{Cl})$ & 2.4 & 1.6 \\
Nickel $(\mathrm{Ni})$ & 1.6 & 0.7 \\
\hline Total & 99.9 & 100 \\
\hline
\end{tabular}

Similar to the quartz sample, the largest area of corrosion on the meteorite sample surface was masked into a grid for laser cleaning tests. Masking material consisted of heavy white cardstock, cut with a $2 \times 3$ grid of $0.5 \mathrm{~cm}^{2}$ squares. To ensure the mask achieved

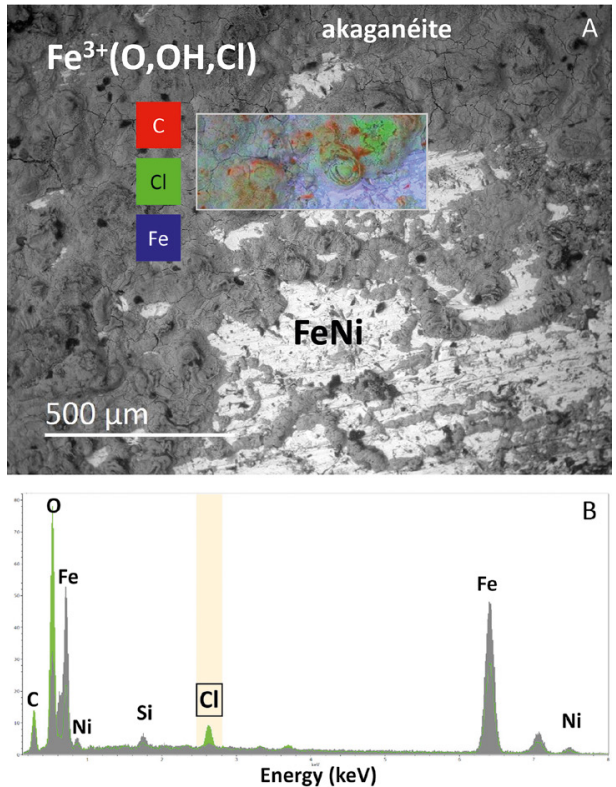

Fig. 3. (A) Backscattered electron image of corrosion on the iron-nickel alloy surface (USNM 676) with a false-color chemical image overlay (carbon, red; chlorine, green; iron, blue), prior to treatment and (B) X-ray spectrum for non-corroded iron- nickel surface (grey) and the chlorine-rich region of corrosion (blue), prior to treatment.

sufficient contact with the sample surface and no ejected material would impact other sample sites, a rare earth magnet secured the grid and mask to the sample.

\subsection{Cleaning Procedures}

All laser cleaning tests were carried out with a state-of-the-art 70-watt, solid-state, high frequency $(10 \mathrm{kHz}-1 \mathrm{MHz})$ conservation laser operating at $1064 \mathrm{~nm}$ [22]. For this setup, the laser had an in-focus spot size of 250 $\mu \mathrm{m}$ when using a $16.0 \mathrm{~cm}$ focal lens; this value was confirmed by measuring a test area on carbon laser paper with the Hirox microscope. The high-frequency pulses of 
the beam are delivered via fiber optic cable to a scanning head that emits a unique circular scan pattern with a conical delivery path that allows the beam to interact with the surface from various angles as the leading edge and trailing edge of the scan move across the object. This optical configuration allows for the scan to conform to shapes and crevices while providing multiple exposures per pass. The continuous path of the circle scan helps ensure that the pulses do not linger in one place for too long. The desired pulse overlap pattern and cleaning speed is determined by the laser parameters and technique employed by the operator. The circular scan pattern used in this experiment measured 2.0 $\mathrm{cm}$ in diameter. Multiple parameters were manipulated during test cleaning to achieve the differing cleaning levels, including pulse energy, pulse duration, pulse frequency, and revolutions per minute (RPM) of the scan. Although a wide range of pulse durations was available to test, in order to maximize the potential for repeatability by others in the field, the most commonly used pulse durations of $10 \mathrm{~ns}$ and $100 \mathrm{~ns}$ were selected.

Test cleaning of quartz was carried out under two environmental conditions: with the specimen submerged underwater, and in ambient conservation laboratory conditions, assisted by the aid of an extraction trunk located approximately $15.0 \mathrm{~cm}$ from the test cleaning site. The former cleaning scenario was used in an effort to increase the efficiency of laser cleaning by reducing potential redeposition of ablated material. Water is commonly used during laser cleaning protocols for benefits such as the added micro-steam-cleaning effect when water is vaporized by laser pulses; additionally, water does not act as a solvent to the contaminant layer and interfere with evaluation of the laser cleaning efficacy. In order to mini- mize the reduction of the fluence of pulses reaching the surface of the crystal due to water absorption of energy, the crystal was submerged only a few millimeters below the surface. For test cleaning carried out in ambient conditions, the specimen was propped at an approximately $40^{\circ}$ angle on a glass stand with white cardstock masking off all areas not to be exposed to the laser energy. Cleaning at the selected parameters was carried out by making 2-3 passes across the test site, moving the scan manually at approximately $1 \mathrm{~cm}$ per second, both vertically and horizontally to ensure equal exposure and achieve the desired aesthetic clean. Unlike low frequency lasers that are commonly used with larger spot sizes, measured in millimeters, and higher pulse energies, a scanning system like this one uses hundreds of thousands of weaker pulses, measured in microns, to allow for even and gentle cleaning. The masks were removed between cleaning tests to evaluate cleaning levels (Tab. 2). For cleaning tests performed with the specimen submerged underwater, the specimen was wrapped in aluminum foil and the test site "windows" were cut out of the foil. An additional foil mask was used across the surface of the specimen to ensure that only one window was exposed to laser energy at a time (Fig. 4).

The sample was then placed on a glass stand in a glass bowl of deionized water. Cleaning at the selected parameters was carried out in a similar manner as before. This procedure produced nearly equal laser exposure and achieved the desired level of cleaning as determined visually.

Test cleaning of the iron meteorite was also carried out in two environmental conditions: the same ambient conservation laboratory conditions as the quartz, and with an argon purge delivered via plastic nozzle at $180 \mathrm{psi}$ with an extraction trunk. The argon purge 
Table 2. Cleaning Parameters per Test Site on Quartz

\begin{tabular}{clcccc}
\hline $\begin{array}{c}\text { Test } \\
\text { Site }\end{array}$ & Environment & $\begin{array}{c}\text { Pulse duration } \\
(\mathrm{ns})\end{array}$ & $\begin{array}{c}\text { Frequency } \\
(\mathrm{KHz})\end{array}$ & $\begin{array}{c}\text { Frequency of } \\
\text { rotation }(\mathrm{RPM})\end{array}$ & $\begin{array}{c}\text { Fluence } \\
\left(\mathrm{J} / \mathrm{cm}^{2}\right)\end{array}$ \\
\hline 1 & Ambient & 250 & 50 & 8,000 & 2.65 \\
2 & Ambient & 10 & 450 & 8,000 & 0.30 \\
3 & Ambient & 50 & 150 & 8,000 & 0.88 \\
4 & Ambient & 80 & 150 & 8,000 & 0.94 \\
5 & Ambient & 150 & 150 & 8,000 & 0.96 \\
6 & Ambient & 250 & 150 & 8,000 & 0.97 \\
7 & Ambient & 250 & 50 & 3,000 & 2.65 \\
8 & Ambient & 250 & 50 & 3,000 & 2.65 \\
9 & Ambient & 250 & 50 & 3,000 & 2.65 \\
10 & Under Water & 250 & 50 & 8,000 & 2.65 \\
11 & Under Water & 250 & 50 & 3,000 & 2.65 \\
12 & Under Water & 10 & 450 & 8,000 & 0.30 \\
13 & Under Water & 50 & 150 & 8,000 & 1.03 \\
14 & Under Water & 80 & 150 & 8,000 & 0.94 \\
15 & Under Water & 150 & 150 & 8,000 & 0.96 \\
16 & Under Water & 250 & 150 & 8,000 & 0.97 \\
\hline
\end{tabular}

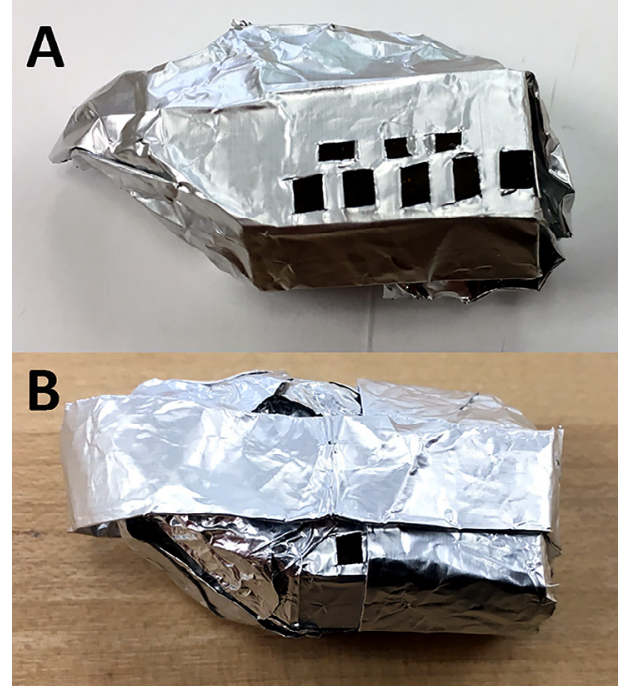

Fig. 4. (A) Quartz sample masked with aluminum foil and (B) quartz sample with the additional aluminum-foil mask for underwater laser cleaning. was used to promote heat dissipation and hinder oxide formation on the metal surface. The sample was propped at a $45^{\circ}$ angle on a glass stand. To have the largest area possible for test cleaning with the limited concentration of corrosion on the surface, six test sites measuring $5.0 \mathrm{~mm}^{2}$ each were masked in heavy white cardstock: three sites for ambient environmental conditions with differing fluences and pulse durations $\left(0.23 \mathrm{~J} / \mathrm{cm}^{2} @\right.$ $10 \mathrm{~ns}, 0.31 \mathrm{~J} / \mathrm{cm}^{2} @ 100 \mathrm{~ns}$, and $1.0 \mathrm{~J} / \mathrm{cm}^{2} @$ 100 ns, respectively), and three for the same fluences with an argon purge. Using two rare earth magnets to hold the heavy white cardstock ensured sufficient contact of the mask to the sample. At each test cleaning site, $2-3$ passes by the conservator with the high-frequency laser were made across the test site both vertically and horizontally in an effort to achieve nearly equal laser exposure. These conditions produced a suitable level of cleaning based upon visual inspection. Masks were removed between cleaning tests to evaluate cleaning levels (Tab. 3). 
Table 3. Cleaning Parameters per Test Site on Iron Meteorite (USNM 676)

\begin{tabular}{clcccc}
\hline $\begin{array}{c}\text { Test } \\
\text { Site }\end{array}$ & Environment & $\begin{array}{c}\text { Pulse duration } \\
(\mathrm{ns})\end{array}$ & $\begin{array}{c}\text { Frequency } \\
(\mathrm{KHz})\end{array}$ & $\begin{array}{c}\text { Frequency of } \\
\text { rotation }(\mathrm{RPM})\end{array}$ & $\begin{array}{c}\text { Fluence } \\
\left(\mathrm{J} / \mathrm{cm}^{2}\right)\end{array}$ \\
\hline A1 & Ambient & 10 & 400 & 8,000 & 0.23 \\
A2 & Argon & 10 & 400 & 8,000 & 0.23 \\
A3 & Ambient & 100 & 400 & 8,000 & 0.31 \\
A4 & Argon & 100 & 400 & 8,000 & 0.31 \\
A5 & Ambient & 100 & 100 & 8,000 & 1.0 \\
A6 & Argon & 100 & 100 & 8,000 & 1.0 \\
\hline
\end{tabular}

\subsection{Documentation and Analysis}

The surfaces of both the quartz and iron meteorite samples were documented prior to, and following laser experiments. Contamination layers were also characterized via visible light and scanning electron microscopy methods outlined below.

Optical microscopy (Hirox KH-8700 with MGX-2500REZ and MGX-1260 lenses with variable lighting attachment) was performed on both the quartz and iron meteorite samples to characterize the thickness of the contamination layers, the nature of the substrates, and to monitor cleaning. Using the variable lighting adapter that employs a rotating mirror to direct the light to the sample, surfaces were observed with the light at $25^{\circ}, 55^{\circ}$, and $75^{\circ}$ angles, highlighting surface non-uniformity and residues.

Scanning electron microscopy with energy dispersive $\mathrm{x}$-ray spectrometry (Hitachi S-3700N, operating at $100 \mathrm{~Pa}$ atmosphere variable pressure for uncoated samples, 15-20 kV, with a Bruker XFlash 4010 detector using a pulse time constant of $1.25 \mu \mathrm{s}$ ) was used to characterize the surfaces of the samples before and after cleaning tests. SEMEDS output documented the morphological and elemental composition of substrates and corrosion products. These results provided an evaluation of relative contaminant concentration before and after test cleaning.
X-ray diffraction (Rigaku D/MAXRAPID XRD) was carried out for corrosion material scraped from the iron meteorite to determine its mineralogical content. An approximately 800 micron thick sample was manually removed from an area of dense corrosion adjacent to the laser testing sites with a scalpel performed at low magnification using a stereomicroscope. The sample was then adhered to a glass fiber with Elmer's glue (PVA emulsion) and exposed to $\mathrm{Cu}$ radiation for 60 minutes using a 300 micron collimator. No discernable diffraction pattern was obtained. This result is likely due to the poor crystallinity of the iron corrosion products.

\section{Cleaning Results and Discussion}

\subsection{Quartz}

Preliminary results show that cleaning tests in ambient conservation laboratory conditions produced macroscopically clean quartz. Cleaning was stopped when the quartz appeared clean to the naked eye. Optical microscopy with coaxial $25^{\circ}$ angle lighting, however, reveals a regular pattern of cleaned circles within an incompletely cleaned field of iron oxide film (Fig. 5).

EDS mapping of the cleaned areas reveals that the diameter of the cleaned circles is roughly $10.0 \mu \mathrm{m}$ and an iron-rich residue is 


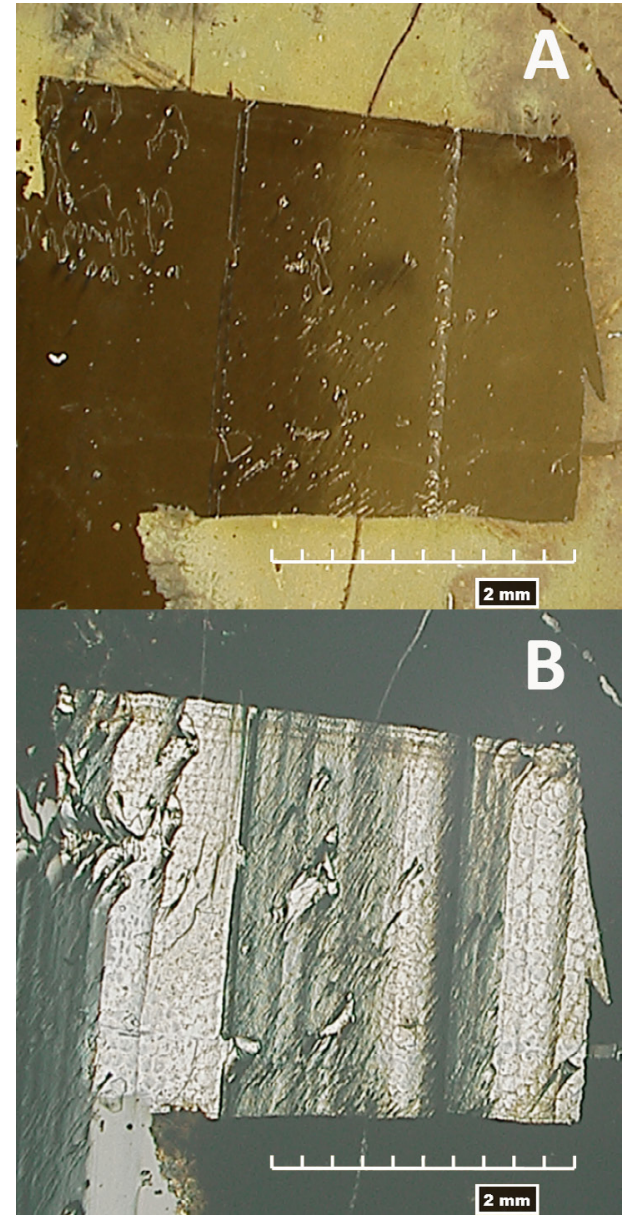

Fig. 5. (A) Spot $1\left(\mathrm{~F}=2.65 \mathrm{~J} / \mathrm{cm}^{2}\right)$ appears clean with ring lighting and (B) Spot 1 under coaxial lighting conditions reveals surface patterning of thin-film residue; whole scales represent $2 \mathrm{~mm}$

left in the areas around the circles (Fig. 6). The circular scan pattern of the beam delivery at $50 \mathrm{kHz}$ and 8,000 RPM allows for significant overlap of each pulse; the residue pattern is inconsistent with this overlap. Furthermore, the fiber-optically delivered beam spot from the laser is $250 \mu \mathrm{m}$ in diameter with the 16 $\mathrm{cm}$ focal lens used in this study, as confirmed by measuring a test area on carbon laser paper with the Hirox microscope. The reason

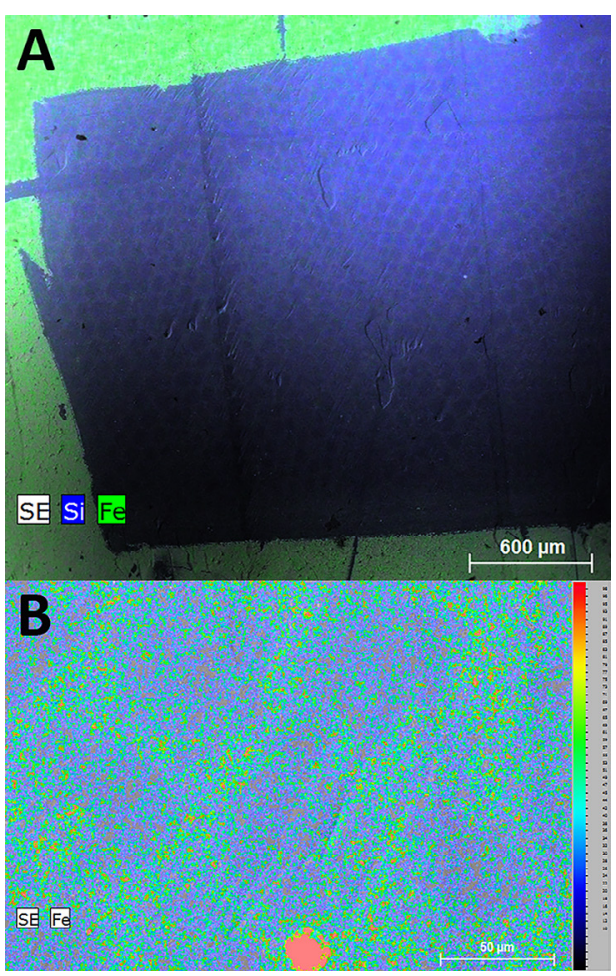

Fig. 6. (A) Secondary electron image with false-color overlay for silicon (blue) and iron (green). There is a residue pattern within spot $1\left(\mathrm{~F}=2.65 \mathrm{~J} / \mathrm{cm}^{2}\right)$ and (B) the log of the intensity (rainbow false color) of $\mathrm{Fe} \mathrm{K}_{a}$ within the only area of significant iron enrichment on the laser-cleaned region of Spot 1; scale in Fig. B represents $50 \mu \mathrm{m}$.

for the residue pattern is not readily apparent considering the instrumentation parameters used. It is unclear if the residue is a result of under cleaning or redeposition of material. It is possible that the $16 \mathrm{~cm}$ focal lens that was used had a contaminant or an optical aberration, or, that additional optical phenomena are occurring as a result of the unique linear and nonlinear optical properties of quartz to create this pattern.

It is also important to note that the quartz is transmissive of $1064 \mathrm{~nm}$ laser pulses, and a cleaning effect on the opposite growth face 
to the cleaning site was also observed. No specific damage to the surface of the crystal was noted, however. Rather, the imperfect surface of the crystal growth face is revealed by the cleaning process. The quartz was not exposed to additional laser pulses after the residue was discovered. It was not tested if additional exposure to more pulses at the same parameters or pulses at other laser parameters would remove the remaining res-

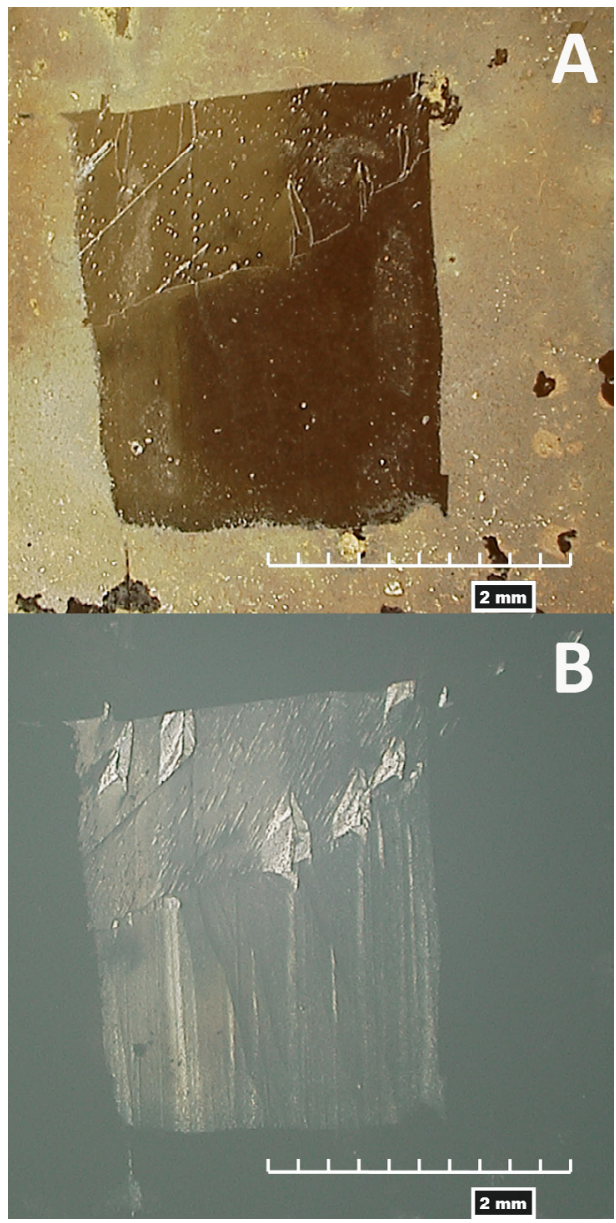

Fig. 7. (A) Spot $10\left(\mathrm{~F}=2.65 \mathrm{~J} / \mathrm{cm}^{2}\right)$ appears clean with ring lighting, and (B) Spot 10 under coaxial lighting conditions reveals no distinct surface patterning of thin-film residue. idue that was revealed with coaxial lighting optical microscopy and EDS.

Cleaning tests performed under water also produced macroscopically clean quartz. Light microscopy revealed no post-cleaning pattern observed in the previous cleaning tests (Fig. 7, 8).

Residual iron oxide film does, however, remain on the surface, as observed through EDS mapping of the cleaned areas. The

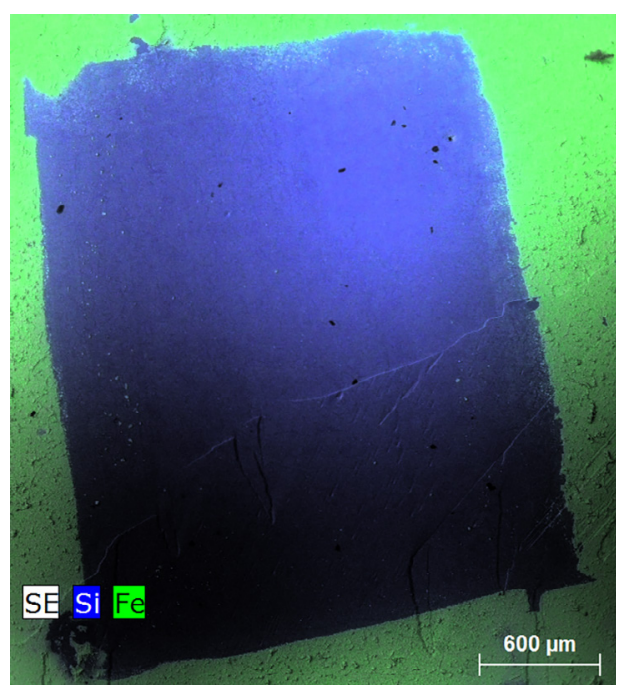

Fig. 8. Secondary electron image with false-color overlay for silicon (blue) and iron (green). There is no residue pattern within spot $10\left(\mathrm{~F}=2.65 \mathrm{~J} / \mathrm{cm}^{2}\right)$.

cleaning effect observed on the opposite growth face to the cleaning site was divested of less material than the test areas cleaned in ambient conditions; this is a result of the energy absorbance of water.

Again, no specific damage to the surface of the crystal is noted however, and the crystal growth-face imperfections are revealed.

On a microscopic scale, oxalic acid cleaning of quartz appears more complete, relative to the laser conditions employed in this study. Although the laser energy did not show 
deleterious effects to the crystal, the oxalic acid method achieved a more completely clean surface. Iron was detectable on the acid-cleaned sample in SEM-EDS (Fig. 9).

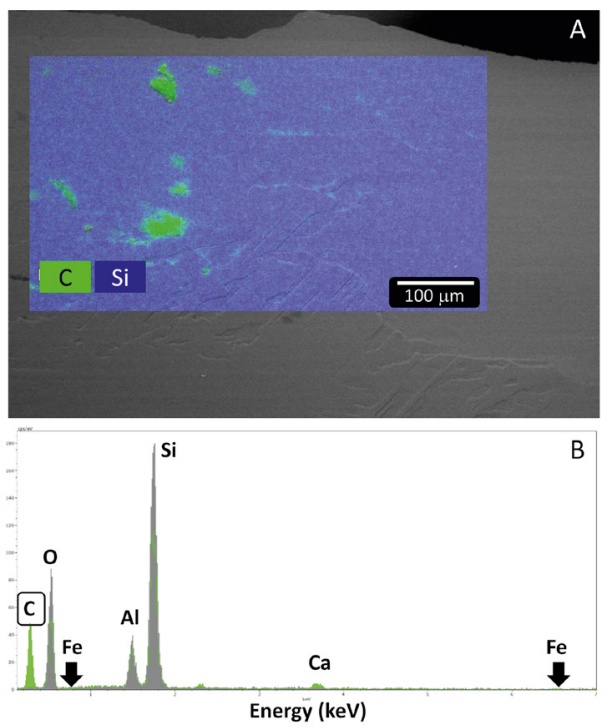

Fig. 9. (A) Backscatter scanning electron image with elemental false-color overlay (silicon, blue; carbon, green), and (B) X-ray spectrum of a carbon-rich area from the same region of interest (green) and the surrounding cleaned area (grey) illustrates how localized carbon is on the surface as a discrete contaminant and iron is not present. However, carbon was detected in localized areas on the acid-cleaned quartz surface and appears to be associated with organic matter from the matrix (e.g. vegetation in the soil) that was not readily removed during treatment with oxalic acid.

\subsection{Meteorite}

Energy imparted by the laser partly removed corrosion products from all test sites of the Canyon Diablo specimen (Fig. 10).

Areas cleaned at a fluence of $0.23 \mathrm{~J} / \mathrm{cm}^{2}$ @ $10 \mathrm{~ns}$, appeared clean with an undisturbed metal substrate. However, the other four test sites cleaned with fluences of $0.31 \mathrm{~J} / \mathrm{cm}^{2} @$ $100 \mathrm{~ns}$ and $1.0 \mathrm{~J} / \mathrm{cm}^{2} @ 100 \mathrm{~ns}$, also appeared

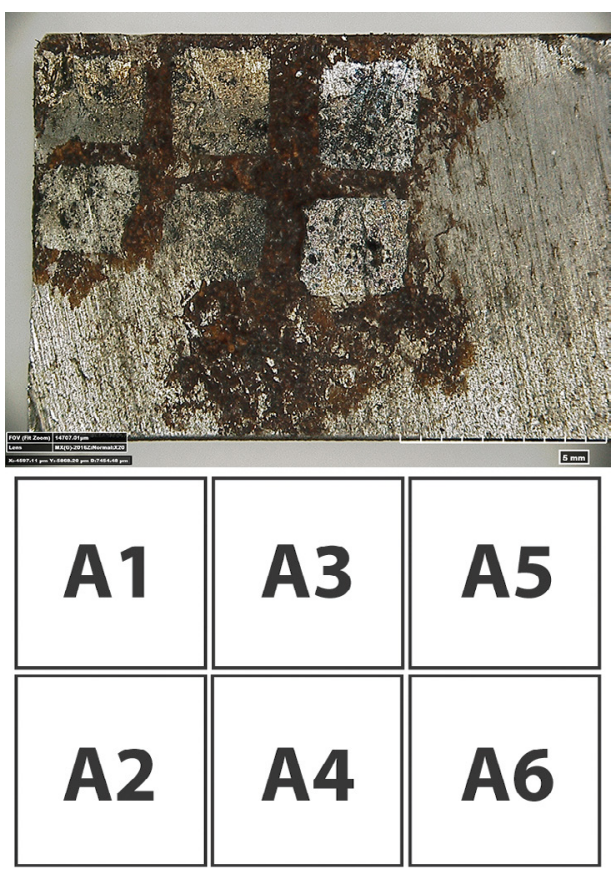

Fig. 10. Photomicrograph of iron meteorite (USNM 676), sawed and corroded surface used for laser test cleaning, after treatment; all test sites successfully removed corrosion products, albeit incompletely. A labeled diagram of the test sites is below.

cleaned, but the substrates were altered by the laser pulses. Additionally, a slight color change to the metal substrate in test areas cleaned in air, versus those cleaned with an argon purge was also noted. There may also be some thin film oxide formation on the metal surface when exposed to air under certain pulsed laser conditions. The argon purge appears to have provided a reducing environment during cleaning, or dissipated heat evolved during ablation through gaseous advection. The observed color differences may also be an artifact of a non-uniform corrosion layer revealing a differently corrosion-affected surface beneath. Further, SEM-EDS mapping illustrated that all of the test sites - those cleaned in air and those with 
an argon purge - exhibited an incompletely cleaned surface. This may be due to an insufficient amount of laser pulses being used to fully clean the surface, or may be caused by: 1) redeposition of ablated material, 2) incomplete ejection of heated corrosion products by the laser, or, 3) both mechanisms operating in tandem. The observed color of the surface metal can also be attributable to the incomplete nature of the cleaning.

Furthermore, the concentration of chlorine appears to be reduced on all sample sites. Comparing the compositional images of the kamacite cleaned with a fluence of $0.31 \mathrm{~J} / \mathrm{cm}^{2} @ 100 \mathrm{~ns}$ surface with an area of incompletely removed corrosion, chlorine is observed to have been removed to within the detection limit of $\mathrm{x}$-ray microanalysis in the laser-cleaned regions. However, chlorine is likely removed only to the depth that the laser energy penetrated the specimen, approximately several microns. In order to evaluate the efficacy of this treatment for chlorine removal, compositional analysis of a cross section is required to monitor the chemical depth profile for chlorine removal. Previous studies have determined that effective bulk chloride removal in iron alloys can only be achieved at temperatures in excess of $400^{\circ} \mathrm{C}[16,17]$. Therefore, given the surface sensitivity of laser cleaning, chloride removal is limited to the uppermost micrometers of the sample surface.

Using the lowest energy density of $0.23 \mathrm{~J} / \mathrm{cm}^{2} @ 10 \mathrm{~ns}$ at the interface of the corrosion layer and cleaned metal substrate, there is a narrow, only microns wide, intermediary boundary between the cleaned area and unaffected corrosion layers (Fig. 11).

Fragile microstructures within the corrosion in this zone are left intact. This suggests that the laser is, indeed, a high-precision tool for selective corrosion removal. Conversely,

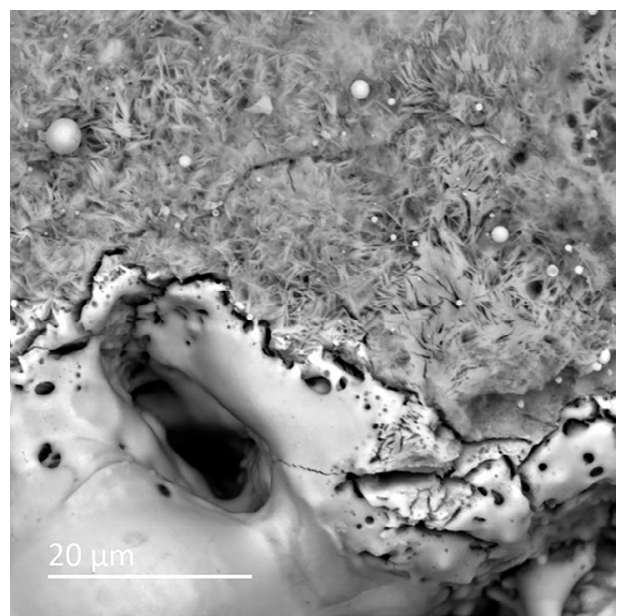

Fig. 11. Detail of the sharp boundary between laser-altered corrosion products and unaffected corrosion on Spot A1.

adjacent to areas of affected corrosion, cleaned but undamaged metal is revealed (Fig. 12). Redeposited spheres of corrosion are also visible on the surface.

At the same low fluence, but with the addition of an argon purge, the kamacite surface is similarly unaffected by the laser

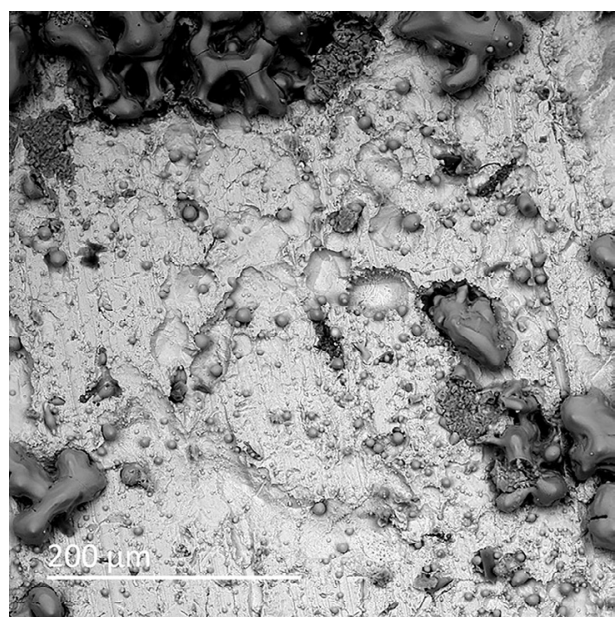

Fig. 12. Spot A1 is incompletely cleaned, but the metal substrate is unaltered by the lowest laser fluence $\left(0.23 \mathrm{~J} / \mathrm{cm}^{2}\right)$. 
energy - demonstrated by the crisp clarity of the shallow corrosion pits. Indeed, the incomplete ejection of corrosion from the surface and resultant formation of iron-oxide spheres is clear, as the corrosion products begin to curl inward toward the center of corrosion pits (Fig. 13). Smaller spheres may also be attributable to redeposition from the laser plume.

At the intermediate energy density used in this study, $0.31 \mathrm{~J} / \mathrm{cm}^{2} @ 100 \mathrm{~ns}$, observations under SEM-EDS revealed additional information related to the laser interaction with the substrate. Again the kamacite surface is unaffected by the laser energy, regardless of treatment in air or with the argon purge. Textural evidence for laser alteration was observed in the form of immiscibility between iron-nickel phosphate and iron-nickel-phosphide, an indication of their molten state during laser cleaning.

Although we have shown that the kamacite appears unaffected at a fluence of $0.23 \mathrm{~J} / \mathrm{cm}^{2}$

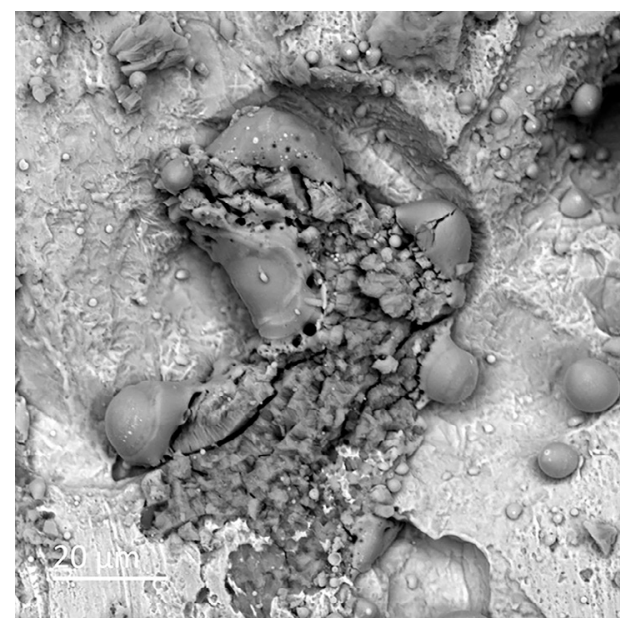

Fig. 13. Incipient melting of corrosion material to form anhydrous spherical particles of iron oxide masses; note the curling of the corrosion product inward from the edges of the pit.
@ $10 \mathrm{~ns}$ and $0.31 \mathrm{~J} / \mathrm{cm}^{2} @ 100 \mathrm{~ns}$, the latter fluence while cleaning in air is enough to preferentially affect the nickel-rich taenite. There was no evidence of taenite alteration on the argon-assisted cleaned area. Moreover, the damage threshold for the kamacite was also determined to be greater than $0.31 \mathrm{~J} / \mathrm{cm}^{2}$ @ $100 \mathrm{~ns}$, but less than $1.0 \mathrm{~J} / \mathrm{cm}^{2} @ 100 \mathrm{~ns}$, regardless of cleaning in air or with an argon purge. The lower damage threshold for the taenite versus the kamacite may be attributable to the mineral intergrowths within the taenite, resulting in a lower melting point of the material [23]; it may also be related to the grain size of the particular class of iron meteorite. A larger sampling of class IAB-MG iron meteorites with larger cleaning test sites is necessary to confirm this observation.

\section{Conclusions}

These initial findings indicate that it is possible to successfully remove iron oxide films from quartz with a high frequency tunable laser system. More testing is recommended to identify optimal parameters and conditions for cleaning quartz specimens. With a homogenous substrate such as quartz, the evaluation of laser cleaning efficacy is possible through SEM-EDS mapping of the treated areas before and after cleaning. It is also an effective tool for understanding the nature of the specimen surface chemistry and morphology following laser cleaning on more complex, heterogeneous samples, such as an iron meteorite.

From a macroscopic appraisal of the cleaned meteorite specimen, an ambient atmosphere coupled with the lowest fluence $\left(0.23 \mathrm{~J} / \mathrm{cm}^{2} @ 10 \mathrm{~ns}\right)$ produced a cleaned surface without affecting the substrate. Follow-up optical microscopy carried out in the 
NMNH's Department of Mineral Science, revealed cleaned areas at the lowest fluence did not have any detectable impact on the metallography. However, the incomplete nature of the laser cleaning required subsequent polishing of the sample for optical microscopy. Although high magnification examination of the test site found the cleaning to be incomplete, the aesthetic affect may be sufficient for non-research specimens or artifacts made of iron meteoritic metals. Cleaning was monitored macroscopically and terminated when the sample visually appeared to be clean; a more thorough cleaning may be possible with additional passes with laser pulses at these parameters, and/or through the incorporation of complementary cleaning techniques such as solvent methods.

Additionally, ranges for damage thresholds of the primary minerals taenite and kamacite were experimentally determined in this study. Taenite appears to have a lower relative damage threshold than kamacite. Neither taenite nor kamacite were affected by laser cleaning with a fluence of $0.23 \mathrm{~J} / \mathrm{cm}^{2}$ @ 10 ns. It should be noted, however, that determination of the damage threshold of the specimen or ablation threshold of the corrosion products were not the primary goals of this study. Rather, the study demonstrates that there are acceptable ranges of parameters to remove corrosion from the meteorite without adversely affecting the underlying substrate.

The effects of the laser observed at the argon-treated sites are believed to be the result of laser ablation under anoxic conditions, but have not been confirmed. From a macroscopic inspection, these sites have the appearance of a cleaner surface; however, the addition of the argon purge did not increase the damage threshold for either kamacite or taenite. A thin-film effect of what may be a native oxide patina appears to form on the samples treated under atmospheric conditions that was not readily observed with the argon-purge treated samples. This film is so thin that SEM-EDS is insufficiently sensitive with regard to chemical depth resolution, and requires a true surface technique, such as $\mathrm{x}$-ray photoelectron spectroscopy (XPS) to determine differences between samples treated in ambient atmosphere versus argon purge conditions.

Finally, the use of SEM-EDS proved to be an effective means for characterizing the surfaces of the samples and the contaminants, and also evaluating their subsequent changes as a result of cleaning treatments. Continued work to incorporate laser cleaning to reduce corrosion and chloride concentration on iron meteorites should employ complementary cleaning techniques already in practice in the treatment of meteorites. Laser cleaning may be able to fully remove corrosion products with proper parameters, or it could be used to successfully reduce the bulk of the surface corrosion, requiring subsequently less mechanical manipulation and polishing of the surface - and therefore less removal of the metal substrate. Only a few of the many possible laser parameter combinations were tested during this study, and these initial findings indicate that more in-depth and diversified experimentation with additional laser parameters is indeed worth exploring.

\section{Acknowledgements}

The authors would like to thank the following colleagues at the Museum Conservation Institute for their support and contributions to this research: Dr. Odile Madden, Dr. Gwénaëlle Kavich, Nicole Little, Dr. Thomas Lam, E. Keats Webb, Janet Douglas, Jessica Johnson, Dr. Robert Koestler, Dr. Paula 
DePriest. Additionally, this project would not have been possible without the support of colleagues in the Department of Mineral Sciences at the National Museum of Natural History: Dr. Timothy McCoy, Julie Hoskin, Timothy Gooding, and Timothy Rose. The authors are also immensely grateful to Dr. Andrzej Dajnowski for lending a GC-1 laser for use in this project and the guidance and support of Dr. Robert Waller, Catharine Hawks, and Marlene Yandrisevits.

\section{References}

[1] A. Dajnowski and B. Dajnowski, "A case study in the removal of paint and corrosion from cast iron using a Nd:YAG laser", in: Lasers in the Conservation of Artworks IX (Proceedings of LACONA IX conference), London, UK, September 7-10, 2011, D. Saunders, M. Strlič, C. Korenberg, N. Luxford, K. Birkhölzer (Eds.), Archetype Publications Ltd, London 2013, pp. 40-44.

[2] A. Dajnowski, "Laser cleaning of the Nickerson Mansion: The first building in the US entirely cleaned using laser ablation", in: Lasers in the Conservation of Artwork,s Proceedings of the International Conference LACONA VII, Madrid, Spain, September 17-21, 2007, J. Ruiz, R. Radvan, M. Oujja, M. Castillejo, P. Moreno (Eds.), CRC Press/Taylor \& Francis, London 2008, pp. 209-214.

[3] F. Landucci, R. Pini, S. Siano, R. Salimbeni, E. Pecchioni, "Laser Cleaning of Fossil Vertebrates: A Preliminary Report", Journal of Cultural Heritage, 1 (2000) S263-S267, DOI: 10.1016/S12962074(00)00141-2.

[4] F. Landucci, E. Pecchioni, D. Torre, P. Mazza, R. Pini, S. Siano, R. Salimbeni, "Toward an Optimsed Laser Cleaning Procedure to Treat Important Paleontological Specimens", Journal of Cultural Heritage, 4 (2003) 106s-110s. DOI: 10.1016/S12962074(02)01185-8.

[5] L. Cornish and C. G. Jones, "Laser Cleaning of Natural History Specimens and Subsequent SEM Examination", in: J. H. Townsend, K, Eremin, A. Adriaens (Eds.) Conservation Science 2002: Papers from the Conference Held in Edinburgh, Scotland, 22-24 May 2002, Archetype Publications Ltd, London 2003, pp. 101-106.
[6] E. Karantoni, E. Malea, N. A. Stefanis, A. Alexandropolou, K. Melessanaki, A. Selimis, P. Pouli, G. Panagiaris, "Studies on the Use of Laser Radiation to Clean Natural History Specimens: Preliminary Results", Poster number 1206 presented at the $17^{\text {th }}$ Triennial Conference of the International Council of Museums Committee for Conservation (ICOM-CC), Melbourne, Australia, 15-19 September 2014, (Proceedings in press).

[7] J. Asmus, "Laser conservation paleontology", Proceedings of SPIE 4402 (2001) 1-7, DOI: 0277786X/01/\$15.00.

[8] K. Dickmann, J. Hildenhagen, J. Studer, E. Musch, "Archaeological ironwork: Removal of corrosion layers by Nd:YAG-laser", in: Lasers in the Conservation of Artworks $V$ (Proceedings of the LACONA V conference), Osnabruck, Germany, September 15-18, 2003, K. Dickmann, C. Fotakis, and J. F. Asmus (Eds.), Springer-Verlag, Berlin 2005, pp. 71-77.

[9] C. Fotakis, D. Anglos, V. Zafiropulos, S. Georgiou, V. Tornari, Lasers in the Preservation of Cultural Heritage: Principles and Applications, Taylor \& Francis, New York 2007, pp. 306-313.

[10] C. Korenberg and A. M. Baldwin, "Laser cleaning of iron: Surface appearance and re-corrosion of model systems", in: Lasers in the Conservation of Artworks VIII (Proceedings of the LACONA VIII conference), Sibiu, Romania, September 21-25, 2009, R. Radvan, J. F. Asmus, M. Castillejo, P. Pouli, and A. Nevin (Eds.), CRC Press, Boca Raton, 2011, pp. 123-133.

[11] S. Siano and R. Salimbeni, "Advances in laser cleaning of artwork and objects of historical interest: The optimized pulse duration approach", Accounts of Chemical Research 43 (2010) 739-750, DOI: 10.1021/ar900190f.

[12] A. W. R. Bevan, "Meteorites", in: F. M. Howie (Ed.), The Care and Conservation of Geological Material: Minerals, Rocks, Meteorites, and Lunar Finds, Butterworth-Heinemann, Oxford 1992, pp. 85-101.

[13] K. Stahl, K. Nielsen, J. Jiang, B. Lebech, J. C. Hanson, P. Norby, J. van Lanschot, "On the akaganéite crystal structure, phase transformations and possible role in post-excavational corrosion of iron artifacts", Corrosion Science 45 (2003) 2563-2575, DOI: 10.1016/S0010-938X(03)00078-7. 
[14] L. Selwyn, Metals and Corrosion: A Handbook for the Conservation Professional, Canadian Conservation Institute, Ottawa 2004.

[15] J. E. Post and V. F. Buchwald, "Crystal structure refinement of akaganéite", American Mineralogist 76 (1991) 272-277, DOI: 0003-004X/91/0102$0272 \$ 02.00$.

[16] J. Scheck, T. Lemke, D. Gebauer, “The Role of Chloride Ions During the Formation of Akaganeite Revisited", Minerals 5 (2015) 778-787, DOI: 10.3390/ $\min 5040524$.

[17] V. F. Buchwald and R. S. Clarke, Jr., "Corrosion of $\mathrm{Fe}-\mathrm{Ni}$ Alloys by $\mathrm{Cl}$-Containing Akaganéite $(\beta-\mathrm{FeOOH})$ : The Antarctic Meteorite Case", American Mineralogist 74 (1989) 656-667, http://rruff. info/doclib/am/vol74/AM74_656.pdf (accessed 8.07.2017).

[18] D. A. Scott and G. Eggert, Iron and Steel in Art: Corrosion, Colorants, and Conservation, Archetype Publications, London 2009.

[19] A. Sjøgren and V. F. Buchwald, "Hydrogen plasma reactions in a D.C. mode for the conservation of iron meteorites and antiquities", Studies in Conservation 36 (1991) 161-171, DOI: 10.2307/1506422.
[20] I. Kotzamanidi, E. Sarris, P. Vassiliou, C. Kollia, G. D. Kanias, G. J. Varoufakis, S. E. Filippakis, "Effect of heat treatment in reducing plasma environments on chloride ion removal and corrosion of oxidised steel artefacts", British Corrosion Journal 34 (1999) 285-291, DOI: 10.1179/000705999101500987.

[21] S. R. Sutton, "Thermoluminescence measurements on shock-metamorphosed sandstone and dolomite from Meteor Crater, Arizona, 2. Thermoluminescence age of Meteor Crater", Journal of Geophysical Research 90:B5 (1985) 3690-3700.

[22] B. Dajnowski and A. Dajnowski, "The Use of new laser technology to precisely control the level of stone cleaning", in: Science and Art: A Future for Stone (Proceedings of the $13^{\text {th }}$ International Congress on the Deterioration and Conservation of Stone), Paisley, Scotland, September 6-10, 2016, J. J. Hughes and T. Howind (Eds.), University of the West of Scotland, Paisley 2016, pp. 719-728.

[23] J. A. Kowalik, D. B. Williams, J. I. Goldstein, "Formation of the lamellar structure in Group IA and IIID iron meteorites", in: Lunar and Planetary Science Conference, $18^{\text {th }}$, Houston, TX, Mar. 16-20, 1987, Proceedings, Cambridge University Press/Lunar and Planetary Institute, Cambridge and Houston, TX, 1988, pp. 493-501. 


\title{
Laser yellowing effect: study of the nanophases created by laser irradiation of synthetic black crusts using transmission electron microscopy (TEM) and electron paramagnetic resonance (EPR) spectroscopy
}

\author{
Marie Godet ${ }^{1,2,3 *}$, Véronique Vergès-Belmin ${ }^{1}$, Christine Andraud ${ }^{2}$, Mandana \\ Saheb $^{4}$, Judith Monnier ${ }^{5}$, Eric Leroy ${ }^{5}$, Julie Bourgon ${ }^{5}$, Laurent Binet ${ }^{6}$ \\ 1 Laboratoire de Recherche des Monuments Historiques (CRC-LRMH USR 3224), 29 rue de Paris \\ 77420 Champs-sur-Marne, France \\ 2 Centre de Recherche et Conservation des Collections (CRC-CRCC USR 3224), 36 rue Geof- \\ froy-Saint-Hilaire - CP 21, 75005 Paris, France \\ 3 SILLTEC, Domaine Technologique de Saclay, 4 rue René Razel, 91400 Saclay, France \\ 4 Laboratoire Interuniversitaire des Systèmes Atmosphériques (LISA, UMR CNRS 7583) Université \\ Paris-Est Créteil and Université Paris-Diderot, 61 avenue du Général de Gaulle, 94010 Créteil Cedex, \\ France \\ 5 Institut de Chimie et des Matériaux Paris-Est (ICMPE, UMR 7182 CNRS-UPEC), Université \\ Paris-Est, 2/8, rue Henri Dunant, 94320 Thiais, France \\ 6 Institut de Recherche de Chimie ParisTech (IRCP), 11 rue Pierre et Marie Curie, 75005 Paris, \\ France \\ * Corresponding author: msgodet@gmail.com
}

\begin{abstract}
Nd:YAG Q-Switched laser cleaning at $1064 \mathrm{~nm}$ can sometimes lead to a more yellow appearance of the stone surface in comparison with other cleaning techniques. The yellow hue can originate from different contributions among which the presence of nano-sized residues generated by the laser interaction with the surface materials to be eliminated. In this study, the nature of such residues has been investigated. The analyzed materials are (i) particles collected from a pure gypsum reference plate; (ii) a synthetic crust, composed of $80 \mathrm{wt} \%$ natural black crust and $20 \mathrm{wt} \%$ synthetic gypsum; (iii) particles ejected from the synthetic crust during laser irradiation. Optical, scanning electron and transmission electron microscopies were used to link color changes at the macro-scale to morphology changes at the submicron- and nano-scales. Chemical composition was also obtained at the nano-scale using TEM coupled with energy dispersive X-ray (EDX) spectroscopy. This multi-scale approach was combined with electron paramagnetic resonance spectroscopy (EPR) analysis at low and room temperatures to examine the possible presence of iron-containing species presenting particular magnetic properties in the sample before and after irradiation. Under laser irradiation, both the crust sample and the ejected gypsum particles take a yellow color. This color shift can be linked with morphology changes occurring at the nano-scale: gypsum crystals from the reference plate show a smooth surface, while those coming from the synthetic black crust are, after irradiation, covered by many spherical nanoparticles and a rough nano-layer ranging from less than $20 \mathrm{~nm}$ to more than 100
\end{abstract}


$\mathrm{nm}$. TEM-EDX analysis reveals that the two types of nanostructures have a chemical composition very similar to that of iron-rich coal fly-ashes called magnetospheres or ferrospheres. It is suggested that laser irradiation induces a transformation of micrometric size fly-ashes present in the black crust into different types of nanostructures having a chemical composition similar to the one of ferrospheres. EPR analysis demonstrates the presence of ferri-ferro-superparamagnetic species both before and after irradiation, thus indicating that nanosized magnetic iron compounds probably corresponding to magnetite or maghemite oxides are present in the black crust, most probably crystallized on the surface or within the fly-ashes microparticles. This study brings to light the contribution of fly-ashes to the laser induced yellowing process.

Keywords: laser, cleaning, black crust, yellowing, fly-ashes, TEM, EPR

\section{Introduction}

Monuments or sculptures generally get a quite different appearance when cleaned by microsandblasting, water based chemicals or Nd:YAG Q-switched laser at 1064nm. The laser-cleaned objects have generally a darker and sometimes a more yellow appearance. A significant number of investigations have been performed to date on the topic [1]: it is now admitted that the phenomenon may be linked to several causes, among others an unveiling of strongly colored surfaces hidden by black surface layers [2]. Another hypothesis raised in the 2000's links the specific color to the presence of nanometer-size particles [3]. These particles clearly originate from the transformation of phases present in the black crust itself, and this would maybe explain why the laser-linked yellowing phenomenon is not ubiquitous, as the composition of black crusts may substantially change from one place to another.

Furthermore, several causes of yellowing may accumulate in some cases: Vergès-Belmin et al. [4] links for instance the strong yellow color of sculptures at Chartres Cathedral, both to the preservation of a past colored surface treatment and the presence over it of a very shallow layer linked to the laser-substrate interaction, and impossible to put in evidence by ordinary investigation tools such as optical microscopy and SEM-EDX.

The black crust contribution to the yellowing process has been recently further investigated by De Oliveira et al. [5]. Diffuse reflectance spectroscopy coupled with Raman analyses were performed on synthetic black crusts elaborated with powdery deposits originating from the Saint-Denis basilica in France. This monument dates back from the $13^{\text {th }}$ century and is located close to Paris, in an area badly impacted by industrial pollution during the $19-20^{\text {th }}$ centuries. The analytical results suggest that the yellow appearance obtained upon laser irradiation is linked to the preservation of goethite $(\alpha-\mathrm{FeOOH})$ or the formation of a mineral having a similar spectral signature in the visible range.

Although this study and those produced previously by different authors [3,6-8] have established some major clues about the black crust contribution to the yellowing phenomenon, the exact nature of the material generated by laser treatment of black crust and contributing to the yellow color is still unknown. Indeed, the neo-formed objects are present in a very low amount - below the detection threshold of many analytical tools - and are nano-sized: they require, to be characterized, the use of a specific and adequate analytical protocol. 
In this study, we follow a previously published methodology [9] to examine the morphology and the chemical composition of the neo-formed objects produced by irradiation of a synthetic black crust. The strategy consists in a multi-scale approach. Optical microscopy (OM) imaging is first performed to characterize the color of the sample at the macro-scale. Scanning electron microscope (SEM) imaging is carried out in a second step to study the morphology at the submicron-scale and eventually transmission electron microscopy (TEM) coupled with energy dispersive $\mathrm{X}$-ray spectroscopy (EDX) analysis is performed to obtain morphology information at the nano-scale in addition to chemical characterization. Electron paramagnetic resonance (EPR) spectroscopy helps to identify the unknown material by giving information on the nature and size of some components of the black crust presenting particular magnetic properties.

\section{Materials and methods}

\subsection{Materials}

Synthetic crusts are elaborated following the same procedure as the one described in De Oliveira et al. [8] but using a natural black crust collected at the Saint-Denis Basilica instead of hematite. The natural crust is a coherent, black, 5-10 mm thick material. It has a framboidal shape and was collected in
2001 in an area of the monument protected from the rain, and situated ca. 30meters high from the ground. Its composition is presented in the Table 1 (De Oliveira et al. [5]).

\section{Sample preparation}

Crust fragments are first crushed into powder and sieved through a $100 \mu \mathrm{m}$ sieve before being mixed with calcium sulphate hemihydrate $\mathrm{CaSO}_{4} \cdot 0.5 \mathrm{H}_{2} \mathrm{O}$ (ALDRICH $97 \%$ ) in the proportions $80: 20 \mathrm{wt} \%$ (natural crust: hemihydrate). A gypsum plate is then prepared from a mixture of hemihydrate and water poured on a $76 \times 26 \mathrm{~mm}^{2}$ glass slide. The dry mixture hemihydrate/black crust is sprinkled over the fresh and still wet plate through a coarse-meshed sieve (about 1 $\mathrm{mm}$ ). The remaining water in the plate will lead to the crystallization of a coherent black gypsum crust the microstructure of which is very close to real one: a gypsum crystals matrix embedding diverse particles, with an irregular surface and a dark grey color. The crust is then left to dry for 24 hours. Apart from its microstructure, this type of sample is interesting because all the contributions from the substrate (natural stone possibly covered by colored layers) - to the laser yellowing effect are avoided.

\section{Laser irradiation}

The crust samples are then irradiated with a Nd:YAG laser (BM industries). The irradiation conditions have been chosen because

Table 1. Saint-Denis Basilica black crust chemical composition

\begin{tabular}{ccccc}
\hline $\mathrm{C}^{*}\left({ }_{\mathrm{wt}} \%\right)$ & $\mathrm{N}^{*}\left({ }_{\mathrm{wt}} \%\right)$ & $\mathrm{S}^{*}\left(_{\mathrm{wt}} \%\right)$ & $\left.\mathrm{Fe}^{* *}{ }_{\mathrm{wt}} \%\right)$ & $\mathrm{CO}^{3-\star * \star}\left({ }_{\mathrm{wt}} \%\right)$ \\
\hline 2.96 & $<0.01$ & 10.83 & 0.74 & $<1$ \\
\hline
\end{tabular}

${ }^{\star} \mathrm{C}-\mathrm{H}-\mathrm{N}-\mathrm{S}$ elementary analyses, ${ }^{* *}$ inductively coupled plasma atomic emission spectroscopy (ICP-AES), ${ }^{* *}$ differential thermal and thermogravimetric analyses coupled with mass spectroscopy (DTA-TGA-MS) 
they are similar to those used by conservators. The laser is operating at a wavelength of $1064 \mathrm{~nm}$ and produces discrete pulses of laser energy up to $0.4 \mathrm{~J}$ with a pulse length of $15 \times 10^{-9}$ seconds ( $15 \mathrm{~ns}$ ). The pulse is delivered using an articulated mirrored arm and a handpiece equipped with a $70 \mathrm{~cm}$ focal converging lens. The fluence used is slowly increasing from 0.2 to $1 \mathrm{~J} . \mathrm{cm}^{2}$ during the treatment with a frequency of $10 \mathrm{~Hz}$ and a duration of irradiation of three minutes per $\mathrm{cm}^{2}$. In other words, about 1800 pulses per square centimeter are used to clean the samples. The surface of the samples is water sprayed once before irradiation, once after $1 \mathrm{~min} 30 \mathrm{~s}$ and once again after $2 \mathrm{~min}$ $45 \mathrm{~s}$. Each spray corresponds to $80 \mathrm{mg}$ of water over ca $7.5 \mathrm{~cm}^{2}$ (approx. $0.01 \mathrm{~g} / \mathrm{cm}^{2}$ ). The ablated particles are collected both on a clean glass slide $(76 \times 26 \mathrm{~mm})$ and on a round adhesive carbon tab (diameter: 6 $\mathrm{mm}$ ) disposed vertically and close to the samples. The slide and the carbon tab are then stored in an airtight box to prevent contamination.

In order to have a reference material, a white synthetic gypsum plate $76 \times 26 \mathrm{~mm}^{2}$ is elaborated and its surface is gently scraped using a scalpel to collect a little amount of reference gypsum particles which are put on a carbon tape for analysis.

\subsection{Methods}

The morphology and color of the crusts before and after irradiation as well as the ablated particulate materials have first been observed with a Digital Microscope 3D VHX-5000 from KEYENCE Corporation.

Scanning electron microscopy (SEM) is then used to study the morphology of the ablated particles at a micron- and submicron-scale. The SEM observation is per- formed with a SEM-FEG MERLIN at $15 \mathrm{kV}$. The adhesive carbon tabs covered by ablated particles or reference ones are metalized with $4 \mathrm{~nm}$ of palladium/platinum and then put directly into the microscope.

Transmission electron microscopy (TEM) analysis is finally used to identify the morphology and the chemical composition of the ablated particles at a nano-scale. TEM analysis is performed at $200 \mathrm{kV}$ with a FEI TECNAI F20 equipped with a STEM device fitted to an EDX spectrometer EDAX R-TEM Sapphire. The EDX capability is used to determine the chemical compositions of the particles. The samples are prepared by rubbing gently the surface of the glass slide with a copper grid covered with a holey amorphous carbon film. As gypsum is a beam sensitive material a cooler sample holder is being used at liquid nitrogen temperature $\left(-172^{\circ} \mathrm{C}\right)$ in order to limit the sample degradation during the analysis.

Electron paramagnetic resonance spectroscopy (EPR) is used to study the possible presence of paramagnetic defects or ferro/ ferri-magnetic materials in the sample before and after irradiation. Pure synthetic gypsum powder has also been analyzed to serve as a reference. The analyses were conducted at two temperatures: ambient (RT) and low $(10 \mathrm{~K})$ to seek for superparamagnetic effect and thus highlight the presence of superparamagnetic species in the samples. EPR is performed on a Bruker ELEXSYS E500 spectrometer working at X band (9.4 $\mathrm{GHz}$ ) and equipped with a SHQ resonator. A $100 \mathrm{kHz}$ modulation of the magnetic field was used for lock-in detection so that the EPR signal appears as absorption derivatives with respect to the magnetic field. A few milligrams of bulk sample are necessary for the analysis. The EPR spectra are normalized to the mass of the samples. 


\section{Results}

\subsection{Optical microscope}

During laser irradiation the surface of the crust sample becomes yellow and a lot of particles are ablated and get on the glass slide.

In the following paragraphs, we will first describe the natural crust sample itself and then the ablated particles.

\section{The crust}

Before irradiation, various micro-sized particles entrapped in a matrix of white or transparent crystals that we interpret as being gypsum are observed on the crust surface (see Fig. 1). These micro-particles are black to yellow and red ochre colored, shapes either rod-like, angular, round or botryoidal and sizes ranging from less than $10 \mu \mathrm{m}$ to more than $50 \mu \mathrm{m}$. The size of the gypsum crystals ranges from a few microns to a few hundred microns with a form of rods, platelets or more irregular sub angular aggregates.

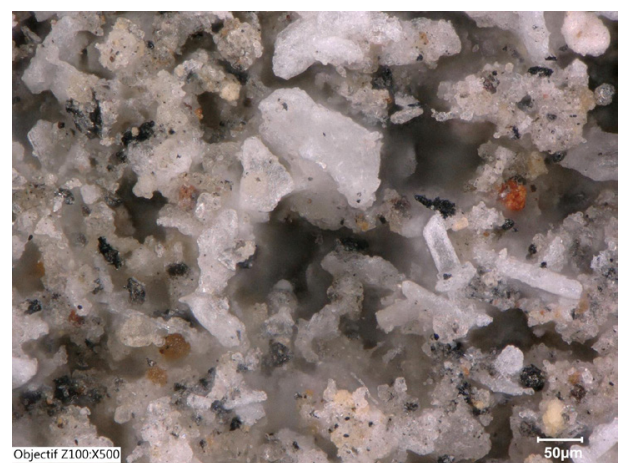

Fig. 1. Optical micrograph of a synthetic black crust before irradiation.

After laser irradiation, a strong yellow discoloration is noticed on the crust surface (see Fig. 2). A large proportion of it has been ablated and the remaining material shows a clearly yellow color. The same micro-sized

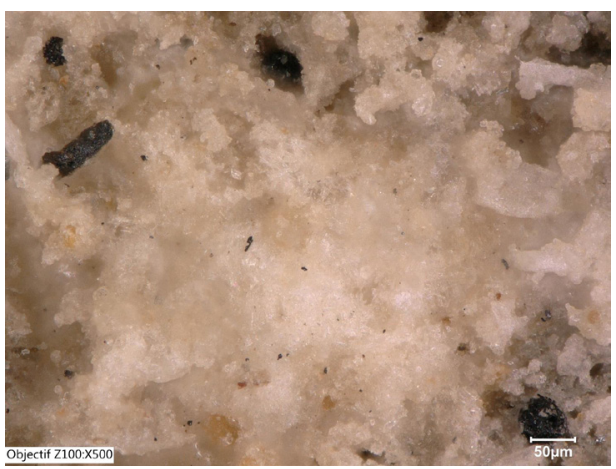

Fig. 2. Optical micrograph of a synthetic black crust after irradiation.

particles as those described before irradiation are still visible but in a smaller amount.

\section{The ablated particles}

The reference gypsum crystals take on the form of white transparent rods or platelets ranging from less than 10 microns to more than a few hundred microns; they often form irregular aggregates measuring a few tens to a few hundred of microns (see Fig. 3).

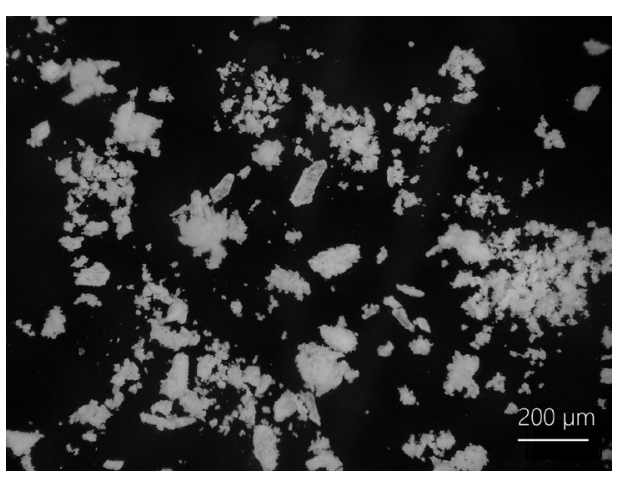

Fig. 3. Optical micrograph of gypsum crystals originating from a synthetic white gypsum plate.

The observation of the laser ablated particles reveals the presence of gypsum crystals having the same yellow color and the same sizes and shapes as the ones of the 
crust they originate from. We can also notice the presence of the same various micro-sized particles as the ones already observed in the natural crust, before and after irradiation (see Fig. 4 and Fig. 5). Though the shapes and sizes of these ablated crystals are similar to the ones of pure gypsum crystals, the color is not the same as we observe yellow ablated crystals whereas the pure crystals are white.

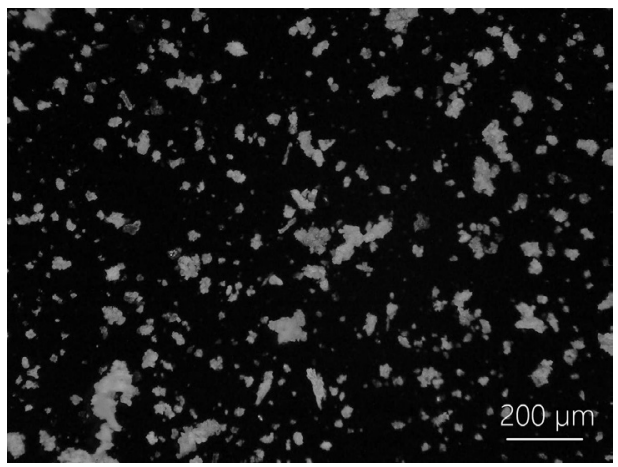

Fig. 4. Optical micrograph of ablated particles during irradiation of synthetic black crust.

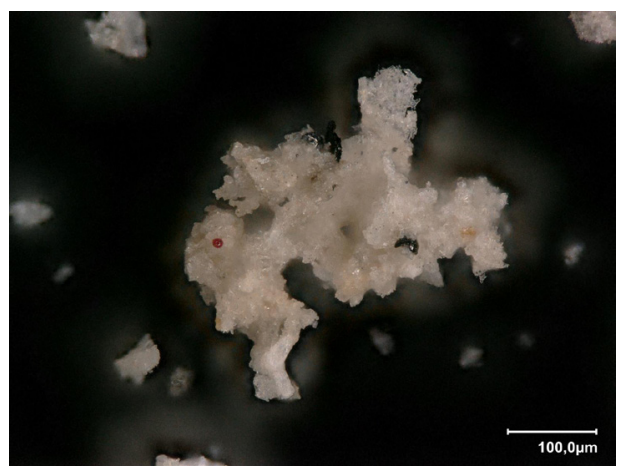

Fig. 5. Optical micrograph of ablated yellow gypsum crystals aggregate and various colored microparticles.

\subsection{Scanning electron microscopy}

SEM observation was conducted on non-irradiated reference gypsum crystals (see Fig.6) and on the ablated particles (see
Fig. 7) in order to determine the morphology differences induced by laser irradiation at the submicron-scale.

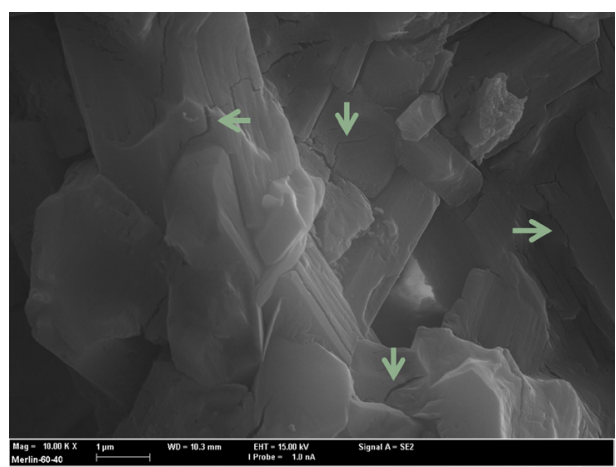

Fig. 6. SEM-SE picture of non-irradiated reference gypsum crystals; green arrow: cracks induced by the electron beam.

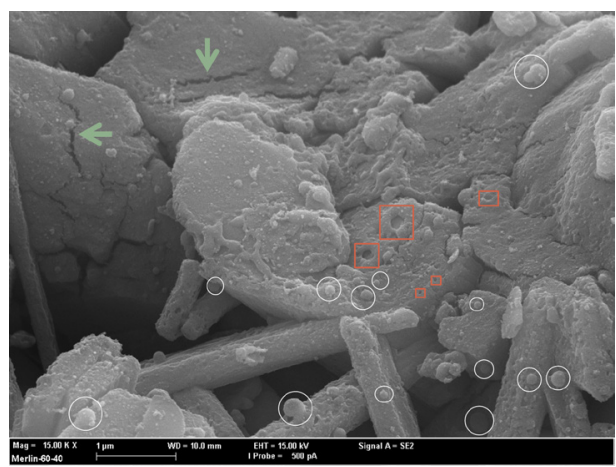

Fig. 7. SEM-SE picture of ablated gypsum crystals after laser irradiation; white circle: spherical nanoparticles; red square: nano-cavities; green arrow: cracks.

\section{Non-irradiated reference gypsum crystals}

The reference gypsum crystals take on the form of smooth rods and platelets entangled together. Their size is a few microns. The cracks observed in some of those crystals are an artifact induced by the electron beam.

\section{The irradiated ablated particles}

After laser irradiation, the surface of the ablated crystals looks significantly different: 
the crystals appear covered by spherical nanoparticles (see Fig. 7) presenting a huge range of sizes from less than a few tens of nanometers to a few hundred nanometers. In addition, the surface of the crystals is not smooth as the reference one but it presents a rough nano-layer. Nano-cavities can be observed at the surface of some crystals. Micro-cavities have also been observed in other areas. The cracks observed in some crystals are again an artifact induced by the beam.

\subsection{Transmission electron microscopy}

TEM analysis was conducted to explore the morphology of the ablated crystals at the nano-scale. Gypsum crystals are irregularly covered by spherical nanoparticles of various sizes ranging from less than $10 \mathrm{~nm}$ to more than $200 \mathrm{~nm}$. A large part of the gypsum crystals presents an irregular surface at the nanoscale (see Fig. 8 and Fig. 9).

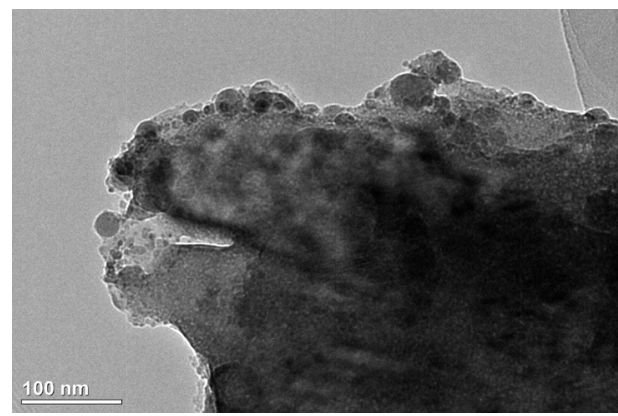

Fig. 8. TEM-BF picture of a surface of an ablated gypsum crystal presenting a rough nanolayer and covered by nanoparticles.

A large amount of isolated nano-spheres is also found on the copper grid. They have probably been detached from the gypsum rods or platelets when we rubbed the copper grid on the glass slide surface. Some nanospheres show a porous morphology with nano-pores measuring less than $50 \mathrm{~nm}$ (see Fig. 10).

The chemical composition of the nanostructures of interest was determined using EDX. All the nanoparticles present similar

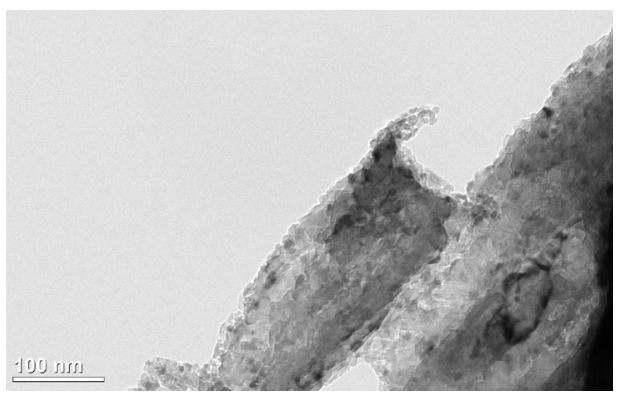

Fig. 9. TEM-BF picture of a surface of an ablated gypsum crystal presenting a rough nanolayer.

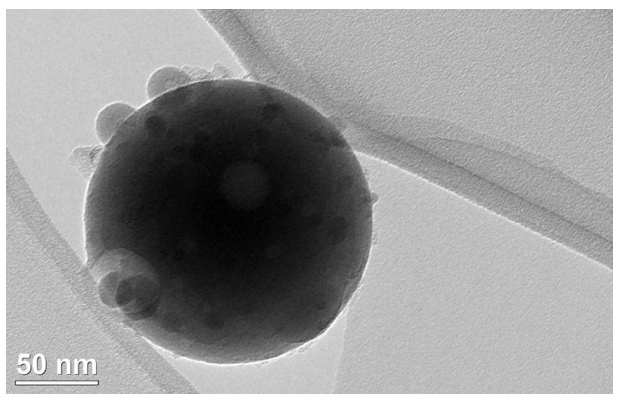

Fig. 10. TEM-BF picture of an isolated and porous nanosphere covered by smaller nanospheres.

compositions with different proportions of each element: a majority of oxygen, silicon, aluminum and iron, and various small amounts of magnesium, potassium, titanium, calcium, sulfur, phosphorus. The rough nanolayer has the same chemical composition (see Tab. 2).

Table 2. Chemical composition of the nanostructures observed on the ablated crystals after irradiation

\begin{tabular}{ll}
\hline Major elements & $\mathrm{O}, \mathrm{Al}, \mathrm{Si}, \mathrm{Fe}$, sometimes $\mathrm{Ca}$ \\
\hline Minor elements & $\mathrm{Mg}, \mathrm{K}, \mathrm{Ti}, \mathrm{Ca}, \mathrm{S}, \mathrm{P}$ \\
\hline
\end{tabular}




\subsection{Electron paramagnetic resonance spectroscopy (EPR)}

Electron paramagnetic resonance spectroscopy (EPR) has been conducted to study the possible presence of paramagnetic defects or ferro/ferri-magnetic materials in the black crust before and after irradiation. The reference gypsum has no EPR signal meaning that there are no paramagnetic defects or ferro/ferri-magnetic materials in the gypsum crystals.
At $10 \mathrm{~K}$, the intensity of the resonance at $\mathrm{g}=2.006$ corresponding to the carbonaceous matter species is much higher than at RT. We note the apparition of a resonance at $\mathrm{g}=4.27$, assigned to isolated $\mathrm{Fe}^{3+}$ in low symmetry sites. The resonance line at $\mathrm{g}=2.16$ has become larger due to the temperature change. In addition, we can notice a shift of this line towards low magnetic field values (see Fig. 12). This broadening and shift of the resonance are characteristic of ferri/ ferro-superparamagnetic materials [12].

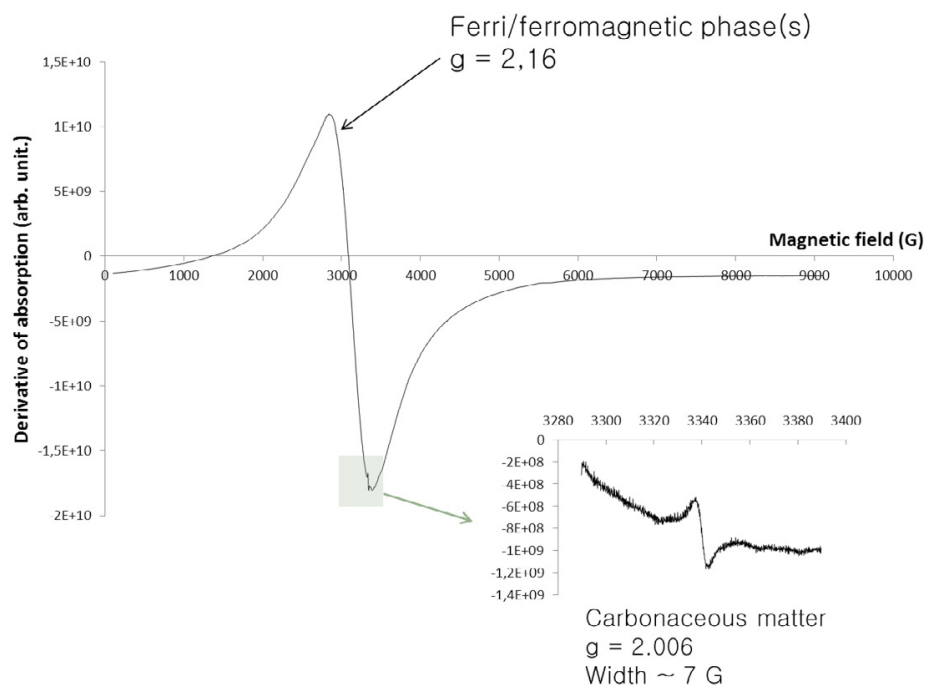

Fig. 11. EPR spectrum of the non-irradiated synthetic black crust at Room Temperature (RT).
Before laser irradiation, we can notice the presence of two main lines on the room temperature EPR spectra of black crust at $g$ $=2.16$ and $g=2.006$ (see Fig. 11). The first consists of a large resonance line given by the presence of ferri/ferromagnetic material corresponding to $\mathrm{Fe}^{3+}$ ions disposed in randomly oriented iron oxides such as magnetite-maghemite particles [10]. The second line at $g=2.006$ has a width of about $7 \mathrm{G}$ and thus it highlights the presence of carbonaceous matter within the black crust [11].
After laser irradiation, the EPR spectrum at RT shows one main line at $\mathrm{g}=2.18$ corresponding to the presence of ferri/ferromagnetic material. The intensity of the resonance is lower than the one of the non-irradiated black crust meaning that there is a lower amount of the ferri/ferromagnetic material after laser treatment. The resonance at $g=2.006$ linked with the presence of carbonaceous matter is no longer visible (see Fig. 13).

At $10 \mathrm{~K}$, we notice the resonance at $\mathrm{g}=$ 4.27 corresponding to isolated $\mathrm{Fe}^{3+}$ in low 

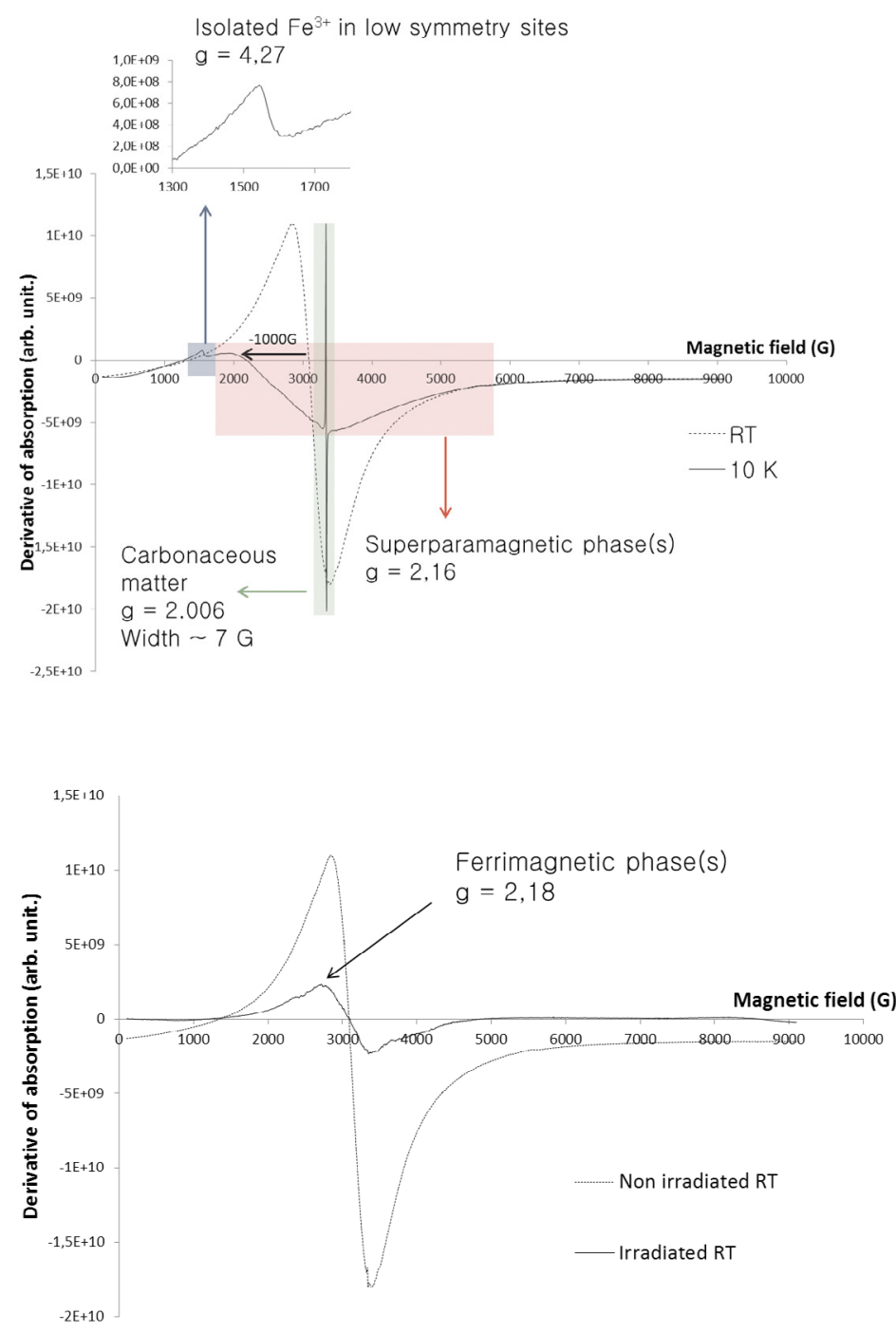

Fig. 12. EPR spectra of the non-irradiated synthetic black crust at RT and $10 \mathrm{~K}$.
Fig. 13. EPR spectra of the irradiated synthetic black crust at RT (in comparison with non-irradiated black crust). symmetry sites, as already seen for the non-irradiated black crust. The resonance line at $\mathrm{g}=2.18$ has also become larger due to the temperature change and we can notice a shift of the line towards low magnetic field values. This broadening and shift of the resonance ascertain the presence of ferri/ ferro-superparamagnetic material within the laser irradiated natural crust. One other line is apparent at $\mathrm{g}=2.006$ corresponding to carbonaceous matter already described in the non-irradiated crust (see Fig. 14).

If we compare the non-irradiated black crust to its irradiated counterpart at $10 \mathrm{~K}$, we can see that the intensities of the resonances corresponding to the ferri/ferromagnetic 


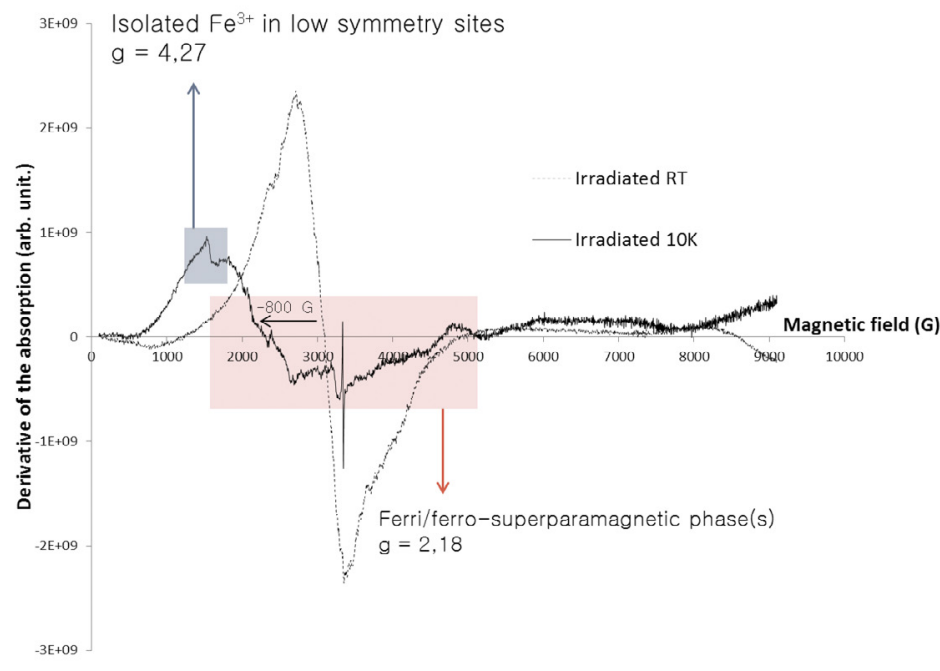

Fig. 14. EPR spectra of the irradiated synthetic black crust at RT and $10 \mathrm{~K}$.

phases $(\mathrm{g}=2.18)$ and to the carbonaceous matter $(\mathrm{g}=2.006)$ have decreased significantly during irradiation (see Fig. 15).

In conclusion, EPR analysis has highlighted the presence of superparamagnetic iron oxides, $\mathrm{Fe}^{3+}$ ion in low symmetry sites and carbonaceous matter in Saint-Denis black crust. After laser irradiation, all three are still present but in lower quantities.

\section{Discussion}

Particles such as those found in the Saint-Denis crust are commonly found in black crusts and have been extensively studied [13-20]: fly-ashes, iron oxides, organic materials, carbonaceous particles, clay minerals, oxalates etc. may all be part of a black crust. Iron compounds are of par-

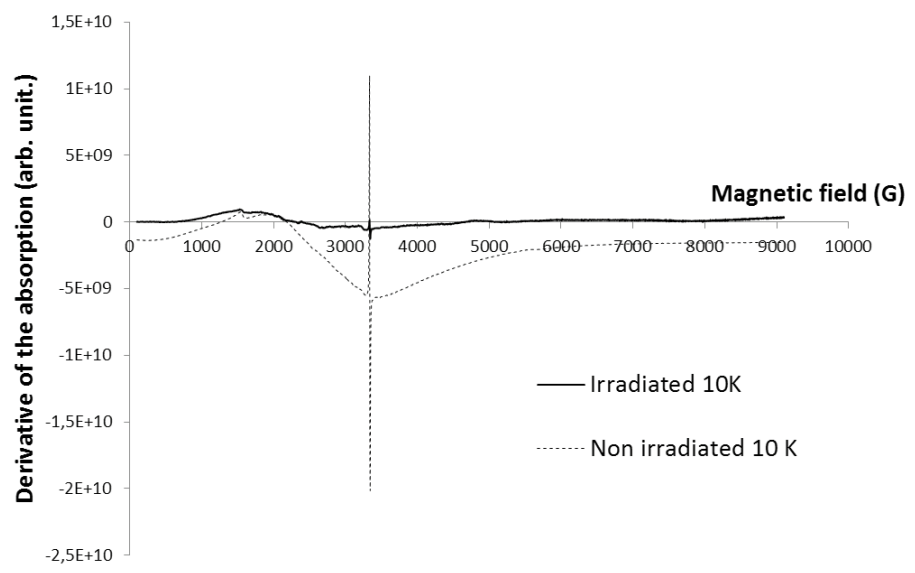

Fig. 15. EPR spectra of the irradiated synthetic black crust at $10 \mathrm{~K}$ (in comparison with non-irradiated black crust). 
ticular interest as they are known to strongly react with the laser beam [21]. They were found to be present in black crusts in the form of hematite $\alpha-\mathrm{Fe}_{2} \mathrm{O}_{3}[5,22]$, goethite a-FeOOH [5], magnetite $\mathrm{Fe}_{3} \mathrm{O}_{4}$ [23], ferrihydrite $\alpha-\mathrm{Fe}_{2} \mathrm{O}_{3} \cdot 1.8 \mathrm{H}_{2} \mathrm{O}$ and jarosite $\mathrm{KFe}_{3}\left(\mathrm{SO}_{4}\right)$ $(\mathrm{OH})_{6}$ [24]. In black crusts, iron is always less than a few weight percent $[20,25,26]$, and in our case, the iron oxide content is $0.74 \mathrm{wt} \%$ [5]. The EPR analysis performed in this study confirms the presence of iron in the Saint Denis crust. It also reveals the existence of isolated $\mathrm{Fe}^{3+}$ in low symmetry sites in addition to ferri or ferro-superparamagnetic species that we interpret as nano-sized - usually smaller than $50 \mathrm{~nm}$ according to Griscom et al. [10] - magnetite $\mathrm{Fe}_{3} \mathrm{O}_{4}$ and/or maghemite $\gamma-\mathrm{Fe}_{2} \mathrm{O}_{3}$. These results are in line with a Mössbauer study by Steger [23] showing that the iron contained in a black crust is partly localized in particles of magnetite $\mathrm{Fe}_{3} \mathrm{O}_{4}$ and partly in a poorly crystallized $\mathrm{Fe}^{3+}$ mineral.

Once the synthetic black crust is irradiated, optical microscopy reveals that the gypsum crystals either at the surface of the crust or ejected from it have turned from white to yellow. This color shift can be correlated with morphology changes observed by SEM on the laser ablated particles: gypsum crystals from the reference plate show a smooth surface, while those coming from the synthetic black crust are, after irradiation, covered by spherical nanoparticles and a rough nano-layer. These observations are very similar to those already made on laser irradiated model crust samples $[3,8,9]$. Actually, the first studies on the interactions between the laser beam and black crust have begun with simplified model crust samples as natural black crusts were considered as too heterogeneous and complex. In their study on laser yellowing, Klein et al.
[3] detected spherical nanoparticles after laser irradiation at the surface of a ternary model crust containing hematite $\alpha-\mathrm{Fe}_{2} \mathrm{O}_{3}$, graphite and gypsum. More recent studies focused on binary model crusts containing only hematite $\alpha-\mathrm{Fe}_{2} \mathrm{O}_{3}$ and gypsum have put in evidence that both the model crusts substrate and the laser ablated material were covered, after irradiation, by spherical nanoparticles and by a rough nano-layer $[8$, 9]. The laser irradiation thus appears to have an analogous effect on the morphology a nano-structuration of the gypsum crystals surface - whether the crystals come from a model or from a natural crust.

TEM-EDX was used in this study, both to image the ablated material at the nano-scale and to analyze its chemical composition on an area of a few nanometers. It is to be noticed that the extremely small size of the neo-formed phases on our samples would not have allowed a proper SEM-EDX anal$y$ sis, since the interaction volume of the electron probe is in the micrometer range for this technique. TEM imaging confirms the presence of spherical nanoparticles of various sizes, and of a rough nano-layer or nano-residues which can be observed on the surface of several gypsum crystals. TEM-EDX analysis reveals that all the nanostructures predominantly contain $\mathrm{O}$, $\mathrm{Si}, \mathrm{Al}, \mathrm{Fe}$ and little amounts of $\mathrm{Mg}, \mathrm{K}, \mathrm{Ti}, \mathrm{Ca}$, $\mathrm{S}, \mathrm{P}$ etc. This composition is very similar to the one of glassy fly-ashes originating from coal combustion plants $[13,14,17,27,28]$, being described as $2-40 \mu \mathrm{m}$ glassy aluminosilicate spheres containing $\mathrm{Mg}, \mathrm{Fe}, \mathrm{Ti}, \mathrm{K}$ as other elements $[19,27]$. More specifically, the nanostructures of interest in the present study have a composition close to that of the so-called magnetospheres or ferrospheres, a particular type of coal fly ashes which are magnetic and enriched in iron [29]. 
An important point to note at this stage is that fly-ashes are essentially defined as micro-sized particles [30]. Seames [31] and Buhre et al. [32] have shown that the PM1 ultra fine fraction of fly-ashes - measuring less than $1 \mu \mathrm{m}$ diameter - represents less than $1_{\text {wt }} \%$ of the total amount of ashes generated by the coal combustion. Yet the TEM analysis we conducted reveals the presence of a large amount of nano-sized fly-ashes covering the ablated gypsum particles. These nanoparticles may have been generated by the interaction between the laser beam and the micrometer-sized fly-ashes present in the black crust before irradiation. This hypothesis is supported by the SEM observation of round cavities at the surface of the gypsum crystals. These micro- and nano-cavities may be the result of the interaction of fly-ashes with the laser beam. A characterization of the natural crust at the nano-scale would thus be useful to check if nanometric fly-ashes type particles are already present before irradiation.

Anyhow, this is the first time that a link between the yellowing phenomenon and the fly-ashes present in black crusts is highlighted. It is not that surprising as fly-ashes contain iron species which are known to contribute to the yellowing effect. Our results also show that the laser-generated nanostructures have a different chemical composition if the irradiated material is mainly a real black crust material as in the present study, or is a model composed of a mixture of hematite and gypsum as in former studies [9]. In the case of model crusts, the nanostructures are found to contain $\mathrm{O}, \mathrm{Fe}, \mathrm{Ca}, \mathrm{S}$ in various proportions whereas for the synthetic black crust, the nanostructures predominantly contain $\mathrm{Si}, \mathrm{Al}, \mathrm{O}, \mathrm{Fe}$.

This seems to indicate that the phases generated during laser irradiation are different depending on if the irradiated material contains iron oxides or fly-ashes. However, in both cases a yellow color is produced at the macro-scale.

\section{Conclusion}

A specific multi-scale analytical methodology has enabled us to reveal for the first time the presence of two types of nanostructures at the surface of laser ablated yellow gypsum crystals - nanospheres and a rough nanolayer - the composition of which is very similar to that of iron rich coal fly-ashes (the so-called magnetospheres or ferrospheres) present in black crusts before irradiation. Our results suggest that laser irradiation induces a transformation of micro-sized fly ashes into nanostructures, having the same chemical composition.

EPR analysis demonstrates the presence of nano-sized magnetic iron compounds $(<50$ $\mathrm{nm}$ ) probably corresponding to magnetite or maghemite in the black crust, the same species being also present - in a lower amount - after irradiation. Such phases would play a key role in the reactivity of fly ashes to laser irradiation, as these species are known to strongly absorb the laser at $1064 \mathrm{~nm}$.

In any event, it is the first time that a contribution from fly-ashes to the laser yellowing effect is highlighted, opening the door for future investigations. De facto further study is currently conducted on new model crusts containing only fly-ashes and gypsum.

\section{References}

[1] P. Pouli, M. Oujja, M. Castillejo, "Practical issues in laser cleaning of stone and painted artefacts: optimisation procedures and side effects", Applied Physics A., 106 (2012) 447-464, DOI: 10.1007/ s00339-011-6696-2. 
[2] V. Vergès-Belmin, C. Dignard, "Laser yellowing: myth or reality?", Journal of Cultural Heritage, 4 (2003) 238-244.

[3] S. Klein, F. Fekrsanati, J. Hildenhagen, K. Dickmann, H. Uphoff, Y. Marakis, V. Zafiropulos, "Discoloration of marble during laser cleaning by Nd:YAG laser wavelengths", Applied Surface Science, 171 (2001) 242-251.

[4] V. Vergès-Belmin, C. De Oliveira, O. Rolland, "Investigations on yellowing as an effect of laser cleaning at Chartres Cathedral, France", in: ICOM-CC $17^{\text {th }}$ Triennial Conference Preprints, J. Bridgland, Melbourne, 2014.

[5] C. De Oliveira, V. Vergès-Belmin, J. Lafait, M. Swider, C. Andraud, A. Tournié, L. Galoisy, "Contribution of goethite to laser-induced stone yellowing", Applied Physics A., 122 (2016), DOI: 10.1007/s00339-016-9818-z.

[6] M. Gavino, B. Hermosin, V. Vergès-Belmin, W. Nowik, C. Saiz-Jimenez, "New insights on the chemical nature of stone yellowing produced after laser cleaning", in: Cultural Heritage Conservation and Environmental Impact Assessment by Non-Destructive Testing and Micro-Analysis, Van Grieken \& Janssens, Antwerp, 2005: pp. 149-157.

[7] P. Pouli, C. Fotakis, B. Hermosin, C. Saiz-Jimenez, C. Domingo, M. Oujja, M. Castillejo, "The laser-induced discoloration of stonework; a comparative study on its origins and remedies", Spectrochimica Acta Part A., 71 (2008) 932-945.

[8] C. De Oliveira, V. Vergès-Belmin, D. Demaille, P. Bromblet, "Lamp black and hematite contribution to laser yellowing: A study on technical gypsum samples", Studies in Conservation, (2015), DOI: 10.1179/2047058415Y.0000000003.

[9] M. Godet, V. Vergès-Belmin, C. Andraud, M. Saheb, J. Monnier, E. Leroy, J. Bourgon, "Laser yellowing of hematite-gypsum mixtures: a multi scale characterization", in: Science and Art: A Future for Stone, John Hughes \& Howind Torsten, Paisley 2016, pp. 785-792.

[10] D.L. Griscom, "Ferromagnetic resonance of precipitated phases in natural glasses", Journal of Non-Crystalline Solids, 67 (1984) 81-118.
[11] A. Barbon, M. Brustolon, "An EPR study on Nanographites", Applied Magnetic Resonance, 42 (2012) 197-210, DOI: 10.1007/s00723-011-0285-6.

[12] R. Berger, J.-C. Bissey, J. Kliava, H. Daubric, C. Estournès, "Temperature dependence of superparamagnetic resonance of iron oxides nanoparticles", Journal of Magnetism and Magnetic Materials, 234 (2001) 535-544.

[13] M. Del Monte, C. Sabbioni, O. Vittori, "Airborne carbon particles and marble deterioration", Atmospheric Environment, 15 (1981) 645-652.

[14] C. Sabbioni, "Contribution of atmospheric deposition to the formation of damage layers", The Science of the Total Environment, 167 (1995) 49-55.

[15] A.G. Nord, A. Svardh, K. Tronner, "Air pollution levels reflected in deposits on building stone", Atmospheric Environment, 28 (1994) 2615-2622.

[16] C. Rodriguez-Navarro, E. Sebastian, "Role of particulate matter from vehicle exhaust on porous building stones (limestone) sulfation", The Science of the Total Environment, 187 (1996) 79-91.

[17] P. Ausset, M. Del Monte, R. Lefèvre, "Embryonic sulphated black crusts on carbonate rocks in atmospheric simulation chamber and in the field: role of carbonaceous fly-ash", Atmospheric Environment, 33 (1999) 1525-1534.

[18] A. Bonazza, C. Sabbioni, N. Ghedini, "Quantitative data on carbon fractions in interpretation of black crusts and soiling on European built heritage", Atmospheric Environment, 39 (2005) 2607-2618.

[19] M. Urosevic, A. Yebra-Rodriguez, E. Sebastian, C. Cardell, "Black soiling of an architectural limestone during two-year term exposure to urban air in the city of Granada (Spain)", The Science of the Total Environment, 414 (2012) 564-575, DOI: 10.1016/j. scitotenv.2011.11.028.

[20] S.A. Ruffolo, V. Comite, M.F. La Russa, C.M. Belfiore, D. Barca, A. Bonazza, G.M. Crisci, A. Pezzino, C. Sabbioni, "An analysis of the black crusts from the Seville Cathedral: A challenge to deepen the understanding of the relationships among microstructure, microchemical features and pollution sources", Science of the Total Environment, 502 (2015) 157-166, DOI: 10.1016/j.scitotenv.2014.09.023. 
[21] S. Siano, F. Fabiani, R. Pini, R. Salimbeni, M. Giamello, G. Sabatini, "Determination of damage thresholds to prevent side effects in laser cleaning of pliocene sandstone of Siena", Journal of Cultural Heritage, 1 (2000) 47-53.

[22] S.S. Potgieter-Vermaak, R.H.M. Godoi, R.V. Grieken, J.H. Potgieter, M. Oujja, M. Castillejo, "Micro-structural characterization of black crust and laser cleaning of building stones by micro-Raman and SEM techniques", Spectrochimica Acta Part A: Molecular and Biomolecular Spectroscopy, 61 (2005) 2460-2467, DOI: 10.1016/j.saa.2004.09.010.

[23] W.E. Steger, H. Mehner, "The Iron in Black Weathering Crusts on Saxonian Sandstones Investigated by Mössbauer Spectroscopy", Studies in Conservation, 43 (1998) 49, DOI: 10.2307/1506636.

[24] A.G. Nord, T. Ericsson, "Chemical analysis of the black layers on building stone", Studies in Conservation, 38 (1993) 25-35.

[25] V. Fassina, L. Lazzarini, G. Biscontin, "Effects of atmospheric pollutants on the composition of black crust deposited on venetian marbles and stones", in: Athens, 1976, pp. 201-210.

[26] P. Maravelaki-Kalaitzaki, "Black crusts and patinas on Pentelic marble from the Parthenon and Erechtheum (Acropolis, Athens): characterization and origin", Analytica Chimica Acta, 532 (2005) 187-198, DOI: 10.1016/j.aca.2004.10.065.

[27] P. Ausset, F. Bannery, M. Del Monte, R. Lefèvre, "Recording of pre-industrial atmospheric environment by ancient crusts on stone monuments", Atmospheric Environment, 32 (1998) 2859-2863.

[28] K. Desboeufs, A. Sofikitis, R. Losno, J.-L. Colin, P. Ausset, "Dissolution and solubility of trace metals from natural and anthropogenic aerosol particulate matter", Chemosphere, 58 (2005) 195-203.

[29] R.J. Lauf, "Characterization of the mineralogy and microchemistry of fly ash", Nuclear and Chemical Waste Management, 5 (1985) 231-236.

[30] Q. Xue, S. Lu, "Microstructure of ferrospheres in fly ashes: SEM, EDX and ESEM analysis", Journal of Zhejiang University-SCIENCE A., 9 (2008) 1595-1600, DOI: 10.1631/jzus.A0820051.

[31] W.S. Seames, "An initial study of the fine fragmentation fly ash particle mode generated during pulverized coal combustion", Fuel Processing Technology, 81 (2003) 109-125, DOI: 10.1016/ S0378-3820(03)00006-7.

[32] B. Buhre, J. Hinkley, R. Gupta, P. Nelson, T. Wall, "Fine ash formation during combustion of pulverised coal-coal property impacts”, Fuel, 85 (2006) 185-193. DOI: $10.1016 /$ j.fuel.2005.04.031. 


\title{
Laser Cleaning of a First Phase Navajo Wool Chief's Blanket by Nd:YAG
}

\author{
Pablo Londero ${ }^{1 \star}$, Ludovica Corda ${ }^{2}$, Daniele Ciofini $^{3}$, Christine Giuntini $^{4}$, \\ Marco Leona ${ }^{4}$
}

1 Institute for the Preservation of Cultural Heritage, Yale University, 300 Heffernan Rd., West Haven, CT 06516

2 Conservator in private practice, 315 King street, Apt. 5H, Port Chester, NY 10573

3 Institute of Applied Physics "Nello Carrara" IFAC, Via Madonna del Piano 10, 50019 Sesto Fiorentino, Florence, Italy

4 The Metropolitan Museum of Art, 1000 Fifth Avenue, New York, NY 10028

* Corresponding author: pablo.londero@yale.edu

\begin{abstract}
A case study was performed for laser cleaning of a soiled First Phase Navajo chief's blanket using the first and second harmonics of a Nd:YAG laser. A cleaning protocol was developed by mapping the impact of wavelength, fluence, pulse number, and surface pre-wetting on churro wool reference samples. Afterward, an approach for applying the protocol to the Navajo blanket was developed, including a number of custom jigs for mounting the object under controlled tension. The most effective laser cleaning parameters were determined to be a wavelength of $532 \mathrm{~nm}$, a fluence of $350 \mathrm{~mJ} / \mathrm{cm}^{2}$, approximately 100 pulses per cross-sectional area of the laser beam, and no pre-wetting. Good results with no observable damage were obtained with $\sim 280$ hours of laser cleaning for the entire blanket, which measured $138 \mathrm{~cm}$ by $208 \mathrm{~cm}$.
\end{abstract}

Keywords: laser cleaning, Navajo, wool, Nd:YAG

\section{Introduction}

Laser cleaning is increasingly used to remove unwanted contamination from the surfaces of historical materials, and has drawn significant interest for such applications on natural and man-made polymers [1-10]. Much of this work involves treatments on paper, such as the abatement of foxing, mold, and soiling [3, $5,6,8]$. Increased attention has been paid to the treatment of textiles woven from natural fibers, and a number of interesting results show the potential as well as limitations of laser cleaning, particularly for the removal of fine soiling and carbonaceous deposits [ 1 , $2,9,10]$.

Studies have shown that 1064 and 532 $\mathrm{nm}$ laser wavelengths, being less absorbed by most natural fibers, are more selective than UV irradiation in removing soiling. UV laser wavelengths can interact strongly with natural fibrous materials, either of vegetal or animal origin, thus resulting to be ineffective and potentially dangerous toward 
the structural integrity of fibers itself [2]. The side effects of this photo-damage are mainly described in terms of discoloration (i.e. yellowing or bleaching) and can be more easily controlled at longer wavelengths, where the interaction is lessened. At $1064 \mathrm{~nm}$ and $532 \mathrm{~nm}$, the laser light often interacts strongly with most inorganic environmental contaminants and weakly with the fiber itself, especially when the latter is not dyed or pigmented. The pollution particles tend to be broadband (black or brown) absorbers with thermal times scales on the order of hundreds of nanoseconds to microseconds [11], and are thus also well-suited to the use of Nd:YAG lasers.

As with many art conservation problems, a challenge for compiling useful data comes from the limitless variety of fabrics that can be created by combining different fibers, yarns and woven structures. A change in any one of these parameters can affect the propagation of laser light, ejected particulates, and any resulting damage to the fabric itself. Furthermore, since these materials involve many different polymers with various aging kinetics, the long-term impact of the treatment can also potentially vary. Additional thorough studies of well-selected fabrics and reference materials are needed in order to build toward the level of knowledge and intuition that already exists for the laser cleaning of materials such as stone and metal. Therefore, the following case study, which purpose-woven churro wool reference material in order to develop safe laser cleaning parameters for cleaning an historical churro wool Navajo blanket, provides a valuable example of laser cleaning on a class of materials of significant historical importance.

Wool harvested from churro sheep gradually became an important textile fiber throughout the American Southwest from the $17^{\text {th }}$ through the early $19^{\text {th }}$ centuries. Purposefully selected for their hardiness in the arid climate of New Spain by the Colonial authorities, the Iberian breed was imported into the Rio Grande valley in the late $16^{\text {th }}$ century. Their long staple fiber and clean fleece was ideally suited to the minimal hand-processing methods practiced by both the Spanish colonists and the Pueblo peoples. After the Pueblo revolt of 1680 , flocks of churro sheep were acquired by other Native American tribes including the Navajo [12].

This work focuses on a case study of laser cleaning of for a privately-owned First Phase Navajo Chief's Blanket mad of churro wool. Cleaning of artificially soiled (hereafter, "sooted") woven churro wool reference material by Nd:YAG was studied under varying parameters including wavelength, fluence, pulse number, and surface pre-wetting. Colorimetric and tensile properties were characterized before and after cleaning, with and without accelerated aging. The resulting data was used to develop a cleaning protocol for the Chief's Blanket. Once the treatment of the historical Navajo weaving was underway, additional techniques were developed as the protocol was adapted to the object.

\section{Materials and Methods}

\subsection{Case Study Description}

The historical object of concern is a privately owned First Phase Navajo Chief Blanket, which was brought to the Department of Scientific Research (DSR) at the Metropolitan Museum of Art (MMA) to investigate a particularly disturbing discoloration. The textile appeared to have been "home-cleaned" by a previous owner, and the original creamy white churro wool now exhibited a dingy 
mottled gray surface, punctuated by irregular splotches of bright white wool. According to the owner, a specialist restorer of traditional southwestern textiles tried to reduce the gray color, but the deeply ingrained soil was resistant to all the currently acceptable cleaning formulas used on this type of historic textile. Instrumental analysis indicated that the soiling was likely due to a carbonaceous deposit. This fact suggested that laser cleaning would be a possible treatment option if it could be demonstrated that the wool substrate would not be damaged.

Our tests focused on laser cleaning at 1064 and $532 \mathrm{~nm}$ with a Quanta System Thunder art High Power Q-switched Nd:YAG Laser for several reasons. The Metropolitan Museum owns such a laser system, which supports 1064, 532 and 355 $\mathrm{nm}$ outputs. However, the $355 \mathrm{~nm}$ output was not deemed to be worth testing as it would be too destructive to the wool substrate at the necessary fluences [2].

\subsection{Reference Sample Preparation}

In order to test the effect of the laser removal on carbonaceous deposits in churro wool, a custom-woven, weft-face churro wool fabric from sheep raised in the Southwest was selected as reference material. A portion of the woven fabric was cut into 12 pieces measuring approximately $15 \mathrm{~cm}$ by $15 \mathrm{~cm}$, and the edges were singed to prevent fraying during testing.

Afterward, laser cleaning experiments were planned according to two distinct application procedures of the artificial sooting material. The first of these involved samples that were sooted very evenly but not as deeply into the weave, the second one, samples that had fine carbon ink soaked into them. While this method placed the soot more unevenly, it also mimicked the Navajo blanket more accurately.

Evenly sooted samples were prepared using lab-made, water-based ink. A custom ink allowed us to apply an ink of pure soot, without the traditional stabilizers such as gum Arabic. $11.5 \mathrm{~mL}$ of DDI water and 125 mg of Kremer Furnace Black were combined in a stoppered glass vial. The vial was then placed in a sonication for four iterations 30 s each, with 10s of vigorous shaking after each sonication. Afterward, a pipet was inserted $\sim 2 / 3$ deep into the volume of ink, and 50\% of the contents were pipetted into a second clean glass vial. The second vial was sonicated and shaken twice as just described, and the contents were then pipetted into the inkwell of an airbrush for application. The ink was sprayed evenly until the reflectance was reduced by $50 \%$. Colorimetric measurements were performed across each sample to ensure $<3 \%$ variation in application. Four additional pieces of test material, $14 \mathrm{~cm}$ by $27 \mathrm{~cm}$, were prepared in the same fashion: the top and lower quarter of the textile were masked and the ink was applied only to the center portion (see Fig. 1a), so that cleaning tests at a given set of parameters could be performed side by side on unsoiled and sooted areas.

Deeply sooted samples were prepared using india ink (Fig. 1b). The ink was sprayed onto the reference wool, then pressed and smeared manually while wearing nitrile gloves. This process was repeated until a relatively even tone was achieved for the sooting throughout the reference samples.

\subsection{Laser Treatments}

Irradiation tests were performed using the fundamental radiation $(1064 \mathrm{~nm})$ and the second harmonic $(532 \mathrm{~nm})$ of a commercial Q-Switched (8 ns) Nd:YAG laser 
(ThunderArt, Quanta System S.p.A.). Laser cleaning performance was initially studied on evenly sooted samples (Fig. 1a). Laser fluence settings were selected to span the cleaning tests were performed on $2 \mathrm{~cm} \times 2$ $\mathrm{cm}$ test areas for every possible combination of selected parameters including wavelength (532 nm and $1064 \mathrm{~nm}$ ), fluence (532 nm, $5 \mathrm{ns:}$

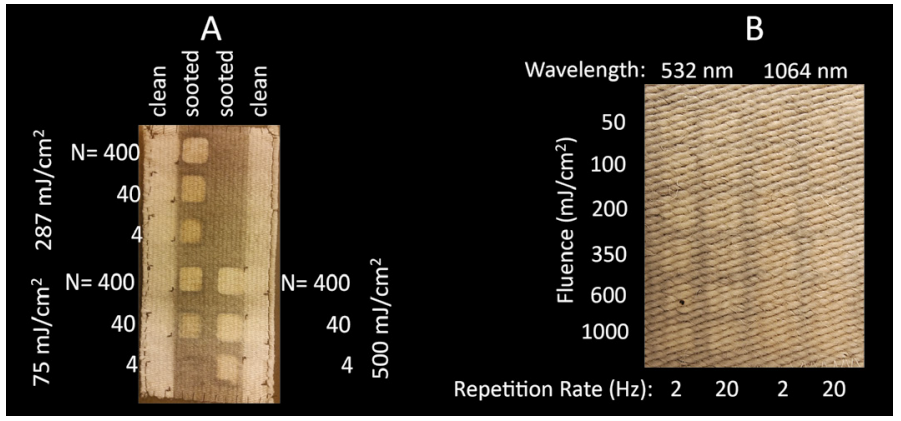

Fig. 1. Examples of reference samples for laser cleaning tests of heavily sooted (A) and deeply sooted (B) churro wool. range between cleaning threshold (judged visually after 10 pulses) and damage threshold, defined as $1 / 4$ of the fluence required to produce visible microscopic damage after 10 pulses. Pulse numbers were selected to explore laser cleaning behavior in both sudden and gradual cleaning regimes. This was deemed particularly important since gradual cleaning with many pulses was desired in order to controllably achieve visual uniformity across the blanket, but it was also important to minimize the risk of damage to the wool. Finally, surfaces were tested with pre-wetting and without, as both approaches have been shown to be beneficial in varying case studies.

In all laser cleaning experiments, laser pulse number was characterized in terms on pulses per laser beam area. This was due to the need to vary the beam diameter in order to obtain some of the greater fluences. The number of pulses per treated area thus varied, but the number of pulses per beam area was a unit of measure proportional to the flux applied over a treated region. A programmable, motorized stage was used to control the number of pulses per laser spot area. Laser
60, 360, $660 \mathrm{~mJ} / \mathrm{cm}^{2} ; 1064 \mathrm{~nm}, 6 \mathrm{~ns}: 75,287$, $\left.500 \mathrm{~mJ} / \mathrm{cm}^{2}\right)$, pulses per beam area $(4,40$, and 400), wool surface (unsoiled and sooted), and pretreatment just before cleaning (pre-wetting and no pre-wetting). A repetition rate of $4 \mathrm{~Hz}$ was selected to achieve reasonable cleaning rates while avoiding generating damage from gradual, continuous heating. This value was arrived at empirically from initial testing in which thermal damage was observed at repetition rates above $10 \mathrm{~Hz}$ and fluences of $350 \mathrm{~mJ} / \mathrm{cm}^{2}$ or higher, which was attributed to heat transfer from contaminants embedded within the purchased reference material.

Based on the results of the study above, additional experiments were planned on deeply sooted samples (Fig. 2a) in order to explore the most viable cleaning parameters under conditions that were less repeatable but more representative of a genuine treatment by a conservator. Instead of a motorized stage, in this experiment, samples were irradiated by scanning manually the laser beam over $1 \times 1 \mathrm{~cm}^{2}$ of surface using a fixed working distance of $12.5 \mathrm{~cm}$ from the target and a spot diameter of about $5 \mathrm{~mm}$ at both the wavelengths. 
Cleaning performance as a function of fluence was systematically tested from $50 \mathrm{~mJ} /$ $\mathrm{cm}^{2}$ up to $1 \mathrm{~J} / \mathrm{cm}^{2}$ for both the wavelengths and the repetition rate was 2 (exp. $50 \mathrm{sec}$ ) and 20 (exp. $5 \mathrm{sec}$ ) Hz. In this way an equal number of laser pulses $(\mathrm{N}=100)$ per spot was delivered. Further tests aimed at assessing the laser-induced color changes on untreated (no sooting to clean) wool were carried out using 350, 600 and $1000 \mathrm{~mJ} / \mathrm{cm}^{2}$ and 2 (exp: $50 \mathrm{sec}$ ), 10 (exp: $10 \mathrm{sec}$ ) and 20 (exp: $5 \mathrm{sec}$ ) $\mathrm{Hz}$, as shown in Fig. 5.

\subsection{Analytical Techniques}

Color measurements were performed using a custom setup consisting of Ocean Optics HL-2000-HP tungsten-halogen source fiber-coupled to an integrating sphere with a 6 mm opening, with the spheres optical output fiber-coupled to an Ocean Optics USB 2000+ spectrometer. Data were collected with a spectral resolution of $2 \mathrm{~nm}$ in the 380-780 $\mathrm{nm}$ range. Calibration was performed by means of $99 \%$ diffuse reflectance standard and CIE $\mathrm{L}^{*} \mathrm{a}^{*} \mathrm{~b}^{*}$ color coordinates were calculated considering $2^{\circ}$ degrees standards observer values. The behavior of test areas in CIE- $L^{*} a b$ color space was characterized in terms of deviations from the original, unsoiled color. In order to properly interpret the colorimetric data, 16 measurements of clean, original textile areas were made in different sections of the reference material, while four colorimetric measurements were taken in laser treated areas. The color variation between the laser cleaned surfaces and the average values for the original surface are expressed as $\Delta \mathrm{L}, \Delta \mathrm{a}$ and $\Delta \mathrm{b}$ and $\Delta \mathrm{E}$ (CIE Lab 2000 color definition).

A final set of accelerated light aging experiments were also prepared, to test the long-term optical response of the wool to the most promising laser cleaning parameters. Cleaned areas of interest were placed in an Atlas ci35 fadeometer at $100 \mathrm{Klx}$ for two weeks, simulating 37.5 years of 50 lux exposure for 10 hours a day. Colorimetric measurements were taken after exposure to compare with pre-existing measurements, using the same setup described above. Accelerated thermal was performed at 60 Celsius and $75 \% \mathrm{RH}$ for three weeks in a Caron 7000-33-1 environmental chamber, approximating 60 years of aging at 20 Celsius and $65 \% \mathrm{RH}$. Longitudinal tensile response for individual fibers was recorded both before and after accelerated aging in accordance with the ASTM D3822-14 protocol, using an Instron 4443 Universal Testing System. Tensile performance of individual fibers was characterized by the offset yield strength, defined as the stress at which the stress-strain curve for axial loading deviates by a strain of $1 \%$ from the linear-elastic line.

Samples for tensile testing were created by performing laser cleaning on both clean and sooted reference sample areas. Sooting was performed in accordance with the protocol for evenly sooted samples, described above. Cleaning of four $2 \mathrm{~cm} \times 2 \mathrm{~cm}$ areas was performed at $350 \mathrm{~mJ} / \mathrm{cm}^{2}$ with 400 pulses per beam area, on both sooted and original areas. After accelerated thermal aging, fifteen fibers were selected from each location. Only those fibers that were most exposed at the surface of the yarns were chosen, as they experienced the greatest amount of sooting and laser radiation and/or therefore the most extreme laser cleaning environment. All fibers were inspected by microscope under transmitted light at 400X magnification for any noticeable laser-induced damage. Fiber lengths of 15.7 $\mathrm{mm}$ were then mounted across plexiglass tabs under mild pretension (as specified in the ASTM standard) using two-component 
epoxy. They were then allowed to dry and acclimated in the same climate-controlled room where the testing was performed, at 23 Celsius and 50\% RH. After testing, the fiber were weighed on a Mettler Toledo Excellence Plus microbalance in order to properly scale the tensile data. The sum of the experiments allowed us to obtain a window of settings for adequately cleaning the blanket without significant damage.

\section{Results and Discussion}

\subsection{Reference Material Study}

In order to properly interpret the colorimetric data, 16 measurements of clean, original textile areas were made in different sections of the reference material. A natural variation of $1.57,0.17,0.8$, and 1.62 was found for $\Delta L$, $\mathrm{D} a, \mathrm{D} b$, and $\Delta \mathrm{E}$, respectively.

The colorimetric results from laser cleaning tests on evenly sooted samples are shown in Figs. 2 and 3. The behavior of test areas in CIE- $L^{\star} a b$ color space was characterized in terms of deviations from the original, unsoiled color (Fig. 2). Changes in value for $\mathrm{L}, a, b$, and the resulting $\Delta \mathrm{E}$ (CIE Lab 2000 definition), between the cleaned surfaces and the average values for the original surface are shown. The measurements reveal a number of clear, instructive patterns. Two broad, general trends are the improved performance of dry samples over pre-wetted ones, and the poor performance of cleaning at $1064 \mathrm{~nm}$ in comparison to the $532 \mathrm{~nm}$ wavelength.

The deterioration of cleaning performance on a wetted surface, as measured by the $\Delta \mathrm{L}$ coordinate is evident at both wavelengths. Cleaning of a dry surface outperforms that of a wet surface for all but the highest fluence and pulse number settings. Performance differences in the $\Delta L$ coordinate between "dry" and "wet" are comparable only at fluences of approximately $0.5 \mathrm{~mJ} / \mathrm{cm}^{2}$ and 400 pulses per beam area. Overall improvement in cleaning at $532 \mathrm{~nm}$ over $1064 \mathrm{~nm}$ is similarly evident. Lightening from soot removal, as characterized by the $\mathrm{L}$ coordinate, requires a high fluence and 400 pulses to achieve the same contrast reduction that the $532 \mathrm{~nm}$ treatment attains at with 40 pulses at nearly one tenth of the fluence.

Other smaller, but still clear, patterns in behavior show that treatments with both $532 \mathrm{~nm}$ and $1064 \mathrm{~nm}$ wavelengths have the capability to induce color change beyond that caused by soot removal. As $532 \mathrm{~nm}$ pulses are applied at greater fluence and number, the wool both shifts to higher values of $L$ and more negative values of $a$, corresponding to a slight lightening and greening in hue. The lightening is evident only at the highest fluence and maximum pulse number of 400 , while the change in hue is perceptible for both medium and high fluence at 400 pulses. It is unlikely that the change is caused by the removal of the added soot, since similar changes are present in clean areas with the same treatment applied. While this color change may partially be due to the removal of additional dirt that is present in the original reference material, it is unlikely to be the cause of the change in hue. This suggests a slight alteration of the material dependent on the pulse peak energy and total pulse number, potentially due either to accumulated brief heating from individual pulses or to previously reported photo-responsive changes in wool's reflective properties that are depend on the illuminating wavelength [13].

For the case of $1064 \mathrm{~nm}$, a certain amount of darkening remains with respect to the original color, even at high fluence and 400 pulses. Additionally, significant and consist- 

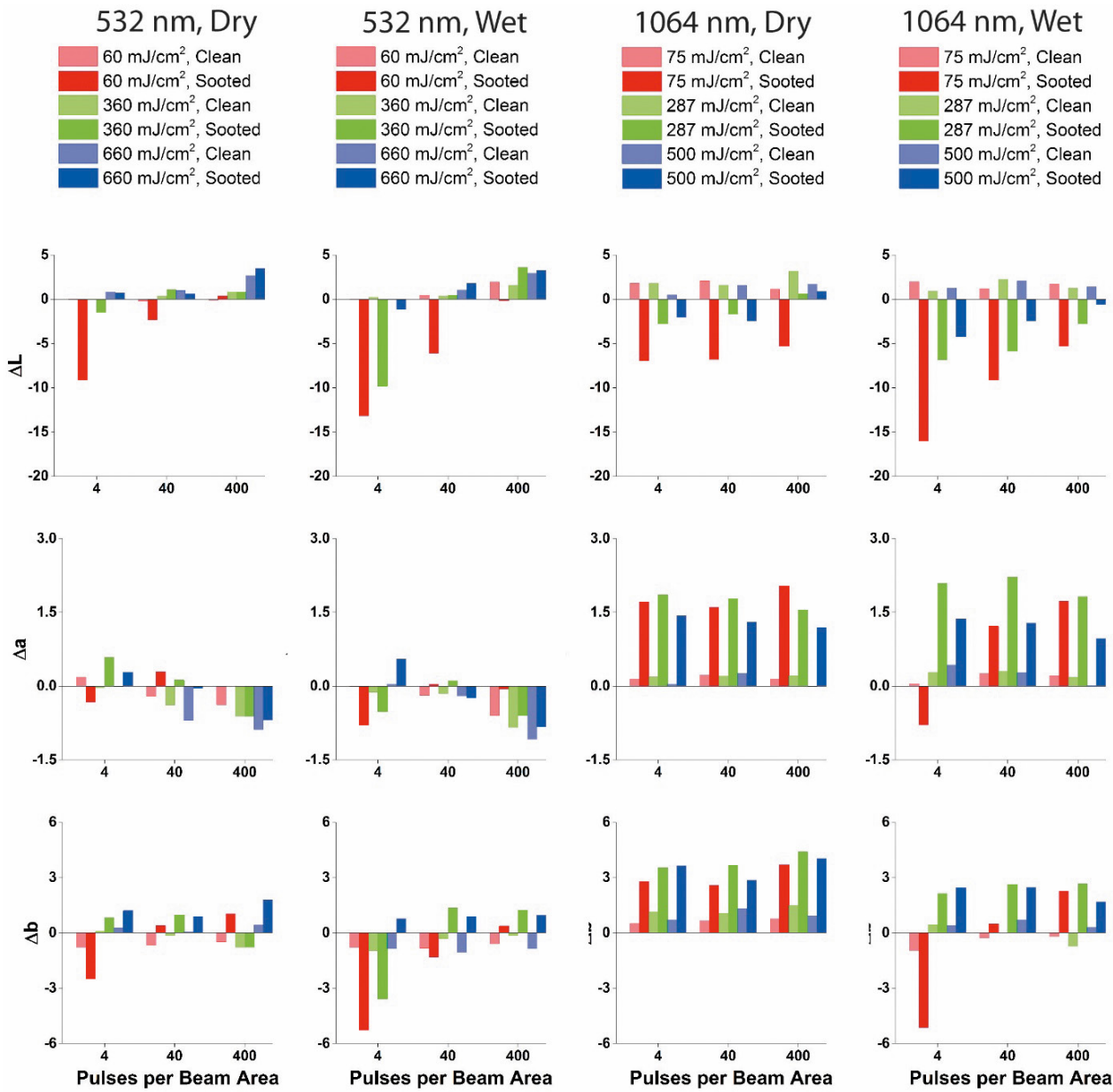

Fig. 2. $\Delta L, \Delta a$, and $\Delta b$ values of colorimetric data for laser cleaning tests of heavily sooted and nearby unsoiled areas. The difference between the laser cleaned area and a nearby original (unsoiled, uncleaned) area is plotted. Wavelength, surface pre-treatment, fluence, and pulse number are varied.

ently positive shifts in coordinates $a$ and $b$ exist at all power levels and pulse number, but only in the areas that were sooted. This last point suggests that the color change, which corresponds to reddening and is clearly visible, is most likely due to remnants or byproducts of the cleaned soot that were not effectively removed by the infrared laser. This was informally supported by cleaning an additional area at medium fluence with
$1064 \mathrm{~nm}$ to generate the red stain, and then removing the red stain by passing over the same area at comparable fluence with $532 \mathrm{~nm}$ pulses. Similar behavior has been observed for removal of carbonaceous deposits on gypsum by $1064 \mathrm{~nm} \mathrm{Nd:YAG} \mathrm{pulses} \mathrm{[14,15].}$ These experiments showed that nanoparticles of remnant soot-derived material are present in colored areas, and that the presence of other organic compounds can enhance the 
development of colored deposits. However, the remaining deposits remain sensitive to and can be removed by shorter wavelengths. It is possible that the sooted wool samples experience similar behavior: $1064 \mathrm{~nm}$ wavelengths generate photochemistry of organic impurities to produce colored substances that do not interact strongly with the ablating laser, or the ablation dynamics create soot-derived nanoparticles during that are not sufficiently absorbing.

Overall, the colorimetric results from the data above suggest that the most interesting range of parameters for laser cleaning of sootlike deposits from churro wool are fluences at or below $360 \mathrm{~mJ} / \mathrm{cm}^{2}$, with tens to hundreds of laser pulses per pulse area, at $532 \mathrm{~nm}$ and without pre-wetting the surface. The analysis
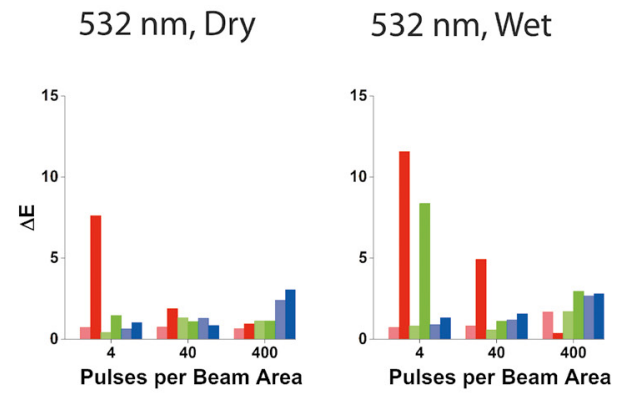

pressed india ink more precisely clarifies the optimal power, pulse number, and repetition rate for laser cleaning deposits on churro wool. As expected, irradiation at $532 \mathrm{~nm}$ generates marked increases of $\Delta L$ in comparison with the $1064 \mathrm{~nm}$ wavelength. In contrast, $\Delta a$ rises as function of laser fluence in major extent at $1064 \mathrm{~nm}$, thus confirming what was previously observed in cleaning tests of heavily sooted samples (Fig. 2). The removal process at $1064 \mathrm{~nm}$ is drastically less efficient than at $532 \mathrm{~nm}$ and the yellow-red color remaining on the surface at $1064 \mathrm{~nm}$ is most likely due to residual ink particles that are not sufficiently absorbing at $1064 \mathrm{~nm}$, as observed with the previously described, more heavily sooted samples. Instead, the 532 $\mathrm{nm}$ laser wavelength produces, just starting

$$
1064 \text { nm, Dry } 1064 \text { nm, Wet }
$$
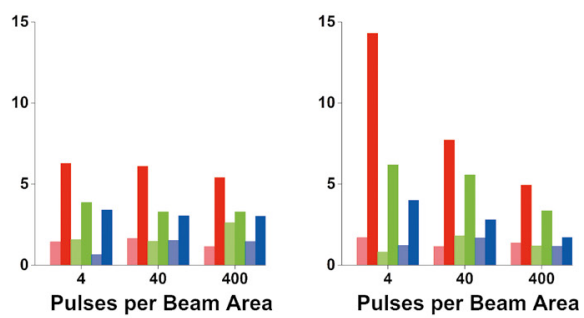

Fig. 3. $\Delta \mathrm{E}$ values for laser cleaning tests of heavily sooted samples, corresponding to the data shown in Fig. 2.

of overall color change from the above measurements $(\Delta \mathrm{E})$, shown in Fig. 3, shows how the general behavior of the laser cleaning experiments supports this conclusion. A consistent color which is within measurement error of the original is obtained throughout this parameter range. We note that previous studies of laser-cleaned animal fibers by SEM have shown morphological change can occur at a threshold that is approximately half that of any visible change in color [2].

The analysis of more focused experiments performed on the samples with soaked and from the lower fluences tested, a high degree of particle removal and it may be therefore considered most suited for reaching appreciable cleaning results on sooted wool textiles. $\Delta b$ increases with increasing fluences as a consequence of soot removal but at the same time, its behavior is similar at both the wavelengths. It is worth noting that the color variations observed in Fig. 4 all have a saturation trend with increasing fluence, and in most cases, become constant around $150-200 \mathrm{~mJ} / \mathrm{cm}^{2}$ at $532 \mathrm{~nm}$ and among $200-350 \mathrm{~mJ} / \mathrm{cm}^{2}$ at $1064 \mathrm{~nm}$. No significant 

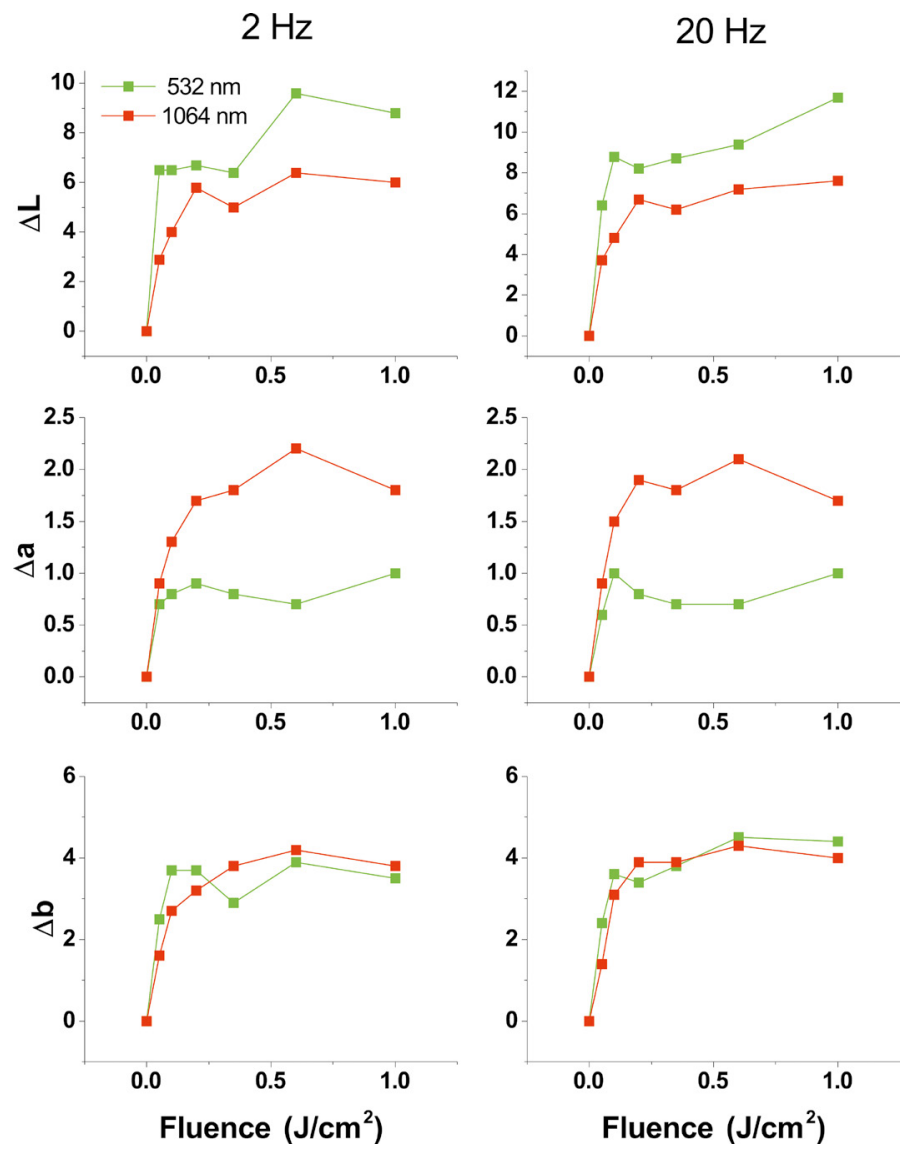

Fig. 4. $\Delta L, \Delta a$, and $\Delta b$ values, with respect to the sooted surface before cleaning, for deeply sooted samples. Using 100 pulses per beam area repetition rate and fluence are varied. Note that improvements in cleaning generally saturate beyond $\sim 350 \mathrm{~mJ} / \mathrm{cm}^{2}$.

differences were observed between $2 \mathrm{~Hz}$ and $20 \mathrm{~Hz}$ operation.

As expected, all the above-mentioned considerations are reflected in the $\Delta \mathrm{E}$ variation, where the slight color difference between the two wavelengths is further demonstrated (Fig. 5).

Data exploring the margin of safety for the optimal parameters above, by varying repetition rate and relatively high fluence rates, is shown in Fig. 6 and Fig. 7. Firstly, $\Delta L, \Delta a$ and $\Delta b$ undergo variation with respect to the original surface that tends to increase more so with the number of laser pulses delivered and less with the fluence and repetition rate. However, variations of
$\Delta L$ appear to be negligible up to $600 \mathrm{~mJ} / \mathrm{cm}^{2}$, either at 2, 10 and $20 \mathrm{~Hz}$.

The opposite trend may be clearly observed in the irradiation tests carried out at $1 \mathrm{~J} / \mathrm{cm}^{2}$. In fact, $\Delta \mathrm{L}$ increases as function of laser pulses and repetition rates, until reaching a constant value of about 3 at 20 $\mathrm{Hz}$. This change is sufficient to be visible to the naked eye, and the irradiated area seems to be brighter. In contrast, $\Delta$ a decreases with increasing laser pulses, beginning at approximately 60 laser pulses per beam diameter. Although the $\Delta \mathrm{b}$ change is quite negligible, it tends to increase, thus suggesting the color change proceeds toward a yellow or green color hue, consistent with the other reference 

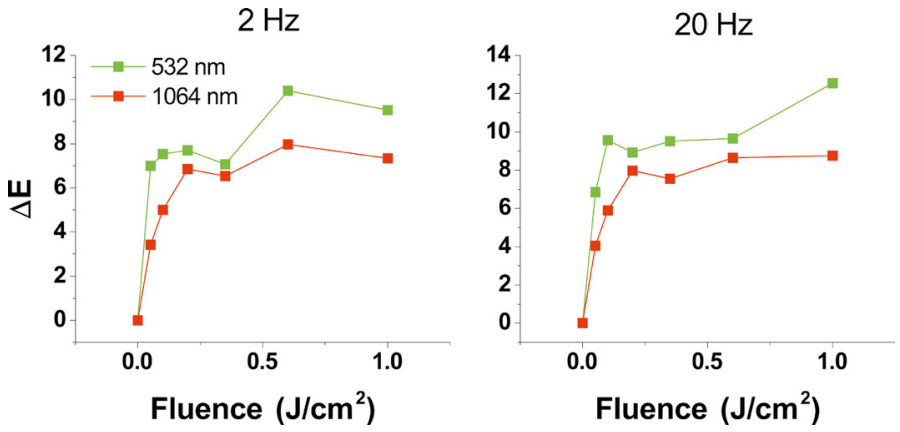

Fig. 5. $\Delta \mathrm{E}$ values for laser cleaning tests of deeply sooted samples, corresponding to the data shown in Fig. 4.
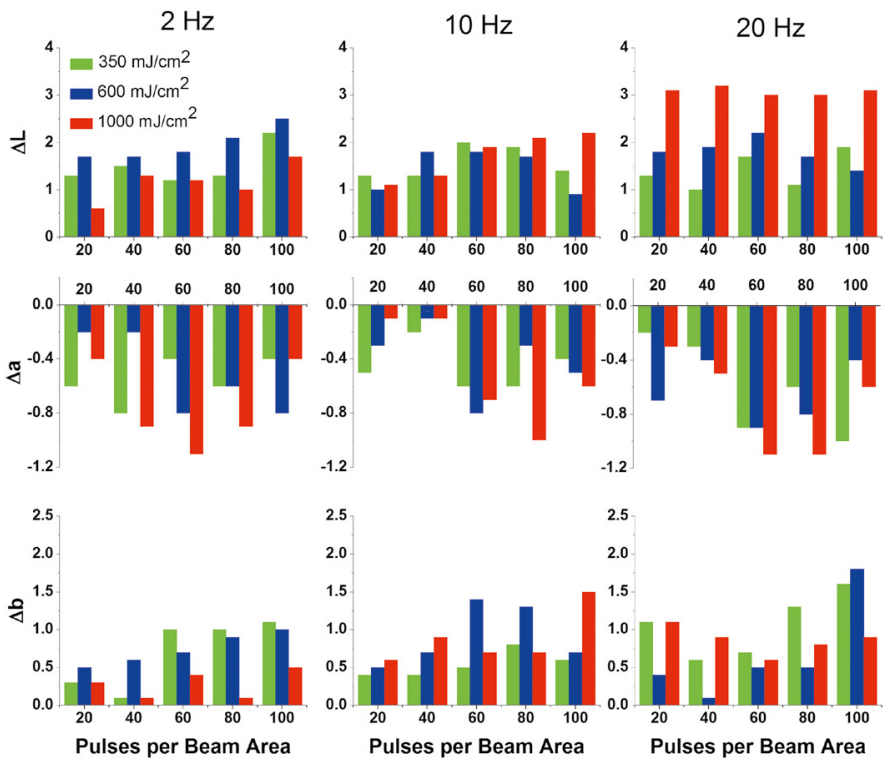

$\begin{array}{lllll}20 & 40 & 60 & 80 & 100\end{array}$

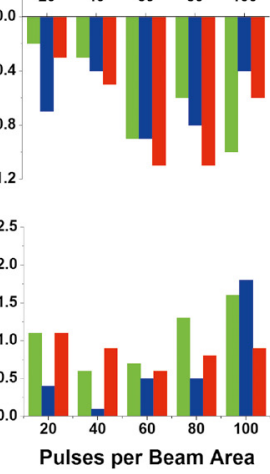

Fig. 6. $\Delta L, \Delta a$, and $\Delta b$ values of colorimetric data for laser cleaning tests of unsoiled samples at $532 \mathrm{~nm}$ with no pre-wetting. The difference between the laser cleaned area and a nearby original (unsoiled, uncleaned) area is plotted. Fluence, pulse number, and repetition rate are varied.

sample results. The variations in $\mathrm{D} a$ and $\mathrm{D} b$ variations are small; however, they present a clear direction for color change as a result of prolonged exposure to laser irradiation.

Trends for $\Delta \mathrm{E}$ under the various conditions are shown in Fig. 7. These are in most cases lower than the material's natural variation of $\Delta \mathrm{E}=1.6$. However, at fluences of $1 \mathrm{~J} / \mathrm{cm}^{2}$ and $20 \mathrm{~Hz}$ color change is the most prominent $(\Delta \mathrm{E} \geq 3)$, independently from the number of pulses. In this case, the occurrence of laser-induced color changes takes place at certain energy density and repetition rate, possibly due to localized cumulative heating. In fact, previous laser irradiation tests aimed at finding macroscopic damage thresholds on single-spot basis, showed that at $350 \mathrm{~mJ} / \mathrm{cm}^{2}$ and $20 \mathrm{~Hz}$ no surface changes were observed after 30 seconds of exposure. Contrarily, a dark brown spot, likely due to cumulative heating, was clearly observable at $600 \mathrm{~mJ} / \mathrm{cm}^{2}, 20 \mathrm{~Hz}$ after 20 seconds and at 1 

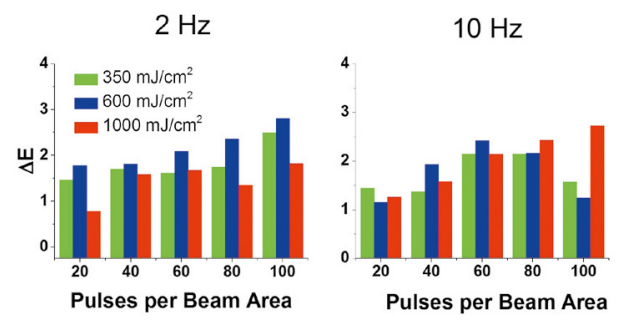

$\mathrm{J} / \mathrm{cm}^{2}, 20 \mathrm{~Hz}$ after 10 seconds. The measured damage thresholds for the untreated wool sample under study are therefore dependent from the total radiant exposure $\left(\mathrm{J} / \mathrm{cm}^{2} \cdot \mathrm{Hz}\right)$ integrated over time.

It is therefore worth noting while some color change is primarily a function of the total number of pulses, which suggests a damage mechanism dependent on the peak energy, other effects take place at very high fluence and repetition rates, which suggests a thermal effect due to constant heating. This second effect, however, is far from the optimal cleaning conditions $\left(350 \mathrm{~mJ} / \mathrm{cm}^{2}\right.$, $10-20 \mathrm{~Hz}$ ). The wool appears to be thermally and mechanically stable to laser irradiation below $600 \mathrm{~mJ} / \mathrm{cm}^{2}$. However, due to the low thermal diffusivity of organic material (for wool thermal conductivity $k$ is $0.03 \mathrm{~W} /(\mathrm{m} \cdot \mathrm{K})$ at $25^{\circ} \mathrm{C}, \rho=1.3 \mathrm{~g} / \mathrm{cm}^{3}$ and $C_{p}=1.357 \mathrm{~J} / \mathrm{g} \cdot \mathrm{K}$, and the calculated thermal diffusivity $D=1.7$ $\cdot 10^{-4} \mathrm{~cm}^{2} / \mathrm{s}$ ) [16-18], it is advisable to move the beam rapidly over the surface to avoid any undesired effect. In addition, thermal damage could be drastically increased if there is prolonged exposure for large absorbing impurities (dirt and pollutants) that remain trapped between fibers, or for melanin-rich or colored fibers.

As described in the section 2.4, accelerated light and thermal aging tests were used to probe for any long-term effects due to laser cleaning. Results from the former are shown in Fig. 8. While the treated areas

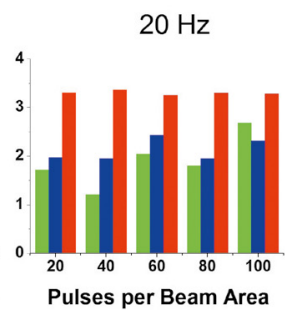

Fig. 7. $\Delta \mathrm{E}$ values for laser cleaning tests of deeply sooted samples, corresponding to the data shown in Fig. 6.

demonstrated an overall color change in comparison to the untreated area that was slightly greater than the one standard deviation of measurement error, the results show no consistent pattern. The unsoiled area cleaned with 40 pulses per beam area showed a greater difference than that cleaned with 400 pulses, while the sooted area cleaned with 40 pulses per beam area showed less difference than that cleaned with 400 pulses. We therefore concluded that the effects were most likely due to slightly greater variation of the initial material in this area, and not the treatment itself. In all of the above cases,

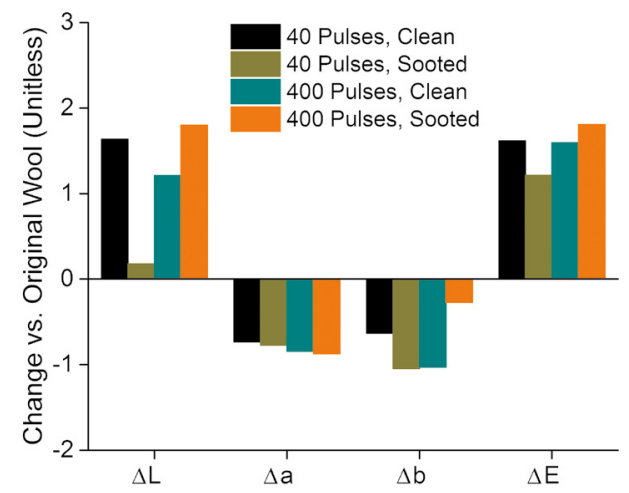

Fig. 8. $\Delta L, \Delta a, \Delta b$, and $\Delta \mathrm{E}$ values for of colorimetric data for laser cleaning tests of the heavily sooted sample laser cleaned at $532 \mathrm{~nm}$ and $350 \mathrm{~mJ} / \mathrm{cm}^{2}$ with no pre-wetting, for pulse numbers of 40 and 400 on both sooted and original (clean) surface areas. The difference between the laser cleaned area and a nearby original (unsoiled, uncleaned) area is plotted. Pulse number is varied. 


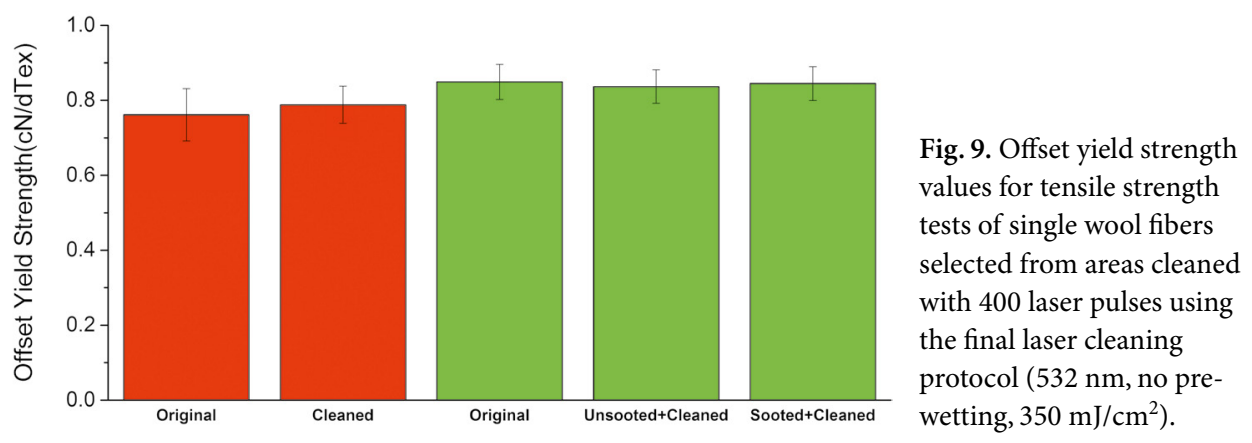

no damage was visible under microscopic examination.

Tensile measurements after accelerated thermal aging are shown in Fig. 9. The offset yield strength does not change appreciably between the original fibers and those that have been sooted and cleaned. This is the case both with and without thermal aging. It is possible that a slight trend of generally increased offset yield strength after thermal aging (red vs. green) is emerging, although more statistics would be needed to verify this. This would likely be due to a slight embrittlement after thermal aging, which is expected behavior for wool fibers.

The cumulative data from both studies shows that optimal laser cleaning of a churro wool blanket should occur at $532 \mathrm{~nm}$ and approximately $350 \mathrm{~mJ} / \mathrm{cm}^{2}$, on a dry surface with up to 100 pulses per beam area. These settings should clean soot-like deposits efficiently without any noticeably color change, whether by eye or using colorimetric instrumentation. One should also expect to see no visible change in the individual fibers, even under 400X magnification.

\subsection{Case Study, Laser Cleaning of a First Phase Navajo blanket}

The physical condition of the Chief Blanket, shown in Fig. 10, was assessed. It was examined by eye at several magnifications, ranging from $5 x$ to $100 x$. The yarn make-up and details of the woven structure were recorded. Although the aesthetic appearance of the blanket was poor, the physical condition was very good. The wool fibers remain strong as do the yarns produced from them. Overall, the woven structure was intact; several internal damages had been expertly rewoven. The selvage twining had also been restored and was intact. The corner tassels were also intact and appear to be in original condition. Importantly, when the blanket was viewed through transmitted light, it was possible to document the small areas where the weft was thinned as a result of abrasion, likely from use and prior cleanings.

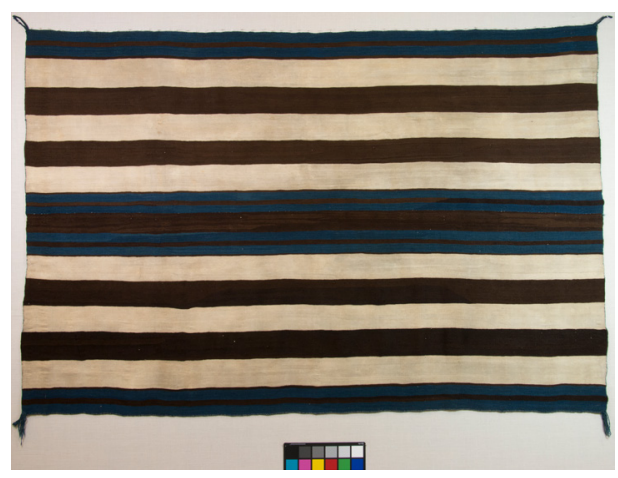

Fig. 10. Image of the "Back" surface of the Navajo blanket. The blanket measures $54-1 / 2$ " $(138 \mathrm{~cm})$ by $82 "(208 \mathrm{~cm})$. Fabrics are elastic objects. These are the maximum dimensions, excluding tassels. 
The major condition issue was the uneven coloration of the undyed white churro wool: it was unevenly stained a sooty gray color on both faces, and these areas were punctuated by a variety of white "stains" where all the gray color had been removed. An example of these conditions is shown in Fig. 11 (left). It was presumed that the uneven gray deposits were also distributed over the blue and brown bands, but were not visible, leaving their appearance unaffected. The likely composition of soiling was determined by reflectance spectroscopy. This method was chosen for several reasons. To begin with, sampling was not permitted. Since the soiling was extremely fine and dilute, and since very little material is needed to produce noticeable color variation, non-invasive vibrational spectroscopy techniques such as Raman spectroscopy and FTIR were only sensitive to the wool. Microscopic examination was also inconclusive: it was unclear whether the particles located were in fact the main contributors to the color variation.

Typical reflectance data is shown in Fig. 11 (right). The contaminant was characterized by measuring the ratio of reflectance from a clean area to that from a soiled area. The resulting spectrum is consistent with sooting: a relatively flat response over a very broad range of wavelengths, with a gradual decrease in reflectance toward the near-infrared [19].

As described in the previous section, the tests run on the hand-woven churro reference fabric indicated that soot removal was more efficient at $532 \mathrm{~nm}$ pulses than at $1064 \mathrm{~nm}$ pulses. During the first hours spent getting familiar with the Nd:YAG laser, the conservators practiced on the churro reference sample and on a large damaged fragment of a Navajo weaving, specifically purchased by the owner for this purpose. Combinations of frequencies $(5,10$, and $20 \mathrm{~Hz})$ and beam size at the surface were tested and the results were compared, in an effort to determine which parameters would deliver a more consistent and effective cleaning. In order to maintain the fluence of the laser beam below $350 \mathrm{~mJ} / \mathrm{cm}^{2}$ and avoid damage to the substrate, the beam diameter at the surface was kept at or above $5-7 \mathrm{~mm}$. The optimum treatment parameter was a setting of $532 \mathrm{~nm}$ at $20 \mathrm{~Hz}$ with a beam size at the surface of approximately $6 \mathrm{~mm}$. This setting ensured the soot particles would be removed evenly (i.e. uniformity of color) within a reasonable amount of time.
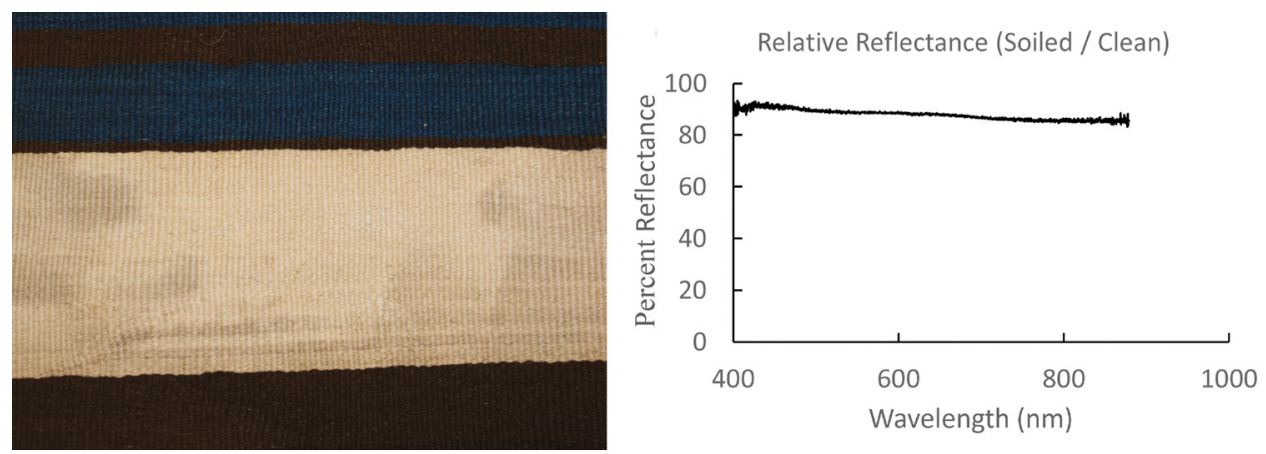

Fig. 11. (Left) Example of the various effects of the soiling, including large areas of discoloring that perhaps indicate a previous cleaning test or attempt, as well as finer variations in darkening throughout. (Right) Typical spectrum for the percent difference in reflectance between soiled and unsoiled areas of the blanket. 
In order to systematically clean the white bands, a label was added to one face of the weaving. The cleaning was begun at the bottom right band. The area to be treated was marked by pins at the left and right sides. Initially, photographs were taken for comparative purposes, but this time-consuming process was discontinued when it became apparent that the protocol was effective. The blue and brown yarns were masked with aluminum foil, pinned into place. The pins were secured into a $1 / 2$ " thick sheet of Ethafoam ${ }^{\circ} 990$ covered with aluminum foil. The aluminum foil would reflect the beam, rather than possibly allowing heat to be absorbed by the underlying foam (see Fig. 12 A). Empirically, it was observed that a small amount of tensioning in the warp direction facilitated the cleaning process. It is presumed that tensioning allows a greater surface area of weft was exposed to the laser beam. It was also determined empirically that tensioning in the weft direction would also facilitate cleaning.

Approximately half of the cleaning was accomplished using the Ethafoam ${ }^{\circ}$ sheet. However, it became clear that some of the soiling remained resistant to the laser, especially in areas near the bright stains. Since the tests with the churro samples revealed that the undyed wool substrate was not affected by the laser at the recommended parameters, and in consultation with the owner, a second pass of the laser was carried out. For this second cleaning, a curved jig was created that would allow more surface area of the wool to be exposed. It was constructed from a section of $31 / 2$ " archival tube, cut in half along the length and secured to a sheet of the Ethafoam ${ }^{\circ}$ 990. This curved form was then covered with aluminum foil. This tool reduced the surface area that could be warp-tensioned during a cleaning session, thus increasing the manipulation of the cloth. However, the curved surface allowed the laser to further penetrate the weft interstices for a more in depth cleaning. This tool, originally used to treat areas that had been already cleaned on the flat foam surface, soon replaced it altogether, when it became apparent that even with more frequent moving and repinning, the cleaning time was reduced. Its use is shown in Fig. 12 B.

There remained areas that retained an eye-catching uneven coloration. The owner approved the testing of a third tool, assembled from 4-inch Styrofoam ${ }^{\text {tw }}$ hemisphere mounted on a cylindrical Ethafoam ${ }^{\circ} 660$ base. It was developed so that small areas of both the warp and weft could be simultaneously tensioned. This tool, called "the dome" (see Fig. 12 C) significantly reduced the area
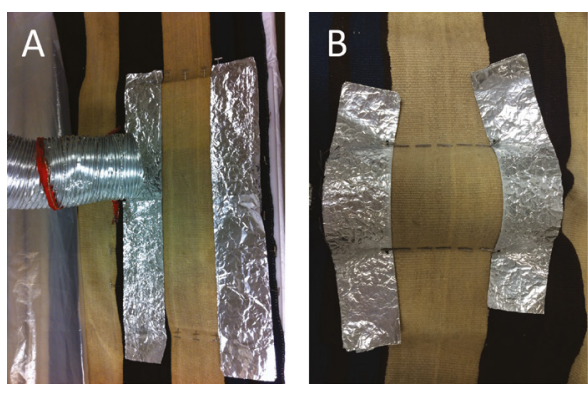

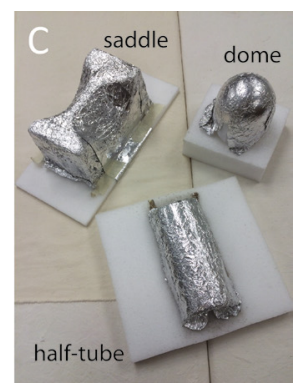

Fig. 12. View of table from top: (A) A section of the blanket prepared for cleaning by stretching horizontally over an Ethafoam ${ }^{\circ}$ sheet; (B) A section of the blanket prepared for cleaning by tensioning horizontally over a half-tube jig;

(C) The three jigs used during laser cleaning of the Navajo blanket. 
that could be cleaned, but allowed the conservators to focus on very specific areas. Again, the evenness of the cleaning was assessed and a fourth and final tool, dubbed "the saddle" (see Fig. 12 C), and made of a tall block of Ethafoam $^{\circ} 660$ that was carved into a shape resembling a narrow horse saddle, was used to focus the cleaning on specific weft interstices and transition lines resulting from the use of the half-tube during the previous cleaning phase. Only about four passes of the weft were exposed during the cleaning and the laser was manipulated to clean around the "crown" of each weft. This final support jig was the most complicated to tension, but it did allow the conservators to remove enough of the resistant soot to even out more of the undyed wool surface. Although it may have been possible to remove more soot, the amount of time under the laser was not equal to the quality of the results, and the cleaning was ended. Afterward, treatment photographs of both faces of the Chief Blanket were taken, along with selected details.

Although it was not possible to do before and after tensile testing of this identically faced tapestry woven Chief Blanket, no damage to the wool was observed at $100 \mathrm{X}$ magnification. Samples were not extracted for higher level magnifications. For both the conservators, scientists and the owner, the look and the handle of the weaving was unchanged. The brown and indigo wools were covered during the treatment and remain unchanged.

Results of the cleaning are shown in Fig. 13. Overall lightening from soot removal is clearly evident when the blanket is folded over to contrast a cleaned area with one that is yet uncleaned (see Fig. 13 A). Additionally, while the white bands remain unevenly colored, the contrast in particularly irregular areas such as that shown in Fig. 11 (left) has been significantly reduced (see Fig. 13 B, C). The eye-catching white stains are not immediately evident, although traces remain, but only to those trained to look for them. The areas where the weft was thin and fragile were unchanged and no losses in these areas occurred during the treatment. Perhaps natural aging will reveal some now-hidden agent of deterioration, but given the amount of pre-treatment testing on churro wool, this event seems unlikely.
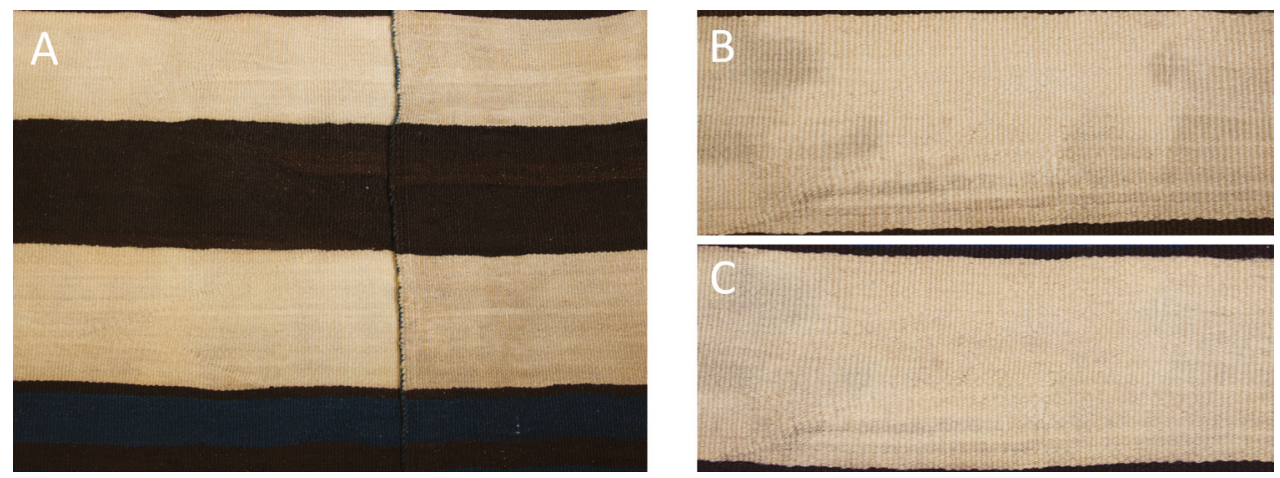

Fig. 13. (A) A section of the blanket is folded over show the difference between cleaned (lighter) and uncleaned (darker) areas of the blanket; (B) A region of the uncleaned blanket with particularly large variation in lightness; (C) The region shown in $12 \mathrm{~B}$, after laser cleaning. 


\section{Conclusion}

We have successfully performed laser cleaning of sooty soiling on a wool First Phase Navajo chief blanket, based on laser parameters that were optimized through an extensive study of woven churro wool reference material. The reference study showed an optimal balance between cleaning effectiveness and negligible damage at settings of $532 \mathrm{~nm}$ and $350 \mathrm{~mJ} / \mathrm{cm}^{2}$, with no pre-wetting of the wool surface. These parameters left no discernable damage even with hundreds of pulses applied per beam area, and accelerated light and thermal aging tests did not exhibit a difference between original and laser-cleaned surfaces. Cleaning of the Navajo blanket resulted in significant overall removal of soiling, exhibited by lightening of the surface color, and reduction of contrast between clean and previously soiled areas. The wool fibers of the cleaned blanket were found to undamaged based on macroscopic, microscopic, and tactile examination of the blanket following treatment.

\section{Acknowledgements}

We gratefully acknowledge Quanta System for providing the Thunder Art Laser to the Metropolitan Museum of Art and Dr. Edgar O. Smith for his support. We would also like to acknowledge Robert Mann and Robert Mann Rugs for providing the reference material.

\section{References}

[1] C. Escudero, M. Martinez, P. Moreno, and A. Arranz, "Study of laser cleaning of ancient fabric with femtosecond pulses", in: Lasers in the conservation of artworks: proceedings of the International Conference LACONA VII, Madrid, Spain, September 17-21, 2007, M. Castillejo, P. Moreno, M. Oujja, R. Radvan,
J. Ruiz (Eds.), CRC Press, Inc., Boca Raton, Florida, 2013, pp. 337-343.

[2] R. Belli, A. Miotello, P. Mosaner, and L. Toniutti, "Laser cleaning of artificially aged textiles", Applied Physics A, 83 (2006) 651-655, DOI: 10.1007/s00339006-3530-3.

[3] D. Ciofini, I. Osticioli, S. Micheli, L. Montalbano, and S. Siano, "Laser removal of mold and foxing stains from paper artifacts: preliminary investigation", Proc. SPIE 9065, (2013), 906512-1 - 906512-11, DOI: $10.1117 / 12.2052820$.

[4] C. Rosati, D. Ciofini, I. Osticioli, R. Giorgi, S. Tegli, and S. Siano, "Laser removal of mold growth from paper," Applied Physics A, 117 (2014) 253-259, DOI: $10.1007 /$ s00339-014-8507-z.

[5] T. Ersoy, T. Tunay, M. Uğuryol, G. Mavili, and S. Akturk, "Femtosecond laser cleaning of historical paper with sizing", Journal of Cultural Heritage, 15 (2014) 258-265, DOI: 10.1016/j.culher.2013.07.002.

[6] S. Pentzien, A. Conradi, and J. Krüger, “The influence of paper type and state of degradation on laser cleaning of artificially soiled paper", in: Lasers in the conservation of artworks: proceedings of the International Conference LACONA VIII, Sibiu, Romania, September 21-25, 2011, R. Radvan, J. F. Asmus, M. Castillejo, P. Pouli, A. Nevin, (Eds.), CRC Press/ Balkema, Leiden, Netherlands, 2011, pp. 59-65.

[7] T. Fourrier, G. Schrems, T. Mühlberger, J. Heitz, N. Arnold, D. Bäuerle, M. Mosbacher, J. Boneberg, and P. Leiderer, "Laser cleaning of polymer surfaces", Applied Physics A, 72, (2001) 1-6, DOI: 10.1007/ s003390000683.

[8] W. Kautek, S. Pentzien, D. Mueller-Hess, K. Troschke, R. Teule, "Probing the limits of paper and parchment laser cleaning by multi-spectral imaging", Proc. SPIE 4402 (2001) 130-138, DOI: 10.1117/12.445655.

[9] H. Sutcliffea, M. Cooperb, J. Farnswortha, "An initial investigation into the cleaning of new and naturally aged cotton textiles using laser radiation", Journal of Cultural Heritage, 1 (2000) S241-S246, DOI: 10.1016/S1296-2074(00)00178-3.

[10] A. Elnaggar, P. Fitzsimons, A. Nevin, K. Watkins, M. Strlic, "Viability of laser cleaning of papyrus: conservation and scientific assessment", Studies in 
Conservation, 60 (2015) S73-S81, DOI: 10.1179/003 9363015Z.000000000211.

[11] F. Liu, M. Yang, F. Hill, D. Snelling, G. Smallwood, "Influence of polydisperse distributions of both primary particle and aggregate size on soot temperature in low-fluence LII", Applied Physics B, 83 (2006) 383-395, DOI: 10.1007/s00340-006-2196-z.

[12] J. Wheat, Blanket Weaving in the Southwest, University of Arizona Press, Tucson, 2003.

[13] H. Launer, "Effect of Light Upon Wool: Part VI: Spectral Conformity in Photobleaching of Wool", Textile Research Journal, 36 (1966) 606-611, DOI: $10.1177 / 004051756603600703$.

[14] C. de Oliveira, P. Bromblet, A. Colombini, V. Vergès-Belmin, "Medium-wave ultraviolet radiation to eliminate laser-induced yellowing generated by the laser removal of lamp black on gypsum", Studies in Conservation, 60 (2015) S34-S40, DOI: 10.1179/0039363015Z.000000000205.
[15] C. de Oliveira, V. Vergès-Belmin, D. Demaille, P. Bromblet, "Lamp black and hematite contribution to laser yellowing: A study on technical gypsum samples", Studies in Conservation, 61 (2016) 136-145, DOI: $10.1179 / 2047058415 Y .0000000003$.

[16] Z. Ye, C. Wells, C. Carrington, N. Hewitt, “Thermal conductivity of wool and wool-hemp insulation", International Journal of Energy Research, 30 (2006) 37-49, DOI: 10.1002/er.1123.

[17] The Engineering ToolBox, http://www.engineeringtoolbox.com/specific-heat-capacity-d_391. html, (accessed 01.10.2016).

[18] Aqua-Calc, http://www.aqua-calc.com/page/ density-table/substance/wool, (accessed 01.10.2016).

[19] R. Levinson, P. Berdahl, H. Akbari, "Solar spectral optical properties of pigments-Part I: model for deriving scattering and absorption coefficients from transmittance and reflectance measurements", Solar Energy Materials and Solar Cells, 89 (2005) 319-349, DOI: 10.1016/j.solmat.2004.11.012. 



\title{
Towards the understanding of the two wavelength laser cleaning in avoiding yellowing on stonework: a micro-Raman and LIBS study
}

\author{
Athanasia Papanikolaou ${ }^{1,2}$, Panayiotis Siozos ${ }^{1}$, Aggelos Philippidis ${ }^{1}$, Kristalia \\ Melessanaki ${ }^{1}$, Paraskevi Pouli ${ }^{1 \star}$ \\ 1 Institute of Electronic Structure and Laser, Foundation for Research and Technology-Hellas (IESL- \\ FORTH), P.O. Box 1385, 71110 Heraklion, Crete, Greece \\ 2 Department of Physics, University of Crete, 70013 Heraklion Crete, Greece \\ * Corresponding author: ppouli@iesl.forth.gr
}

\begin{abstract}
The synchronous use of Infrared (IR at $1064 \mathrm{~nm}$ ) and Ultraviolet (UV at $355 \mathrm{~nm}$ ) wavelengths of a Q-Switched Nd:YAG laser has been shown to efficiently confront the issue of discoloration, which may be observed upon laser ablation of environmental encrustation from marble surfaces, and thus this methodology is effectively employed in various laser cleaning applications. In order to further investigate the mechanisms that influence this result, a series of tests were undertaken on fragments of marble with environmental homogeneous compact crust. Through a number of irradiation tests applying a variety of laser parameters (individual use of IR and UV beams, as well as, their synchronous use) the different ablative processes have been investigated. In this paper, micro-Raman and Laser Induced Breakdown Spectroscopy (LIBS) were employed in order to examine the role of each laser beam, as well as their combination, and so to approach the reasons that induce yellow discoloration. The results of the above studies will be presented with the aim to further describe the two wavelength laser cleaning methodology and its principle, thus elucidating its effectiveness.
\end{abstract}

Keywords: Laser cleaning, pollution encrustation, yellowing, double wavelength, laser spectroscopy

\section{Introduction}

\subsection{The yellowing effect}

Outdoor Cultural Heritage (CH) monuments made of stone (marble, limestone etc.) are susceptible to a number of harmful environmental factors such as micro climate, acid rain, humidity and airborne particles from exhaustions of cars and factories. Due to these compounds a hard, dark-coloured crust is often developed on their surface. In an effort to remove the crust from the surface of the marble IR lasers were used but yellowing of the treated surfaces often appears as an undesirable side effect.

The discoloration effect has been extensively studied and a number of hypotheses explaining its origin have been developed through the last years [1-9]. A brief presentation of the prevailing explanations upon the yellowing hue on the irradiated surface is given here: 
- The revealing of pre-existing layers: According to this hypothesis, IR radiation removes the encrustation layer and reveals coloured pre-existing layers or "patinas". These layers may be either intentionally applied (i.e. to protect the original surface and/or as a preparation layer before the application of paints) or they may have been developed naturally (due to weathering effect). In both cases they are considered historical and must be safeguarded as they may contain valuable information, as for example sculpted details and tooling traces, as well as historical evidence about materials and techniques used (traces of colours/pigments etc.) [10].

- The change of the original colour of the stone surface; this alteration may be caused due to the migration of water soluble organic compounds from the encrustation to the underlying stone, which is favoured in environments with high humidity and rain. As a result, the surface revealed upon laser cleaning show a colour different to the expected one $[11,12]$.

- The chemical transformation of iron components after the irradiation with IR. According to this hypothesis, although the percentage of iron oxides in the encrustation is indeed very low $(\sim 0.6 \%[13])$, their chemical transformation may result into yellowing of the treated surfaces, which can be significant. Studies performed by different research groups have detected the presence of nanospheres rich in iron on the surface of technical samples containing hematite and graphite, after irradiation with IR [14], or the reduction of iron components present in pollution crusts, such as $\mathrm{Fe}_{2} \mathrm{O}_{3}$, into magnetite $\left(\mathrm{Fe}_{3} \mathrm{O}_{4}\right)$ [15]. Recently it has been suggested that both hematite $\left(\mathrm{Fe}_{2} \mathrm{O}_{3}\right)$ and goethite $(\alpha-\mathrm{FeO}(\mathrm{OH}))$ exist at the encrustation [9] and that yellowing can possibly be attributed to the transformation of hematite $\left(\mathrm{Fe}_{2} \mathrm{O}_{3}\right)$ to goethite $(\alpha-\mathrm{FeO}(\mathrm{OH}))$ or maybe maghemite $\left(\gamma-\mathrm{Fe}_{2} \mathrm{O}_{3}\right)$.

- The selective vaporization of black components and the preservation of a gypsum matrix on the marble's surface after the irradiation at $1064 \mathrm{~nm}$ below the ablation threshold. The dark coloured particles highly absorb the IR radiation and are removed from the surface leaving behind a gypsum matrix with very small holes in their place. The incident, on the surface, light is then differentially scattered (according to Rayleigh scatter) causing a shift to the cleaned surface towards yellow [16].

In order to approach this delicate issue, namely the discoloration of the treated surface of polluted marble following irradiation with IR laser beams, research efforts have been focused on the identification of the composition of the environmental encrustation $[13,17]$ in relation to the series of cleaning tests at different laser parameters $[5,9,18]$. Physicochemical analyses and evaluation of the treated surfaces and/or the material removed $[7,12,15,19]$ were also employed to further investigate the yellowing issue.

\subsection{The double wavelength methodology}

With the aim to overcome the challenge of laser induced yellowing, a laser cleaning methodology has been developed [20], in which two wavelengths from the same laser system are used simultaneously to effectively remove the encrustation from the marble's surface without changes to its colour, chemistry or delicate structure. The success of this methodology relies on the blending of an IR and a UV beam, at a specific ratio and fluence values, which provides the opportunity to efficiently remove the encrustation. 
To further investigate the yellowing effect and understand the mechanisms of the two-wavelength blending, a series of studies were undertaken. They involved tests using different laser parameters (wavelengths, laser pulse duration, fluence values etc.) on technical samples simulating black crusts, as well as on real fragments.

It should be noted that pollution accumulations on outdoors stonework are particularly complex systems and their composition, morphology and stratigraphy may vary among monuments of different materials; located in different countries (depending on their climatic conditions, pollution level and history) and representing various historic eras. This is an important parameter to take into account when studying laser cleaning and interpreting analytical data for evaluation purposes.

This research has been focused on the investigation of fragments of minor historic value taken from the Athenian Acropolis. Analytical techniques provided the chemical and molecular composition of the samples before and after laser irradiation in order to understand the physicochemical processes taking place while using two laser wavelengths separately or simultaneously.

\section{Materials and methods}

\subsection{Samples}

Irradiation tests were performed on a marble fragment with real environmental homogeneous thin compact crust. The fragment is part of a freshly-cut marble complement added onto the Parthenon Frieze in Athens, Greece, during the restoration actions by Balanos in 1960s. During this restoration marble pieces (of the same quarry in Pentelic mountain) have been added to replace miss- ing or damaged parts of the sculpted Frieze blocks. These complements bear the same accumulations with the rest of the Frieze and thus have been chosen for the purpose of this study [4].

\subsection{Experimental parameters}

The laser irradiation tests were performed with a Q-Switched Nd:YAG nanosecond (ns) laser (a customised system of Spectron, series SL850) emitting in the first and third harmonic wavelengths, in the infrared (IR, 1064nm) and ultraviolet (UV, $355 \mathrm{~nm}$ ) respectively. The laser provides the opportunity to emit each of the wavelengths separately and simultaneously. When the two beams are used simultaneously, the operator has the opportunity to select the fluence (F, measured in $\mathrm{J} / \mathrm{cm}^{2}$ ) output of each beam in order to get different ratios of the fluence of the IR $\left(\mathrm{F}_{\mathrm{IR}}\right)$ and the UV $\left(\mathrm{F}_{\mathrm{UV}}\right)$ beams. Thus it is possible to select a final output ratio $\mathrm{F}_{\mathrm{IR}} /$ $F_{U V}=1 / 4$ or $4 / 1$ and therefore get a different cleaning result. It should be noted that the decision on the ratios presented in this paper is the result of extensive past studies [5].

A marble fragment covered with environmental homogeneous thin compact crust was irradiated in a variety of laser parameters (fluence value, number of pulses for each beam, as well as their combination). Three clearly distinctive in colour and effectiveness, cleaning results have been chosen for this study and are indicated in Fig. 1. Specifically, the area on the left side of Fig. 1 was irradiated at $1064 \mathrm{~nm}$ with 5 pulses of fluence $\mathrm{F}=0.8 \mathrm{~J} / \mathrm{cm}^{2}$. The middle area was irradiated with the same number of pulses at $355 \mathrm{~nm}$ and $\mathrm{F}=0.2 \mathrm{~J} / \mathrm{cm}^{2}$. A combination of the two beams (in synchronous mode) in the same fluence values (in ratio $\mathrm{F}_{\mathrm{UV}} / \mathrm{F}_{\mathrm{IR}}=1 / 4$ ) was used for the area shown in the right side of 
Fig. 1. The fluence values shown correspond to the cleaning thresholds of this crust in both wavelengths. The tests were performed in dry conditions and the laser was operating in low repetition rate $(3 \mathrm{~Hz})$. high sensitivity with low dark counts. The objective lens used for these analyses was $\times 20$ and the beam power on the sample was adjusted at $30 \mathrm{~mW}$. Typical exposure time on the CCD was 30 s per scan and the spectra

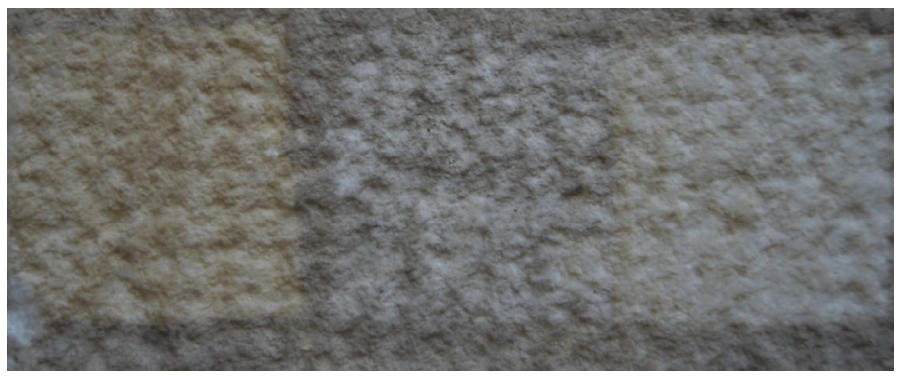

Fig. 1. Detail of marble

fragment with environmental homogeneous thin compact crust after laser irradiation tests: (left) $1064 \mathrm{~nm}, \mathrm{~F}=0.8$ $\mathrm{J} / \mathrm{cm}^{2}$, (middle) $355 \mathrm{~nm}$, $\mathrm{F}=0.2 \mathrm{~J} / \mathrm{cm}^{2}$ and (right) their synchronous application, $\mathrm{F}_{\mathrm{UV}} / \mathrm{F}_{\mathrm{IR}}=1 / 4$ (each square is $\left.2 \times 2 \mathrm{~cm}^{2}\right)$.

\subsection{Analytical Documentation}

\section{Raman spectroscopy}

Raman analysis was applied before and after laser irradiation on the real marble samples with the aim to detect possible variations on the molecular composition of the surface. The mobile Raman spectrometer (HE 785, Horiba-Jobin Yvon, France) was employed with excitation at $785 \mathrm{~nm}$ provided by a cw (continuous wave) diode laser coupled to an optical head ([21, 22]). A light-emitting diode (LED) and a high resolution colour camera (video microscope) are also part of the optical head and offer a very clear view of the area under investigation, necessary for positioning the beam on individual areas. The scattered radiation is collected through the objective lens, passes through an edge filter that cuts off Rayleigh scattering, and finally is focused into an optical fiber that is fed into a compact spectrograph, equipped with a concave grating, which provides spectral coverage in the range of $120-3395 \mathrm{~cm}^{-1}$ at a spectral resolution of about $10-15 \mathrm{~cm}^{-1}$. The detector, a Synapse TM CCD (1024 $\times$ 256 pixels), is Peltier-cooled and features recorded correspond to an average of 2 consecutive scans of the same point.

\section{LIBS Spectroscopy}

Laser induced breakdown spectroscopy (LIBS) was used aiming to detect and analyse the chemical composition of the samples surface before and after the laser treatment with a variety of parameters. The mobile LIBS spectrometer (LMNTII+), developed and constructed at IESL-FORTH, was used for this study [21,22]. The instrument uses a compact passively Q-switched Nd:YAG laser emitting pulses at $1064 \mathrm{~nm}(10 \mathrm{~mJ} /$ pulse, $10 \mathrm{~ns}$ ). Pulses are focused by means of plano-convex lens $(f=+75 \mathrm{~mm})$ on the surface of the object. The plasma emission is collected through the same lens and transmitted via an optical fiber that splits it into a dual spectrometer unit (Avaspec-2048-2-USB2, Avantes) that records emission spectra across a wavelength range extending from 200 to $660 \mathrm{~nm}$, with resolution of about $0.2-0.3 \mathrm{~nm}$. The laser along with necessary optics and a miniature CCD camera are integrated in a light-weight optical probe head. The camera offers a magnified view of the object surface 
during the analysis and permits accurate aiming of the laser beam with the aid of a cross-hair indicator superimposed on the image. All LIBS spectra were collected with a time-delay of $1.3 \mu$ s with respect to the laser pulse and for an integration time of 1 ms. A nearly circular spot with a diameter of about $150-300 \mu \mathrm{m}$ is probed by the laser beam.

\section{Results and discussion}

\subsection{Visual evaluation}

Visual observation of the sample shows that the areas treated with IR irradiation appear yellow, areas treated with UV irradiation are grey while the ones treated with both beams, simultaneously, are closer to the colour expected in this case.

\subsection{Raman evaluation}

Upon microRaman analysis calcite $\left(\mathrm{CaCO}_{3}-157,285\right.$ and $\left.1088 \mathrm{~cm}^{-1}\right)$ and/or gypsum $\left(\mathrm{CaSO}_{4} \cdot 2 \mathrm{H}_{2} \mathrm{O}-1009 \mathrm{~cm}^{-1}\right)$ were detected on the homogeneous thin compact crust as well as on all the surfaces treated either with IR and UV, separately or simultaneously. An intense fluorescent signal (Fig. 2) was observed on the crust and the area irradiated at $1064 \mathrm{~nm}$, though this is clearly reduced on areas irradiated at $355 \mathrm{~nm}$ or with the combination of 1064 and $355 \mathrm{~nm}$. The origin of the fluorescence can be attributed to organic compounds previously detected on the homogeneous thin compact crust [6]. Indeed, studies on pollution accumulations (on marble and limestone) by means of GC-MS detected a number of components, part of which show intense fluorescence.

The presence of these fluorescent compounds indicates that either these com-

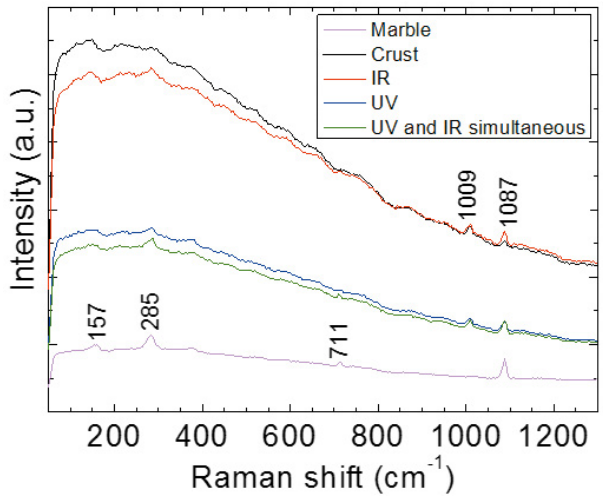

Fig. 2. Raman spectra acquired from the marble fragment presented in Fig. 1. Crust and areas treated with IR exhibit an intense fluorescence, while areas irradiated with UV or the combination of the 2 beams have a lower fluorescence, which is closer to the one of marble.

pounds were not affected, and thus they were not removed, upon IR irradiation, or they could possibly be induced by the laser beam as indicated by Oliveira et al. in their study [18]. Further analysis is currently performed in order to respond to this question. Among the arguments that support the first hypothesis (fluorescing compounds not removed by the IR laser beam) is that the fluorescing signal was detected on the sample prior to its irradiation/cleaning and the intensities of the Raman spectra recorded from the IR treated and reference areas are comparable. No additional fluorescence signal was detected after the IR radiation and thus the hypothesis that the detected fluorescence is due to IR laser irradiation seems unlikely for our samples. On the other hand, the reduction of the fluorescence after UV irradiation implies the removal of this fluorescent material from the surface of the sample. This difference in the Raman spectra of the irradiated areas suggests that the ablation occurs in a different and probably preferential/selective way for the two wavelengths. 


\subsection{LIBS analysis}

LIBS analysis on the same areas of the sample detected the presence of a number of elements with most important $\mathrm{Ca}, \mathrm{Al}, \mathrm{Fe}$, $\mathrm{Mg}, \mathrm{Si}$ and $\mathrm{Na}$. Characteristic spectra from these measurements are presented in Fig. 3. In order to detect and investigate the possible alterations in the elemental composition of the laser irradiated areas, either separately or simultaneously, the ratio of the chemical elements $\mathrm{Fe}$ and $\mathrm{Ca}$ was studied. The major components of the encrustation are $\mathrm{Fe}, \mathrm{Si}$ and $\mathrm{Mg}[17,23]$ but on the basis of previous research indicating that Fe holds a significant role in laser yellowing, our study was focused on the Fe concentration and behaviour for different irradiation parameters. In order to
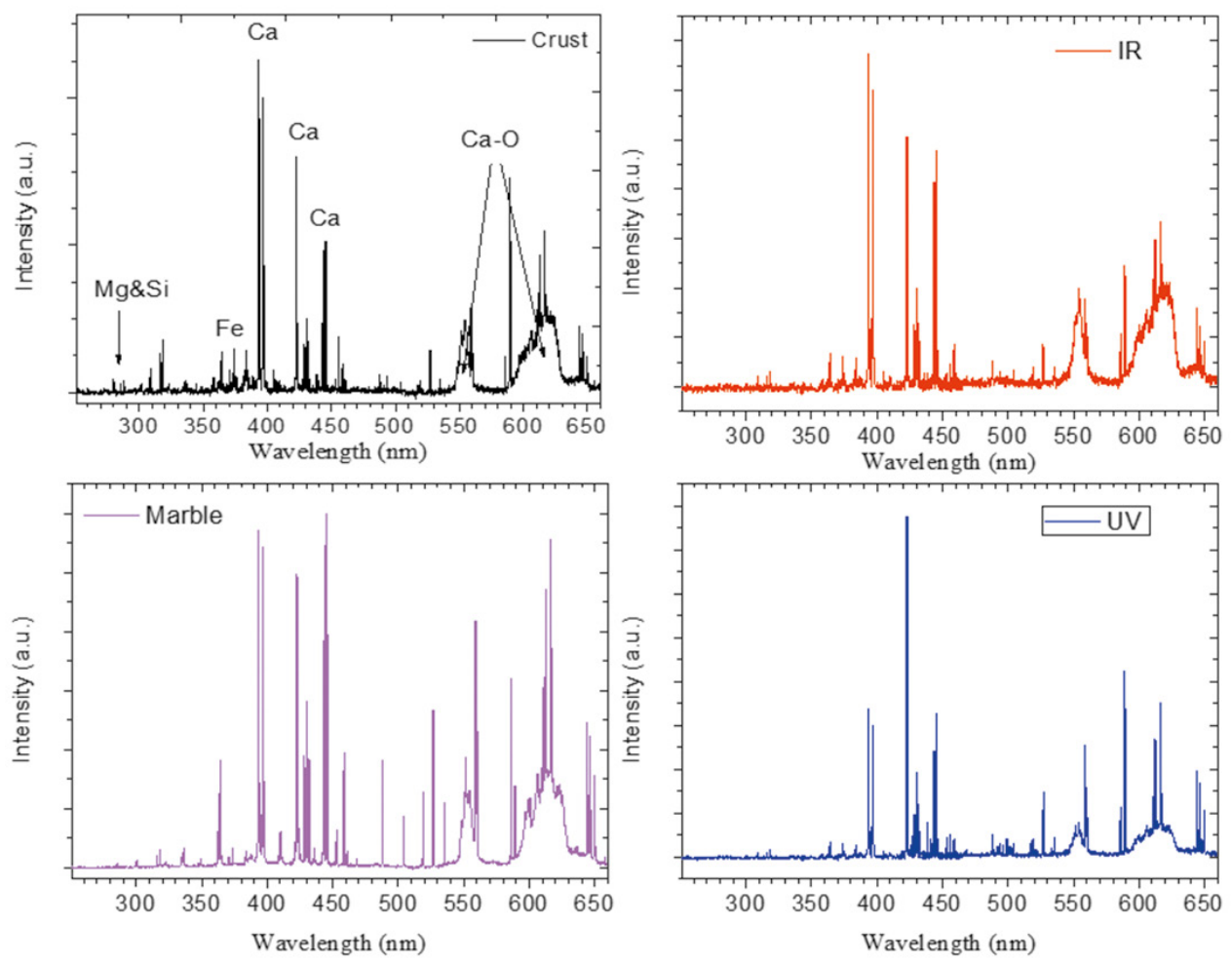

achieve accurate results, the detected signal intensity of the Fe peak was normalized with another element that exhibits a constant concentration. The selected component was Ca since its presence is homogenous among the sample. Therefore, data analysis was performed by investigating the ratio of $\mathrm{Fe} / \mathrm{Ca}$ for each irradiation parameter.

The proper selection of spectral lines was very important for achieving accurate results. The spectral lines chosen for this study are $\mathrm{Fe} 438.354 \mathrm{~nm}$ and $\mathrm{Ca} 445.478 \mathrm{~nm}$. Calcium (Ca) exhibits a number of emission spectral lines but $445.478 \mathrm{~nm}$ was chosen because it originates from a highly excited state with big excitation energy and therefore

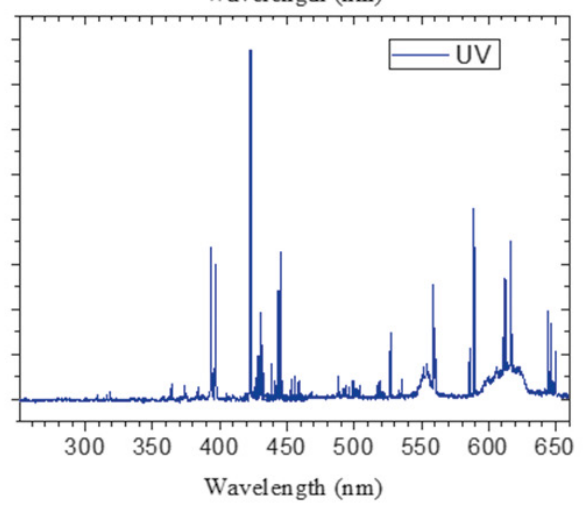
low self-absorption possibility. The available

Fig. 3. LIBS Spectra obtained from the marble fragment prior (crust and marble reference) and after laser irradiation (with IR and UV beams). 
spectral lines for iron are at 302.064, 371.994, 374.556 and $385.991 \mathrm{~nm}$ in the UV range and $438.354,440.475 \mathrm{~nm}$ in the visible region of the LIBS spectrum. The spectral lines at $371.994,374.556$ and $385.991 \mathrm{~nm}$ coincide with the molecular emission band of the $\mathrm{CN}$, and thus cannot be used. Furthermore, the line at $302 \mathrm{~nm}$ is too weak to provide reliable results. Therefore, the line at $438 \mathrm{~nm}$ has been used for the analysis because it has a higher intensity than the one at $440 \mathrm{~nm}$. Of significant importance is the average value, taken from 10 different spots/points on the studied areas, of the ratio corresponding to the first LIBS pulse of each irradiation condition. It was decided to take into account for our measurements only the first LIBS pulse as, due to ablation, any additional pulse will represent a different/altered surface. Figure 4 shows the average value of $\mathrm{Fe} / \mathrm{Ca}$ ratio for each irradiation parameter (described before in the caption of Fig. 1). A significant proportion of iron $(\mathrm{Fe})$ to calcium $(\mathrm{Ca})$, and therefore a greater concentration of iron, is tion than the area treated simultaneously with IR and UV. In addition, the reference marble has the lowest proportion of $\mathrm{Fe} / \mathrm{Ca}$ and it is comparable to the area treated with IR and UV simultaneously. The above lead to the conclusion that IR radiation removes preferentially the iron compounds, and thus that the ablation occurs in a selective way. On the other hand, the selective removal of the iron compounds does not occur upon UV irradiation, while the simultaneous use of both wavelengths shows the most efficient iron removal and results into a surface very similar, in iron concentration, to the one of the marble.

\section{Conclusions}

In summary, analytical investigation on a marble fragment with thin environmental homogeneous compact crust, which was irradiated with different laser beams and parameters, allows us to further under-

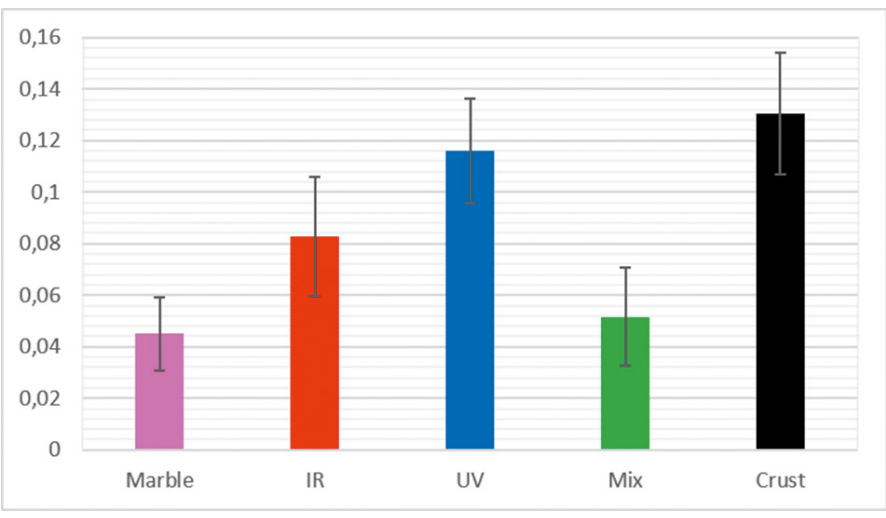

Fig. 4. The average Fe/ Ca ratio obtained from the first pulse of the LIBS spectra accumulated from 10 different spots for each of the irradiated areas. The error bars correspond to the standard deviation of the Fe/ Ca ratio. detected in areas with crust deposits and areas treated with UV radiation. Furthermore, the area treated with IR presents a lower ratio value, compared to the one irradiated with UV or the crust, but higher iron concentra- stand the ablation mechanisms that can be significant upon irradiation with different laser wavelengths. Optical observation confirmed the different ablation processes that take place upon irradiation with IR and 
UV wavelengths, and/or their combination, and thus to the final colour of the treated surface. Raman spectroscopy showed reduced fluorescence on the areas treated with UV wavelength (or where UV was involved), suggesting a selective removal of organic/fluorescing compounds from the surface of the sample in this laser ablation regime. On the contrary ablation with IR, at the fluence values employed in this study, does not remove the bulk of the crust and possibly these fluorescing particulates that are trapped therein. The hypothesis that this ablative regime may cause the presence of these types of fluorescing has been also examined. In addition, LIBS analysis detected a lower Fe concentration in the area treated with IR suggesting the selective removal of iron particles from the marbles surface, in contrast with areas irradiated with UV wavelengths.

Taking all the above into account, it is suggested that the ablation mechanisms occurring during the irradiation of environmental homogeneous compact crust from stonework are closely dependant to the beam wavelength. On the basis of the different absorption properties of the involved materials to the applied laser wavelength, it has been shown that different removal processes vary significant in each case. Our hypothesis is that upon UV irradiation organic fluorescing components are preferentially removed while upon IR irradiation Fe-rich particulates are effectively ablated, as shown by our LIBS measurements. By combining the two beams simultaneously dark coloured particles and organic/fluorescing compounds are equally removed from the crust, resulting thus into a homogeneous and efficient cleaning procedure. Of course, the ratio of the two beams is an important parameter in the whole process.
It has also to be noted that in our experiments the aim was to understand the ablative mechanisms rather than clean our samples. Therefore, for the purpose of our study it was important to irradiate the crust, but not remove it totally, in order to have a base for investigation. This remaining material, of different colour and composition, was analysed and studied. Further experiments are in progress, focused on the study of different ablation parameters through technical simulation samples and real marble fragments, in order to elucidate the ablation mechanisms and processes.

\section{References}

[1] P. Pouli, M. Oujja, and M. Castillejo, "Practical issues in laser cleaning of stone and painted artefacts: optimisation procedures and side effects", Applied Physics A, 106 (2012) 447-464, DOI: 10.1007/ s00339-011-6696-2.

[2] P. Pouli, V. Zafiropulos, and C. Fotakis, "The Combination of Ultraviolet and Infrared Laser Radiation for the Removal of Unwanted Encrustation from Stonework; a Novel Laser Cleaning Methodology" in: Proceedings of the $10^{\text {th }}$ International Congress on Deterioration and Conservation of Stone, Stockholm, Sweden, June 27-July 2, 2004, Daniel Kwiatkowski and Runo Lofvendahl (Eds.), ICOMOS, Sweden, 2004, pp. 315-322, ISBN: 9163114585.

[3] G. Marakis, P. Pouli, V. Zafiropulos, and P. Maravelaki-Kalaitzaki, "Comparative study on the application of the first and the third harmonic of a Nd:YAG laser system to clean black encrustation on marble", Journal of Cultural Heritage, 4 (2003) 83-91, DOI: 10.1016/S1296-2074(02)01208-6.

[4] P. Pouli E. Papakonstantinou, K. Frantzikinaki, A. Panou, G. Frantzi, C.Vasiliadis, and C. Fotakis, "The two-wavelength laser cleaning methodology; theoretical background and examples from its application on $\mathrm{CH}$ objects and monuments with emphasis to the Athens Acropolis sculptures", Heritage Science, 4 (2016) 9-20, DOI: 10.1186/s40494-016-0077-2. 
[5] P. Pouli, K. Frantzikinaki, E. Papakonstantinou, V.Zafiropulos, and C. Fotakis, "Pollution Encrustation Removal by Means of Combined Ultraviolet and Infrared Laser Radiation : The Application of this Innovative Methodology on the Surface of the Parthenon West Frieze" in: $5^{\text {th }}$ International Conference on Lasers in the Conservation of Artworks, LACONA V'03 Proceedings, Osnabrück, Germany, Sept. 15-18, 2003, K. Dickmann, C. Fotakis, and J. F. Asmus, (Eds.), Springer, Berlin Heidelberg, 2005,(100) pp. 333-340, DOI: $10.1007 / 3-540-27176-7 \_41$.

[6] P. Pouli, C. Fotakis, B. Hermosin, C. Saiz-Jimenez, C. Domingo, M. Oujja, and M. Castillejo., "The laser-induced discoloration of stonework; a comparative study on its origins and remedies" Spectrochimica Acta Part A: Molecular and Biomolecular Spectroscopy, 71 (2008) 932-945, DOI: 10.1016/j. saa.2008.02.031.

[7] C. de Oliveira, V. Vergès-Belmin, D. Demaille, and P. Bromblet, "Lamp black and hematite contribution to laser yellowing: A study on technical gypsum samples", Studies in Conservation, 61 (2016) 136-145, DOI: 10.1179/2047058415Y.0000000003.

[8] V. Vergès-Belmin and C. Dignard, "Laser yellowing: myth or reality?", Journal of Cultural Heritage, 4 (2003) 238-244, DOI: 10.1016/S12962074(02)01203-7.

[9] C. de Oliveira V. Vergès-Belmin, J. Lafait, M. Swider, C. Andraud, A. Tournié, and L. Galoisy, "Contribution of goethite to laser-induced stone yellowing”, Applied Physics A, 122 (2016) 467-476, DOI: 10.1007/s00339-016-9818-z.

[10] V. Vergès-Belmin, C. Pichot, and G. Orial, “Elimination de croutes noires sur marbre et craie : a quel niveau arreter le nettoyage?" in: Conservation of stone and other materials: proceedings of the international RILEM/UNESCO congress, UNESCO headquarters, Paris, June 29-July 1, 1993, E.\&F.N. Spon, London, UK, 1993, pp 534-541, ISBN:0419184104.

[11] S.S. Potgieter-Vermaak, R.H.M. Godoi, R. Grieken, J. H. Potgieter, M. Oujja, and M. Castillejo, "Micro-structural characterization of black crust and laser cleaning of building stones by micro-Raman and SEM techniques", Spectrochimica Acta Part A: Molecular and Biomolecular Spectroscopy, 61 (2005) 2460-2467, DOI : 10.1016/j.saa.2004.09.010.
[12] M. Gavino, B. Hermosin, M. Castillejo, M. Oujja, E. Rebollar, V. Vergès-Belmin, W. Nowik, and C. Saiz-Jimenez, "Black crusts removal: the effect of stone yellowing and cleaning strategies" in: Air Pollution and Cultural Heritage (Proceedings of the International workshop on Air Pollution and Cultural Heritage), Seville, Spain, December 1-3, 2003, C. Saiz-Jimenez (Ed.), London: AA Balkema, 2004, pp 239-245, DOI: 10.1201/b17004-37.

[13] P. Maravelaki-Kalaitzaki, "Black crusts and patinas on Pentelic marble from the Parthenon and Erechtheum (Acropolis, Athens): Characterization and origin", Analytica Chimica Acta, 532 (2005) 187-198, DOI: 10.1016/j.aca.2004.10.065.

[14] S. Klein, F. Ferksanati, J. Hildenhagen, K. Dickmann, H. Uphoff, Y. Marakis, and V. Zafiropulos, "Discoloration of marble during laser cleaning by Nd:YAG laser wavelengths", Applied Surface Science, 171 (2001) 242-251 DOI: 10.1016/S01694332(00)00706-6.

[15] M. Gracia, M. Gaviño, V. Vergès-Belmin, B. Hermosin, W. Nowik, and C. Sáiz-Jiménez, "Mössbauer and XRD Study of the Effect of Nd:YAG-1064 nm Laser Irradiation on Hematite Present in Model Samples", in: Lasers in the Conservation of Artworks: LACONA V Proceedings, Osnabrück, Germany, Sept. 15-18, 2003, K. Dickmann, C. Fotakis, and J. F. Asmus, (Eds.), Springer, Berlin Heidelberg, 2005, pp. 341-346, DOI: 10.1007/3-540-27176-7_42.

[16] V. Zafiropulos, P. Pouli, V. Kylikoglou, P. Maravelaki-Kalaitzaki, B. S. Luk'yanchuk, and A. Dogariu, "Synchronous Use of IR and UV Laser Pulses in the Removal of Encrustation: Mechanistic Aspects, Discoloration Phenomena and Benefits", in: Lasers in the Conservation of Artworks: LACONA V Proceedings, Osnabrück, Germany, Sept. 15-18, 2003, K. Dickmann, C. Fotakis, and J. F. Asmus, (Eds.), Springer, Berlin Heidelberg, 2005, pp. 311-318,. DOI: 10.1007/3-540-27176-7_38.

[17] P. Maravelaki-Kalaitzaki, D. Anglos, V. Kilikoglou, and V. Zafiropulos, "Compositional characterization of encrustation on marble with laser induced breakdown spectroscopy", Spectrochimica Acta Part B:Atomic Spectroscopy, 56 (2001) 887-903, DOI: 10.1016/S0584-8547(01)00226-9. 
[18] C. de Oliveira, P. Bromblet, A. Colombini, and $\mathrm{V}$. Vergès-Belmin, "Medium-wave ultraviolet radiation to eliminate laser-induced yellowing generated by the laser removal of lamp black on gypsum", Studies in Conservation, 60 sup1 (2015) S34-S40, DOI: 10.1179/0039363015Z.000000000205.

[19] C. De Oliveira, V. Vergès-Belmin, D. Demaille, and P. Bromblet, "Lamp black and hematite contribution to laser yellowing: A study on technical gypsum samples.", Studies in Conservation, 61 (2016) 136-145, DOI: 10.1179/2047058415Y.0000000003.

[20] K. Frantzikinaki, G. Marakis, A. Panou, C. Vasiliadis, E. Papakonstantinou, P. Pouli, T. Ditsa, V. Zafiropulos, and C. Fotakis, "The Cleaning of the Parthenon West Frieze by Means of Combined IRand UV-Radiation", in: Lasers in the Conservation of Artworks (Proceedings of LACONA VI conference), Vienna, Austria, September 21-25, 2005, J. Nimmrichter, W. Kautek, and M. Schreiner, (Eds.) Springer
Verlag, Berlin Heidelberg 2007, pp. 97-104, DOI: 10.1007/978-3-540-72310-7_12.

[21] P. Westlake et al., "Studying pigments on painted plaster in Minoan, Roman and Early Byzantine Crete. A multi-analytical technique approach", Analytical and Bioanalytical Chemistry, 402 (2012) 1413-1432, DOI: 10.1007/s00216-011-5281-z.

[22] Z.E. Papliaka, A. Philippidis, P. Siozos, M. Vakondiou, and K. Melessanaki, "A multi-technique approach, based on mobile / portable laser instruments, for the in situ pigment characterization of stone sculptures on the island of Crete dating from Venetian and Ottoman period", Heritage Science, 4 (2016) 1-18, DOI: 10.1186/s40494-016-0085-2.

[23] P. V Maravelaki, V. Zafiropulos, V. Kilikoglou, M. Kalaitzaki, and C. Fotakis, "Laser-induced breakdown spectroscopy as a diagnostic technique for the laser cleaning of marble", Spectrochimica Acta Part B: Atomic Spectroscopy, 52 (1997) 41-53, DOI: 10.1016/S0584-8547(96)01573-X. 


\title{
The potential of OCT for assessing laser assisted removal of deposits from ceramic tiles
}

\author{
Magdalena Iwanicka ${ }^{1 \star}$, Jędrzej Musiela ${ }^{2}, J^{2}$ adwiga W. Łukaszewicz ${ }^{1}$, \\ Henryk Stoksik ${ }^{3}$, Marcin Sylwestrzak ${ }^{4}$ \\ 1 Institute for the Study, Restoration and Conservation of Cultural Heritage, Nicolaus Copernicus \\ University, ul. Sienkiewicza 30/32, 87-100 Toruń, Poland \\ 2 Restauro Sp. z o.o., ul. Wola Zamkowa 6, 87-100 Toruń, Poland \\ 3 Faculty of Ceramics and Glass, The Academy of Art and Design, Plac Polski 3/4, 50-156 Wrocław, \\ Poland \\ 4 Institute of Physics, Faculty of Physics, Astronomy and Informatics, Nicolaus Copernicus University, \\ ul. Grudziądzka 5, 87-100 Toruń, Poland \\ * Corresponding author: magiwani@gmail.com
}

\begin{abstract}
Optical Coherence Tomography (OCT), the technique of non-invasive imaging of transparent and semi-transparent sub-surface layers was employed to monitor the state of glazes of historic tiles before and after laser ablation trials. The laser used was Nd:YAG Thunder Art system from LightForArt (El. En. S.p.A.).

In this paper, OCT cross-sectional images of both fragments and preserved historic tiles before, during and after laser ablation are shown to examine the process of the removal of surface deposits as well as mid-twentieth century conservation coatings. In case of some of the ceramic fragments (donated to science) the destruction of the glaze layer after a few steps of laser cleaning was induced intentionally and then visualized with OCT.

The experiments performed confirmed that OCT is a potential diagnostic tool for the assessment and monitoring of laser removal of deposits from semi-transparent glazes on historic tiles, as well as enabled to define and identify specific risk factors for these objects, such as pre-existing cracks or delaminations within the glaze layer.
\end{abstract}

Keywords: optical coherence tomography (OCT), laser, ablation, cleaning, glazed ceramics, SEM-EDS

\section{Introduction}

Ceramic artefacts from archaeological sites suffer from a vast range of deteri oration phenomena, some of which are occurring in glaze layers. Such problems, caused mainly by long exposure to environmental factors, are: cracks, delaminations, pitting, all resulting eventually in the formation of losses within the glaze. Also surface dirt and old conservation coatings contribute to the complexity of present conservation issues. The cleaning of such objects is both difficult and controversial, since some conservators believe 
that the corroded glaze layer should not be removed. "Wet" cleaning or solvent cleaning of such artefacts is possible; nevertheless, it poses danger of transferring particles of unwanted materials into the porous structure of the object. Therefore, since during laser cleaning deposits evaporate from the surface and do not penetrate into fine cracks, laser ablation seems to be an adequate alternative to conventional cleaning. However, in some cases, laser cleaning may also be not safe for the object. Pre-existent destruction phenomena in glaze layer, such as delaminations and cracks, as well as raised temperature on the surface of glaze (lead based glazes have low melting point) may result in damages.

The aim of this work was to evaluate the applicability of Optical Coherence Tomography for following and controlling the process of laser removal of unwanted mineral deposits and secondary layers. As for our knowledge it is the first time that OCT (here in combination with SEM-EDS) has been used for progress assessment of laser cleaning tests on glazed ceramic objects.

It is important to note that the main intention of the authors of this experiment was to employ OCT as an aid for free-hand cleaning by a commercial laser system, which would be used in the typical restoration studio. Therefore, the emphasis was put on the early damage detection in glaze layers, which was possible thanks to the OCT data and not on developing new approaches to laser cleaning of ceramic tiles.

\section{Materials and methods}

\subsection{Investigated ceramic objects}

A group of medieval archeological ceramic tiles with various deterioration problems (both fragments and whole preserved tiles) with glazes semi-transparent to infra-red radiation were chosen from the collection of the District Museum in Torun, Poland. In this study, we present the results of experiments on three objects, all found in the archaeological site at the Teutonic Order Castle in Torun during the excavation works in 1958-1966. Their exact place of origin is, however, uncertain, since the castle ruins had been used as a wasteyard for the whole old city area from the middle $15^{\text {th }} \mathrm{c}$., when the castle was demolished, up to late $19^{\text {th }}$ century. Two tile fragments of uncertain time of origin (17 $7^{\text {th }}$ c.?) and one piece (with depiction of St. Dorothy, $15^{\text {th }} \mathrm{c}$.) were examined by means of macroXRF mapping and SEM-EDS scanning (on microsamples) in order to establish the technology of origin (stratigraphy and glaze composition) as well as surface morphology and state of preservation. The pieces were subsequently treated to laser cleaning tests with the aid of OCT as a tool to follow and assess the ablation process.

\subsection{Optical Coherence Tomography}

Optical Coherence Tomography (OCT) [1,2] is an optical interferometric non-contact and non-invasive technique of depth-resolved imaging within media scattering and/or absorbing near-infrared light moderately. A narrow beam of infrared light (spectral range: $770-970 \mathrm{~nm}$ ) penetrates the object and is scattered at its structural elements. Then it is brought to interference in a Michelson interferometer and a structure of the object along the beam (an A-scan) is reconstructed from the detected signal. This procedure is repeated line-by-line for the whole examined cross-section.

OCT's ability of scanning areas (up to 12 $\times 12 \mathrm{~mm}^{2}$ ) in order to obtain information about sequence, continuity and thickness of 
the surface and subsurface layers makes it especially suitable for assessment of removal of unwanted layers [3-7]. For examination whose results will be presented herein, a high resolution (ca $2 \mu \mathrm{m}$ axial resolution in material, $15 \mu \mathrm{m}$ lateral resolution) spectral domain OCT instrument utilising near infrared $(770-970 \mathrm{~nm})$ radiation of $0.8 \mathrm{~mW}$ power beam at the object was utilised.

Thanks to the motor-driven translation of the OCT head away from the examination region for a time of the laser ablation test and then back to the exactly same position, scans obtained before and after laser ablation can be correlated easily and compared in a straightforward manner. The OCT tomograms (Figs. 4-6) are shown in false colour scale with areas not-scattered or not reachable for light shown in black. Low scattering centres are shown in blue whereas high scattering - in warm colours (from green to red). The uppermost region in all tomograms is just an air above the object. Then the air-glaze interface is seen as a strong green line. Non-scattering glaze layer below is mostly black and finally the surface of strongly absorbing ceramic body is visible in bluish hue. Surface deposits and cracks strongly scatter the light and are well visible in green. Notice that the tomograms are vertically stretched for better readability. Scale bars in all the tomograms shown in the figures are equivalent to $200 \mu \mathrm{m}$ (in air) in both directions.

\subsection{Laser ablation tests}

For laser ablation of unwanted material an Nd:YAG Thunder Art system from Light For Art (El.En. S.p.A.) was operated at $1064 \mathrm{~nm}$. Laser pulses of 8 ns length and spot size $\approx$ $7 \mathrm{~mm}$ were used with repetition rate $7 \mathrm{~Hz}$. Different energy values and fluency ranges were used for different pieces:
Tile fragment $\# 1: \mathrm{E} \approx 600-700 \mathrm{~mJ}$; fluency $\approx 1.6-1.8 \mathrm{~J} / \mathrm{cm}^{2}$

Tile fragment $\# 2: \mathrm{E} \approx 500-610 \mathrm{~mJ}$; fluency $\approx 1.3-1.6 \mathrm{~J} / \mathrm{cm}^{2}$

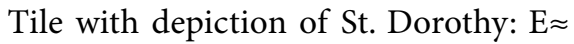
$350-370 \mathrm{~mJ}$; fluency $\approx 0.91-0.96 \mathrm{~J} / \mathrm{cm}^{2}$

\subsection{Additional analytical techniques MacroXRF scanning}

MacroXRF scanning is an imaging and semi-quantitative technique of elemental analysis which enables extended surface scans over thousands of square centimetres during a single examination. For this study, the M6 JetStream scanning system from Bruker Nano $\mathrm{GmbH}$ was employed [8]. It permits large surface scans - up to $70 \mathrm{~cm} \times 55 \mathrm{~cm}$ with lateral resolution up to $50 \mu \mathrm{m}$. It comprises a X-ray lamp working at $50 \mathrm{kV} / 600 \mu \mathrm{A}$ conditions with Rh anode and a polycapillary focusing optics. It is equipped with the $30 \mathrm{~mm}^{2}$ Silicon Drift Detector (SDD) with $\mathrm{Zr}$ window. An open-air system permits imaging elements of atomic numbers not lower than 16 (sulphur).

\section{SEM-EDS scanning}

Scanning electron microscope (SEM) from LEO Electron Microscopy Ltd, UK, model $1430 \mathrm{VP}$ was used for imaging of topography of the samples. Energy dispersive X-ray spectrometer (EDS) Quantax 200 (detector XFlash 4010) from Bruker AXS was utilised for elemental mapping of the samples' cross-sections.

\section{Results}

\subsection{Tile fragment \#1}

The fragment listed in this contribution as \#1 (MOT - P 72 from the District Museum 
in Torun, $17^{\text {th }}$ c.?) has a lead-based, copper tinted glaze on whitish engobe (Fig 1,2). The piece was laser cleaned in few spots using similar laser parameters (ca 600-700 mJ, fluency: $1.6-1.8 \mathrm{~J} / \mathrm{cm}^{2}$ ). Afterwards, macro XRF scanning was performed and finally four microsamples were collected, two from spots in uncleaned areas and two from spots located in cleaned areas showing different visual properties after the ablation tests (Fig. 1). calcium-rich layer on top of the glaze. The discontinuity of the surface marked by red and blue arrows in Fig. $2 \mathrm{~b}$ indicate that this layer is not a mere secondary deposit, but in fact a disintegrated upper layer of glaze, which accumulated calcium ions either from the surrounding or as a result of leaching of the deeper layers of the glaze.

Two different results of laser ablation tests on this object are presented in Figs. 3 and 4.

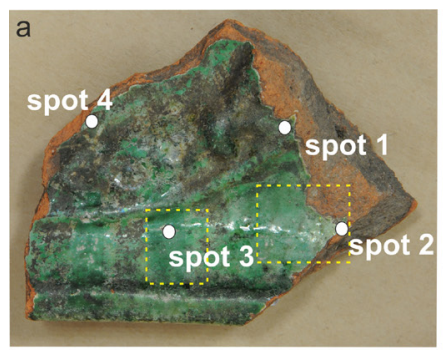

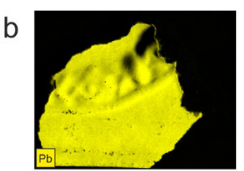

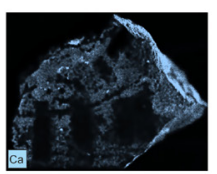

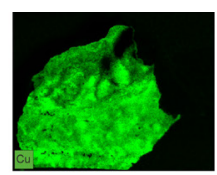

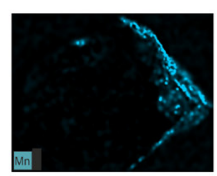

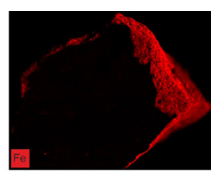

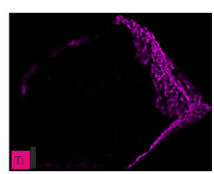

Fig. 1. a. Tile fragment \#1 in visible light after laser ablation tests. Dashed lines mark areas of laser ablation tests. White dots mark sample collection spots. b. Macro XRF scans after laser ablation tests.

Photography (Fig. 2a) of the cross-section of the sample collected from an uncleaned area (spot no 4, in Fig. 1a) reveals the stratigraphy of the tile piece: ceramic body with whitish engobe and green glaze. SEM image and EDS map (Fig. 2b,c) give insight into the
Both areas were cleaned with the same laser parameters in two steps. Only results after the second step are shown.

The area (around spot 2 in Fig. 1) shown in Fig. 3 was cleaned to a satisfactory extent, leaving glossy glaze layer. OCT tomograms a

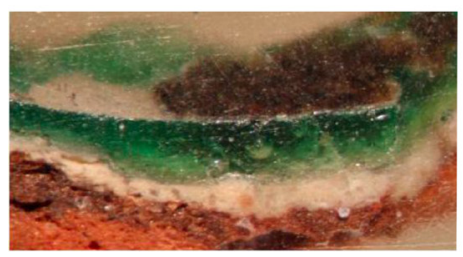

b

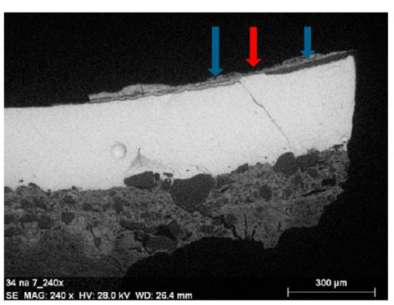

C

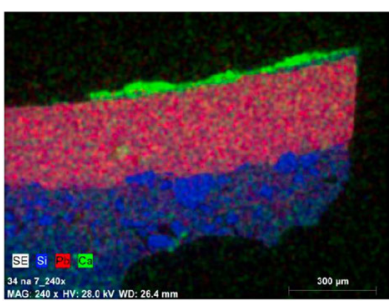

Fig. 2. Tile fragment \#1, spot no 4, uncleaned. a. Cross-section in reflected light microscopy: body, engobe and uneven green glaze are visible. b. SEM image. The arrows show primary level of glaze (red) as well as declined level (blue) due to disintegration processes and formation of black crusts. c. EDS map: Ca-rich layer of corrosion (marked green) and surface deposit (black crusts) is visible on top of the glaze layer (containing $\mathrm{Pb}$ - marked red). Si (shown in blue) indicates the engobe. 
a

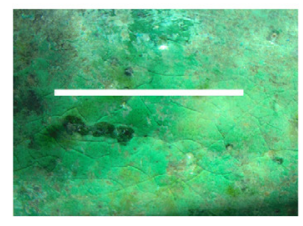

b

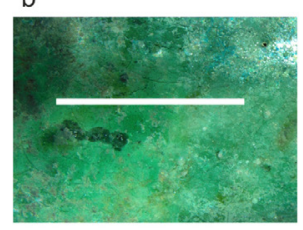

C

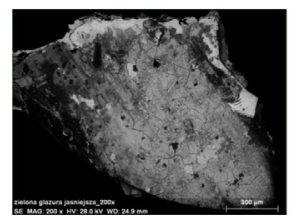

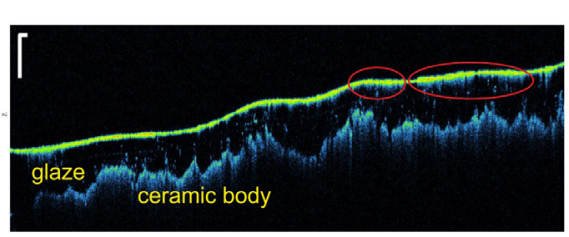

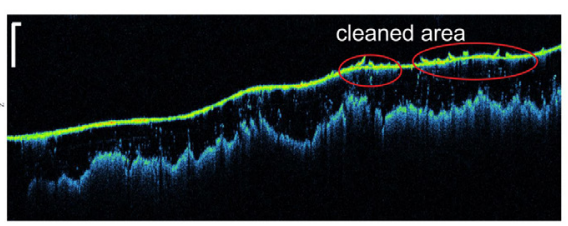

d

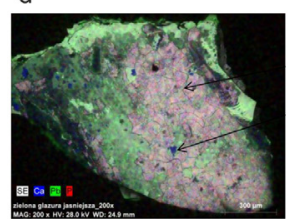

Fig. 3. Tile fragment \#1, spot no. 2 . The area cleaned to a desired extent, showing glossy glaze layer. a. Optical microscopy and OCT images collected before laser ablation. The white line in the microphotograph shows location of the OCT scan. Red ovals in the OCT tomogram mark surface deposits addressed during following laser ablation tests. b. Optical microscopy and OCT images collected after laser ablation, step 2 ( $\mathrm{E}=680$ $\mathrm{mJ})$. Red ovals marks surface deposits detached from the glaze. c. SEM image, after cleaning. d. EDS map, after laser ablation. Arrows mark calcium deposits. give insight into the laser ablation process. It is evident that the deposits (marked with red ovals in Fig. 3a), semi-transparent to the infra-red and thus coded in greenish hue in the tomograms get detached from the surface during laser treatment and are discernible as loose flakes (the tomogram was recorded before dry wiping of the surface) in Fig. 3b. In the SEM image (Fig. 3c) of the sample collected afterwards (spot no. 2, Fig. 1a) there are neither surface deposits nor ablation-related abrasions/losses visible. EDS map (Fig. 3d) shows concentration of calcium only locally and in the craquelure. All the results (microscopic photography, OCT cross-sectional images, SEM image and EDS map) show convergent results: the investigated area was effectively cleaned without damage to the glaze.

In the second experiment (Fig. 4) performed in a different area (around spot 3 in Fig. 1) surface deposits were completely removed, yet leaving the glaze matte and the surface rough, which is clearly visible in the OCT image (Fig. 4b). SEM image (Fig. 4c) and EDS map (Fig. 4d) reveal the removal of the disintegrated upper layer of glaze.

Dissimilar cleaning results observed in two areas (Fig. 3 and Fig. 4) of the tile fragment \#1 could have been due to the differences in the state of preservation of the glaze layer. Since we had found corroded glaze on this tile (see Fig. 2b, c), we could assume that the stage of deterioration varied in two cleaned areas. Another factor that should be taken into consideration is that the thickness (and IR absorption) of mineral deposit had varied in two ablated areas (which is visible in OCT images - see Figs. $3 a$ and $4 a$ ), which could have influenced the results of laser tests. 
a
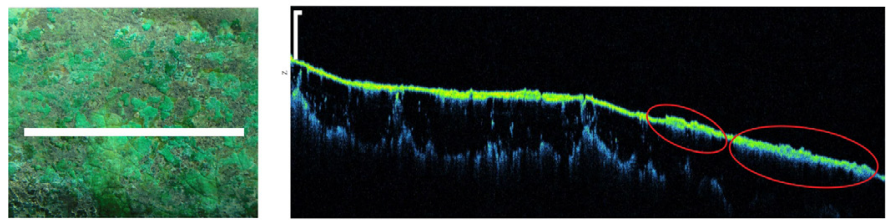

b
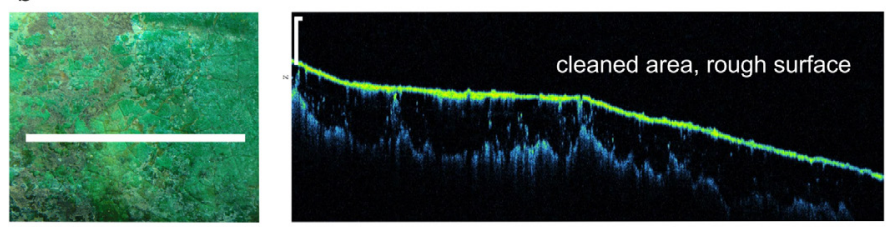

C

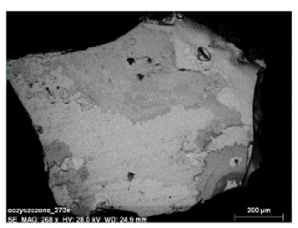

d

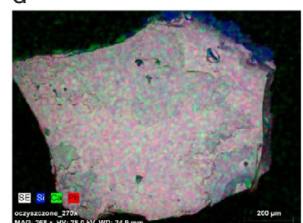

Fig. 4. Tile fragment \#1, spot no. 3. Area overcleaned, showing matte glaze layer with calcium-rich corrosion layer removed. a. Optical microscopy and OCT images before laser ablation. White line in the microphotograph shows location of the OCT scan. Red ovals in the OCT tomogram mark surface deposits addressed during following laser ablation. b. Optical microscopy and OCT images after laser ablation, step $2(\mathrm{E}=700 \mathrm{~mJ})$. c. SEM image, after laser ablation. d. EDS map, after laser ablation.

\subsection{Tile fragment \#2}

Tile fragment \#2 (MT/ZK-K-56, Fig. 5a) was executed with a similar technology as tile fragment \#1. For this piece only optical coherence tomography was used for the eval- uation of the laser ablation process. Surface deposits in this case were non-transparent to infra-red. This is why the ceramic body was not visible from under the glaze in the OCT tomogram collected before laser ablation tests (Fig. 5b). However, after the a
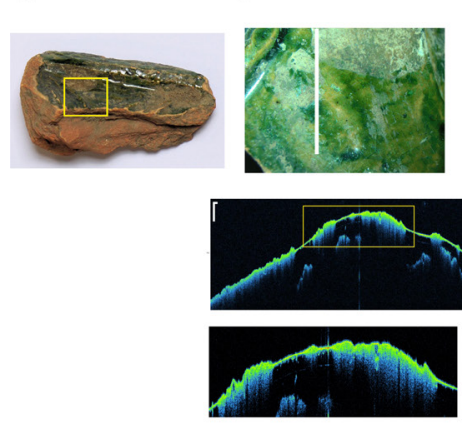

C

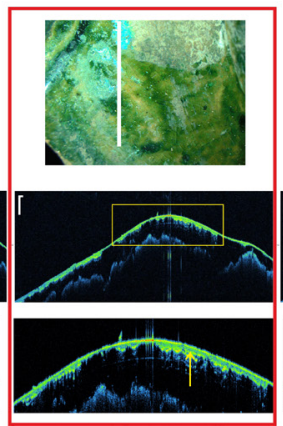

d
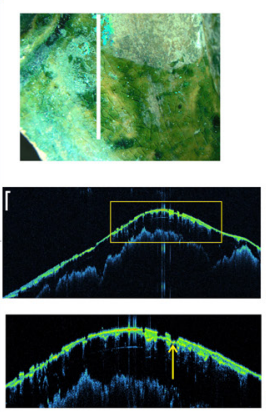

e
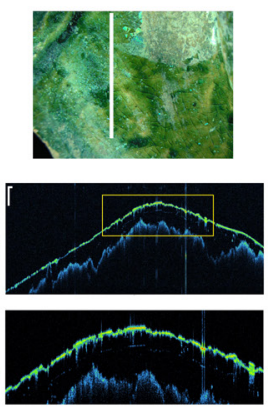

Fig. 5. a. Tile fragment \#2 in visible light. b-e. Microscopic photographs and OCT tomograms from the consecutive stages of laser ablation tests (b. before cleaning; $c$. after cleaning step 1, E=500 mJ; d. after cleaning step 2, E=560 mJ; e. after cleaning step 3, E=610 mJ); position of the OCT scans marked on the photographs with the white line. Red rectangle marks the most satisfactory result in the authors' opinion. Yellow arrows mark delaminations and following losses in the upper layer of glaze. 
first step of cleaning (Fig $5 \mathrm{c}$ ) the deposits were removed and both the glaze surface and glaze-body interface were clearly visible. Moreover, it was visible in the cross-sectional OCT image (Fig 5c) that the upper layer of glaze was delaminated (yellow arrows in Fig $5 c, d$ ) which was indicated by the high intensity of signal reflected at that interface. In step 2 and 3 intentional overcleaning was performed, and, as it had been anticipated, the delaminations became the source of losses and eventually of the rough surface (Fig. 5e).

\subsection{Tile with depiction of St. Dorothy}

Late $15^{\text {th }} \mathrm{c}$. tile with lead-based, copper tinted glaze (Fig. 6,7) was subject to some undocumented conservation treatments, resulting in the presence of a thick layer of hardly soluble synthetic resin coating. Additionally, OCT examination revealed the presence of dirt embedded under the coating (Fig. 6a).

The evaluation of the laser cleaning by means of OCT assessment was performed in a flat area of the background (red rectangle a

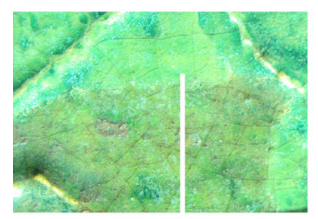

b

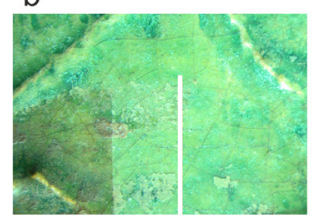

C

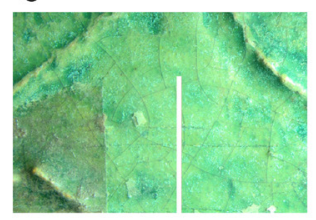

d

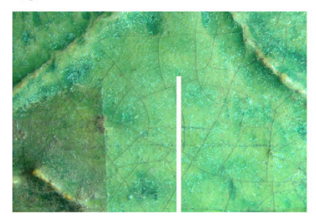

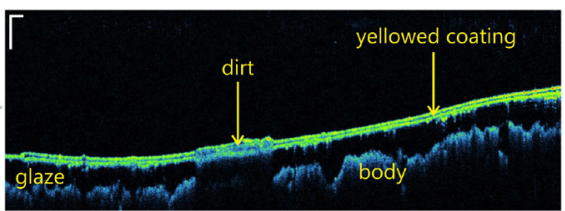
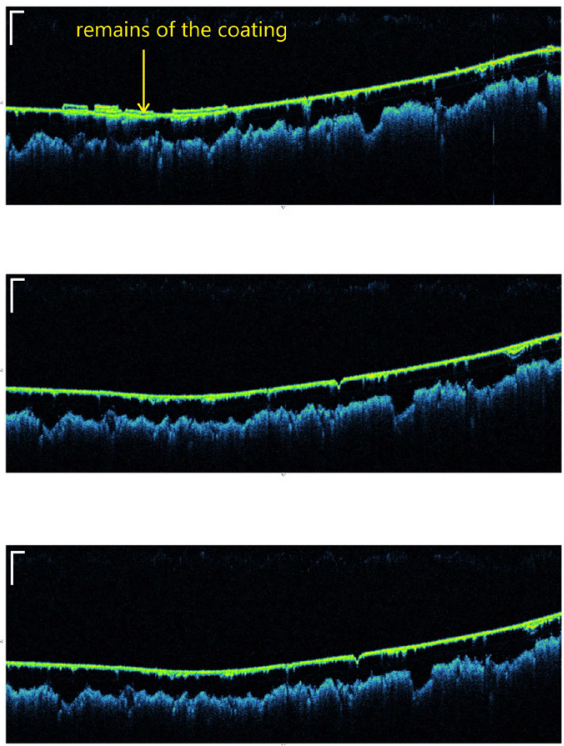
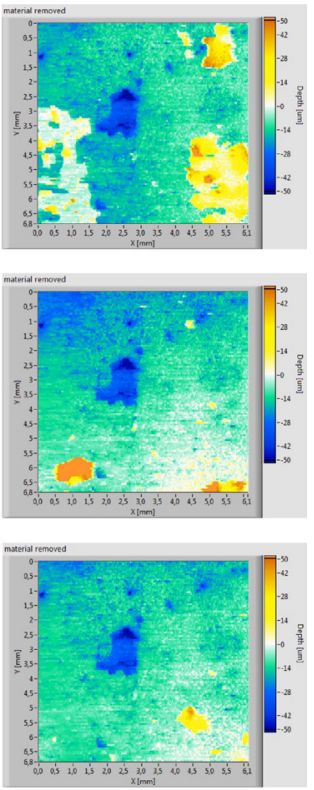

Fig. 6. The tile with a depiction of St. Dorothy. From the left: microscopic photographs, OCT tomograms and material removal maps from the consecutive stages of laser cleaning; positions of the OCT scans are marked on the photographs with white lines. a. before cleaning; b. after cleaning step 1, E=370 mJ; c. after cleaning step 2, E=350 mJ; d. after final swabbing with white spirit). 

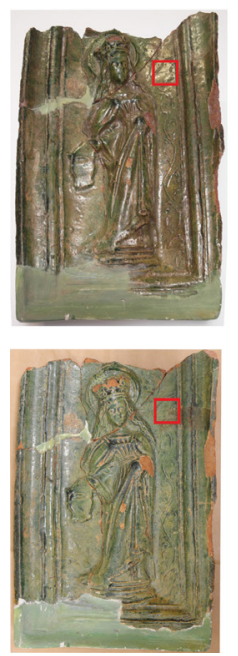
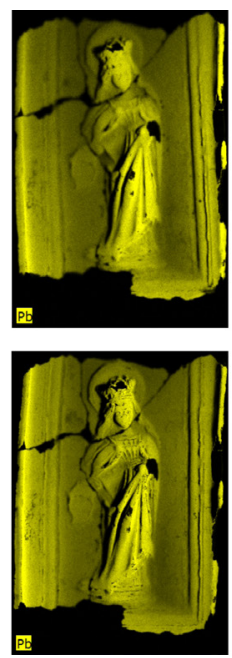
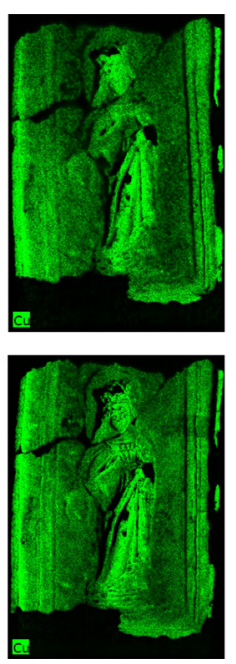
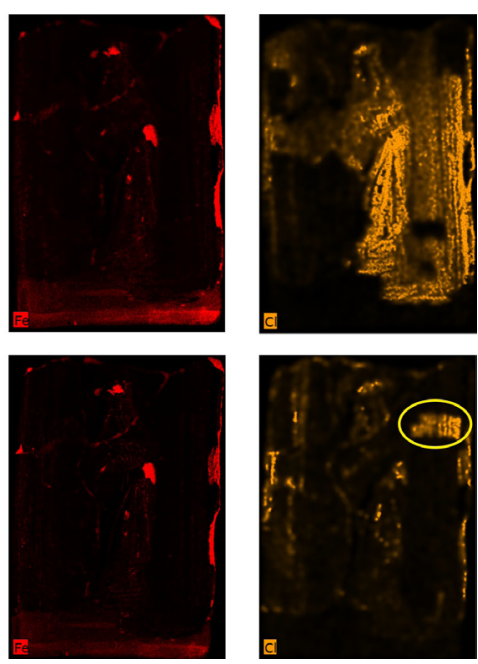

Fig. 7. The tile with a depiction of St. Dorothy. The tile in visible light with macro XRF scans before (upper row) and after (lower row) laser cleaning. Red rectangles in visible light photographs mark spot of the cleaning evaluation shown in Fig. 6. Yellow oval at chlorine map marks reference area, intentionally left uncleaned.

in visible light photographs in Fig. 7). OCT tomograms obtained before laser ablation (Fig. 6a) unfolded the good state of preservation of the glaze layer, lacking cracks or delaminations. After the first step of ablation (Fig. 6b), it is clear that the resin is mostly removed; however, some detached flakes still remain on the surface. These are removed during the second step (Fig. 6c) and the final wiping of the glaze with white spirit (Fig. 6d). Swabbing is routinely performed by the restorers at the end of the laser treatment; therefore, it had been included in the experiment. No damage to the glaze was recorded due to the laser treatment, as well as no decrease in the thickness of the glaze, which had been theoretically possible due to the evaporation of lead compounds with low melting point. It is also worth noting that in the chosen cross-sectional OCT image there is no visible difference between the surface after cleaning step 2 (Fig. 6c) and after swabbing with white spirit (Fig. 6d). Some loose particles of removed resin can be seen in the surface map (Fig. 6c) and it is evident that they are displaced during the swabbing (Fig. 6d).

For the steps 1,2 and after final wet cleaning additional maps of the removed material were created from the OCT data cubes. In order to do so, in the first place the surface elevation maps were generated from OCT data for every step and the OCT data cubes were corrected for refractive distortion. Since the removal of a coating usually causes some translation of the object, careful alignment of data sets was necessary, using the ceramic body, non-altered during the cleaning process, as a reference. Finally, the aligned surface elevation maps were subtracted to get a map of a removed material. In the maps cold colours (green, blue) show the decline of the surface level due to coating removal. Warm colours (yellow, orange) present the rise in the surface level - in this case resulting 
from the loose flakes of the ablated resin still present on the surface of the glaze.

Additionally, macro XRF scans of the object collected before and after cleaning (Fig. 7) aided in evaluation of the results of the ablation process. Chlorine maps (orange) show the effect of synthetic resin coating (chlorine-rich) removal, whereas lead (yellow) and copper (green) represent the state of glaze preservation before and after cleaning. The iron distribution map (red) shows, on the other hand, the areas of glaze losses, since the iron signal is detected from the ceramic body. The range of glaze losses did not grow larger after the laser treatment. The authors believe that such comparison is more informative than a mere analysis of the photographs of the tile, since the presence of the yellowed synthetic resin could have obscured visual analysis. The resin is "transparent" for X-ray radiation; therefore, minor losses of glaze present before cleaning and covered with the resin were still detectable in macro XRF scans.

\section{Discussion and conclusions}

In this study, we presented the first attempt to use optical coherence tomography as a progress assessment tool for laser ablation tests of semi-transparent glazes on historic tiles. According to the authors' knowledge, there are no systematic research results available on the absorption properties of historic glazes of various compositions. However, some indication on absorption properties of the glazed objects may be derived from the OCT results. To utilise these data for ablation experiments, it was necessary to choose laser wavelength from the same spectral range as used for OCT probing light. Here, from the OCT data, a conclusion was drawn that mineral deposits (on tile fragments \# 1 and \#2) were definitely more absorbing IR than the glazes. In case of the removal of the old resin coating, we had found a layer of dirt between the lacquer and the glaze. Therefore, some self-termination effect during the cleaning was expected. On the other hand, in case of fragments \#1 and \#2, where no strong absorption of the IR light in the glazes was found and deep penetration was thus envisaged, the authors intentionally wanted to create some destruction phenomena within the glazes to investigate the potential of OCT to detect such problems (intentional destruction was performed for the tile fragment \#2).

In conclusion, OCT proved suitable for the glazes transparent to near infrared. The thickness of glaze layer can be measured fast, instantly and non-invasively in many spots. The continuity of the surface can be evaluated prior to and after laser ablation and surface profiles can be compared in a straightforward way.

OCT tomograms and varnish removal maps do not only enable the evaluation of the amount of material removed as well as recording of the possible ablation-related damage, but they also help to define and identify specific risk factors for glazed ceramic objects. Such factors are pre-existing cracks or delaminations within the glaze layer, as well as areas of reliefs which are covered with much thinner layer of glaze than the flat background. The authors believe that such areas should be approached with greater caution.

\section{Acknowledgements}

The authors would like to thank Mrs Romualda Uziembło, Senior Curator and Head of the Archaeological Department of the District Museum in Torun for providing objects as well as valuable 
information on their archaeological background. Particular thanks should be given to dr Grażyna Szczepańska (Faculty of Chemistry NCU) for SEMEDS analyses. This research has been co-financed by H2020 IPERION CH project (contract number: 654028) and conducted with the use of the research infrastructure of Interdisciplinary Centre for Modern Technology of Nicolaus Copernicus University in Toruń, Poland, financed by Regional Operational Programme for Kujawsko-Pomorskie Voivodship (Project No.: RPKP.05.04.00-04-001/10).

\section{References}

[1] P. Targowski, M. Iwanicka, "Optical Coherence Tomography: its role in the non-invasive structural examination and conservation of cultural heritage objects-a review", Appl. Phys. A 106, (2012), 265-277, DOI: $10.1007 / \mathrm{s} 00339-011-6687-3$.

[2] Complete list of papers on application of OCT to examination of artwork may be found at http:// www.oct4art.eu: Optical coherence tomography for examination of works of art, (accessed 8.07.2017).

[3] J. Striova, R. Fontana, M. Barucci, A. Felici, E. Marconi, E. Pampaloni, M. Raffaelli, C. Riminesi, "Optical devices provide unprecedented insights into the laser cleaning of calcium oxalate layers", Microchemical Journal, 124, (2016), 331-337, DOI: 10.1016/j.microc.2015.09.005.

[4] M. Iwanicka, P. Targowski, E.A. Kwiatkowska, M. Sylwestrzak, "Application of optical coherence tomography for monitoring some conservation treatments", in: Lasers in the Conservation of Artworks IX (Proceedings of Lacona IX conference),
London, UK, September 7-10, 2011, D. Saunders, M. Strlič, C. Korenberg, N. Luxford, K. Birkhölzer (Eds.), Archetype Publications Ltd, London 2013, pp. 19-25.

[5] M. Iwanicka, D. Kończalska, P. Targowski, B.J. Rouba, "Non-Contact and Non-Invasive Monitoring of Overpaint Removal with Optical Coherence Tomography", in: New Insights into the Cleaning of Paintings: Proceedings from the Cleaning 2010 International Conference, M.F. Mecklenburg, A.E. Charola, R.J. Koestler (Eds.), Smithsonian Contributions to Museum Conservation III, Washington D.C. 2013 (extended abstract), pp. 27-29.

[6] P. Targowski, R. Ostrowski, J. Marczak, M. Sylwestrzak, E.A. Kwiatkowska, "Picosecond laser ablation system with process control by Optical Coherence Tomography", Proc. SPIE 7391, (2009), 7391G, DOI: $10.1117 / 12.827286$.

[7] M. Góra, P. Targowski, A. Kowalczyk, J. Marczak, A. Rycyk, "Fast spectral optical coherence tomography for monitoring of varnish ablation process", Proceedings of the international conference LACONA VII: - Lasers in the Conservation of Artworks, Madrid, Spain, September 17-21, 2007, M. Castillejo, P. Moreno, M. Oujja, R. Radvan, J. Ruiz (Eds.), CRC Press, Taylor \& Francis Group, London 2008, pp. 23-27.

[8] M. Alfeld, J. Vaz Pedrozo, M. van Eikema Hommes, G. Van der Snickt, G. Tauber, J. Blaas, M. Haschke, K. Erler, J. Dik, K. Janssens, "A mobile instrument for in situ scanning macro-XRF investigation of historical paintings", Journal of Analytical Atomic Spectrometry 28, (2013), 760, DOI: 10.1039/ c3ja30341a. 


\title{
The Hanford Nuclear Plant: Radiation Ablation Meets History, Cultural Heritage, and Nuclear Decontamination ${ }^{\mathrm{a}}$
}

\author{
John F. Asmus ${ }^{1 \star}$, Vadim A. Parfenov ${ }^{2,3}$, Jessica P. Elford ${ }^{1}$ \\ 1 Department of Physics, University of California, San Diego, 9500 Gilman Dr., La Jolla, CA, 92093 - \\ 0360, USA \\ 2 Department of Quantum Electronics and Opto-Electronic Devices, St. Petersburg Electrotechnical \\ University; 5, Prof. Popova ul., 197376, St. Petersburg, Russian Federation \\ 3 Department of Laser Technological Systems, St. Petersburg National Research University of Infor- \\ mation Technologies, Mechanics and Optics, 49, Kronverksky pr., 197101, St. Petersburg, Russian \\ Federation \\ * Corresponding author: jfasmus@ucsd.edu
}

\begin{abstract}
The Hanford Nuclear Reservation in the state of Washington (USA) has 12 shutdown atomic reactors that were constructed about 70 years ago and established for the production of plutonium. The facility also encompasses five chemical-processing complexes for the extraction and refining of plutonium. Major activities associated with the closing of Hanford (at the end of the "Cold War") first centered on disassembling the reactors, the chemical plants, and the waste storage facilities. A remediation program was initiated to drain the leaking tanks and ponds so that the toxic wastes could be buried elsewhere and/or transferred to more secure double-shell reservoirs. This poses problems when disposing of refuse materials and hardware from the site. It has been experimentally determined that this hazardous surface contamination may be ejected by means of radiation ablation. In this procedure the dislodged surface contamination is freed to float in reactor-core water and is then captured and concentrated by the filters of the fluid circulation systems. The final phase of the project has been assistance in designing and fabricating a monumental Nuclear Reliquary to mark both the successful decontamination of the reservation and the return of the land of the Hanford site to its natural state. The artist-in-residence (James Acord) of Imperial College, London, created a small $500 \mathrm{~kg}$ trial statue (Monstrance for a Grey Horse) of the eventual kilometer-scale Hanford monument. It is currently installed in the Jimi Hendrix Seattle Rock and Roll Museum to symbolize "nuclear alchemy" in the form of an embedded capsule of benign ruthenium that had been transmuted through neutron capture from radioactive technetium $\left({ }^{99} \mathrm{Tc}\right)$. The full-scale Hanford Monument was designed prior to the death of James Acord and will again have a transmuted ruthenium core to symbolize the decommissioning of the Hanford Works. The many tons of material comprising the bulk of the Stonehenge-like structure celebrating the decontamination of the Hanford Reservation will be atomic reactor components cleaned through radiation ablation. Optical radiation ablation is favored in numerous surface-divestment applications due to inherent self-limiting and selectivity features. However, in nuclear decontamination the most profound advantage may be in cost reduction through secondary waste reduction. No abrasives (e.g.
\end{abstract}

${ }^{a}$ Presented at LACONA IX conference, The British Museum and University College London, 7-10 September 2011. 
sandblasting) or significant volumes of liquids (e.g. hydro blasting) are used. Furthermore, at high optical irradiation fluxes most toxic organic waste chemicals are destroyed and converted into innocuous gases (e.g. water vapor and carbon dioxide), and no additional secondary waste is formed. Submerged flashlamp irradiation was found to be the most economical approach for the radiation ablation.

Keywords: Decontamination, ablation, laser cleaning, flashlamp, nuclear waste, Hanford, nuclear reactor

\section{Hazardous radioactive contamination at Hanford}

The USA's nuclear weapons program known as the "Manhattan District" was established shortly after Prof. Albert Einstein wrote to President Franklin Roosevelt regarding the technical feasibility of an atomic bomb. The Los Alamos Scientific Laboratory (LASL, then LANL) was established to design and fabricate nuclear weapons. The Oak Ridge facility was established in order to refine uranium in order to concentrate ${ }^{235} \mathrm{U}$ for uranium gun-design devices such as Little Boy. The Hanford Works facility was established in eastern Washington State in order to produce plutonium $\left({ }^{239} \mathrm{Pu}\right)$ for the Fat Man Implosion weapon.

When the Hanford nuclear reactor facility was established and expanded throughout the 1940s and 1950s, long-term storage and disposal of radioactive waste was not recognized as a significant problem. With the enactment of the SALT treaties and by the end of Cold War tensions, it was recognized that the Hanford Reservation was no longer needed. When plans for its closing were drawn up and assessed, it was established that very serious nuclear waste decontamination and disposal issues had to be confronted [1].

Major activities associated with the closing of Hanford first centered on disassembling the reactors, the chemical plants, and the waste storage facilities. Radioactive parts and assemblies were to be transported across state boundaries to the Yucca Mountain long-term underground storage caverns in the western wilderness of the state of Nevada. However, a number of developments conspired to obstruct this plan. First, there was a political impasse. The citizens of the State of Nevada objected to the importation of dangerous toxic substances into their state. Second, there was a related financial problem associated with the preparation and transportation of a prodigious mass of contaminated materials. Finally, it was discovered that significant quantities of radioactive wastes were leaking from storage containment vessels and ponds into the soils and aquifers beneath Hanford (Fig. 1). It was discovered that the liquid wastes were seeping toward the Columbia River. Upon reaching the river, the contamination would next be swept onto the extremely important Kennewick archaeological dig, which may hold secrets of the human migration to North America. Major population centers such as Portland and Vancouver are further downstream, and together with Kennewick may have to be evacuated as in the cases of Chernobyl and Fukushima. Consequently, funds that were destined to close Hanford and move the nuclear wastes to Nevada had to be redirected toward the prevention of the radioactive polluting of a major river. This entails moving the wastes to more secure newer double shell reservoirs, decontaminating the old containment facilities, and stopping the underground seepage toward the river. 


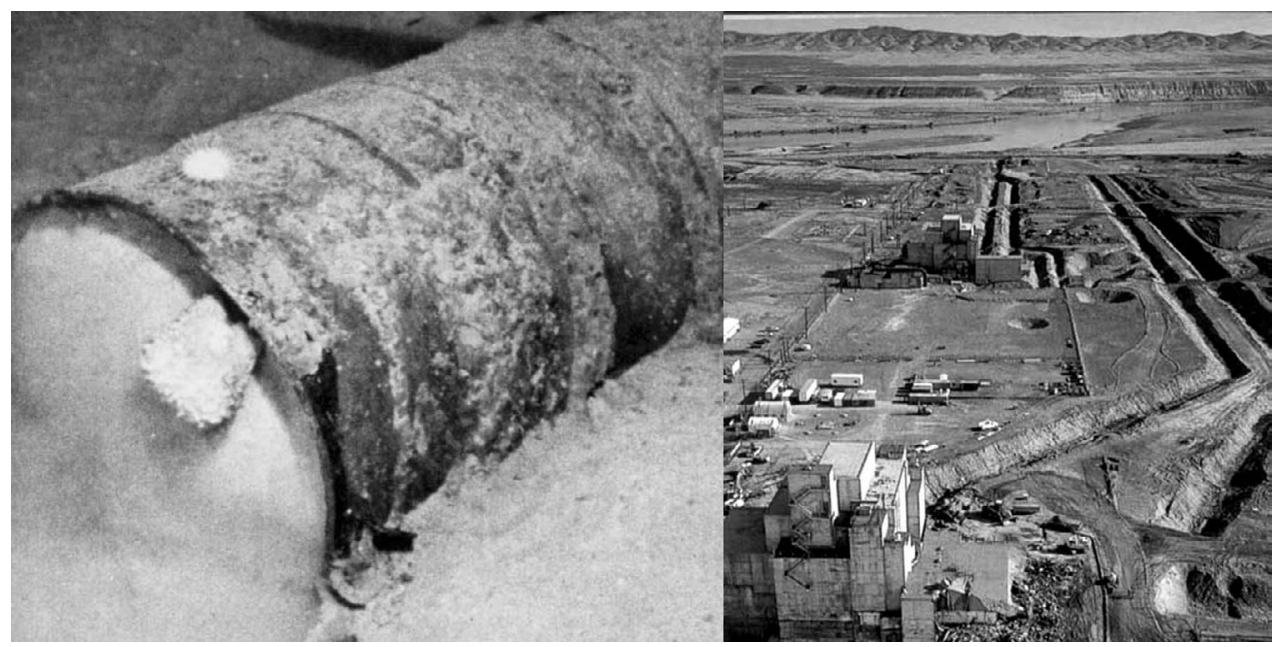

Fig. 1. A corroded and leaking nuclear waste storage drum (left). Hanford nuclear reactor and its earthen waste drainage ditches with leaking liners (right).

In anticipation of the eventual complete decontamination of the entire nuclear facility and the removal of radio-active isotopic waste materials to secure storage sites it was expected that the Hanford Reservation would be rededicated as an environmental-cleanup-demonstration National Park. The park's centerpiece would be a monument designed by Hanford's "nuclear artist-in-residence," Mr James Acord (Imperial College, London.) The monument is to be fabricated from reactor components that have been decontaminated either by conventional or radiation-ablation (flashlamp) procedures. In preparing for the full-scale monument, Acord created a small $500 \mathrm{~kg}$ trial statue (Monstrance for a Grey Horse) of the eventual kilometer-scale Hanford monument. This anticipatory piece is currently installed in Paul Allen's “Jimi Hendrix Seattle Rock and Roll Museum" in order to symbolize "nuclear-alchemy" decontamination, in the form of an embedded capsule of benign ruthenium that was transmuted through neutron capture from radioactive technetium $\left({ }^{99} \mathrm{Tc}\right)$.

\section{Nuclear reactor accidents}

Human errors, natural events, and engineering deficiencies have also led to the spread of hazardous radioactive contamination. A portion of the Hanford problem has been traced to the accidental opening of a valve that drained radioactive and toxic chemicals directly into the aquifer. This tragedy was exacerbated by the tardy realization of the human mistake. Apparently, the Chernobyl disaster is traced to a similar human error. The Fukushima explosion and partial meltdown can be attributed to the natural catastrophe (earthquake and associated tsunami) and/or to an inadequate engineering safety margin and emergency safety plan.

Chernobyl released from $1 / 100$ to $1 / 1000$ of the total amount of radioactivity released by nuclear weapons testing during the 1950s and 1960s. Approximately $100,000 \mathrm{~km}^{2}$ of land was significantly contaminated with fallout, with the worst hit regions being in Belarus, Ukraine and Russia. Four square kilometers of pine forest directly downwind 
of the reactor turned reddish-brown and died. In addition, the radioactive contamination of aquatic systems is also a problem in the aftermath of the accident. However, the greatest problem of the Chernobyl incident pertains to health as it may have resulted in $10,000-200,000$ additional human deaths in the period between 1990 and 2001. Therefore, the Chernobyl accident made it quite clear (perhaps, for the first time in human history) that the contaminants released after a severe nuclear accident may spread over large areas, and thereby come to form a significant external radiation hazard not only on-site in the place of a disaster, but also in surrounding areas of high population density. In any event, all require major decontamination efforts for the safety of the human populations as well as to return the local environments to habitability and productivity. We anticipate that the radiation-ablation decontamination procedure developed at Hanford may be useful under such circumstances.

\section{Kennewick Man downstream from the Hanford spill}

In 1966 the skeletal remains of a 9,300-yearold Caucasian male were discovered on the shore of the Columbia River near the town of Kennewick, Washington. The skull (and bones at a later time) was found by two college students while watching hydroplane races. The office of the Benton County Coroner determined that the skeleton was not the result of a contemporary homicide, but was the consequence of the natural death of an "Ancient One" at least 9,200 years earlier. The remains of this five-foot ten-inch male (1.8 meters) were determined to be $90 \%$ complete and are among the oldest ever found in North America. Furthermore, a two-inch $(5 \mathrm{~cm})$
"Clovis" spear point was found imbedded in his pelvis. The bone exhibited signs of healing indicating that he had survived a hostile attack.

Predictably, the discovery of Caucasian remains that predated the earliest Asian remains found in North America turned asunder the generally accepted theory of the human migration across the Siberian-Alaskan land bridge at the end of the last Ice Age. The discovery of the Caucasian Kennewick Man seemingly lent support to theories postulating that seafarers who originated in or near Europe initially populated North America. Nevertheless, Native American Indian tribes (of Asian origin) claimed Kennewick Man as an ancestor and requested custody of the remains in order to perform a traditional religious burial. This issue became the subject of a court battle between anthropologists who wished to learn all they could about the relic and the U.S. Army Corps of Engineers (owner of the Columbia River shoreline at Kennewick) wanting to have it reburied in accordance with tribal wishes in order to put an end to the controversy. The local tribes (Umatilla, Nez Perce, Yakima, Wannapum, Warm Springs Nation, and the Colville Nation) maintained that the skeleton should be reburied without further study. They had no explanation as to how their "ancestor" could be Caucasian.

At the heart of this controversy is the rewriting of American pre-Columbian history. Consequently, a group of anthropologists joined in the lawsuit in 1996. This caused five of the Indian tribes to fight the anthropologists in court contending that the repatriation law covered Kennewick Man, and that scientific examinations disrespected Native American beliefs about the sanctity of their dead. In 2002 Judge Jelderks ruled in favor of the anthropologists. The U.S. Ninth 
Circuit of Appeals upheld that ruling in 2004. Subsequently, the relics have been placed in temporary storage in the basement of the Burke Museum. Twenty-two anthropologists have studied the skeleton and have performed thousands of measurements by means of radiocarbon dating, MRI, and DNA analysis. It was determined that he died in his 30 s, but not from the spear wound. In light of the ongoing litigation and the possibility that the remains of the Kennewick skeleton will eventually be reburied (especially because the bones have already been studied so extensively) it is imperative to decontaminate Hanford before radioactivity reaches the burial site and other relics yet to be found.

\section{Flashlamp ablation of nuclear contamination}

The disassembly, cleanup, decontamination, and decommissioning of the Hanford facility is an enormous task in its entirety. Soils and groundwater are polluted with radioactive isotopes and toxic chemicals, as are chemical processing plants, chemical handling equipment, and storage areas. Contaminated nuclear reactors together with spent fuel, fuel cores, and water-cooling and moderating systems present a wide range of technical and logistical cleanup issues. If large, heavy, and bulky equipment can be economically cleaned, only the concentrated hazardous waste substances will require expensive transportation to, and long-term storage in, secure facilities such as Yucca Mountain. There are several advantages of light ablation surface treatments over conventional stripping and cleaning techniques. Some of these are inherent in the technology and benefit any suitable application. Other advantages are specific to the area of radioactive contamination removal and relate to special problems faced at the Hanford site [as well as other U.S. Department of Energy (DOE) and commercial nuclear facilities]. Optical radiation ablation is favored in numerous surface-divestment applications due to inherent self-limiting and selectivity features. However, in nuclear decontamination the most profound advantage may be in cost reduction through secondary waste reduction. No abrasives or significant volumes of liquids are used. Moreover, at high optical irradiation fluxes most toxic organic waste chemicals are destroyed and converted into innocuous gases, and no additional secondary waste is formed. Fig. 2 (left) shows, as an example, the flashlamp removal of contaminated rust from a steel beam. Figure 2 (right) is a pho-
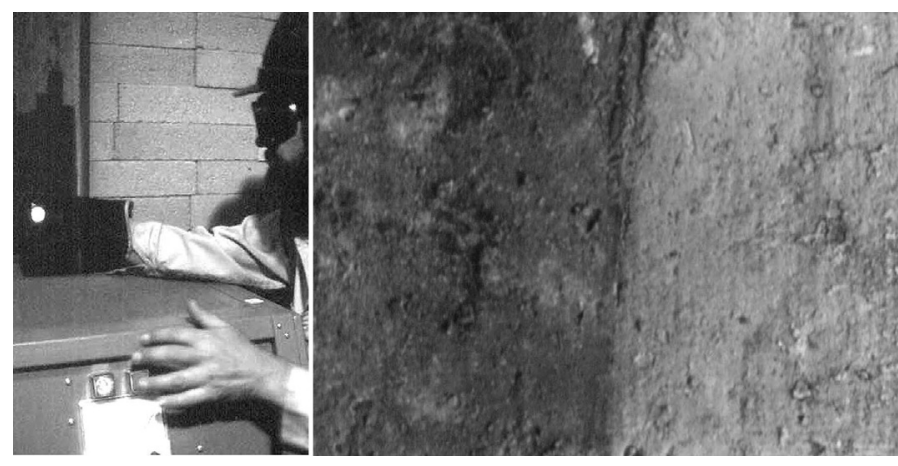

Fig. 2. Xenon flashlamp surface ablation in air of contamination on a steel beam (left). Concrete surface contamination on left and flashlamp divested region on the right side. (right) 
tograph revealing flashlamp divestment of a contaminated crust from concrete through radiation spallation (under water). Figure 3 presents measurements of the rates at which

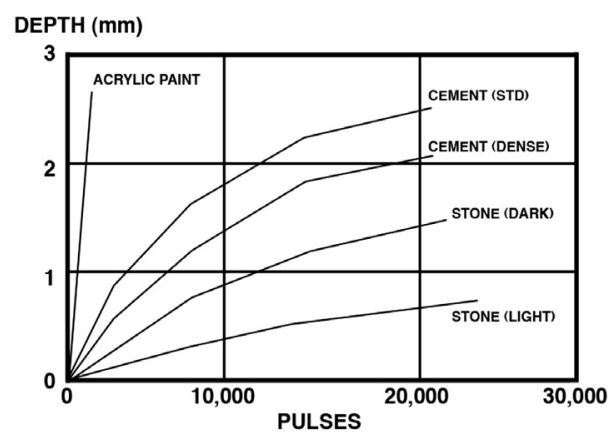

Fig. 3. Experimental flashlamp ablation rates for the materials of nuclear reactor fuel water pools at an incident underwater optical flux of $8 \mathrm{~J} / \mathrm{cm}^{2}$.

high-power flashlamp radiation ablates the contaminated surfaces of materials encountered at water-moderated reactor vessel walls. Flashlamp, rather than laser, radiation was employed in these investigations due to the enormous areas to be processed and the over-riding importance of cost effectiveness.

The following specific problem areas at Hanford have been identified for possible application of flashlamp ablative decontamination [2].

1. Cell wall decontamination.

2. Waste Encapsulation Storage Facility (WESF) cell decontamination prior to transuranic (TRU) monitor test installation. The purpose would be to reduce gamma background to prevent interference with neutron detection instrument testing and operation.

3. Cell decontamination.

4. The Reduction Oxidation (REDOX) Plant is scheduled to be the first canyon building onsite to undergo complete decon- tamination and decommissioning. The $\mathrm{B}$ Plant may also require limited cell decontamination in the next few years to accommodate WESF support operations.

5. The areas to be decontaminated include the tank exterior surfaces and cell walls. This will involve both paint stripping and concrete spalling.

6. Double shell tank-waste retrieval pumps and equipment. After water or chemical flushing the interiors of the pipe and pumps, and the exterior may be irradiated to further reduce contamination and corrosion.

7. Hot-cell and glove box laboratory instruments.

8. Lead brick shielding used in laboratories, hoods, canyons, and cells that have developed corrosion films that have collected radioactive surface contaminants, (characterized as mixed wastes).

9. Fast Flux Test Facility (FFTF) equipment maintenance and field operations.

10. Single-shell tank clean-out equipment.

11. Core sampling equipment. Maintenance tools. Organic destruction.

12. Destruction of complex concentrated organics from the thin-film reactor process. Repair of hot-cell electrical equipment that is intolerant to water/acid/salt decontamination.

13. Fuel/Capsule storage pools.

As most of the decontamination operations listed above are favorably performed under water (in order to contain the ejected contaminants), submerged flashlamp irradiation experiments were performed. Figure 4 (employing laser, rather than flashlamp ablation for photographic clarity) reveals how optical irradiation under water constrains the blow off plasma, thereby increasing the surface impulse. 


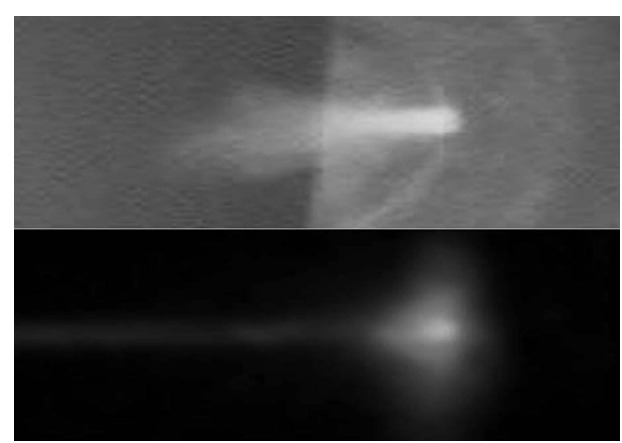

Fig. 4. Blow-off plasma luminosity from laser irradiation $\left(1.06 \mu \mathrm{m}, 250 \mu \mathrm{s}, 8 \mathrm{~J} / \mathrm{cm}^{2}\right)$ of concrete in air (top) and underwater (bottom).

Figure 5 demonstrates that performing the optical ablation underwater enhances the strength of the impulse over that generated in air. Furthermore, underwater ablation facilitates capturing the contamination in the water

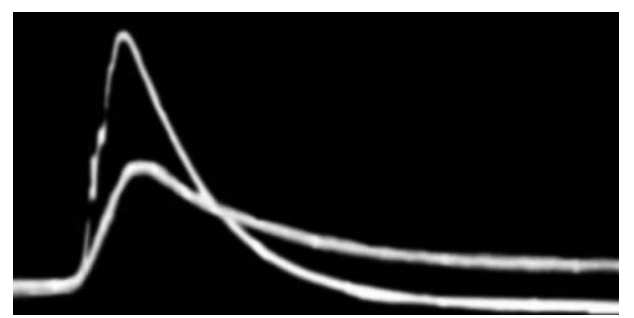

Fig. 5. Impulse from laser irradiation of a surface in air (lower trace) and underwater (upper trace)

so that filtration systems readily collect and concentrate the radioactive waste. Observing the system in operation above a reactor pool is seen in Fig. 6. The technologies (including flash ablation) to clean up the various contamination zones at Hanford have been developed and tested. Unfortunately, radioactive seepage and migration continues as the political roadblock to the use of Yucca Mountain for long-term storage remains unresolved. However, as the radioactive contamination threat to high population areas grows, a political accommodation must be reached.

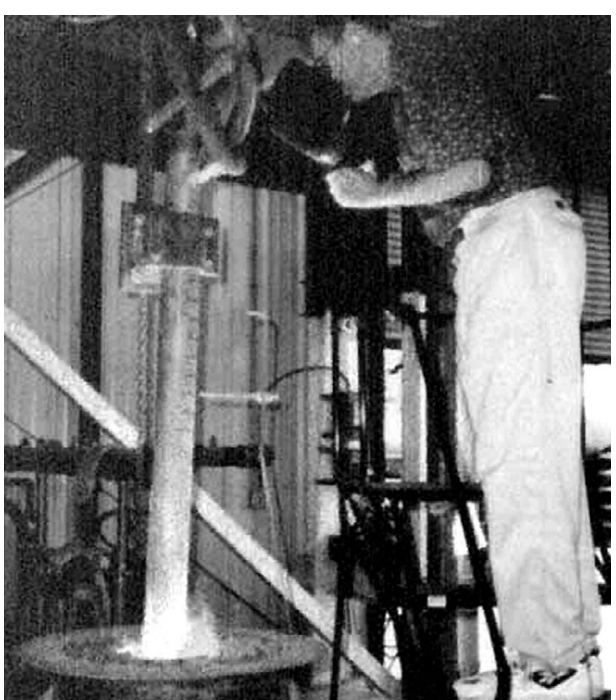

Fig. 6. Observing the flashlamp ablation process taking place within a nuclear reactor fuel core water pool.

\section{New trends in laser ablation technologies for nuclear decontamination}

In the case of decontamination at the Hanford nuclear facilities flashlamp ablation was more effective than laser processes. However, sometimes, the potential of laser cleaning may be very useful. Laser cleaning is an effective method of removal of soiling particles of different materials and sizes, as well as films and coatings from a solid surface [3]. This is a nontoxic and inexpensive technology and can be used for removal of various contaminants, including those that cannot be removed by the conventional techniques. As laser cleaning employs low levels of radiation intensity, contamination removal can be realized not only by means of evaporation, but also in the solid phase. In this case thermal influence on the substrate is very small. Variations in laser output parameters on a large scale allow one to choose the generation regime specifically for removal 
of every type of surface contamination. Laser cleaning also has other advantages, such as operation at a distance, absence of mechanical damage of the surface, and cost effectiveness.

For the removal of nuclear contamination one can use direct laser ablation. Such an approach has been realized in the framework of the project to decontaminate the Hanford nuclear reactors. Regarding new trends in the development of laser cleaning techniques for nuclear decontamination, we point out an attractive approach to the problem of removal of nuclear contaminants which has been proposed by a group of scientists with the National Research University of Information Technologies, Mechanics \& Optics (St. Petersburg, Russia). They have further developed a technology which is called LIFT (laser-induced films transfer). The main idea of this approach is surface cleaning through a transparent film. This film is a collector which inhibits soiling of the surface [4].

In a number of cases of laser cleaning the necessity of avoiding re-soiling of the surface arises. The most striking example of such a task is the decontamination of components and details of nuclear power installations. It is known that applying nanosecond pulses of laser radiation (for instance, Nd:YAG laser) allows one to remove surface corroded layers up to $200 \mathrm{~nm}$ thickness without melting or evaporation of the substrate. The experts of the above-mentioned Russian university developed the method of nuclear decontamination, by which the laser beam affects the surface through the thin film, which is transparent at a wavelength of $1.06 \mu \mathrm{m}$.

The main advantage of this technique is that the film separates the "dirty" machining zone from the "clean" and also adsorbs the products of cleaning on to the glue film surface that faces the treated object. This facilitates the collection and concentration of the radioactive waste.

\section{Conclusions}

The story of discovery of Kennewick Man stresses the very real problem of nuclear decontamination in the USA, which is a part of a global problem of our civilization. The solution to this problem may be realized through the use of radiation ablation technologies (based either on laser or flash-lamp cleaning).

Within the programs of decommissioning of the Hanford facility it was found that the hazardous surface contamination may be removed by light ablation: literally blowing it off as a cloud of plasma, using photons. It was shown that this is cost-effectively accomplished through surface decontamination by irradiation with an underwater xenon flashlamp. In this case the dislodged surface contamination floats in the water and can then be captured and concentrated by the filters of the fluid circulation systems for safe disposal elsewhere. There are several advantages of light ablation surface treatments over conventional stripping and cleaning techniques, one of which is that it generates no secondary waste to be disposed of. Therefore, further development of light ablation technologies is a task requiring systematic practical field investigation.

As for radioactive seepage from Hanford, it will reach the Kennewick region in a few decades if the present advance rate continues. At that point millions of inhabitants in Portland, Vancouver, and smaller cities will face evacuation as in the case of the Chernobyl and Fukushima disasters. This prospect may break the political impasse and a site will be selected to receive the Hanford waste and the 
radiation ablation decontamination of the facility will proceed in haste. The excavation of the area around the Kennewick Man discovery will proceed and the anthropological mystery of the populating of the Americas may be resolved through acceleration of the ongoing investigations. In conclusion we should mention that sometimes absolutely diverse disciplines unpredictably intersect. In this case study of the Kennewick Man, radiation ablation met history and cultural heritage, and it is really a very surprising mismatch. However, in the past two decades laser cleaning technology was very frequently used in artworks conservation, making the application of laser techniques in cultural heritage preservation [5] an entirely routine approach. However, the discovery of the Kennewick Man (and the creation of the James Acord decontamination monument) is exceptional, because here issues connected with use of lasers in nuclear decontamination were surprisingly "mixed" with cultural heritage and anthropology.

\section{References}

[1] U.S. Department of Energy, Disposal of U.S. Department of Energy, Disposal of Hanford defense high-level transuranic and tank wastes, Public Document, DOE/EIS-0113, vol. 5 (December 1987), available from https://energy.gov/nepa/downloads/ eis-0113-final-environmental-impact-statement-0 (accessed 8.07.2017).

[2] Westinghouse Hanford Company, Light Aided (Laser) Decontamination, Westinghouse Document, WHC-SD-WM-TI-518 (1992).

[3] B. S. Luk'yanchuk (Ed.), Laser cleaning, B.S. Luk'yanchu World Scientific, Singapore 2002.

[4] V.P. Veiko, T.Y. Mutin, V.N. Smirnov, E.A. Shakhno, "Laser decontamination of radioactive nuclides polluted surface”, Laser Physics, 21 (2011) 608-613, DOI: 10.1134/S1054660X11050264.

[5] C. Fotakis, D. Anglos, V.Zafiropulos et al., Lasers in the Preservation of Cultural Heritage. Principles and Applications, CRC Press, Taylor \& Francis Group, Boca Raton, USA 2007. 



\section{Coherent light-based methods for imaging, documentation, examination and analysis of objects and structures}





\title{
Surface micro-profilometry for the assessment of the effects of traditional and innovative cleaning treatments of silver
}

\author{
Claudia Daffara ${ }^{1 \star}$, Nicola Gaburro ${ }^{1}$, Giacomo Marchioro ${ }^{1}$, Alessandro Romeo ${ }^{1}$, \\ Giulia Basilissi ${ }^{2}$, Andrea Cagnini ${ }^{2}$, Monica Galeotti ${ }^{2}$ \\ 1 Department of Computer Science, University of Verona, Strada Le Grazie 15-37134 Verona, Italy \\ 2 Opificio delle Pietre Dure, Viale F. Strozzi 1-50129 Firenze Italy \\ * Corresponding author: claudia.daffara@univr.it
}

\begin{abstract}
In the complex procedure for the conservation of a work of art, the effective monitoring of the surface treatment is an important step in order to establish a conservation protocol. The evaluation of the effects of the cleaning requires the inspection of the outermost layer with complementary diagnostic techniques to obtain information on both materials and surface morphology.

In this interdisciplinary work, a custom optical micro-profilometry device based on laser conoscopic holography was used for testing the performance of different mechanical procedures for the cleaning of silver alloy objects.

Tarnishing is the main alteration phenomenon for silver alloy artworks. Even if such modification does not affect the long-term preservation of the objects, it heavily influences aesthetical features, making the removal of tarnishing one of the most important conservation treatments of the case. The mechanical cleaning procedure based on abrasive powders suspended in a liquid matrix is of widespread use. Recently, a novel dry cleaning procedure based on the use of erasers was proposed as alternative method and tested for both the efficacy of tarnishing removal and effects on the surface appearance using optical microscopy and scanning electron microscopy. However, the employed laboratory techniques cannot provide an objective measure of the roughness at micrometric scale over the sample.

In this work, the effects on the surface structure of traditional and innovative silver treatments was investigated with multiscale profilometry, using scanning conoscopic holography and atomic-force microscopy. 3D surface metrology was carried out using standard areal amplitude parameters and a multiscale approach.

Preliminary results are presented, addressing two aspects: 1) the validation of laser optical micro-profilometry for the diagnostics of silver objects, which are challenging due to the highly specular surface; 2) the validation of the dry-cleaning treatment based on rubbers, by adding to the previous study the complimentary analysis of the micro-surface. The results obtained on silver mockups confirm that the proposed dry cleaning methods produce surface variations less or similar to traditional methods. The optical micro-profilometry results are of particular significance since this technique is portable with wide field capability, thus allowing an in-situ use and a real monitoring of actual artwork surfaces.
\end{abstract}

Keywords: optical micro-profilometry, conoscopic holography, silver cleaning, surface analysis, artworks 


\section{Introduction and background}

The degradation of silver is an important topic in conservation science. Many ancient artefacts were made of silver but this precious metal is more sensitive to degradation compared to other noble metals such as gold and for this reason requires special care to preserve the original surface. Silver degradation has been studied in different environments [1] and extensive studies have been carried out regarding the embrittlement of buried silver objects $[2,3]$. One of the main degradation processes affecting silver artefacts is the corrosion due to atmosphere gases [4]. This is nowadays one of the most critical issue for objects conserved in collections.

Tarnishing is the main alteration phenomenon for silver alloy artworks due to the interaction of silver with sulphur-containing compounds in the environment. Such modification heavily influences aesthetical features, making the removal of tarnishing one of the most important conservation treatments of the case. Different cleaning procedures are proposed, based on mechanical, electrochemical, chemical and physical methods [5]. The mechanical procedure utilizing an abrasive powder suspended in a liquid matrix is of widespread use due to its simplicity and effectiveness. Despite these advantages, issues are raised concerning the cleaning of objects in which more materials coexist near to or above silver (enamels, other metals, and organics). In these cases, the use of water- or solvent-based means is often not advisable. To address this point, the Opificio delle Pietre Dure has recently introduced a dry cleaning process based on the use of erasers as alternative to traditional mechanical methods [6]. Several rubber-based methods have been tested regarding the efficacy of tarnishing removal and the effects on the surface appearance. Preliminary tests have proven that such innovative procedure effectively removes tarnishing and has been recently used in the final phase of the cleaning of the silver "agemina" letters in the North Door by Lorenzo Ghiberti of the Baptistery of Florence in Italy.

The evaluation of the effects of the cleaning requires the outermost layer of the artwork to be inspected with complementary diagnostic techniques to gain information on both materials and surface morphology. Different studies regarding the choice of the best cleaning methodology have been carried out using routine benchtop techniques on tarnished silvers mock-ups $[7,8]$ to control mass loss and surface variation after the cleaning process. However, most of these techniques can analyse only a small area of the sample and cannot be brought in situ.

In this work, we used a newly developed optical micro-profilometer based on 3D scanning conoscopic holography that can be brought in the place where artefacts are conserved for an in-situ monitoring [9]. Laser conoscopic holography is a non-contact technique that has been developed in the last decades [10]. It provides distance measurements in non-invasive way in a versatile working range allowing the acquisition of artwork surfaces at different scales with micrometric resolution. This technique has proven its potential on different applications, e.g. archaeology [11], laser cleaning [12], painting conservation [13]. Silver material, which is highly specular, is particularly challenging.

Here, laser conoscopic holography was applied to monitor the surface in different mechanical procedures for the cleaning of silver alloy objects, including traditional 
treatments and the innovative dry-cleaning methods based on rubbers.

This interdisciplinary work addresses two aspects, one related to the instrumental tool, the other related to the specific conservation application. The aims are:

- to validate laser optical micro-profilometry as an effective diagnostic tool for the analysis of silver objects, which are challenging due to the highly specular surface;

- to further validate a new cleaning treatment for silver, adding to the previously carried out study [6] the complimentary analysis of the micro-surface.

\section{Materials and methods}

\subsection{Silver cleaning samples}

A set of silver alloy mock-ups was prepared reproducing different mechanical cleaning treatments. The original untreated silver surface was finished using abrasive paper of different grain sizes, with the polishing carried out manually along a fixed direction.
Two samples were treated with traditional mechanical methods (calcium carbonate and sodium hydrogen carbonate suspended in water) and five samples were treated with the innovative dry cleaning method based on erasers. Five rubbers were employed with different characteristics and abrasive power. The cleaning treatments were performed by ten crisscross applications each. After scrubbing, the surface was washed with acetone, where the erasers were used, and with water where abrasive powders were used. The Table 1 reports the silver cleanings samples specifications. The rubber-based cleaning tools contain abrasive particles of different size and distributions.

The efficacy of removal of silver sulphide was studied using optical microscopy and SEM in a previous work [6] while this study is aimed at investigating the effects on the surface trough a quantitative roughness analysis. In fact, though laboratory microscopy techniques, which are being used for the inspection of surface morphology alteration, are proven to detect the single defects locally,

Table 1. Silver cleaning treatments

\begin{tabular}{cll}
\hline Sample & Cleaning treatment & Abrasive medium \\
\hline A & $\begin{array}{l}\text { Sodium hydrogen carbonate suspended } \\
\text { in water }\end{array}$ & Sodium bicarbonate, grain size $300-500 \mu \mathrm{m}$ \\
\hline B & Calcium carbonate suspended in water & Calcium carbonate, grain size about $100 \mu \mathrm{m}$ \\
\hline C & Rubber Staedtler refill & $\begin{array}{l}\text { Calcium carbonate, grain size } 1-30 \mu \mathrm{m}, \\
\text { organic matrix medium with S and Cl }\end{array}$ \\
\hline D & Rubber Cretacolor Monolith & $\begin{array}{l}\text { Calcium carbonate, grain size } 1-50 \mu \mathrm{m} \text { with } \\
\text { higher density and same composition of } \\
\text { sample C }\end{array}$ \\
\hline E & Rubber pencil Perfection Faber-Castel & $\begin{array}{l}\text { Calcium carbonate, grain size } 1-35 \mu \mathrm{m} \text { with } \\
\text { homogeneous density. No S and Cl }\end{array}$ \\
\hline F & Dental wheel rubber Wilpas & $\begin{array}{l}\text { Silicon rubber. Zirconium of grain size 1-10 } \\
\mu \mathrm{m} \text { with homogeneous distribution }\end{array}$ \\
\hline G & Dental pointed rubber Identoflex & $\begin{array}{l}\text { Silicon rubber. Aluminum oxide of grain size } \\
1-10 \mu \mathrm{m} \text { with homogeneous distribution }\end{array}$ \\
\hline
\end{tabular}


they cannot provide an objective measure of the surface features at micrometric scale over the sample.

In this experiment, the cleaning treatments were applied directly to the silver surface without inducing artificial tarnishing in order to focus on the effect of the mechanical abrasive process on the surface.

\subsection{Surface measurements}

The measured roughness is not an intrinsic property but is dependent on the bandwidth of the measurement [14]. The distribution of size and shape of the asperities in the digitized surface is limited by the sampling step, which determines the shortest spatial structure, and by the sampling length, which determines the longest one. The information collected should refer to the range of surface features determined by the specific application.

For acquiring the surface at micrometric level we have used a custom optical micro-profilometer that has been implemented in the framework of the Scan4Reco European project [15]. The system is based on a laser conoscopic holography probe mounted on two linear micrometric stages $\left(30 \times 30 \mathrm{~cm}^{2}\right.$ scan size). While the motion system scans the sample, the probe measures the distance to the surface, thus collecting a sequence of heights that allows the reconstruction of the surface map. Measurement range, lateral resolution, and standoff distance vary with the probe-lens coupling and can be tailored to the scale of the object (from sub-millimetres to centimetres). For the silver cleaning samples a ConoPoint-3R sensor (by Optimet) coupled to a $25 \mathrm{~mm} \mathrm{~N}$ lens was used, with a measurement range of $1 \mathrm{~mm}$ and a standoff distance of $16 \mathrm{~mm}$. The probe has a lateral resolution of 5 micron determined by the laser spot and a repeatability of 0.06 micron. In this study, the surface data has been acquired using a stage step of 5 micron. The entire size of the samples has been acquired.

The samples have been measured also with Atomic Force Microscopy (AFM) to provide supplementary surface data at sub-micron scale. AFM allows the measurement of fine details of the surface but in a small scan size. AFM measurements have been carried out with a NT-MDT -SMENA A with a goldcoated silicon tip from the same company in semi-contact mode. Five scans have been taken for each sample with a scan length of 60 micron (256 sampled points).

\subsection{Surface data analysis}

The surface geometrical structure has a multi-scale nature being a superimposition of a large number of scales of roughness. The common approach in surface metrology [16] is to describe a surface profile in terms of components of different bandwidth along the scan length: the roughness, i.e. irregularities at smaller wavelengths, the waviness, i.e. more widely spaced variations, and the shape (Fig. 1). Such features are usually ascribed to deviations from an "ideal" surface; for instance, the roughness can be put in relation with the manufacturing process inherent to the material, while the waviness with the quality of the process, e.g. presence of vibration. The cut-off value, i.e. the length of the scale at which the roughness is isolated, should be determined by the application by putting in relation these scale-limited features to the process in which are generated. However, in the cultural heritage field the artistic materials are not created in controlled processes such as in engineering and the artwork is being subjected to events that make 

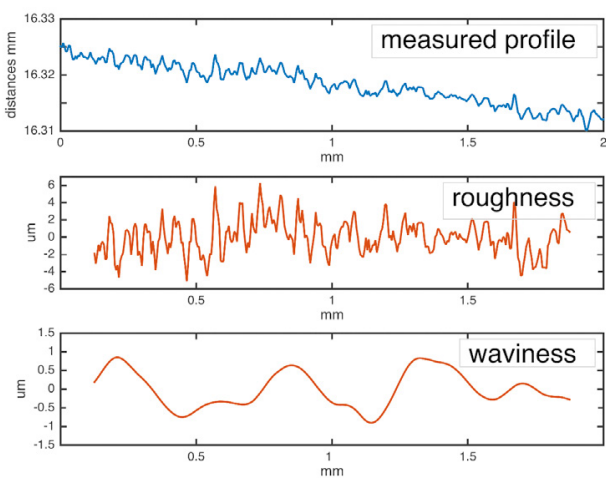

Fig. 1. Example of a profile measured by the optical profilometer and the roughness and waviness components for a cut-off of 250 micron ( $2 \mathrm{~mm}$ detail from sample A).

its surface history complex (and unknown) including materials' degradation and manual treatments.

From the scanning profilometry measurements we obtain a 2D map of heights, hence, a $3 \mathrm{D}$ points cloud that models the real surface in the bandwidth of the measurement. This digital representation is processed for extracting the parameters that describe the surface quality according to the interested features. Inspection of surface structure includes the detection of local defects and global roughness. Here, we are mainly concerned with the roughness aspect and even if the optical micro-profilometry is shown capable to detect fine local defects in silver, e.g. scratches, strokes, they are taken into account only in relation to the roughness amplitude contribution without their localization.

Different kinds of metrological parameters are available for roughness evaluation [17] and the ability of discriminating the topography can depend on this choice. In this study, surface evaluation is performed using areal parameters recommended by the standards [18], in terms of:
1) global features based on the average behaviour of the surface amplitude, by calculating the roughness parameter $S q$ (root mean square of the height distribution);

2) isolated events (i.e. defects), by calculating the extreme values parameters: $S p$ (maximum height of peaks), $S v$ (maximum height of valleys), and $S z$ (maximum height of the surface);

3) a multiscale approach, by inspecting the variation of the roughness features with the scale.

In order to characterize the surface topography, the first step was the removal of the form from the measured data. The analysis of the surface was then carried out with a multiscale approach as follows. As a first step, the roughness was analysed in a local representative region, and then the variation of the roughness distribution over the sample was evaluated. As a second step, the separation of the roughness and the waviness components was performed on the whole sample for different values of cut-off (Fig. 2). Roughness parameters were then calculated both on the total surface than on the scale-limited components. As a third step, the variation of the roughness parameters with the observation scale, i.e. the scan area, was inspected.

\section{Results}

\subsection{Micro-profilometry}

The surface texture generated by the different cleaning procedures is shown in Fig. 3 for comparison. For each sample it is reported the $3 \mathrm{D}$ plot of a central ROI after form removal and mean subtraction. The structure of the surface presents peaks, deep valleys, and plateaus due to the different cleaning treatment, with the amplitude density func- 


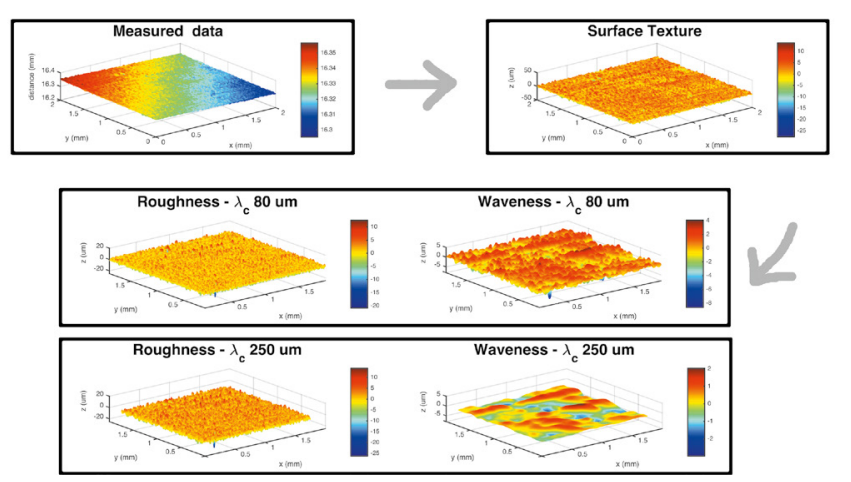

Fig. 2. Scheme of surface processing $\left(2 \times 2 \mathrm{~mm}^{2}\right.$ detail from sample A): from measured surface data to surface texture after form removal with polynomial best fit; extraction of roughness and waviness by Gaussian filtering with different cut-off values (example with 80 and 250 micron).
A

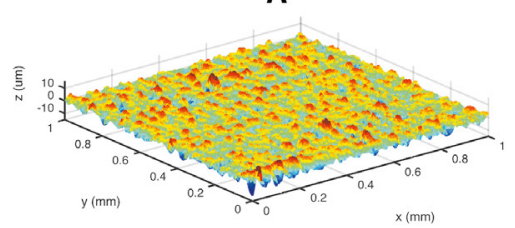

C

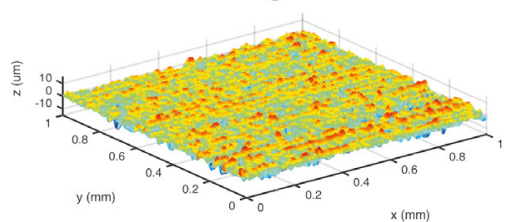

E

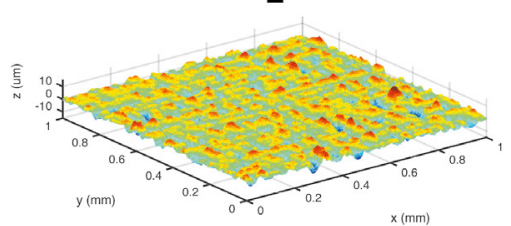

G

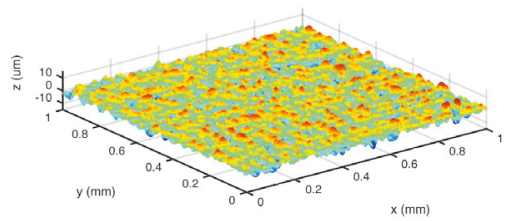

B
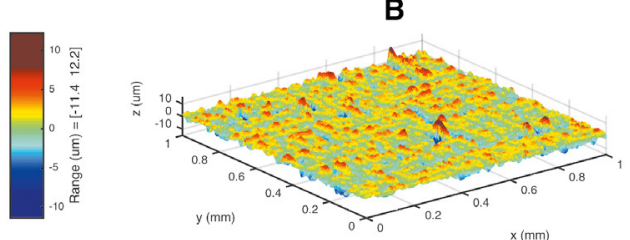

D
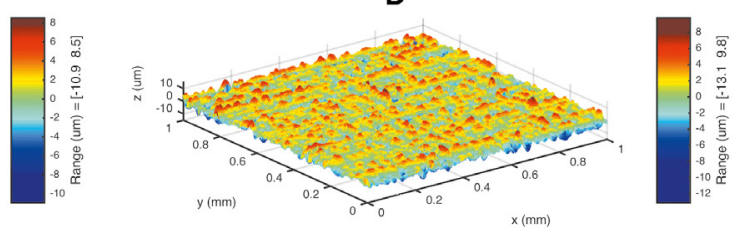

F

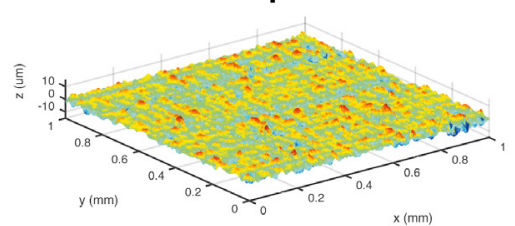

Non treated

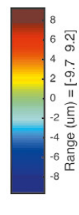

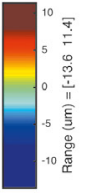

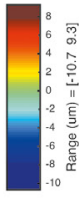

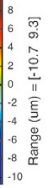

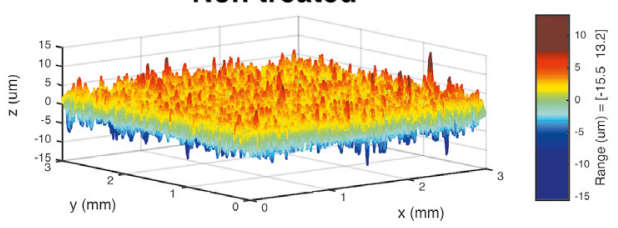

Fig. 3. 3D mesh plot of the micro-surface texture of each cleaning sample $\left(1 \times 1 \mathrm{~mm}^{2} \mathrm{ROI}\right)$ and of the nontreated silver sample $\left(3 \times 3 \mathrm{~mm}^{2} \mathrm{ROI}\right)$. 
tion (ADF) in a local ROI of nearly Gaussian characteristic (Fig. 4).

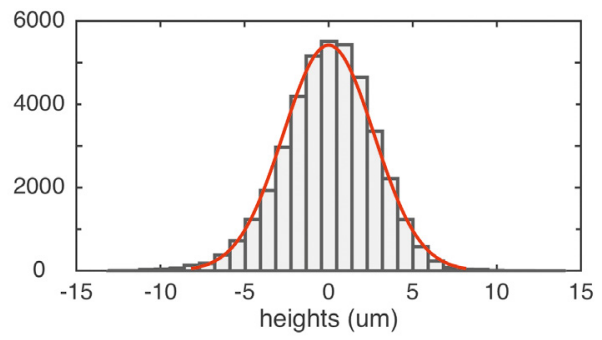

Fig. 4. Example of amplitude distribution of a local ROI $\left(1 \times 1 \mathrm{~mm}^{2}\right)$ in sample A with the fitted normal distribution.

In the first phase of the analysis, the $S q$ roughness was evaluated in a central ROI of $2 \times 2 \mathrm{~mm}^{2}$, after removing the outliers and averaging three different measurements taken with the sample rotated in different positions. In general, the roughness generated by the erasers is similar (order of micron) to the one generated by the abrasive powders showing that the dry cleaning process is compatible with the traditional methods (Fig. 5). The roughness is higher for the sample cleaned with the sodium bicarbonate $(\mathrm{A})$ and lower for the two samples treated with the dental tools ( $F$ and $G$ ) containing zirconium grains;

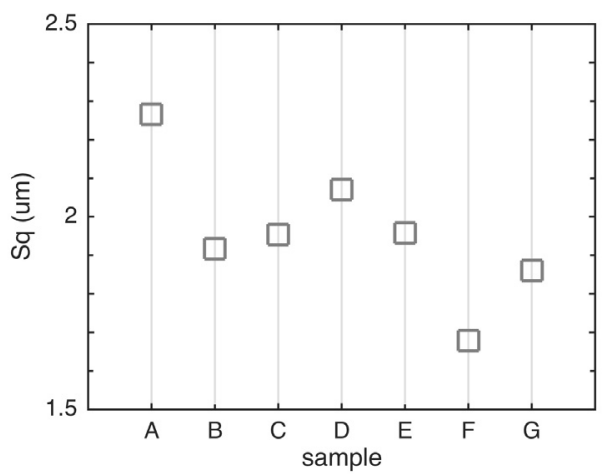

Fig. 5. Roughness $S q$ of the cleaning samples calculated in a representative ROI of $2 \times 2 \mathrm{~mm}^{2}$. the rubber tools containing grains of calcium carbonate $(\mathrm{C}, \mathrm{D}$, and $\mathrm{E})$ generate a roughness similar to the one generated by the calcium carbonate powder (B). The differences in the $S q$ roughness are compatible with the sizes of the abrasive grains observed with SEM and reported in Table 1. In an abrasion process there is expected a decrease of the amplitude roughness with the decrease of the abrasive grain size [19].

The roughness amplitude in the above-evaluated local ROIs is found to follow a nearly Gaussian distribution, as expected in relation to the underlying abrasion process. Abrasion is a very complex mechanism that involves phenomena related to the microstructural properties of the abrasive and the abraded materials and to the kind of process applied. The final roughness is influenced by a large number of factors, above all the hardness, the morphology and the size of the abrasive particles [20]. In principle, when an invasive mechanical cleaning is applied homogeneously to the surface, the overall roughness is lowered and if the process is applied for enough time the final roughness tends to a stationary value that is characteristics of the cleaning method, which is determined by the size and distribution of the abrasive particles contained in the cleaning tool. However, the cleaning process may not be homogeneous, some area may be cleaned for longer time or with higher pressure compared to others and this can lead to an inhomogeneous surface with a higher roughness (Fig. 6).

If the sampling area is too small compared to the inhomogeneity of the surface, these differences cannot be measured properly. Inhomogeneity of the surface roughness over the sample can be inspected by looking at the distribution of the averaged roughness obtained by dividing the sample in sub-re- 
Original surface $\mathrm{Rq}=0.142$

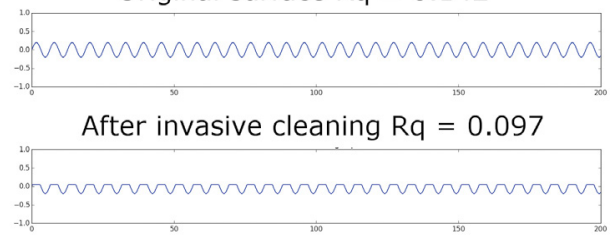

After inhomogeneous cleaning $\mathrm{Rq}=0.108$

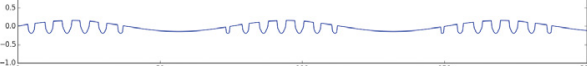

Fig. 6. Effect of inhomogeneous cleaning on the roughness. In the example, obtained using synthetic data in arbitrary units, the final roughness is given by the sum of contributes from the peak abrasion and from the waviness due to the inhomogeneous process.

gions of $50 \times 50$-sampled points (squares of 250 micron side). Here, the dry cleanings are shown to generate a homogeneous roughness, with the coefficient of variation (standard deviation normalized by the mean) of the roughness distribution compatible with the traditional methods (Fig. 7). As example, the averaged roughness map obtained for

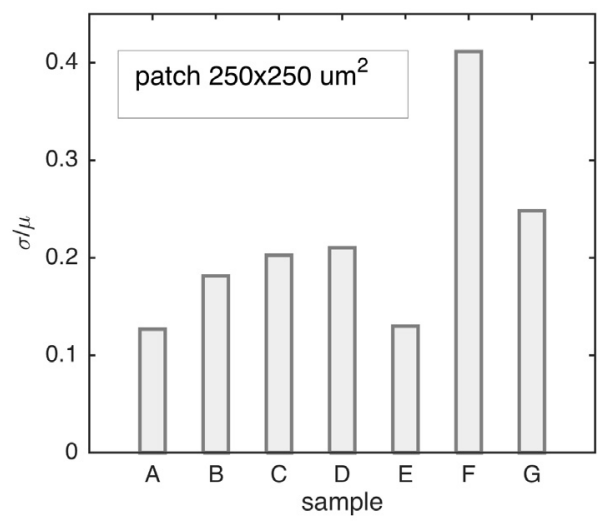

Fig. 7. Relative standard deviation (std dev/mean) of the averaged roughness distribution after dividing the sample in sub-regions of $250 \times 250 \mu \mathrm{m}^{2}$ for different treatment methods (Table 1 ). The rubber-based cleanings generate a surface roughness with a dispersion compatible to the traditional powders method $(A, B)$, with exclusion of the dental wheel (F). the samples treated with traditional and dry cleaning is shown in Fig. 8.

In the second phase of the analysis, the roughness and the waviness have been separated over the whole sample using Gaussian filtering for the following sequence of cut-off: $50,100,200,250,500,800$, and 1000 microns. The multiscale analysis shows a similar roughness behaviour for the dry cleaning treatments and the traditional methods. In addition, the roughness calculated in the scale-limited components points out three
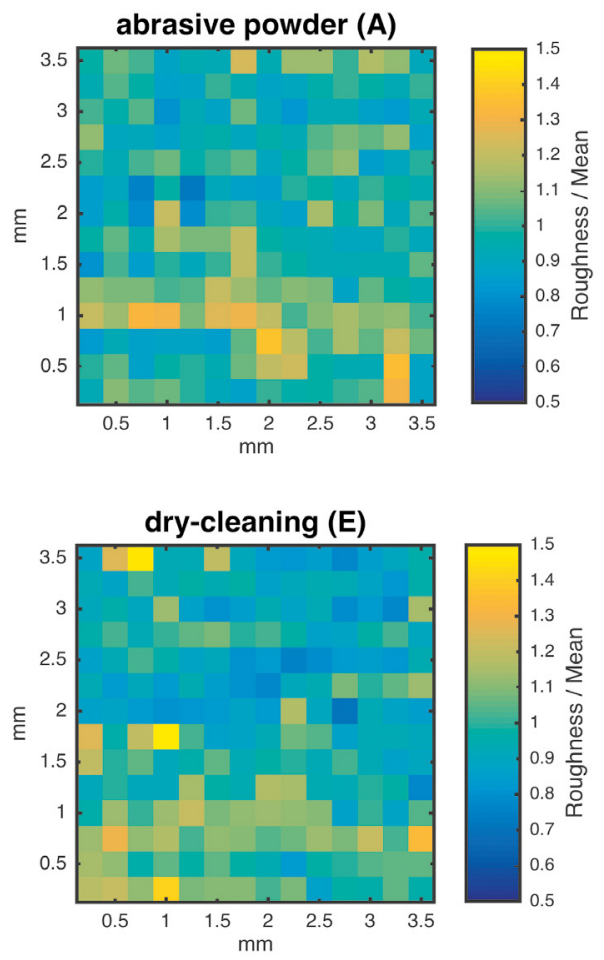

Fig. 8. Map of the roughness averaged in cells of 250 $\times 250 \mu \mathrm{m}^{2}$ and normalized by the mean of the cells for comparison: sodium bicarbonate abrasive powder (A) and dry cleaning with the rubber "Perfection" (E). The interval $0.5-1.5$ is plotted, with all the values outside assigned to the extremes of the colour map, to separate the effects of isolated peaks and valley and inspect the homogeneity. 
distinct groups (Fig. 9 and Fig. 10): the samples treated with abrasive powders (A and $B)$, which have the higher roughness at the various cut-off, the samples treated with the calcium carbonate rubbers $(\mathrm{C}, \mathrm{D}$, and $\mathrm{E})$

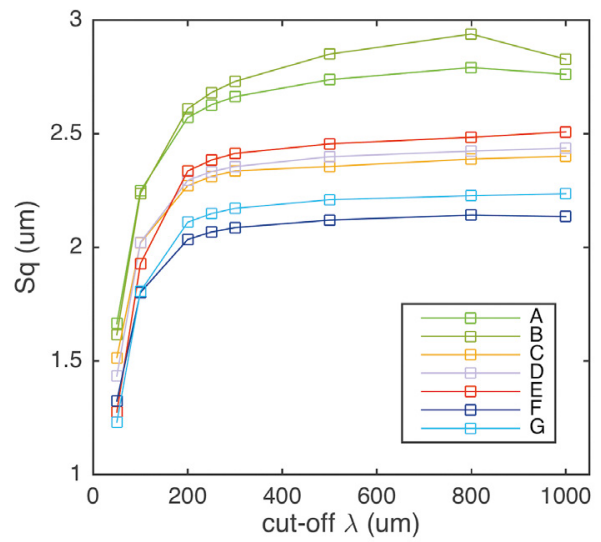

Fig. 9. Roughness $S q$ of the samples calculated for different scale-limited components using a Gaussian filter and a sequence of cut-off in the range from 50 $\mu \mathrm{m}$ to $1 \mathrm{~mm}$.

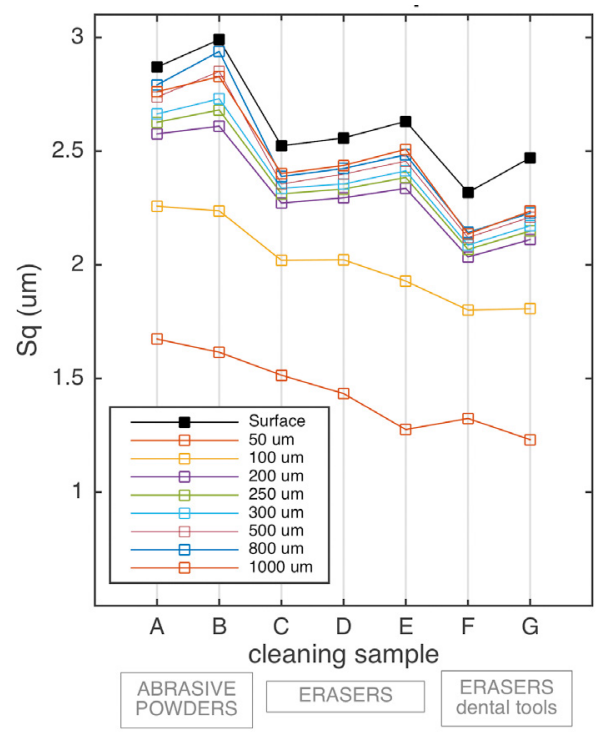

Fig. 10. Roughness $S q$ of the total surface and scale-limited components plotted versus the method of treatment (Table 1). with intermediate values of roughness, and the dental tools based on zirconium grains ( $F$ and $G$ ), which have the lower level of roughness. At the lower cut-off the contribute should be put in relation with the abrasive medium while at the longer scale with the waviness contribute induced by the kind of process over the sample.

The examined treatments have the same nature of a statistical abrasive process but the mechanical removal of the material at microscopic level is different. In fact, in the cleaning with the abrasive powder the abrasive particles are free, suspended in a liquid, while in the dry cleaning with the rubbers the abrasive grains are bonded to the tool. The observed differences in the roughness in the scale-limited components therefore depend on both microscopic and macroscopic factors, from the kind of abrasive mechanism, the kind of abrasive particles, to the manual procedure applied by the restorer (pressure, path in which the tool is moved, etc.).

Due to the multiscale nature of the surface the roughness may increase with the size of the evaluation area, but roughness variations may be ascribed also to the surface treatment that accumulates defects resulting in isolated peaks or valleys. Independent scratches or marks with low periodicity increase the global roughness. In order to consider this aspect, in the third phase of the analysis, the main roughness amplitude parameters were evaluated as function of the scan size using a multiscale approach [21]. To compute the multi-scale roughness it has been implemented an algorithm as follows. The surface is subdivided in square sub-regions of specific side; for each patch the extreme roughness amplitudes $S p$ (representing the peaks), $S v$ (representing the valley), $S z$ (representing the maximum amplitude range), together with the $S q$ roughness, are calculated; then 
the roughness parameters are averaged on the patches. This step is repeated varying the side of the patch. The roughness parameters are plotted versus the patch size, giving an insight of their variation with the observation scale (Fig. 11).

The plots show a similar behaviour for the dry cleaning methods and the traditional abrasive powders. The maximal and minimal roughness amplitudes increase with the scale due to the higher probability of encountering higher peaks or deeper valleys. Differences
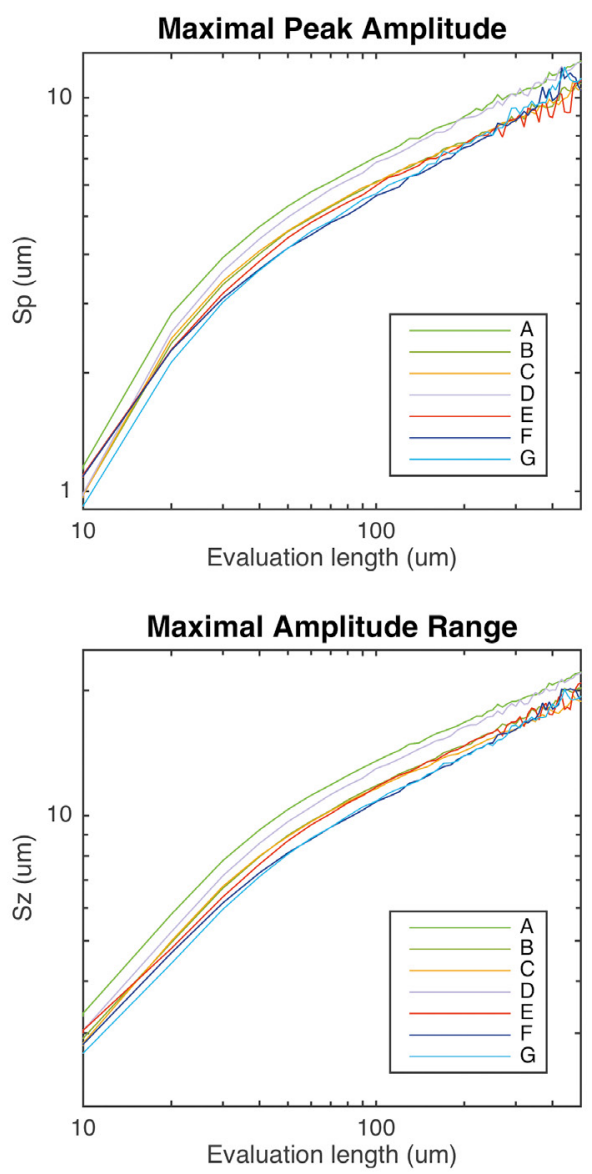

are related to the kind of abrasive particles and to the kind of process applied. The extreme amplitudes are higher for the sample treated with the sodium bicarbonate powder of larger grain size (400 micron), and lower for the samples treated with the rubbers containing the smaller zirconium grains (10 micron). Among the rubbers, the "Cretacolor Monolith" (D) exhibits higher values of both maximal and minimal roughness. The other dry cleaning methods based on rubber containing calcium carbonate show similar
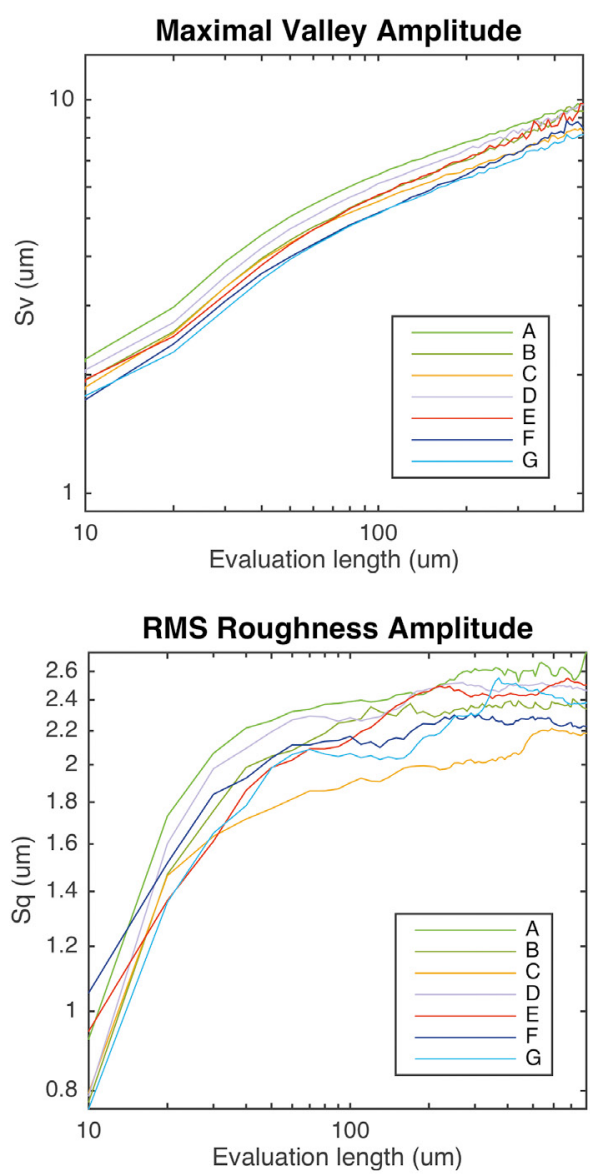

Fig. 11. Variation of the roughness parameters with the scan size (log-log plot): extreme amplitudes of peaks $S p$ (top-left), of valleys $S v$ (top-right), maximal surface heights $S z$ (bottom-left), RMS roughness $S q$ (bottom-right). 
values than the traditional powder method. Differently from the extreme amplitude roughness parameters, the $S q$ was found to tend to a constant value with the scale, meaning that there is a long-wavelength behaviour in relation to the underlying process.

\subsection{AFM analysis}

AFM measurements provided the supplementary evaluation of the sub-micron roughness locally (scan size $60 \times 60 \mu \mathrm{m}^{2}$ ). AFM surface map reveals the anisotropic surface structure with a predominant linear pattern in both the treated and non-treated samples (Fig. 12). This lay is due to the fact that the original surface of the silver sample has been
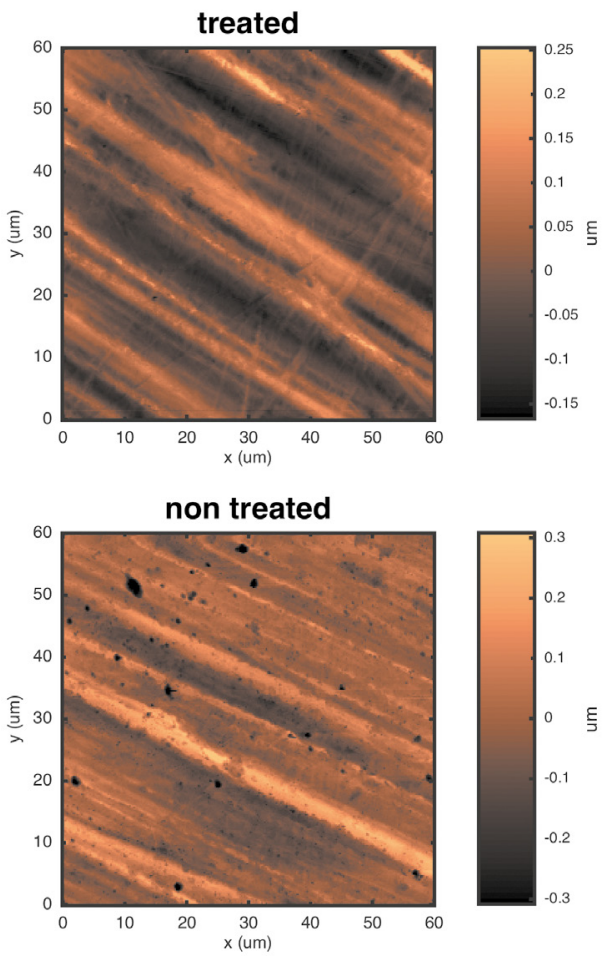

Fig. 12. AFM surface map (256pts, $60 \times 60 \mu \mathrm{m}^{2}$ scan size): treated (rubber) and non-treated silver sample. finished using sand paper along a single direction, thus engraving a regular grating in the surface. In the AFM surface map we can see the scratches due to the manufacturing process itself and the smaller scratches due to the cleaning process.

The roughness was evaluated by averaging $S q$ from five measurements taken for each samples. The comparison is reported in Fig. 13; here, the high values of uncertainty given by the error bars are due to the intrinsic variability of the roughness across the sample. Roughness is normally higher

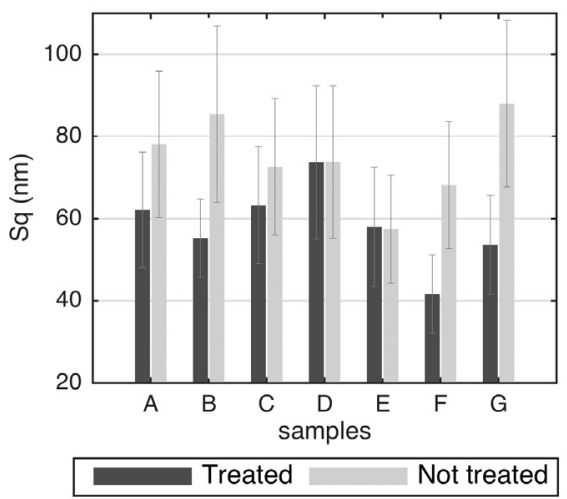

Fig. 13. AFM roughness of treated and non-treated silver sample for the different cleaning procedures. Roughness was obtained by averaging $S q$ from five different AFM scans for each sample; bar error is the standard deviation of the mean

for the reference sample and lower for the cleaned sample. Among the dry cleanings, the rubber-based treatments $\mathrm{D}$ and $\mathrm{E}$ exhibit the lower difference between the reference roughness and the sample roughness, the dental tools $F$ and $G$ produce higher surface variations.

\subsection{Discussion}

Most of the techniques used for retrieving the micro-surface of an object allow exam- 
ining only a small portion of the sample. An advantage of the set-up used in this work is the capability to measure objects with sizes up to $30 \times 30 \mathrm{~cm}^{2}$, together with keeping micrometric resolution, thus allowing the evaluation of low-periodicity features as waviness and shape deformation beside the roughness. Measuring the waviness requires such high depth precision as roughness but large scan sizes in order to evaluate the widely spaced periodicity variations.

Measuring the surface with the scanning micro-profilometer can provide a more effective way to estimate the cleaning inhomogeneity of the sample. In this case, the standard deviation of the heights distribution can generally provide an insight of the homogeneity. A visual evaluation of the surface topography map can also help to identify patterns left by the cleaning tool (Fig. 14).

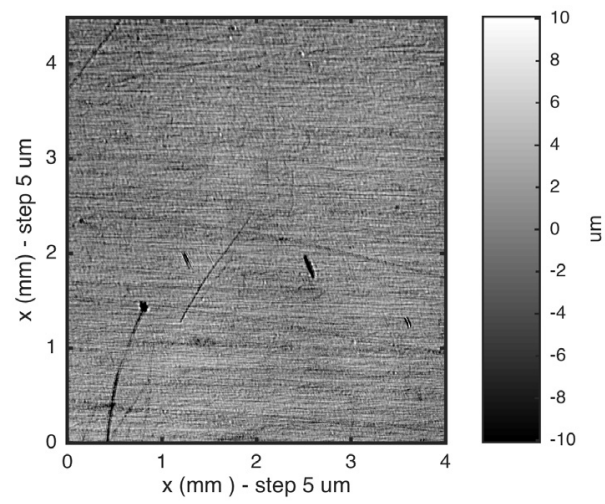

Fig. 14. Micro-profilometry surface map (2D intensity plot of the heights): detail showing the marks and scratches left by the cleaning procedure.

What is measured with the AFM technique is the roughness of the material, hence the invasiveness of the cleaning, while little information can be achieved regarding the homogeneity of the treatment. In this case, it is expected that a lower roughness ascribe a more invasive cleaning method.
Micro-profilometry and AFM allowed the evaluation of roughness features of different length scales depending on the bandwidth of the device. An (ideal) selection criterion should be to choose the cleaning method that best preserves the original roughness at the different scales.

\section{Conclusions}

The effectiveness of laser conoscopic holography in the analysis of the cleaning of silver, has been demonstrated, namely:

1. the technique is able to measure specular surfaces;

2. the bandwidth of the measurement yields to the representation of a relevant number of asperities, i.e. the distribution of shape and size of irregularities allows a reliable description of the main structures of the surface;

3 . the length scales of the measured roughness features (waviness and roughness) is relevant for the application, and some aspects of the surface topography are put in relation with the kind of material processing.

The optical micro-profilometer provides a more effective way to estimate the quality of the cleaning process in relation to the roughness inhomogeneity of the sample, including defects accumulation.

The multiscale approach allows a more accurate surface analysis. Anyway, understanding which is the relevant analysis scale is difficult. Given the multiscale nature of the roughness, as well as the multiscale capability of the optical profilometric probes, complementary measurement techniques with different bandwidths should be used for the characterization of the surface for a more comprehensive evaluation of the effective- 
ness of the cleaning method. To this respect, a further work is being planned to integrate optical micro-profilometry and AFM.

Concerning the aspect of surface conservation, the results obtained on a set of untarnished silver samples treated with both wet and dry cleaning methods point out that the rubber-based treatment produces a surface characterized by a roughness smaller or similar to traditional methods.

The optical scanning profilometry results are of particular significance since this technique allows an in-situ use for monitoring real artwork surfaces.

\section{Acknowledgements}

This work was supported by the Scan4Reco project funded by EU Horizon 2020 Framework Programme for Research and Innovation under Grant Agreement no 665091 .

\section{References}

[1] M.B. Mcneil, B.J. Little, "Corrosion Mechanisms for Copper and Silver Objects in Near-Surface Environments", Journal of the American Institute for Conservation, 31 (1992) 355-366, DOI: 10.2307/3179729.

[2] R.J.H. Wanhill, "Brittle Archaeological Silver: A Fracture Mechanisms and Mechanics Assessment", Archaeometry, 45 (2003) 625-636, DOI: 10.1046/j.1475-4754.2003.00133.x.

[3] R.J.H. Wanhill, "Case Histories of Ancient Silver Embrittlement", Journal of Failure Analysis and Prevention, 11 (2011) 178-185, DOI: 10.1007/ s11668-010-9429-5.

[4] J.P. Franey, G.W. Kammlott, T.E. Graedel, "The corrosion of silver by atmospheric sulfurous gases", Corrosion Science, 25 (1985) 133-143, DOI: 10.1016/0010-938X(85)90104-0.

[5] V. Costa, "The deterioration of silver alloys and some aspects of their conservation", Reviews in Con- servation, 2 (2001) 18-34, DOI: 10.1179/sic.2001.46. Supplement-1.18.

[6] G. Basilissi, A. Cagnini, "Ricerca e sperimentazione di nuove metodologie di pulitura dell'argento e messa a punto di idonei formulate protettivi", in: Proc. Lo stato dell'arte 14, L'Aquila, Italy, October 20-22, 2016, Nardini Editore, Firenze 2016, pp. 99-107.

[7] T. Palomar, B. Ramírez Barat, E. García, E. Cano, "A comparative study of cleaning methods for tarnished silver", Journal of Cultural Heritage, 17 (2016) 20-26, DOI: 10.1016/j.culher.2015.07.012.

[8] T. Palomar, M. Oujja, I. Llorente, B. Ramirez Barat, M.V. Canamares, E. Cano, M. Castillejo, "Evaluation of laser cleaning for the restoration of tarnished silver artifacts", Applied Surface Science, 387 (2016) 118-127, DOI: 10.1016/j.apsusc.2016.06.017.

[9] R. Fontana, M.C. Gambino, M. Greco, L. Marras, M. Materazzi, E. Pampaloni, L. Pezzati, "High-precision surface analysis of the roughness of Michelangelo's David", Proc. SPIE 5146 (2003) 236-243, DOI: 10.1117/12.501252.

[10] G. Y. Sirat, "Conoscopic holography. I. Basic principles and physical basis", Journal of the Optical Society of America A, 9 (1992) 70-83, DOI: 10.1364/ JOSAA.9.000070.

[11] P. Carcagni, C. Daffara, R. Fontana, M.C. Gambino, M. Mastroianni, C. Mazzotta, E. Pampaloni, L. Pezzati, "Optical micro-profilometry for archaeology”, Proc. SPIE 5857 (2005) 58570F, DOI: 10.1117/12.612527.

[12] C. Colombo, C. Daffara, R. Fontana, M.C. Gambino, M. Mastroianni, E. Pampaloni, M. Realini, A. Sansonetti, "Evaluation by Laser Micro-Profilometry of Morphological Changes Induced on Stone Materials by Laser Cleaning", in: Laser in the Conservation of Artworks (Proceedings of LACONA VI Conference), Vienna, Austria, September 21-25, 2005, M. J. Nimmrichter, W. Kautek, M. Schreiner (Eds.), Springer proceedings in physics 116, Springer, Berlin 2007, pp. 523-526, DOI: 10.1007/978-3-540-72310-7_62.

[13] R. Fontana, A. Dal Fovo, J. Striova, L. Pezzati, E. Pampaloni, M. Raffaelli, M. Barucci, "Application of non-invasive optical monitoring methodologies to follow and record painting cleaning processes", Ap- 
plied Physics A, 121 (2015), 957-966, DOI: 10.1007/ s00339-015-9505-5.

[14] Bharat Bhushan, "Surface Roughness Analysis and Measurement Techniques", in: Modern Tribology Handbook, CRC Press, Boca Raton, London, New York, Washington D.C. 2000, pp. 49-119, DOI: $10.1201 / 9780849377877$.

[15] N. Gaburro, G. Marchioro, C. Daffara, "A versatile optical profilometer based on conoscopic holography sensors for acquisition of specular and diffusive surfaces in artworks", Proc. SPIE 10331 (2017) 103310A, DOI: 10.1117/12.2270307.

[16] D.J. Whitehouse, Handbook of Surface Metrology, Institute of Physics Publishing, Bristol U.K. 1994.

[17] R. Leach (Ed), Characterisation of Areal Surface Texture, Springer, Heidelberg 2013.
[18] ISO 25178-2, Geometrical Product Specifications (GPS) - Surface texture: Areal - Part 2): Terms, Definitions and Surface Texture Parameters, International Organization for Standardization 2012.

[19] J.J. Coronado, "Effect of Abrasive Size on Wear" in: M. Adamiak (Ed.), Abrasion Resistance of Materials, InTech, 2012, pp. 167-184.

[20] K. Kato, K. Adachi, "Wear Mechanism" in: Bharat Bhushan (Ed.), Modern Tribology Handbook, CRC Press, Boca Raton, London, New York, Washington D.C. 2000, pp. 273-300, DOI: 10.1201/9780849377877.

[21] M. Bigerelle, T. Mathia, S. Bouvier, "The multiscale roughness analysis and modelling of abrasion with the grit size effect on ground surface", Wear, 286-287 (2012) 124-135, DOI: 10.1016/j. wear.2011.08.006. 


\title{
Nonlinear optical imaging techniques (NLO) for painting investigation
}

\author{
Alice Dal Fovo ${ }^{\star}$, Raffaella Fontana, Jana Striova, Enrico Pampaloni, Marco Barucci, \\ Marco Raffaelli, Raffaella Mercatelli, Luca Pezzati, Riccardo Cicchi
}

Istituto Nazionale di Ottica - CNR-INO, Largo Fermi 6, 50125, Firenze, Italy

* Corresponding author: alice.dalfovo@ino.it

\begin{abstract}
Nonlinear optical imaging techniques (NLO imaging), i.e. multiphoton excitation fluorescence (MPEF), second and third harmonic generation (SHG, THG), are high-resolution imaging modalities which may provide non-destructive determination of thickness and composition within multi-layer objects as a function of depth. NLO techniques have been recently introduced into artworks analyses, giving promising results. As regards paintings, the guiding idea is to exploit the low absorption in the near-infrared (NIR) of most materials, using a laser light in this spectral region which may generate non-linear interactions within materials. The objective is to make feasible the in-depth profiling of thin films on the basis of refractive index changes, variation of optical activity and presence of fluorophores. Such information is definitely useful for the analysis of painted objects and can be also crucial for the monitoring of restoring operations. In this study, we present the preliminary results obtained through the application of NLO imaging techniques on a series of single- and multi-layers systems simulating real egg-tempera wood panel paintings. This research is part of the wider Iperion $\mathrm{CH}$ Project, whose WP6 entails the evaluation of NLO-imaging to obtain highly resolved en-face and 3D images of small areas on paintings. To that purpose, a set of multi-layer painted samples, simulating real paintings, was designed and realized. They are currently under analysis with different operating systems and modalities within the partnership. In order to assess the reliability of NLO measurements, data were complemented with other well-established techniques, such as Fourier Transform Infrared Spectroscopy (FTIR) and X-Ray Fluorescence (XRF) for the chemical characterization of materials, Optical Coherence Tomography (OCT) for the determination of layers thickness and laser scanning micro-profilometry for the study of surface morphology. In addition, colorimetric analyses, i.e. reflectance spectroscopy in the visible region, were performed in order to chromatically characterize pigments and lakes. Finally, the transparency of the different materials was investigated by means of Scanning Multispectral VIS-NIR Reflectography.

The preliminary results suggest that the $740 \mathrm{~nm}$ wavelength, while enabling the visualization of the painting surfaces micromorphology, is not effective for the determination of thickness through the detection of the non-linear signals.
\end{abstract}

Keywords: non-invasive techniques, non-linear imaging, multi-photon excitation fluorescence, paintings, monitoring 


\section{Introduction}

Multiphoton excitation fluorescence (MPEF), second and third harmonic generation (SHG, THG), and fluorescence lifetime imaging microscopy (FLIM) are high-resolution non-linear imaging modalities, which may provide non-destructive determination of thickness and composition within multi-layer objects. NLO techniques are widely used as diagnostic tools in the biological field and have been applied to artworks analyses for the identification of layers corrosion in metal-based objects [1], for the visualization and characterization of wood microstructures [2] and for the study of synthetic glue and varnish protective layers [3-5]. In regard to paintings, only few tests have been performed by means of femtosecond pumpprobe microscopy on painted mock-ups and historic artworks [6, 7], encouraging further investigations. The guideline of this research is to examine inner layers by exploiting the low absorption of most painting materials in the near-infrared region, thus obtaining the in-depth profiling of thin films, the micrometric surface mapping and the detection of fluorescence chromophores and specific molecular species. Such information may turn useful for the analysis of painted objects, as well as for the monitoring of restoring operations, like the cleaning process, that modify surfaces micrometric morphology and coating layers thickness.

NLO techniques are based on physical processes in which a molecule can be excited by simultaneous interaction with two or more photons in the same quantum event. SHG and THG are second and third order non-linear scattering phenomena in which two or three photons of $\omega$ angular frequency are simultaneously converted in a photon of $2 \omega$ or $3 \omega$ angular frequency, respectively. SHG enables the detection of high degree-organized structures without inversion symmetry, such as stacked membranes and arranged proteins (e.g. collagens), for the visualisation of wood microstructure, specifically crystalline cellulose $[2,3]$. On the other hand, THG allows to resolve transparent interfaces, on the basis of local differences in refractive index and dispersion [8]. In Two-Photon-Excited Fluorescence (TPEF) the detection of fluorescence emitted after the simultaneous absorption of two infrared photons [9] yields to the identification of chromophores. Finally, fluorescence lifetime imaging microscopy (FLIM) measures time-decay of the fluorescence intensity exhibited by fluorescent emitting molecules [10], yielding information on the molecular microenvironment of a molecule and on its energy exchanges.

The use of non-linear techniques offers several advantages to the three-dimensional imaging, mainly because of the non-linear properties of the high-intensity excitation source employed - i.e. a tightly focused femtosecond (fs) pulsed laser. Firstly, non-linear interaction occurs only at, or near, the focal point of the beam. Secondly, long wavelengths, particularly in the near-infrared spectral region, allow deeper penetration in most pictorial materials and are not scattered as much as shorter wavelengths. Thirdly, since in non-linear processes the excitation volume is proportional to a power of the laser intensity, the axial resolution achievable is greatly increased. Finally, photo-damage and photo-bleaching are drastically reduced with respect to confocal and wide-field fluorescence imaging, which is desirable in $\mathrm{CH}$ studies.

In this work we present the preliminary results obtained through the application of NLO imaging techniques with a custom-made laser-scanning non-linear micro- 
scope developed by INO-CNR (Cicchi R. et al.) [10]. The analyses were performed on a series of single- and multi-layers systems, specifically designed for NLO imaging applications, simulating the real egg-tempera wood panel paintings. Such samples are distributed in a round robin approach within the partnership and are currently under analysis with different operating systems and modalities. This research is part of the wider IperionCH Project whose WP6 entails the evaluation of NLO-imaging to obtain highly resolved en-face and 3D images .

\section{Materials and Methods}

\subsection{Description of samples}

The samples analyzed in this study were designed to create favourable and variable condition to detect NLO signals in order to assess the applicability and the efficiency of non-linear microscopy techniques on a given number of cases. In this section, the design of the samples is reported firstly, followed by the description of material used.

Each sample is a multi-layer system simulating a real painting, showing a sequence of layers of different thickness, obtained by increasing the number of the superimposed stratifications.

We prepared three sets of samples - red, yellow and blue - labelled R, Y and B, respectively (Fig. 1). For each colour three versions, $\mathrm{A}, \mathrm{B}$ and $\mathrm{C}$, were realized, showing the same sequence of stratification and materials but different layers' thickness. Each set (R, Y, B) is composed of 24 square samples $(3 \mathrm{~cm} \times$ $3 \mathrm{~cm}$ ), for a total number of 72 squares.

The stratifications sequence is the following: wooden support (a "sandwich" structure made of fir and poplar), preparation layer (gypsum and animal glue), underdrawings (lead and tin stylus, natural carbon, iron-gall
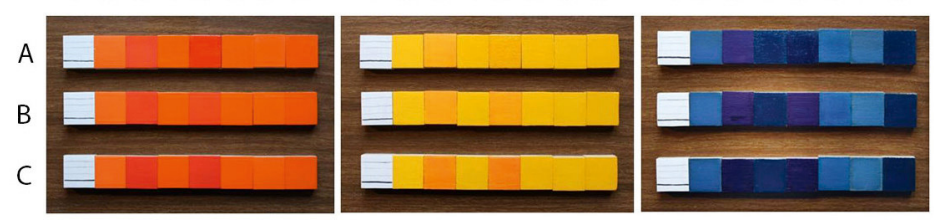

\begin{tabular}{lccl}
\hline $\begin{array}{l}\text { Colour } \\
\text { sets }\end{array}$ & Versions & $\begin{array}{c}\text { Number of square } \\
\text { samples }\end{array}$ & Acronym \\
\hline \multirow{3}{*}{ Red (R) } & A & 8 & R_A1, A2, A3, A4, A5, A6, A7, A8 \\
\cline { 2 - 4 } & B & 8 & R_B1, B2, B3, B4, B5, B6, B7, B8 \\
\cline { 2 - 4 } & C & 8 & R_C1, C2, C3, C4, C5, C6, C7, C8 \\
\hline \multirow{3}{*}{ Yellow (Y) } & A & 8 & Y_A1, A2, A3, A4, A5, A6, A7, A8 \\
\cline { 2 - 4 } & B & 8 & Y_B1, B2, B3, B4, B5, B6, B7, B8 \\
\cline { 2 - 4 } & C & 8 & Y_C1, C2, C3, C4, C5, C6, C7, C8 \\
\hline \multirow{3}{*}{ Blue (B) } & A & 8 & B_A1, A2, A3, A4, A5, A6, A7, A8 \\
\cline { 2 - 4 } & B & 8 & B_B1, B2, B3, B4, B5, B6, B7, B8 \\
\cline { 2 - 4 } & C & 8 & B_C1, C2, C3, C4, C5, C6, C7, C8 \\
\hline
\end{tabular}

Fig. 1. Multi-layers samples simulating real paintings with the relative acronyms. 
ink), first pictorial layer (egg tempera with three different pigments), second pictorial layer (three different lakes) and superficial protective layers (natural and synthetic varnishes, egg, and multi-layer coatings). The stratification sequence for version A - sets R, $\mathrm{Y}, \mathrm{B}$ - which is the first that has been analyzed for this study, is shown in Fig. 2 and described in the following scheme:

We chose the wood as a support because, besides being historically and physically suitable for egg-tempera, it also gives the opportunity to visualize wood microstructures by means of NLO signals, specifically crystalline cellulose (SHG signal) and lignin (2PEF signal) in the wood cell walls. binders, such as linseed oil). We chose three different pigments by Kremer (Germany) - i.e. lead minium (red lead, $\mathrm{Pb}_{3} \mathrm{O}_{4}$ ) - cod. 42500; cadmium yellow medium (Cadmium sulfide, CdS) - cod. 21051; Egyptian blue (blue copper silicate chemically classified as the mineral cuprorivaite, $\mathrm{CaCuSi}_{4} \mathrm{O}_{10}$ ) - cod. 10060 - in order to reproduce the optical properties of three representative painting layers. The selected pigments were widely used throughout history and show some specific optical properties, which hopefully make the study of their non-linear response relevant.

Minium is an oxidation product of lead minerals, originally extracted in the river

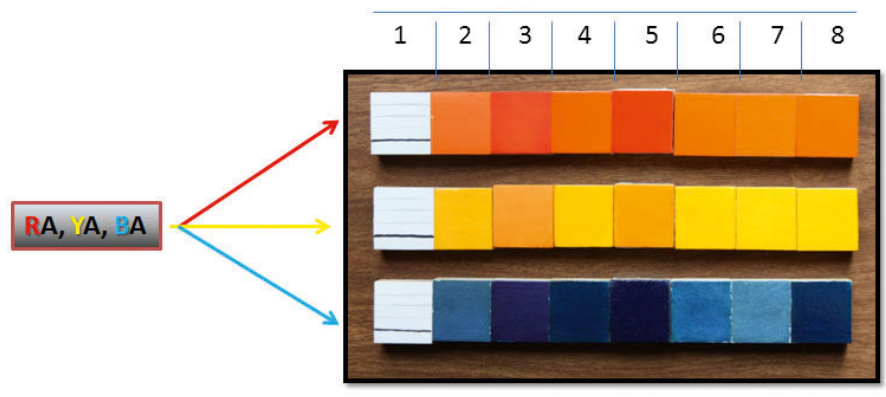

Fig. 2. Version A (basic layers thickness) of R, Y, B sets.

Scheme of the stratifications:

- 1: preparation + underdrawings

- 2: preparation + underdrawings + egg-tempera

- 3: preparation + underdrawings + egg-tempera + lake

- 4: preparation + underdrawings + egg-tempera + varnish (dammar)

- 5: preparation + underdrawings + egg-tempera + lake + varnish (dammar)

- 6: preparation + underdrawings + egg-tempera + varnish (synthetic varnish)

- 7: preparation + underdrawings + egg-tempera + protein layer (white egg)

- 8: preparation + underdrawings + egg-tempera + multi-layer coating (white egg + dammar)

The presence of the underdrawings on the preparation layer enables the evaluation of the layers' transparency. We selected the egg as a binder because of its drying speed, good stability, compatibility with most pigments, and reduced fluorescence (compared to oily
Minius in North-Western Spain, anciently produced by smelting lead ore and later prepared by heating lead white (basic lead carbonate) in air. Nowadays, it is obtained by heating litharge or white lead for some hours at high temperature (about $480^{\circ} \mathrm{C}$ ). Minium 
is an intense orange red pigment compatible with oil and egg binders, quite unstable and liable to discoloration in the presence of air pollutants, such as hydrogen sulfide, and may darken when exposed to humidity and light. Such a behaviour is limited when the pigment is dispersed in oil medium. However, given the high level of toxicity of red lead, minium has been gradually removed from the market and replaced with others, which are safer.

Cadmium sulfide refers to a number of yellow-orange-red compounds sharing a common structure based on CdS. By substituting $\mathrm{Cd}$ or $\mathrm{S}$ with various elements including $\mathrm{Zn}, \mathrm{Hg}$, and $\mathrm{Se}$, a variety of hues can be obtained. Such chromatic properties are due to $\mathrm{CdS} 2.42 \mathrm{eV}$ band gap that can be modulated by the progressive substitution of Cd with one of the former elements (e.g. Zn to obtain pale yellow cadmium zinc sulfide pigments [11]). Cadmium yellow, cadmium red and cadmium orange are brilliant pigments with good permanence and tinting power, which have been widely used since 1840, especially by painters like Claude Monet, Vincent Van Gogh, Edvard Munch and Jackson Pollock. Initially, Cadmium yellow (Cadmium sulfide, CdS) was prepared with an acid solution of cadmium salt (either chloride or sulfate) which was heated with hydrogen sulfide gas until a powder was generated to obtain hues ranging from lemon yellow to deep orange. In the past few years, once Cadmium's toxicity had been determined, the production of Cadmium pigments gradually decreased and they were partially replaced by azo-pigments, which have the advantage of being both cheaper and non-toxic. Cadmium sulfide is not very toxic either (median lethal dose $\mathrm{LD}_{50}>5000 \mathrm{mg}$ / $\mathrm{kg}$ ) when used as a pigment, although intense exposure to cadmium vapours from welding is harmful. Since Cadmium yellow optical behaviour has been thoroughly researched, we presumed it would have been significant for NLO investigations.

Egyptian blue is a copper silicate chemically classified as the mineral cuprorivaite $\left(\mathrm{CaCuSi}{ }_{4} \mathrm{O}_{10}\right)$, rarely found in nature but synthetically obtained since ancient times. Indeed, it is the first synthetic pigment that we know of, having been produced since the $4^{\text {th }}$ Egyptian Dynasty (around 2500 BC). Thanks to its chemical composition, Egyptian blue is stable in all media and does not undergo any colour change when applied to various organic media. Strong light does not influence its colour either, as it may be observed in samples which have survived for millennia in both dry and damp environments and still exhibit an intense hue with no evidence of photobleaching. Recently, a further remarkable property of this pigment has been found, namely the exceptionally high level of emission quantum efficiency in the near infrared $\left(\lambda_{\max }=\right.$ $\left.910 \mathrm{~nm} ; \Phi_{\mathrm{EM}}=10.5 \%\right)$ and the long excited lifetime $(107 \mu \mathrm{s})$, which makes cuprorivaite an excellent candidate as a luminescent label for a number of applications [12]. In view of this, we decided to investigate Egyptian blue non-linear behaviour.

A few samples (numbers 3 and 5 of each set) present an additional pictorial layer over the egg tempera, made of three different lakes, Old Holland Classic Oil Colors by Kremer (Ruby lake - R set -, Quinacridon-Anthanthrone, colour index: PR209-PR168; Blue lake - B set -, Phthalocyanine, colour index: PB15; Indian yellow-orange lake extra - Y set -, Azo condensation-Diketo Pyrrolo Pyrrole, colour index: PY95-PR251), in order to simulate real restoring interventions, and more specifically the pictorial retouching through the superposition of layers. Some other samples (numbers $4,5,6,7,8$ ) are covered 
with several protective layers: natural resin (dammar, by Zecchi) or synthetic varnish (picture varnish gloss, by Zecchi), protein white egg - layer, and a multilayer coating (white egg + dammar), so to replicate real cases of varnished paintings. The variability of paint and protective layers thickness and composition was chosen/established in order to evaluate the NLO-signals as a function of depth and of chemical composition.

\subsection{Measurements}

NLO measurements were performed using a custom-made laser-scanning nonlinear microscope, originally developed by Cicchi R. et al. for biological applications at INO-CNR [10]. The excitation source is a mode-locked Ti:Sapphire laser (Mira 900 F, Coherent Inc., Santa Clara, CA, US). The beam is focused onto the specimen by an objective (Nikon Plan Apo 20x, NA 0.75 Air). The filter used for the SHG detection is a narrow bandpass filter centred at $370 \pm 10 \mathrm{~nm}$ HQ420BP (Chroma Technology Corporation). For this study, the excitation wavelength was set to $740 \mathrm{~nm}$, with a pulse duration of $120 \mathrm{fs}$ and a repetition rate of $80 \mathrm{MHz}$, with a power of about $5 \mathrm{~mW}$ on the specimen. Non-linear signals coming from the sample were revealed by two different detection systems (Fig. 3-4): one for intensity images and the other for lifetime images. Two-photon-fluorescence intensity images are provided using a photomultiplier tube (Hamamatsu H7422), with a 190-900 nm spectral sensitivity, by assigning to each pixel an intensity value (grey level), that is proportional to the amount of fluorescence photons reaching the detector from each surface point scanned by the laser. TPEF images were acquired with a field of view of $200 \times 200 \mu^{2}$, a resolution of $512 \times 512$ pixels, z-step of $2 \mu \mathrm{m}$ for an

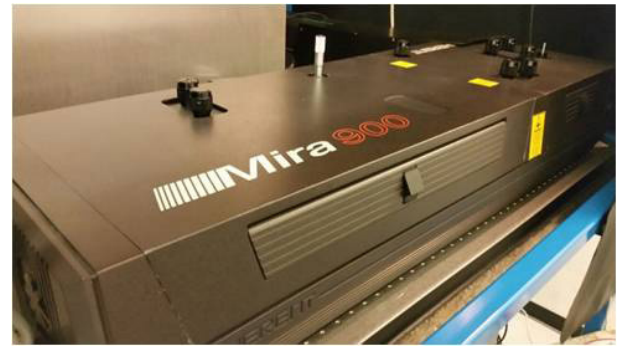

Fig. 3. Laser excitation source Mira 900 F, Coherent Inc., Santa Clara, CA, US.

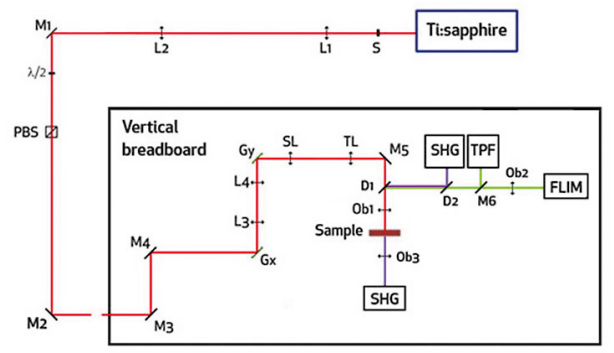

Fig. 4. Scheme of the experimental setup of the laser-scanner nonlinear microscope: laser source (Ti:Sapphire), optics (L 1-4, lens; TL, tube lens; SL, scanning lens; M 1-6, mirrors; Ob 1-3, shutters; Gx, Gy, galvanometric mirrors) and detection system (SHG, TPF, FLIM detectors).

acquisition time of about $5 \mathrm{sec}$. Using ImageJ (National Institutes of Health, Bethesda, VA, US) “Orthogonal Views" plug-in, 3D reconstructions from the stacks were obtained.

Fluorescence lifetime imaging microscopy (FLIM) was performed using a fast photon-counting detector (Becker \& Hickl PMH-100), with 400-600 nm spectral sensitivity. The output data, i.e. the decay curves and the related FLIM images, are reconstructed pixel-by-pixel from individual time measurements. Based on Time-Correlated Single-Photon Counting (TCSPC) principle, the arrival time of the individual photons is recorded with respect to the excitation pulse. Data processing and display are performed 
by means of SPC-Image software (Becker \& Hickl GmbH, Germany). The SPC-Image is coded in a false-colour scale image, in which each point represents the average fluorescence lifetime calculated through mono, bi- or tri-exponential fit of the related fluorescence decay curve, which can be visualized positioning the cursor in points of interest on the image.

Finally, a fast 16-channel photon counting photomultiplier (PML-16C Becker \& Hickl $\mathrm{GmbH}$ ) provides 16 fluorescence decay curves at 16 different narrow spectral bands for each point of the FLIM image. In this mode, the emitted light is sent to a spectrograph, it reaches the 16-channel photomultiplier with a transit time spread typical value of $150 \mathrm{ps}$, and then it is processed by the FLIM (Fluorescence Lifetime Imaging Microscopy) apparatus. Therefore, for each point of the measured area, 16 FLIM images are provided, containing the xy maps of the fluorescence decay, which are recorded with a spectral resolution of $12,5 \mathrm{~nm}$ within 400-600 $\mathrm{nm}$ instrument's operating range, thus enabling the detection of specific emitting molecular species.

In order to assess the reliability of NLO measurements, in cooperation with Opificio delle Pietre Dure (OPD- Scientific Laboratory), UV-Vis cross-section images on micro-samples taken from the edge of each sample were obtained and they were used as a reference for thickness measurements, except in cases where the polyester resin used for embedding the samples was found to dissolve the protective layers (fresh dammar or synthetic resin). Besides that, in some cases the infiltration of the polyester through the superficial porosity perceptibly increased the layer's thickness, thus affecting again the thickness evaluation. X-Ray Fluorescence (XRF) was also performed in order to chemically characterize the pigments (OPD).

Data were also complemented with well-established non-invasive techniques, such as Optical Coherence Tomography (OCT) $[13,14]$ for the determination of layers thickness, and laser scanning micro-profilometry for the study of surface morphology [15-17].

As regards OCT measurements, two different devices were used: the first one, a Time-Domain confocal OCT prototype (INO-CNR) [18], with operating wavelength $1550 \mathrm{~nm}$, which combines confocal microscope optics with the OCT technique setup (axial resolution: $10 \mu \mathrm{m}$, lateral resolution: 2,5 $\mu \mathrm{m}$ ); the second one, a Thorlabs Ganymede Series Spectral-Domain OCT System, with operating wavelength $900 \mathrm{~nm}$ (axial resolution: $3 \mu \mathrm{m}$, imaging depth: 1,9 $\mathrm{mm})$. As the tomographic images acquired with SD-OCT Thorlabs @900 nm were affected by a strong surface scattering, the interference signal turned out to be too weak to enable the layers' thickness evaluation. For that reason, those OCT images are not reported, whereas the images obtained with the Td-OCT prototype are discussed in the results section.

The microprofilometer $(1 \mu \mathrm{m}$ axial resolution, $20 \mu \mathrm{m}$ lateral resolution, $8 \mathrm{~mm}$ dynamic range) produces a topographic map of the measured surface that is subsequently processed by means of purposely developed software and displayed as simulated raking light image.

The optical behaviour of pigments and lakes in VIS and NIR regions was examined by applying colorimetric analyses and Fibre Optics Reflectance Spectroscopy (FORS). Colorimetric analysis and reflectance spectrometry in the visible range were performed using a CM-2500c Konica Minolta 
Spectrophotometer, with $45^{\circ} / 0^{\circ}$ illumination/ observation configuration, $360 \mathrm{~nm}$ to 740 $\mathrm{nm}$ spectral sensitivity, and $10 \mathrm{~nm}$ spectral resolution. Three acquisitions were made for each sampled point, calculating both their average and the relative standard deviations. Fibre optics reflectance spectra were acquired with the Spectroanalyser Carl Zeiss UV-Vis-NIR (acquisition range between 304 and $1700 \mathrm{~nm}$ ). The final spectra were obtained as an average of 3 acquisitions for each measurement. Finally, the transparency of the different materials was investigated by means of Scanning Multispectral VISNIR Reflectography whose output is a set of aberration-free monochromatic images (16 Vis + 16 NIR images; spatial sampling $250 \mu \mathrm{m}$; spectral resolution $20-30 \mathrm{~nm}$ in the Vis, and $50-100 \mathrm{~nm}$ in the NIR) in the range 380-2500 nm [19-21].

\section{Results and discussion}

The first aim of the study was to verify the capability to assess the layers' thickness through NLO signals. We started by evaluating the thickness of the egg-tempera layer for the red, yellow, blue pigments. It is worth underlining that the results presented here are preliminary and request deeper investigations.

Measurements were initially conducted on samples R_A2,Y_A2, B_A2 (egg tempera without superficial varnish, Fig. 5, 6, 7) in order to collect only non-linear signals coming from the painting with no interference due to the fluorescence of varnishes.

The painted layers thickness estimated through cross section analysis is consistent with that obtained with OCT measurements. In specific, the red-tempera layer thickness resulted between 35 and $45 \mu \mathrm{m}$ (Fig. 8, 9); the yellow tempera $45-55 \mu \mathrm{m}$ (Fig. 10, 11); the blue tempera $120-150 \mu \mathrm{m}$ (Fig. 12, 13).

With regard to these results, the layers' thickness measured through NLO proves to be considerably underestimated in all cases: the results provided by TPEF imaging were around $5 \mu \mathrm{m}$ for the red sample (Fig. 14), 4 $\mu \mathrm{m}$ for the yellow sample (Fig. 15) and $10 \mu \mathrm{m}$ for the blue sample (Fig. 16). Even with the increase of the beam power, no deeper detection was obtained. The fact that no signal over $15 \mu \mathrm{m}$ depth was detected by NLO, even if the two-photon excitation fluorescence is very likely to occur at greater depth, can be explained by the following hypothesis: the $740 \mathrm{~nm}$ excitation wavelength used for NLO measurements, while enabling an adequate penetration inside the material, generates TPEF signals which are likely to undergo absorption and attenuation effects before reaching the external surface.

To support this explanation, the optical properties of the three pigments have been compared with the spectra obtained by FORS and the multispectral reflectance images acquired on samples R2, Y2, B2 in the spectral range of interest (Fig. 17).

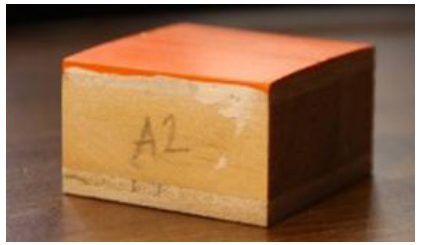

Fig. 5. Sample R_A2.

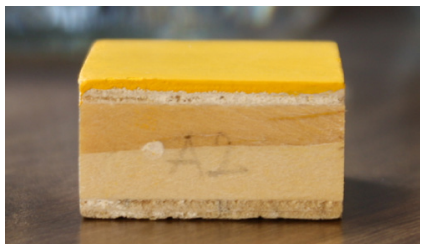

Fig. 6. Sample Y_A2.

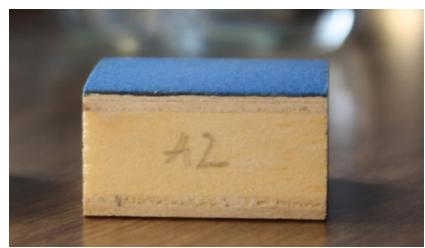

Fig. 7. Sample B_A2. 


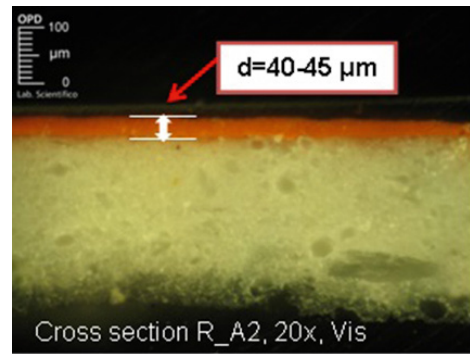

Fig. 8. Cross section R_A2, 20x, Vis (OPD).

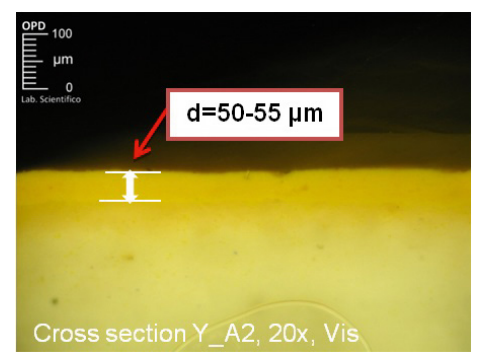

Fig. 10. Cross section $Y_{-}$A2, 20x, Vis (OPD).

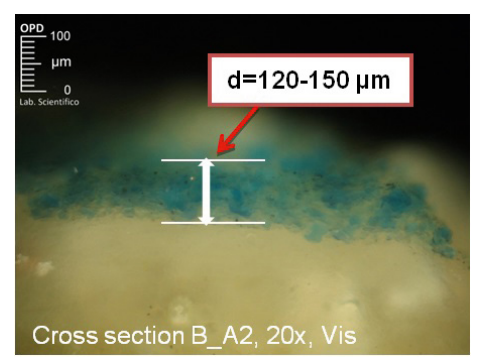

Fig. 12. Cross section B_A2, 20x, Vis (OPD).

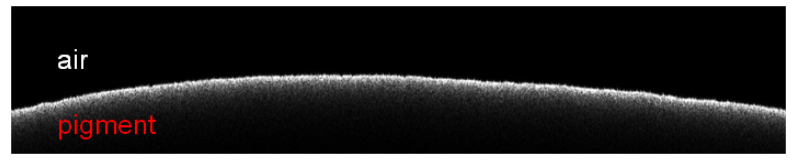

TdOCT profile@1550nm

$\mathrm{y}$ axis $=25 \mathrm{~mm}$

$\mathrm{z}$ axis $=1 \mathrm{~mm}$

Egg tempera layer thickness: $35-45 \mu \mathrm{m}$

Fig. 9. Sample R_A2 TdOCT profile $\left(\lambda_{\text {in }}=1550 \mathrm{~nm}\right)$.

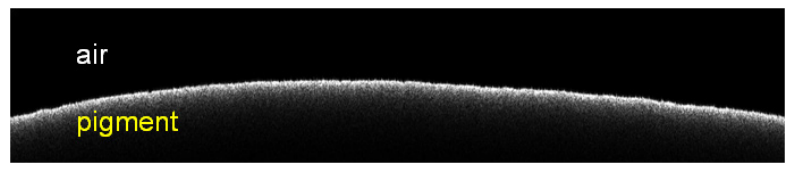

$$
\begin{aligned}
& \text { TdOCT profile @1550nm } \\
& \mathrm{y} \text { axis }=25 \mathrm{~mm} \\
& \mathrm{z} \text { axis }=1 \mathrm{~mm} \\
& \text { Egg tempera layer thickness: } 45-55 \mu \mathrm{m}
\end{aligned}
$$

Fig. 11. Sample Y_A2 TdOCT profile $\left(\boldsymbol{\lambda}_{\text {in }}=1550 \mathrm{~nm}\right)$.

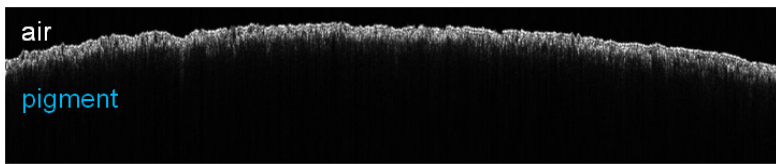

$$
\begin{aligned}
& \text { TdOCT profile @1550nm } \\
& \mathrm{y} \text { axis }=25 \mathrm{~mm} \\
& \mathrm{z} \text { axis }=1 \mathrm{~mm} \\
& \text { Egg tempera layer thickness: } 120-140 \mu \mathrm{m}
\end{aligned}
$$

Fig. 13. Sample B_A2 TdOCT profile $\left(\lambda_{\text {in }}=1550 \mathrm{~nm}\right)$.
All samples, especially the red and the yellow ones, show an intense and broad absorption band in the spectral detection range of non-linear microscopy, 400-600 $\mathrm{nm}$, thus suggesting the auto-absorption of the fluorescence radiation emitted as a consequence of the non-linear interaction inside the material. Within the spectral range of interest, minium and Cd-yellow show a similar behaviour: in both cases there is an absorption band between 350 and $550 \mathrm{~nm}$, and a following rapid increase of the reflectance values (probably due to the reflecting gypsum background), which reaches the $90 \%$ at $650 \mathrm{~nm}$ for minium and at $600 \mathrm{~nm}$ for yellow, remaining between 100\% and $80 \%$ up to $1700 \mathrm{~nm}$. With reference to the Egyptian blue, the reflectance spectrum 

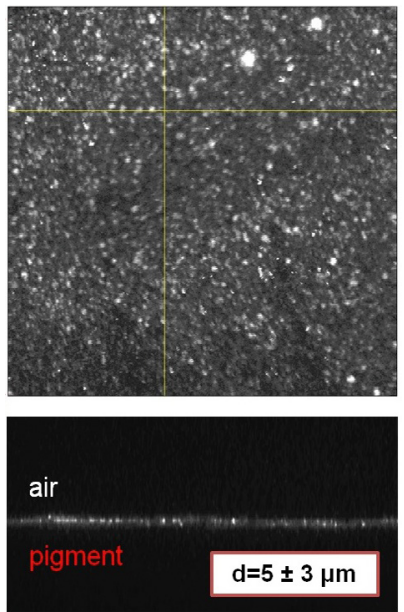

Fig. 14. Sample R_A2 grayscale TPEF intensity image (square 200 $\times 200 \mu \mathrm{m})$ and $\mathrm{z}$-stack profile $(\mathrm{z}=$ $100 \mu \mathrm{m})$.
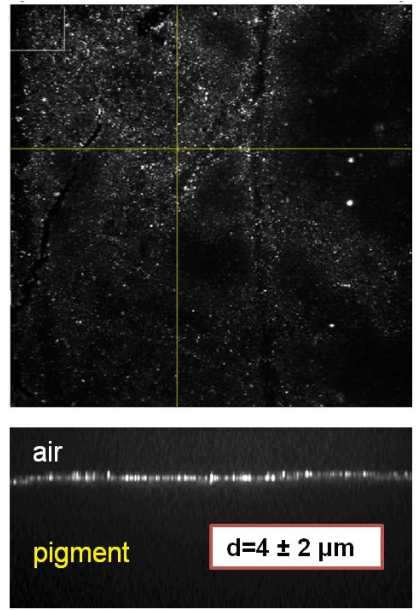

Fig. 15. Sample Y_A2 grayscale TPEF intensity image (square 200 $\times 200 \mu \mathrm{m})$ and $\mathrm{z}$-stack profile $(\mathrm{z}=$ $100 \mu \mathrm{m})$.
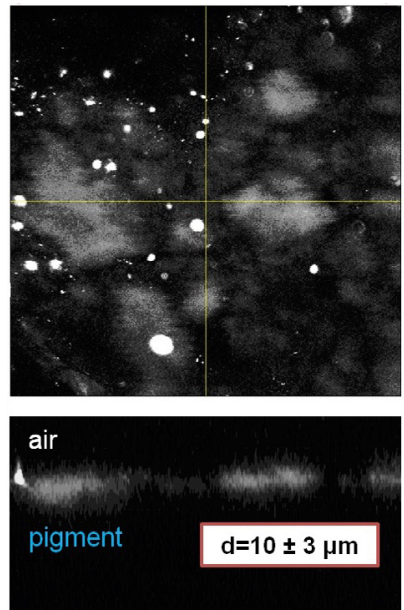

Fig. 16. Sample B_A2 grayscale TPEF intensity image (square 200 $\times 200 \mu \mathrm{m})$ and $\mathrm{z}$-stack profile $(\mathrm{z}=$ $100 \mu \mathrm{m})$.

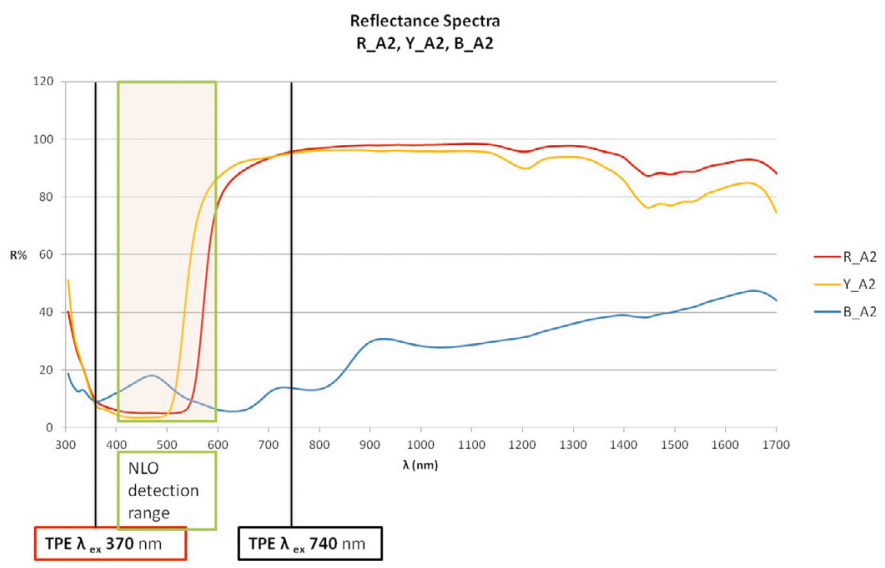

Fig. 17. Samples R_A2, Y_A2, B_A2 FORS Reflectance Spectra. The non-linear detection range (400-600 $\mathrm{nm}$ ) is highlighted in the green rectangle, as well as the TPEF excitation wavelength $(740 \mathrm{~nm})$. shows low reflectance at $740 \mathrm{~nm}$ (about 14\%) and $\mathrm{R}=40 \%$ at $1550 \mathrm{~nm}$. This behaviour is observed also in the images obtained with the Multispectral Vis-Nir Scanner (INO-CNR), shown in Fig. 18. Concerning the discrimination of the protective layers on the other samples, no significant results were found, except for samples R7, Y7, B7, which are covered with a protective layer made of white egg. The intensity fluorescence images (Fig. 19) show different micro-morphologies for the three pigments, which may be related to the drying process and the pigment particle size.

In any case, we were not able to evaluate the thickness of the superficial layers, or to discriminate the interfaces, probably because 

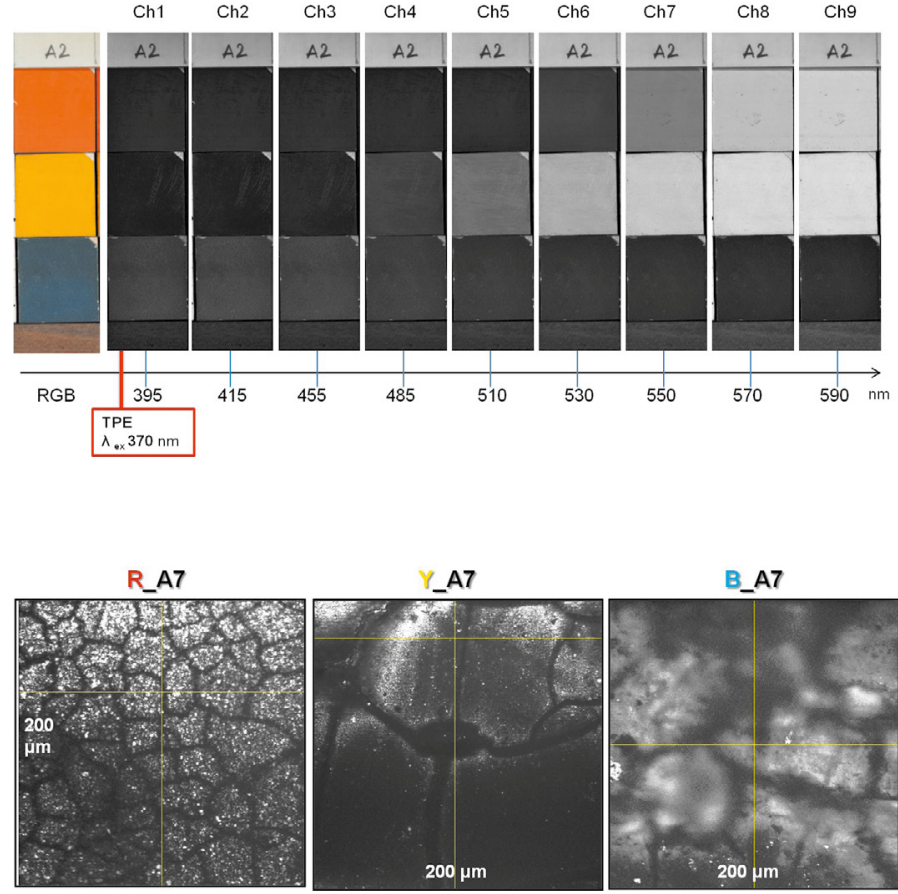

Fig. 19. Samples R_A7, Y_A7, B_A7 gray scale TPEF intensity image (square $200 \times$ $200 \mu \mathrm{m})$. of the transparency of the protective layers to the wavelength used. The discrimination of the three pigments was possible by means of FLIM by revealing different fluorescence lifetime, resulting in different trends in the decay curves. The evaluated average values of fluorescence lifetime for red lead, cadmium yellow and Egyptian blue are 240 ps, 170 ps, and over $30 \mathrm{~ns}$, respectively. It is worth noticing that the blue pigment fluorescence lifetime is well above the repetition rate of the laser (12 ns), thus resulting in a constantly linear trend of the decay curve (which is not reported). By contrast, the red and the yellow pigments (Fig. 20,21) are characterized by a significantly short fluorescence lifetime that is comparable or smaller than the acquisition time window of the system (which is approx. 200 ps). In conclusion, the FLIM analysis revealed relevant differences between the three pigments fluorescence lifetimes, especially for the blue pigment. The physi-

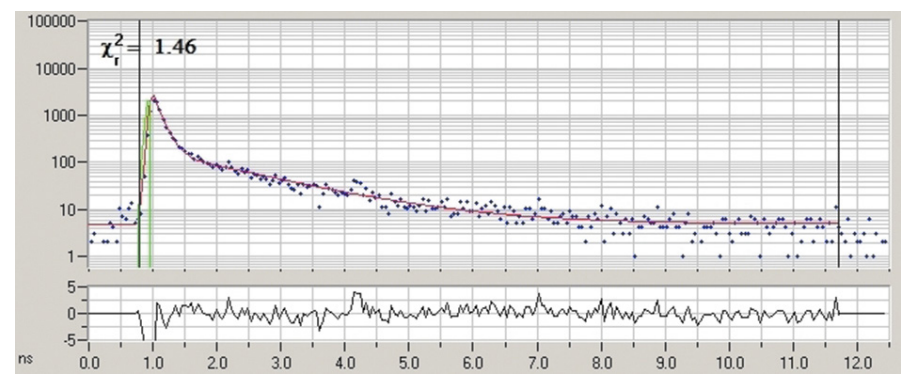

Fig. 20. Sample R_A2 Fluorescence lifetime decay curve. The time (ns) is reported on the $\mathrm{x}$ axis and the counts are reported on the $y$ axis. Recorded data (dots), monoexponential fit (red continuous line). 


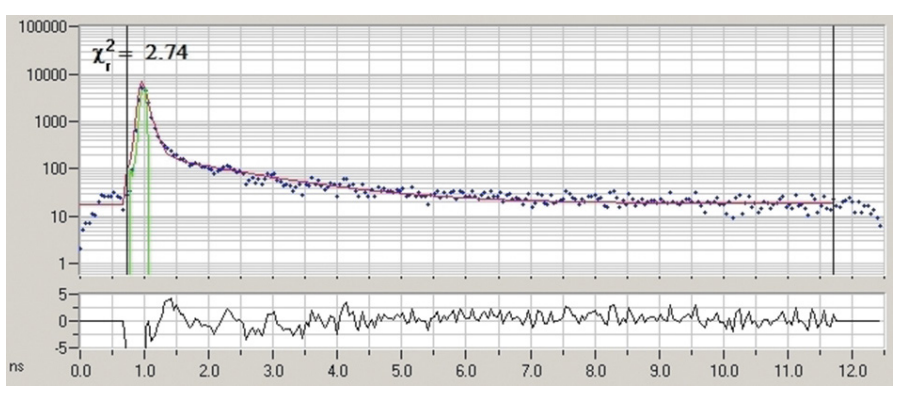

Fig. 21. Sample Y_A2 Fluorescence lifetime decay curve. The time (ns) is reported on the $\mathrm{x}$ axis and the counts are reported on the $y$ axis. Recorded data (dots), monoexponential fit (red continuous line). cal-chemical explanation of such different trends needs to be further investigated.

\section{Conclusions}

The purpose of this work is to describe whether and if so, to what extent NLO imaging techniques prove useful in probing multilayer painted objects, in evaluating the capability to collect non-linear signals and determine the layers thickness. In this respect, analyses were applied on a series of single- and multi-layers systems simulating real egg-tempera wood panel paintings. The preliminary results suggest that the $740 \mathrm{~nm}$ wavelength, while enabling the visualization of the painting surfaces micromorphology, is not effective for the determination of thickness through the detection of non-linear signals. On the basis of the combined analysis reported, we assume that a longer excitation wavelength may prove more effective. In collaboration with other research groups, further non-linear analyses with different devices and modalities are currently being conducted, as well as the linear fluorescence behaviour of each pigment is under investigation.

\section{Acknowledgements}

The research leading to these results was funded by the EU Community's H2020- Research Infrastructure programme under the IPERION CH Project (GA n. 654028), and in collaboration with Opificio delle Pietre Dure (Firenze).

\section{References}

[1] F. Faraldi, G.J. Tserevelakis, G. Filippidis, G.M. Ingo, C. Riccucci, C. Fotakis, "Multi photon excitation fluorescence imaging microscopy for the precise characterization of corrosion layers in silver-based artifacts" Applied Physics A, 111 (2013) 177-181, DOI: 10.1007/s00339-013-7548-z.

[2] G. Latour, J.P. Echard, M. Didier, M.C. SchanneKlein, "In situ 3D characterization of historical coatings and wood using multimodal nonlinear optical microscopy”, Optics Express, 20 (2012): 24623-24635, DOI: 10.1364/OE.20.024623.

[3] G. Filippidis, M. Massaouti, A. Selimis, E.J. Gualda, J.-M. Manceau, S. Tzortzakis, "Nonlinear imaging and $\mathrm{THz}$ diagnostic tools in the service of Cultural Heritage", Applied Physics A, 106 (2012), 257-263, DOI: 10.1007/s00339-014-8357-8.

[4] G. Filippidis, J. E. Gualda, K. Melessanaki, C. Fotakis, "Nonlinear imaging microscopy techniques as diagnostic tools for art conservation studies", Optics Letters 33 (2008) 240-242, DOI: 10.1364/ OL.33.000240.

[5] A. Nevin, D. Comelli, I. Osticioli, G. Filippidis, K. Melessanaki, G. Valentini, R. Cubeddu, C. Fotakis, "Multi-photon excitation fluorescence and third-harmonic generation microscopy measurements combined with confocal Raman microscopy for the analysis of layered samples of varnished oil films", Applied Physics A, 100 (2010) 599-606, DOI 10.1007/s00339-010-5644-x. 
[6] T. E. Villafana, W. Brown, W. S. Warren, and M. Fischer, "Ultrafast Pump-Probe Dynamics of Iron Oxide Based Earth Pigments for Applications to Ancient Pottery Manufacture", Proc. SPIE 9527, (2015) 952709, DOI: 10.1117/12.2184758.

[7] T.E. Villafana, P.W. Brown, J.K. Delaney, M. Palmerc, W. S. Warren, and M. C. Fischer, "Femtosecond pump-probe microscopy generates virtual cross-sections in historic artwork", Proc. of the National Academy of Sciences of the USA, 111 (2014) 1708-1713, DOI: $10.1073 /$ pnas.1317230111.

[8] G. Filippidis, K. Melessanaki and C. Fotakis, "Second and third harmonic generation measurements of glues used for lining textile supports of painted artworks", Analytical and Bioanalytical Chemistry (2009), DOI: 10.1007/s00216-009-3060-x.

[9] G. Filippidis, G. J. Tserevelakis, A. Selimis, C. Fotakis, "Nonlinear imaging techniques as non-destructive, high-resolution diagnostic tools for cultural heritage studies", Applied Physics A, 118 (2015) 417-423, DOI: 10.1007/s00339-014-8357-8.

[10] R. Mercatelli, F. Ratto, F. Rossi, F. Tatini, L. Menabuoni, A. Malandrini, R. Nicoletti, R. Pini, F. S. Pavone, R. Cicchi, “Three-dimensional mapping of the orientation of collagen corneal lamellae in healthy and keratoconic human corneas using SHG microscopy", Journal of Biophotonics, 10 (2017) 75-83, DOI: 10.1002/jbio.201600122.

[11] F. Rosi, C. Grazia, F. Gabrieli, A. Romani, M. Paolantoni, R. Vivani, B.G. Brunetti, P. Colomban, C. Miliani, "UV-Vis-NIR and micro Raman spectroscopies for the non destructive identification of $\mathrm{Cd}_{1-}$ ${ }_{\mathrm{x}} \mathrm{Zn}_{\mathrm{x}} \mathrm{S}$ solid solutions in cadmium yellow pigments", Microchemical Journal, 124 (2015) 856-867, DOI: 10.1016/j.microc.2015.07.025.

[12] G. Accorsi, A. Verri, M. Bolognesi, N. Armaroli, C. Clementi, C. Miliani, and A. Romani, "The exceptional near-infrared luminescence properties of cuprorivaite (Egyptian blue)", Chemical Communications (2009) 3392-3394, DOI: 10.1039/b902563d.

[13] P. Targowski, B. Rouba, M. Wojtkowski, A. Kowalczyk, "The application of optical coherence tomography to nondestructive examination of museum objects", Studies in Conservation, 49 (2004), 107-114.
[14] T. Arecchi, M. Bellini, C. Corsi, R. Fontana, M. Materazzi, L. Pezzati, A. Tortora, "A new tool for painting diagnostics: Optical coherence tomography" Optics and Spectroscopy 101 (2006), 23-26, DOI: 10.1134/S0030400X06070058.

[15] R. Fontana, M.C. Gambino, M. Greco, L. Marras, M. Materazzi, E. Pampaloni, L. Pezzati, "High precision surface analysis of roughness of Michelangelo's David", Proc. SPIE 5146 (2003) 236-243, DOI: 10.1117/12.501252.

[16] R. Fontana, M.C. Gambino, M. Greco, L. Marras, M. Materazzi, E. Pampaloni, L. Pezzati, P. Poggi, "Integrating 2D and 3D data for diagnostics of panel paintings”, Proc. SPIE 5146 (2003) 88-98, DOI: 10.1117/12.501247.

[17] R. Fontana, C. Mazzotta, M.C. Gambino, M. Greco, E. Pampaloni, L. Pezzati, "High-resolution 3D survey of artworks", Proc. SPIE 5457 (2004) 719-726, DOI: 10.1117/12.545660.

[18] R. Fontana, M. Barucci, E. Pampaloni, L. Pezzati, C. Daffara, "A study of surface optical properties for characterizing the cleaning process of paintings", Proc. SPIE 8790 (2013) 87900O, DOI: 10.1117/12.2020738.

[19] C. Bonifazzi, P. Carcagni, R. Fontana, M. Greco, M. Mastroianni, M. Materazzi, E. Pampaloni, L. Pezzati, D. Bencini, "A scanning device for VIS-NIR multispectral imaging of paintings", Journal of Optics A: Pure and Applied Optics, 10 (2008), 064011 , DOI: 10.1088/1464-4258/10/6/064011.

[20] P. Carcagni, Patria A. Della, R. Fontana, M. Greco, M. Mastroianni, M. Materazzi, E. Pampaloni, L. Pezzati, "Multispectral imaging of paintings by optical scanning", Optics and Lasers in Engineering, 45 (2007), 360-367, DOI: 10.1016/j. optlaseng.2005.02.010.

[21] R. Fontana, M. Barucci, E. Pampaloni, J. Striova, L. Pezzati, "From Leonardo to Raffaello: insights by Vis-IR Reflectography”, in: Acta Artis Academica 2014 Proceedings of the $5^{\text {th }}$ interdisciplinary Conference of ALMA, Interpretation of Fine Art's Analysis in Diverse Contexts, D. Hradil, J. Hradilová (Eds.), Academy of Fine Arts in Prague, Prague 2014, pp.: 15-26, ISBN 978-80-87108-48-2. 



\title{
A moderate microsampling in Laser Ablation Inductively Coupled Plasma Mass Spectrometry analysis of cultural heritage objects: a review
}

\author{
Barbara Wagner*, Olga Syta, Marek Sawicki \\ Faculty of Chemistry, Biological and Chemical Research Centre, University of Warsaw, \\ ul. Żwirki i Wigury 101, 02-089 Warsaw, Poland \\ * Corresponding author: barbog@chem.uw.edu.pl
}

\begin{abstract}
Laser Ablation Inductively Coupled Plasma Mass Spectrometry (LA-ICP-MS) is one of modern instrumental methods whose usage in the elemental analysis of cultural heritage objects has recently noticeably increased. The method requires almost no sample preparation and permits the direct analysis of solid samples, availing only minute amount of the material. Although they seem to be microdestructive, ablation processes remain usually within the scale acceptable for art historians, conservators, archaeologists and art curators; therefore, the capability to perform a multi-elemental, ultra trace and isotopic analysis can be introduced to studies of cultural heritage objects. The lack of appropriate matrix-matched certified reference materials or fractionation effects influencing the final quantitative results to a different extent is widely reported among the main limitations of LA-ICP-MS. Despite these constrains, LA-ICP-MS is a method that can be flexibly tuned to collect the desired elemental information and this paper brings together information about the successful application of this method in the analysis of diverse historical materials enabling researchers to build valuable knowledge about cultural heritage objects from all over the world.
\end{abstract}

Keywords: LA-ICP-MS, cultural heritage, archaeometry, works of art, elemental analysis

\section{Introduction}

The interest in archaeometric investigations and conservation science is growing every year and guidelines for selecting the optimum instrumental method are discussed in the literature. Van Grieken and Janssens [1] recalled Lahanier et al. [2], who published a list including a few desirable features of the best method of choice for the analysis of cultural heritage objects. The list included the multielemental character of direct measurements, low limits of detection, high sensitivity, versatility, universality, and a relatively short time of the analysis. The non-destructive character of the measurements was also pointed out by Lahanier.

The need to protect cultural heritage objects gives the priority to non-destructive measurements, although many of currently available methods can successfully utilize microscopic mass of samples [1,3]. Laser Ablation Inductively Coupled Plasma Mass Spectrometry (LA-ICP-MS) is one of modern 
instrumental methods whose need for sample consumption is reduced to absolute minimum [4-6]. All LA-ICP-MS measurements benefit from very sensitive elemental analysis and low limits of detection (down to $\mathrm{ngg}^{-1}$ ) [7-10], which allow the successful use of this method during provenance investigations [11-30], material identification [31-35], studies devoted to reconstructions of past technologies [36-42] and conservation research [43-46].

In this paper, we would like to discuss possible research scenarios based on the use of LA-ICP-MS, depending on the size of objects and agreement for sampling as well as a possibility of transportation to the laboratory. The number of papers devoted to the use of LA-ICP-MS for conservation science and archaeometry may be taken as a good indicator for the growing interest in the use of this method for the analysis of cultural heritage objects (Fig. 1).

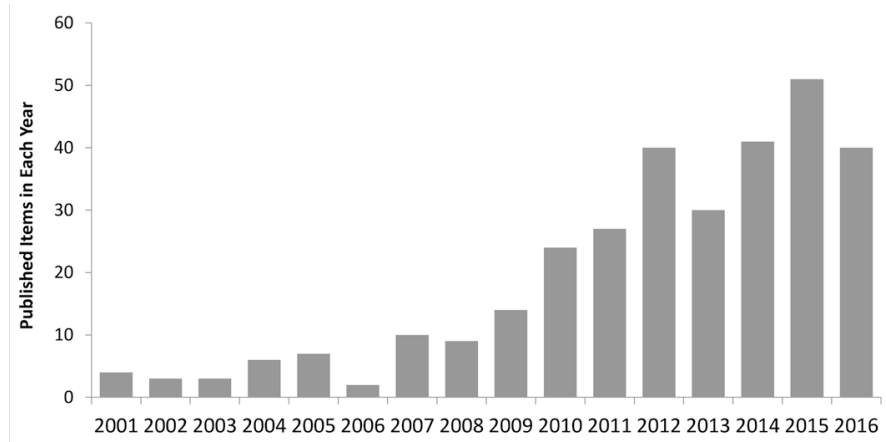

limits of detection (LOD) to the level of about $10 \mathrm{ng} \cdot \mathrm{g}^{-1}$ [47-49]. The later introduction of a commercially available instrumentation gave a new tool for: (i) direct analysis of solid; (ii) microanalysis; (iii) surface mapping and (iv) depth profiling of investigated materials $[10,50]$. The method has proven to be applicable for the analysis of literally all solids while elemental or isotopic information is needed.

Over 30 years of experience with laser ablation ICP-MS has resulted in rich literature about fundamentals and applications of the method based on a reliable recognition of its advantages and limitations $[4,50]$. The selection of cultural heritage as the research area has not changed the general procedural rules of using LA-ICP-MS; however, various analytical scenarios for using LA-ICP-MS during investigations of valuable objects were proposed.
Fig. 1. The number of papers published per each year according to the report generated by Web of Science.

\section{Introduction to LA-ICP-MS}

LA-ICP-MS was mentioned for the first time in 1985, when Gray described his preliminary study of using laser ablation with ICP-MS during investigation of rock samples [47]. His work extended the previous use of laser ablation combined with ICP-OES lowering

\section{LA-ICP-MS setup}

Typical LA-ICP-MS systems utilize laser ablation of solids in a neutral gas (argon and/or helium) atmosphere; the possibility to ablate solids in air is reported rarely [51]. While standard set-up is being used, a sample or an object is located in a moveable 
$\mathrm{x}-\mathrm{y}-\mathrm{z}$ stage and closed in a tightly sealed ablation cell continuously flushed with a carrier gas. A surface of a sample exposed to the laser beam can be observed in the real time with a CCD camera, allowing selection of the investigated area and proper laser beam focusing. The ablated aerosol is then transported by the carrier gas to the plasma and mass spectrometer (Fig. 2), where ions are separated according to their mass to-charge the investigated material, as well as parameters of the applied laser set up. Melting and boiling points, or ionization potentials of elements of interest, might also have an influence on ablation processes leading to their different course during the micro-sampling of solids [55-57]. Occurring differences were defined as fractionation, meaning the sum of non-stoichiometric processes changing the analytical response of signals if compared

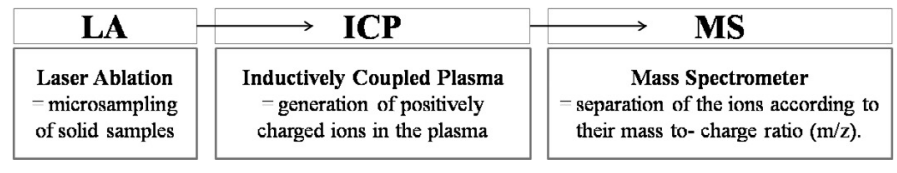

Fig. 2. The scheme of a typical LA-ICP-MS system. ratio $(\mathrm{m} / \mathrm{z})[5,50,52]$. There are no specific technical solutions of LA-ICP-MS setups especially designed for cultural heritage objects investigations; however, some systems facilitating microsampling of precious items were suggested in the literature $[53,54]$.

Laser ablation is effective practically for all solid materials; nevertheless, the course of the process depends on the number of factors related to chemical and physical properties of to the real stoichiometry of investigated samples [58]. The reliable quantification is challenging, yet achievable by using shorter lasers wavelengths (Table 1), shorter pulse durations and optimized power densities according to the actual knowledge about LA-ICP-MS measurements [8, 52, 59-66].

Lasers emitting shorter wavelengths (193 $\mathrm{nm}$ ) together with shorter pulse durations (fs) can reduce fractionation and would be

Table 1. Selected lasers used in LA-ICP-MS measurements

\begin{tabular}{cccc}
\hline Wavelength $(\mathrm{nm})$ & \multicolumn{2}{c}{ Laser type } & Pulse duration \\
\hline 1064 & Nd:YAG & Solid state & $\mathrm{ns}$ \\
$650-1100$ & Ti:Sapphire & Solid state & $\mathrm{fs}$ \\
694 & Ruby & Solid state & $\mathrm{ns}$ \\
532 & $\mathrm{Nd}: Y A G$ & Solid state & $\mathrm{ns}$ \\
355 & $\mathrm{Nd}: Y A G$ & Solid state & $\mathrm{ns}$ \\
308 & $\mathrm{XeCl}$ & Gas excimer & $\mathrm{ns}$ \\
$266^{*}$ & $\mathrm{Nd}: Y A G$ & Solid state & ns \\
248 & KrF & Gas excimer & ns \\
$213^{*}$ & $\mathrm{Nd}: Y A G$ & Solid state & ns \\
193 & $\mathrm{Nd}: Y A G$ & Solid state & ns \\
$193^{*}$ & ArF & Gas excimer & ns \\
157 & $\mathrm{~F}_{2}$ & Gas excimer & ns
\end{tabular}

*) The lasers most commonly used to ablate cultural heritage objects for ICP-MS analysis. 
preferably used during quantitative elemental analysis; however, the ablation with the use of $\mathrm{Nd}: Y A G$ ns lasers emitting either $\lambda=266 \mathrm{~nm}$ or $\lambda=213 \mathrm{~nm}$ is still popular. Femtosecond lasers are less commonly used [16] for investigations of cultural heritage objects than the nanosecond ones.

To minimize the effects of fractionation Brostoff et al. [16] used fs-LA-ICP-MS in provenance studies of rare ancient gold artifacts. The main groups of the objects were distinguished on the basis of elemental composition and variable content of $\mathrm{Ag}, \mathrm{Pd}, \mathrm{Pt}$ or $\mathrm{Sn}, \mathrm{Cu}$ and $\mathrm{Zn}$. The multielemental information obtained by means of LA-ICP-MS was important to support historical, technical and stylistic evaluation of the investigated gold objects and was used for matching the fragments by origin of manufacture with a good precision and accuracy.

The appropriate calibration for quantification of multielemental LA-ICP-MS measurements remains a challenging task mainly due to the strong dependence of ablation processes on manifold physicochemical properties of solids. Properly selected reference materials with the known content of elements of interest allow the reliable calibration and accurate determination of the elemental composition of investigated objects (Table 2). Three possible calibration approaches can be used to quantify LA-ICP-MS signals. The most popular calibration approach is based on the use of solid matrix-matched reference materials [61, 67], while dual introduction of standard solutions together with the ablated matter [68-70] or direct ablation of liquid standards [71-73] are less commonly applied.

There are many commercially available and well characterized solid reference materials $[43,74,112,113,134-138]$ which can be used in LA-ICP-MS investigations (Table 2).
Only these of the composition and the structure properly matching analyzed materials can offer accurate quantification by ensuring the consistency of ablation processes for both: the sample and the standard [14]. A careful selection of the matrix-matched standards would provide accurate results of LA-ICP-MS measurements together with internal standardization (IS), which is recommended to reduce matrix effects and correct differences in ablation efficiency of samples $[61,139]$. If no adequate standards are available, non-matrix matched calibration approaches could be applied with normalization to $100 \%$ procedure [140-143] and allow for collecting information about major, minor and trace elemental composition of investigated objects. In all cases, the results of LA-ICP-MS measurements should be checked according to the quality control protocol.

\section{LA-ICP-MS in relation to destructiveness and invasiveness of investigations of cultural heritage objects}

Many authors pointed out that LA-ICP-MS causes only those damages that are not visible to the naked eye $[32,54,76,93,113,144$, 145]. A laser beam energy may be focused on an area with diameters of about a few up to several hundred micrometers limiting the post-ablation marks to the selected location. The spot ablation means the micro-sampling of the material from a single point, while rastering or line/multiline ablation allows assessment of elemental/isotopic spatial distribution over a surface of a sample [179]. An opportunity for selective analysis of surface or sub-surface domains is particularly important during investigations of heterogeneous objects, corroded or covered with patina. 
Table 2. Reference materials used in LA-ICP-MS quantitative analysis of cultural heritage objects

\begin{tabular}{|c|c|}
\hline Sample & Reference materials used \\
\hline $\begin{array}{l}\text { Glass } \\
{[13,18,22,26,74-92]}\end{array}$ & $\begin{array}{l}\text { National Institute of Standards and Technology: 610, 612, } 614 \text { (trace elements in } \\
\text { glass) } \\
\text { Corning Museum of Glass: A, B, C, D } \\
\text { U.S. Geological Survey: BCR-2G, BHVO-2G, BIR-1G (basalt glasses) } \\
\text { Society of Glass Technology: 2, 3, 4, 5, 6, 7, 8, } 9\end{array}$ \\
\hline $\begin{array}{l}\text { Obsidian } \\
{[12,93-98]}\end{array}$ & $\begin{array}{l}\text { National Institute of Standards and Technology: 610, } 612 \\
\text { Corning Museum of Glass: B, D } \\
\text { U.S. Geological Survey: BCR-2G } \\
\text { Natural obsidians from: Glass Butte (USA), Sierra de Pachuca (Mexico), Wekwok } \\
\text { (Papua New Guinea) }\end{array}$ \\
\hline $\begin{array}{l}\text { Ceramic } \\
{[23,29,30,33,35,36} \\
40,53,99-111]\end{array}$ & $\begin{array}{l}\text { National Institute of Standards and Technology: } 679 \text { (brick clay), 1633a (coal fly } \\
\text { ash); 610, 612, 614, } 1412 \text { (glasses) } \\
\text { Corning Museum of Glass: B, D } \\
\text { Missouri University Research Reactor Center: New Ohio Red Clay } \\
\text { U.S. Geological Survey: BIR1 (basalt); AGV2 (andesite); GSP2 (granodiorite); } \\
\text { RGM1 (rhyolite); BCR-2G, BHVO-2G (basalt glasses) } \\
\text { National Institute for Metallurgy: NIM-L (lujavrite) } \\
\text { Institute for Reference Materials and Measurements: BCR-126A (lead glass) } \\
\text { ANRT reference material: FK-N (potash feldspar) }\end{array}$ \\
\hline $\begin{array}{l}\text { Metal } \\
{[14,67,112-124]}\end{array}$ & $\begin{array}{l}\text { National Institute of Standards and Technology: 500, C1123, C1252, C1257 } \\
\text { (copper); 685, 8053, 8054, 8059, 8062, 8063, 8065, 8068, 8074, } 8077 \text { (gold); } 481 \\
\text { (gold-silver alloy); 610, 612 } \\
\text { Federal Institute for Materials Research and Testing: } 211,227,228,378 \text { (bronzes) } \\
\text { Centre de Developpement des Industries de Mise en Forme des Materiaux: B10, } \\
\text { B12 (bronzes) } \\
\text { Bureau of Analysed Samples: 50.03-4, 50.04-4, 51.13-4, 71.32-4 (bronzes) } \\
\text { MBH Analytical: 32X SN1, 32X SN2, 32X SN3 (bronzes); 31X TB2, 31X TB4 } \\
\text { (brasses) } \\
\text { Swerea Nordic CRM Working Group: JK 27A (stainless steel), JK 2D (pure iron) } \\
\text { U.S. Geological Survey: GSE-1G (lead isotope ratio) }\end{array}$ \\
\hline $\begin{array}{l}\text { Human fossil } \\
{[125-128]}\end{array}$ & $\begin{array}{l}\text { National Institute of Standards and Technology: } 987 \text { (strontium isotope ratio); } \\
610,612 \\
\text { New York State Department of Health: RM05-1, RM05-2, RM05-4 (lead-dosed } \\
\text { caprine bone pellets) } \\
\text { China National Analysis Center: GBW0760 (human hair) }\end{array}$ \\
\hline $\begin{array}{l}\text { Mortar } \\
{[129-133]}\end{array}$ & $\begin{array}{l}\text { National Institute of Standards and Technology: 1d (argillaceous limestone); 610, } \\
611,612,613 \\
\text { U.S. Geological Survey: BCR-2G }\end{array}$ \\
\hline
\end{tabular}

Imaging techniques allow for clear mapping of a distribution of selected elements in forms of $2 \mathrm{D}[53,90,147,174]$ or $3 \mathrm{D}[180]$ models. Van Elteren with co-workers [180] applied a 3D LA-ICP-MS mapping procedure to the analysis of historic glass to study degradation mechanisms of glass weathering. Previous analytical protocols required 
cross-sectional fragments of glass for the analysis, which was omitted in this project. LA-ICP-MS measurements are relatively fast; therefore, many analyses were repeated in a reasonable period of time.

Statements indicating a low degree of changes due to LA-ICP-MS analysis include such wording as quasi-destruction-free [113], virtually non-destructive [19, 32, 54, $76,93]$ or relatively/almost non-destructive $[12,29,144-146]$. Other papers [35, 77, 125, 147-150] state that LA-ICP-MS as a micro-destructive method provides multielemental information with minimal sample damage and should rather be understood as an attempt to present the method in the best light, not as a precise term describing effects of ablation.

Possible controversies with assigning attributes of destructiveness or invasiveness to any analytical protocol have been considered in works of Wouters [3, 151], who suggested to use the term intervention instead. The degree of intervention might be described at three levels: (i) molecular (low change), (ii) microscopic (medium change) and (iii) visual (high change). Wouters nomenclature is rational and useful to clarify the scale of changes provoked by application of instrumental methods to the measurements of cultural heritage objects.

According to Wouters [3, 151], microscopic interventions/medium changes to cultural heritage objects can be expected if the ablation was executed directly from the analyzed item. Ablation is usually performed inside ablation cells; therefore, a size of an object to be analyzed is important $[7,53$, 54, 152-157]. Variable constructions of ablation cells were described in the literature, allowing the best selection of analytical approach for particular size and shape of objects.

\section{Degree of intervention induced by LA-ICP-MS measurements}

The development of methodology for studying cultural heritage objects is paired with specific restrictions in order to keep the analyzed objects possibly intact, to remain them within the molecular or microscopic degrees of intervention. These requirements can be met during the application of LA-ICP-MS; however, depending on the size and uniqueness of investigated objects, different scenarios for conducting laser ablation can be proposed (Fig. 3).

\section{CULTURAL HERITAGE OBJECTS}

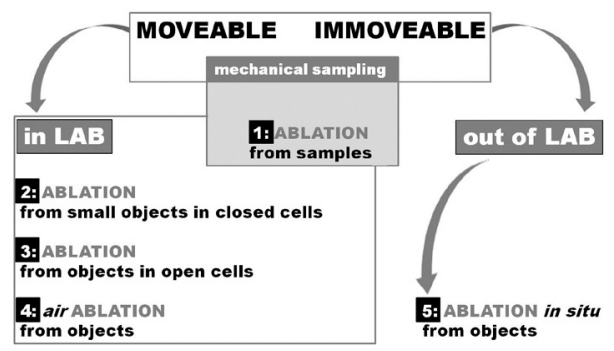

Fig. 3. Various scenarios for using LA-ICP-MS during investigations (the description is given in the text).

\subsection{Scenario 1: mechanical sampling followed by laser ablation from the taken fragments}

The most common scenario of using LA-ICP-MS for the analysis of cultural heritage objects concerns ablation of samples mechanically taken from artifacts (Fig. 4). Mechanical sampling enables the investigations of the chemical composition of immoveable or large moveable objects which cannot be transported to a laboratory. The step of mechanical sampling takes responsibility of the scale of intervention to the object of interest because the same sam- 

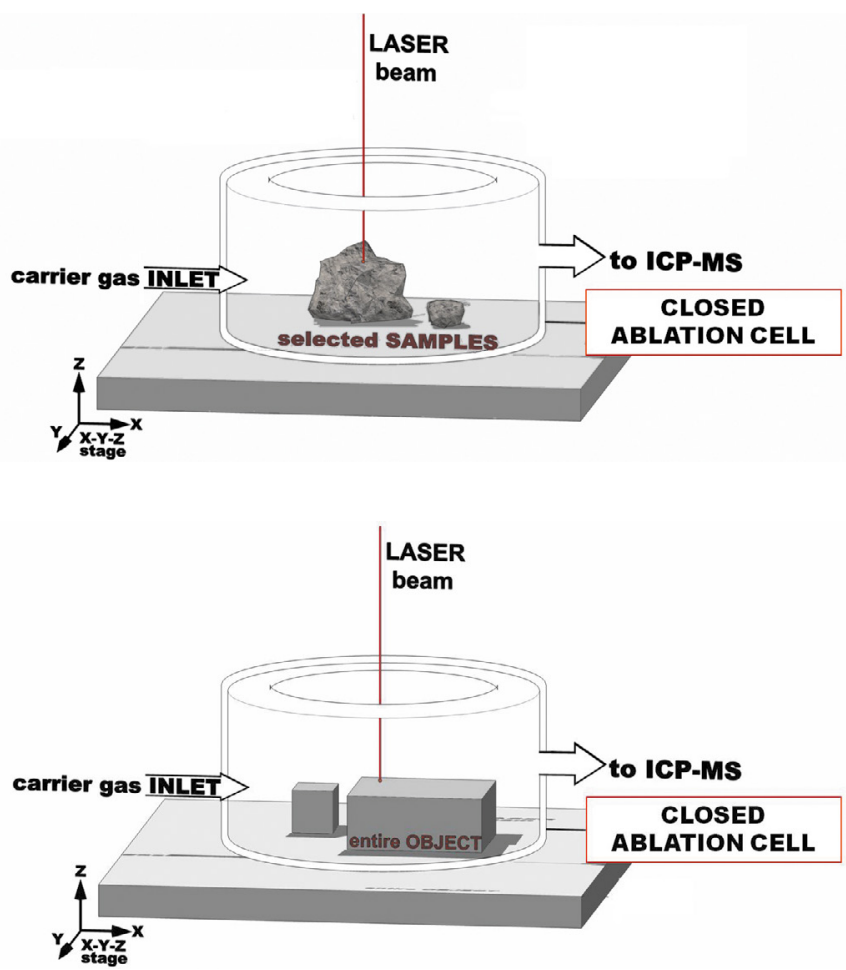

Fig. 4. Scenario 1: ablation executed in closed cell from samples.

Fig. 5. Scenario 2: ablation executed directly from the object, which fits to a closed ablation cell. pling, not the process of laser ablation, is the most destructive step in the whole analytical procedure.

Collected fragments can be additionally analyzed by other methods [74, 96, 101, $102,105,115,126,129,135,158-165]$ to broaden the knowledge about their chemical composition. Small samples are often taken from historical glass to be analyzed by means of SEM-EDX and LA-ICP-MS [38, 74, 79, $84-89,135,166-169]$. In this case, small fragments are embedded in resin blocks and prepared in accordance to EDX analysis requirements (polishing, carbon coating). They can be analyzed in the same form by means of both methods. The results from SEM-EDX analysis may be used first to select and then to determine the content of the internal standard $\left(\mathrm{CaO}\right.$ or $\left.\mathrm{SiO}_{2}\right)$. These two methods provide results with a satisfactory correlation for major and minor elements, while LA-ICP-MS additionally allows for determination of trace elements.

The incorporation of LA-ICP-MS into research involves the necessity to sample large objects. The inner volume of the standard closed cell prerequisites the size of an item which can fit it during ablation; therefore, objects of small sizes can be located inside a cell, allowing direct laser ablation from the object (Fig. 5).

\subsection{Scenario 2: direct laser ablation from small objects}

The size of an item plays an important role in the analytical procedure including LA-ICP-MS investigations when no agreement can be obtained for the mechanical sampling of the object. The commercially 
available ablation cells are characterized by the defined dimensions and only objects that are small enough to be placed inside the ablation cells can be ablated directly $[11,12$, $78,93,106,117,127,170-173]$. This scenario allows not only to lower the level of intervention to the minimum, but also the most time-consuming step of samples preparation is eliminated and LA-ICP-MS measurements can be performed much faster $[18,19]$.

The shortening of the analysis time is a beneficial side-effect of the scenario 2 and is helpful for investigations of objects which are found in large quantities, like glass beads from archaeological sites all around the world [76]. Robertshaw et al. [18] analyzed more than 360 glass beads from the southern Africa, while Dussubieux et al. [19] reported the results for 486 items of archaeological glass beads from India, Sri Lanka, Bangladesh, Thailand, Malaysia, Indonesia, Cambodia, Vietnam and Turkey. Thanks to availability to a number of data on a large group of similar objects, it was possible to conduct statistical surveys and extract information about the existence of variable sub-groups representing several glass-making centers. The hypothesis about the existence of a few glass making centers in different locations was supported by the results of LA-ICP-MS investigations of mineral soda-alumina glass and allowed to distinguish the sub-groups on the basis of concentrations of selected elements (Ca, $\mathrm{Mg}$, $\mathrm{U}, \mathrm{Ba}, \mathrm{Sr}, \mathrm{Zr}$ and $\mathrm{Cs})$.

\subsection{Scenario 3: the use of open ablation cells}

Objects that are too large to be placed inside the ablation cell can be analyzed with the use of so called "open" or "moveable" ablation cells (Fig. 6) [53, 54, 152-153, 174]. Small open ablation cells are adhered to the surface of an object, maintaining the degree of inter- vention of LA-ICP-MS measurements at the microscopic level $[53,54,175]$.

The use of open ablation cells allows to avoid mechanical sampling from valuable objects and thus expands the range of applications of LA-ICP-MS. The use of such scenario is more time-consuming than the previously described ones. The object has to be handled with the highest caution to avoid damage caused by mounting at the $\mathrm{x}-\mathrm{y}-\mathrm{z}$ stage for the time of ablation. Open cells are usually characterized by small intrinsic volumes that permit the shortening of the wash-out-time from the cell; therefore, the investigations of elemental distribution with the use of this scenario are facilitated. Various designs of open cells were proposed [53, 54, 152, 153, 175, 176]; however, the optimum construction should allow their use irrespective of the particular size or shape of the object and would be insensitive to the surface irregularity.

Devos, Moor and Lienemann [54] described the successful use of ablation open cell during elemental analysis of silver artifacts. The open cell was used because the objects of interest were too large to fit into a standard ablation cell and the mechanical sampling of them was not allowed. Contents of $\mathrm{Ag}, \mathrm{Zn}, \mathrm{Cd}, \mathrm{Sn}, \mathrm{Sb}, \mathrm{Au}, \mathrm{Pb}$ and $\mathrm{Bi}$ were determined and allowed to discriminate one forgery among the originals. A crater-to-crater repeatability of the normalized signals was reported below $10 \% \operatorname{RSD}(n=3)$ for most of the selected elements with detection limits within the sub-ppm to ppm range.

Low limits of detection are the most preferred advantages of LA-ICP-MS measurements. Recently, sampling systems with no ablation cell have been developed for a direct atmospheric ablation $[67,177]$, according to the scenario 4 (Fig. 7), and also allow to maintain limits of detection at the 


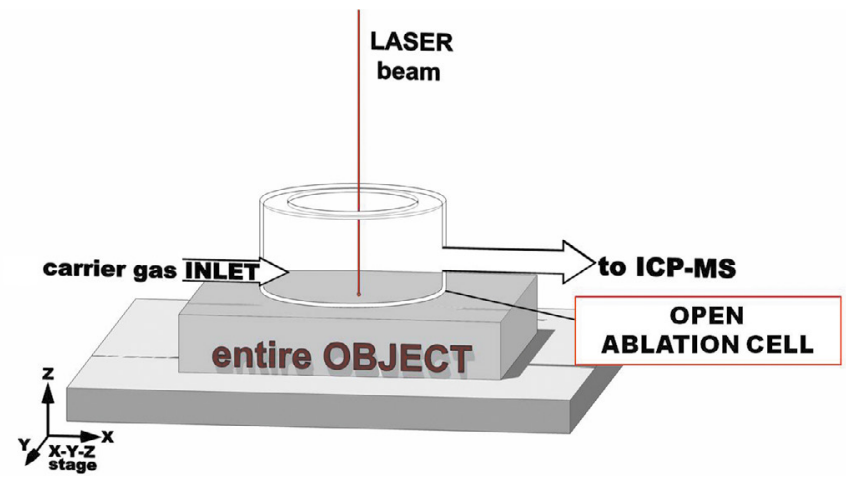

Fig. 6. Scenario 3: ablation executed in an open cell attached to a surface of an object.

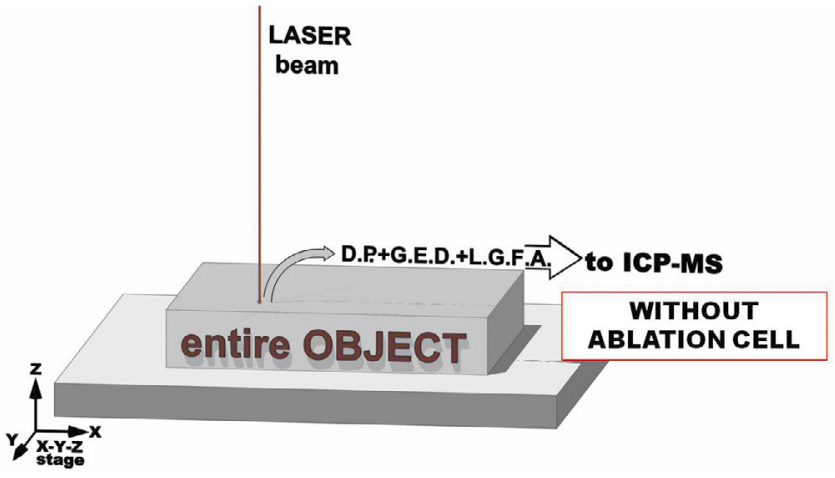

Fig. 7. Scenario 4: air ablation without using any ablation cell. D.P. $=$ diaphragm pump; G.E.D. = gas exchange device; L.G.F.A. $=$ laminar gas flow adapter [177]. low level for most of the elements. This kind of technical solution seems very promising for all cultural heritage objects investigations in the near future, releasing the LA-ICP-MS measurements from having to be run only in a sealed ablation cell.

\subsection{Scenario 4: direct laser ablation from cultural heritage objects in ambient air}

The innovative direct sampling by laser ablation in ambient air during LA-ICP-MS measurements was described by Kovacs et al. [67] in 2010. The open set-up was used for ablation with no cell at all. The exchange of air to argon by means of a gas exchange device was required together with a diaphragm pump, which then permitted the transpor- tation of the laser-generated aerosol into the ICP-MS. The level of oxides formation was monitored carefully as it was considered a possible limitation of this method. Oxide formation and count rates of the gas blank or potential spectral interferences from polyatomic ions were satisfactory and the accuracy of the obtained results was similar to the one achieved with closed transport systems.

In 2013 Dorta et al. [178] described laser ablation sampling in air under atmospheric pressure coupled with MC-ICP-MS. A lead isotope ratio analysis was performed, using two brass certified reference materials, galena and zircon. The precision and accuracy of the measurements were similar to the obtained by conventional LA-MC-ICP-MS in a sealed cell and helium atmosphere. The direction of 
development of such systems enabling the ablation in ambient air directly from the surface of unique objects seems very promising. Such instrumentation would cancel most of limitations for dimensions of objects easily analyzed by means of LA-ICP-MS. There is still a need to transport objects to the laboratory; therefore, the simplicity of using LA-ICP-MS method will remain mostly for moveable objects.

\subsection{Scenario 5: use of portable laser ablation sampling device}

The use of a portable laser sampling device facilitates the analysis of any immoveable cultural heritage objects (Fig. 8). These objects were the last category, which could not be investigated by means of LA-ICP-MS without mechanical sampling until the paper transported to a laboratory. The portable LA sampling device has already been successfully applied to the elemental analysis of ancient Chinese ceramics [177] and the determination of lead isotopic composition [196]. Taking into account all demands of the conservation science or archaeometric investigations, the possible use of the portable LA set up has a huge potential to be used in the future analysis of all cultural heritage objects, which cannot be examined by any other technique without invasive sampling.

The portable laser ablation sampling utilizes all advantages of microscopic degree of intervention caused by laser ablation. Some essential problems, such as polyatomic interferences caused by filter material during the subsequent re-ablation in the lab, element-dependent sensitivity enhancement, signal fluctuations and elemental fractiona-

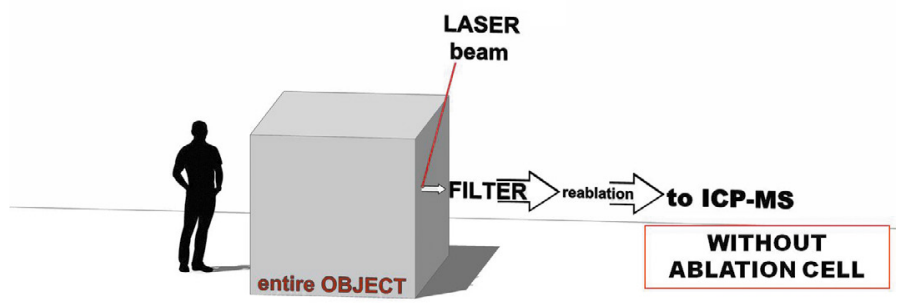

Fig. 8. Scenario 5: air ablation with collection of particulate matter on filters and subsequent re-ablation inside a closed cell. by Glaus et al. [177] reported the use of the portable sampling device applicable to the ablation of any object regardless of its size and location. Ablated particles are collected on special filters. Filters protected from contamination are transported to a laboratory, where they can be examined either by means of LA-ICP-MS with re-ablation or by any other instrumental method.

This approach allows the in situ microsampling of all objects (apart from the underwater cultural heritage) in spite of their dimensions and possibility for being tion have been observed [177]; however, these limitations do not diminish the attractiveness of the proposed method for the elemental and isotopic analysis of immoveable cultural heritage objects.

\section{Multi-technique approach incorporating LA-ICP-MS}

The results of sensitive LA-ICP-MS elemental/isotopic measurements can be used to support the use of other instrumental meth- 
ods such as Scanning Electron Microscopy with Energy Dispersive X-Ray Spectrometry (SEM-EDS) $[12,31,38,74,79,112,118,130$, 135, 166, 170, 181, 182], Electron Probe Microanalysis (EPMA) [75, 80, 81, 97, 112, 167, 183-185], X-Ray Fluorescence (XRF) $[16,31,35,44,93,94,98,119,120]$, Proton Induced X-Ray Emission [22, 41, 95, 114, 186, 187] or Neutron Activation Analysis (NAA) $[23,36,99,100,107,108,114,146$, 188-192]. Leroy et al. [193] reported the first application of confocal Synchrotron Radiation micro X-Ray Fluorescence (SR-mXRF) with LA-ICP-MS. They examined microscopic slag inclusions in mediaeval armours attributed to a Lombard provenance from stylistic considerations. The potential of coupling SR-m-XRF with LA-ICP-MS during quantification of trace elements in slag inclusions was evaluated with respect to drawbacks and advantages of each method.

LA-ICP-MS was merged with instrumental methods providing molecular information about the determined compounds: Raman spectroscopy (RS) [79, 121, 168, 194], Fourier Transformed Infrared Spectroscopy (FTIR) [131, 194, 195] or Gas Chromatography Mass Spectrometry (GC/MS) [195] during glass, ceramic, bones and wall-paintings investigations.

Di Bella et al. [82], Gallo et al. [83], Walton et al. [169] described the application of X-ray Powder Diffraction (XRPD) together with LA-ICP-MS for the historic glass analysis. The combination of Computed Tomography (CT) and LA-ICP-MS was reported by A. Kreiter et al. [109]. The authors used CT to assess differences in the porosity, number and size of inclusions in three visible distinguishable layers of a ceramic figurine. The LA-ICP-MS analysis was carried out to quantify major, minor and trace elements in the abovementioned figurine layers. The application of these complementary methods led to the answer to a question whether the analyzed object was formed over time using material from the same resource.

\section{Conclusions}

The interest in the use of laser ablation ICP-MS in archaeometry and restoration/ conservation science is constantly increasing; however, the non-destructive character of the measurements pointed out by Lahanier [2] as one of the requirements for instrumental methods most suitable for the analysis of cultural heritage objects cannot be fully attributed to LA-ICP-MS.

The possibility to investigate cultural heritage objects by means of LA-ICP-MS should be always verified according to conservators recommendations, taking into account dimensions of the analyzed objects and the possibility of samples collections, their number and size. The mechanical sampling is one of the suggested solutions, while the other is the possibility to perform the direct laser ablation from cultural heritage objects. The use of open ablation cells or direct air ablation was proposed when the mechanical sampling is impossible or unacceptable. Such direct ablation offers many advantages and expands the usefulness of LA-ICP-MS for the analysis of cultural heritage objects. The real milestone of the development of LA-ICP-MS was the use of mobile LA systems allowing for microsampling independently from a size and the mobility of the analyzed object.

\section{Acknowledgements}

This work was a part of the broader research project funded by the National Science Center of Poland, No 2011/01/B/ST4/00478. It was partially realized 
at the Biological and Chemical Research Centre, University of Warsaw, established within the project co-financed by European Union from the European Regional Development Fund under the Operational Program Innovative Economy, 2007-2013.

\section{References}

[1] R. Van Grieken, K. Janssens, Non-destructive testing and microanalysis for the diagnostics and conservation of cultural heritage, Taylor \& Francis/ Balkema Publishers, Rotterdam 2005.

[2] C. Lahanier, "Scientific methods applied to the study of ART objects", Mikrochimica Acta, 2 (1991) 245-254, DOI: 10.1007/BF01245512.

[3] J. Wouters, "Surrealism in science: the difference between analysis and analysis" in: J.J. Boon, E.S.B. Ferreira (Eds.), Reporting Highlights of the De Mayerne Programme, The Hague, 2006, pp. 13-20.

[4] M. Resano, E. Garcia-Ruiz, F. Vanhaecke, "Laser ablation-inductively coupled plasma mass spectrometry in archaeometric research", Mass Spectrometry Review, 29 (2010) 55-78 - DOI: 10.1002/mas.20220.

[5] B. Giussani, D. Monticelli, L. Rampazzi, "Role of laser ablation-inductively coupled plasma-mass spectrometry in cultural heritage research: A review", Analytica Chimica Acta, 635 (2009) 6-21, DOI: 10.1016/j.aca.2008.12.040.

[6] G. Spoto, G. Grasso, "Spatially resolved mass spectrometry in the study of art and archaeological objects", Trac-Trends in Analytical Chemistry, 30 (2011) 856-863, DOI: 10.1016/j.trac.2011.01.016.

[7] C. C. Garcia, H. Lindner, K. Niemax, "Laser ablation inductively coupled plasma mass spectrometry-current shortcomings, practical suggestions for improving performance, and experiments to guide future development", Journal of Analytical Atomic Spectrometry, 24 (2009) 14-26, DOI: 10.1039/ B813124B.

[8] J. Koch, D. Guenther, "Review of the State-of-theArt of Laser Ablation Inductively Coupled Plasma Mass Spectrometry", Applied Spectroscopy, 65 (2011) 155-162, DOI: 10.1366/11-06255.

[9] S. A. Junk, "Ancient artefacts and modern analytical techniques - Usefulness of laser ablation
ICP-MS demonstrated with ancient gold coins", Nuclear Instruments \& Methods in Physics Research Section B, 181 (2001) 723-727, DOI: 10.1016/S0168583X(01)00366-4.

[10] R. E. Russo, X. Mao, J. J. Gonzalez, V. Zorba, J. Yoo, "Laser Ablation in Analytical Chemistry", Analytical Chemistry, 85 (2013) 6162-6177, DOI: 10.1021/ac4005327.

[11] G. H. Fontaine, K. Hametner, A. Peretti, D. Guenther, "Authenticity and provenance studies of copper-bearing andesines using $\mathrm{Cu}$ isotope ratios and element analysis by fs-LA-MC-ICPMS and ns-LAICPMS", Analytical and Bioanalytical Chemistry, 398 (2010) 2915-2928, DOI: 10.1007/s00216-010-4245-z.

[12] D. Barca, G. Lucarin, F. G. Fedele, "The provenance of obsidian artefacts from the WĀDI ATH-THAYYILAH 3 Neolithic side (eastern Yemen Plateau) by LA-ICP-MS", Archaeometry, 54 (2012) 603-622, DOI: 10.1111/j.1475-4754.2011.00643.x.

[13] C. M. Jackson, P. T. Nicholson, "The provenance of some glass ingots from the Uluburun shipwreck", Journal of Archeological Science, 37 (2010) 295-301, DOI: 10.1016/j.jas.2009.09.040.

[14] R. Kovacs, S. Schlosser, S. P. Staub, A. Schmiderer, E. Pernicka, D. Guenther, "Characterization of calibration materials for trace element analysis and fingerprint studies of gold using LA-ICP-MS", Journal of Analytical Atomic Spectrometry, 24 (2009) 476-483, DOI: 10.1039/B819685K.

[15] A.-M. Desaulty, P. Dillmann, M. L'Heritiera, C. Mariet, B. Gratuze, J. L. Joron, P. Fluzin, "Does it come from the Pays de Bray? Examination of an origin hypothesis for the ferrous reinforcements used in French medieval churches using major and trace element analyses", Journal of Archeological Science, 36 (2009) 2445-2462, DOI: 10.1016/j.jas.2009.07.002.

[16] L. B. Brostoff, J. J. Gonzalez, P. Jett, R. E. Russo, "Trace element fingerprinting of ancient Chinese gold with femtosecond laser ablation-inductively coupled mass spectrometry", Journal of Archeological Science, 36 (2009) 461-466, DOI: 10.1016/j. jas.2008.09.037.

[17] I. Scharlotta, "Micro-sampling of human bones for mobility studies: diagenetic impacts and potentials for elemental and isotopic research", Journal of 
Archeological Science, 37 (2010) 1929-1941, DOI: 10.1016/j.jas.2013.07.014.

[18] P. Robertshaw, M. Wood, E. Melchiorre, R. S. Popelka-Filcoff, M. D. Glascock, "Southern African glass beads: chemistry, glass sources and patterns of trade", Journal of Archeological Science, 37 (2010) 1898-1912, DOI: 10.1016/j.jas.2010.02.016.

[19] L. Dussubieux, B. Gratuze, M. Blet-Lemarquand, "Mineral soda alumina glass: occurence and meaning", Journal of Archeological Science, 37 (2010) 1646-1655, DOI: 10.1016/j.jas.2010.01.025.

[20] P. Robertshaw, N. Benco, M. Wood, L. Dussubieux, E. Melchiorre, A. Ettahiri, "Chemical analysis of glass beads from medieval AL-BASRA (Morocco)", Archaeometry, 52 (2010) 355-379 DOI: 10.1111/j.1475-4754.2009.00482.x.

[21] S. M. Fitzpatrick, H. Takamiya, H. Neff, W. R. Dickinson, "Compositional analysis of Yayoi-Heian period ceramics from Okinawa: Examining the potential for provenance study", Geoarchaeology-an International Journal, 21 (2006) 803-822 - DOI: 10.1002/gea.20140.

[22] Z. Smit, K. Janssens, E. Bulska, B. Wagner, M. Kos, I. Lazar, "Trace element fingerprinting of facon-de-Venise glass", Nuclear Instruments \& Methods in Physics Research Section B, 239 (2005) 94-99, DOI: 10.1016/j.nimb.2005.06.182.

[23] J. H. Sterba, F. Munnik, N. J. G. Pearce, "Raising the temper-mu-spot analysis of temper inclusions in experimental ceramics", Journal of Radioanalytical and Nuclear Chemistry, 291 (2012) 25-35, DOI: 10.1007/s10967-011-1208-6.

[24] I. De Raedt, K. Janssens, J. Veeckman, L. Vincze, B. Vekemans, T. E. Jeffries, "Trace analysis for distinguishing between Venetian and facon-de-Venise glass vessels of the $16^{\text {th }}$ and $17^{\text {th }}$ century", Journal of Analytical Atomic Spectrometry, 16 (2001) 1012-1017, DOI: 10.1039/b102597j.

[25] T. Zhu, W. Sun, H. Zhang, H. Wang, G. Kuang, L. Lv, "Study on the provenance of Xixun Qingbai wares from the Northern Song Dynastyof China", Archaeometry, 54 (2012) 475-488 - DOI: 10.1111/j.1475-4754.2011.00636.x.

[26] A. Shortland, N. Rogers, K. Eremin, “Trace element discriminants between Egyptian and
Mesopotamian Late Bronze Age glasses", Journal of Archeological Science, 34 (2007) 781-789 - DOI: 10.1016/j.jas.2006.08.004.

[27] M. F. Guerra, C. O. Sarthre, A. Gondonneau, J. N. Barrandon, "Precious metals and provenance enquiries using LA-ICP-MS", Journal of Archeological Science, 26 (1999) 1101-1110 - DOI: 10.1006/ jasc.1999.0405.

[28] L. M. Mallory-Greenough, J. D. Greenough, G. Dobosi, J. V. Owen, "Fingerprinting ancient Egyptian quarries: Preliminary results using laser ablation microprobe-inductively coupled plasma-mass spectrometry", Archaeometry, 41 (1999) 227-238, DOI: 10.1111/j.1475-4754.1999.tb00979.x.

[29] S. L. Eckert, W. D. James, "Investigating the production and distribution of plain ware pottery in the Samoan archipelago with laser ablation-inductively coupled plasma-mass spectrometry (LA-ICP-MS)", Journal of Archeological Science, 38 (2011) 2155-2170, DOI: 10.1016/j.jas.2011.03.009.

[30] N. Sharratt, M. Golitko, P. R. Williams, L. Dussubieux, "Ceramic Production During the Middle Horizon: Wari and Tiwanaku Clay Procurement in the Moquegua Valley, Peru", Geoarchaeology-an International Journal, 24 (2009) 792-820, DOI: 10.1002/gea.20288.

[31] D. Miriello, D. Barca, A. Bloise, A. Ciarallo, G. M. Crisci, T. De Rose, C. Gattuso, F. Gazineo, M. F. La Russa, "Characterisation of archaeological mortars from Pompeii (Campania, Italy) and identification of construction phases by compositional data analysis", Journal of Archeological Science, 37 (2010) 2207-2223, DOI: 10.1016/j.jas.2010.03.019.

[32] R. J. Speakman, H. Neff, "Evaluation of painted pottery from the Mesa Verde region using laser ablation-inductively coupled plasma-mass spectrometry (LA-ICP-MS)", American Antiquity, 67 (2002)137-144, DOI: 10.2307/2694882.

[33] M. E. Beck, H. Neff, "Hohokam and Patayan interaction in southwestern Arizona: Evidence from ceramic compositional analyses", Journal of Archeological Science, 34 (2007) 289-300, DOI: 10.1016/j. jas.2006.05.003.

[34] L. Rampazzi, B. Rizzo, C. Colombo, C. Conti, M. Realini, U. Bartolucci, M. P. Colombini, A. Spiriti, 
L. Facchin, "The stucco decorations from St. Lorenzo in Laino (Como, Italy): The materials and the techniques employed by the "Magistri Comacini", Analytica Chimica Acta, 630 (2008) 91-100, DOI: 10.1016/j.aca.2008.09.052.

[35] J. Perez-Arantegui, M. Resano, E. Garcia-Ruiz, F. Vanhaecke, C. Roldan, J. Ferrero, J. Coll, “Characterization of cobalt pigments found in traditional Valencian ceramics by means of laser ablation-inductively coupled plasma mass spectrometry and portable X-ray fluorescence spectrometry", Talanta, 74 (2008) 1271-1280, DOI: 10.1016/j.talanta.2007.08.044.

[36] D. V. Hill, R. J. Speakman, M. D. Glascock, "Chemical and mineralogical characterization of Sasanian and Early Islamic glazed ceramics from the Deh Luran Plain, southwestern Iran", Archaeometry, 46 (2004) 585-605, DOI: 10.1111/j.14754754.2004.00175.x.

[37] M. Senn, U. Gfeller, B. Guenette-Beck, P. Lienemann, A. Ulrich, "Tools to qualify experiments with bloomery furnaces", Archaeometry, 52 (2010) 131-145 - DOI: 10.1111/j.1475-4754.2009.00461.x.

[38] S. Cagno, M. Mendera, T. Jeffries, K. Janssens, "Raw materials for medieval to post-medieval Tuscan glassmaking: new insight from LA-ICP-MS analyses", Journal of Archaeological Science, 37 (2010) 3030-3036, DOI: 10.1016/j.jas.2010.06.030.

[39] I. Caterina, T. Maurizio, S. Giuseppe, "Archaeometallurgy in Messina: iron slag from a dig at block p, laboratory analyses and interpretation", Mediterranean Archaeology \& Archaeometry, 8 (2008) 49-60.

[40] S. Duwe, H. Neff, “Glaze and slip pigment analyses of Pueblo IV period ceramics from east-central Arizona using time of flight-laser ablation-inductively coupled plasma-mass spectrometry (TOFLA-ICP-MS)", Journal of Archaeological Science, 34 (2007) 403-414, DOI: 10.1016/j.jas.2006.06.001.

[41] Z. Smit, P. Pelicon, G. Vidmar, B. Zorko, M. Budnar, G. Demortier, B. Gratuze, S. Sturm, M. Necemer, P. Kump, M. Kos, "Analysis of medieval glass by X-ray spectrometric methods", Nuclear Instruments \& Methods in Physics Research Section B, 161 (2000) 718-723, DOI: 10.1016/S0168-583X(99)00947-7.

[42] M. P. Coustures, D. Beziat, F. Tollon, C. Domergue, L. Long, A. Rebiscoul, “The use of trace element analysis of entrapped slag inclusions to establish ore - bar iron links: examples from two Gallo-Roman iron-making sites in France (Les Martys, Montagne Noire, and Les Ferrys, Loiret)", Archaeometry, 45 (2003) 599-613, DOI: 10.1046/j.14754754.2003.00131.x.

[43] B. Wagner, E. Bulska, W. Sobucki, "Magnesium distribution in paper subjected to deacidification investigated by means of Laser Ablation Inductively Coupled Plasma Mass Spectroscopy" Journal of Cultural Heritage, 9 (2008) 60-65, DOI: 10.1016/j. culher.2007.11.001.

[44] J. Malesic, M. Kojc, V. S. Selih, "Assessment of the effect of various bleaching agents on papers with foxing stains", Restaurator-International Journal for the Preservation of Library and Archival Material, 29 (2008) 142-154, DOI: 10.1515/rest.2008.010.

[45] B. Wagner, E. Bulska, A. Drewniak, W. Sobucki, D. Rams, "LA-ICPMS investigations of a long-term effect of indoor air pollution on paper", Analytical Chemistry (Warsaw), 54 (2009) 1253-1264.

[46] B. Wagner, E. Bulska, "On the use of laser ablation inductively coupled plasma mass spectrometry for the investigation of the written heritage", Journal of Analytical Atomic Spectrometry, 19 (2004) 1325-1329, DOI: 10.1039/B408384A.

[47] A. L. Gray, "Solid sample introduction Laser ablation for inductively coupled plasma source mass spectrometry", Analyst, 110 (1985) 551-556.

[48] M. Thompson, J. E. Goulter, F. Sieper, "Laser ablation for the introduction of solid samples into an inductively coupled plasma for atomic emission spectrometry", Analyst, 106 (1981) 32-39.

[49] J. W. Carr, G. Horlick, "Laser vaporization of solid metal samples into an inductively coupled plasma”, Spectrochimica Acta Part B, 37 (1982) 1-15, DOI: 10.1016/0584-8547(82)80003-7.

[50] D. Gunther, S. E. Jackson, H. P. Longerich, "Laser ablation and arc/spark solid sample introduction into inductively coupled plasma mass spectrometers", Spectrochimica Acta Part B, 54 (1999) 381-409, DOI: 10.1016/S0584-8547(99)00011-7.

[51] R. Kovacs, K. Nishiguchi, K. Utani, D. Guenther, "Development of direct atmospheric sampling for laser ablation-inductively coupled plasma-mass spec- 
trometry", Journal of Analytical Atomic Spectrometry, 25 (2010) 142-147, DOI: 10.1039/B924425E.

[52] D. Gunther, B. Hattendorf, "Solid sample analysis using laser ablation inductively coupled plasma mass spectrometry", Trac-Trends in Analytical Chemistry, 24 (2005) 255-265, DOI: 10.1016/j. trac.2004.11.017.

[53] B. Wagner, W. Jedral, "Open ablation cell for LA-ICP-MS investigations of historic objects", Journal of Analytical Atomic Spectrometry, 26 (2011) 2058-2063, DOI: 10.1039/C1JA10137D.

[54] W. Devos, C. Moor, P. Lienemann, "Determination of impurities in antique silver objects for authentication by laser ablation inductively coupled plasma mass spectrometry (LA-ICP-MS)", Journal of Analytical Atomic Spectrometry, 14 (1999) 621-626, DOI: 10.1039/A900073I.

[55] S. M. Eggins, L. P. J. Kinsley, J. M. G. Shelley, "Deposition and element fractionation processes during atmospheric pressure laser sampling for analysis by ICP-MS", Applied Surface Science, 127 (1998) 278-286, DOI: 10.1016/S0169-4332(97)00643-0.

[56] P. M. Outridge, W. Doherty, D. C. Gregoire, "The formation of trace element-enriched particulates during laser ablation of refractory materials", Spectrochimica Acta Part B, 51 (1996) 1451-1462, DOI: 10.1016/0584-8547(96)01542-X.

[57] P. M. Outridge, W. Doherty, D. C. Gregoire, "Ablative and transport fractionation of trace elements during laser sampling of glass and copper", Spectrochimica Acta Part B, 52 (1997) 2093-2102, DOI: 10.1016/S0584-8547(97)00112-2.

[58] B. J. Fryer, S. E. Jackson, H. P. Longerich, "The design, operation and role of the laser-ablation microprobe coupled with an inductively coupled plasma; mass spectrometer (LAM-ICP-MS) in the earth sciences", The Canadian Mineralogist, 33 (1995) 303-312.

[59] J. Gonzalez, X. L. Mao, J. Roy, S. S. Mao, R. E. Russo, "Comparison of 193, 213 and $266 \mathrm{~nm}$ laser ablation ICP-MS", Journal of Analytical Atomic Spectrometry, 17 (2002) 1108-1113, DOI: 10.1039/ B202122F.

[60] I. Horn, D. Gunther, “The influence of ablation carrier gasses $\mathrm{Ar}, \mathrm{He}$ and $\mathrm{Ne}$ on the particle size distribution and transport efficiencies of laser ablation-induced aerosols: implications for LA-ICP-MS", Applied Surface Science, 207 (2003) 144-157, DOI: 10.1016/S0169-4332(02)01324-7.

[61] R. E. Russo, X. L. Mao, H. C. Liu, J. Gonzalez, S. S. Mao, "Laser ablation in analytical chemistry a review”, Talanta, 57 (2002) 425-451, DOI: 10.1016/ S0039-9140(02)00053-X.

[62] T. E. Jeffries, N. J. G. Pearce, W. T. Perkins, A. Raith, "Chemical fractionation during infrared and ultraviolet laser ablation inductively coupled plasma mass spectrometry-implications for mineral microanalysis", Analytical Communications, 33 (1996) 35-39, DOI: 10.1039/AC9963300035.

[63] H. Wiltsche, D. Guenther, "Capabilities of femtosecond laser ablation ICP-MS for the major, minor, and trace element analysis of high alloyed steels and super alloys", Analytical and Bioanalytical Chemistry, 399 (2011) 2167-2174, DOI: 10.1007/ s00216-010-4605-8.

[64] R. Glaus, R. Kaegi, F. Krumeich, D. Guenther, "Phenomenological studies on structure and elemental composition of nanosecond and femtosecond laser-generated aerosols with implications on laser ablation inductively coupled plasma mass spectrometry", Spectrochimica Acta Part B, 65 (2010) 812-822, DOI: 10.1016/j.sab.2010.07.005.

[65] M. Waelle, J. Koch, D. Tabersky, K. Hametner, N. D. Zhigadlo, S. Katrych, J. Karpinski, D. Guenther, "Analyses of lithium-doped and pure magnesium diboride using ultraviolet nano- and femtosecond laser ablation inductively coupled plasma mass spectrometry", Journal of Analytical Atomic Spectrometry, 25 (2010) 193-195, DOI: 10.1039/B914547H.

[66] J. Koch, A. von Bohlen, R. Hergenroder, K. Niemax, "Particle size distributions and compositions of aerosols produced by near-IR femto- and nanosecond laser ablation of brass", Journal of Analytical Atomic Spectrometry, 19 (2004) 267-272, DOI: 10.1039/B310512A.

[67] R. Kovacs, K. Nishiguchi, K. Utani, D. Guenther, "Development of direct atmospheric sampling for laser ablation-inductively coupled plasma-mass spectrometry", Journal of Analytical Atomic Spectrometry, 25 (2010) 142-147, DOI: 10.1039/B924425E. 
[68] D. Gunther, H. Cousin, B. Magyar, I. Leopold, "Calibration Studies on Dried Aerosols for Laser Ablation-Inductively Coupled Plasma Mass Spectrometry", Journal of Analytical Atomic Spectrometry, 12 (1997)165-170, DOI: 10.1039/A604531F.

[69] J. J. Leach, L. A. Allen, D. B. Aeschliman, R. S. Houk, " $\mathrm{C}$ alibration of Laser Ablation Inductively Coupled Plasma Mass Spectrometry Using Standard Additions with Dried Solution Aerosols", Analytical Chemistry, 71 (1999) 440-445, DOI: 10.1021/ac980855i.

[70] L. Halicz, D. Gunther, "Quantitative analysis of silicates using LA-ICP-MS with liquid calibration", Journal of Analytical Atomic Spectrometry, 19 (2004) 1539-1545, DOI: 10.1039/B410132D.

[71] D. Gunther, R. Frischknecht, H. J. Muschenborn, C. A. Heinrich, "Direct liquid ablation: a new calibration strategy for laser ablation-ICP-MS microanalysis of solids and liquids", Fresenius Journal of Analytical Chemistry, 359 (1997) 390-393, DOI: 10.1007/s002160050594.

[72] B. J. Masters, B. L. Sharp, "Universal Calibration Strategy for Laser Ablation Inductively Coupled Plasma Mass Spectrometry Based on the Use of Aqueous Standards With Modified Absorption Coefficients", Analytical Communications, 34 (1997) 237-239, DOI: 10.1039/A704726F.

[73] F. Boue-Bigne, B. J. Masters, J. S. Crighton, B. L. Sharp, "A calibration strategy for LA-ICP-MS analysis employing aqueous standards having modified absorption coeficients", Journal of Analytical Atomic Spectrometry, 14 (1999) 1665-1672, DOI: 10.1039/A905479K.

[74] B. Wagner, A. Nowak, E. Bulska, J. Kunicki-Goldfinger, O. Schalm, K. Janssens, “Complementary analysis of historical glass by scanning electron microscopy with energy dispersive X-ray spectroscopy and laser ablation inductively coupled plasma mass spectrometry", Microchimica Acta, 162 (2008) 415-424, DOI: 10.1007/s00604-007-0835-7.

[75] R. Arletti, C. Giacobbe, S. Quartieri, G. Sabatino, G. Tigano, M. Triscari, G. Vezzalini, “Archaeometrical investigation of Sicilian Early Byzantine glass: chemical and spectroscopic data", Archaeometry, 52 (2010) 99-114, DOI: 10.1111/j.1475-4754.2009.00458.x.
[76] L. Dussubieux, C. M. Kusimba, V. Gogte, S. B. Kusimba, B. Gratuze, R. Oka, "The trading of ancient glass beads: new analytical data from South Asian and East African soda-alumina glass beads", Archaeometry, 50 (2008) 797-821, DOI: 10.1111/j.1475-4754.2007.00350.x.

[77] M. Bertini, A. Shortland, K. Milek, E. M. Krupp, "Investigation of Iron Age north-eastern Scottish glass beads using element analysis with LA-ICP-MS", Journal of Archaeological Science, 38 (2011) 2750-2766, DOI: 10.1016/j.jas.2011.06.019.

[78] L. Dussubieux, P. Robertshaw, M. D. Glascock, "LA-ICP-MS analysis of African glass beads: Laboratory inter-comparison with an emphasis on the impact of corrosion on data interpretation", International Journal of Mass Spectrometry, 284 (2009) 152-161, DOI: 10.1016/j.ijms.2008.11.003.

[79] A. M. de Francesco, R. Scarpelli, F. del Vecchio, D. Giampaola, "Analysis of early medieval glass from excavations at Piazza Bovio, Naples (Italy)", Archeometry, 56 (2014) 137-147, DOI: 10.1111/arcm.12070.

[80] T. Purowski, B. Wagner, E. Bulska, O. Syta, P. Dzierzanowski, "Glassy faience from the Hallstatt C period in Poland: a chemicophysical study", Journal of Archaeological Science, 50 (2014) 288-304, DOI: 10.1016/j.jas.2014.06.022.

[81] C. M. Jackson, S. Cottam, "A green thought in a green shade'; Compositional and typological observations concerning the production of emerald green glass vessels in the $1^{\text {st }}$ century A.D.", Journal of Archaeological Science, 61 (2015) 139-148, DOI: 10.1016/j.jas.2015.05.004.

[82] M. Di Bella, S. Quartieri, G. Sabatino, F. Santalucia, M. Triscari, “The glass mosaics tesserae of 'Villa del Casale' (Piazza Armerina, Italy): a multi-technique archaeometric study", Archaeological and Anthropological Sciences, 6 (2014) 345-362, DOI: 10.1007/s12520-013-0172-1.

[83] F. Gallo, A. Silvestri, G. Molin, "Glass from the Archaeological Museum of Adria (North-East Italy): new insights into Early Roman production technologies", Journal of Archaeological Science, 40 (2013) 2589-2605, DOI: 10.1016/j.jas.2013.01.017.

[84] S. Cagno, P. Cosyns, A. Izmer, F. Vanhaecke, K. Nys, K. Janssens, “Deeply colored and black-ap- 
pearing Roman glass: a continued research", Journal of Archaeological Science, 42 (2014) 128-139, DOI: 10.1016/j.jas.2013.11.003.

[85] K. Hellemans, A. Vincke, S. Cagno, D. Herremans, W. De Clercq, K. Janssens, "Composition and state of alteration of $18^{\text {th }}$-century glass finds found at the Cistercian nunnery of Clairefontaine, Belgium", Journal of Archaeological Science, 47 (2014) 121-133, DOI: 10.1016/j.jas.2014.03.039.

[86] J. J. Kunicki-Goldfinger, I. C. Freestone, I. McDonald, J. A. Hobot, H. Gilderdale-Scott, T. Ayers, "Technology, production and chronology of red window glass in the medieval period - rediscovery of a lost technology", Journal of Archaeological Science, 41 (2014) 89-105, DOI: 10.1016/j.jas.2013.07.029.

[87] V. Devulder, A. Gerdes, F. Vanhaecke, P. Degryse, "Validation of the determination of the $\mathrm{B}$ isotopic composition in Roman glasses with laser ablation multi-collector inductively coupled plasma-mass spectrometry", Spectrochimica Acta Part B, 105 (2015) 116-120, DOI: 10.1016/j.sab.2014.08.038.

[88] D. Barca, D. Miriello, A. Pecci, L. Barba, A. Ortiz, L.R. Manzanilla, J. Blancas, G.M. Crisci, "Provenance of glass shards in archaeological lime plasters by LA-ICPMS: implications for the ancient routes from the Gulf of Mexico to Teotihuacan in Central Mexico,' Journal of Archaeological Science, 40 (2013) 3999-4008, DOI: 10.1016/j.jas.2013.05.016.

[89] S. Panighello, E. F. Orsega, J. T. van Elteren, V.S. Selih, "Analysis of polychrome Iron Age glass vessels from Mediterranean I, II and III groups by LA-ICP-MS", Journal of Archaeological Science, 39 (2012) 2945-2955, DOI: 10.1016/j.jas.2012.04.043.

[90] S. Panighello, A. Kavčič, K. Vogel-Mikuš, N. H. Tennent, A. Wallert, S. B. Hočevar, J. T. van Elteren, "Investigation of smalt in cross-sections of $17^{\text {th }}$ century paintings using elemental mapping by laser ablation ICP-MS", Microchemical Journal, 125 (2016) 105-115, DOI: 10.1016/j.microc.2015.11.015.

[91] B. Siquin, Q. H. Li, F. X. Gan, "Analysis of Some Chinese Potash Glass by Laser Ablation-Inductively Coupled Plasma-Atomic Emission Spectrometry and Laser Ablation-Inductively Coupled Plasma-Mass Spectrometry", Chinese Journal of Analytical Chemistry, 41 (2013) 1328-1333, DOI: 10.1016/ S1872-2040(13)60678-7.
[92] J. T. van Elteren, N. H. Tennen, V. S. Šelih, "Multi-element quantification of ancient/historic glasses by laser ablation inductively coupled plasma mass spectrometry using sum normalization calibration", Analytica Chimica Acta, 644 (2009) 1-9, DOI: 10.1016/j.aca.2009.04.025.

[93] L. Khalidi, B. Gratuze, S. Boucetta,"Provenace of obsidian excavated from late chalcolithic levels at the sites of Tell Hamoukarand Tell Brak, Syria", Archaeometry, 51 (2009) 879-893, DOI: 10.1111/j.14754754.2009.00459.x.

[94] L. C. Kellett, M. Golitko, B. S. Bauer, "A provenance study of archaeological obsidian from the Andahuaylas region of southern Peru", Journal of Archaeological Science, 40 (2013) 1890-1902, DOI: 10.1016/j.jas.2012.11.014.

[95] C. Reepmeyer, G. Clark, "Post-colonization interaction between Vanuatu and Fiji reconsidered: the re-analysis of obsidian from Lakeba island, Fiji", Archaeometry, 52 (2010) 1-18, DOI: 10.1111/j.14754754.2009.00465.x.

[96] W. Ambrose, C. Allen, S. O'Connor, M. Spriggs, N. V. Oliveira, C. Reepmeyer, "Possible obsidian sources for artifacts from Timor: narrowing the options using chemical data", Journal of Archaeological Science, 36 (2009) 607-615, DOI: 10.1016/j. jas.2008.09.022.

[97] F.H. Brown, B.P. Nash, D.P. Fernandez, H.V. Merrick, R.J. Thomas, "Geochemical composition of source obsidians from Kenya", Journal of Archaeological Science, 40 (2013) 3233-3251, DOI: 10.1016/j.jas.2013.03.011.

[98] X. Terradas, B. Gratuze, J. Bosch, R. Enrich, X. Esteve, F. X. Oms, G. Ribé, "Neolithic diffusion of obsidian in the western Mediterranean: new data from Iberia", Journal of Archaeological Science, 41 (2014) 69-78, DOI: 10.1016/j.jas.2013.07.023.

[99] B. A. Alex, D. L. Nichols, M. D. Glascock, "Complementary compositional analysis of formative period ceramics from Teotihuacan valley", Archaeometry, 54 (2012) 821-834, DOI: 10.1111/j.14754754.2011.00652.x.

[100] H. Neff, "Analysis of Mesoamerican Plumbate Pottery Surfaces by Laser Ablation-Inductively Coupled Plasma-Mass Spectrometry (LA-ICP-MS)", 
Journal of Archaeological Science, 30 (2003) 21-35, DOI: $10.1006 /$ jasc.2001.0801.

[101] M. Golitko, J. V. Dudgeon, H. Neff, J. E. Terrell, "Identification of post-depositional chemical alteration of ceramics from the north coast of Papua New Guinea (Sanduan province) by time-offlight - laser ablation - inductively coupled plasma - mass spectrometry (TOF-LA-ICP-MS)", Archaeometry, 54 (2012) 80-100, DOI: 10.1111/j.14754754.2011.00612.x.

[102] E. E. Cochrane, H. Neff,"Investigating compositional diversity among Fijian ceramics with laser ablation-inductively coupled plasma-mass spectrometry (LA-ICP-MS): implications for interaction studies on geologically similar islands", Journal of Archaeological Science, 33 (2006) 378-390, DOI: 10.1016/j.jas.2005.08.003.

[103] J. D. Robertson, H. Neff, B. Higgins, "Microanalysis of ceramics with PIXE and LA-ICP-MS", Nuclear Instruments \& Methods in Physics Research Section B, 189 (2002) 378-381, DOI: 10.1016/S0168583X(01)01093-X.

[104] L. G. Cecil, H. Neff, "Postclassic Maya slips and paints and their relationship to socio-political groups in El Peten, Guatemala", Journal of Archaeological Science, 33 (2006) 1482-1491, DOI: 10.1016/j. jas.2006.02.005.

[105] M. Resano, J. Perez-Arantegui, E. Garcia-Ruiz, F. Vanhaecke, "Laser ablation-inductively coupled plasma mass spectrometry for the fast and direct characterization of antique glazed ceramics", Journal of Analytical Atomic Spectrometry, 20 (2005) 508-514, DOI: 10.1039/B500691K.

[106] M. Resano, P. Marzo, J. Perez-Arantegui, M. Aramendia, C. Cloquet, F. Vanhaecke, "Laser ablation-inductively coupled plasma-dynamic reaction cell-mass spectrometry for the determination of lead isotope ratios in ancient glazed ceramics for discriminating purposes", Journal of Analytical Atomic Spectrometry, 23 (2008) 1182-1191, DOI: 10.1039/b802266f.

[107] N. J. Wallis, G. D. Kamenov, "Challenges in the analysis of heterogeneous pottery by LA-ICP-MS: a comparison with INAA", Archaeometry, 55 (2013) 983-909, DOI: 10.1111/j.1475-4754.2012.00718.x.
[108] P. Van Valkenburgh, S. J. Kelloway, L. Dussubieux, J. Quilter, M. D. Glascock, "The production and circulation of indigenous lead-glazed ceramics in northern Peru during Spanish colonial times", Journal of Archaeological Science, 61 (2015) 172-185, DOI: 10.1016/j.jas.2015.06.006.

[109] A. Kreiter, D. J. Riebe, W. A. Parkinson, Á. Peto, M. Tóth, P. Pánczél, E. Bánffy, "Unique in its chaîne opératoire, unique in its symbolism: undressing a figurine from the $6^{\text {th }}$ Millennium BC Körös culture, Hungary", Journal of Archaeological Science, 44 (2014) 136-147, DOI: 10.1016/j.jas.2014.01.027.

[110] R. Scarpelli, A.M. De Francesco, M. Gaeta, D. Cottica, L. Toniolo, "The provenance of the Pompeii cooking wares: Insights from LA-ICP-MS trace element analyses", Microchemical Journal, 119 (2015) 93-101, DOI: 10.1016/j.microc.2014.11.003.

[111] P. Comodi, A. Buccianti, A. Zucchini, M. Merletti, M. Bergamini, S. Nazzareni, "Insights into the provenance of Roman moulds and poincons found at Scoppieto (Terni, Italy)", Archeometry, 56 (2014) 58-77, DOI: 10.1111/arcm.12068.

[112] L. Dussubieux, A. Deraisme, G. Frot, C. Stevenson, A. Creech, Y. Bienvenu, "LA-ICP-MS, SEM-EDS and EPMA analysis of eastern North American copper-based artefacts: impact of corrosion and heterogeneity on the reliability of the LA-ICP-MS compositional results", Archaeometry, 50 (2008) 643-657, DOI: 10.1111/j.1475-4754.2007.00367.x.

[113] L. Dussubieux, L. Van Zelst, "LA-ICP-MS analysis of platinum-group elements and other elements of interest in ancient gold", Applied Physics A, 79 (2004) 353-356, DOI: 10.1007/s00339-004-2532-2.

[114] M. F. Guerra, T. Calligaro, "Gold traces to trace gold”, Journal of Archaeological Science, 31 (2004) 1199-1208, DOI: 10.1016/j.jas.2002.05.001.

[115] M. Resano, M. P. Marzo, R. Alloza, C. Saenz, F. Vanhaecke, L. Yang, S. Willie, R. E. Sturgeon, "Laser ablation single-collector inductively coupled plasma mass spectrometry for lead isotopic analysis to investigate evolution of the Bilbilis mint", Analytica Chimica Acta, 677 (2010) 55-63, DOI: 10.1016/j. aca.2010.07.032.

[116] E. E. Cochrane, H. Neff,"Investigating compositional diversity among Fijian ceramics with laser 
ablation-inductively coupled plasma-mass spectrometry (LA-ICP-MS): implications for interaction studies on geologically similar islands", Journal of Archaeological Science, 33 (2006) 378-390, DOI: 10.1016/j.jas.2005.08.003.

[117] C. Bendall, D. Wigg-Wolf, Y. Lahaye, H. M. Von Kaenel, G. P. Brey, "Detecting changes of Celtic gold sources through the application of trace element and $\mathrm{Pb}$ isotope laser ablation analysis of Celtic gold coins", Archaeometry, 51 (2009) 598-625, DOI: 10.1111/j.1475-4754.2008.00423.x.

[118] V. Lyubomirova, R. Djingova, I. Kuleff, "Comparison of analytical techniques for analysis of archaeological bronze", Archaeometry, 57 (2015) 677-686, DOI: 10.1111/arcm.12138.

[119] D. Walaszek, M. Senn, A. Wichser, M. Faller, B. Wagner, E. Bulska, A. Ulrich, "Minimally-invasive Laser Ablation Inductively Coupled Plasma Mass Spectrometry analysis of model ancient copper alloys", Spectrochimica Acta Part B, 99 (2014) 115-120, DOI: 10.1016/j.sab.2014.06.023.

[120] L. Dussubieux, H. Walder, "Identifying American native and European smelted coppers with pXRF: a case study of artifacts from the Upper Great Lakes region", Journal of Archaeological Science, 59 (2015) 169-178, DOI: 10.1016/j.jas.2015.04.011.

[121] L. C. Giannossa, S. Loperfido, M. Caggese, G. E. De Benedetto, R. Laviano, L. Sabbatini, A. Mangone, "A systematic characterization of fibulae from Italy: from chemical composition to microstructure and corrosion processes", New Journal of Chemistry, 37 (2013) 1238-1251, DOI: 10.1039/c2nj40362e.

[122] F. Nocete, R. Sáez, M.R. Bayona, J.M. Nieto, A. Peramo, P. López, J.I. Gil-Ibarguchi, N. Inácio, S. García, J. Rodríguez, "Gold in the Southwest of the Iberian Peninsula during the 3rd Millennium BC", Journal of Archaeological Science, 41 (2014) 691-704, DOI: 10.1016/j.jas.2013.10.006.

[123] V. Mozna, J. Pisonero, M. Hola, V. Kanicky, D. Günther, "Quantitative analysis of Fe-based samples using ultraviolet nanosecond and femtosecond laser ablation-ICP-MS", Journal of Analytical Atomic Spectrometry, 21 (2006) 1194-1201, DOI: 10.1039/ B606988F.
[124] B. Zhian, Y. Wenting, Y. Honglin, L. Xu, C. Kaiyun, Z. Chunlei, "Non-matrix-matched determination of lead isotope ratios in ancient bronze artifacts by femtosecond laser ablation multi-collector inductively coupled plasma mass spectrometry", International Journal of Mass Spectrometry, 402 (2016) 12-19, DOI: 10.1016/j.ijms.2016.03.001.

[125] A. Cucina, J. Dudgeon, H. Neff,"Methodological strategy for the analysis of human dental enamel by LA-ICP-MS", Journal of Archaeological Science, 34 (2007) 1884-1888, DOI: 10.1016/j.jas.2007.01.004.

[126] J. Farell, D. Amarasiriwardena, A. H. Goodman, B. Arriaza, "Bioimaging of trace metals in ancient Chilean mummies and contemporary Egyptian teeth by laser ablation-inductively coupled plasma-mass spectrometry (LA-ICP-MS)", Microchemical Journal, 106 (2013) 340-346, DOI: 10.1016/j.microc.2012.09.005.

[127] L. Bartkus, D. Amarasiriwardena, B. Arriaza, D. Bellis, J. Yanez, "Exploring lead exposure in ancient Chilean mummies using a single strand of hair by laser ablation-inductively coupled plasma-mass spectrometry (LA-ICP-MS)", Microchemical Journal, 98 (2011) 267-274, DOI: 10.1016/j.microc.2011.02.008.

[128] M. Willmes, L. Kinsley, M. H. Moncel, R. A. Armstrong, M. Aubert, S. Eggins, R. Grün, "Improvement of laser ablation in situ micro-analysis to identify diagenetic alteration and measure strontium isotope ratios in fossil human teeth", Journal of Archaeological Science, 70 (2016) 102-116, DOI: 10.1016/j.jas.2016.04.017.

[129] D. Barca, C. M. Belfiore, G. M. Crisci, M. F. La Russa, A. Pezzina, S. A. Ruffolo, "A new methodological approach for the chemical characterization of black crusts on building stones: a case study from the Catania city centre (Sicily, Italy)", Journal of Analytical Atomic Spectrometry, 26 (2011) 1000-1011, DOI: $10.1039 /$ C0JA00226G.

[130] L. Moreau, M. Brandl, P. Filzmoser, C. Hauzenberger, E. Goemaere, I. Jadin, H. Collet, A. Hauzeur, R. W. Schmitz, "Geochemical Sourcing of Flint Artifacts from Western Belgium and the German Rhineland: Testing Hypotheses on Gravettian Period Mobility and Raw Material Economy", Geoarchaeology: An International Journal, 31 (2016) 229-243, DOI: 10.1002/gea.21564. 
[131] S. A. Ruffolo, V. Comite, M. F. La Russa, C. M. Belfiore, D. Barca, A. Bonazza, G. M. Crisci, A. Pezzino, C. Sabbioni, "An analysis of the black crusts from the Seville Cathedral: A challenge to deepen the understanding of the relationships among microstructure, microchemical features and pollution sources", Science of the Total Enviroment, 502 (2015) 157-166, DOI: 10.1016/j.scitotenv.2014.09.023.

[132] A. A. Evans, Y. B. Wolframm, R. E. Donahue, W. A. Lovis, "The phytolith archaeological record: strengths and weaknesses evaluated based on a quantitative modern reference collection from Greece", Journal of Archaeological Science, 34 (2007) 2161-2169, DOI: 10.1016/j.jas.2006.10.017.

[133] D. Miriello, D. Barca, A. Pecci, R. de Luca, G. M. Crisci, "Plasters from different buildings of the sacred precinct of Tenochtitlan (Mexico City): characterization and provenance", Archaeometry, 57 (2015) 100-127, DOI: 10.1111/arcm.12074.

[134] F. Cattin, B. Guenette-Beck, P. Curdy, N. Meisser, S. Ansermet, B. Hofmann, R. Kuendig, V. Hubert, M. Woerle, K. Hametner, D. Guenther, A. Wichser, A. Ulrich, I. M. Villa, M. Besse, "Provenance of Early Bronze Age metal artefacts in Western Switzerland using elemental and lead isotopic compositions and their possible relation with copper minerals of the nearby Valais", Journal of Archaeological Science, 38 (2011) 1221-1233, DOI: 10.1016/j. jas.2010.12.016.

[135] V. Van der Linden, P. Cosyns, O. Schalm, S. Cagno, K. Nys, K. Janssens, A. Nowak, B. Wagner, E. Bulska, "Deeply coloured and black glass in the northern provinces of the Roman Empire: differences and similarities in chemical composition befora and after AD 150", Archaeometry, 51 (2009) 822-844, DOI: 10.1111/j.1475-4754.2008.00434.x.

[136] L. Dussubieux, in Archaeological Chemistry: Analytical Techniques and Archaeological Interpretation, M. D. Glascok, R. J. Speakman, R. S. Popelka Filcoff (Eds.), 968 (2007) 336-348.

[137] B. Wagner, A. Nowak, E. Bulska, K. Hametner, D. Guenther, "Critical assessment of the elemental composition of Corning archeological reference glasses by LA-ICP-MS", Analytical and Bioanalytical Chemistry, 402 (2012) 1667-1677, DOI: 10.1007/ s00216-011-5597-8.
[138] M. Pakiela, M. Wojciechowski, B. Wagner, E. Bulska, "A novel procedure of powdered samples immobilization and multi-point calibration of LA ICP MS", Journal of Analytical Atomic Spectrometry, 26 (2011) 1539-1543, DOI: 10.1039/C0JA00201A.

[139] P. Budd, J. Montgomery, A. Cox, P. Krause, B. Barreiro, R. G. Thomas, "The distribution of lead within ancient and modern human teeth: Implications for long-term and historical exposure monitoring", Science of Total Environment, 220 (1998) 121-136, DOI: 10.1016/S0048-9697(98)00244-7.

[140] D. Gunther, A. von Quadt, R. Wirz, H. Cousin, V. J. Dietrich, "Elemental Analyses Using Laser Ablation-Inductively Coupled Plasma-Mass Spectrometry (LA-ICP-MS) of Geological Samples Fused with Li2B4O7 and Calibrated Without Matrix-Matched Standards", Microchimica Acta, 136 (2001) 101-107, DOI: $10.1007 / \mathrm{s} 006040170038$.

[141] H. P. Longerich, S. E. Jackson, D. Gunther, "Laser Ablation Inductively Coupled Plasma Mass Spectrometric Transient Signal Data Acquisition and Analyte Concentration Calculation", Journal of Analytical Atomic Spectrometry, 11 (1996) 899-904, DOI: 10.1039/JA9961100899.

[142] B. Gratuze, "Obsidian Characterization by Laser Ablation ICP-MS and its Application to Prehistoric Trade in the Mediterranean and the Near East: Sources and Distribution of Obsidian within the Aegean and Anatolia", Journal of Archaeological Science, 26 (1999) 869-881, DOI: 10.1006/ jasc.1999.0459.

[143] Y. Liu, Z. Hu, S. Gao, D. Guenther, J. Xu, C. Gao, H. Chen, "In situ analysis of major and trace elements of anhydrous minerals by LA-ICP-MS without applying an internal standard", Chemical Geology, 257 (2008) 34-43, DOI: 10.1016/j.chemgeo.2008.08.004.

[144] D. Barca, A. M. De Francesco, G. M. Crisci, "Chemical and mineralogical examination of metallurgical ceramics from a Late Bronze Age copper smelting site in Cyprus", Journal of Cultural Heritage, 34 (2007) 141-150, DOI: 10.1016/j.jas.2006.04.005.

[145] M. I. Szynkowska, K. Czerski, T. Paryjczak, E. Rybicki, A. Wlochowicz, “Testing Textiles Using the LA-ICP-MS-TOF Method", Fibres \& Textiles in Eastern Europe, 14 (2006) 87-90. 
[146] G. Sarah, B. Gratuze, J.-N. Barrandon, "Application of laser ablation inductively coupled plasma mass spectrometry (LA-ICP-MS) for the investigation of ancient silver coins", Journal of Analytical Atomic Spectrometry, 22 (2007) 1163-1167, DOI: 10.1039/B704879C.

[147] V. S. Selih, J. T. van Elteren, "Quantitative multi-element mapping of ancient glass using a simple and robust LA-ICP-MS rastering procedure in combination with image analysis", Analytical and Bioanalytical Chemistry, 401 (2011) 745-755, DOI: 10.1007/s00216-011-5119-8.

[148] M. Bertini, A. Izmer, F. Vanhaecke, E. M. Krupp, "Critical evaluation of quantitative methods for the multi-elemental analysis of ancient glasses using laser ablation inductively coupled plasma mass spectrometry", Journal of Analytical Atomic Spectrometry, 28 (2013) 77-91, DOI: 10.1039/C2JA30036B.

[149] D. Walaszek, M. Senn, M. Faller, L. Philippe, B. Wagner, E. Bulska, A. Ulrich, "Metallurgical and chemical characterization of copper alloy reference materials within laser ablation inductively coupled plasma mass spectrometry: method development for minimally-invasive analysis of ancient bronze objects", Spectrochimica Acta Part B, 79-80 (2013) 17-30, DOI: 10.1016/j.sab.2012.11.001.

[150] M. S. Walton, A. Shortland, S. Kirk, P. Degryse, "Evidence for the trade of Mesopotamian and Egyptian glass to Mycenaean Greece", Journal of Archaeological Science, 36 (2009) 1496-1503, DOI: 10.1016/j.jas.2009.02.012.

[151] J. Wouters, "Protecting Cultural Heritage. Reflections on the Position of Science in Multidisciplinary Approaches", Chemistry International, 30 (2008) 4-8, https://www.iupac.org/publications/ ci/2008/3001/1_wouters.html (accessed 14.10.2016).

[152] K. Smith, K. Horton, R. J. Watling, N. Scoullar, "Detecting art forgeries using LA-ICP-MS incorporating the in situ application of laser-based collection technology", Talanta, 67 (2005) 402-413, DOI: 10.1016/j.talanta.2005.06.030.

[153] P. Arrowsmith, S. K. Hughes, "Entrainment and Transport of Laser Ablated Plumes for Subsequent Elemental Analysis", Applied Spectroscopy, 42 (1988) 1231-1239, DOI: 10.1366/0003702884430100.
[154] C. C. Garcia, H. Lindner, K. Niemax, "Transport efficiency in femtosecond laser ablation inductively coupled plasma mass spectrometry applying ablation cells with short and long washout times", Spectrochimica Acta Part B, 62 (2007) 13-19, DOI: 10.1016/j.sab.2006.11.005.

[155] D. Autrique, A. Bogaerts, H. Lindner, C. C. Garcia, K. Niemax, "Design analysis of a laser ablation cell for inductively coupled plasma mass spectrometry by numerical simulation", Spectrochimica Acta Part B, 63 (2008) 257-270, DOI: 10.1016/j. sab.2007.11.032.

[156] J. Pisonero, D. Fliegel, D. Guenther, "High efficiency aerosol dispersion cell for laser ablation-ICP-MS", Journal of Analytical Atomic Spectrometry, 21 (2006) 922-931, DOI: 10.1039/ B603867K.

[157] E. Marin, A. Padró, A. Miquel, J. F. Garcia, "Characterization of Paintings by Laser Ablation-Inductively Coupled Plasma-Mass Spectrometry", Analytical Letters, 48 (2015) 167-179, DOI: 10.1080/00032719.2014.921823.

[158] M. Aubert, I. S. Williams, K. Boljkovac, I. Moffat, M.-H. Moncel, E. Dufour, R. Gruen, "In situ oxygen isotope micro-analysis of faunal material and human teeth using a SHRIMP II: a new tool for palaeo-ecology and archaeology", Journal of Archaeological Science, 39 (2012) 3184-3194, DOI: 10.1016/j.jas.2012.05.002.

[159] A. Simonetti, M. R. Buzon, R. A. Creaser, "Insitu elemental $\mathrm{Sr}$ isotope investigation of human tooth enamel by laser ablation-(MC)-ICP-MS: successes and pitfalls", Archaeometry, 50 (2008) 371-385, DOI: 10.1111/j.1475-4754.2007.00351.x.

[160] C. L. King, N. Tayles, K. C. Gordon, "Re-examining the chemical evaluation of diagenesis in human bone apatite", Journal of Archaeological Science, 38 (2011) 2222-2230, DOI: 10.1016/j.jas.2011.03.023.

[161] M. Gil, R. Green, M. L. Carvalho, A. Seruya, I. Queralt, A. E. Candeias, J. Mirao, "Rediscovering the palette of Alentejo (Southern Portugal) earth pigments: provenance establishment and characterization by LA-ICP-MS and spectra-colorimetric analysis", Applied Physics A, 96 (2009) 997-1007, DOI: $10.1007 / \mathrm{s} 00339-009-5134-1$. 
[162] K. Polikreti, C. Christofides, "Composition profiles and time evolution of buried marble patinas by LA-ICP-MS and laser-induced micro-photo-luminescence", Archaeometry, 52 (2010) 937-948, DOI: 10.1111/j.1475-4754.2010.00531.x.

[163] J. Baker, S. Stos, T. Waight, "Lead isotope analysis of archaeological metals by multiple-collector inductively coupled plasma mass spectrometry", Archaeometry, 48 (2006) 45-56, DOI: 10.1111/j.14754754.2006.00242.x.

[164] M. Ponting, J. A. Evans, V. Pashley, "Fingerprinting of Roman mints using laser-ablation MC-ICP-MS lead isotope analysis", Archaeometry, 45 (2003) 591-597, DOI: 10.1046/j.14754754.2003.00130.x.

[165] T. Carter, G. Poupeau, C. Bressy, N. J. G. Pearce, "A new programme of obsidian characterization at Catalhoyuk, Turkey" Journal of Archaeological Science, 33 (2006) 893-909, DOI: 10.1016/j. jas.2005.10.023.

[166] S. Panighello, E. F. Orsega, J. T. van Elteren, V.S.Selih, "Analysis of polychrome Iron Age glass vessels from Mediterranean I, II and III groups by LA-ICP-MS", Journal of Archaeological Science, 39 (2012) 2945-2955, DOI: 10.1016/j.jas.2012.04.043.

[167] N. Schibille, "Late Byzantine Mineral Soda High Alumina Glasses from Asia Minor: A New Primary Glass Production Group", Plos One, 6 (2011), DOI: 10.1371/journal.pone.0018970.

[168] Y. Su, L. Qu, H. Duan, N. Tarcea, A. Shen, J. Popp, J. Hu, "Elemental analysis-aided Raman spectroscopic studies on Chinese cloisonné wares and painted enamels from the Imperial Palace", Spectrochimica Acta Part A, 153 (2016) 165-170, DOI: 10.1016/j.saa.2015.08.005.

[169] M. Walton, K. Eremin, A. Shortland, P. Degryse, S. Kirk, "Analysis of late Bronze Age glass axes from Nippur - a new cobalt colourant", Archaeometry, 54 (2012) 835-852, DOI: 10.1111/j.14754754.2012.00664.x.

[170] S. Schlosser, A. Reinecke, R. Schwab, E. Pernicka, S. Sonetra, V. Laychour, "Early Cambodian gold and silver from Prohear: composition, trace elements and gilding", Journal of Archaeological Science, 39 (2012) 2877-2887, DOI: 10.1016/j.jas.2012.04.045.
[171] B. Arriaza, D. Amarasiriwardena, L. Cornejo, V. Standen, S. Byrne, L. Bartkus, B. Bandak, "Exploring chronic arsenic poisoning in pre-Columbian Chilean mummies", Journal of Archaeological Science, 37 (2010) 1274-1278, DOI: 10.1016/j.jas.2009.12.030.

[172] S. Byrne, D. Amarasiriwardena, B. Bandak, L. Bartkus, J. Kane, J. Jones, J. Yanez, B. Arriaza, L. Cornejo, "Were Chinchorros exposed to arsenic? Arsenic determination in Chinchorro mummies' hair by laser ablation inductively coupled plasma-mass spectrometry (LA-ICP-MS)", Microchemical Journal, 94 (2010) 28-35, DOI: 10.1016/j.microc.2009.08.006.

[173] S. R. Copeland, M. Sponheimer, J. A. LeeThorp, P. J. le Roux, D. J. de Ruiter, M. P. Richards, "Strontium isotope ratios in fossil teeth from South Africa: assessing laser ablation MC-ICP-MS analysis and the extent of diagenesis", Journal of Archaeological Science, 37 (2010) 1437-1446, DOI: 10.1016/j. jas.2010.01.003.

[174] O. Syta, K. Rozum, M. Choińska, D. Zielińska, G. Z. Żukowska, A. Kijowska, B. Wagner, "Analytical procedure for characterization of medieval wall-paintings by X-ray fluorescence spectrometry, laser ablation inductively coupled plasma mass spectrometry and Raman spectroscopy", Spectrochimica Acta Part B, 101 (2014) 140-148, DOI: 10.1016/j. sab.2014.08.003.

[175] D. Asogan, B. L. Sharp, C. J. P. O'Connor, D. A. Green, R. W. Hutchinson, "An open, non-contact cell for laser ablation-inductively coupled plasma-mass spectrometry", Journal of Analytical Atomic Spectrometry, 24 (2009) 917-923, DOI: 10.1039/B904850B.

[176] M. B. Fricker, D. Kutscher, B. Aeschlimann, J. Frommer, R. Dietiker, J. Bettmer, D. Guenther, "High spatial resolution trace element analysis by LA-ICP-MS using a novel ablation cell for multiple or large samples", International Journal of Mass Spectrometry, 307 (2011) 39-45, DOI: 10.1016/j. ijms.2011.01.008.

[177] R. Glaus, J. Koch, D. Guenther, "Portable Laser Ablation Sampling Device for Elemental Fingerprinting of Objects Outside the Laboratory with Laser Ablation Inductively Coupled Plasma Mass Spectrometry", Analytical Chemistry, 84 (2012) 5358-5364, DOI: 10.1021/ac3008626. 
[178] L. Dorta, R. Kovacs, J. Koch, K. Nishiguchi, K. Utani, D. Günther, "Determining isotope ratios using laser ablation sampling in air with MC-ICPMS", Journal of Analytical Atomic Spectrometry, 28 (2013) 1513-1521, DOI: 10.1039/C3JA50126D.

[179] D. Barca, D. Miriello, A. Pecci, L. Barba, A. Ortiz, L. R. Manzanilla, J. Blancas, G. M. Crisci, "Provenance of glass shards in archaeological lime plasters by LA-ICP-MS: implications for the ancient routes from the Gulf of Mexico to Teotihuacan in Central Mexico", Journal of Archaeological Science, 40 (2013) 3999-4008, DOI: 10.1016/j. jas.2013.05.016.

[180] J. T. van Elteren, A. Izmer, M. Sala, E. F. Orsega, V.S. Selih, S. Panighello, F. Vanhaecke, "3D laser ablation-ICP-mass spectrometry mapping for the study of surface layer phenomena - a case study for weathered glass", Journal of Analytical Atomic Spectrometry, 28 (2013) 994-1004, DOI: 10.1039/ c3ja30362d.

[181] S. Panighello, E. F. Orsega, J. T. van Elteren, V.S. Selih, "Analysis of polychrome Iron Age glass vessels from Mediterranean I, II and III groups by LA-ICP-MS”, Journal of Archaeological Science, 39 (2012) 2945-2955, DOI: 10.1016/j.jas.2012.04.043.

[182] D. Barca, E. Basso, D. Bersani, G. Galli, C. Invernizzi, M. F. La Russa, P. P. Lottici, M. Malagodi, S. A. Ruffolo, "Vitreous tesserae from the calidarium mosaics of the Villa dei Quintili, Rome. Chemical composition and production technology", Microchemical Journal, 124 (2016) 726-735, DOI: 10.1016/j.microc.2015.10.037.

[183] W. Devos, M. Senn-Luder, C. Moor, C. Salter, "Laser ablation inductively coupled plasma mass spectrometry (LA-ICP-MS) for spatially resolved trace analysis of early-medieval archaeological iron finds", Fresenius Journal of Analytical Chemistry, 366 (2000) 873-880, DOI: 10.1007/s002160051588.

[184] T. Purowski, P. Dzierzanowski, E. Bulska, B. Wagner, A. Nowak, "A study of glass beads from the Hallstatt C-D from southwestern Poland: implications for glass technology and provenance", Archaeometry, 54 (2012) 144-166, DOI: 10.1111/j.1475-4754.2011.00619.x.

[185] J. Henderson, S. Chenery, E. Faber, J. Kröger, "The use of electron probe microanalysis and laser ablation-inductively coupled plasma-mass spectrometry for the investigation of $8^{\text {th }}-14^{\text {th }}$ century plant ash glasses from the Middle East", Microchemical Journal, 128 (2016) 134-152, DOI: 10.1016/j.microc.2016.03.013.

[186] R. Bugoi, B. Constantinescu, C. Neelmeijer, F. Constantin, "The potential of external IBA and LA-ICP-MS for obsidian elemental characterization", Nuclear Instruments \& Methods in Physics Research Section B, 226 (2004) 136-146, DOI: 10.1016/j. nimb.2004.04.185.

[187] Z. Šmit, T. Milavec, H. Fajfar, T. Rehren, J.W. Lankton, B. Gratuze, "Analysis of glass from the post-Roman settlement Tonovcov gra (Slovenia) by PIXE-PIGE and LA-ICP-MS", Nuclear Instruments and Methods in Physics Research B, 311 (2013) 53-59, DOI: 10.1016/j.nimb.2013.06.012.

[188] C. M. Sinopoli, Dueppen, S., Brubaker, R., Descantes, C., Glascock, M.D., Griffin, W., Neff, H., Shoocongdej, R., Speakman, R.J., "Characterizing the Stoneware 'Dragon Jars' in the Guthe Collection: Chemical, Decorative, and Formal Patterning", Asian Perspectives, 45 (2006) 240-282, DOI: 10.1353/ asi.2006.0026.

[189] A. B. Stahl, M. d. D. Cruz, H. Neff, M. D. Glascock, R. J. Speakman, B. Giles, L. Smith, "Ceramic production, consumption and exchange in the Banda area, Ghana: Insights from compositional analyses", Journal of Anthropological Archaeology, 27 (2008) 363-381, DOI: 10.1016/j.jaa.2008.04.001.

[190] R. Liu, H. Zhou, L. Zhang, Z. Zhong, W. Zeng, H. Xiang, S. Jin, X. Lu, C. Li, "Paleoproterozoic reworking of ancient crust in the Cathaysia Block, South China: Evidence from zircon trace elements, $\mathrm{U}-\mathrm{Pb}$ and $\mathrm{Lu}-\mathrm{Hf}$ isotopes", Chinese Science Bulletin, 54 (2009) 1543-1554, DOI: 10.1007/s11434-0090096-4.

[191] M. F. Guerra, "The circulation of South American precious metals in Brazil at the end of the $17^{\text {th }}$ century", Journal of Archaeological Science, 31 (2004) 1225-1236, DOI: 10.1016/j.jas.2004.03.018.

[192] L. G. Cecil, "Central Peten blue pigment: A maya blue source outside of Yucatan, Mexico", Journal of Archaeological Science, 37 (2010) 1006-1019, DOI: 10.1016/j.jas.2009.12.001. 
[193] S. Leroy, R. Simon, L. Bertrand, A. Williams, E. Foy, P. Dillmann, "First examination of slag inclusions in medieval armours by confocal SR- $\mu$ XRF and LA-ICP-MS", Journal of Analytical Atomic Spectrometry, 26 (2011) 1078-1087, DOI: 10.1039/ C0JA00261E.

[194] C. L. King, N. Tayles, K. C. Gordon, "Re-examining the chemical evaluation of diagenesis in human bone apatite", Journal of Archaeological Science, 38 (2011) 2222-2230, DOI: 10.1016/j.jas.2011.03.023.

[195] L. Rampazzi, B. Rizzo, C. Colombo, C. Conti, M. Realini, U. Bartolucci, M. P. Colombini, A. Spiriti,
L. Facchin, "The stucco decorations from St. Lorenzo in Laino (Como, Italy): The materials and the techniques employed by the "Magistri Comacini"', Analytica Chimica Acta, 630 (2008) 91-100, DOI: 10.1016/j.aca.2008.09.052.

[196] R. Glaus, L. Dorta, Z. Zhang,Q. Ma, H. Berke, D. Günther, "Isotope ratio determination of objects in the field by portable laser ablation sampling and subsequent multicollector ICPMS", Journal of Analytical Atomic Spectrometry, 28 (2013) 801-809, DOI: 10.1039/C3JA30379A. 


\title{
A preliminary study of contemporary binders by Time Resolved Laser Induced Fluorescence (TR-LIF) spectroscopy: characterization of the painting Nascita Della Forma by Nato Frascà
}

\author{
Martina Romani ${ }^{\star}$, Marco Marinelli, Alessandra Pasqualucci, \\ Gianluca Verona-Rinati \\ INFN-Department of Industrial Engineering, University of Rome "Tor Vergata", Via del Politecnico 1, \\ 00133, Rome, Italy \\ * Corresponding author: martina.romani@uniroma2.it
}

\begin{abstract}
In this work, an original study of contemporary binders by Time Resolved Induced Fluorescence Spectroscopy (TR-LIF) is presented. Specifically, fluorescence emission spectra of three classes of binders, commonly employed in contemporary artworks, such as acrylic, vinyl and natural ones, were investigated. The obtained results allow identifying each family through the study of their characteristic emission wavelengths and decay times. Finally, the capability of the TR-LIF technique was tested on a real artwork: Nascita Della Forma (Birth of the Form) by Nato Frascà (1962), housed in the MACRO-Museo d'Arte Contemporanea di Roma (Museum of Contemporary Art in Rome). The above-mentioned painting is characterized by several craquelures, probably due to the overlapping of materials with different chemical and physical properties. The spectra acquired on the selected painting are compared to the ones recorded from laboratory samples, in order to characterize the binders used by the artist.
\end{abstract}

Keywords: contemporary artworks, contemporary binders, Laser Induced Fluorescence Spectroscopy, Time Resolved Laser Induced Fluorescence Spectroscopy, UV Fluorescence Imaging Analysis

\section{Introduction}

A major problem encountered in the conservation of contemporary paintings is the identification of their material composition. This is due to the lack of information provided by the artists in this respect [1]. Indeed, during the $19^{\text {th }}$ century, several new synthetic materials became available on the market, often mixed in tubes and characterized by unknown compositions [2]. Among all these products, acrylic and vinyl polymers probably represent the most used binders in contemporary paintings due to their advantages of fast drying, high resistance and easy coating [3]. However, despite the great popularity of such polymeric binders, many artists still preferred to use the traditional ones, as linseed oil or dammar varnish, and it was usual to find these binders employed 
together with the synthetic ones [4-6]. The use of different components at the same time often results in common damages on the painted surface, such as craquelures, i.e. a traditional degradation of acrylic paint on an oil one [4]. As a consequence, the binder identification is mandatory in order to plan appropriate conservation and restoration strategies.

Laser Induced Fluorescence (LIF) is a non-destructive technique adopted in the investigation, characterization and preservation of cultural heritage thanks to its peculiar advantages of high sensitivity, non-invasiveness and prompt response $[7,8]$. In particular, after an absorption of UV radiation, such a technique allows recording characteristic emissions produced by luminescent materials, thus providing compositional information about the principal components of the pictorial layers [9-11]. However, a limit of LIF analysis shows up when a mix of multi-material samples is investigated, making it difficult to assign each spectral signatures to specific components. Moreover, synthetic binders often present intense fluorescence bands that could cover possible weak emissions from other compounds [12]. Therefore, the emission wavelength alone is not enough to obtain a complete characterization of the analyzed materials.

In this work, the use of the Time Resolved Laser Induced Fluorescence spectroscopy (TR-LIF) is proposed to overcome the above-mentioned issue. Such a technique allows discriminating among emissions from different compounds and analyzing the time evolution of their fluorescence spectra. In particular, in TR-LIF the characterization of binders is performed by studying their typical emission wavelengths and decay times [13-16]. The experimental results confirm the capability to isolate specific contribu- tions from the investigated constituents by a TR-LIF analysis.

In order to verify the reliability of our laboratory results, the painting named Nascita Della Forma (Birth of the Form) by Nato Frascà (1962), hosted in MACRO-Museo d'Arte Contemporanea di Roma (Museum of Contemporary Art in Rome), was analyzed. The picture, in well-defined sections of its surface, is characterized by several craquelures, probably due to the use of different mixtures selected by the artist himself. The results obtained in the present study allowed updating the technical documentation about the artwork and giving useful information to restorers in order to adopt proper conservation and restoration strategies.

\section{Materials and methods}

\subsection{Materials}

\section{Laboratory samples}

In this work, nine synthetic and natural binders are investigated. They were chosen for their common employ in contemporary artworks as binding media [17]. As reported in Table 1 , they are divided in three different classes, according to their chemical composition:

- Acrylic resins: Primal B60-A, Acrytal C12, Primal AC 35, Plextol D 498;

- Vinyl resins: Vinavil, Vinyl Glue, Vinil Pritt;

- Natural binder: linseed oil, dammar varnish.

Each binder was applied on a black cardboard support to perform a preliminary study. Such support was chosen in order to isolate the spectral behavior of pure binders. This is because the negligible fluorescence emission of the cardboard and the low 
penetration depth of the UV radiation allow performing a superficial analysis of the pictorial layers, excluding any contribution arising from the support itself [18].

\section{The painting Nascita Della Forma}

The capability of the TR-LIF technique was tested on the painting Nascita Della Forma (Birth of the Form) by Nato Frascà, 1962, preserved at MACRO (Fig. 1). This work of art was realized in 1962 in Palermo (Sicily), before the establishment of Gruppo 1, by Frascà and other artists $[19,20]$. The above-mentioned painting is classified by the artist himself as "oil on canvas", as reported in the technical documentation of the museum. However, the pictorial layer is actually characterized by several craquelures, a typical degradation caused by the overlapping of different binders (Fig. 2). In particular, such a degradation is observable in two specific areas, corresponding to the orange and light blue pigments. Restorers supposed that these

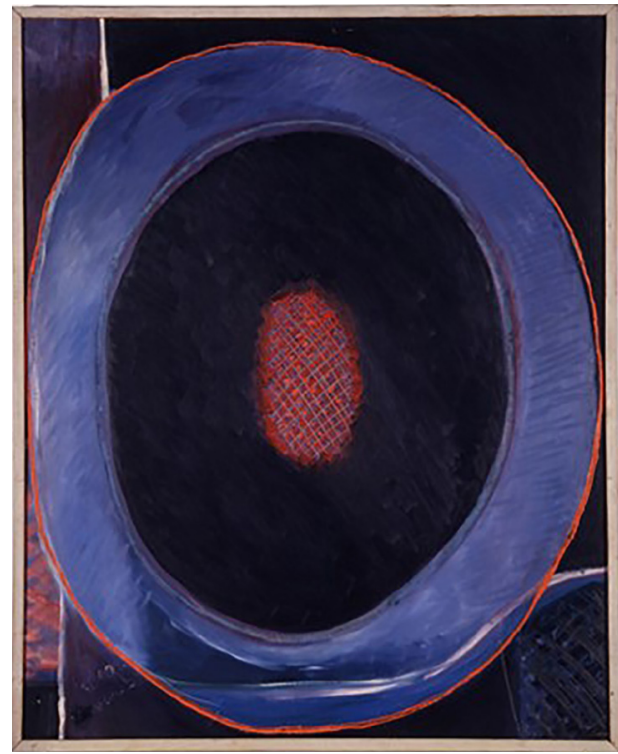

Fig. 1. Nato Frascà, Nascita Della Forma (Birth of the Form), 1962, MACRO.

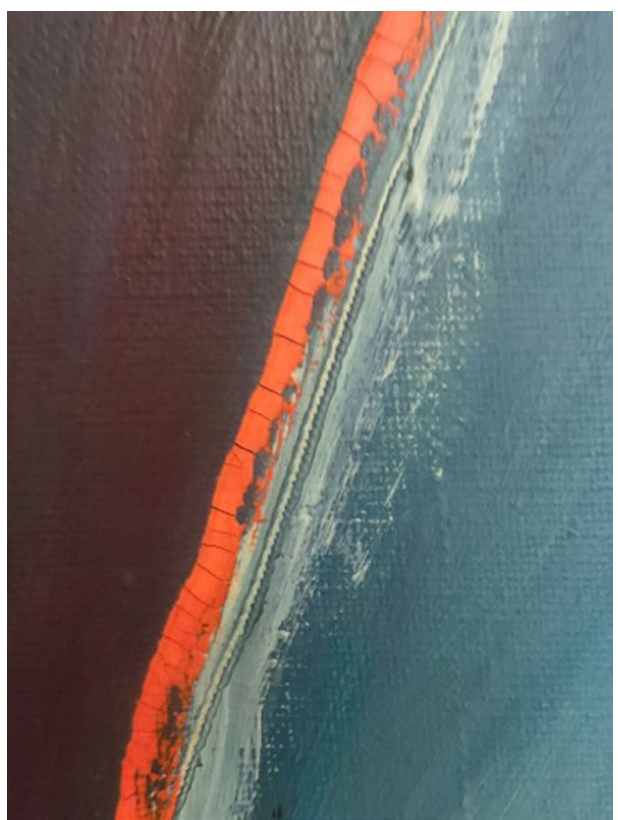

Fig. 2. Nato Frascà, Nascita Della Forma (Birth of the Form), detail of craquelures.

pigments were probably combined with a different binder as compared to the one chosen for the other mixtures used in the painting. Indeed, an aim of this work is to characterize such a binder, which might be responsible of the observed surface degradation.

\subsection{Methods}

\section{TR-LIF set up}

The TR-LIF experimental set up (Fig. 3) allows the acquisition of fluorescence spectra emitted during a well-defined time window with respect to the laser pulse. In particular, both the gate delay (i.e. delay time from the laser pulse and the acquisition start) and the gate window (i.e. aperture time) can be properly selected with a resolution of few ns.

In this work, emission spectra were acquired by using $220 \mathrm{~nm}$ as excitation wavelength, with an average laser pulse energy of 
Table 1. Summary of the investigated samples and TR-LIF analysis results: emission peak wavelengths and estimated decay times

\begin{tabular}{|c|c|c|c|c|}
\hline Class & Sample & Description & LIF analysis: no delay & LIF analysis: $30 \mathrm{~ns}$ delay \\
\hline \multirow{4}{*}{ 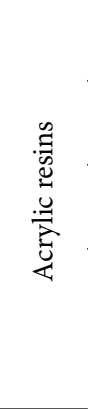 } & $\begin{array}{l}\text { Primal } \\
\text { B60-A }\end{array}$ & $\begin{array}{l}\text { Ethylacrilate-co-methacrylate } \\
\text { made by Rhom and Has }\end{array}$ & $\begin{array}{l}280 \mathrm{~nm} \text {; intermediate } \\
294 \mathrm{~nm} \text {; fast }\end{array}$ & $280 \mathrm{~nm}$; intermediate \\
\hline & $\begin{array}{l}\text { Acrytal } \\
\text { C12 }\end{array}$ & $\begin{array}{l}\text { Aqueous acrylic resin dispersion } \\
\text { made by Iridon }\end{array}$ & $280 \mathrm{~nm}$ : intermediate & $280 \mathrm{~nm}$; intermediate \\
\hline & $\begin{array}{l}\text { Primal } \\
\text { AC } 35\end{array}$ & $\begin{array}{l}\text { Methylmethacrylate made by } \\
\text { Kremer Pigmente }\end{array}$ & $280 \mathrm{~nm}$; intermediate & 310-350: slow \\
\hline & $\begin{array}{l}\text { Plextol } \\
\text { D498 }\end{array}$ & $\begin{array}{l}\text { Aqueous pure acrylic dispersion } \\
\text { of a copolymer based on ethyl } \\
\text { acrylate and methyl methacrylate } \\
\text { by Kremer Pigmente }\end{array}$ & $280 \mathrm{~nm}$; intermediate & $310-350 \mathrm{~nm}$; slow \\
\hline \multirow{3}{*}{ 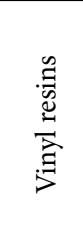 } & Vinavil & Vinyl Acetate by Vinavil & $\begin{array}{l}280 \mathrm{~nm} \text { : intermediate } \\
320-360 \mathrm{~nm} \text { : slow }\end{array}$ & $\begin{array}{l}280 \mathrm{~nm} \text { : intermediate } \\
320-360 \mathrm{~nm} \text {; slow }\end{array}$ \\
\hline & Vinyl Glue & $\begin{array}{l}\text { Emulsion of polyvinyl acetate and } \\
\text { polyvinyl alcohol }\end{array}$ & $\begin{array}{l}280 \mathrm{~nm} \text {; intermediate } \\
320-360 \mathrm{~nm} \text {; slow }\end{array}$ & $\begin{array}{l}280 \mathrm{~nm} \text {; intermediate } \\
320-360 \mathrm{~nm} \text { : slow }\end{array}$ \\
\hline & Vinil Pritt & $\begin{array}{l}\text { Ethylene-vinyl acetate (EVA) by } \\
\text { Henkel Italia (Milan, Italy) }\end{array}$ & $\begin{array}{l}280 \mathrm{~nm} \text {; intermediate } \\
320-360 \mathrm{~nm} \text {; slow }\end{array}$ & $\begin{array}{l}280 \mathrm{~nm} \text {; intermediate } \\
320-360 \mathrm{~nm} \text {; slow }\end{array}$ \\
\hline \multirow{2}{*}{ 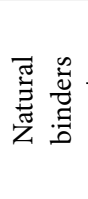 } & Linseed oil & $\begin{array}{l}\text { Refined linen seed extract by } \\
\text { Maimeri (Milan, Italy) }\end{array}$ & $270-310 \mathrm{~nm}$ : fast & - \\
\hline & $\begin{array}{l}\text { Dammar } \\
\text { varnish }\end{array}$ & $\begin{array}{l}\text { Natural dammar resin with } \\
\text { turpentine by Maimeri (Milan, } \\
\text { Italy) }\end{array}$ & $270-310 \mathrm{~nm}$; fast & - \\
\hline
\end{tabular}

about $3 \mu \mathrm{J}$ and a $20 \mathrm{~Hz}$ repetition rate. Such a laser wavelength was chosen because the emission of the studied binders exhibit characteristic features in the $250-360 \mathrm{~nm}$ spectral region [21], which can be fully appreciated by using a shorter excitation wavelength. On the contrary, longer wavelengths would result in a partial loss of spectral information from the investigated materials. The laser beam is focused on the sample surface with a spot size about $0.3 \times 1 \mathrm{~mm}^{2}$ (Fig. 3). For each sample two spectra were acquired. The first one was taken immediately after the laser pulse (no delay), while the other with a 30 ns delay ( 30 ns delay). In both cases, 20 ns gate window was used. In order to achieve a good statistic, each acquired spectrum was integrated over 2000 laser pulses. The above gate window and delay time were selected after preliminary TR-LIF measurements, performed to assess the typical time evolution of the investigated samples emission spectra. A rough estimation of the decay times was then calculated, as reported in the following (see section 3). However, it should be pointed out that this work can be considered a preliminary study about common contemporary binders, so that only a qualitative classification of their decay times is reported. For this reason, a more detailed characterization of the samples is recommended to achieve a comprehensive database and further studies are currently ongoing in our laboratory.

It is worth noting that the TR-LIF results reported in the present work were obtained by a bench setup facility available in our 


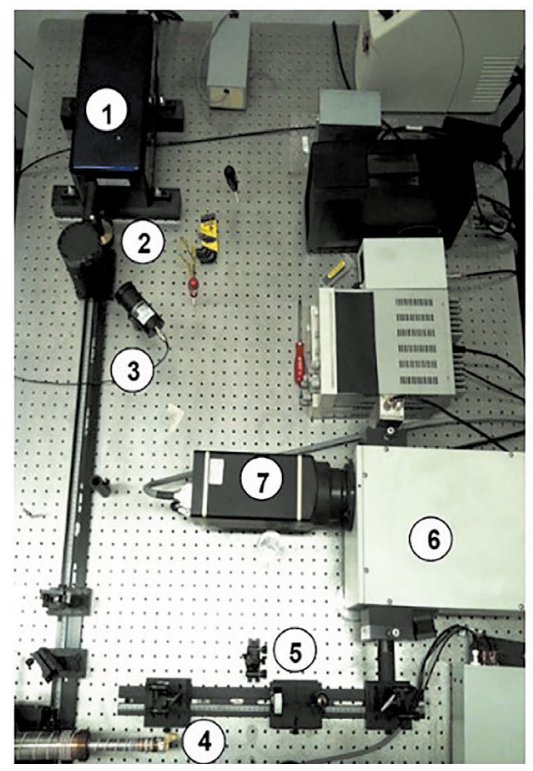

\begin{tabular}{|l|l|}
\hline Excitation wavelength & $220 \mathrm{~nm}$ \\
\hline Duration of the laser pulse & $3 \mathrm{~ns}$ \\
\hline Energy & $3 \mu \mathrm{J}$ \\
\hline Investigated area & $0.3 \times 1 \mathrm{~mm}^{2}$ \\
\hline Repetition rate & $20 \mathrm{~Hz}$ \\
\hline Laser fluence & $1 \mathrm{~mJ} / \mathrm{cm}^{2}$ pulse \\
\hline Gate aperture time & $20 \mathrm{~ns}$ \\
\hline
\end{tabular}

Fig. 3. TR-LIF experimental set-up and measurements conditions employed in this work: (1) $210-2400 \mathrm{~nm}$ optical parametric Oscillator Nd:YAG tunable laser, producing approximately 3 ns wide pulses (Opolette laser by Opotek); (2) Beam splitter; (3) Pyrometer for beam intensity monitoring; (4) Sample holder; (5) Off-axis parabolic mirrors for beam focalization and photoluminescence light recoil; (6) Spectrometer (SpectraPro 3001, Roper Scientific); (7) PI-MAX Roper Scientific gated multi-channel-plate/ CCD camera.

lab. A new portable setup is currently being realized by our group, so to allow in situ characterization of artworks.

\section{UV Fluorescence Imaging Analysis System}

Ultraviolet (UV) fluorescence imaging analysis was performed by using a NIR-converted Nikon D7100 camera with a Silicon sensor (Fig. 4). The UV fluorescence images were acquired by illuminating the painted surface with an UV lamp, characterized by an excitation wavelength of about $400 \mathrm{~nm}$. When recording images, the camera was equipped with two superimposed filters: a long pass filter at $500 \mathrm{~nm}$ and an infrared (IR) cut filter at $680 \mathrm{~nm}$. The above-mentioned filters system allows excluding any possible interference from the UV and IR regions respectively during the acquisitions [22].

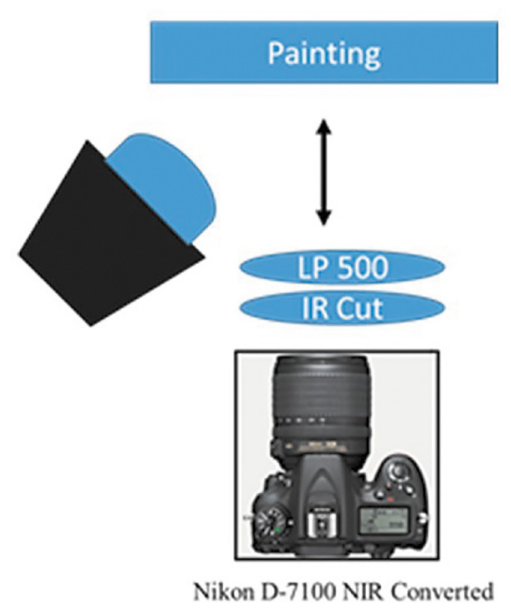

Fig. 4. UV Fluorescence imaging analysis system: Nikon D7100-NIR converted; sensor type: Si; spectral band: 370-1100nm; resolution: 24.1 MegaPixel; UV lamp centered at about $400 \mathrm{~nm}$; filters LP 500 and IR Cut. 


\section{Results}

As mentioned above, the TR-LIF spectra of each analyzed sample were measured in order to study the characteristic fluorescence emissions and decay times from specific binders. The duration of the gate aperture and delay time were chosen after preliminary TR-LIF measurements to investigate the typical time evolution of the studied emissions. The decay time is estimated for each fluorescence band as:

$$
\tau=\frac{\left(\mathrm{t}_{2}-\mathrm{t}_{1}\right)}{\ln \left(I_{1} / \mathrm{I}_{2}\right)}
$$

where and represent the recorded light powers integrated in the time intervals (; $+\Delta \mathrm{t})$ and $(;+\Delta \mathrm{t})$, respectively. It is worth highlighting that this equation is valid only for single-exponential decay and it is used to obtain a rough estimation of the decay times of the investigated sample. However, as mentioned in section 2.2.1, the spectra acquired with no delay and $30 \mathrm{~nm}$ delay can be considered sufficient to obtain such a qualitative estimation. Specifically, the emission decay times are classified in three different categories: fast, intermediate and slow. In particular, the decay time is considered fast if it is shorter than $9 \mathrm{~ns}$, intermediate if it is included between 9 and $15 \mathrm{~ns}$ and slow if it is longer than $15 \mathrm{~ns}$.

It should be pointed out that some spectra reported below and acquired with no delay showed a relatively weak peak at $440 \mathrm{~nm}$. This contribution is due to the laser source, which was not totally filtered out. In addition, a large emission band between $400-500 \mathrm{~nm}$ is observed for all the analyzed samples. However, similar bands are already reported in literature for several organic compounds, so that they cannot be considered as distinctive signatures of the investigated materials
[23]. For the above reasons, both spectral features will not be discussed in detail in the following part of the text.

\subsection{Laboratory results}

The emission of the cardboard support was studied at first in order to exclude any significant contribution during the fluorescence acquisitions. In Fig. 5, the spectra obtained

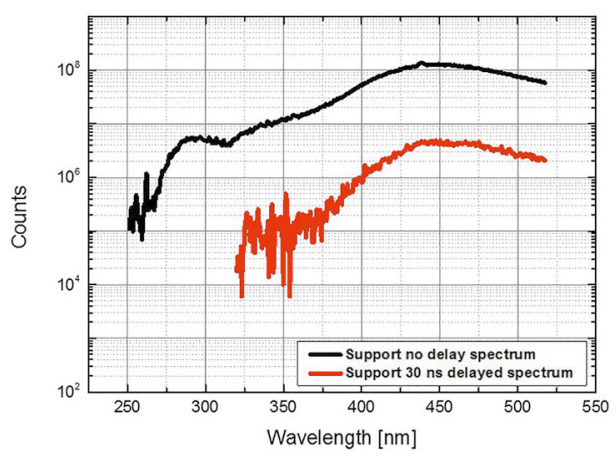

Fig. 5. TR-LIF cardboard support spectra: no delay spectrum (black line), $30 \mathrm{~ns}$ delay spectrum (red line).

from the cardboard are shown: the black line corresponds to spectrum acquired immediately after the laser pulse, no delay spectrum, while the red line refers to the one recorded after $30 \mathrm{~ns}, 30 \mathrm{~ns}$ delayed spectrum. In the former curve (no delay) it is possible to appreciate a weak and wide band at about $270-300 \mathrm{~nm}$. Such an emission is not present in the delayed spectrum, because of its fast decay time, which is shorter than 9 ns. Moreover, a large band between $400-500 \mathrm{~nm}$ is also observed, as previously discussed [23]. The cardboard contribution can thus be considered as negligible due to the lack of meaningful emissions in the UV region, where the contemporary binders fluorescence emission is usually expected [24]. 
In Fig. 6, all the spectral features obtained for the four analyzed acrylic binders are reported altogether. The most intense fluorescence emission is observed for the Primal B60-A (black line), showing two bands in the no delay spectrum, centered at about $280 \mathrm{~nm}$ and at $294 \mathrm{~nm}$ respectively. However, in the delayed spectrum (Fig. 6B; black line), the latter emission shows a fast reduction of its fluorescence intensity (approximately one order of magnitude in $20 \mathrm{~ns}$ ) and a drift of the peak position towards shorter wavelength. This behavior could be explained considering two superimposed spectral contributions centered at $280 \mathrm{~nm}$ and $294 \mathrm{~nm}$ respectively, the second one being characterized by a faster decay time.

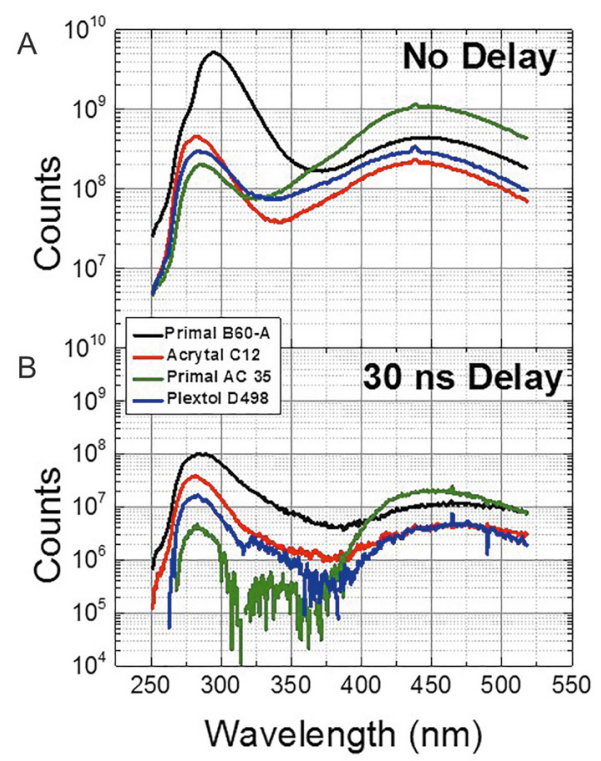

Fig. 6. TR-LIF spectra of the analyzed acrylic binders: Primal B60-A (black line), Acrytal C12 (red line), Primal AC 35 (green line), Plextol D498 (blue line).

The same emission centered at $280 \mathrm{~nm}$, already evident for the Primal B60-A and characterized by an intermediate decay time, is observed in the other three analyzed acrylic binders as well: Acrytal C12 (red lines), Primal AC35 (green lines) and Plextol D498 (blue lines). In addition, the Primal AC35 and the Plextol D498 spectra show a further band between $310-350 \mathrm{~nm}$, only appreciable in the delayed spectra due to its slow decay time. These different behaviors of Primal B60-A (the fast emission at 294 nm), Primal AC35 and Plextol D498 (the slow emission at $310-350 \mathrm{~nm}$ ) are probably due to several compounds possibly contained into the binders themselves. Indeed, in Fig. 6 it is possible to appreciate that all the studied binders are characterized by an intense emission at $280 \mathrm{~nm}$, described by an intermediate decay time.

The spectral behaviors obtained from the vinyl binders are reported in Fig. 7. In particular, lines presented in Fig. 7A correspond to the spectra acquired immediately after the laser pulse, while the delayed ones are showed in Fig. 7B. All the curves exhibit the same emission band at about $280 \mathrm{~nm}$, already observed for the acrylic class. Such an emission is characterized by an intermediate decay time, as it is possible to appreciate from the delayed spectra in Fig. 7B. In addition, differently from the acrylic ones, the vinyl binders show a further emission band in all the delayed acquisitions, in the $320-360 \mathrm{~nm}$ range. Such a band is also characterized by a slow decay time, longer than $15 \mathrm{~ns}$. Then, the presence at once of both the emission bands in the fluorescence spectra $(280 \mathrm{~nm}$ and $320-360 \mathrm{~nm}$ ) could be considered characteristic for the vinyl resins class.

At last, the spectral features observed for the selected natural binders, linseed oil and dammar varnish are reported in Fig. 8A and $8 \mathrm{~B}$, respectively. It must be pointed out that both the spectral signatures appear very similar between them and to the support ones. Indeed, they show a weak intensity emission 


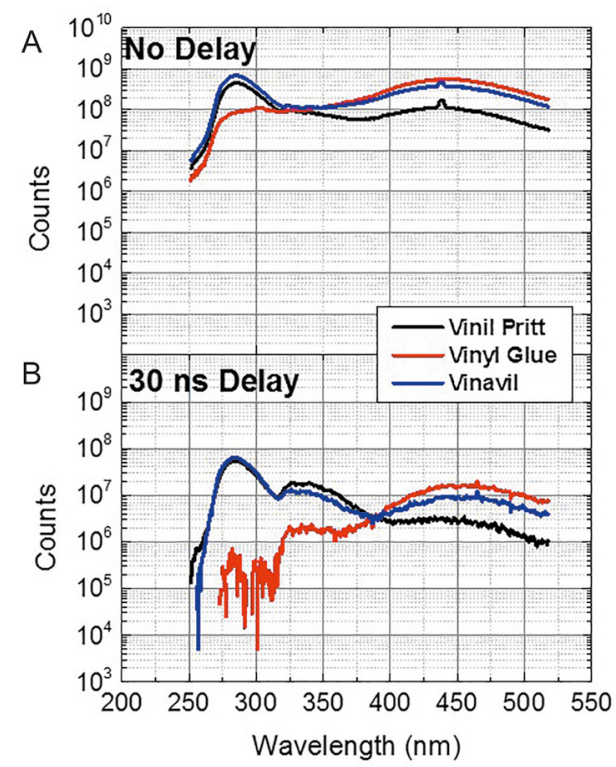

Fig. 7. TR-LIF spectra of the investigated vinyl binders: Vinil Pritt (black line), Vinyl Glue (red line), Vinavil (blue line).

in the UV region if compared to the ones observed for the acrylic and vinyl classes. It should be noticed that the above-mentioned natural binders are almost completely absorbed by the cardboard, so that an extremely thin binder layer is present on the support surface. This implies that the fluorescence emission from the support is probably not negligible in this case, making it difficult to extract characteristic information from the obtained results. For the above reason, it might be interesting to perform a systematic investigation on these natural binders on different supports and as a function of the laser excitation wavelength.

One of the turning points of this research regards the potential capability of the TR-LIF to identify signatures from mixed compounds (i.e.: binders and pigments). Therefore, each of the nine binders previously analyzed was mixed with some selected

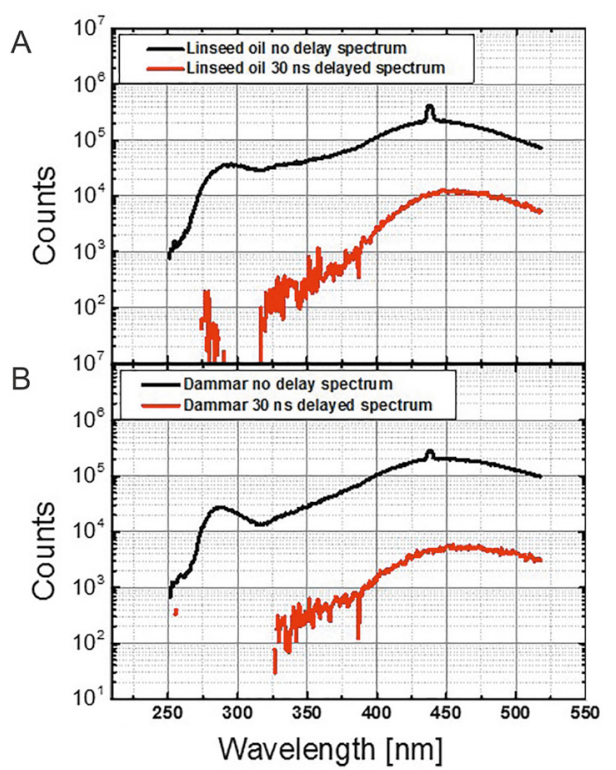

Fig. 8. TR-LIF spectra of the studied natural binders: A) linseed oil, B) dammar varnish in turpentine. For each graph, the black line represents the no delay spectrum and the red line the 30 ns delay spectrum.

pigments, usually employed in contemporary painted artworks. In Fig. 9, the spectra obtained from a mixture of Zinc white and an acrylic binder is reported, as an example. In this graph, it is possible to recognize the characteristic acrylic emission peak centered at $280 \mathrm{~nm}$. Moreover, a further peak at $380 \mathrm{~nm}$ is appreciable, corresponding to the Zinc band gap, as reported in literature [25, 26]. The delayed spectrum (red line) shows that the studied compounds are defined by an intermediate (acrylic binder) and decay time (Zinc oxide), respectively. In addition, the obtained results proved that there is no evidence of quenching effects or spectral interactions among the single constituents of the studied mixture. Indeed, the measured spectra consist in a linear combination of the emissions obtained from a mixture of pure binder and pigment powder. 


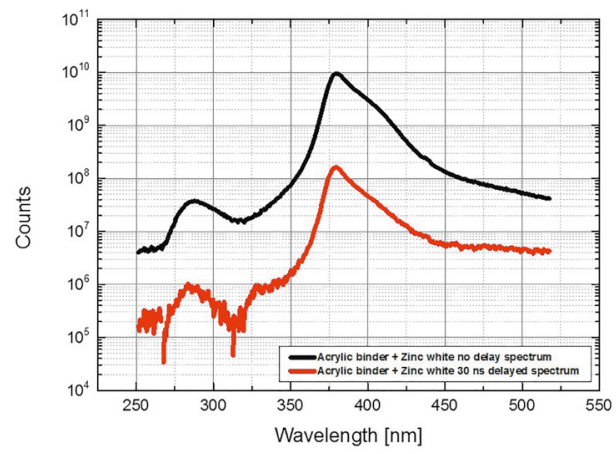

Fig. 9. TR-LIF spectrum of an acrylic binder mixed with Zinc white with no delay (black line) and with 30 ns delay (red line).

\subsection{Case Study}

The laboratory results were then compared to the ones acquired on the painting Nascita Della Forma (Birth of the Form), by Nato Frascà (1962), in order to characterize the binders employed by the artist on its painted surface, classified by himself as "oil on canvas" (Fig. 1). As shown in the magnification (Fig. 2 ), the surface is characterized by a degradation of painted layer, corresponding to the fluorescent orange and light blue pigments. Such a behavior suggests a probable use of different binders in the corresponding areas, as compared to the one chosen for the painted surface. At first, the fluorescence image of the pictorial layers was recorded in order to map fluorescence emissions of different compounds, thus guiding the TR-LIF measurements. In Fig. 10, it is possible to appreciate how the orange and light blue pigments are characterized by an intense fluorescence that other pigments do not show. These behaviors are indicating a possibly different composition of the above-mentioned paints [27].

The TR-LIF acquisitions were recorded on the painted surface by using the same measurements conditions applied for the laboratory samples. The obtained spectra are reported in Fig. 11, where the black line corresponds to no delay spectrum, while the red one is for $30 \mathrm{~ns}$ delayed spectrum. Both the curves exhibit a band centered at $280 \mathrm{~nm}$, which is also characterized by an intermediate decay time. A further intense peak at 380 $\mathrm{nm}$, described by a fast decay time, which is compatible with the emission from the Zinc oxide reference sample shown in Fig. 9 and with previous results reported in literature $[25,26]$. This is also confirmed by additional XRF measurements performed on the same orange and light blue pigments, not reported here.

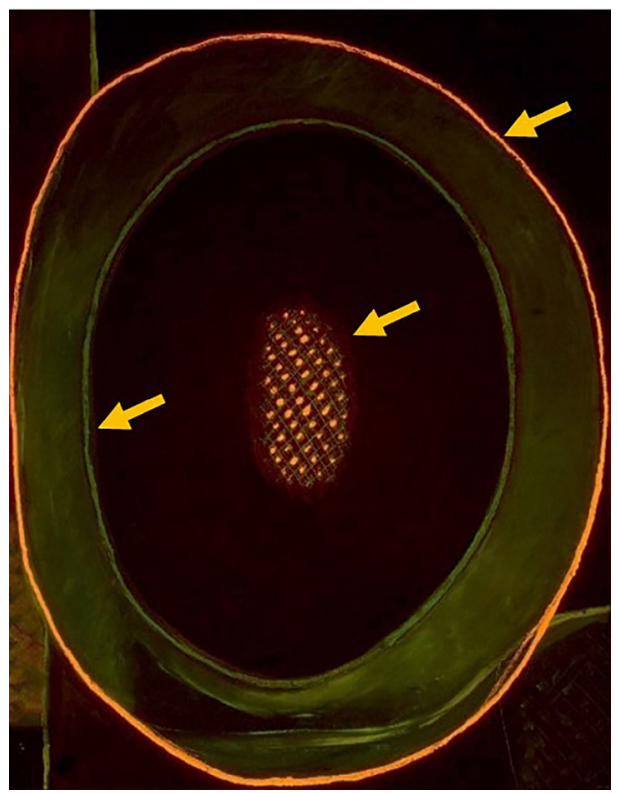

Fig. 10. Fluorescence image of the painting Nascita Della Forma (Birth of the Form).

A comparison between the results discussed in section 3.1 with the ones obtained from Nascita Della Forma (Birth of the Form) suggests that the binder used for the two selected paints is an acrylic one due to the characteristic emission band at $280 \mathrm{~nm}$. Such 


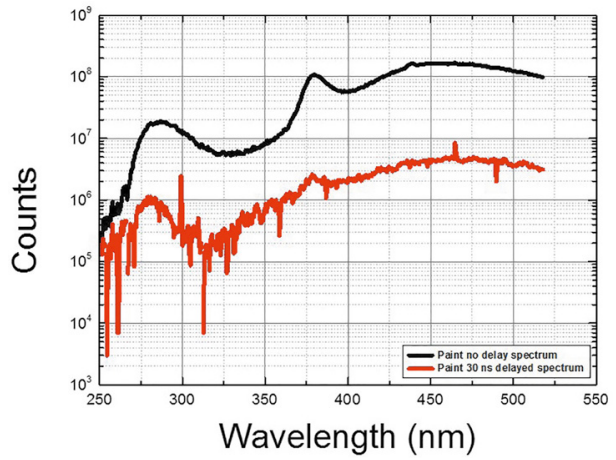

Fig. 11. TR-LIF spectra acquired on the painting Nascita Della Forma (Birth of the Form): no delay spectrum (black line), 30 ns delay spectrum (red line).

a hypothesis is also supported by observing the degradation corresponding to the orange and light blue pigments, characterized by the presence of several cracks [28].

\section{Conclusion}

In this work, the TR-LIF spectroscopy is demonstrated to be a valid tool to study different classes of binders employed in the contemporary art. Distinctive spectral features were observed from acrylic, vinyl and natural binders. Specifically, an emission band centered at $280 \mathrm{~nm}$, and defined by an intermediate decay time, characterized all the spectra from acrylic binders. The same band was also observed for the investigated vinyl compounds, which additionally showed a further emission band between 320-360 $\mathrm{nm}$ in the $30 \mathrm{~ns}$ delayed spectra, defined by a slow (>15 ns) decay time. This last distinctive feature can be used in order to distinguish the vinyl compounds from the acrylic ones. No peculiar fluorescent emissions were observed instead from the natural binders investigated in the present study due to the similarity of their spectral emissions to the cardboard support ones. Such a behavior was probably due to an unfitting excitation wavelength. Finally, the laboratory results were compared with the ones acquired on the contemporary painting Nascita Della Forma (Birth of the Form) by Nato Frascà. The study of UV fluorescence images before and the TR-LIF measurements after allowed identifying the acrylic binders employed in the painted surface, thus providing an important information in view of a proper conservation of the investigated artwork.

It should be pointed out that further studies are mandatory in order to establish the specific acrylic and vinyl fluorophores responsible for the observed fluorescence emissions. Nevertheless, the achieved classification represents a good starting point towards the realization of a dedicated database for contemporary materials and allows improving technical information about artworks. In conclusion, the TR-LIF spectroscopy analysis should be considered a valid tool for the investigation, characterization and preservation of contemporary artworks.

\section{Acknowledgments}

This study was made possible thanks to a partnership with the MACRO-Museo d'Arte Contemporanea di Roma (Museum of Contemporary Art in Rome). The authors wish to thank the Director of the MACRO, Dr. Federica Pirani, and the art experts of the MACRO, Dr. Antonia Rita Arconti and Dr. Paola Coltellacci for their helpful collaboration.

\section{References}

[1] C. Cucci, L. Bigazzi, M. Picollo, "Fiber Optic Reflectance Spectroscopy as a non-invasive tool for investigating plastics degradation in contemporary art 
collections: A methodological study on an expanded polystyrene artwork", Journal of Cultural Heritage, 14 (2013) 290-296, DOI: 10.1016/j.culher.2012.08.003.

[2] T. Learner, "A review of synthetic binding media in twentieth-century paints", The Conservator, 24,(2000), 96-103, DOI: 10.1080/01410096.2000.9995156.

[3] E. Jablonski, T. Learner, J. Hayes, M. Golden, "Conservation concerns for acrylic emulsion paints", Studies in Conservation, 48 (2003) 3-12, DOI: 10.1179/sic.2003.48.Supplement-1.3.

[4] G.C. Scicolone, Il restauro dei dipinti contemporanei, Nardini Editore, Florence 2004.

[5] M. Matteini, A. Moles, La chimica nel restauro. I materiali dellarte pittorica, Nardini Editore, Florence 2007.

[6] L. Mayer, G. Myers, "A Note on the Early Use of Dammar Varnish", Studies in Conservation, 47 (2002) 134-138, DOI: 10.1179/sic.2002.47.2.134.

[7] D. Anglos, C. Balas, C. Fotakis, "Laser spectroscopic and optical imaging techniques in chemical and structural diagnostics of painted artwork", American Laboratory, 31 (1999) 60-67.

[8] A. Nevin, S. Chater, D. Anglos, C. Fotakis, "Analysis of protein-based binding media found in paintings using laser induced fluorescence spectroscopy", Analytica Chimica Acta, 573-574 (2006) 341-346, DOI: 10.1016/j.aca.2006.01.027.

[9] T. Miyoshi, M. Ikeya, S. Kinoshita, T. Kushida, "Laser-Induced Fluorescence of Oil Colours and Its Application to the Identification of Pigments in Oil Paintings", Japanese Journal of Applied Physics, 21 (1982) 1032-1036, URL: http://iopscience.iop. org/1347-4065/21/7R/1032.

[10] A. Romani, C. Celementi, C. Miliani, G. Favaro, "Fluorescence Spectroscopy: A Powerful Technique for the Noninvasive Characterization of Artwork", Accounts of Chemical Research, 43 (2010) 837-846, DOI: 10.1021/ar900291y.

[11] V. Lazic, F. Colao, R. Fantoni, A. Palucci, V. Spizzichino, I. Borgia, B. G. Brunetti, A., Sgamellotti, "Characterisation of lustre and pigment composition in ancient pottery by laser induced fluorescence and breakdown spectroscopy", Journal of Cultural Heritage, 4 (2003) 303-308, DOI: 10.1016/S12962074(02)01212-8.
[12] A. Nevin, I. Osticioli, D. Anglos, A. Burnstock, S. Cather, E. Catellucci, "Raman Spectra of Proteinaceous Materials Used in Paintings: A Multivariate Analytical Approach for Classification and Identification", Analytical Chemistry, 79 (2007) 6143-6151, DOI: $10.1021 / \mathrm{ac} 070373 \mathrm{j}$.

[13] A. Nevin, D. Comelli, G. Valentini, D. Anglos, A. Burnstock,S. Cather, R. Cubeddu, "Time-resolved fluorescence spectroscopy and imaging of proteinaceous binders used in paintings", Analytical and Bioanalytical Chemistry, 388 (2007) 1897-1905, DOI: 10.1007/s00216-007-1402-0.

[14] A. Nevin, D. Anglos, "Assisted interpretation of Laser-Induced Fluorescence spectra of egg-based binding media using total emission fluorescence spectroscopy", Hindawi Publishing Corporation Laser Chemistry, (2006) 1-5, DOI: 10.1155/2006/82823.

[15] R. Ortiz, P. Ortiz, F. Colao, R. Fantoni, M. A. Gómez-Morón, M. A. Vázquez, "Laser spectroscopy and imaging application for the study of cultural heritage murals", Construction and Building Materials, 98 (2015) 35-43, DOI: 10.1016/j.conbuildmat.2015.08.067.

[16] T. Miyoshi, "Fluorescence from Oil Colours, Linseed Oil and Poppy Oil under N2 Laser Excitation", Japanese Journal of Applied Physics, 24 (1985) 371-372.

[17] P. Symour, The Artist's Handbook, Arcturus Publishing Limited, London 2003.

[18] M. Romani, F. Colao, R. Fantoni, M. Guiso, M.L.Santarelli, "Hyperspectral fluorescence for organic pigment characterization in contemporary artwork", Journal of Applied and Laser Spectroscopy, 1 (2014) 29-36.

[19] F. Sossi, Dallocchio al cervello: Gruppo 1 di Roma: Carrino, Frascà, Uncini, Magna Grecia, Taranto 1965.

[20] G. Di Genova, Generazione anni 30, in Storia dellarte italiana del'900: per generazioni, Bora, Bologna 2000.

[21] M. Marinelli, A. Pasqualucci, M. Romani, G. Verona-Rinati, "Time resolved laser induced fluorescence for characterization of binders in contemporary artworks", Journal of Cultural Heritage, 23 (2017) 98-105, DOI: 10.1016/j.culher.2016.09.005. 
[22] A. Cosentino, "Identification of pigments by multispectral imaging; a flowchart method", Heritage Science, 2 (2014) 1-12, DOI: 10.1186/2050-7445-2-8.

[23] D. Comelli, A. Nevin, G. Verru, G. Valentini, R. Cubeddu, "Time-resolved fluorescence spectroscopy and fluorescence lifetime imaging for the analysis of organic materials in wall painting replicas" in: F. Piqué, G. Verri (Eds.), Organic materials in Wall Paintings, Project Report, Getty Conservation Institute 2015, pp. 83 - 96, http://www.getty.edu/conservation/publications_resources/pdf_publications/ pdf/omwp_report.pdf (accessed 22.08.2017).

[24] D. Comelli, G. Valentini, A. Nevin, A. Farina, L. Toniolo, R. Cubeddu, "A portable UV-fluorescence multispectral imaging system for the analysis of painted surfaces", Review of Scientific Instruments, 79 (2008), DOI: 10.1063/1.2969257.

[25] I. Borgia, R. Fantoni, C. Flamini, T. M. Di Palma, A. Giardini Guidoni, A. Mele, "Luminescence from pigments and resins for oil paintings induced by laser excitation", Applied Surface Science, 127-128 (1998), 95-100, DOI: 10.1016/ S0169-4332(97)00616-8.

[26] D. W. Bahnemann, C. Kormann, M. R. Hoffmann, "Preparation and Characterization of Quantum Size Zinc Oxide: A Detailed Spectroscopic Study", The Journal of Physical Chemistry, (1987) 91 3789-3798, DOI: 10.1021/j100298a015.

[27] M. Bacci, M. Picollo, G. Trumpy, M. Tsukada, D. Kunzelman, "Non-Invasive Identification of White Pigments on $20^{\text {th }}$-Century Oil Paintings by Using Fiber Optic Reflectance Spectroscopy", Journal of the American Institute for Conservation, 46 (2007) 27-37, DOI: 10.1179/019713607806112413.

[28] P. M. Whitmore, D. Moran, C. Bailie, "Shrinkage Stresses in Art and Conservation Coatings Based on Synthetic Polymers", Journal of the American Institute for Conservation, 38 (1999) 429-441, DOI: 10.1179/019713699806113402. 


\title{
LIBS, optical and multivariate analyses of selected $17^{\text {th }}$-century oil paintings from the Museum of King Jan III's palace at Wilanów
}

\author{
Agnieszka Pawlak ${ }^{1 \star}$, Wojciech Skrzeczanowski² ${ }^{2}$ Krzysztof Czyż ${ }^{2}$ \\ 1 Museum of King Jan III’s Palace at Wilanów, ul. Stanisława Kostki Potockiego 10/16, 02-958 Warsaw, \\ Poland \\ 2 Military University of Technology, ul. Gen. Sylwestra Kaliskiego 2, 00-908 Warsaw, Poland \\ * Corresponding author: apawlak@muzeum-wilanow.pl
}

\begin{abstract}
:
The paper presents measurement results of LIBS and optical microscopy investigations applied to five $17^{\text {th }}$-century oil paintings, belonging to the collection of the Museum of King Jan III's Palace at Wilanów. The analysis devoted to ground layers at depths of about 200-300 $\mu \mathrm{m}$ (40-60 laser pulses) allowed to find stratigraphy distributions of elements characteristic for ground layers. During the latest investigations, the researchers concentrated mainly on the comparison of some specific elements like $\mathrm{Li}, \mathrm{Ba}$, Ti which could indicate the origin of the pigment and help in the process of classification of the paintings. LIBS spectra analyses were supported by statistical factorial analyses which visibly confirmed conclusions drawn from spectral and optical microscopy research.
\end{abstract}

Keywords: painting analysis, LIBS, multivariate analysis, oil paintings, ground and colour layers, Wilanów Palace Museum

\section{Introduction}

The Palace in Wilanów is a Polish suburban royal residence located in the south-west area of Warsaw. It was built in the years 1677-1696 as a summer residence for King Jan III Sobieski and his wife Maria Kazimiera. Designed by Augustin Locci, the building combines the character of an Italian garden villa and a French palace in the style of Louis XIV.

King Jan III was a comprehensively educated connoisseur of arts and an attentive artists' patron. He engaged foreign artists for his commissions. Among them there were French and Italian painters. Moreover, the ruler cared for national artists and funded scholarships in the St. Luke's Academy in Rome, which were dedicated to talented Polish painters. The aim of his activity was to educate and form a masterclass of artists for a planned academy, nevertheless the project was never completed. His protégés were Jan Reisner and Jerzy Eleuter Szymonowicz Siemiginowski, both trainees of the Roman Accademia. Jan Sobieski also engaged famous Polish portrait painters such as Daniel Schultz and Jan Tretko named Trycjusz. Many foreign artists worked for the Polish king, e.g. French painters: Claude Callot, Alexandre-Francois Desportes, Henri Gascar; Italian artists, e.g: 
Michelangelo Palloni and Martin Altomonte as well as a Dutch painter Ferdynand van Kessel.

The erudite king was also a staunch art collector who looked for art objects all around Europe and even further. Unfortunately, after his death, the rich and valuable collection (which we know following the Inventory of 1696) was divided between his heirs. In result, along with the years passing by, it was scattered, and finally, almost completely dispersed.

Since the end of WWII, art historians have worked on determining what pieces of art were parts of the King's collection. Continuous efforts are made to recreate the royal collection. Although a lot of frescoes or ceiling paintings decorating the palace have survived until today, it is extremely difficult to find easel paintings belonging to the collection of Jan III Sobieski, including those which were created at his request. Over the years they have been subject to exchanges or trade transactions, transformation and more or less skilful conservation works. Moreover, they were usually not signed. Today, it is hard to recreate the collection.

Nowadays, the collection of paintings at Museum of King Jan III's Palace in Wilanów consists of 2291 paintings on canvas and wooden panels. Some of them could come from the royal collection.

The mission of conservators and art historians who currently take care of the collection housed at the Wilanów Palace Museum is to explain the secrets of the objects gathered there. All of them would like to discover information about the workshops in which the paintings were created as well as about their authors.

That is why in 2012 a large and interdisciplinary project entitled "Monumentum Sobiescianum" was initiated by the curators at the Wilanów Palace Museum. Its topic is in -depth research related to King Jan III and his relatives.

In the framework of "Monumentum Sobiescianum", a separate research is conducted, focusing on the recognition of the techniques and technologies of the works painted by the court painters. One part of this project is concentrated on unsigned portraits of Jan III and his family, which were probably painted during the King's lifetime and belonged to the royal collection. In the study, different methods have been used. Beside conventional micro-chemical tests and visual inspection of stratigraphic samples by light microscopy, we have applied modern analytical instrumentation, in particular LIBS but also SEM-EDS, XRPD as well as $\mu$ X-Ray Powder Diffraction analyses.

Since 2012 fifteen portraits of the King and his family have been examined - nine of them by Laser-Induced Breakdown Spectroscopy (LIBS).

We have studied stratigraphy distributions of various elements over grounds and paint layers, which allowed us to find similarities and differences of analyzed paintings.

In the current study, we have concentrated on the comparison of the composition of ground layers, essentially some specific elements like $\mathrm{Li}, \mathrm{Ba}, \mathrm{Ti}$, and tried to classify the paintings according to their content.

\section{Materials and methods}

\subsection{Investigated paintings}

The paper describes the examinations made in 2016 on material collected during two campaigns of investigations conducted in the years 2012 [1] and 2014 [2].

In 2012 and 2014, nine oil paintings, portraits of King Jan III Sobieski and his 
family, were tested with the use of LIBS method and digital 3D microscopy. In 2016, we selected five of them, painted on canvas, to compare the composition of the ground layers. Results were compared with cross-sections, SEM/EDS and $\mu$ XRDP data available for some paintings. The obtained results allowed us to find stratigraphy distributions of elements in selected parts of paintings as well as to identify pigments used in the tested points.

The artworks chosen for present examination are unsigned; however, we know that they were painted during the King's lifetime. All belong to the historical collection of the Wilanów palace. Two official portraits of the King wearing archaized vestments may have been created in Venice (Italy)- the bigger one is described as the original painting whereas the smaller one is considered to be a copy from the same period.

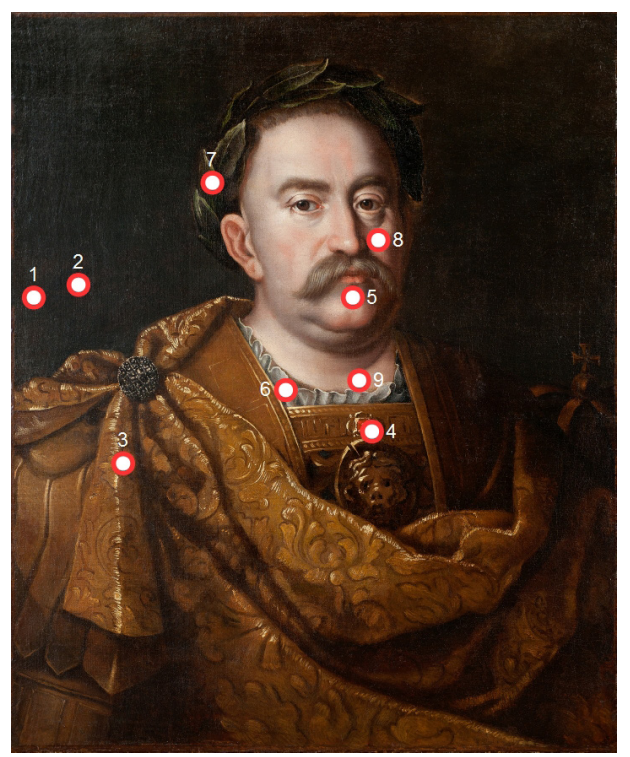

Fig.1. Wil.1348, Portrait of King Jan III, unknown author, oil/canvas, $72.8 \mathrm{~cm} \times 60.5 \mathrm{~cm}$, photo by W. Holnicki.
Two equestrian portraits of Queen Marie and King Jan III were attributed to the court painter Jerzy Eleuter Szymonowicz Siemiginowski and the portrait of Teresa Kunegunda, the royal daughter, is attributed to the French painter Pierre Mignard.

The ground layers in all five pictures are colourful: from yellow-orange to reddish brown, through brown-yellow, orange-red and light red. The clay-based coloured grounds are characteristic for the baroque epoch in which they replaced white grounds based on chalk and gypsum. Iron oxides and aluminosilicates dominate their chemical composition. These colourful grounds differ from each other by natural additions - products of the natural weathering of rocks. They are mainly composed of minerals rich in Fe-oxides, Si-oxides and Al-oxides (goethite, kaolinite and quartz) $[3,4]$. Beside various additions of pigments like minium, calcium

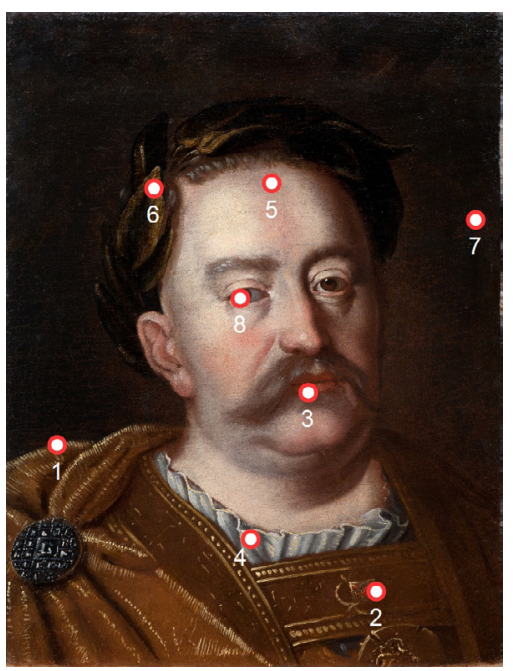

Fig. 2. Wil.1197, Portrait of King Jan III, unknown author, oil/canvas, $48.0 \mathrm{~cm} \times 37.0 \mathrm{~cm}$, photo by W. Holnicki. 


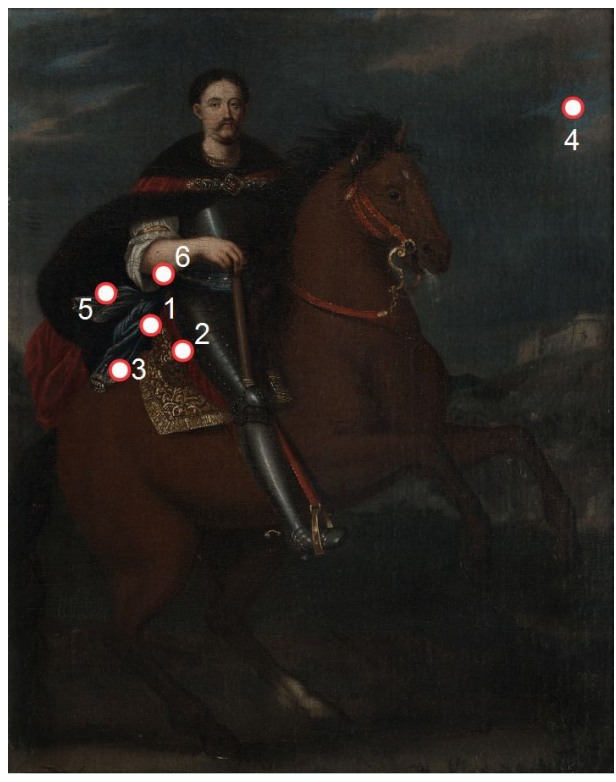

Fig. 3. Wil.1685, Equestrian portrait of King Jan III, oil/canvas, $51 \mathrm{~cm} \times 40 \mathrm{~cm}$, photo by Z. Reszka.

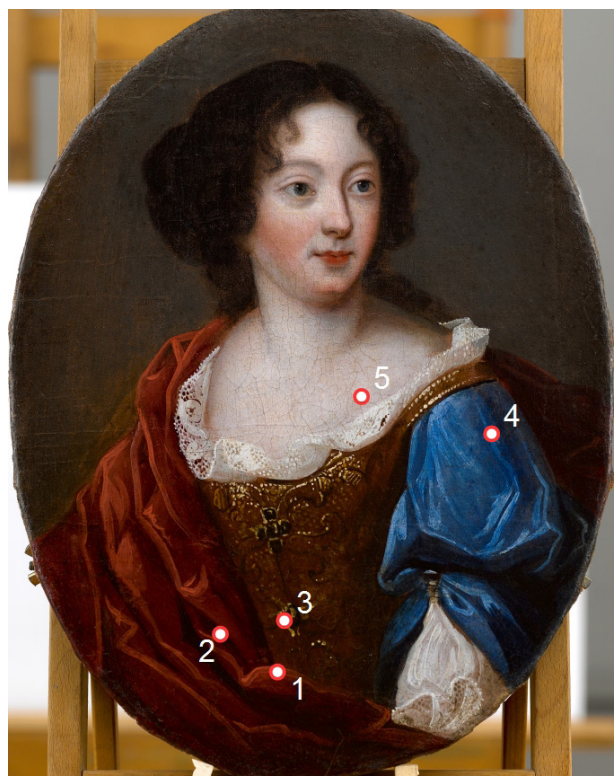

Fig. 5. Wil.1200, Portrait of Teresa Kunegunda Sobieska, oil/canvas, $39 \mathrm{~cm} \times 30 \mathrm{~cm}$, photo by A. Indyk.

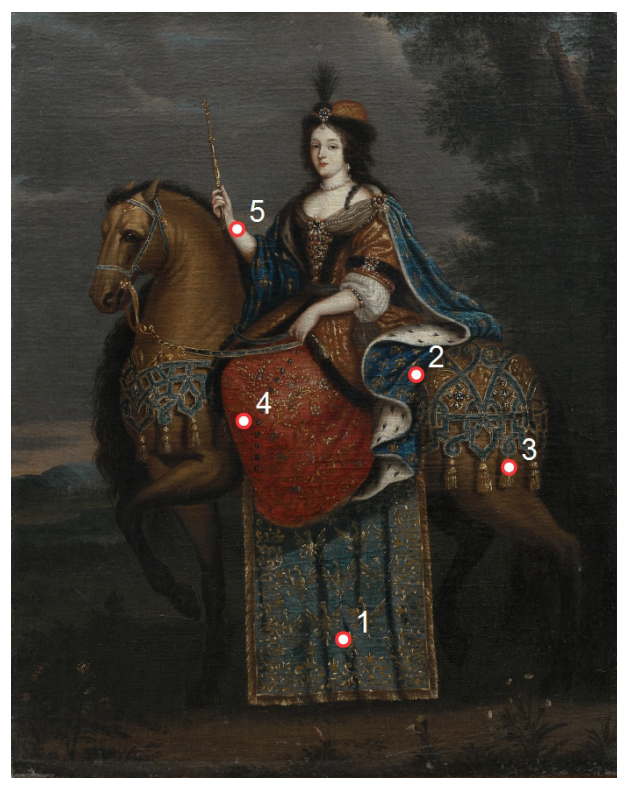

Fig.4. Wil.1686, Equestrian portrait of Queen Maria Kazimiera, oil/canvas, $51 \mathrm{~cm} \times 40 \mathrm{~cm}$, photo by Z. Reszka.

carbonate, led white, iron red pigments, they can also differ from each other on deposits containing such elements as $\mathrm{Ba}, \mathrm{Li}$ and $\mathrm{Ti}$. For example, anatase $\left(\mathrm{TiO}_{2}\right)$ was detected by X-ray diffraction in some grounds of baroque paintings by researchers from the Czech Republic [4]. That is why we selected four elements - Li, Ba, Ti and Fe for further analysis of our paintings:-Li, $\mathrm{Ba}$ and $\mathrm{Ti}$ were selected as specific additions in grounds which could help in finding differences in production materials or workshops, and $\mathrm{Fe}$ as a representative of elements typically present in grounds composition (like $\mathrm{Al}, \mathrm{Mg}$, $\mathrm{Si}$ or $\mathrm{Ca}$ and few others).

In the presented study we tried to classify the paintings basing on differences in $\mathrm{Ti}, \mathrm{Ba}$ and $\mathrm{Li}$ contents in ground layers (Ca served as a reference element which allowed to carry out relative calculations and to compare results from both research campaigns 
- in 2012 and 2014). We made an attempt to distinguish between investigated paintings via analysis of these additions to find clay provenance/origin.

\subsection{Methodology and instrumentation}

The primary analytical tool used in this study was Laser Induced Breakdown Spectroscopy (LIBS) [5], which allowed for the qualitative identification of the chemical composition and the superficial layer stratigraphy of artworks. The choice of LIBS as a primary diagnostic tool was made by the curators of the Palace Museum at Wilanów, mainly due to the low destructivity of the diagnostic method and the short time available for tests before starting conservation procedures. LIBS, in short, relies on using high power focused laser energy to cause the ablation of a small amount of surface material, resulting in plasma that emits both continuum and line radiation. Analysis of the spectra from this line radiation allows us to identify elements found in the sample, as each element emits a unique spectral signature.

A laser source in the LIBS arrangement consisted of a $266 \mathrm{~nm}$ UV Brio laser (from Quantel/BigSky) with $8 \mathrm{~mJ}$ or $10 \mathrm{~mJ}$ energy in $4 \mathrm{~ns}$ pulses. Stronger laser pulses, which would have resulted in a brighter plasma and a stronger signal, were not used in order to minimize the risk of micro-damages to oil paintings. The laser beam was directed through a $100 \mathrm{~mm}$ focal lens to focus the laser energy into a fine spot for ablation on painting and ground layers. The ESA 4000 spectrometer was used to collect spectral information in the $200-780 \mathrm{~nm}$ range during ablation. In order to capture useful line radiation, data collection lasted $5 \mu$ s and began with a 500 ns delay after the laser pulse, allowing the initial continuum emission from the plasma to disappear. For stratigraphy needs, laser pulses and spectra collection were repeated in the same area until the signal decay, which indicated that the end of a ground layer was reached. The number of laser pulses was related to a thickness of painting/ground layer and typically amounted between $25-150$ pulses.

During each measurement campaign the experimental conditions were kept constant, which allowed us to compare results for different spots in all investigated paintings. Spectral line intensities of the analysed elements were used as markers representing the content of these elements. The lines selected for each element and taken for calculations were throughout the same and are shown in Table 1.

However, in the 2012 campaign the laser pulse energy was $10 \mathrm{~mJ}$, and in 2014-8 $\mathrm{mJ}$. In order to compare the results, we used relative contents of chosen elements $(\mathrm{Li}, \mathrm{Ti}$, $\mathrm{Fe}$, and $\mathrm{Ba}$ ) represented by selected spectral lines referred to calcium content because "absolute" (counts) and relative (for example $\mathrm{Ti} / \mathrm{Ca}$ ) dependencies are almost the same. Calcium was selected as a reference element

Table1. Spectral lines selected for comparisons

\begin{tabular}{|c|c|c|c|c|c|}
\hline Element & $\mathrm{Ca} \mathrm{I}$ & Li I & Ti I & Fe I & $\mathrm{Ba}$ II \\
\hline Wavelength, nm & 445.478 & 670.783 & 498.173 & 438.351 & 455.403 \\
\hline Transition probability, $10^{7} \times \mathrm{s}^{-1}$ & 8.70 & 3.69 & 6.60 & 5.00 & 11.10 \\
\hline $\begin{array}{l}\text { Transition } \\
\text { (upper level - lower level) }\end{array}$ & $\begin{array}{l}3 p^{6} 4 s 4 d- \\
3 p^{6} 4 s 4 p\end{array}$ & $1 s^{2} 2 p-1 s^{2} 2 s$ & $\begin{array}{c}3 d^{3}\left({ }^{4} F\right) 4 p- \\
3 d^{3}\left({ }^{4} F\right) 4 s\end{array}$ & $\begin{array}{c}3 \mathrm{~d}^{7}\left({ }^{4} \mathrm{~F}\right) 4 \mathrm{p}- \\
3 \mathrm{~d}^{7}\left({ }^{4} \mathrm{~F}\right) 4 \mathrm{~s}\end{array}$ & $6 p-6 s$ \\
\hline
\end{tabular}


since it was present over all paintings in all spots and almost in all depths.

In all stratigraphy results we present depths in terms of laser pulse numbers, since due to lack of other absolute depth-resolved diagnostics like, for example, optical coherent tomography [6], only such relative spatial resolution was possible to apply. Nevertheless, when comparing after LIBS microscopy measurements we can assume a depth of about $3-5 \mu \mathrm{m}$ as created by one laser pulse (depending on a paint/ground layer).

The second diagnostic tool was digital microscopy. Microscopic observations were made using the digital Hirox 6700 3D microscope. Every time before the LIBS test, the surface of the painting was examined by the microscope to precisely choose the point of the laser shot (LIBS experiment). After LIBS measurement, the microscopic analysis of the surface was carried out again to find a crater profile and its characteristics (depth, size, shape, etc.) in order to evaluate the scale of surface destruction. In Fig. 7 the examination of a paint layer using digital 3D microscope is presented. The 3D microscopy reveals relatively low destruction of painting layers caused by LIBS technique. The scale
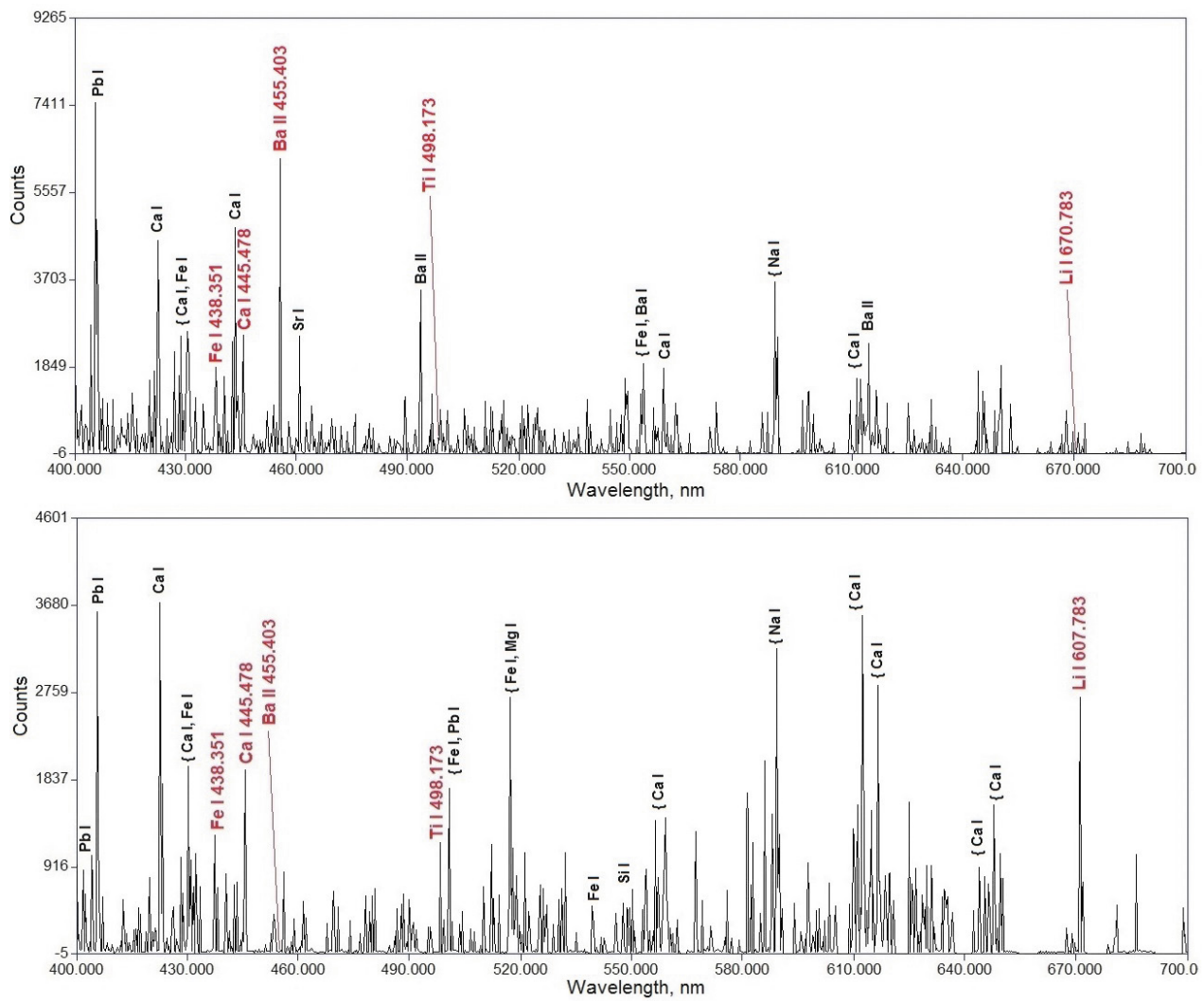

Fig. 6. Typical spectra registered during LIBS experiment: upper spectrum for painting Wil.1200, measurement point 2 , depth at $54^{\text {th }}$ laser pulse, and lower spectrum for painting Wil. 1685 , measurement point 5 , depth at $47^{\text {th }}$ laser pulse. Spectral peaks selected for calculations are marked in red, while other intense peaks - in black. 
of LIBS destructiveness is shown in Figs. 7 and 8.

The results allowed us to find stratigraphy distributions of elements in selected parts of paintings as well as to identify pigments used in tested points. Finally, we selected 33 measurement points in 5 paintings. From 25 up to over 150 LIBS spectra were taken in the selected points. Totally, over 2000 LIBS spectra were analyzed. Two typical exemplary spectra with marked peaks of selected elements are shown in Fig. 6 for

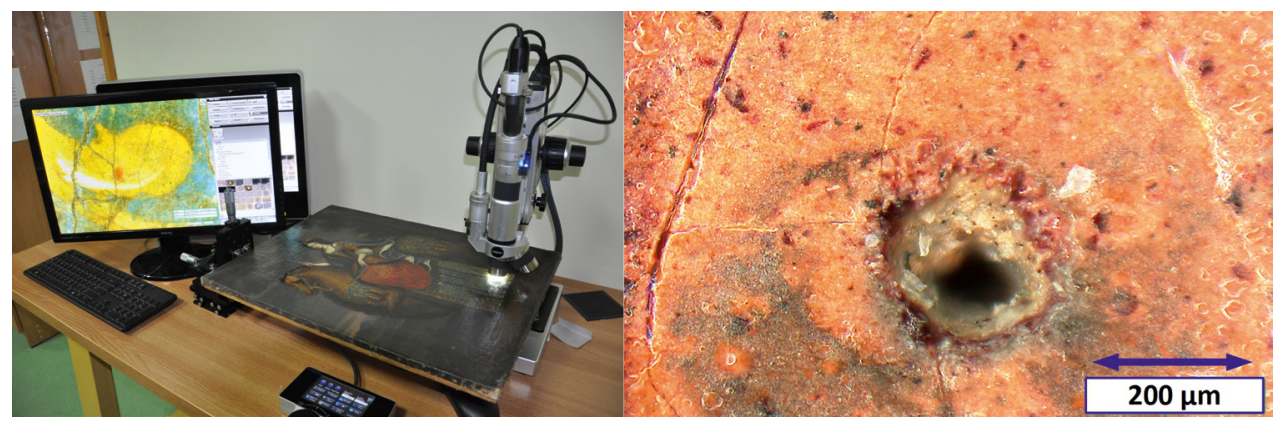

Fig. 7. Examination of a paint layer using digital 3D microscope: a) experimental stage, b) crater image showing destructive scale, photos by A. Pawlak, K. Czyż.
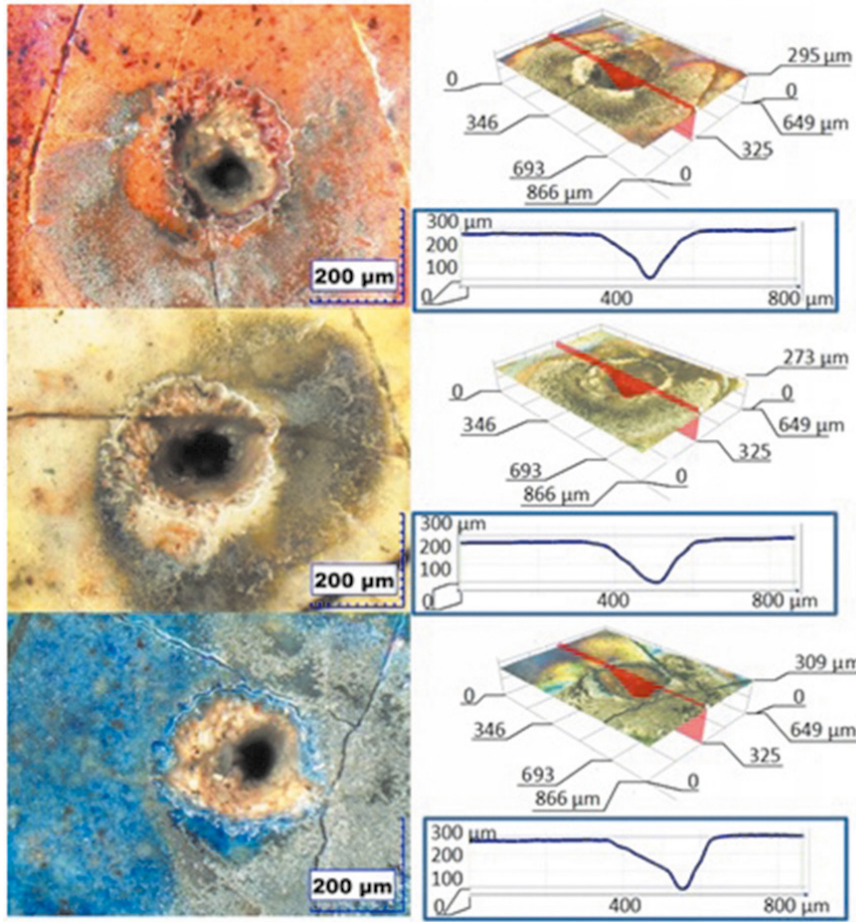

- Magnification 350x,

- Crater after 150 laser shots,

- Depth: $240 \mu \mathrm{m}$

- Radius $\sim 175 \mu \mathrm{m}$

- Magnification 350x,

- Crater after 120 laser shots,

- Depth: $300 \mu \mathrm{m}$

- Radius $\sim 150 \mu \mathrm{m}$

- Magnification 350x,

- Crater after 120 laser shots,

- Depth: $300 \mu \mathrm{m}$

- Radius $\sim 150 \mu \mathrm{m}$

Fig. 8. Digital 3D microscopy for various paint layers and the scale of LIBS destructiveness, photo by K. Czyż. 
paintings Wil.1200 and Wil.1685. The location of measurement points was shown in Figs. 1 to 5.

Evident differences and similarities were found in grounds deposited on particular paintings. During these latest investigations, we concentrated on comparison of some elements like: $\mathrm{Ba}, \mathrm{Li}$, Ti, identified in grounds layers. Basing on this, we tried to classify the paintings following the character of the ground layers. SEM/EDS and XRPD data available for some paintings were useful in aiding interpretation of LIBS spectra.

The analysis was additionally supported by statistical analytical techniques- in particular, a multivariate factorial analysis(FA).

The FA involves the reduction of a number of input variables, which may be correlated in a variety of ways, into a lower number of sometimes unobservable variables called factors. The reduction of variables results in the loss of some information from entry data, meaning that the first most important factors cannot account for all changes in the data. Nevertheless, the method provides a good analysis of entry data, simplifies complex sets of data and finds relationships in the data that may not be obvious or even have a physical interpretation. Moreover, it still provides a greater understanding of probabilities and correlations. It is commonly accepted that in order to assure an accurate analysis of the data, the first two factors should account for about $70 \%$ of the variance in the entry data $[7,8]$. The FA analysis was conducted with the STATISTICA ver.10 software [9] by entering a matrix of data constructed from several dozen modified LIBS spectra, from which stratigraphic information was previously obtained. A similar approach to the $17^{\text {th }}$-century paintings research was published in [10].

\section{Results}

\subsection{Stratigraphy results}

We compared ground layers in five paintings in terms of presence of such elements as barium $(\mathrm{Ba})$, lithium $(\mathrm{Li})$, titanium $(\mathrm{Ti})$ and iron $(\mathrm{Fe})$, which is the main component of all these ground layers (see Fig.9). The presented results are an average of all measurement points selected for the paintings and are shown as ratios of these four elements to calcium. Error bars are standard deviations of peak intensity ratios of specified element pairs $(\mathrm{Li} / \mathrm{Ca}, \mathrm{Ti} / \mathrm{Ca}, \mathrm{Fe} / \mathrm{Ca}$, and $\mathrm{Ba} / \mathrm{Ca})$.

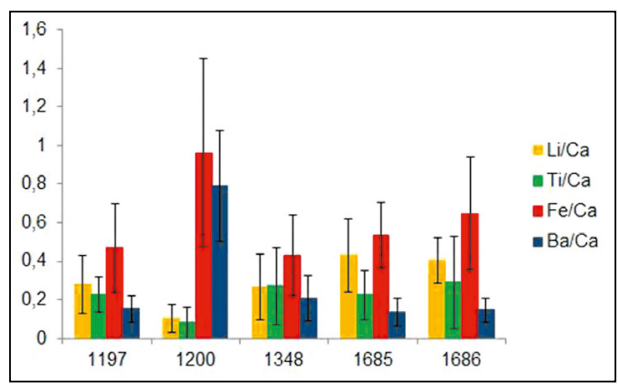

Fig. 9. Relative $\mathrm{Li} / \mathrm{Ca}, \mathrm{Ti} / \mathrm{Ca}, \mathrm{Fe} / \mathrm{Ca}$ and $\mathrm{Ba} / \mathrm{Ca}$ content for 40-60 laser pulse depth.

The charts show the similarity of grounds in terms of Li and Ba content in both equestrian portraits of the king and the queen (Wil.1685 and Wil.1686). Barium content in the ground layers of the portrait of Teresa Kunegunda (Wil.1200) is completely different. The barium signal is several times stronger from that recorded in the equestrian portraits of the king and the queen, and in the two portraits of the king (Wil.1197 and Wil.1348), which could mean that its barium content is much higher than in the other paintings. Lithium (signal) content, on the other hand, is several times higher in the equestrian portraits, and in the king's portraits as compared to the image of Teresa 
Kunegunda. But if we compare this Li signal in equestrian portraits and in the king's portraits, we can observe that we obtained comparable values in both equestrian portraits of the king and the queen (Wil.1685 and Wil.1686), and in both portraits of the king (Wil.1197 and Wil.1348), but the Li signal in equestrian portraits is stronger than the same signal in portraits of the king.

Regarding the titanium signal, it is almost the same in four portraits except for Teresa Kunegunda (Wil.1200) where both $\mathrm{Ti}$ and Li signals were weak.

We can note that the Teresa Kunegunda portrait (Wil.1200) undoubtedly differs from the other four pictures by higher content of barium and low contents of $\mathrm{Li}$ and $\mathrm{Ti}$. The details of these differences are shown in Figs. $10-12$. It can be seen in these figures that after stronger signals from shallow superfi-

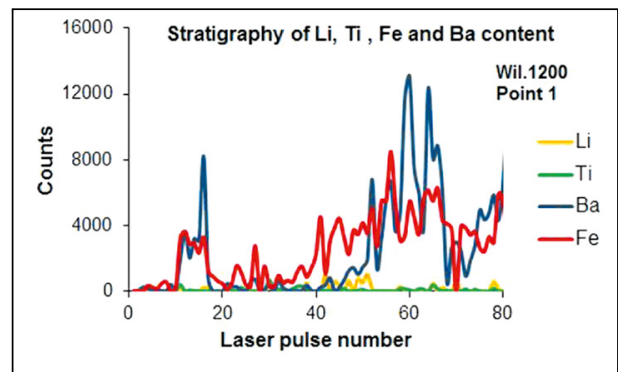

Fig.10. Li, Ti, Ba and Fe stratigraphy in point 1 of the painting 1200 (Teresa Kunegunda)

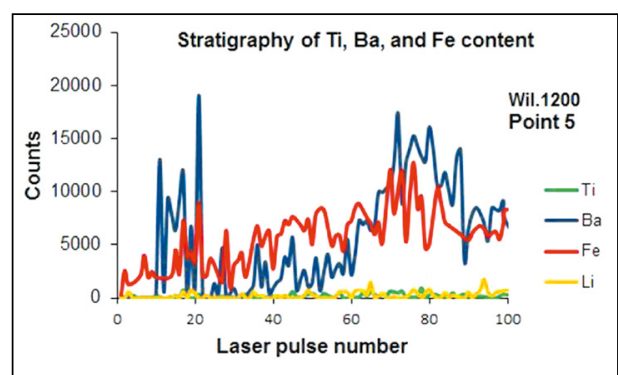

Fig. 11. Ti, Ba, Fe, and Li stratigraphy in point 5 of the painting 1200 (Teresa Kunegunda).

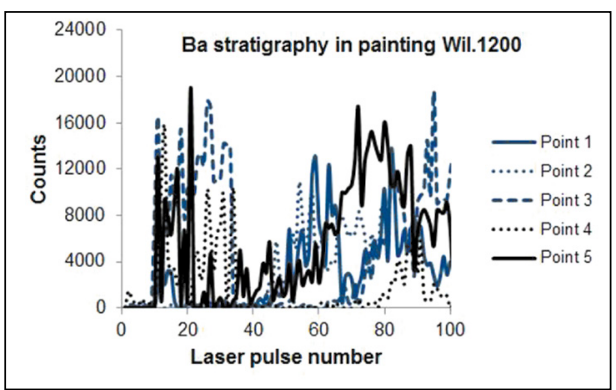

Fig. 12. Ba stratigraphy in various points of the painting Wil.1200 (Teresa Kunegunda portrait).

cial paint layer (typically within 1-30 laser pulses) a certain signal fall is observed, next followed by gradual increase of the analysed signal intensity (usually from $30^{\text {th }}-40^{\text {th }}$ laser pulse), which sometimes spreads over 200-300 laser pulses. This signal rise can be interpreted as a start of a ground layer. Since in some measurement points both painting and ground layer are very thin (see Fig. 17), we selected for a further analysis signal depths between 40 and 60 laser pulses as representatives of upper parts of ground layers. Similar signal behaviour is visible in Figs. 13-15, 16 (left) and 17 (right).

The stratigraphy distributions in Fig. 12 reveal that the barium content in points $1200.1,1200.2,1200.3,1200.4$ and 1200.5 , shown in Fig. 9 as an average factor for the entire painting for depths 40-60 laser pulses, can be clearly observed outside the depth of 40-60 pulses, and show a clear barium signal in the surface parts for all tested points of the painting. Moreover, in this painting the $\mathrm{Ba}$ signal is stronger than $\mathrm{Li}$ one.

Synthetic barium sulfate $\left(\mathrm{BaSO}_{4}\right)$ has been used since the earlier $19^{\text {th }}$ century as a white pigment but $\mathrm{Ba}$ was identified by Alain R. Duval in coloured grounds of paintings created by French artists between 1620 and 1680 in Paris $[11,12]$. The author of that study argued that it was used as an 


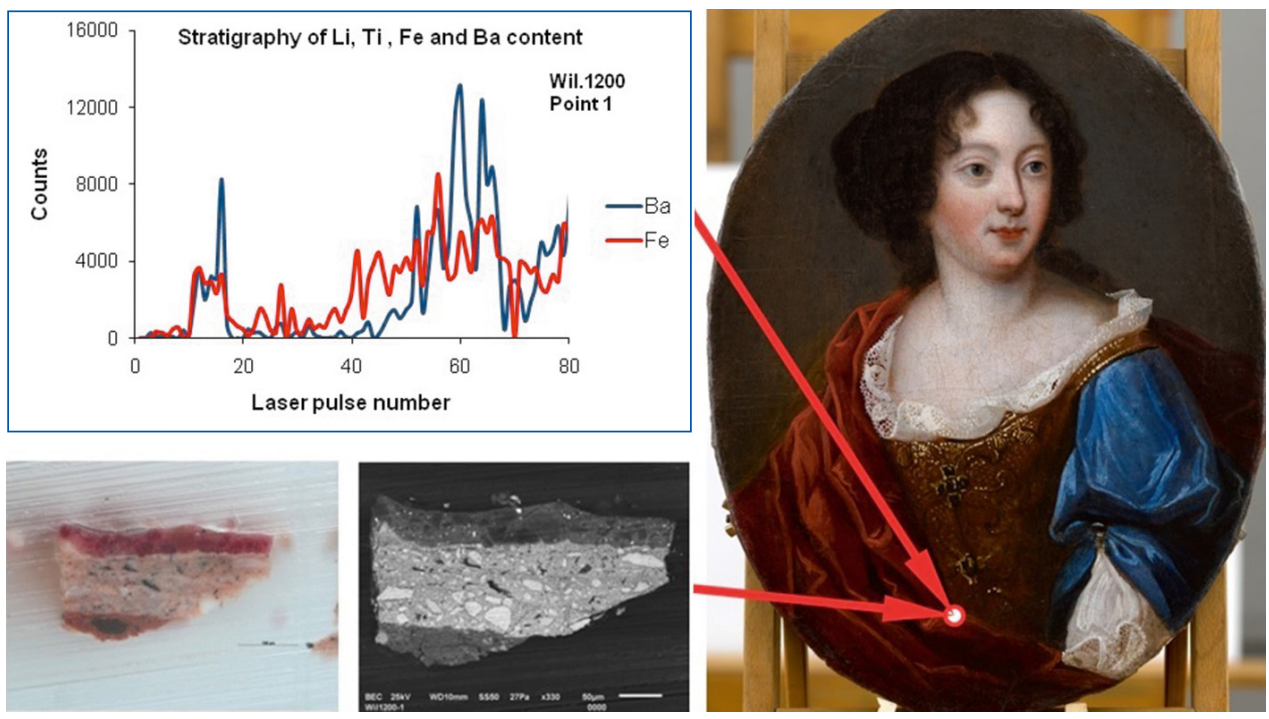

Fig. 13. Ba stratigraphy in point 1 of the painting 1200 (Teresa Kunegunda portrait), photos of cross-sections by Z. Cermakova, J. Hradilowa ALMA laboratory.

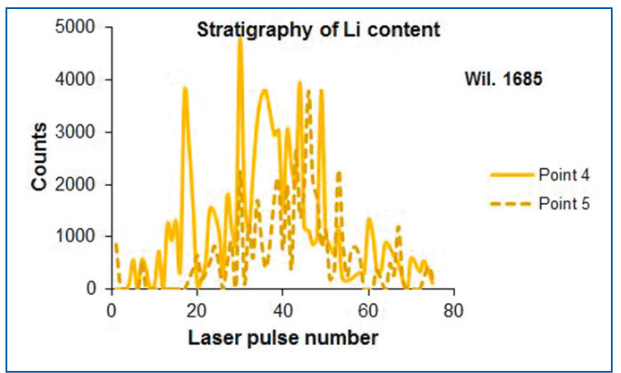

Fig. 14. Li stratigraphy in points 4 and 5 of the painting Wil.1685 (equestrian portrait of the king).

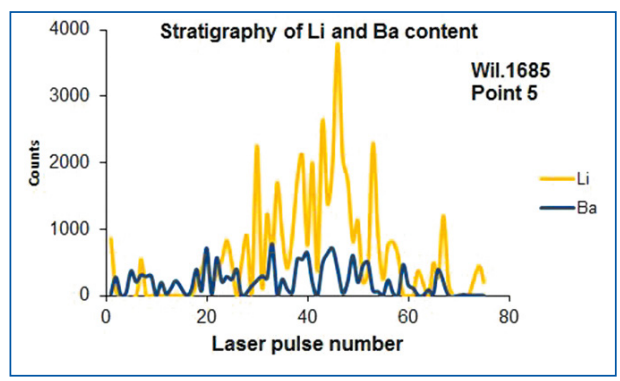

Fig. 15. Li and Ba stratigraphy in point 5 of the painting Wil.1685 (equestrian portrait of the king). extender in grounds supplied by an unidentified dye provider operating in Paris in the $17^{\text {th }}$ century. Having examined grounds in 155 paintings by $17^{\text {th }}$ and $18^{\text {th }}$ century French painters, the study further established that barite was present in grounds layers prepared on the basis of earth pigments rich in iron oxides. It could have been added to increase the weight of the merchandise (in oil binding agents, $\mathrm{BaSO}_{4}$ is completely colourless) or probably as a natural impurity. SEM-EDS analyses of Teresa Kunegunda Sobieska's portrait [13] confirm these observations, as in this portrait $\mathrm{Ba}$ was identified within a particle surrounded with a red particle of red iron. Examples of SEM-EDS analyses for point 1 in the Teresa Kunegunda portrait are shown in Fig. 13.

In the equestrian portraits (Wil.1685 and Wil.1686) the lithium signal was much stronger than the less evident barium one, which is shown in Figs. 16 and 17 (left), and was several times higher as compared to that 


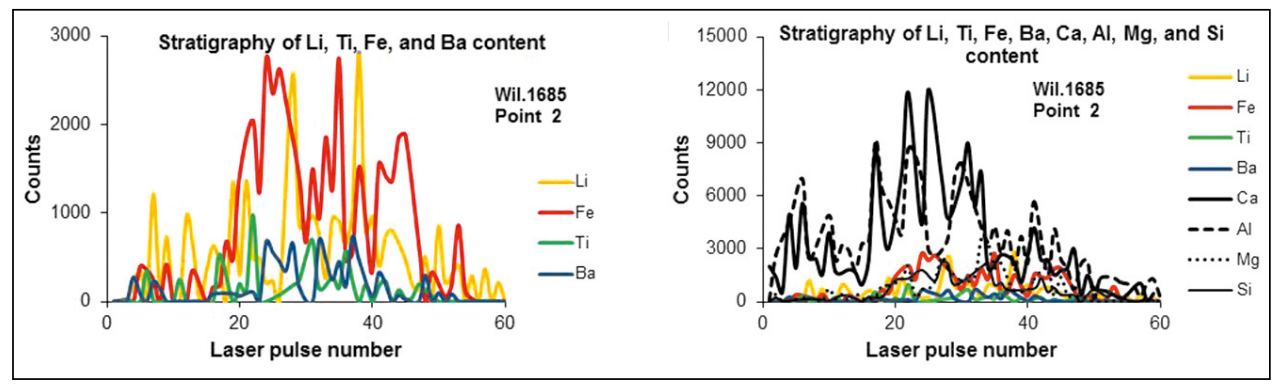

Fig. 16. $\mathrm{Li}, \mathrm{Ti}, \mathrm{Fe}$, and $\mathrm{Ba}$ stratigraphy (left) and $\mathrm{Li}, \mathrm{Ti}, \mathrm{Fe}, \mathrm{Ba} \mathrm{Ca}, \mathrm{Al}, \mathrm{Mg}$, and $\mathrm{Si}$ (right) for point 2 of the painting Wil.1685 (equestrian portrait of the king).

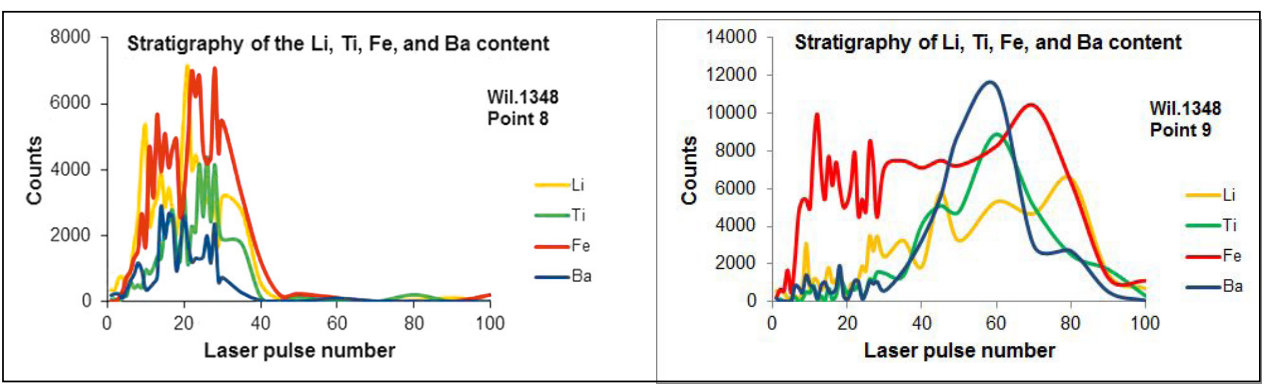

Fig. 17. Li, Ti, Fe and Ba stratigraphy in points 8 and 9 of the painting 1348 (portrait of the King, points 8 - left cheek and 9 - neck refer to carnations).

found in the portrait of Teresa Kunegunda (Wil.1200) - we can see this in Figs. 14 and 15.

Lithium, on the other hand, could be an element found, e.g., in a mineral known as spodumene $\left(\mathrm{Li}_{0.85} \mathrm{Mg}_{0.24} \mathrm{Fe}_{0.91} \mathrm{Si}_{2} \mathrm{O}_{6}\right)$, which had been identified during a previous study conducted using $\mu$-XRPD $[14,15]$ on samples taken from the painting Lato (Summer) plafond by Jerzy Eleuter Szymonowicz Siemiginowski. In Fig. 16 (right) it is shown that the signals from all elements of spodumene like: Li; Si; Mg; Fe were identified in paint and ground layers in the equestrian portrait of the king (Wil.1685).It is not out of the question that, if found in subsequent studies, this mineral content could be a benchmark that could help in painting classification. The same applies to barite content in the grounds and painting layers.

A clear Ti signal was identified in the ground layers of the two portraits of the king. It was not so evident in the case of Teresa Kunegunda portrait and a bit weaker in two equestrian portraits. At the present stage of our study it is not possible to identify the compound being a source of $\mathrm{Ti}$ in these grounds. In the paper by T. Grygar [4] Ti was identified in red-orange bolus grounds found in four baroque paintings. In the $17^{\text {th }}$ century synthetic pigments containing titanium oxide (titanium white) were yet not known, that is why we can suppose that the presence of titanium compounds in grounds can be related to natural admixtures to clays, which are the major components of bolus 
grounds. These clays can be products of the intense weathering of laterite rocks, rich in aluminium and iron compounds, containing, among others, titanium oxide - the anatase. Laterite rocks arise in result of weathering of silicate rocks in a hot and humid climate. This can be an indication for searching such materials among painting products. The authors of the study [4] claim that Ti-contents must be a "fingerprint" of the individual ochres and characteristic of their mineralogical origin.

Moreover, during our work we noted a few unexpected facts. For example, we observed significant differences in the characteristics of painting layers that seemed very similar at first sight. This is shown in two different points for carnations in the bigger painting of the King (Wil.1348), where the paint layer is two times thicker in the neck than in the cheek (see Fig. 17). Additionally, one can observe a significantly higher Ba signal in the thicker layer (point 9), even stronger than the Li signal. This was not observed in other measurement points in the King's portraits.

These occasional, specific observations made the entire analysis very time-consuming, but fortunately sometimes revealed new potential directions for our future painting research with the use of other diagnostic methods and equipment.

\subsection{Factorial analysis}

As it was already mentioned in subsection 2.2 , the comparative study of lithium, barium, iron and titanium content in the grounds was concluded with the multivariate statistical analysis, namely Factorial Analysis (FA), which was applied to find similarities/differences between paint and ground layers and to confirm (or not) the conclusions drawn from the stratigraphy distributions. The results of this statistical approach are shown in Fig. 18. The figure represents the entire chemical composition(not only $\mathrm{Li}, \mathrm{Ti}, \mathrm{Fe}$, and $\mathrm{Ba}$ ) reflected by LIBS spectra in all five paintings taken from depths of 40-60 laser pulses (about $200 \sim 300 \mu \mathrm{m}$ ). Each spectrum is marked as a separate point in the chart, wherein the LIBS spectra for the individual painting are marked with the same colour. The data suggest that the paintings may come from three separate workshops. The first one for equestrian portraits of the King and Queen; the second workshop for the King's portraits and the third one for Teresa Kunegunda portrait. We can observe a certain similarity to the composition of equestrian portraits Wil.1685 and Wil.1686 and the King's portraits Wil.1197 and Wil.1348 in painting undercoats, which corresponds to the similarity of complete LIBS spectra at the depths of 40-60 laser pulses. Thus, the factorial analysis, a mathematical-statistical method, confirms the observations of ours senses and historical examinations. This can be a good point to start further classification of the paintings.

\section{Conclusions}

Finally, the results showed that the LIBS measurements can be used as an efficient method to complement micro-chemical analysis as well as a method to identify and examine artworks.

The presence in grounds of such characteristic elements as $\mathrm{Ti}, \mathrm{Li}$ and $\mathrm{Ba}$ could possibly serve as a basis for classifying paintings.

It should be noticed, however, that in such complex tasks as research of complicated and ambiguous paint layers of oil paintings, the LIBS method is sometimes not sufficient - 


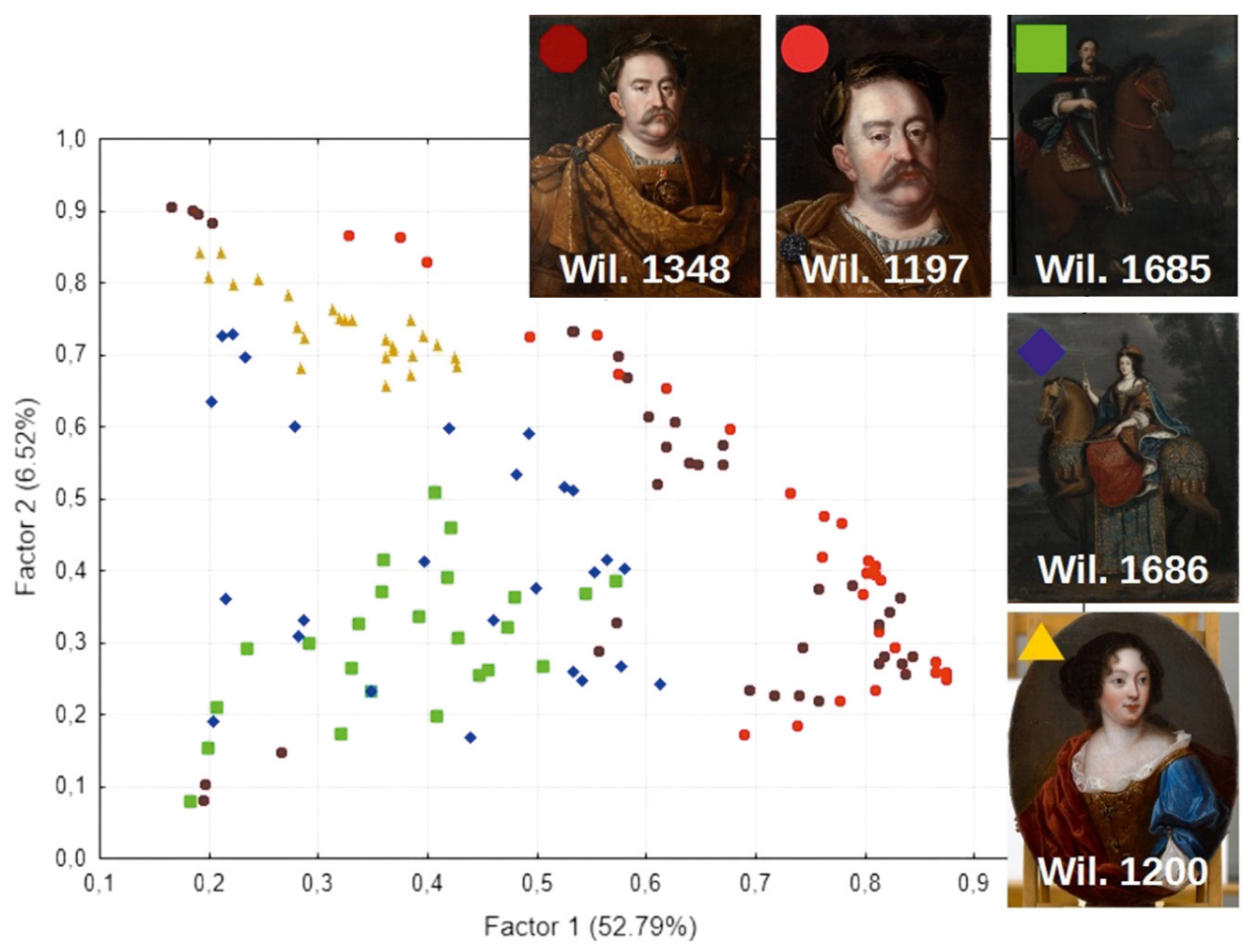

Fig. 18. Factorial analysis of LIBS spectra for depths of 40-60 laser pulses.

it should be complemented by other tests and analyses, for example SEM/EDS and XRPD.

The presented results suggest that:

- the two equestrian portraits of the King and the Queen could be painted by one author, most likely a court painter;

- the two King's portraits probably came from the same workshop but not necessarily the same one as the equestrian portraits;

- the Daughter's portrait clearly exhibits differences in chemical composition of the ground (high $\mathrm{Ba}$ and low Li and Ti concentration) as compared to the other artworks-it was probably created in France.

The research should be and will be continued. In the future research we, are going to expand our investigations to a new set of paintings and try to combine LIBS and microscopy measurements with Raman spectroscopy and other optical, non-destructive optical diagnostic methods (like visible fluorescence spectroscopy or XRF). This should enrich results and new data will be of significant meaning for identification and assignment of major production workshops, art schools, and/or painters performing during the epoch of King Jan III Sobieski.

\section{Acknowledgements}

The authors are deeply grateful to the following co-workers: Sylwia Pawełkowicz-Svorová from the Conservation Laboratory for her SEM/EDS data allowing comparisons with presented LIBS data, Dr Alicja Rafalska-Łasocha and Jowita Dranka from 
the Structural Powder Diffractometry Group at the Faculty of Chemistry of the Jagiellonian University in Kraków, directed by prof. Wiesław Łasocha, for XRPD analysis.

Presented research has been realized in the framework of the contract PBN/03-203/2014/WAT (519/ $\mathrm{KO} / \mathrm{AP} / 2014$ ) with the financial support of the Museum of King Jan III's Palace at Wilanów

\section{References}

[1] W. Skrzeczanowski, A. Pawlak, "Investigations of pigments found in artworks using LIBS method", Report No PBN/03-18)9/2012/WAT,2012, manuscript (in Polish).

[2] W. Skrzeczanowski, A. Pawlak, "Investigations and analyses of pigments in five oil paintingson canvas using 3D Digital microscopy and LIBS method", Report No PBN/03-203/2014/WAT, 2014, manuscript (in Polish).

[3] T. Grygar, J. Hradilova, D. Hradil., P. Bezdicka, "Clay and Iron pigments in the history of painting", Applied Clay Science 22 (2003), 223-236, available online at https://www.researchgate.net/ publication/222554679_Clay_and_iron_pigments_in_the_history_of_painting, DOI: 10.1016/ S0169-1317(03)00076-0 (accesed 29.06.2017).

[4] T. Grygar, J. Hradilova, D. Hradil, P. Bezdicka, S. Baradjieva, "Analysis of earthy pigments in grounds of Baroque paintings", Analytical and Bioanalytical Chemistry 375 (2003) 1154-1160, DOI: 10.1007/ s00216-002-1708-x.

[5] A. Miziolek, V. Palleschi, I. Schechter, Laser-induced breakdown spectroscopy. Cambridge University Press, Cambridge 2006.

[6] E. Kaszewska, M. Sylwestrzak, J. Marczak, W. Skrzeczanowski, M. Iwanicka, E. Szmit-Naud, D. Anglos, and P. Targowski, "Depth-Resolved Multilayer Pigment Identification In Paintings: Combined Use of Laser-Induced Breakdown Spectroscopy (LIBS) and Optical Coherence Tomography (OCT)",
Applied Spectroscopy, 8 (2013)960-972, DOI: 10.1366/12-06703

[7] I. T. Jolliffe, Principal Component Analysis, second ed., Springer-Verlag, New York 2002.

[8] N. H. Timm, Applied Multivariate Analysis. Springer-Verlag, New York 2002.

[9] STATISTICA10 PL, 2011.

[10] E. Modzelewska, A. Pawlak, A. Selerowicz, W. Skrzeczanowski, J. Marczak, "Use of the LIBS method in oil paintings examination, based on examples of analyses conducted at the Wilanow Palace Museum", Proc. SPIE 8790 (2013) 879005, DOI: $10.1117 / 12.2020694$

[11] A. R. Duval, "Les preparation colorées des tableaux de l'école française des dix-septième et dix-huitième sciècles", Studies in Conservations, 37 (1992) 239-258, DOI: 10.1179/sic.1992.37.4.239

[12] A. R. Duval, "Les enduits de preparation de Nicolas Poussin”, Techné, 1, (1994) 35-41.

[13] S. Pawełkowicz, P. Bauerova, J. Hradilova, Z. Cermakova, P. Svora, A. Borkowski, K. Małek, E. Staniszewska, "Report on the stratigraphy and identification tests of the Teresa Kunegunda Sobieska portrait Wil. 1200", August 2013, manuscript (in Polish).

[14] M. Grzesiak-Nowak, A. Rafalska-Łasocha, J. Dranka, A. Pawlak, S. Pawełkowicz, W. Łasocha, "X-ray micro-diffraction and SEM-EDS analyses of pigments and grounds in three paintings from the Museum of King Jan III's Palace at Wilanów collection", OpusculaMusealia, 23 (2015), http://www. ejournals.eu/Opuscula-Musealia/2015/Volume-23/ art/7225/ (accessed 03.06.2016,in Polish).

[15] J. Dranka, "The use of X-ray micro-diffraction in the research of cultural heritage objects", Bachelor dissertation performed in the Structural Powder Diffractometry Group at the Faculty of Chemistry of the Jagiellonian University in Krakow, directed by prof. W. Łasocha, 2014, manuscript (in Polish). 


\title{
Elemental analysis of Chinese red seal inks on xuan paper by ArF laser-induced plume fluorescence
}

\author{
Bruno Yue Cai ${ }^{1 *}$, Vincent Motto-Ros ${ }^{2}$, Vincent Detalle ${ }^{3}$, Judy T.S. Lum ${ }^{4}$, \\ Kelvin S.Y. Leung ${ }^{4}$, Nai-Ho Cheung ${ }^{5}$
}

1 ANA Artwork Material Analysis Company Limited, 1/F Winner Mansion, 691A, Nathan Road, Mong Kok, Hong Kong, China

2 Université Claude Bernard Lyon 1 and the CNRS (UMR5306), Campus LyonTech- La Doua, Bâtiment Kastler, 10 rue Ada Byron, 69622 Villeurbanne CEDEX, France.

3 Centre de Recherche et de Restauration des Musées de France (C2RMF), Louvre Museum, Paris, France

4 Department of Chemistry, Room T1214, Cha Chi-ming Science Tower, Ho Sin Hang Campus, Hong Kong Baptist University, Hong Kong, China

5 Department of Physics, Room T924, Cha Chi-ming Science Tower, Ho Sin Hang Campus Hong Kong Baptist University, Hong Kong, China

* Corresponding author: brunocai@artworkanalysis.com

\begin{abstract}
Chemical analysis of Chinese red seal ink on xuan paper is useful for the authentication of Asian artwork. The analysis has to be nondestructive and has to accommodate artworks of all sizes. We use ArF laser-induced plume fluorescence to analyze six commercial Chinese red seal inks on xuan paper. Two nano-second laser pulses are required for the analysis. The first $355 \mathrm{~nm}$ laser pulse desorbs a thin layer of the ink to create a plume; the second $193 \mathrm{~nm}$ laser pulse induces multi analytes in the plume to fluoresce. Partial-least-square discriminant analysis of the fluorescence spectra unambiguously sorts the six inks while the sampled area is not visibly damaged even under the microscope. The laser probe can handle samples of arbitrary size and shape, is air compatible, and no sample pretreatment is necessary.
\end{abstract}

Keywords: Invisible Damage, Laser plume analysis, Inks

\section{Introduction}

Red seal imprints are present practically on all Chinese paintings and calligraphies. This is a tradition that dates back to the Qin and Han dynasties over two thousand years ago [1]. In general, each artwork is decorated with multiple seal imprints. They signify, respectively, the author, his alias, his studio, the collectors, well-wishes, idioms and various other historic information [1]. This practice is also common in Japan, Korea, Vietnam and Central Asia [2]. Examination of the seal imprints is therefore critically important in 
establishing the provenance and authenticity of Asian artworks.

For a long time, appraisal of seal imprints has been visually performed by experts who focus on the appearance, style and context of the imprints [3]. While this kind of visual examination is very useful, an objective scientific analysis of the inks can be a powerful complement [4].

The ingredients of the Chinese red seal ink are well documented [1]. The making of the ink paste has a long history. By the Yuan dynasty $(\sim 1300 \mathrm{AD})$, the recipe has evolved to the current oil-based version [1]. Typically, the red pigments are fine powder made from minerals such as cinnabar $(\mathrm{HgS})$, minium $\left(\mathrm{Pb}_{3} \mathrm{O}_{4}\right)$, kermesite $\left(\mathrm{Sb}_{2} \mathrm{~S}_{2} \mathrm{O}\right)$ and other shades of red, but modern inexpensive ones may use red dyes instead [1]. The binder is vegetable oil, usually castor oil, which also acts as a dispersant. Fine powder of kaolin $\left[\mathrm{Al}_{2} \mathrm{Si}_{2} \mathrm{O}_{5}(\mathrm{OH})_{4}\right]$, baryte $\left(\mathrm{BaSO}_{4}\right)$ or other clay is added as filler to give body; and ground mugwort fiber is introduced as an absorbent. The oil, powder and fiber are thoroughly mixed to form the paste. Some manufacturers also add preservatives such as borneol and perfumes $[1,5]$.

Red seal ink can be chemically analyzed by a host of techniques. For the organics, chromatographic separation such as thinlayer [6], high-performance [7], and gas chromatography [7], followed by fluorescence or mass spectrometry are very effective methods of analysis. Transmission Fourier-transform infra-red (FTIR) spectroscopy is also useful in identifying specific bonds [8] To perform these analyses, ink samples have to be scraped from the specimen. They are therefore destructive and cannot be applied to the analysis of valuable artworks.

Alternative techniques that are nondestructive have been deployed. Notable examples include micro attenuated-total-reflectance FTIR ( $\mu$ ATR-FTIR) spectroscopy [9], and laser Raman spectroscopy $[6,10]$. Both techniques are sensitive to the vibrational signature of the inorganic pigments [11]. When these vibrational analysis are performed together with elemental analysis, numerous red inks can be sorted unambiguously [8]. Yet once again, the destructiveness of the elemental analysis step is a concern. For example, energy dispersive $\mathrm{x}$-ray (EDX) analysis will require the extraction of ink sample from the artwork for insertion into the analysis chamber [8]. The invasiveness can be reduced if techniques of higher sensitivity are adopted, such as time-of-flight secondary ion mass spectrometry (TOF-SIMS) [12]. When the analyte elements are present at $\mathrm{mg} / \mathrm{g}$ level and higher, the nondestructive portable $\mathrm{x}$-ray fluorescence ( $\mathrm{pXRF}$ ) probe can be used [13].

We have used pXRF to analyze laser printed black toners on photocopier paper and Chinese black ink on xuan paper $[14,15]$. We showed that it is a good screening tool when the analyte elements are abundant (percent range) and when the specimen is optically thick. However, for the elemental analysis of seal imprints on artwork the inked area is likely to be sparse; and for authentication purposes, the signature elements are probably present at low concentrations. In such cases, we have shown that pXRF is best supplemented by an analytical technique that we named plume-LIF, or PLIF for short [14, 15]. PLIF stands for laser-induced-fluorescence of plumes. The plumes are produced by pulsed laser sampling of the specimen. We have demonstrated that PLIF can simultaneously detect multi-elements, including trace ones [18]. It can pin-point down to $\mu \mathrm{m}$ accuracy, causes no visible damage, is 
air-compatible, requires no sample pretreatment, and can accommodate samples of any size [19].

Therefore, PLIF is ideal for the elemental analysis of red seal inks on artworks. In this proceeding, we report such a study that demonstrates the successful sorting of six Chinese red seal inks based on nondestructive PLIF analysis.

\section{Materials and methods}

\subsection{Materials}

Six Chinese red seal inks coded C1 to C6 were selected. They were all acquired from the well-known ink paste manufacturer Xilengyinshe in Shanghai, China. Details are summarized in Table 1. Their prices ranged from entry grade to midrange. C2 and C5 are particularly similar in color. They are chosen to test the discrimination power of our analysis. Each ink was applied evenly on Chinese xuan paper and allowed to air dry in a dust-free environment for two days at room temperature before use. The inked paper was mounted flat on a 1 " $\times 3$ " glass slide for PLIF analysis.

\subsection{PLIF analysis}

The experimental setup for two-laser-pulse PLIF analysis was reported previously [15]. Briefly, the first pulse from a Nd:YAG laser (Continuum Surelite II, $3^{\text {rd }}$ harmonic, 355 $\mathrm{nm}, 10 \mathrm{~Hz}, 9 \mathrm{~ns}$ ) was focused on the sample to desorb a minute amount $(\leq \mathrm{ng}$ ) of the ink mass. The focal spot at the target surface was $25 \mu \mathrm{m}$ radius $\left(1 / \mathrm{e}^{2}\right)$ and the laser fluence was about $200 \mathrm{~mJ} \mathrm{~cm} \mathrm{~cm}^{-2}$. The sample was translated to avoid repetitive ablation of the same area.

$51 \mu$ s later, the desorbed plume was intercepted transversely by the second laser pulse from an ArF laser (GAM Ex5, 193 nm, 10 $\mathrm{Hz}, 8 \mathrm{~ns})$. The ArF laser beam was focused to a point $8 \mathrm{~mm}$ in front of the plume. The spot size at the plume was $640 \mathrm{~mm} \times 280 \mathrm{~mm}$ and its center was $145 \mu \mathrm{m}$ from the sample surface. The ArF fluence was about $440 \mathrm{~mJ}$ $\mathrm{cm}^{-2}$. The ArF laser pulse vaporized the ink particulates in the plume to produce analyte atoms. The trailing portion of the same 193$\mathrm{nm}$ laser pulse induced the various analytes to fluoresce [20].

Fluorescence emissions were collected and imaged onto an optical fiber bundle

Table 1. The Chinese red seal inks and paper used in this study

\begin{tabular}{lll}
\hline Ink & Brand name & Manufacturer \\
\hline C1 & Qinghua quangming zhusha & Xilengyinshe \\
C2 & Qinghua jingmian zhusha & Xilengyinshe \\
C3 & Qinghua meili zhusha & Xilengyinshe \\
C4 & Fencai jianzhu zhusha & Xilengyinshe \\
C5 & Fencai jingzhi shangpin zhubiao & Xilengyinshe \\
C6 & Fencai tezhi zhenpin zhusha & Xilengyinshe \\
\hline & & \\
\hline xuan & Brand name & Manufacturer \\
Ripe & Yuxing fan xuan & Anhui Sheng Jing Xian Yuquan \\
\end{tabular}


whose other end was mounted at the entrance slit of a $0.5 \mathrm{~m}$ spectrometer (Acton SpectraPro-500) equipped with a gateable intensified charge-coupled device (ICCD, Andor iStar DH738F-63). The ICCD was gated on with the firing of the $193-\mathrm{nm}$ pulse and stayed on for $200 \mathrm{~ns}$. The full width of each captured spectrum was $160 \mathrm{~nm}$ and the instrumental resolution was about $1 \mathrm{~nm}$. This resolution was preserved in all off-line spectral smoothing. Three spectral segments centering respectively on 340,500 and 640 $\mathrm{nm}$ covered the whole spectral range used in this study.

The probed area was viewed by an in-line CCD camera in real-time at $64 \times$ magnification to reveal the sample damage caused by each laser shot.

\subsection{AA and ICPMS analysis}

The elemental compositions of the ink pastes and blank xuan paper were analyzed by atomic absorption (AA) and inductively coupled plasma mass spectrometry (ICPMS) to establish analyte concentration for reference. It was performed by adding $7 \mathrm{~mL}$ concentrated $\mathrm{HNO}_{3}$ (Aristar, $\mathrm{BDH}, \mathrm{UK}$ ) to weighed portion of the seal paste. Digestion was carried out in a temperature programmed ETHOS One Advanced Microwave Digestion System (Milestone S.r.l., Sorisole, Italy). After cooling, the digest was diluted to $25 \mathrm{~mL}$ with MilliQ (18.2 M $\Omega \mathrm{cm}$ Millipore, Billerica, MA, USA) water for subsequent elemental determination by ELAN DRC II ICP-MS (PerkinElmer, Waltman, MA, USA) or Spectra AA 220FS Atomic Absorption Spectrometer (Varian, Agilent Technologies, Santa Clara, CA, USA). Elemental content was quantified against calibrated response curves prepared from $1000 \mathrm{gL}^{-1}$ stocks (VHG, Manchester, $\mathrm{NH}$, USA).

\subsection{Chemometrics}

The chemometric analysis was performed using SIMCA (version 14.0).

\section{Results and discussion}

\subsection{PLIF spectra}

We captured more than a thousand single-shot PLIF spectra of the six inks. Fig. 1 shows spectra of inks C1 (black), C2 (blue), C3 (brown), C4 (green), C5 (orange) and C6 (gray) on xuan. The display format is explained in the figure caption. Each trace is the average of 100 single-shot spectra and consists of three 160-nm-wide regions stitched together. The spectral area of each region is normalized to remove shot-to-shot fluctuations. For the purpose of graphical clarity, the vertical scale of the spectra in the $350 \mathrm{~nm}$ to $410 \mathrm{~nm}$ region was shrunk $3 \times$ to keep the strong $\mathrm{Pb}$ I lines within view. The prominent spectral features are labeled by color-coded lines at the bottom. They demonstrated the multi-analyte detection capability of PLIF. None of the spectral features was visible when either laser beam was blocked. This testifies to the synergy of the two laser pulses.

Based on Fig. 1, four observations can be drawn. First, the spectral features among the six inks are similar, making the sorting very challenging. The line identities are given at the bottom of the plot. Lead is from the abundant kermesite. Chromium and antimony are probably from pigments used to shade the red. Aluminum is presumably from the filler. Carbon dimer is from the oil binder. Sodium and calcium are most likely from the mugwort fiber or vegetable oil.

Second, the examination of the fine spectral differences shows that $\mathrm{C} 6$ has the most 


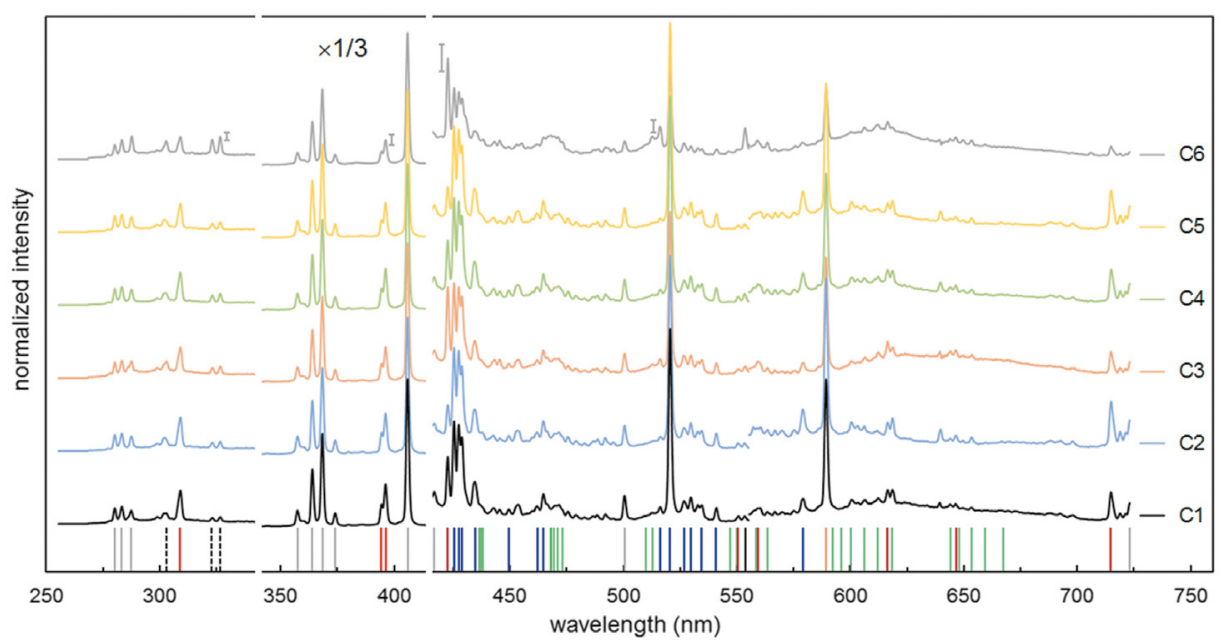

Fig. 1. Normalized PLIF spectra of six red seal inks, C1 to C6. Each trace is the average of 100 single-shot normalized spectra, with the leading pixels zeroed to indicate the baseline. The standard deviations of four selected line intensities of the $\mathrm{C} 6$ trace are indicated by error bars. The traces are offset vertically for graphical clarity. The vertical scale of the $350 \mathrm{~nm}$ to $410 \mathrm{~nm}$ region is shrunk $3 \times$ to keep the strong $\mathrm{Pb}$ I lines within view. Identities of the more prominent spectral lines are color-coded at the bottom: Al I (red), Ba I (black), Ca I (brown), Cr I (blue), Na I (orange), $\mathrm{Pb}$ I (gray), Sb I (black, dotted), and $\mathrm{C}_{2}$ band heads (green).

intense $\mathrm{Sb}$ (323.3 and $326.8 \mathrm{~nm}$ ), Ca (422.7 $\mathrm{nm})$, and $\mathrm{C}_{2}(516.5 \mathrm{~nm}$ band head) features and can be readily sorted. The remaining five inks can then be sorted based on C4 having the brightest Al lines (309.2, 394.4 and 396.2 $\mathrm{nm}$ ) while $\mathrm{C} 5$ the dimmest, and $\mathrm{C} 1$ has the weakest $\mathrm{Sb}$ lines. Then between the last two inks $\mathrm{C} 2$ and $\mathrm{C} 3$, the latter has a significantly brighter Ca line $(422.7 \mathrm{~nm})$. While these distinguishing features are not the brightest spectral lines, their intensities are still many times above the noise and are therefore reliable. This is evident from the relative heights of the error bars drawn against them (Fig. 1, C6 trace).

Third, inks C2 and C5 are not only similar in color, their spectra are also very similar. Careful study shows that $\mathrm{C} 5$ has weaker Al I lines than $\mathrm{C} 2$.

Fourth, the spectral intensities displayed in Fig. 1 do not seem to correlate with the abundance of the elements (Table 2). For example, $\mathrm{C} 6$ has about ten times less lead than $\mathrm{C} 1$ and $\mathrm{C} 2$ but its $\mathrm{Pb}$ I lines are not that much weaker. The discrepancy can be partially explained by the fact that the spectra shown in Fig. 1 are normalized. The intensities are magnified relative to the empirical PLIF spectra by the factors shown in Table 3. As can be seen, the Pb I lines of C6 are magnified about three times more than those of $\mathrm{C} 1$ and $\mathrm{C} 2$. Another complication is the fact that AA and ICPMS analyzed ink pastes rather than ink imprints on xuan. Fractionation of the ink chemicals at the seal imprinting step and during the plume formation process can occur.

\subsection{Chemometric analysis}

Based on the PLIF spectra, we used partialleast-square discriminant analysis (PLSDA) 
Table 2. Concentrations ( $\mathrm{mg} / \mathrm{g}$ ) of the more abundant elements present in the six Chinese red seal ink pastes and the xuan paper used in this study, as measured by atomic absorption (AA) and inductively-coupled plasma mass spectrometry (ICPMS)

\begin{tabular}{lccccccc} 
& \multicolumn{9}{c}{$\mathrm{mg} / \mathrm{g}$} \\
\cline { 2 - 8 } & \multicolumn{9}{c}{$\mathrm{AA}$} & & & ICPMS \\
\cline { 2 - 8 } & $\mathrm{Pb}$ & $\mathrm{Hg}$ & $\mathrm{Cr}$ & $\mathrm{Ca}$ & $\mathrm{Al}$ & $\mathrm{Sb}$ & $\mathrm{Ba}$ \\
\hline xuan & - & 0.004 & 0.006 & 1.34 & 0.578 & - & 0.003 \\
ink C1 & 148 & 6.94 & 18.1 & 7.46 & 2.14 & 0.965 & 0.387 \\
ink C2 & 93.9 & 20.5 & 6.94 & 5.88 & 1.92 & 1.02 & 0.407 \\
ink C3 & 39.8 & 12.0 & 1.75 & 10.6 & 0.981 & 0.457 & 0.995 \\
ink C4 & 57.0 & 44.7 & 8.86 & 13.2 & 1.14 & 7.00 & 1.51 \\
ink C5 & 53.7 & 2.94 & 5.01 & 10.3 & 1.32 & 1.11 & 1.31 \\
ink C6 & 10.7 & 3.09 & 1.29 & 21.3 & 0.444 & 0.208 & 1.18 \\
\hline
\end{tabular}

Table 3. The normalized spectra shown in Fig. 1 are magnified relative to the empirical PLIF spectra. Tabulated are the magnification factors for the corresponding inks for the three respective spectral segments centering at 340,500 and $640 \mathrm{~nm}$

\begin{tabular}{lccc} 
& \multicolumn{3}{c}{ Scaling factor } \\
\hline ink & $340 \mathrm{~nm}$ & $500 \mathrm{~nm}$ & $640 \mathrm{~nm}$ \\
\hline C1 & 1.48 & 0.56 & 1.00 \\
C2 & 1.02 & 0.40 & 1.34 \\
C3 & 2.20 & 0.75 & 1.06 \\
C4 & 1.27 & 0.52 & 1.15 \\
C5 & 1.43 & 0.53 & 1.10 \\
C6 & 3.97 & 0.98 & 1.23 \\
\hline
\end{tabular}

to sort the six inks. For that purpose, we captured on different days two independent sets of spectral data from sample replicates. One set is used to train the PLSDA model while the other set is used to test the model. The training set consists of 200 single-shot panoramic spectra covering the three spectral regions $(1024 \times 3$ pixels $)$ for each of the six inks. In PLSDA language, we have a supervised six-class model with each ink belonging to one class; $1024 \times 3$ variables, $200 \times 6$ training observations. The test set consists of 100 single-shot panoramic spectra for each of the six inks, that is, a total of $100 \times$
6 test observations. The class identity of each of the observations is known.

For data pre-processing, we normalized the total intensity of each 1024-pixel single-shot spectrum to 5,000 to remove the shot-to-shot fluctuations. We then mean-centered the data values for each of the $1024 \times 3$ variables. No outliers are discarded from either the training or the test set.

The sorting accuracy of the $100 \times 6$ test observations is shown in Table 4 in the form of a confusion matrix. As can be seen, the sorting accuracy is $100 \%$ for inks C2, C3 and C6. C4 has the lowest accuracy of $87 \%$. It is mainly confused for $\mathrm{C} 1(8 / 100)$ and $\mathrm{C} 2$ $(4 / 100)$. Overall, the sorting accuracy is $96.2 \%$.

\subsection{Coefficient spectra}

The weight coefficients for the $1024 \times 3$ variables used by the PLSDA model are plotted in Fig. 2 in the form of coefficient spectra. Several features can be noted. First, none of the inks can be unambiguously identified by a single spectral feature. Multiple features have to be weighed. Analytical techniques that can detect multi analytes simultaneously 
Table 4. Confusion matrix of the sorting of six Chinese red seal inks on xuan

\begin{tabular}{|c|c|c|c|c|c|c|c|c|}
\hline \multirow{2}{*}{$\begin{array}{l}\text { Test } \\
\text { class }\end{array}$} & \multirow{2}{*}{$\begin{array}{l}\text { \# test } \\
\text { observations }\end{array}$} & \multicolumn{6}{|c|}{ Model class } & \multirow{2}{*}{ Correct } \\
\hline & & $\mathrm{C} 1$ & $\mathrm{C} 2$ & $\mathrm{C} 3$ & $\mathrm{C} 4$ & C5 & C6 & \\
\hline $\mathrm{C} 1$ & 100 & 92 & 4 & 1 & 3 & 0 & 0 & $92 \%$ \\
\hline $\mathrm{C} 2$ & 100 & 0 & 100 & 0 & 0 & 1 & 0 & $100 \%$ \\
\hline $\mathrm{C} 3$ & 100 & 0 & 0 & 100 & 0 & 0 & 0 & $100 \%$ \\
\hline $\mathrm{C} 4$ & 100 & 8 & 4 & 0 & 87 & 0 & 1 & $87 \%$ \\
\hline C5 & 100 & 0 & 1 & 0 & 1 & 98 & 0 & $98 \%$ \\
\hline C6 & 100 & 0 & 0 & 0 & 0 & 0 & 100 & $100 \%$ \\
\hline & & & & & & & avg & $96.2 \%$ \\
\hline
\end{tabular}

are therefore preferred and PLIF is such a technique. Second, the weighed features correspond to the prominent lines of $\mathrm{Pb}, \mathrm{Cr}$, $\mathrm{Ca}, \mathrm{Sb}, \mathrm{Al}, \mathrm{Na}, \mathrm{Ba}$, and $\mathrm{C}_{2}$ identified in Fig. 1. They are also indicated at the bottom of Fig. 2. Third, because mean-centering was used in the data pre-processing, intensities below the mean will be weighed negatively. An example is the Sb I 323.3 and $326.8 \mathrm{~nm}$ doublet of $\mathrm{C} 1$. Fourth, the coefficient spectra of C2, C3 and C6 are less noisy. This is consistent with their high sorting accuracy (100\%). In contrast, the coefficient spectrum of C4 is the noisiest. The corresponding sorting accuracy is also the lowest (87\%).

\subsection{Invisible damage}

We investigated the damage to the sample caused by the $355 \mathrm{~nm}$ laser ablation. We used ink $\mathrm{C} 1$ on xuan as a typical specimen. Micrographs of the craters are shown in

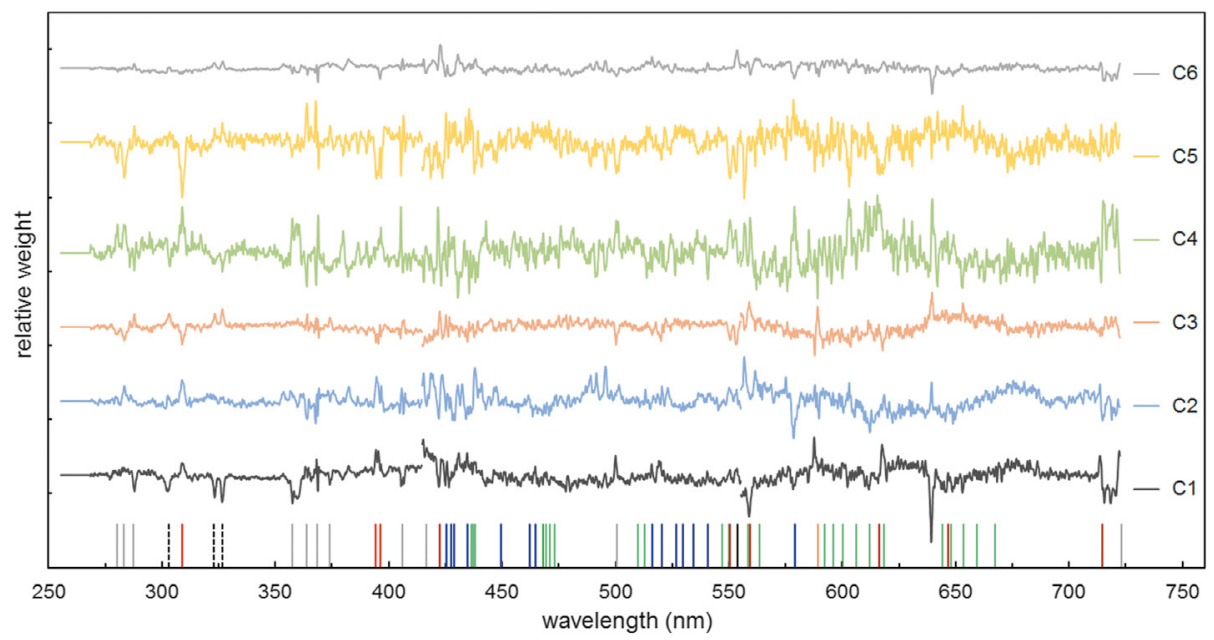

Fig. 2. PLSDA coefficient spectra of the six red seal inks, $\mathrm{C} 1$ to C6. The traces are offset vertically for graphical clarity. The leading pixels of each trace are set to zero to indicate the baseline. Identities of the prominent spectral lines are color-coded at the bottom: Al I (red), Ba I (black), Ca I (brown), Cr I (blue), Na I (orange), $\mathrm{Pb}$ I (gray), Sb I (black, dotted), and $\mathrm{C}_{2}$ band heads (green). 

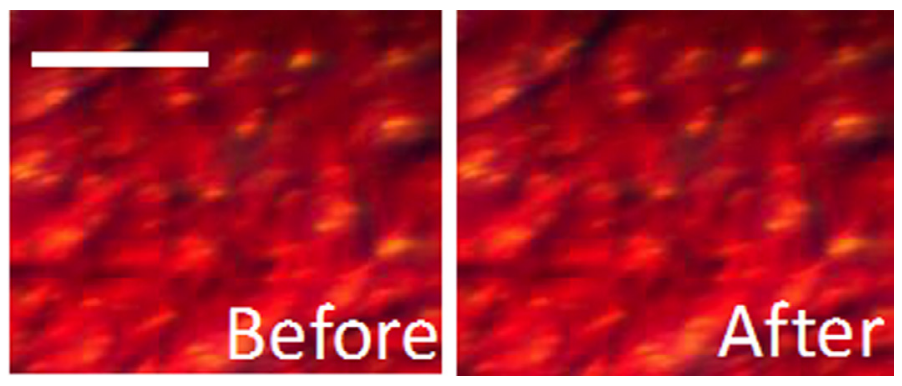

Fig. 3. Micrographs of the craters. The left panel shows the region of interest before the ablation. The right panel shows the same region after ablation by one $355 \mathrm{~nm}$ laser pulse at $200 \mathrm{~mJ} / \mathrm{cm}^{2}$ which is the fluence used in the PLIF analysis. Scale bar represents $100 \mu \mathrm{m}$.
Fig. 3. The left panel shows the region of interest before the ablation. The right panel shows the same region after ablation by one $355 \mathrm{~nm}$ laser pulse at $200 \mathrm{~mJ} / \mathrm{cm}^{2}$ which is the fluence used in the PLIF analysis. As can be seen, damage was not visible even under $64 \times$ magnification.

\section{Conclusions}

We analyzed six commercial Chinese red seal inks that were coated on xuan paper. The inks are from the same manufacturer which is well known in China. Two of the six inks were identical in color and were purposely selected to present analytical challenges. We analyzed the samples by a two-laser-pulse PLIF scheme performed on a high precision optical setup. The first $355 \mathrm{~nm}$ laser pulse ablated a thin layer of the ink; the second $193 \mathrm{~nm}$ laser pulse induced multi analytes in the desorbed ink to fluoresce. Based on the fluorescence spectra, we could tell all the inks apart by partial-least-square discriminant analysis, including the two challenging ink samples. Meanwhile, the sampled area was not visibly damaged even under $64 \times$ magnification.

We plan to extend the PLIF analysis to more seal inks from different manufacturers and different eras in order to establish a comprehensive spectral library. At the same time, we will use pXRF, PLIF and $\mu$ ATR-FTIR to analyze seal inks and to determine the age of the print.

\section{Acknowledgements}

We thank Dr. Xueshi Bai of Centre de Recherche et de Restauration des Musées de France and Dr. Delphine Syvilay of Laboratoire Recherche des Monuments Historiques for useful discussions. This work is supported by ANA Artwork Material Analysis Company Limited under the Hong Kong Innovation and Technology Commission Technology Start-up Supporting Scheme 2016/2017.

\section{References}

[1] S. Deng, Zhuankexue (A study in the art of seal carving), People's Fine Arts Publishing, Beijing 1979. In Chinese.

[2] L. Long, A Ricci Dictionary of Seals, An English and French Dictionary of Chinese Seals and of the Art of Seal Carving, posted by Association Ricci, Paris, http://www.grandricci.org/paraitre_sceaux_leaflet. pdf (accessed 26.10.2016).

[3] Q.Ye, Gu xiyin yu gu xiyin jianding (Ancient seals and how to appraise them), Cultural Relics Publishing House, Beijing 1997. In Chinese.

[4] T. Learner, Analysis of Modern Paints, The Getty Conservation Institute, Los Angeles 2004.

[5] Instructions for the preparation of the red seal ink paste, in Chinese, http://zhidao.baidu.com/ link?url=wm5qRj6i2 cr9hz-GnGNCBS4Pu3_lgJCbGlTfPUClYCSeOo_MkkqJ7N-M7Mbdw0Yn1cD14_ 
DFfWU8DTZLD4chIXf42Vt8GE6itDbUSkDze2S (accessed 26.10.2016).

[6] Raza and B. Saha, "Application of Raman spectroscopy in forensic investigation of questioned documents involving stamp inks", Science and Justice, 53 (2013) 3338, DOI: 10.1016/j.scijus.2012.11.001.

[7] Y.-T. Yao, J. Song, J. Yu et al., "Differentiation and dating of red ink entries of seals on documents by HPLC and GC/MS", Journal of Separation Science, 32 (2009) 291927, DOI: 10.1002/jssc.200900110.

[8] Vila and J.F. Garcia, "Analysis of the chemical composition of red pigments and inks for the characterization and differentiation of contemporary prints", Analytical Letters, 45 (2012) 127285, DOI: 10.1080/00032719.2012.673100.

[9] W. Dirwono, J.S. Park, M.R. Agustin-Camacho, et al., "Application of micro-attenuated total reflectance FTIR spectroscopy in the forensic study of questioned documents involving red seal inks", Forensic Science International, 199 (2010) , DOI: 10.1016/j.forsciint.2010.02.009.

[10] J. Lv, S. Liu, Y.L.J. Feng, Y. Liu and S. Zhou, "Differentiation of inks used for seals by confocal Raman microscopy and Fourier transform infrared microscopy", Spectroscopy, 30 (2015) 33 http://www.spectroscopyonline.com/ differentiation-inks-used-seals-confocal-raman-microscopy-and-fourier-transform-infrared-microscopy?pageID $=1$ (accessed 8.07.2017).

[11] S. Vahur, U. Knuutinen, and I. Leito, "ATRFTIR spectroscopy in the region of $5030 \mathrm{~cm}-1$ for identification of inorganic red pigments", Spectrochimica Acta A, 73 (2009) 7671, DOI: 10.1016/j. saa.2009.03.027.

[12] J. Lee, C. Lee, K. Lee, and Y. Lee, “TOF-SIMS study of red sealing-inks on paper and its forensic applications", Applied Surface Science, 255 (2008) 152526, DOI: 10.1016/j.apsusc.2008.05.094.

[13] F. Rosi, A. Burnstock, K.J. Van den Berg, C. Miliani, B.G. Brunetti, and A. Sgamellotti, "A non-invasive XRF study supported by multivariate statistical analysis and reflectance FTIR to assess the composition of modern painting materials", Spectrochimica Acta A, 71 (2009) 165662, DOI: 10.1016/j. saa.2008.06.011.

[14] P.C. Chu, B.Y. Cai, Y.K. Tsoi, R. Yuen, K.S.Y. Leung, and N.H. Cheung, "Forensic analysis of laser printed ink by $\mathrm{x}$-ray fluorescence and laser-excited plume fluorescence", Analytical Chemistry, 85 (2013) 431315, DOI: 10.1021/ac400378q.

[15] Y. Cai, Z. Huang, M.H.C. Cheung, et al., "Elemental analysis of Chinese black inks on xuan paper by ArF laser-excited plume fluorescence", Analytical Chemistry, 88 (2016) 10970978, DOI: 10.1021/acs. analchem.6b02628.

[16] S.K. Ho and N.H. Cheung,"Minimally destructive and multi-element analysis of aluminum alloys by ArF laser-induced atomic emissions", Journal of Analytical Atomic Spectrometry, 22 (2007) 2997, DOI: 10.1039/B611899B.

[17] S.K. Lau and N.H. Cheung, "Minimally Destructive and Multi-Element Analysis of Steel Alloys by Argon Fluoride Laser-Induced Plume Emissions", Applied Spectroscopy, 63 (2009) 8338, DOI: $10.1366 / 000370209788700973$.

[18] P.C. Chu, W.L. Yip, Y. Cai, and N.H. Cheung, "Multi-element analysis of ceramic and polymeric samples by ArF laser excited atomic fluorescence of ablated plumes", Journal of Analytical Atomic Spectrometry, 26 (2011) 121216 DOI: 10.1039/ C1JA10026B.

[19] Y. Cai, P.C. Chu, S.K. Ho, and N.H. Cheung, "Multi-element analysis by ArF laser excited atomic fluorescence of laser ablated plumes: Mechanism and applications", Frontiers of Physics, 7 (2012) 6778 DOI: $10.1007 / \mathrm{s} 11467-012-0264-x$.

[20] X. Wang, Z. Huang, P.-C. Chu, et al., "The mechanism of ArF laser-induced fluorescence of dense plume matter", Journal of Analytical Atomic Spectrometry, 31 (2016) 236374, DOI: 10.1039/ C6JA00290K. 



\section{Case studies}





\title{
Using the new G.C. Laser Cleaning System for cleaning and surface preparation for re-gilding of a large outdoor bronze monument of Alexander Hamilton
}

\author{
Andrzej Dajnowski ${ }^{1 \star}$, Bartosz A. Dajnowski ${ }^{1,2}$ \\ 1 CSOS Inc., 900 S. Des Plaines Ave, Forest Park, IL 60130, USA \\ 2 GC Laser Systems Inc., 900 S. Des Plaines Ave, Forest Park, IL 60130, USA \\ * Corresponding author: adajnowski@csosinc.com
}

\begin{abstract}
In this paper, the conservation of the Alexander Hamilton Monument by John Angel from the Chicago Park District and the Ferguson Monument Fund will be discussed. The monument was installed in 1952 and was re-gilded in 1983. By 2015, over $80 \%$ of the gilding had failed and fallen off. Successful gilding of outdoor monuments requires very thorough surface preparation in order to remove all chlorides and active corrosion products. Inter-granular corrosion is a particular problem that is often difficult to resolve. In order for gilding to last, a bronze has to be as corrosion-free as possible. Traditional surface preparation techniques such as media blasting and ultra high-pressure washing carry the risk of causing physical changes to surface topography of the metal, closing surface pores and embedding salts. They may also leave active chlorides behind. In comparison, laser cleaning is more environmentally friendly as there is no contaminated media waste disposal and the precision and control during cleaning is unparalleled. The results of laser cleaning and surface preparation for re-gilding using the new tunable $1064 \mathrm{~nm}$ pulsed G.C. Laser Cleaning Systems, specifically designed for art conservation treatments, will be presented.
\end{abstract}

Keywords: laser cleaning, gilding, corrosion, primer, oil size, bronze

\section{Introduction}

The monument to Alexander Hamilton was commissioned by Kate Sturges Buckingham in the 1930's in Chicago and sculpted by John Angel [1]. Although one monument to Hamilton already existed in Chicago, Sturges strongly believed that the first Secretary of the Treasury and the man who created the financial foundation of the new republic deserved more recognition for what he had done for the country. In her will she donated
$\$ 1$ million dollars to the trustees of the Art Institute of Chicago for the creation of the monument to Hamilton. She also established a timeline of 10 years after which the money would no longer be available. The monument was likely gilded by that time and kept in indoor storage from the 1930s to the 1950s. There is no available information on the condition of the sculpture, the storage environment, or the surface preparation for the first gilding. However, it is known that war and the economical situation in the USA at 


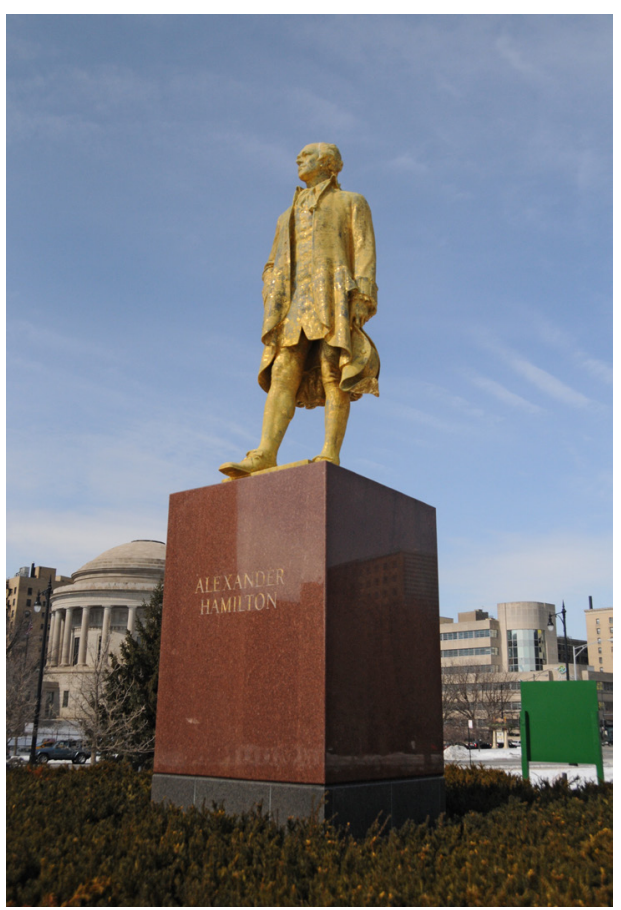

Fig. 1. Hamilton Monument before treatment (Lincoln Park, Chicago).

the time caused the delay of the installation. Although the sculpture was finished in clay in 1939 and cast in 1940, it was not installed until $1952[1,2]$. It is recognized that the sculpture was re-gilded in 1983 and within a decade the new re-gilding application had severely failed. The intent for this sculpture's appearance is for it to be gold, so its deteriorated condition was not acceptable.

The selection of an appropriate technique for treatment was discussed. In order to help ensure the longevity of the treatment, it was agreed that it was necessary to remove all of the copper alloy corrosion products from the bronze surface. The main goal of this treatment was to re-gild the sculpture and provide a stable surface finish that will last as long as possible outdoors. Laser cleaning was chosen as the safest and most controllable method for removing corrosion products from the bronze $[4,5,10]$. Other methods such as abrasive cleaning can leave behind or even embed chlorides inside metal pores and carry the risk of altering the surface. This is the first time a historic monumental bronze had been prepared for traditional oil re-gilding with laser ablation.

\section{Examination}

When examined from distance on a sunny day in 2015, the monument of Alexander Hamilton appeared to be golden in color. However, the yellow tone of the sculpture was not actual gold, but rather yellow due to the yellow zinc primer that covered the surface. The 3.91 meter tall, $1,587 \mathrm{~kg}$ bronze was heavily corroded and most of the gilding was missing. Furthermore, the corrosion was masked by the bright yellow tone of the primer and the reflection of the remaining traces of gold.

Upon closer examination, it was obvious that the surface of the sculpture was very deteriorated. Portable XRF, Raman, FTIR, and a $3 \mathrm{D}$ microscopy were used to analyze and examine the condition of the sculpture. The bronze was covered by green and black corrosion products, deteriorated yellow primer, and some traces of gold leaf. Once the sculpture was moved indoors to the CSOS conservation facility, the poor condition of the surface became more apparent (Fig. 2). The re-gilding done in 1983 had severely failed by the 1990s and sunlight reflecting off the yellow surface made the sculpture appear to be in better condition than it was. Poor preparation of the surface and failure to remove active corrosion products prior to re-gilding likely contributed to the fast deterioration of the new gilding. Corrosion 

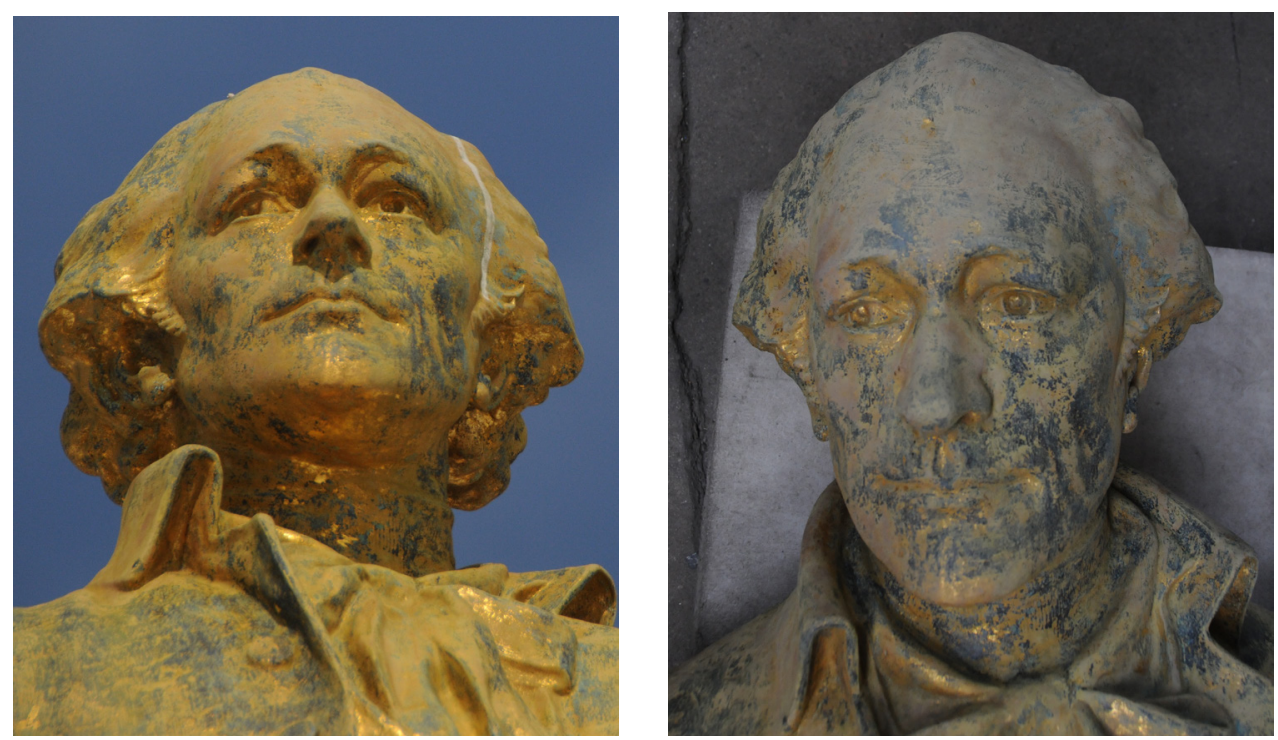

Fig. 2. Detail of the face. Left image taken on a sunny day while the sculpture was still in the park and the image on the right taken indoors without the presence of direct sunlight.

products found on the surface were examined using Raman and FTIR. They were identified as brochantite, copper acetate, antlerite, atacamite, paratacamite, copper oxalate; moreover, some gypsum was found.

\subsection{Raman scattering}

A Horiba XploRA PLUS Raman spectrometer was used with a $785 \mathrm{~nm}$ (red) diode laser operating at a laser power of $1 \%(300 \mathrm{~mW}$ total power). The instrument was frequency calibrated with a silicon reference standard. Data were collected over a range of 100 $\mathrm{cm}^{-1}$ to $2400 \mathrm{~cm}^{-1}$, and the spectral resolution of the instrument is $3 \mathrm{~cm}^{-1}$. A $50 \times$ objective was used for all data collection (J. Mass).

\subsection{FTIR}

A size 11 steel blade scalpel was used to remove small (millimeter sized) samples for molecular analysis by FTIR (Fourier trans- form infrared spectroscopy). Samples were mounted on a diamond half-cell support for transmission mode. Data were acquired and analyzed with a Thermo Scientific Nicolet iN-10 FTIR microscope controlled by OMNIC Picta software. A total of 128 scans were collected over a spectral range of $4000-650 \mathrm{~cm}^{-1}$ with a spectral resolution of 4 $\mathrm{cm}^{-1}$. A set of databases, including the IRUG (Infrared and Raman Users Group) database and a number of commercial databases, were used for identification (J. Mass).

\section{3. $X R F$}

The XRF used for the analysis was tripod-mounted and it was software controlled by a motorized X-Y stage. The resolution of the Elio XRF that was used is $<140 \mathrm{eV}$ at $\mathrm{Mn}$ $K a$ with spatial resolution based on beam size $\approx 1 \mathrm{~mm}$. The instrument used for this study was equipped with an $\mathrm{Rh} \mathrm{X}$-ray tube, and the data collected was normalized against 
standards to remove Rh peaks. XRF analysis of the metal established that the sculpture is bronze, that it is composed of $89.21 \%$ copper, $5.99 \%$ of tin, $2.24 \%$ zinc, $2.13 \%$ of lead, with trace amounts of $\mathrm{Ni}, \mathrm{Fe}, \mathrm{Ti}$ and $\mathrm{Cr}$ in it (error of 0.08 to $1.04 \%$ ). The alloy composition measurements were performed on an area of bare metal free of corrosion, primer and gold.

Figure 3 shows the surface of the deteriorated gilding and active corrosion products. The area photographed is approximately 1.0 $\mathrm{mm}$ wide. The gold and primer are cracked and separated from the actively corroding metal substrate.

The examination of samples of the gilding revealed that there are two double layers of dence that the gilding performed in 1983 was done without complete corrosion removal and without complete removal of the old primer. According to the historical information, the sculpture was "cleaned for one week by two workers" [2]. First of all, the surface was likely washed and mechanically cleaned. Secondly, the new zinc chromate primer was applied in two layers on top of the old gilding and then the surface of the bronze was re-gilded. This kind of surface preparation is not sufficient for the treatment of an outdoor sculpture that is to be gilded and exposed to Chicago weather. If the sculpture was kept in a controlled indoor environment, then perhaps the re-gilding would have survived in better condition. In the case of outdoor

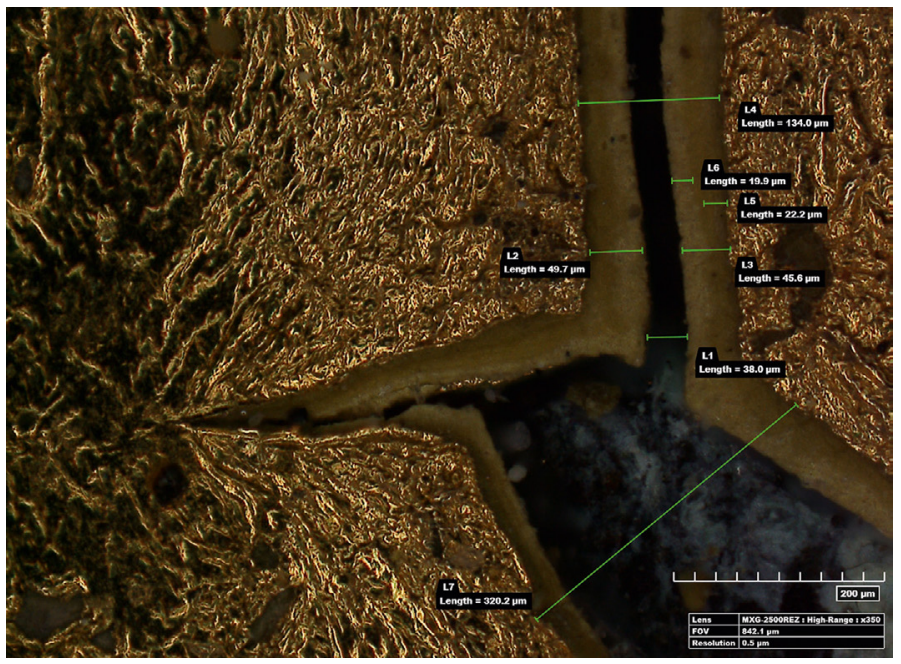

Fig. 3. 3D Micrograph illustrating the deterioration of the gilding and active corrosion. primer separated by a layer of gold in the middle. The top of the cross-section (Fig. 4) shows small areas of gold that was preserved on the top of the original primer. The dark bottom layer (under the primer) was composed typically of the green corrosion that was found in all of our samples. This sample illustrates that corrosion was undercutting the gold and primer layers. It provides evi- gilded bronzes, leaving deteriorated layers of primer and any surface corrosion under a new gilding campaign is a recipe for failure in a short period of time. The preservation of original and stable surface layers is usually preferred in conservation; however, it must also be taken into consideration that repeated re-treatments are not desirable for an object, and omitting or simply hiding inherent prob- 


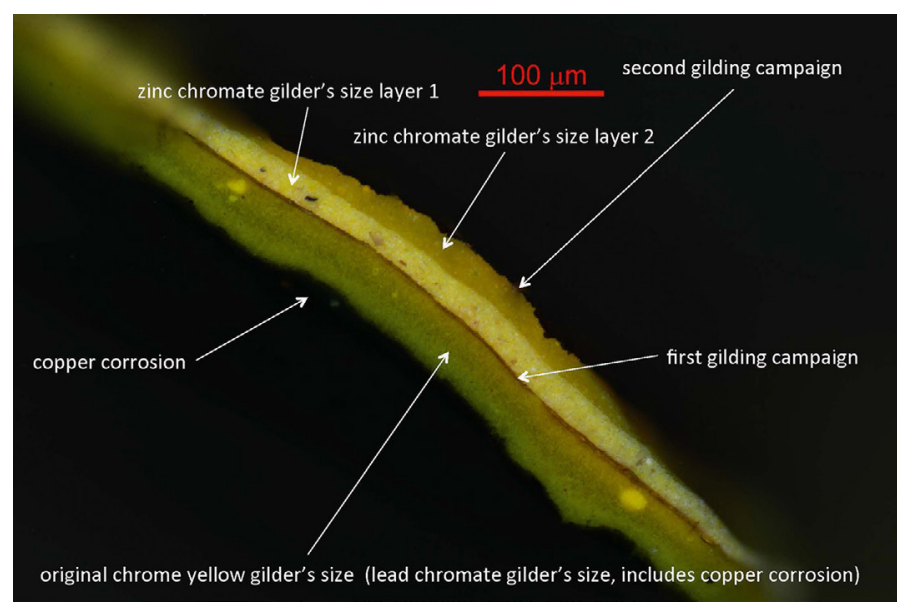

Fig. 4. Cross-section microscopy image of a sample of the gilding where layers from different gilding campaigns are visible (prepared by J. Mass and A. Finnefrock). lems on a surface is ultimately irresponsible. The removal of all corrosion products [3] and the deteriorated primer to a completely clean bronze surface was the only way to ensure a stable foundation onto which new gilding could be applied to last for decades. It must be noted that all aspects of this project were discussed with the owner. The importance of using laser ablation for this project was significant, because it allowed conservators to safely remove all surface deposits without disturbing surface topography of the bronze [4]. In particular, the G.C. Laser systems used for this project allowed for precise control over parameters and efficient cleaning of this large bronze.

\section{Treatment}

Historically, the surfaces of bronze sculptures that were about to be re-gilded were cleaned of corrosion using chemical cleaning, high pressure washing, sand blasting, or glass bead pinning to a bare bronze surface [6, 7]. Mechanical cleaning methods change the surface topography of the cleaned metal and repeated campaigns can result in the loss of surface details over time $[8,9]$. Chemical cleaning processes are messy and difficult to control. Moreover, they present the risk of contamination of the surroundings [7]. Consequently, laser cleaning was chosen as an effective alternative to other methods due to the high level of precision and control it offers, and because a properly calibrated laser system can remove corrosion products without damaging the underlying surface of the metal. Laser cleaning was proposed to the Art Institute of Chicago and Chicago Park District as an alternative to media blasting and was approved. To the authors' knowledge, this is the first time in conservation history that laser cleaning was used to prepare a monumental bronze for re-gilding. G.C. Laser Systems were used on this project due to their ease of use, high level of tunability, and unique circular scan pattern that ensures even an efficient distribution of energy across the surface. These systems are capable of producing a high frequency of laser pulses ranging from $10 \mathrm{kHz}-1,000 \mathrm{kHz}$. During this treatment we used the following $1064 \mathrm{~nm}$ laser systems: GC-1, GC-50, GC-100. 100ns to $250 \mathrm{~ns}$ pulses were used, depending on the area being cleaned. The GC- 1 allows for 

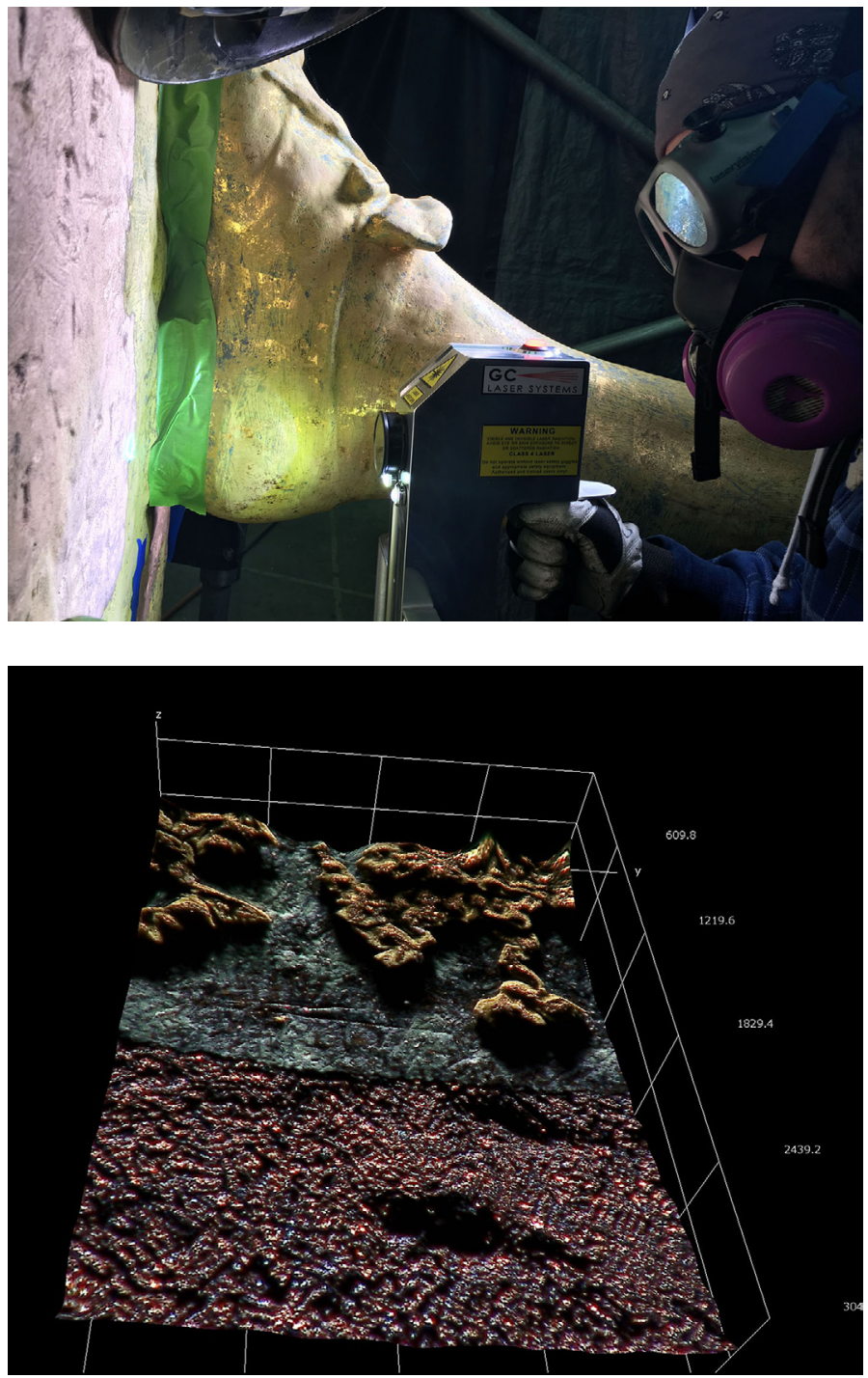

Fig. 5. Laser cleaning of the bronze surface performed by CSOS laser technician Christopher Ciaston. The laser has built-in lights to illuminate the workspace. An extraction trunk at the top left of the image is collecting fumes.

Fig. 6. Laser cleaning is able to remove old gilding, primer, and active corrosion products from the surface and uncover the dendritic structure of the bronze surface. Dimensions of examined area are: depth $3.05 \mathrm{~mm}$ and width $2.29 \mathrm{~mm}$. pulse duration to be selected by the operator. These lasers allowed for very precise and consistent surface cleaning.

Examination with a Hirox 3D microscope shows that the laser effectively removes failed gilding, old primer, and corrosion products to uncover the original dendritic crystal features of the bronze surface. Some of this dendritic pattern is visible in the green corrosion, but after laser cleaning it is very clearly uncovered. The laser is able to clean the surface without damaging the dendritic features of the original metal. A layer of cuprite can also be preserved.

During testing it was established that a 100 ns pulse with a pulse energy of 0.1 $\mathrm{mJ}$ and fluence of around $0.5-1.5 \mathrm{~J} / \mathrm{cm}^{2}$ removed the surface corrosion down to 


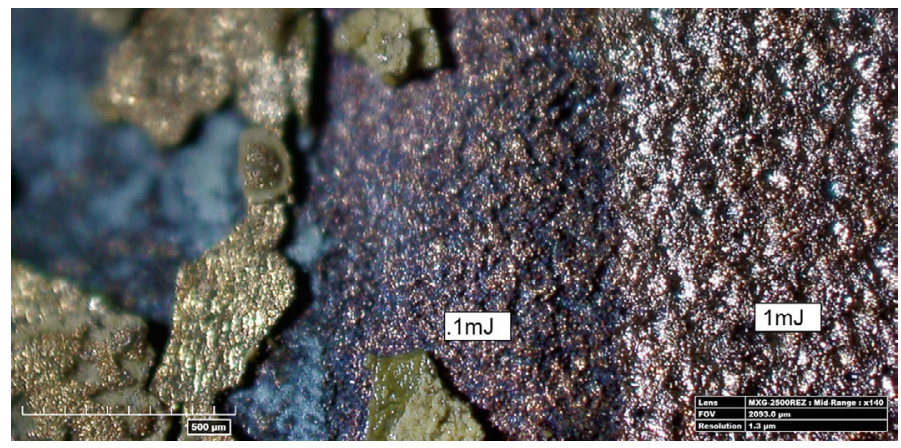

Fig. 7. Image illustrating the surface before laser cleaning on the left, the surface cleaned with $0.1 \mathrm{~mJ}$ pulses in the middle and $1 \mathrm{~mJ}$ pulses on the right.

a cuprite layer, but did not always remove all of the primer and gold material. In order to ensure all active corrosion, products were removed, 100ns-250ns pulses with a higher pulse energy of $1 \mathrm{~mJ}$ per pulse were used at a fluence range of $3-5.5 \mathrm{~J} / \mathrm{cm}^{2}$. Various optics were used to reach different focal depths with an in focus spot size of 200 microns with a pulse frequency ranging from $50 \mathrm{kHz}-300$ $\mathrm{kHz}$, depending on the area being cleaned and the size of the scan. Because this sculp- ture was going to be painted with a primer, it was desirable to clean down to a pure raw bronze surface to ensure that all corrosion products were removed. Examination with an Elio Portable XRF showed that chlorides were effectively removed from the laser cleaned area (Fig. 8).

The XRF was used to generate a false color map for chlorine and the laser cleaned square section is cool blue, indicating that chlorine is not present.

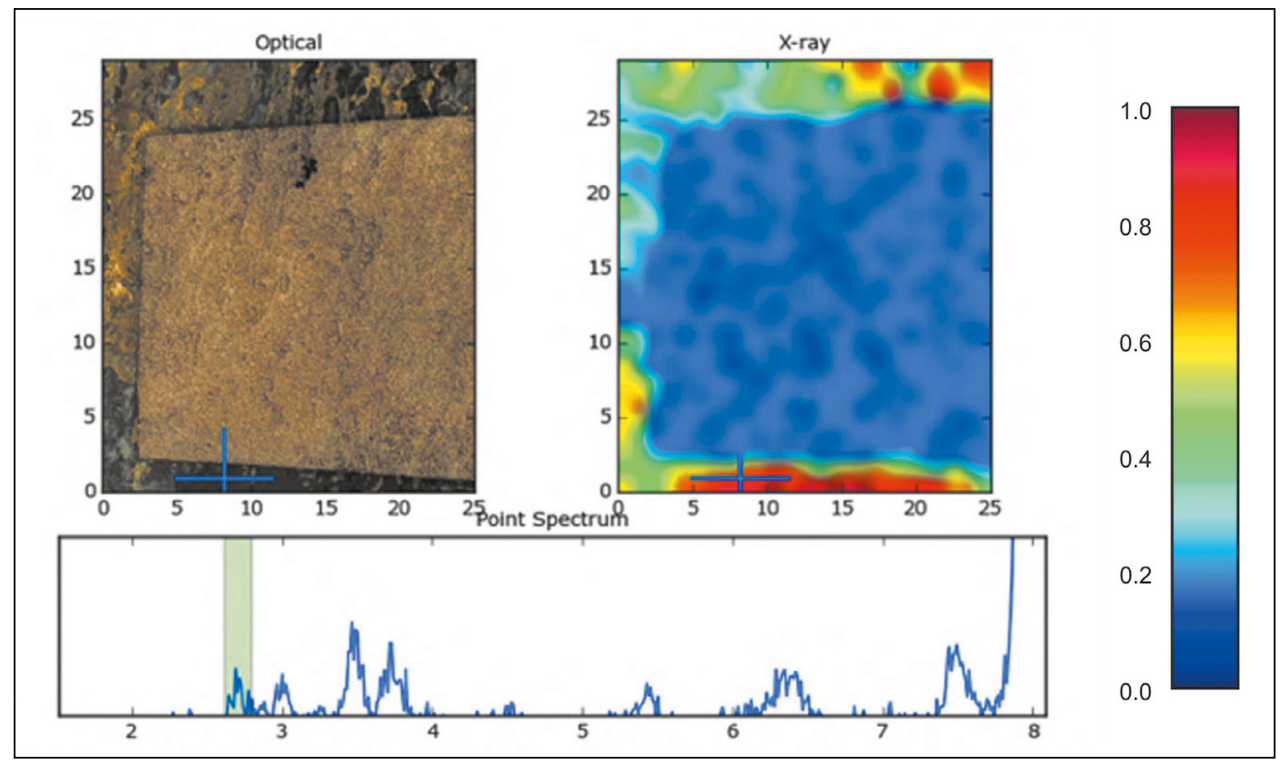

Fig. 8. Elio XRF chlorine distribution shown as a false color map. The laser cleaned area is cool blue, indicating low concentration of chlorine (image by N. Barbi). 


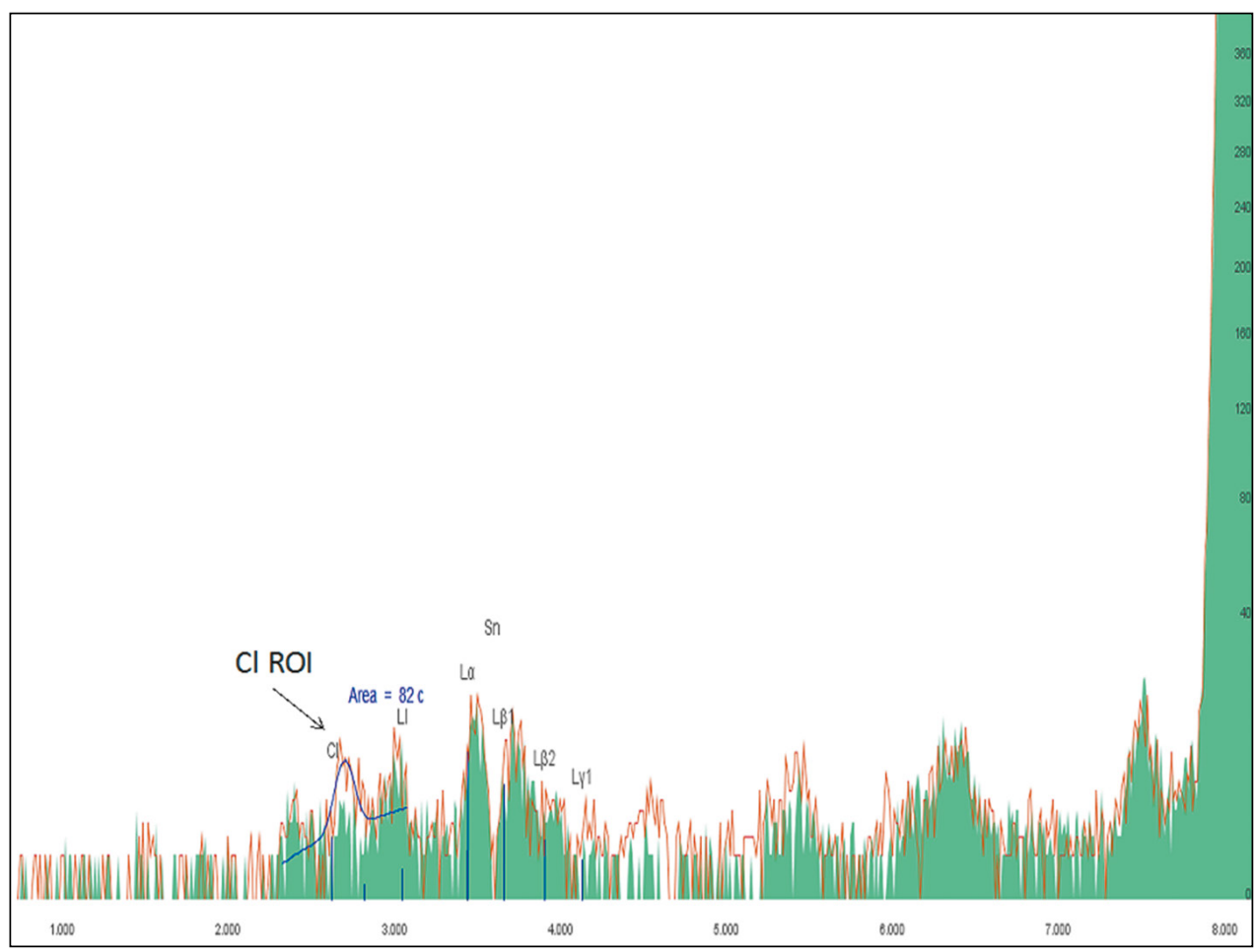

Fig. 9. Identification of chlorine by Elio XRF. The red spectrum is from the red region in the map and the green spectrum is from the blue region in the map in Fig. 8 (N. Barbi).

Each region of interest (ROI) in the XRF spectrum (Fig. 9) was mapped according to the false color scale: the red represents areas of the maximum intensity and blue represents the minimum intensity of the $\mathrm{Cl}$ signal. The scales of axes of images are given in millimeters. In the spectrum in Fig. 9 the horizontal axis scale is expressed in $\mathrm{keV}$, and the vertical axis is intensity. The intensity at the $100 \%$ value corresponds to 30 counts. Each spectrum was collected for 1 second (operator selected).

The signal of $\mathrm{Cl}$ is very week, because the concentration of $\mathrm{Cl}$ is low and spectrum integration time is short (1 second). Nevertheless, the spectral comparisons can reveal pixel to pixel differences, and by using maps, the human vision system is very effective at picking out the overall patterns. This pattern recognition skill of the human brain is one reason maps are so powerful. Fig. 9 illustrates 1-second spectra, one from a red area in the map (high intensity) and one from a blue area (low intensity). The difference in the $\mathrm{Cl}$ $\mathrm{ROI}$ can be readily seen. The fitting calculates a peak integral of 82 counts, the square root of which is about 9 , with a sigma of $11 \%$ (N. Barbi). The data indicates that chlorides have been successfully removed by the laser cleaning process.

The upper portion of Fig. 10. presents 3D image of the area cleaned with laser. The lower diagram shows surface topography of the cleaned metal. It should be noted that there are no topographic changes in the cleaned zone. The above image illustrates that 


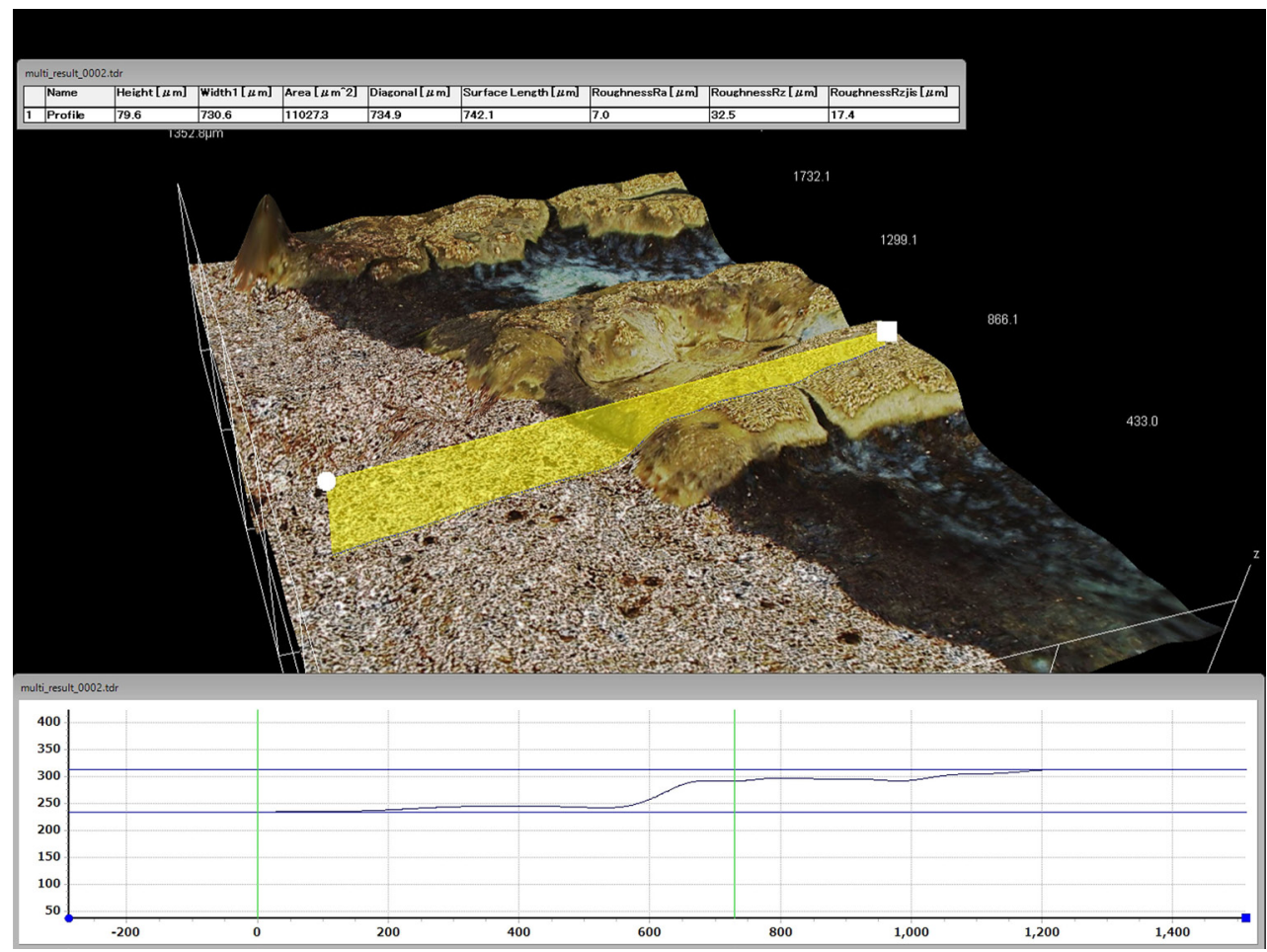

Fig. 10. Hirox 3D depth profile of the laser cleaned area shows that approximately 50 microns of corrosion and old gilding are removed by the laser.

it is possible to remove the corrosion products using laser ablation process and keep the original surface intact. In addition, the profile shows that after laser cleaning there was no change in the surface topography.

During treatment, in order to make sure that the bronze was free of any hidden copper chlorides, the surface of the sculpture was sprayed with pure distilled water after laser cleaning to encourage any remaining chlorides to emerge. Some porous areas with deep chloride penetration developed new green corrosion spots 24 hours after being exposed to water approximately 1 to $2 \mathrm{~mm}$ in diameter and were laser cleaned again. The surface was visually inspected each day. This process was repeated as necessary in areas where chlorides continued to emerge from the metal until these areas were completely clean and stable.

The sculpture after laser cleaning was degreased with the use of acetone and ethyl alcohol. Two coats of epoxy based zinc chromate primer manufactured by PPG were applied to the entire surface of the sculpture.

The primer was allowed to cure for five days and 12 hour Lefranc oil size was applied to the areas that were going to be gilded. Rosanobel double thickness 23.75 karat gold was used for gilding the monument. The head, shoulders, base and upper surfaces of the sculpture were gilded twice. The application of second layer of gold to those areas will provide longer lasting protection for the surface of the bronze. 


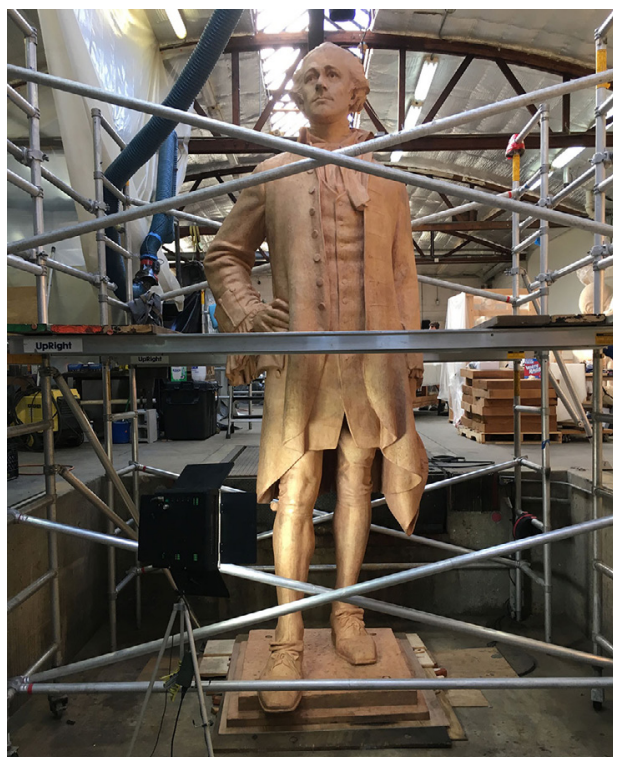

Fig. 11. Hamilton Monument entirely cleaned of the surface corrosion with the use of the laser ablation process.

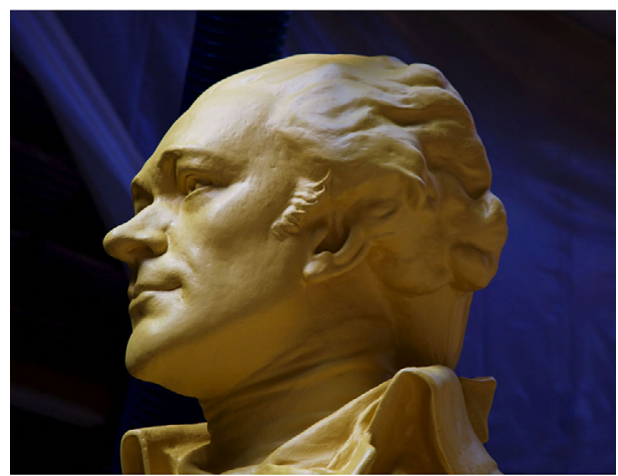

Fig. 12. Face of the sculpture after coating with zinc chromate.
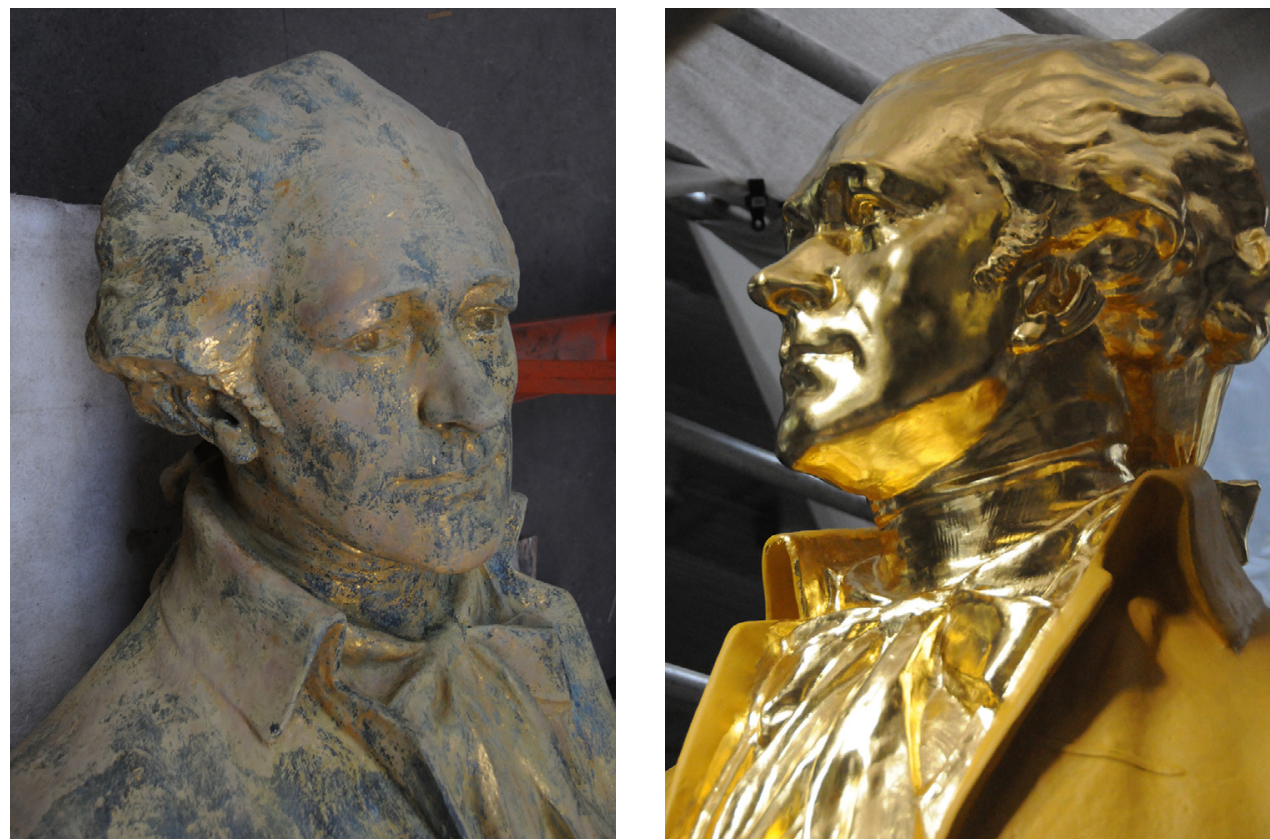

Fig. 13. On the left - the image of the sculpture before treatment, on the right - gilded face and chest of the figure. The jacket is primed before gilding. 


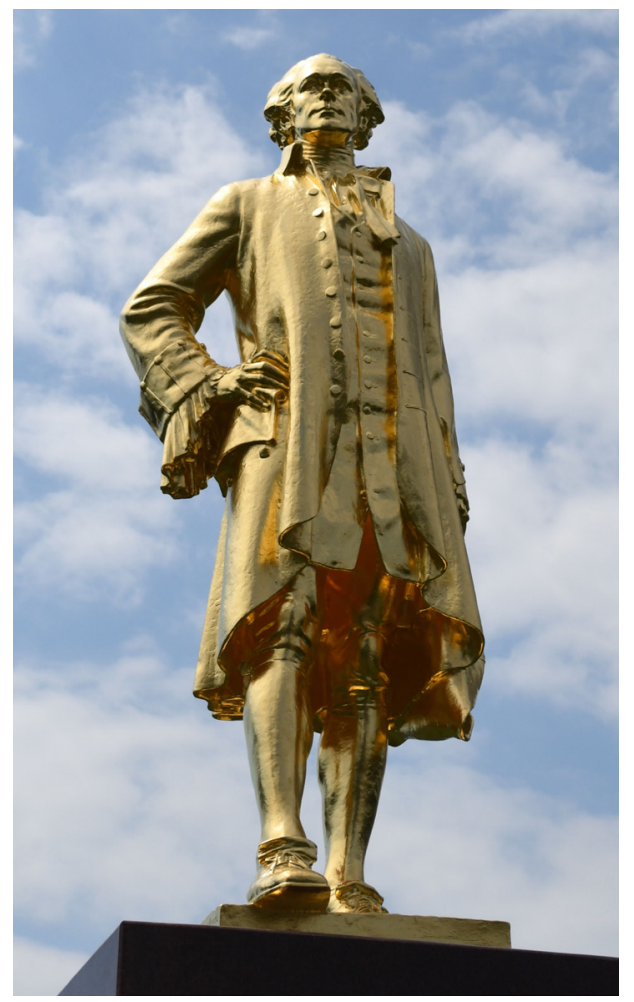

Fig. 14. Hamilton sculpture after gilding reinstalled in Lincoln Park.

\section{Conclusions}

The laser cleaning process provided us with a clean surface of metal that was corrosion-free and undamaged. After laser cleaning, any chlorides that were deeper in the metal were encouraged to activate and emerge by being sprayed with distilled water. Any chlorides that bloomed after being exposed to water were removed with the laser. This process was repeated as necessary until all active chlorides were successfully removed. The very high level of laser parameter control using G.C. Laser Systems allowed us to completely remove corrosion while preserving the topography of the surface. The bronze was corrosion-free, which will provide long-lasting treatment results. Obviously, the sculpture will require periodic maintenance such as washing with deionized water to remove contaminants, e.g. winter salts and bird droppings from the surface. According to our knowledge, this was the first use of laser ablation process for the preparation of a large monument for gilding. The high precision of corrosion removal with G.C. lasers provided a very clean surface of bronze for gilding. The overall treatment of the iconic Hamilton monument proved to be successful. The monument is being held for safe storage over the winter at CSOS and will be reinstalled in Lincoln Park in Chicago in the spring of 2017. At the moment, laser ablation cleaning appears to be the best alternative to traditional cleaning methods for preparing bronze sculptures for gilding.

\section{Acknowledgements}

We owe many thanks for help and support during this project to: Susanne Schnepp, Art Institute of Chicago, Michael Fus, Chicago Park District, Jennifer Mass, Ph.D. Scientific Analysis of Fine Art, LLC, Adam Finnerfrock, Ph.D., Scientific Analysis of Fine Art, LLC, Nicholas C. Barbi, Ph.D., nSynergies Inc., and CSOS staff: Christopher Ciaston, Artur Konarzewski, Cathereen Lee, Robert Zarycki, Tadeusz Mlynarczyk, Jonathan Murach.

\section{References}

[1] I. Bach, M. Lackritz Gray, A guide to Chicago's Public Sculpture, The University of Chicago Press, Chicago 1983, p. 150.

[2] J. Riedy, Chicago Sculpture, University of Illinois Press, Urbana 1981, pp. 14, 16, 224.

[3] L.B. Brostoff, Coating Strategies for the Protection of Outdoor Bronze Art and Ornamentation, University of Amsterdam, Amsterdam 2003, pp. 3-4.

[4] A. Dajnowski, "Laser as a Cleaning Tool for the Treatment of Large Scale Bronze Monuments", in: 
Lasers in the Conservation of Artworks - Lacona VII Proceedings, M. Castillejo et al. (Eds.), Taylor \& Francis Group, London 2008, pp. 303-308.

[5] S. Siano, R. Salimbeni, "Advances in Laser Cleaning of Artwork and Objects of Historical Interest: The Optimizes Pulse Duration Approach", Acc. Chem. Res., 43 (2010) 739-750, DOI: 10.1021/ar900190f.

[6] T. Drayman-Wiseser, Gilded Metals History, Technology and Conservation, Archetype Publishing Ltd, London 2000, p. 337, 349.

[7] D.A. Scott, Copper and Bronze in Art Corrosion, Colorants, Conservation, Getty Trust Publications, Santa Monica CA 2002, pp. 122-144.
[8] W.T. Chase, N. F. Veloz, "Some Considerations in Surface Treatment of Outdoor Metal Sculptures", in: Preprints of Papers Presented at the Thirteenth Annual Meeting: Washington, D.C., 22-26 May 1985, American Institute for Conservation of Historic and Artistic Works, A.I.C. 1985, pp. 23-25.

[9] T. Draysman-Weisser, "Dialogue/89, The Conservation of Bronze Sculpture in the Outdoor Environment: A Dialogue Among Conservators, Curators, Environmental Scientist, and Corrosion Engineers", NACE, Huston, Texas, USA, 1992.

[10] M. Giust, G.M. Radke, The Gates of Paradise: From the Renaissance Workshop of Lorenzo Ghiberti to the Restoration Studio, Giunti Industrie Grafiche S.p.A., Prato 2012. 


\title{
Experiences at the Academy of Fine Arts of Brera in Milan, Italy: the application of laser-technology on three case studies of the historical heritage
}

\author{
Elisa Isella ${ }^{1 \star}$, Donatella Bonelli ${ }^{1}$, Silvia Cerea ${ }^{1}$, Francesca Mancini ${ }^{1}$, Veronica \\ Ruppen ${ }^{1}$, Alessandra Botteon ${ }^{2}$, Antonio Sansonetti ${ }^{2}$ \\ 1 Brera Fine Arts Academy, Milan, Italy - Via Brera 28, 20121, Milan \\ 2 Institute for Conservation and Valorization of Cultural Heritage ICVBC - CNR, Milan, Italy - Via \\ Roberto Cozzi, 53, 20125 Milan \\ * Corresponding author: elisa.isella@libero.it
}

\begin{abstract}
Scientific research regarding the conservation of gypsum plaster heritage is quite scarce; cleaning is often a problematic issue on this kind of material due to its water sensitivity and mechanical features. Therefore, laser cleaning is often a good option as some recent paper has reported.

The research presented here focused on laser cleaning on three gypsum plaster casts belonging to the collection of Brera Fine Arts Academy and dated back to the $19^{\text {th }}$ century; recalling the names of the original marble statues, the items are the Flora Farnese, The Velletri Pallas and the Barberini Faun. The three casts had been exposed for many decades in the entrance hall of the Academy in a semi-confined environment. Unfortunately, the continuous passage of students soiled the plaster surface with a grey greasy layer localized especially on the lower parts and on the surfaces with a strong horizontal component. It was possible to identify a superficial grime and traces of the original patina. Marks of different nature (felt, pen, pencil, scratches) were also present. Cleaning tests were carried out using Thunder Art Nd:YAG able to emit both at $1064 \mathrm{~nm}$ and at $532 \mathrm{~nm}$. The effects of laser cleaning were studied with a diagnostic campaign carried out both before and after the cleaning operation. To define the aspects and the nature of the surface microsamples were sampled and analysed with XRD, XRF, then observed with a scanning electron microscope SEM, and with optical microscopy.

Several tests were carried out on the Flora Farnese, calibrating the fluence in order to match the ablation threshold avoiding any damage (from $\mathrm{FL}=1-1,45 \mathrm{~J} / \mathrm{cm}^{2}$ at $1064 \mathrm{~nm}$ to $\mathrm{FL}=0,5-0,7 \mathrm{~J} / \mathrm{cm}^{2}$ at $532 \mathrm{~nm}$ with repetition rate from $6 \mathrm{~Hz}$ to $10 \mathrm{~Hz}$ ) in particular on these surfaces the removal of cement splashes constituted a hard task. A comparison in between tests at $1064 \mathrm{~nm}$ and at $532 \mathrm{~nm}$ were performed. Both tests were evaluated with the aid of humidification with free water (applied with brush) and with the use of AgarArt ${ }^{\oplus}$ rigid gel. On the basis of laser-plaster interaction observed on the Flora, the tests on the other casts excluded the IR radiation, because of a yellowing effect. For what regards the cast of the Pallas, one of the low relieves in the wooden support base was involved. The laser cleaning allowed the perfect removal of the outer layers of scialbo.

For the cast of the Faun, laser cleaning proved to be the appropriate system to eliminate greasy deposits and dark grey stains visible on the irregular surface, maintaining the original morphology. Hence it was possible to avoid invasive mechanical and chemical systems, minimizing the interaction with the matter and saving working time. The aim of this work is to provide a contribution to the scientific
\end{abstract}


researches by the point of view of a Fine Arts Academy, which is starting to build a database for the conservation products and methods about such a known and used material as plaster, which is not sufficiently studied.

Keywords: Nd:YAG, $532 \mathrm{~nm}$ radiation, plaster casts, guano, Newberyite, ablation threshold, safety and security

\section{Introduction}

Cleaning artwork mainly composed by gypsum is a hard challenge even for skilled conservators. Gypsum is partly soluble and moreover, it is very sensitive to any kind of wet cleaning; furthermore, it is not mechanically resistant to abrasive systems and its resistance is dramatically decreased even when it is slightly wet. However, the interaction between the cleaning system and the gypsum surface is influenced by secondary components, especially when there are organic additives such as animal glues or other protein materials. Recently the Agar cleaning has proved to be efficacious on this kind of artworks, even though some research is still needed in this field. Laser cleaning allows to remove soiling, but at the same time avoiding damages to the artwork as proved by some recent papers [1].

This research focuses on the application of laser cleaning on three gypsum plaster casts of the historic collection of the Fine Arts Academy of Brera, dating back to the $19^{\text {th }}$ century; they are the Flora Farnese, the Velletri Pallas and the Barberini Faun. Laser cleaning was applied to remove the compact grayish deposits present on the surface and partially penetrated into the gypsum. The Nd:YAG laser source was chosen for the advantages offered by its versatility and broad operating range of laser pulse durations. This instrument was employed to complete a predominately mechanical ablative process with the emission of short impulses in Q-switch to avoid the excessive heating of the surface usually associated with long pulse duration. Furthermore, based on the research of basic laser interaction mechanisms, recent bibliographic references $[2,3]$ have suggested to operate in the visible spectrum at $\lambda=532$ $\mathrm{nm}$ to avoid the undesirable yellowing of the substrate.

\section{The Brera Academy of Fine Arts}

The survey here presented was carried out at the Conservation School in the Academy of Fine Arts, an Italian Institute that is based in Milan and was founded in 1776 by Empress Maria Teresa of Austria. The Brera building hosts various institutional bodies such as the Ministry of Culture, the Fine Art Gallery, the Botanical Garden, the Astronomical Observatory and the Braidense Library; they are all enclosed together to create a network. The overall collection is worldwide renowned. It goes back to the $19^{\text {th }}$ century and consists in different kinds of works like paintings, ancient books, drawings, sculptures and plaster casts. The Conservation School was founded in 1997.The Historical Heritage of Brera Academy is composed by 900 gypsum plaster casts positioned in several of school spaces, both public and reserved to the students. All the conservation activities are devoted at the aim to create the new gypsum gallery in the original old basement of the Academy. The Heritage Collection could 


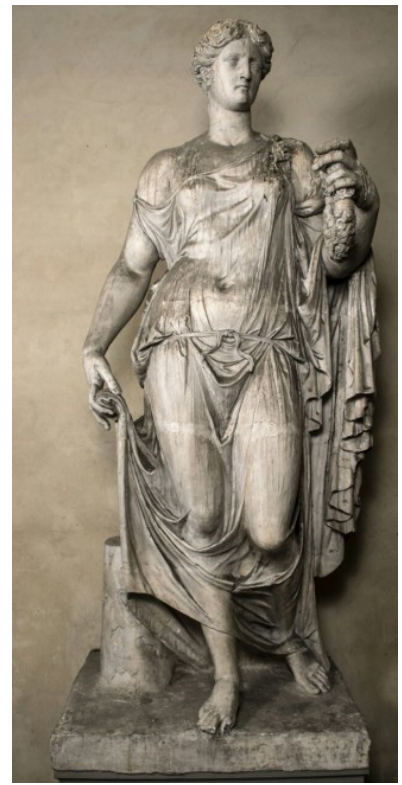

Fig. 1. Flora Farnese before the restoration.

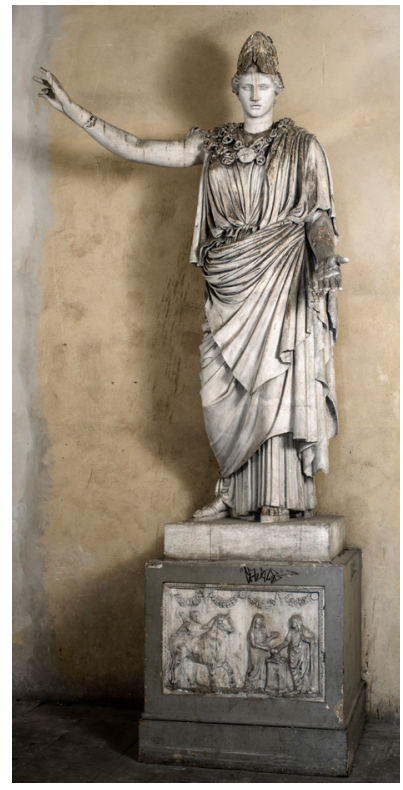

Fig. 2. Velletri Pallas before the restoration.

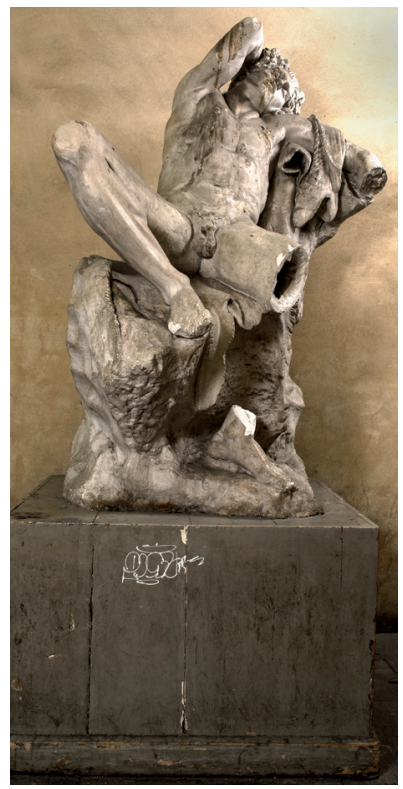

Fig. 3. Barberini Faun before the restoration. be split into two parts: the $19^{\text {th }}$ and the $20^{\text {th }}$ century artworks, different for size, materials and techniques.

The oldest artwork category group includes items of huge dimensions, which were modelled by using the original marbles; on the contrary, the recent heritage is mainly formed by prototypes useful to the artist for the final marble sculpture.

\section{The case studies}

The Flora Farnese, Velletri Pallas and Barberini Faun (Figs. 1-3) are three gypsum plaster casts dating back to the end of the $18^{\text {th }}$ century: their sizes constitute a challenge for the conservators, being in the range from $2,04 \mathrm{~m}$ to $3,55 \mathrm{~m}$ approximately. They were made from original marble statues which are currently conserved in three different museums: the Archaeological Museum of Naples (Flora Farnese), the Louvre Museum (Pallas of Velletri) and the Munich Glyptothek (Barberini Faun).

These three sculptures were located for the last decades in the entrance hall of the Academy, in a large hallway leading to the Academy classrooms. This semi-confined environment favours the ventilation and acts on the average of the temperature and the relative humidity, which replicates seasonal standards. Urban colonies of pigeons and the constant flow of visitors and students in the corridors created different processes and patterns of decay.

Plaster casts surfaces were covered by a thick layer of different exogenic material, composed of pigeon dropping (guano) (Fig. 4), solid particles coming both from soil and pollution. As a result of the contact with human hands, a greasy, grey layer had 


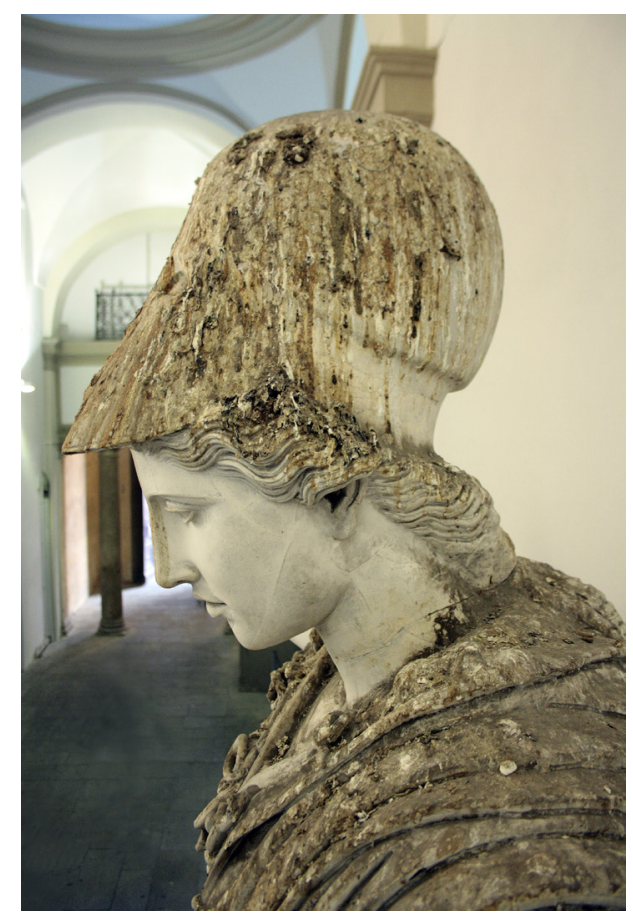

Fig. 4. The Velletri Pallas helmet covered with a thick and uneven layer of guano.

been formed on the lower part of the plaster casts; moreover, the artworks were damaged by different acts of vandalism, which caused the fracture of exposed extremities. The most

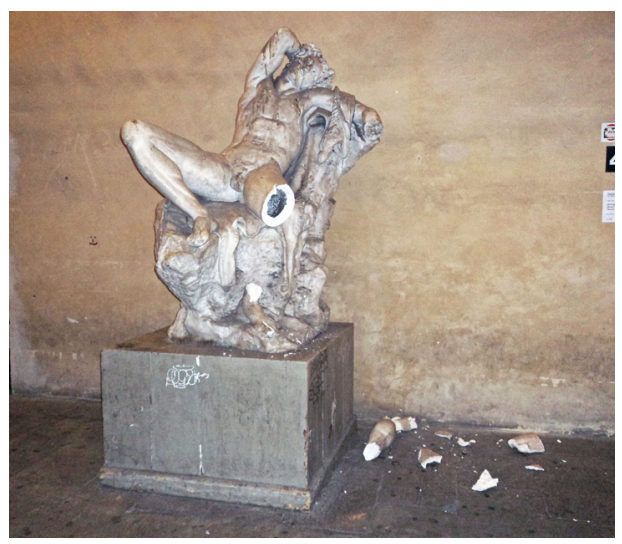

Fig. 5. Barberini Faun with fragments of the left leg after the vandalism act. recent act of vandalism was inflicted on the Barberini Faun in March 2014: the left leg was detached due to an inappropriate move caused by a student (Fig. 5), whereas the Pallas was intentionally splattered with blue paint (Fig. 6).

Previous restoration and masonry building maintenance severely worsened the state of the conservation of the three sculptures; especially the surface of Flora which was damaged by splashes of a grey mortar. An in-depth study of the composition of the gypsum plaster matrix highlighted interesting details about the execution of the three casts; specific technical diagnostic methods were used leading to the current phase of conservation.

Through X-ray diffraction (XRD) and thermal analysis (TGA-DTA), it was possible to identify gypsum as the principal component, with a presence up to $90 \%$; the rest is composed of traces of bassanite, quartz and calcium oxalates. There is also a minimum percentage of organic substances, specifically traces of protein highlighted by infrared

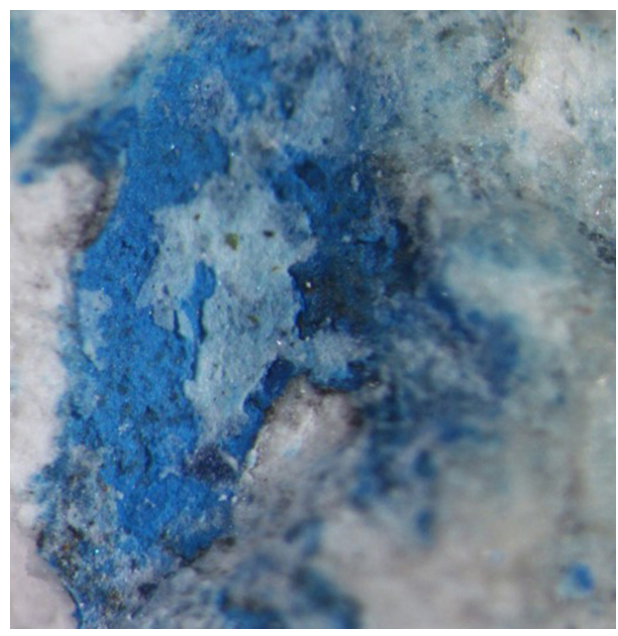

Fig. 6. The vandalism act on the Pallas surface: a detail of the blue stain splattered, observed at the stereomicroscope. 

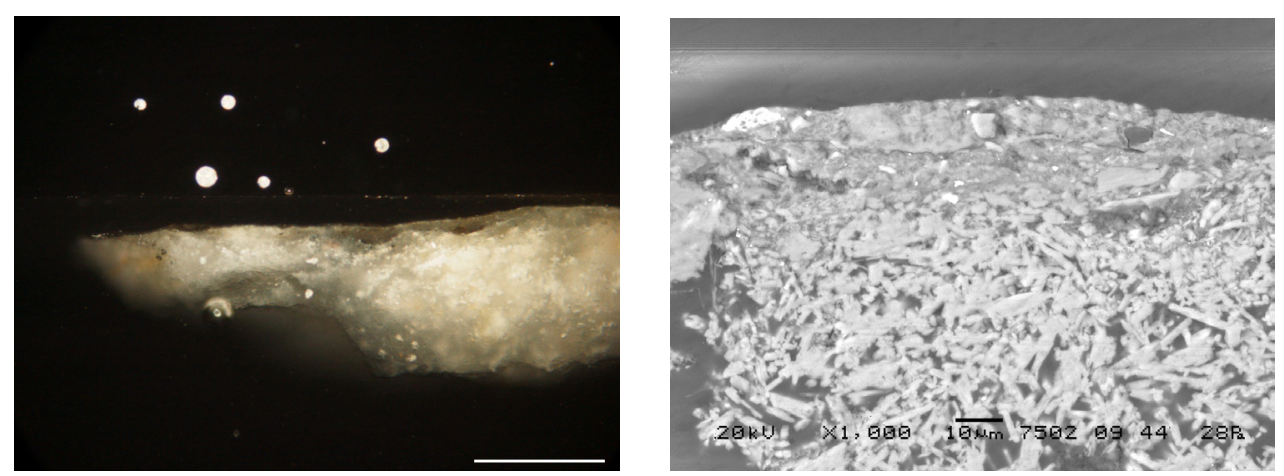

Figs. 7 and 8. Optical and SEM images in backscattered electrons of the uncleaned area. They show the stratigraphic sequence of the plaster.

spectroscopy (FTIR). The optical and the scanning electron microscope (SEM) images (Figs. 7 and 8) provided the stratigraphic sequence of the plaster surface.

On the outer gypsum surface it was possible to observe a grey layer that at the naked eye appeared to be a scialbo. However, this hypothesis could not be confirmed by further analysis; in fact, no specific distinctive layer was present, but phosphorus, magnesium and silicon have been detected by EDS spectra on the surface and the near/surface region of the
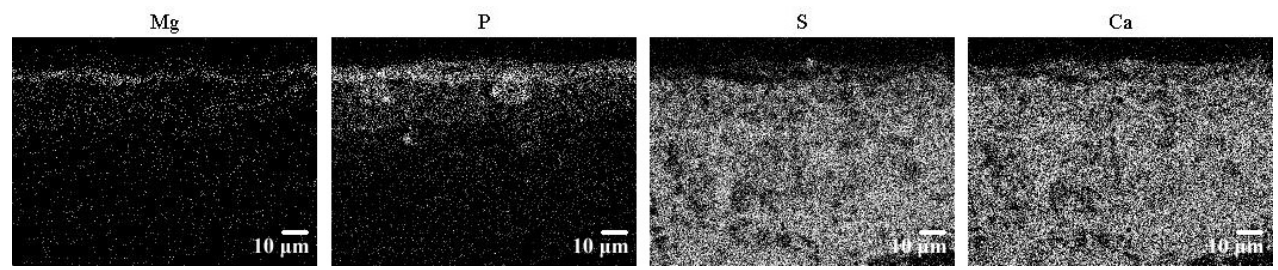

Fig. 9. SEM - EDS maps of magnesium, phosphorus, silicon and calcium.

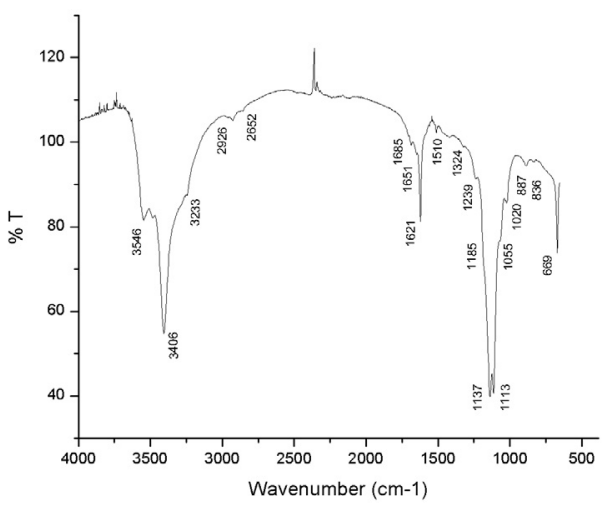

Fig. 10. FITR spectrum of Newberyite after irradiation. gypsum matrix. It is possible to see tracks of these elements in the maps (Fig. 9).

FTIR spectrum (Fig. 10) shows the absorption pattern of unknown material sampled from the grey layer. It displays a good overlapping referring to the mineral Newberyte. This is a magnesium hydrogen phosphate which crystallizes with three molecules of water $\left[\mathrm{Mg}\left(\mathrm{HPO}_{4}\right) \cdot 3 \mathrm{H}_{2} \mathrm{O}\right]$. The hydrogen phosphate ion is reported to be prevalent in weakly basic conditions, but it is still present in a significant amount at $\mathrm{pH}$ 7 [4]. Different forms of magnesium phosphates have been detected as crystalline forms 
in animal's guano. Depending on the guano age, the presence of humidity, the content of ammonium ion, a different mineral phase is formed: Struvite $\left[\mathrm{MgNH}_{4} \mathrm{PO}_{4} \cdot 6 \mathrm{H}_{2} \mathrm{O}\right]$, occurs in fact in great abundance in the moist depth of guano, while on the contrary, Newberyte occurs in the older and drier parts of the deposits. Its colour is described as greyish or grey/brown [5]. As a general rule, the hydrogen and di-hydrogen phosphates are slightly more soluble than the corresponding phosphates [6]. The low solubility product of newberyte $1,510^{-6}$ at $25^{\circ} \mathrm{C}$ (Taylor, 1963), compared to the $\mathrm{K}_{\mathrm{sp}}$ of gypsum, $10^{-4.58}$ allows to suppose that the mechanism explains the current localization of phosphate and magnesium in the near-surface region [7]. Subsequent cycles of condensation phenomena, solubilize a greater amount of gypsum in the outer surface, leaving the Newberyite intact; the following re-precipitation of gypsum in a drier season, moves it forward to the outer profile of the surface. Newberyite has been detected in cave minerals, in guano and in kidney stones. In the field of cultural heritage, the scientific literature reported an occurrence of this substance on ivory surfaces [8]. In this paper, the first detection on gypsum statues was presented, as to the authors knowledge.

\section{Previous treatments and preliminary laser test}

The conservation works began in October 2014. The preliminary cleaning phases were the following:

1. Dry cleaning (Fig. 24)

2. Wet cleaning (Fig. 11)

3. Agar Gel cleaning (Fig. 11 and Fig. 25)

Processing with a dry cleaning on a decayed gypsum surface, using a synthetic rubber or a sponge, kneads the soiling onto the gypsum matrix. This is a drawback, because it produces an uneven cleaning level, which gives the impression of a stained surface. Wetting the surface with a water based system and adding water to the gypsum material with a brush or a poultice did not help at all; on the contrary, it enhanced the possibility of permanent damages.

The Agar gel was not effective inside the scratches and the poultice seemed to damage the morphology of the plaster surface (Fig. 11).

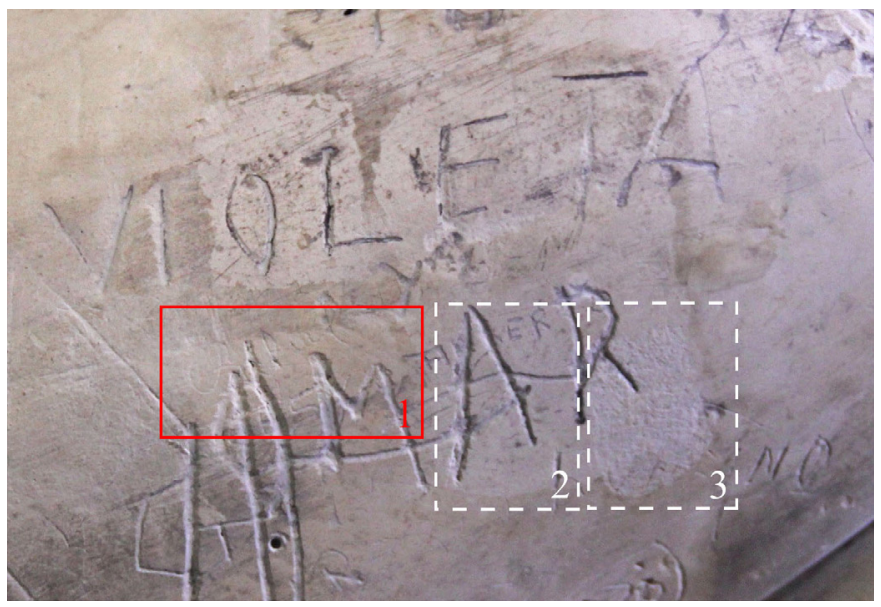

Fig. 11. Laser test (1) gel test (2) solvent pad test (3). 


\section{The laser cleaning}

Eventually, laser cleaning tests were performed (Figs. 12-13). As already mentioned, with gels and poultice solvent (hydro-alcoholic solution) the cleaning resulted in an unsatisfactory surface respect to the laser, which on the contrary, proved a correct balance between harmfulness and effectiveness.

Several laser tests (THUNDER ART - El. En. - Florence, Italy) were carried out on the Flora Farnese, calibrating the fluence (FL) in order to match the ablation threshold avoiding damages from $\mathrm{FL}=1 \div 1,45 \mathrm{~J} / \mathrm{cm}^{2}$ at $1064 \mathrm{~nm}$ to $\mathrm{FL}=0,5 \div 0,7 \mathrm{~J} / \mathrm{cm}^{2}$ at 532 $\mathrm{nm}$ with repetition rates within the range 6 $\mathrm{Hz}$ to $10 \mathrm{~Hz}$. A comparison in between tests was performed at $1064 \mathrm{~nm}$ and at $532 \mathrm{~nm}$. Both tests were evaluated with some support like wetting with clear water (applied with

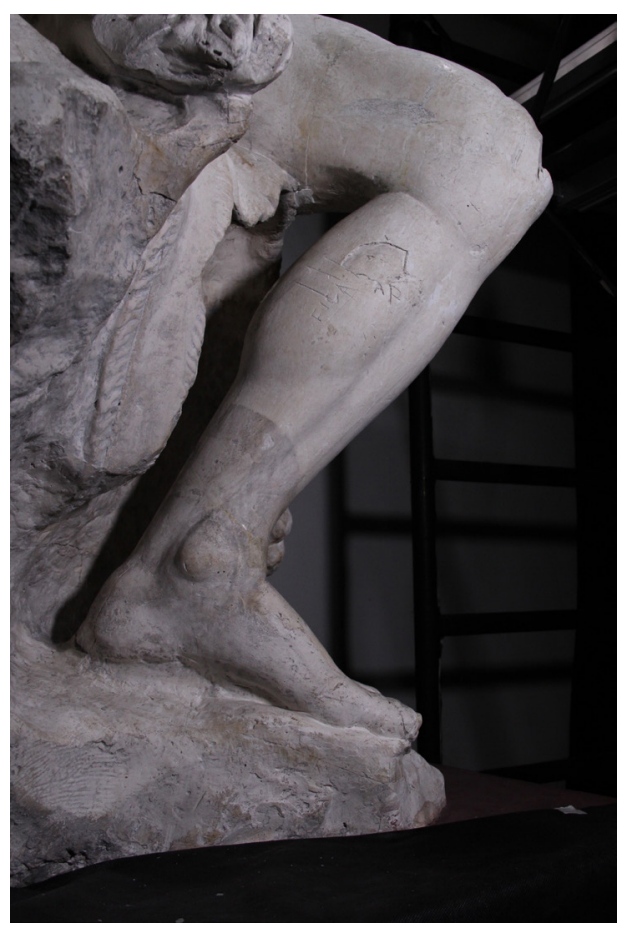

Fig. 12. Laser test at $1.5 \mathrm{~J} / \mathrm{cm}^{2}$. a brush) and using the AgarArt ${ }^{\circ}$ rigid gel as an intermediary agent. A yellowing effect was observed on the Flora surfaces, which led to the exclusion of the radiation $1064 \mathrm{~nm}$, on the other two casts.

As it is possible to see in the maps of the elements, the ablation at FL over $1.9 \mathrm{~J} / \mathrm{cm}^{2}$ removed almost completely the magnesium and phosphorus formations, clearly indicating an overcleaning (Fig. 17). The visual results displayed in Fig. 15 highlighted in fact an unnatural white surface, where a portion of the near-surface region has been removed with the outcome that it damaged the surface of the gypsum work (Figs. 14-17).

In conclusion, analyses were carried out to evaluate the differences between the untreated and cleaned laser zones; they allowed to observe the partial lowering of the Newberyte formation avoiding its com-

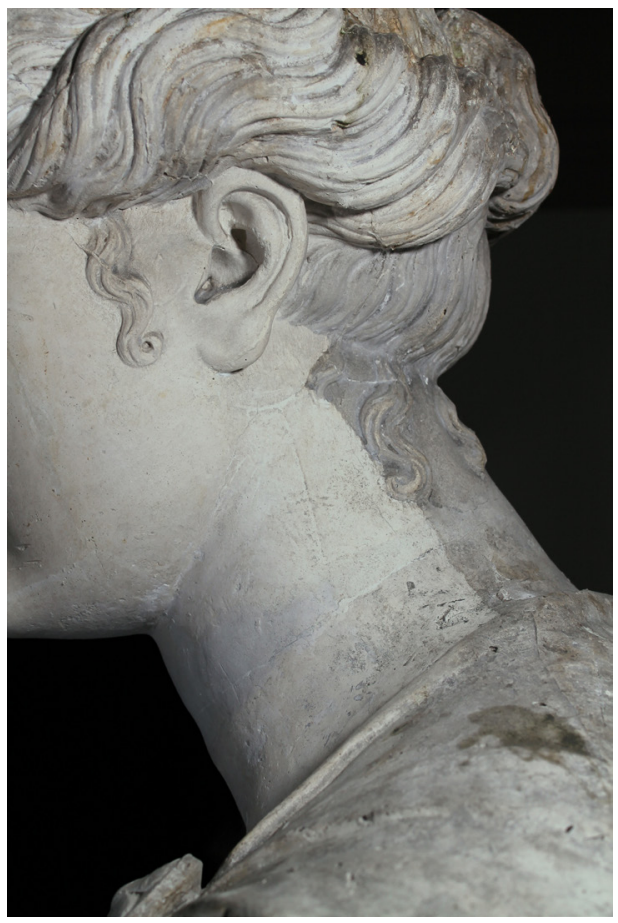

Fig. 13. Laser test at $0.7 \mathrm{~J} / \mathrm{cm}^{2}$. 
plete removal, except for the over cleaning case.

In order to remove the grey layer, which covered a wide range of the surface, it was decided to complete the cleaning with the laser THUNDER ART (El. En. - Florence, Italy; wavelength $=532 \mathrm{~nm}$; spot size $10 \mathrm{~mm}$; pulse duration $\approx 8$ ns. (Fig. 26).

Working with the THUNDER ART Laser allowed the completion of the complex phase

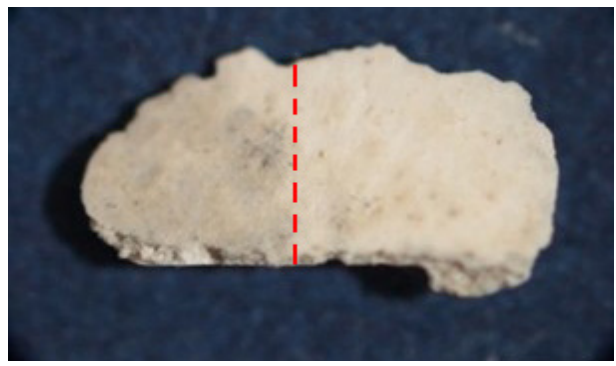

Fig. 14. The sample before and after a cleaning test (the grey layer has become thinner).

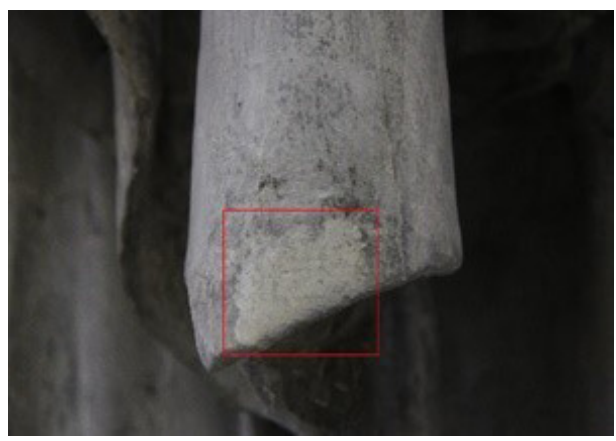

Fig. 15. The laser removal shows an overcleaning level.

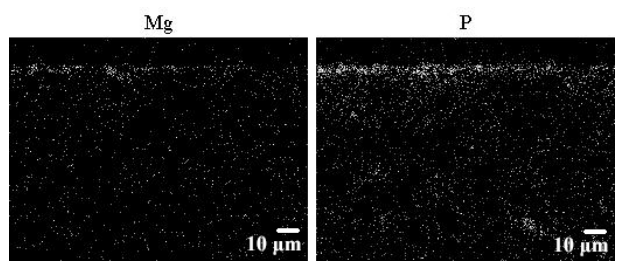

Fig. 16. Ablation threshold. These maps show that the Newberyite has decreased after the laser cleaning. of cleaning by optimizing working time, but also achieving better results compared to the traditional cleaning methods previously tested. Thanks to its high selectivity, the laser system allowed full control and respect of the surface characteristics. Moreover, the laser beam offered the possibility to clean the undercut areas such as the internal folds of clothing, the flowers garland, and other particularly delicate surfaces like the carnation. The cleaning operations on the three plaster casts necessitated approximately fifteen working days.

As a first step, the lower parts of the casts were irradiated, specifically the bases made of plaster because of their easy access. The plaster bases of the Pallas and the Flora have a smooth and simple morphology and it was possible to reach an excellent level of cleaning by using a fluence $=1.7 \mathrm{~J} / \mathrm{cm}^{2}$. Regarding the Faun, it was necessary to use a range of fluence from 1 to $1.7 \mathrm{~J} / \mathrm{cm}^{2}$ (higher with respect to preliminary the tests) because the plaster base had an irregular rocky surface, which carries the working marks, transferred on the plaster from the original marble. During this first laser tests, it was obligatory to take into consideration the cleaning of the bas-relief located in the front of the wooden base structure on which the plaster cast of the Pallas stands (Figs. 18-19). Fluence $=0.7 \mathrm{~J} / \mathrm{cm}^{2}$ was used to remove the homogenos grey layer of the background, while the figures were treated with fluence $=$

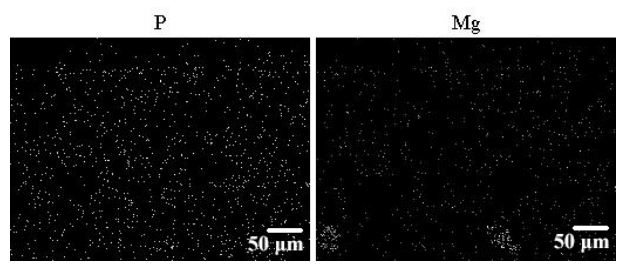

Fig. 17. Overcleaning. These maps show that the Newberyite is no longer present. 


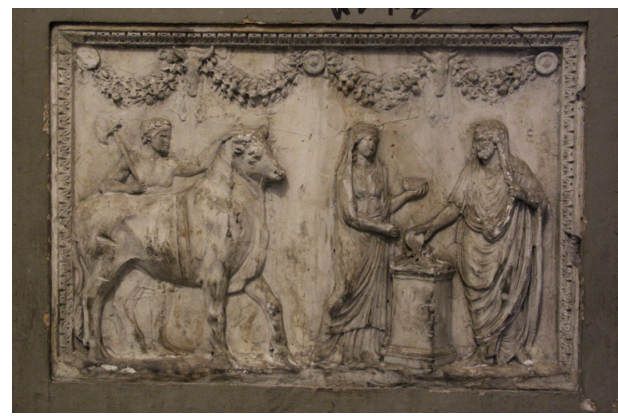

Fig. 18. Low relief before irradiation.

$0.8 \mathrm{~J} / \mathrm{cm}^{2}$, also increasing the value on stains up to a maximum of $1 \mathrm{~J} / \mathrm{cm}^{2}$. It was necessary to modulate the values of fluence and the repetition frequency, instead of using fixed parameters, to be able to clean effectively the draped dresses of the two female figures.

To clean the tunic of the Pallas which is folded on the hips forming a large draping triangle, it was needed to use a range of fluence that varied from $1 \mathrm{~J} / \mathrm{cm}^{2}$, to $1.4 \mathrm{~J} /$ $\mathrm{cm}^{2}$, and a repetition frequency of $12 \mathrm{~Hz}$.

Laser cleaning proved to be particularly effective to eliminate the accumulated black particles in the areas underneath the vertical folds and under the laying zones of the richly decorative elements of the breastplate (depicting snakes and the head of Medusa) (Figs. 20-21).

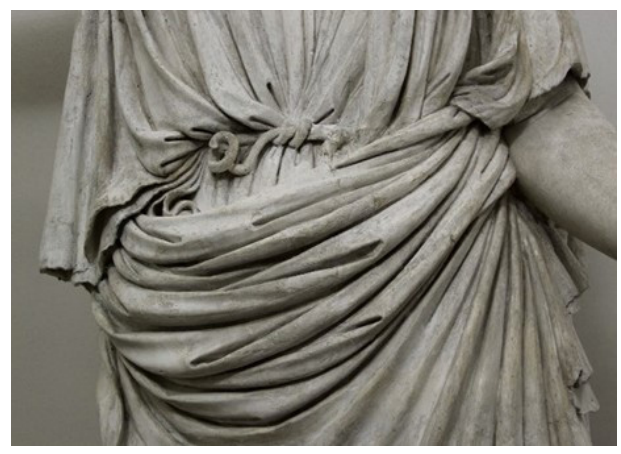

Fig. 20. The tunic of the Pallas before laser treatment.

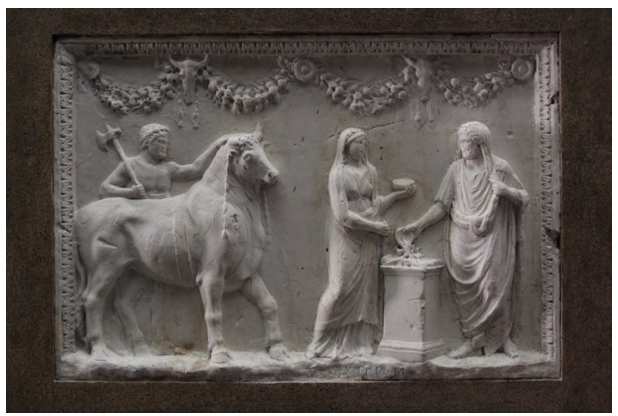

Fig. 19. Low relief after irradiation at $0.7 \mathrm{~J} / \mathrm{cm}^{2}$.

The technical difficulty in these two cases was keeping the hand tool as perpendicular as possible to the modelled surface; on the contrary, extra care had to be taken of the Flora plaster cast in order to be able to execute a uniform cleaning, respecting the chiaroscuro effects, which give plasticity to the figure. Her left forearm holds the elegant draping of the himation (a type of clothing); this area was irradiated with values of fluence from $0.9 \mathrm{~J} / \mathrm{cm}^{2}$ to $1.4 \mathrm{~J} / \mathrm{cm}^{2}$ at a repetition frequency of $18 \mathrm{~Hz}$ (Figs. 22-23). Excellent results were achieved on the wreath of flowers, where blackened areas were successfully removed in the spaces among the flowers.

In correspondence with the anatomical parts, it was necessary to be very careful and delicate and to use the right manual skills. In

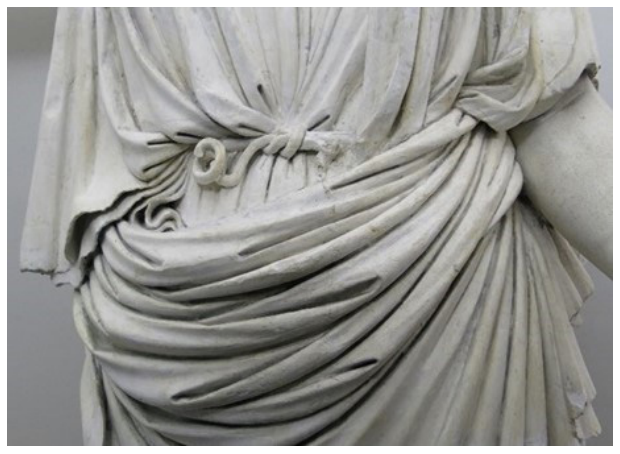

Fig. 21. The tunic of the Pallas after laser treatment. 


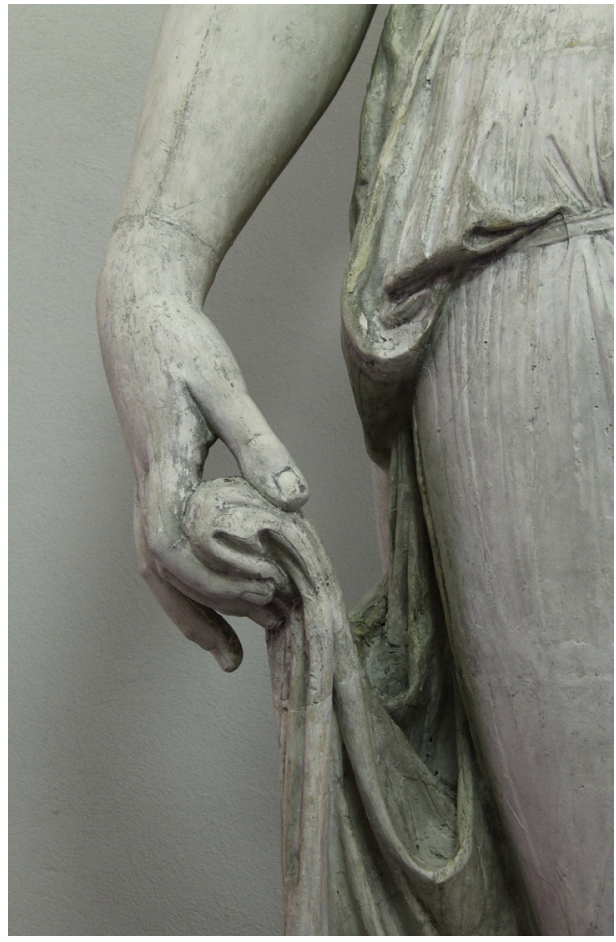

Fig. 22. Particular of the hand before laser cleaning.

particular, the nudity of the Faun regained the full lapse definition of details, which previously were hidden by deposits irregularly distributed on the modelled surface.

The expression on his face and the natural rendering of the abdominal band re-emerged using the laser with a range of fluence starting with $0.7 \mathrm{~J} / \mathrm{cm}^{2}$ through $1.5 \mathrm{~J} / \mathrm{cm}^{2}$, with a repetition frequency of $7 \mathrm{~Hz}$ (Fig. 26). To remove the grey layer, which was easily evident on the faces of the other two casts, the laser parameters varied its distance from the hand tool to the modelled surface. The values in the interval between $0.5 \mathrm{~J} / \mathrm{cm}^{2}$ and $1.1 \mathrm{~J} / \mathrm{cm}^{2}$ were used to obtain a modulated cleaning based on the state of the conservation of different surfaces.

The left leg of the Faun is considered as a "special issue" because of the obvious

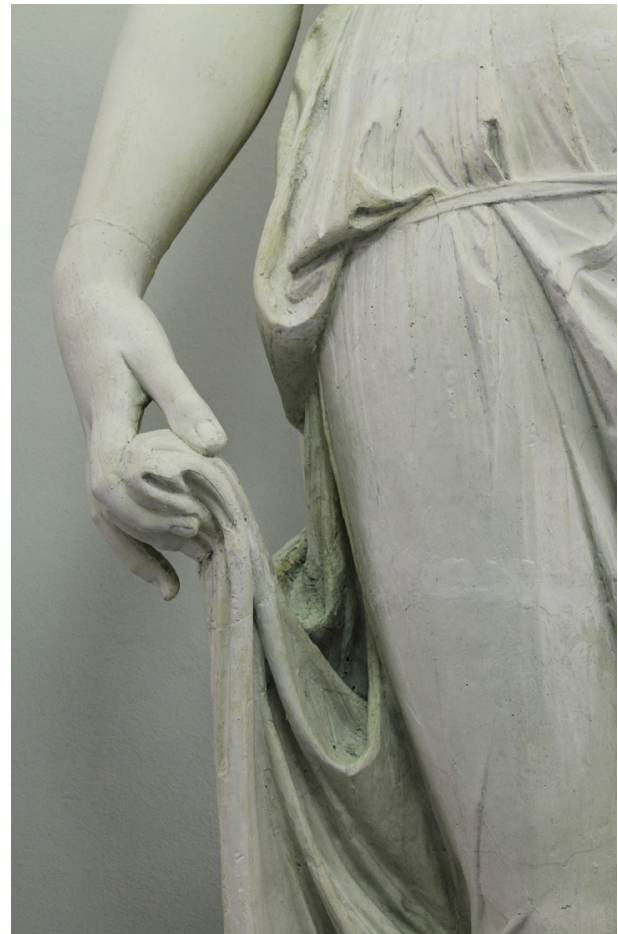

Fig. 23. Particular of the hand after laser cleaning.

signs of deposits due to the interaction with the environment, the plaster material and the problems related to its previous recent reconstruction. The signs of the deposits were removed with fluence from $1 \mathrm{~J} / \mathrm{cm}^{2}$ to 1.7 $\mathrm{J} / \mathrm{cm}^{2}$, while the marks in correspondence of the integration filling stucco needed to be increased up to the high value of $3 \mathrm{~J} / \mathrm{cm}^{2}$.

In the previously mentioned target areas, produced by the first laser tests, a blackening was observed due to a focused laser irradiation; continuing the irradiation in the same points with increased fluence $\left(1.7 \mathrm{~J} / \mathrm{cm}^{2}\right.$ to $3 \mathrm{~J} / \mathrm{cm}^{2}$ ) allowed removing the blackening. This phenomenon was possibly provoked by the presence of an acrylic resin, binding a pigment and previously applied to uniform the colour of the surface by a visual point of view. 


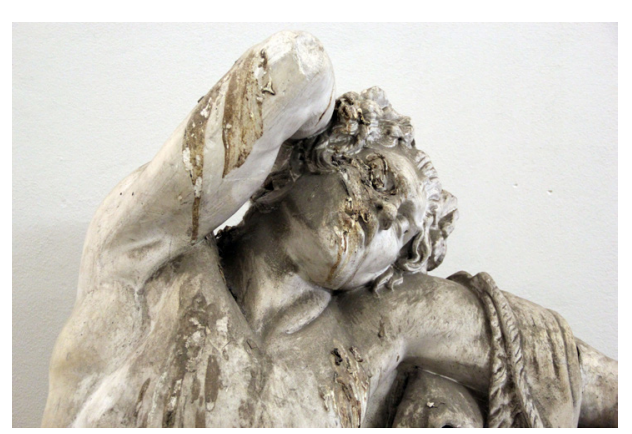

Fig. 24. Dry cleaning.

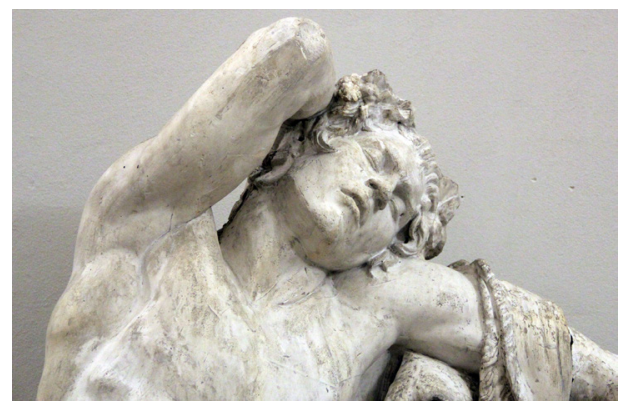

Fig. 25. Gel cleaning.

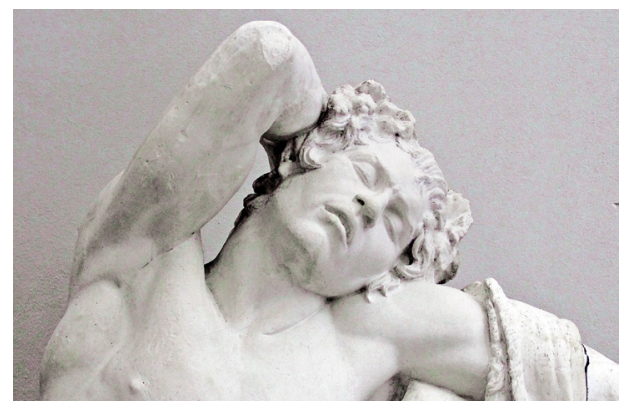

Fig. 26. Laser cleaning.
On the trunk of the Flora, using a fluence of 1.1 and of $1.7 \mathrm{~J} / \mathrm{cm}^{2}$, was the only possibility to obtain the appropriate cleaning level, not achievable with any other cleaning method. In fact, the presence of mortar patches on the side and the back of the trunk damaged heavily the sculpture; the laser cleaning system proved an extreme ability to remove unwanted materials. Through a careful calibration of the laser cleaning system, it was possible to reveal the subtle chromatic variations and the chiaroscuro effects, enhancing the legibility of the plaster cast surface.

The repetition rate changed importantly as to the different plaster casts, ranging from 7 to $18 \mathrm{~Hz}$; in fact, it was used as a valid tool to face the uneven level of deposits on the various surfaces and to obtain the correct restitution of the surface brightness.

All the values of the fluence quoted in the text are shown together with the correspondence with plaster areas in the following table (Tab. 1).

A very important aspect to stress is the crucial importance of safety, not only for the work of art but, in this special case for the students, for the workers and the public. All the students were trained specifically in order to use the laser in safe conditions. They have employed Individual Protection Devices during the restoration phases; they also

Table 1. Synoptic overview of used fluences $\left[\mathrm{J} / \mathrm{cm}^{2}\right]$

\begin{tabular}{|c|c|c|c|c|}
\hline \multirow{2}{*}{ Velletri Pallas } & Carnation & Dress & Basement & Base Low Rilief \\
\hline & $0.5 \rightarrow 1.1$ & $1 \rightarrow 1.4$ & 1.7 & $0.7 \rightarrow 1.0$ \\
\hline \multirow{2}{*}{ Flora Farnese } & Carnation & Dress & Basement & Tree Trunk \\
\hline & $0.5 \rightarrow 1.0$ & $0.9 \rightarrow 1.4$ & 1.7 & $1.1 \rightarrow 1.7$ \\
\hline \multirow{4}{*}{ Barberini Faun } & Carnation & Base & & \\
\hline & $0.7 \rightarrow 1.5$ & \multirow{3}{*}{$1.0 \rightarrow 1.7$} & & \\
\hline & Left Leg & & & \\
\hline & $1.0 \rightarrow 1.7$ AND 3 & & & \\
\hline
\end{tabular}


underwent a medical examination before and after the project. The importance of the detailed work plan is crucial when working in a crowded hallway: in fact, students and visitors frequently pass the hallway and we were obliged to set-up the site with strict laser safety barriers. The conservation site is under the supervision of the Ministry of Culture, which is located on the top floor of the Brera building; inspectors of the Ministry had a close supervision of the entire conservation process.

\section{Conclusion}

The Conservation School of Brera Academy is in charge of the maintenance and conservation of its own Cultural Heritage. The three case studies here presented were a pilot project for future works, taking into account the overall difficulties in cleaning gypsum based materials due to its solubility and low mechanical properties. Newberyite was highlighted in the near surface region as a new mineral formation, which possibly comes from guano; it is supposed to be related with a superficial grey layer, but further investigation is still needed. The laser cleaning technology was applied with success after preliminary treatments, with the aim to lower the grey layer already mentioned. The casts have been cleaned with THUNDER ART @ 532 nm, in the QS regime, ranging the fluence from 1.1 to 1.7 $\mathrm{J} / \mathrm{cm}^{2}$ and the repetition rate from 12 to 20 $\mathrm{Hz}$ in the average. The performance of laser cleaning treatments proved to be effective in removing the grey layer from the substrate.

\section{References}

[1] M. Anzani, L. Borgioli, A. Brunetto, A. Rabbolini, A. Sansonetti, J. Striova, "Sperimentazione di pulitura laser con intermediazione di gel di agar" in: APLAR 3 - Applicazioni LASER nel Restauro, Bari 18-19 june, 2010, Il prato 2011, pp. 45-58.

[2] S. Siano, J. Agresti, I. Cacciari, D. Ciofini, M. Mascalchi, I. Osticioli, A. A. Mencaglia, "Laser cleaning in conservation of stone, metal and painted articafts: state of the art and new insights on the use of the Nd:YAG lasers", 106 (2012) 419-446, DOI: 10.1007/ s00339-011-6690-8.

[3] A. Sansonetti, C. Colombo, M. Realini, M. Palazzo, M. De Marchi, "Laser cleaning of stucco's fragments from an early middle age bas-relief" in: Lasers in the Conservation of Artworks VII, Proceedings of International Conference Lacona VII Madrid, Spain, 17-21 Sep. 2007, Marta Castillejo, Pablo Moreno, Mohamed Oujja, Roxana Radvan, Javier Ruiz (Eds.), CRC Press 2008, pp. 243-248.

[4] Z. Mesikova, P. Sulcova, M. Trojan, "Syntesis and characterization of Newberyte", J. of Thermal Analysis and Calorimetry, 88 (2007) 103-106.DOI: 10.1007/ s10973-006-8099-8.

[5] R.L. Frost, S.J. Palmer, R. Pogson, "Raman spectroscopy of newberyite $\mathrm{Mg}\left(\mathrm{PO}_{3} \mathrm{OH}\right) \cdot 3 \mathrm{H}_{2} \mathrm{O}$ - a cave mineral", Spectrochimica Acta Part A., 79 (2011). DOI: 10.1016/j.saa.2011.04.035.

[6] M. Hanhoun, L. Montastruc C. Azzaro-Pantel, B. Biscans, M. Freche, L. Pibouleau, “Temperature impact assessment on struvite solubility product: a thermodynamic modeling approach", Biochemical Engineering Journal, 167 (2011) 50-58, DOI: 10.1016/j.cej.2010.12.001.

[7] A.W. Taylor, A.W. Frazier, E.L. Gurney, J.P. Smith, "Solubility products of di-and trimagnesium phosphates and the dissociation of Magnesium phosphate solutions", Transactions Faraday Soc., $1963,59,1585-1589$.

[8] A. Freund, G. Eggert, H. Kutzker, B. Barbier, "On the occurrence of magnesium phosphates on ivory", Studies in Conservation, 47 (2002) 155-160. 


\title{
Decontamination of biocidal loaded wooden artworks by means of laser and plasma processing
}

\author{
Birgit Angelika Schmidt ${ }^{1}$, Simone Pentzien ${ }^{1}$, Andrea Conradi ${ }^{1}$, Jörg Krüger ${ }^{1 \star}$, \\ Constanze Roth ${ }^{2}$, Oliver Beier ${ }^{2}$, Annett Hartmann ${ }^{2}$, Bernd Grünler ${ }^{2}$ \\ 1 Bundesanstalt für Materialforschung und-prüfung (BAM), Unter den Eichen 87, 12205 Berlin, \\ Germany \\ 2 INNOVENT e.V., Prüssingstraße 27 B, 07745 Jena, Germany \\ * Corresponding author: joerg.krueger@bam.de
}

\begin{abstract}
Many wooden artworks are contaminated by DDT (dichlorodiphenyltrichloroethane) as a result of a surface treatment by means of the liquid preservative Hylotox-59 ${ }^{\circ}$. It was used until the end of the 1980s. DDT crystal structures are formed on the wood surfaces by the "blooming" of chlorine compounds. In addition to an aesthetic disturbance, it is assumed that DDT represents a health risk. Even decades after applying, the toxins in the wood preservatives are still detectable because they are of low volatility in many wood samples. Contaminated waste wood with natural biocide ageing, gilded and wood carved elements of an old picture frame and wooden samples with paint layers were provided by the Schlossmuseum Sondershausen. Non-contact procedures using laser and plasma appear reasonable to remove the DDT crystals. During the experiments, health and safety issues for the operator have to be taken into account.

The removal of DDT was evaluated employing femtosecond and nanosecond laser radiation and cold atmospheric plasma techniques with different working gases (air, nitrogen, and argon). Before laser application, a chlorine measurement representing the DDT density on the wooden surface is done by X-ray fluorescence (XRF) analysis as reference. After laser processing, the XRF analysis is used again at the same surface position to determine the depletion rate. Additionally, a documentation and characterization of the sample surface is performed before and after laser and plasma treatment using optical microscopy (OM). For plasma processing with various systems a chlorine measurement is done by gas chromatographic-mass spectrometry (GCMS) analysis.
\end{abstract}

Keywords: decontamination, DDT, wooden artworks, femtosecond laser, nanosecond laser, cold atmospheric pressure plasma

\section{Introduction}

The main focus of this paper is the so-called Golden Carriage from the Schlossmuseum Sondershausen (see Fig. 1). It belongs to the collection of the Thuringian Foundation of
Palaces and Gardens. The Golden Carriage is a princely state coach of the Grand Carrosse type developed in France since the second half of the $17^{\text {th }}$ century. The type of the vehicle, its design and partly also its equipment indicate that this coach was built around 1710 in Paris [1]. 


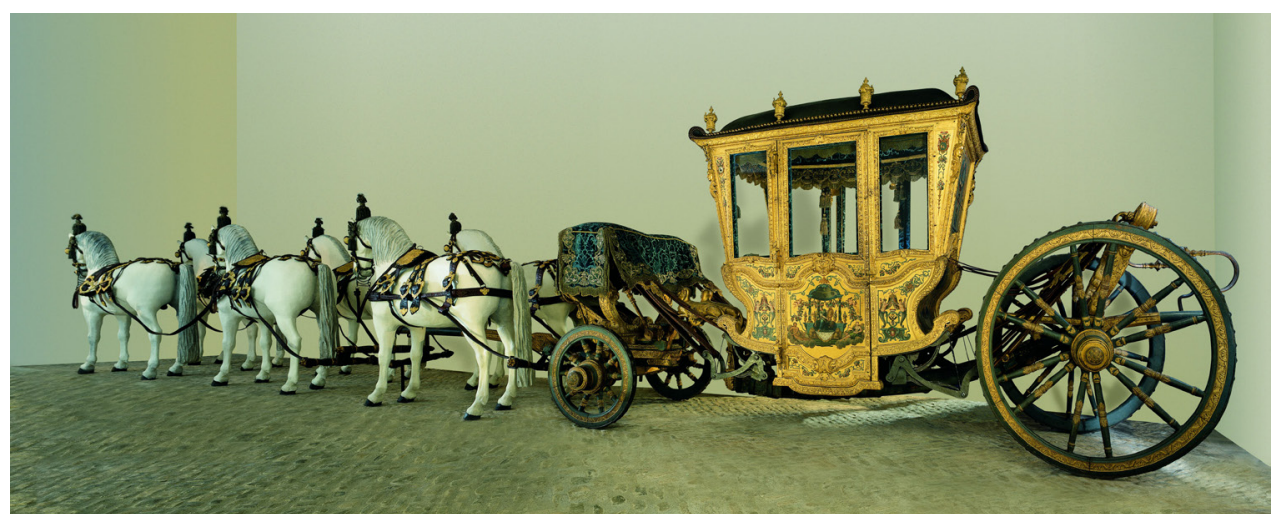

Fig. 1. The Golden Carriage. Copyright: Schlossmuseum Sondershausen. Photograph by U. Kneise.

Based on records [2], the use of Hyloto $x-59^{\odot}$, a DDT and lindane containing preparation against vermins, was firstly documented on construction parts of the Golden Carriage in 1969. Hylotox-59 ${ }^{\odot}$ as a liquid wood preservative was extensively used until the end of the 1980s in the former GDR. At that time, it was a very common wood preservative. It contains 3.5\% DDT and $0.5 \%$ lindane in a solvent mixture.

Studies have shown that the amount of biocide-contaminated objects in German cultural institutions like museums is very high, especially in the textile and natural historical fields [3].

From today's point of view, biocidal substances and agents represent a high risk with respect to a health hazard and environmental impact as well as potential object damage [4]. Therefore, it is of great importance to protect employees of collections and visitors of exhibitions in developing methods for reducing or even removing the toxic components from the contaminated materials.

In addition to dry processes for removing the crystalline efflorescence on surfaces, today a variety of wash procedures is used, among which the vacuum washing method is the most famous one. Furthermore, decontamination is performed by means of supercritical carbon dioxide and also allows a deep cleaning. Especially for profiled or carved surfaces and objects of art that cannot be exposed to moisture, the non-contact laser technology is a suitable alternative [5].

DDT crystal structures are formed on surfaces by the "blooming" of chlorine compounds as can be seen on the magnification of a surface of a wooden sample in Fig. 2. The aim of the studies presented here is to remove those DDT crystal structures from the surface. The removal of DDT was evaluated by employing femtosecond and nanosecond laser radiation and cold atmospheric plasma techniques as an alternative

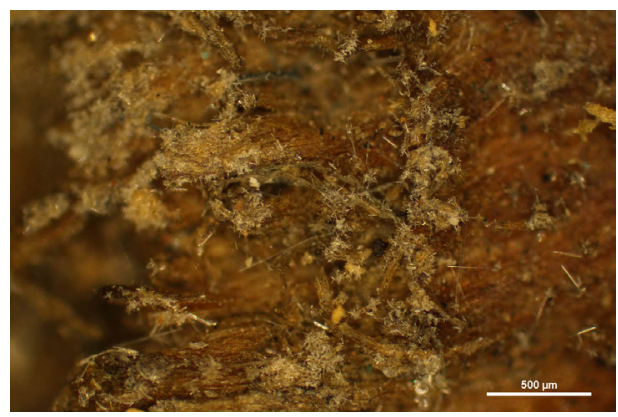

Fig. 2. Optical micrograph of DDT crystal structures ("blooming") on a contaminated wooden sample; scale bar represents $500 \mu \mathrm{m}$ 
or in addition to mechanical or chemical cleaning methods.

Experiments were performed on test samples as well as on original construction elements of the Golden Carriage. The results should give a guideline whether these methods can successfully be performed on these artworks.

\section{Materials and methods}

\subsection{Description of samples}

The Schlossmuseum Sondershausen provided three different types of samples. Contaminated wood samples with natural biocide ageing namely two strips which served as the backing protection of an easel painting (Fig. 3) and an original construction component of the Golden Carriage (Fig. 4) were investigated. In addition to crystalline DDT efflorescence, they also showed a light soiling, which proved to be an advantage for the use of lasers.

Furthermore, three gilded and stucco carved decorative elements of an old picture
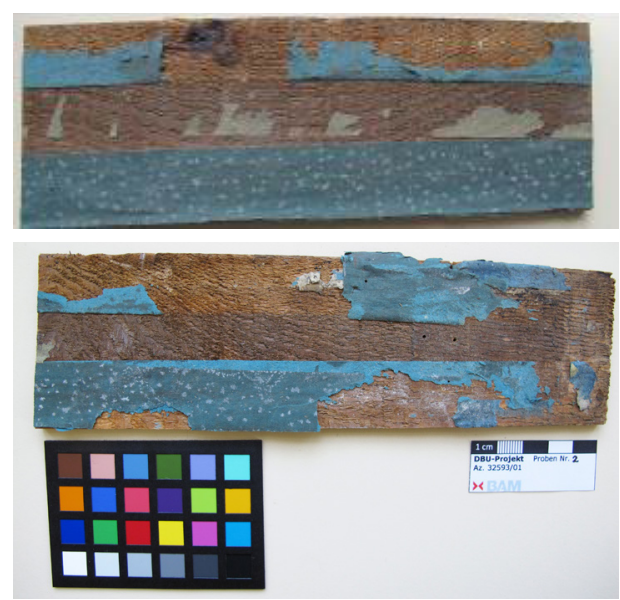

Fig. 3. Contaminated wood from the backing of an easel painting.

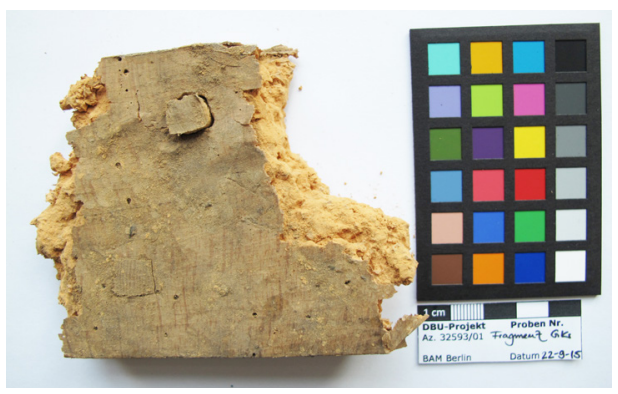

Fig. 4. Contaminated wood from the Golden Carriage.

frame (Fig. 5) as well as two wooden samples with different paint layers (Fig. 6) were tested.

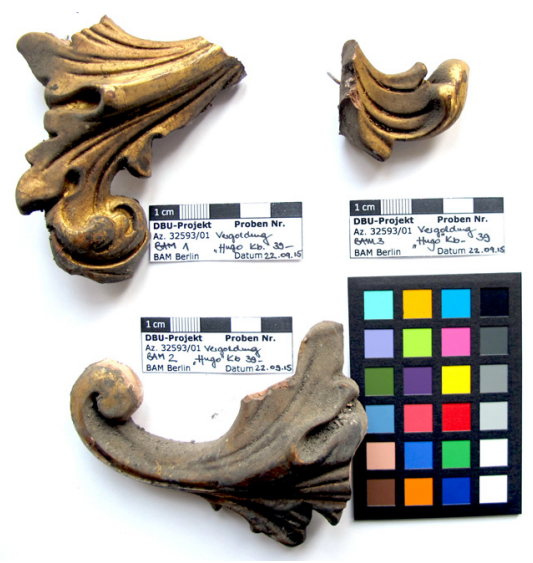

Fig. 5. Gilded, stucco carved objects.

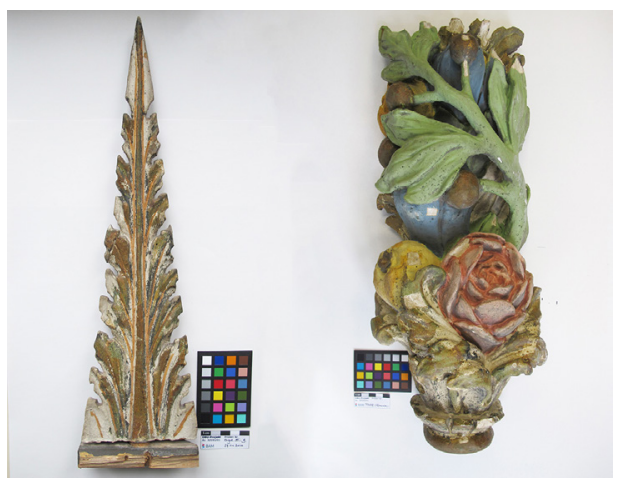

Fig. 6. Wood with paint layers. 


\subsection{Experimental methods}

In the following, details of the contactless laser and plasma treatment are described. Before and after laser application, a chlorine measurement is done by $\mathrm{X}$-ray fluorescence (XRF) analysis at exactly the same surface position. Chlorine depletion rates can be calculated using these data. Additionally, a documentation and characterization of the sample surface is done before and after laser and plasma treatment using optical microscopy.

\section{Energy dispersive $X$-ray fluorescence}

The X-ray fluorescence analysis is a non-destructive spectroscopic method, which is used for the qualitative and quantitative determination of the elemental composition of sample material. The surface of the object is thereby irradiated with X-rays. Element-specific secondary X-rays are emitted. Here, the X-ray spectra were recorded by means of a Fischer Scope XDAL XRF (Helmut Fischer GmbH + Co KG, Sindelfingen, Germany) equipped with a tungsten anode and operated at $10 \mathrm{keV}$ with a nickel filter. The X-ray spot diameter amounted to 0.7 $\mathrm{mm}$ and the data acquisition time was 60 seconds.

\section{GC MS measurements}

GC MS (gas chromatograph/mass spectrometer) is a combined analyzing system. The gas chromatograph serves to separate the substance mixture to be examined and the mass spectrometer for the identification and, if appropriate, also the quantification of the individual components. At INNOVENT the DDT content of samples was measured by using Headspace GC MS. The samples were heated in headspace vials and the evaporated phase was analyzed.

\section{Ultra short pulsed laser system}

The Ti:sapphire laser system (Compact Pro, Femtolasers) provides 30-fs laser pulses at $800 \mathrm{~nm}$ wavelength and a repetition rate of $1000 \mathrm{~Hz}$. Laser pulse energies of up to $1 \mathrm{~mJ}$ can be generated. The laser pulse energies were measured by means of a pyroelectric detector (Coherent). The laser pulses were focused by a spherical dielectric mirror of $500 \mathrm{~mm}$ focal length. A laser beam radius $\left(1 / e^{2}\right)$ of about $40 \mu \mathrm{m}$ was set on the sample surface. In contrast to the short pulse laser system, the object was moved relative to the laser beam employing linear $\mathrm{x}-\mathrm{y}-\mathrm{z}$ translation stages. Laser-treated areas of up to $5 \mathrm{~mm} \times 8$ $\mathrm{mm}$ were reached. The number of pulses per spot $\left(\mathrm{N}_{\mathrm{eff}}\right)$ was changed between 5 and 10 .

\section{Short pulsed laser system}

The BAM prototype laser cleaning workstation was described in detail by Kautek and Pentzien [6]. Some important parameters should be given here. The samples were exposed to a pulsed Q-switched Nd:YAG-laser (DINY $p Q$, IB Laser) generating 7-ns laser pulses at $532 \mathrm{~nm}$ wavelength and 10-ns laser pulses at $1064 \mathrm{~nm}$ wavelength at a pulse repetition frequency of $500 \mathrm{~Hz}$. Pulse energies were measured using an energy meter (Nova, Ophir). The spatial beam profile was Gaussian. Beam radii $\left(1 / e^{2}\right)$ of about $140 \mu \mathrm{m}(532$ $\mathrm{nm}$ wavelength) and $141 \mu \mathrm{m}(1064 \mathrm{~nm})$ on the sample surface were achieved. The laser beam was scanned over the samples reaching a field size of $2 \mathrm{~mm} \times 2 \mathrm{~mm}$. The maximum laser fluence in the focal spot $\left(\mathrm{F}_{0}\right)$ was varied by more than one order of magnitude for both wavelengths. The number of pulses per spot $\left(\mathrm{N}_{\mathrm{eff}}\right)$ was changed between 1 and 100 . The laser cleaning system is computer controlled and equipped with an exhaust system to remove ablation products. The cleaning action can be monitored on a computer 
screen. The laser-processing compartment fulfils Laser Class I conditions.

\section{Atmospheric Pressure Plasma}

In general, plasma is referred to as the fourth aggregate state besides solid, liquid and gaseous. Plasma is an ionized gas containing charged particles, such as ions and electrons. However, uncharged and partially charged components are also being included. One can differentiate for the different plasma types for example between low pressure and atmospheric pressure plasma as well as cold plasmas or discharges in thermal equilibrium. In the project INNOVENT decides to work with cold atmospheric pressure plasma jets, because it is a very variable, mobile and cost efficient technology.

The temperature of cold atmospheric pressure plasma is between 30 to $600^{\circ} \mathrm{C}$. The plasma intensity can be adjusted across the entire spectrum and investigations of plasmas itself were already made in restoration projects [7]. INNOVENT compared two different sources namely the TIGRES Plasma MEF (Tigres GmbH, Marschacht, Germany) (see Fig. 7) and kINPen 09 (neoplas tools $\mathrm{GmbH}$, Greifswald, Germany). This source is very easy to handle, has a gentle performance and is already in use for dermatological treatments [8], while the TIGRES source offers more variations in parameters due to its technical design. Here, results obtained utilizing TIGRES plasma MEF are presented.

The parameters gas type, number of treatments (run) and the distance between the treatment lines (pattern distance) were varied. The process gases during the experiments for the TIGRES plasma MEF were nitrogen, air or a nitrogen-hydrogen-mixture ( $5 \%$ hydrogen). Other parameters were kept constant, the velocity of the substrate relatively to the stationary plasma jet was set to $50 \mathrm{~mm} / \mathrm{s}$, the plasma-substrate distance was $10 \mathrm{~mm}$ and the electrical power $300 \mathrm{~W}$. An evaluation of the chlorine residues was performed with headspace GCMS for DDT peak areas.
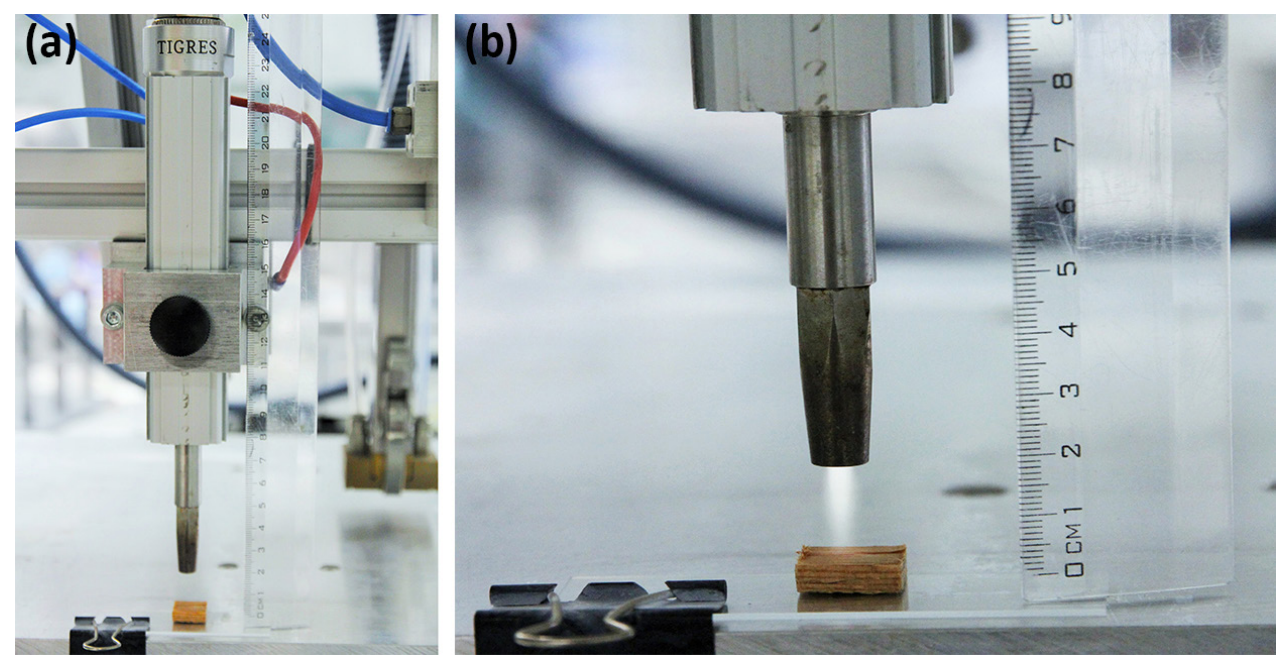

Fig. 7. Total view of TIGRES plasma MEF (a). Visualization of the discharge using air as process gas (b). Photographer: INNOVENT e.V. 


\section{Results}

Before cleaning experiments were performed, the laser damage thresholds of the sample materials had to be identified. They depend on pulse duration, wavelength and number of pulses per spot. Based on previous cleaning investigations for the restoration of a medieval wooden panel chamber, laser fluences should be limited to $<1.5 \mathrm{~J} / \mathrm{cm}^{2}$ for the use of $1064 \mathrm{~nm}$ laser radiation (and $6 \mathrm{~ns}$ pulse duration) to avoid microscopic wood damage [9]. The use of energy densities $>100$ $\mathrm{J} / \mathrm{cm}^{2}$ leads to significant ablation of beech model material [10]. Therefore, the task is the laser action caused a reduction of the chlorine concentration on the surface as can be seen by a comparison of the chlorine concentration values between Fig. $8 \mathrm{~b}$ and Fig. $8 \mathrm{a}$ within the white frame.

\subsection{Ultra short pulsed laser treatment on contaminated wood}

Test series on contaminated wooden surfaces using ultra short laser pulses led to a chlorine depletion rate of about $75 \%$ at a laser fluence of $\mathrm{F}_{0}=0.13 \mathrm{~J} / \mathrm{cm}^{2}$ and an effective number of pulses per spot of $\mathrm{N}_{\mathrm{eff}}=10$. Figure 9 depicts the relative change of the chlorine content
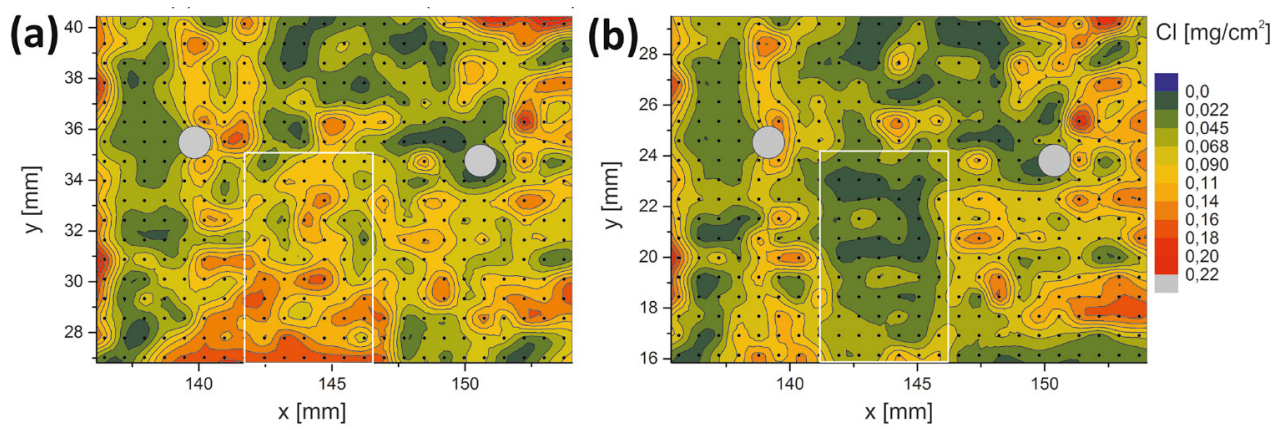

Fig. 8. Chlorine distribution of a selected area on a wood sample before (a) and after (b) laser treatment ( $30 \mathrm{fs}$, $800 \mathrm{~nm}, \mathrm{~F}_{0}=0.13 \mathrm{~J} / \mathrm{cm}^{2}, \mathrm{~N}_{\text {eff }}=10$ ).

to find a laser working range, that means energy density $\left(\mathrm{F}_{0}\right)$ and effective number of pulses per spot $\left(\mathrm{N}_{\mathrm{eff}}\right)$, ideally to be above the cleaning threshold and below the damage threshold of the sample material. As mentioned above, XRF analysis was performed for the determination of the chlorine content before (Fig. 8a) and after laser treatment (Fig. 8b).

Figure 8 shows a comparison of the absolute chlorine concentration of a predefined surface area before and after laser treatment. The white frame marks the area where laser treatment was performed. It is obvious that

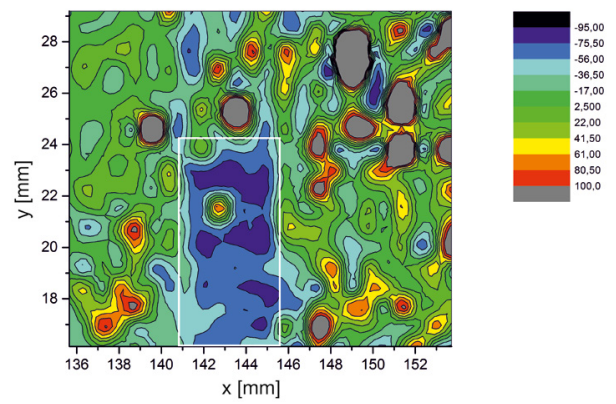

Fig. 9. Relative chlorine distribution plot of a wood sample. The values were obtained by a pointwise calculation according to (XRF signal after laser XRF signal before laser) / (XRF signal before laser) $\times 100 \%$. 
expressed as a percentage. In the violet range indicating the above mentioned depletion rate, the laser treatment was done.

Figure 10 illustrates the original surface contamination on a wood sample (Fig. 10a). Clearly, DDT crystals can be observed on the surface as white efflorescence. Figure $10 \mathrm{~b}$ shows an OM of the sample surface after
The picture in Fig $11 \mathrm{~b}$ depicts the cleaning result in the red frame, while Fig. 11a shows the surface before.

Compared with the ultrashort pulse laser at $800 \mathrm{~nm}$, a slightly lower chlorine depletion rate can be observed with the short pulse laser system. Moreover, based on the micrographs (see Figs. 10 and 11), it can be seen
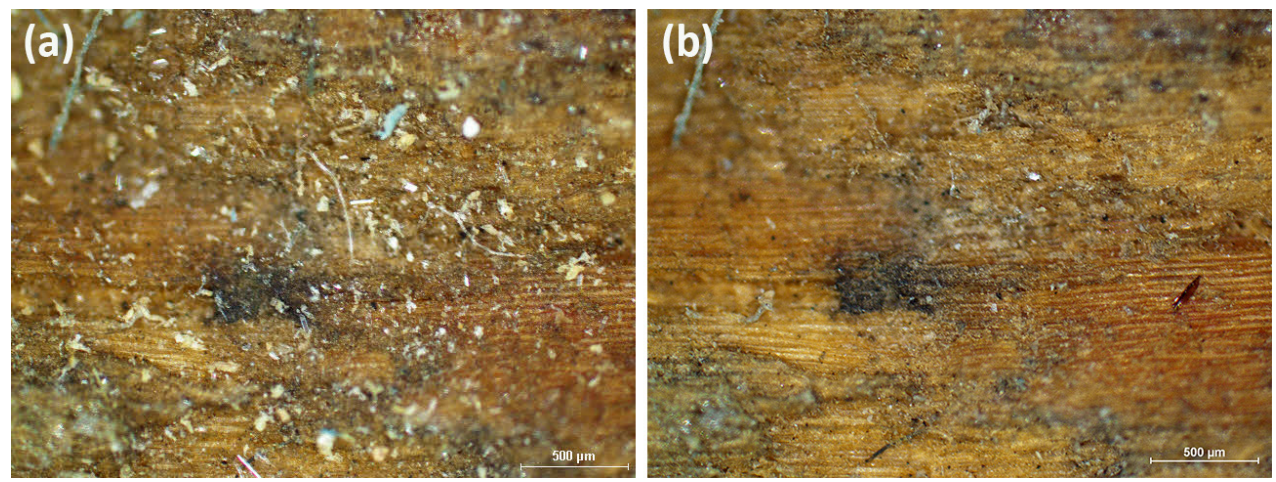

Fig. 10. Optical micrographs of a wooden sample before (a) and after (b) laser irradiation ( $30 \mathrm{fs}, 800 \mathrm{~nm}$ ); scale bars represent $500 \mu \mathrm{m}$.

femtosecond laser treatment of the whole area. The successful cleaning of the surface is demonstrated. Almost no DDT crystals remain on the surface.

\subsection{Short pulsed laser treatment on contaminated wood}

With the short pulsed laser system, it was possible to achieve chlorine depletion rates in the range of $55-70 \%$ employing laser energy densities from $0.4-0.8 \mathrm{~J} / \mathrm{cm}^{2}$ and pulse numbers per spot of 5 and 10. These parameters are above the cleaning threshold and below the damage threshold of the tested material. In addition to the XRF-analysis, OM micrographs of the original surface were created and were compared with micrographs after laser action. that in the femtosecond case a better removal of DDT crystals was achieved compared to the nanosecond laser use.

\subsection{Short pulsed laser treatment on gilded objects}

Several experiments on gilded wooden samples were carried out but even good cleaning results achieved no chlorine reduction due to the fact that there is only a small amount of chlorine on the sample. Therefore, only a removal of dust is needed in this special case. XRF measurements confirmed the low amount of chlorine, too. A uniform cleaning result was obtained on the sample surface with an intact gold layer using laser pulses with $\mathrm{F}_{0}=0.2 \mathrm{~J} / \mathrm{cm}^{2}$ and $\mathrm{N}_{\text {eff }}=5$ (Fig. 12a). If the laser energy density is increased by 


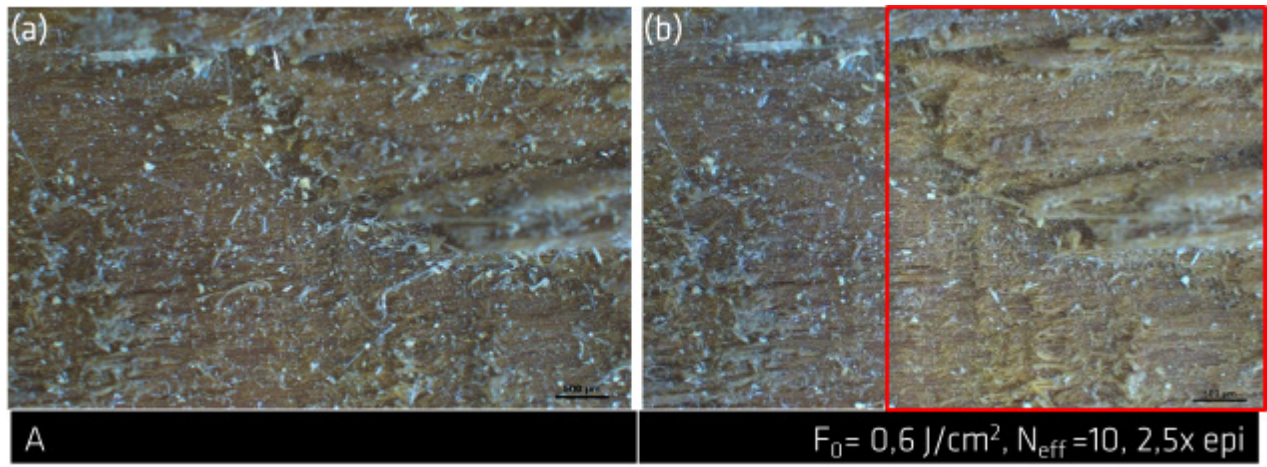

Fig. 11. Optical micrographs of a wooden sample before (a) and after (b) laser irradiation (10 ns, $1064 \mathrm{~nm})$; scale bars represent $500 \mu \mathrm{m}$.

a factor of two only for the same number of pulses per spot, the sensitive gold layer is damaged (Fig. 12b). be determined microscopically due its low layer thickness. The second layer from 1886 is designed as a wood imitation on

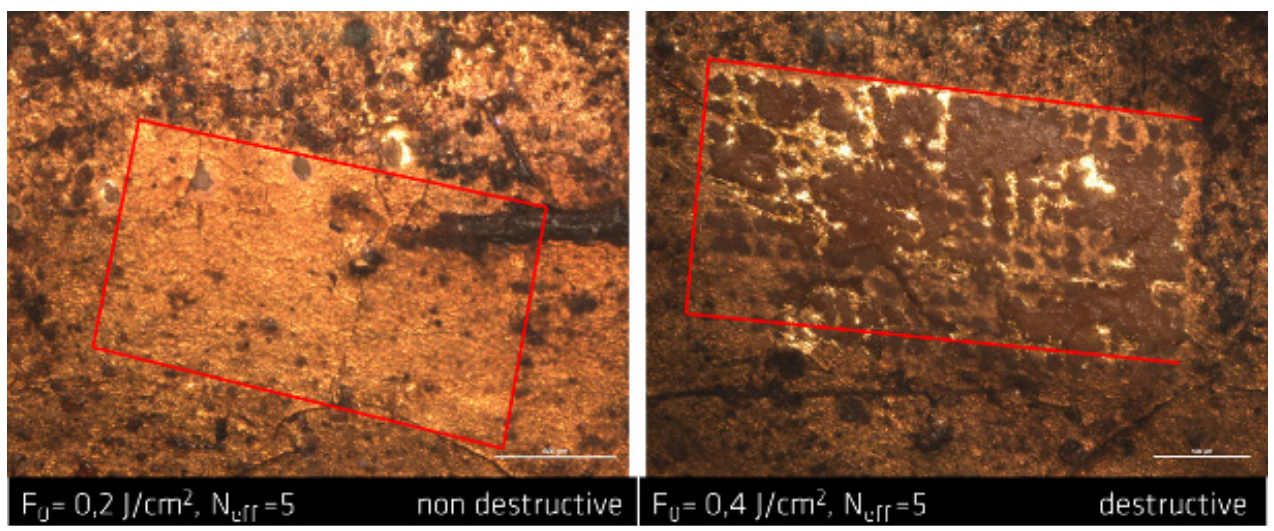

Fig. 12. Optical micrographs of a golden sample after a non destructive cleaning experiment (a) and after a destructive cleaning experiment (b) with laser pulses at a pulse length of $10 \mathrm{~ns}$ at 1064 wavelength; scale bars represent $500 \mu \mathrm{m}$.

\subsection{Short pulsed laser treatment on wood with paint layers}

Investigations were done on wood craft samples with paint layers from the baroque era. The first layer, performed in 1703 , is made of pure white on a chalk ground (probably a polished white layer) and also contains chalk and blanc fixe. It can only an ocher, oil-based primer. The nowadays still visible third layer was performed in 1934 and consists of different oil colours on a yellowish-white, oil-based primer. An OM micrograph (Fig. 13) clearly identifies DDT crystals, therefore cleaning attempts were made. For all different colour regions (brown, white, gold, green, and black), the damage thresholds of the paint layers were 


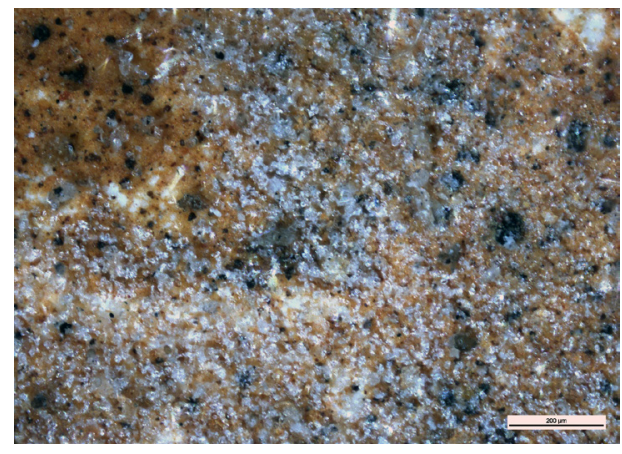

Fig. 13. Optical micrograph of DDT crystal structures ("blooming") on a contaminated wooden sample with paint layers; scale bar represents $200 \mu \mathrm{m}$.

determined. In many cases, these damage thresholds are comparable or even lower than the cleaning threshold to remove DDT of about $0.3 \mathrm{~J} / \mathrm{cm}^{2}$. As a conclusion and with the exception of only two coloured areas (brown and white), it was not possible to remove the DDT crystals without causing damage to the paint layers beneath.

\subsection{Atmospheric pressure plasma on contaminated wood}

In the first series of tests, appropriate parameters for cleaning of wood surfaces were investigated.

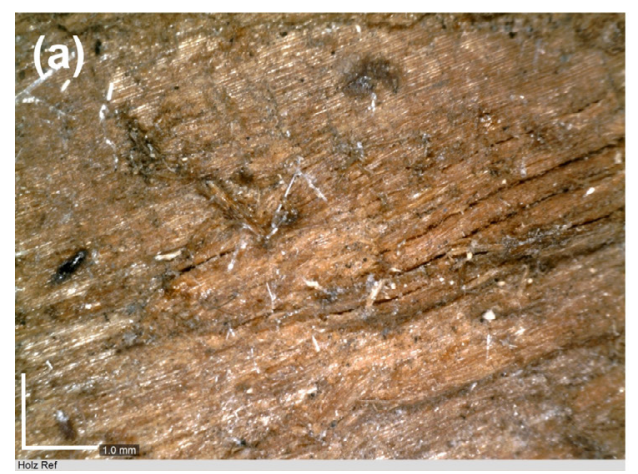

The prerequisites were minimal loading of the surface by the plasma treatment and a significant (damage-free) cleaning effect. Figure 14b shows the plasma cleaning result of a wooden sample from the Schlossmuseum Sondershausen by optical microscopy. Here, nitrogen was used as a process gas with only one plasma treatment run on the surface.

As can be seen after the plasma treatment, a strong decrease of visible DDT crystallites (the white needles in Fig. 14a) at the wooden surface is obtained (Fig. 14b). But it has to be mentioned that for these samples no reproducible depletion quantities could be determined since the original chlorine loading by the wood preservative was not documented or directly known. GC MS measurements revealed here from sample to sample significant differences in DDT concentrations allowing no clear statements for the different treatment conditions. Therefore, new samples were prepared from beech veneers, which had a defined chlorine loading (DDT). INNOVENT has chosen beech veneer, because the Golden Carriage is mainly made of beech. With these samples the plasma treatments were carried out first and the GC MS measurements followed. The results for different types of process gases

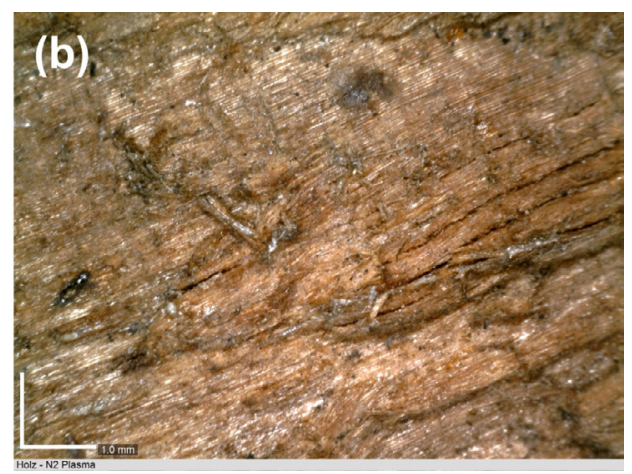

Fig. 14. OM of contaminated wood before (a) and after (b) plasma treatment with nitrogen as process gas; scale bars represent $1 \mathrm{~mm}$. 
(air, nitrogen, nitrogen/hydrogen mixture), a pattern distance of $1 \mathrm{~mm}$ or $3 \mathrm{~mm}$ and the number of treatment runs are shown in Table 1. For each parameter six samples were plasma treated and measured by GC MS. The depletion rate was compared to untreated reference samples measured on the same day to minimize environmental effects.

By varying the type of gas, the distance between the treatment lines (pattern distance) und number of treatments (run) different results could be achieved. At first, all gases show a chlorine (DDT) depletion. For air, the standard deviation was the highest for the same amount of treated samples. Here it could be observed that besides samples with high depletion rates with more than $50 \%$ also low rates of max. $20-30 \%$ occur. In contrast, for nitrogen the standard deviation is lower under the same conditions and trend to decrease with higher numbers of plasma treatments (increase of interaction times - higher energy input). Best depletion results were achieved for nitrogen with a 1 $\mathrm{mm}$ pattern distance and ten treatment runs.
With these parameters a depletion of DDT total volume between $60-70 \%$ is possible and at the same time comparable cleaning results from sample to sample are obvious. First experiments with the nitrogen-hydrogen gas mix show that there is already a similar effect to that of nitrogen in three runs, which will be further investigated.

\section{Conclusions}

The removal of unwanted DDT crystals from wooden artworks as a result of a biocidal treatment in the 1980s with advanced non-contact laser and plasma techniques was performed. Short and ultrashort pulsed lasers and cold atmospheric plasma were used. On wooden samples, a depletion of the chlorine concentration of $55-70 \%$ and $75 \%$ was achieved for 1064-nm nanosecond pulses and $800-\mathrm{nm}$ femtosecond laser pulses, respectively. For the application of 30-fs laser pulses, no crystalline DDT residues remain on the sample surfaces. Using the TIGRES

Table 1. Evaluation results of headspace GC MS peak areas for DDT for different plasma treatment parameters

\begin{tabular}{lcccc}
\hline $\begin{array}{l}\text { Type } \\
\text { of Gas }\end{array}$ & $\begin{array}{c}\text { Pattern } \\
\text { distance }\end{array}$ & Run & $\begin{array}{c}\text { Mean value decontamination } \\
\text { rate / total volume DDT }\end{array}$ & $\begin{array}{c}\text { Standard variation of decontam- } \\
\text { ination rate / total volume DDT }\end{array}$ \\
\hline $\mathrm{N}_{2}$ & 3 & 3 & $50 \%$ & $14 \%$ \\
\hline $\mathrm{N}_{2}$ & 3 & 5 & $35 \%$ & $14 \%$ \\
\hline $\mathrm{N}_{2}$ & 3 & 7 & $37 \%$ & $10 \%$ \\
\hline $\mathrm{N}_{2}$ & 3 & 10 & $65 \%$ & $8 \%$ \\
\hline $\mathrm{N}_{2}$ & 1 & 5 & $60 \%$ & $6 \%$ \\
\hline $\mathrm{N}_{2}$ & 1 & 7 & $65 \%$ & $8 \%$ \\
\hline $\mathrm{N}_{2}$ & 1 & 10 & $70 \%$ & $9 \%$ \\
\hline $\mathrm{N}_{2} \mathrm{H}_{2}$ & 3 & 1 & $29 \%$ & $10 \%$ \\
\hline $\mathrm{N}_{2} \mathrm{H}_{2}$ & 3 & 2 & $52 \%$ & $6 \%$ \\
\hline $\mathrm{N}_{2} \mathrm{H}_{2}$ & 3 & 3 & $51 \%$ & $10 \%$ \\
\hline Air & 3 & 3 & $52 \%$ & $21 \%$ \\
\hline Air & 1 & 3 & $56 \%$ & $14 \%$ \\
\hline Air & 1 & 5 & $51 \%$ & $18 \%$ \\
\hline Air & 1 & 7 & $47 \%$ & \\
\hline
\end{tabular}


plasma MEF with nitrogen as working gas depletion rates of $60-70 \%$ could be achieved with a relatively high number of treatment runs. Initial experiments with nitrogen-hydrogen gas mix show that similar effects can be achieved after three runs.

Gilded wooden objects with high soiling but low DDT contamination can be cleaned successfully with nanosecond laser pulses. Based on colour changes, cleaning of only a few selected wooden samples with paint layers is possible.

\section{Acknowledgements}

The authors would like to thank Mario Sahre and Thorid Lange (BAM 6.7) for the XRF measurements and Karolina Barnikol (INNOVENT) for GCMS measurements. Fruitful discussions with Antje Pohl are gratefully acknowledged. This work was financed by the Deutsche Bundesstiftung Umwelt DBU (German Federal Environmental Foundation).

\section{References}

[1] B. Bärnighausen, H. Bärnighausen, Die Goldene Kutsche von Schloß Sondershausen. Amtlicher Führer Special, Deutscher Kunstverlag GmbH München, Berlin 2001,pp. 7.

[2] B. Bärnighausen, H. Bärnighausen, Die Goldene Kutsche von Schloß Sondershausen. Amtlicher Führer Special, Deutscher Kunstverlag GmbH München, Berlin 2001, pp. 37.

[3] H. Tello, B. Paz, „Über den Einsatz von Bioziden in naturkundlichen, botanischen und musealen Sammlungen - Rückblick und Ausblick“, in: P. Zalewski (Ed.), Biozidbelastete Kulturgüter- Grundsätzliche Hinweise und Texte zur Einführung in die Problematik. Bericht über das EU-/ ESF-Projekt „Kleine und Mittlere Unternehmen und Wissenschaft im Dialog. Dekontamination von Kulturgütern“, Frankfurt (Oder), 2014, pp. 107-124.
[4] H. Tello, „Die Problemlage der Kontamination von Kunst- und Kulturgütern hervorgerufen durch den Einsatz von Bioziden im Land Brandenburg", in: P. Zalewski (Ed.), Biozidbelastete KulturgüterGrundsätzliche Hinweise und Texte zur Einführung in die Problematik. Bericht über das EU-/ ESF-Projekt „Kleine und Mittlere Unternehmen und Wissenschaft im Dialog. Dekontamination von Kulturgütern“, Frankfurt (Oder), 2014, pp. 19.

[5] E. Jelen, K. Püschner, "Dekontamination biozidbelasteter Kunst- und Kulturgüter aus Holz", in: $\mathbf{L a}$ seranwendung in Restaurierung und Denkmalpflege. Grundlagen-Chancen-Perspektiven (Proceedings), Osnabrück, Germany, January 30-31, 2009, G. Wiedemann, U. Klotzbach, U. Bauer-Bornemann (Eds.), Fraunhofer IRB Verlag, Stuttgart 2009, pp. 165-170.

[6] W. Kautek, S. Pentzien, "Laser cleaning system for automated paper and parchment cleaning", in: Springer Proceedings in Physics 100 (2005), Proceedings LACONA $V$, Osnabrück, Germany, September 15-18, 2003, K. Dickmann, C. Fotakis, J. F. Asmus (Eds.), pp.403-410,DOI: 10.1007/3-540-27176-7_51.

[7] PANNA - Plasma and Nano for new age soft conservation, closed Project in EU Frame Programme 7 (FP 7), Project ID: 282998, 2011-2014. http://cordis. europa.eu/project/rcn/101319_en.html (accessed: 26.06.2017).

[8] T. von Woedtke, K.-D. Weltmann, „Grundlagen der Plasmamedizin“, Der MKG-Chirurg, Ausgabe 4/2016, https://www.springermedizin.de/grund lagen-der-plasmamedizin/10981948, (accessed: 26.06.2017).

[9] G. Wiedemann, M. Schulz, J. Hauptmann, H.-G. Kuscha, S. Müller, M. Panzner, H. Wust, "Laser cleaning applied in the restoration of a medieval wooden panel chamber at Pirna", Journal of Cultural Heritage 1 (2000), pp. S247-S258, DOI: 10.1016/ S1296-2074(00)00133-3.

[10] M. Panzner, G. Wiedemann, K. Henneberg, R. Fischer, T. Wittke, "Experimental investigation of the laser ablation process on wood surfaces", Applied Surface Science 127-129 (1998), pp. 787-792, DOI: 10.1016/S0169-4332(97)00743-5. 



\title{
Laser-based techniques for a multidisciplinary action aimed at the restitutive restoration of $S$. Costanzo church in Ronciglione (Italy)
}

\author{
Valeria Spizzichino $^{1 \star}$, Luisa Caneve ${ }^{1}$, Massimiliano Ciaffi ${ }^{1}$, Roberta Fantoni ${ }^{1}$, \\ Massimo Francucci ${ }^{1}$, Massimiliano Guarneri ${ }^{1}$, Antonio Palucci ${ }^{1}$, Gaetano \\ Terranova $^{1}$, Franca Persia ${ }^{2}$, Angelo Tati ${ }^{2}$, Maria Fernanda Falcon Martinez ${ }^{3}$, \\ Chiara Giuffrida ${ }^{3}$, Francesca Scirpa ${ }^{3}$, Laura Bartoli ${ }^{4}$, Alessandro Zanini ${ }^{4}$
}

1 ENEA - Italian National Agency for New Technologies, Energy and Sustainable Economic Development, Via Enrico Fermi, 45, 00044 Frascati (Rome), Italy

2 ENEA - Italian National Agency for New Technologies, Energy and Sustainable Economic Development, Via Anguillarese, 301, 00123 Rome, Italy

3 Freelance conservator

4 El.En. S.p.A. Via Baldanzese, 1750041 Calenzano Firenze - Italy

* Corresponding author: valeria.spizzichino@enea.it

\begin{abstract}
In the framework of the COBRA (Conservation of Cultural Heritage through Radiation and Enabling Technologies) project a multidisciplinary study was carried out in order to restore the mural painting of the dome of the S. Costanzo church in Ronciglione (Italy). Large difficulties were met during the restitutive restoration of the original renaissance fresco decorating the dome, patronized by the Italian Ministry of Cultural Heritage (SABAP RM MET). In fact, two and sometimes three layers of paint, which lays on an extremely crumbled substrate were detected on the surface, in addition to large, deep and complex degradation forms with the presence of efflorescence. To solve such a critical situation, researchers, conservators and laser cleaning system suppliers collaborated to plan the most appropriate intervention. Laser-based diagnostics were employed to characterize different areas of the fresco surface before and after cleaning tests. In particular, ENEA offered its prototypes of LIF (Laser Induced Fluorescence) and RGB-ITR (Red Green Blue Imaging Topological Radar, an innovative 3D colour laser scanner) in addition to a laser Raman system and an infrared camera for thermographic measurements for both the material and degradation recognition and a micro-structural study. The results obtained allowed pointing at the most suitable restoration and conservation strategies. The cleaning tests were performed with both classical methods and lasers made available by El.En. group. Different operative parameters such as pulse duration, repetition rate, energy were tested. The laser employed made it possible to overpass the difficulties encountered by other cleaning techniques: degradation and painting layers were removed selectively, allowing bringing to light the original surface.
\end{abstract}

Keywords: Laser Induced Fluorescence, Raman spectroscopy, laser cleaning, Imaging Topological Radar 


\section{Introduction}

In order to face a diagnostic/conservation/ restoration project, a broad collaboration among different experts is needed. In particular, art historians, conservators, scientists and administrations (museum's managers, employees at departments of National Cultural Heritage) have to work in strict collaboration to obtain satisfactory results. In fact, their jobs and their expertise are complementary. Moreover, all of them have questions to ask and answers to offer. The term "archaeometry" was coined in 1958 as a name of the internal bulletin of the Research Laboratory for Archaeology and the History of Art of Oxford to provide an answer to the Humanist's questions: what is that? How was it realized? What is the material provenance? What kind of materials are used? In the same period, between the years 1957 and 1959, the first lasers were created and patented. Since then, archaeometry and lasers had developed on different ways up to 1970 s when their paths crossed [1]. Since that time, several spectroscopic and optical laser techniques have been applied to artworks and demonstrated their effectiveness, mainly as case studies and only for "scientific" purposes. The time is ripe for a more synergistic and multidisciplinary approach in artwork diagnostics and conservation. The availability of low cost and well-tuned laser instrumentation may help to fulfil this request [2-5]. In this context, ENEA (Italian National Agency for New Technologies, Energy and Sustainable Economic Development) is carrying out a project called COBRA (Conservation of Cultural Heritage through Radiation and Enabling Technologies), funded by Latium Region (lr13, n.1031), which is addressed to the transfer of innovative technologies and methodologies from research to $\mathrm{CH}$ end users and SME involved in the conservation. In the frame of the project COBRA, ENEA offers technologies and expertise in creating digital services, mobile and fixed laboratories to respond to end users' requirements and to the requests of the specialized market. The first case-study was a multidisciplinary action carried out in order to restore the mural painting of the dome of the S. Costanzo church in Ronciglione (Italy). To solve the large difficulties met during the restitutive restoration of the original renaissance fresco decorating the dome, researchers, conservators and suppliers of lasers for cleaning collaborated to plan the most appropriate intervention. Several areas of the treated surface were analysed and characterized by laser-based diagnostics before and after cleaning tests. LIF (Laser Induced Fluorescence) and Raman spectroscopy were applied to the recognition of both constituent materials and degradation forms. A micro-structural study was performed with an RGB-ITR (Red Green Blue Imaging Topological Radar). Thermal Infrared Technology was applied to detect humidity in the walls, detachments, fissures. Both classical chemical methods and lasers made available by El.En. group were tested for the cleaning of the degraded painted surface. After several evaluations, the most suitable laser and operation parameters were selected for every area. In the places where no other techniques worked, the laser cleaning allowed bringing to light the original surface.

The characteristics and critical issues of the dome decoration, details of the apparatus used and the first results obtained are presented in the following sections. 


\section{The decoration of the dome of S. Costanzo}

Historical documentation traces the church's origin back to the $16^{\text {th }}$ century. During its lifetime, the decorative setting has gone through many changes, and the current general appearance spans from the baroque style of the altar to the neoclassic one of the nave. The dome shared its fate: the ongoing restoration work is showing the visible coffered decoration of the $18^{\text {th }}$ century, concealing other more ancient decorations. A long history of infiltration and carelessness had produced several damages such as the decay of mortar, accretions, brittleness, losses and flaking. The paintings appeared to be in very poor condition, but yet several different and fine decorative pieces were still visible. An earlier overview highlighted the presence of two/three layers of paint, irregularly overlapped one to each other, laying on a plaster substrate extremely disaggregated and with the presence of efflorescence. In Fig. 1 examples of the deteriorated surfaces are presented.

On a disaggregated mortar, filled with soluble salt deposits and altered materials due to past infiltrations, there was a fresco with a glory of angels, of very fine manufacture, yet highly damaged and overpainted. It presented painting holes, secco paintings and strong decohesion. On such layer, there was a polychromy at lacunars motif, painted without any preparatory layer and engraving the decorative pattern directly on the fresco layer. In some areas, there was a third layer having another polychromy with the same lacunar motif of underlying layer. The degradation of the polychrome multi-layer was so dramatic that it could not sustain neither the pressure of a lancet nor the weight of a compress. Then, issues encountered in consolidation, in identification of biodeteriogens and of different pictorial techniques that were used required accurate investigations and the use of the least invasive techniques. Moreover, due to the large quantity of areas to be evaluated rapidly (see Fig. 2), fast imaging techniques were preferred in order to reduce the time of the analysis.

After the first phase of the study of the dome, of the decoration and of its degradation, the open questions were emphasised. In fact, to face the consolidation and the cleaning of the surface correctly, leading to the restitution of the original renaissance fresco, the conservators needed to know the techniques (secco or tempera) used for the outer layers, have knowledge regarding the presence of biodeteriogens, detachment and crack location. Moreover, since the first tests of consolidation by acrylic resins showed the lack of homogeneity in the distribution

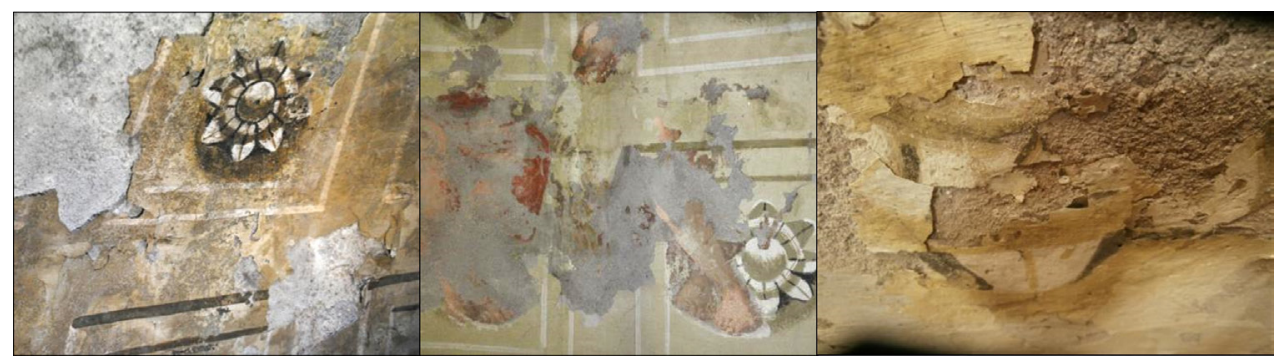

Fig. 1. Deterioration of decorate surface of the dome. 


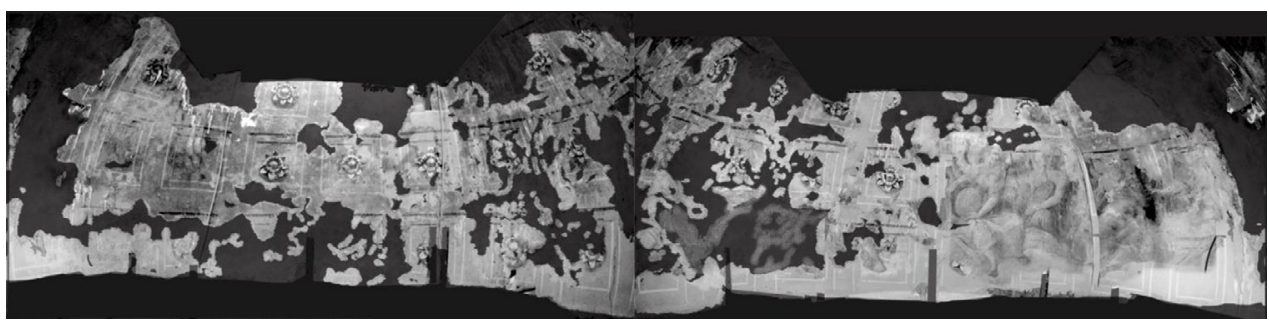

Fig. 2. The $360^{\circ}$ view of the dome showing the major intervention areas (in light gray). The circumference at $80 \mathrm{~cm}$ is $14 \mathrm{~m}$. The total dome area is $37 \mathrm{~m}^{2}$ of which $22 \mathrm{~m}^{2}$ with painting remains.

of the consolidants in the surface, their penetration level and migration directions had to be evaluated. Finally, the possibility to perform a laser cleaning and its efficiency were evaluated.

As in many real cases, referring to large artworks, the main requirements were the ability to work in situ (in uncontrolled experimental conditions), in quasi-real time conditions, in order to quickly find a solution to specific problems, mapping large areas, without sampling. To meet them, the techniques presented in next session were selected.

\section{Techniques}

For the characterization of constituent materials and degradation forms Laser Induced Fluorescence (LIF) and Raman spectroscopy were used $[5,6]$. For the latter, a portable $i$-Raman spectrometer (B\&W TEK Inc., USA) provided with a $785 \mathrm{~nm}$ excitation laser source and a CCD detector thermoelectrically cooled at $10^{\circ} \mathrm{C}$ was chosen. The spectrometer having a resolution of $3 \mathrm{~cm}^{-1}$ covers the spectral range $789-1048 \mathrm{~nm}$, corresponding to Raman shifts of $75-3200 \mathrm{~cm}^{-1}$. The excitation source is a GaAlAs solid state diode laser with a power scalable in the range 3-300 $\mathrm{mW}$. The laser power used during the measurements was set to a few milliwatts to prevent pigment photodecomposition. Typical acquisition conditions were 1 to 10 accumulations with exposure times of 1-5 s each. For measurements a probe, equipped with a quartz window and a spot diameter of $85 \mu \mathrm{m}$, was used. It has two optical fibres: the first is to deliver the excitation source and has a $105 \mu \mathrm{m}$ core, the second is for the collection and is characterized by a $200 \mu \mathrm{m}$ core. For LIF measurements a scanning prototype able to collect hyperspectral fluorescence images on large areas, realized at the ENEA in the Diagnostic and Metrology Laboratory of Frascati, was used. The system performs a point by point scanning, suitable for rapid investigations on large areas. A diode pumped Nd:YAG laser source is utilized to generate the UV radiation at $266 \mathrm{~nm}$ with repetition rate of $20 \mathrm{~Hz}$, pulse duration of $8 \mathrm{~ns}$ and energy up to $1.5 \mathrm{~mJ}$, which is well below the damage threshold previously tested on fresco's specimens. Each scan is monitored by a portable computer in which a specific program developed in LabView allows setting experimental parameters and controlling data acquisition. In the main control panel, data are shown both as $2 \mathrm{D}$ monochromatic images and LIF spectra, from $270 \mathrm{~nm}$ to 750 $\mathrm{nm}$ with a $2.5 \mathrm{~nm}$ spectral resolution, for each pixel. Additionally, the LIF scanning system can be utilized as hyperspectral analyser, with 
the laser switched off, to collect reflectance images upon the availability of an intense standard light source. For locating discontinuities and detachments thermovision and RGB-ITR (Red Green Blue Imaging Topological Radar) measurements were performed and compared. For thermographic measurements a commercial infrared camera FLIR T440 with frame rate of $60 \mathrm{~Hz}$, field of view $25^{\circ} \times 19^{\circ}$, a minimum focus distance of $0.4 \mathrm{~m}$, thermal sensitivity $<0.045^{\circ} \mathrm{C}$ at $30^{\circ} \mathrm{C}$, a focal plane array (FPA) uncooled microbolometer $320 \times 240$ pixels as a detector and a spectral range between 7.5 and $13 \mu \mathrm{m}$ was used. The RGB-ITR is a scanner prototype developed in DIM laboratory at ENEA Frascati: the main feature of this system is the capability to collect simultaneously both colour and structural information by the use of three amplitude modulated lasers and respective photodiodes [7].

\section{Diagnostic Results}

Measurements were performed mainly in the western and southern sectors of the dome, which appear to be the most interesting and rich in original fresco remains. With the LIF system some different areas of about $0.25 \mathrm{~m}^{2}$ each were scanned with a spatial resolution of about $1 \mathrm{~cm}$. This value is a compromise between the need of shortening acquisition times and those of providing answers to the specific questions asked by conservators. All collected spectra were processed and used to build fluorescence images, as the ones shown in Fig. 3. To generate every image, spectral data were filtered at the selected wavelength and normalized by the maximum obtained at such wavelength on the whole scan. In particular, in Fig. 3, fluorescence images at $300 \mathrm{~nm}$ and $700 \mathrm{~nm}$ are reported. They are of great importance because they are indicative of the presence of acrylic consolidants on the one hand and of photosynthetic microorganisms on the other, according to literature [8] and a database prepared thanks to laboratory research conducted on known samples. In this way, characteristics not visible by naked eye were highlighted. The obtained results confirmed the presence of preferential migration directions for consolidants injected in the surface for the consolidation action (Fig.3, centre), providing detailed maps which can help conservators in the evaluation of the consolidation technique.

The fluorescence spectra of the whole surface are dominated by the presence of large bands centred at $450 \mathrm{~nm}$ and $500 \mathrm{~nm}$ due to the presence of $\mathrm{CaCO}_{3}$ and $\mathrm{CaSO}_{4}$. No signals that can be referred to organic

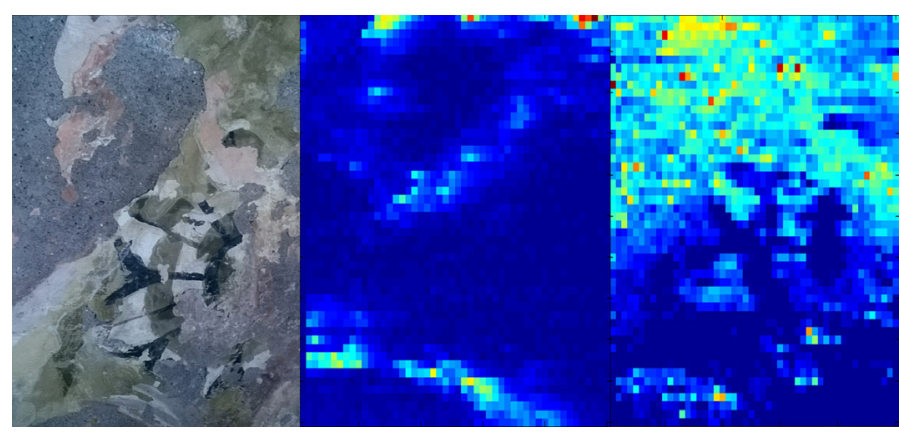

Fig. 3. An area examined by LIF: standard image in visible range (left), fluorescence map at $300 \mathrm{~nm}$, indicative of acrylic consolidants (centre), fluorescence map at $700 \mathrm{~nm}$, indicative of photosynthetic microorganisms (right). In the maps the colour is from blue for low intensities to red for higher intensities at the selected wavelength. 
binders, in agreement with a database built in laboratory from known samples prepared according to Cennino Cennini's recipes [9], were found on the surface. This observation would exclude the use of a tempera technique for the repainting of the dome, but rather it would suggest the use of lime painting. These results were confirmed by Raman spectroscopy, which allowed to recognize pigments and constituent materials. In the outer layers in the dark spots a black carbonaceous pigment from animal sources (ivory black or/and bone black) was individuated (Fig. 4 left) [10]. In the original fresco in red and pink areas, red ochre, mixed with $\mathrm{CaCO}_{3}$ and $\mathrm{CaSO}_{4} \times 2 \mathrm{H}_{2} \mathrm{O}$ was found (Fig. 4 right). Moreover, characteristic signals from calcium carbonate and sulphate were found on the whole surface without any signals that could be assigned to organic binders, in the limits of detections of the specific instrument used. In fact, spectral bands that can be assigned to aliphatic modes (near $3000 \mathrm{~cm}^{-1}$ and between 900 and $1500 \mathrm{~cm}^{-1}$ ) or to carboxylic group and unsaturations (1600-1800 $\mathrm{cm}^{-1}$ region) typical of most common historical binding media (fresh and aged) were not detected $[11,12]$.
The RGB-ITR was used for digitalizing before and after the laser cleaning intervention. This kind of digitalization had a dual purpose: the first was to have a historical memory of the drawings before the cleaning of the surface, the second to have two 3D multispectral maps of the interested areas for a semi-automatic detection of possible colour or structural modifications caused by the laser-cleaning intervention. This result was obtained by superimposing the data collected by different instruments (like LIF, RAMAN and thermography) on RGB-ITR $3 \mathrm{D}$ colorimetric meshes. The RGB-ITR was tested only on a limited portion of the dome: the rest of the structure was digitalized by the use of a low-cost structured light sensor called COBRAKIN. This latter sensor is also able to collect high definition photos by the use of a standard camera: this information was affected by the environmental lights, because it was impossible to create a photographic light setup. For calibrating these images the RGB-ITR colorimetric information will be used. An example of the images obtained is presented in Fig. 5. In this figure, a thermogram of a selected area (in the black box) is presented, too. Thermog-

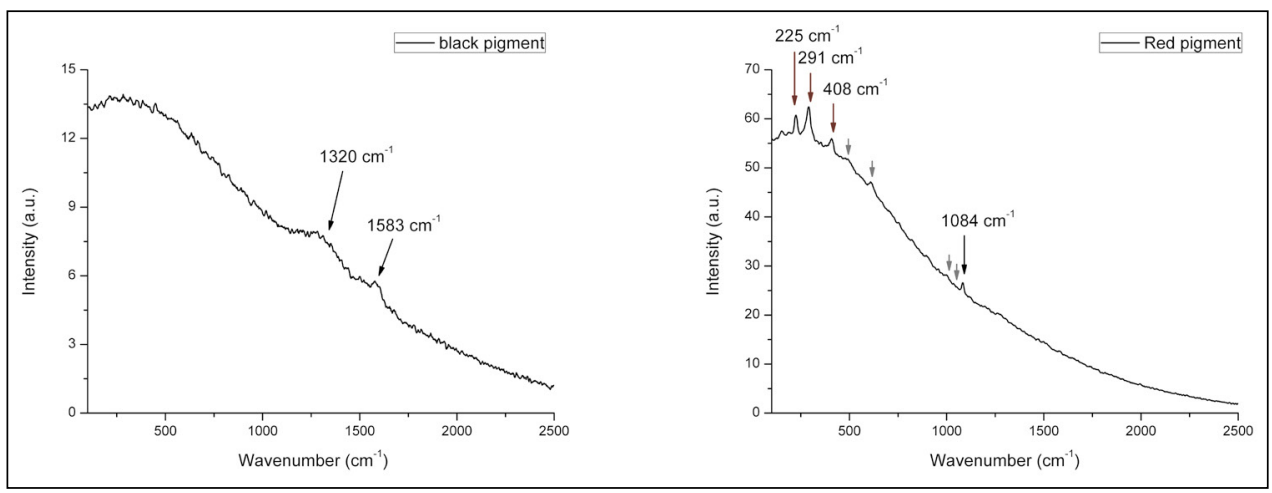

Fig. 4. Raman spectra for: black pigment in the outer layers (left) and for red pigment in the original fresco (right). In the latter Raman lines from calcite (at $1084 \mathrm{~cm}^{-1}$ ), red ochre (at 225, 291, $408 \mathrm{~cm}^{-1}$ ) [13] and $\mathrm{CaSO}_{4} \times \mathrm{H}_{2} \mathrm{O}(492,621,1007$, and $1135 \mathrm{~cm}-1)$ are clearly visible. 


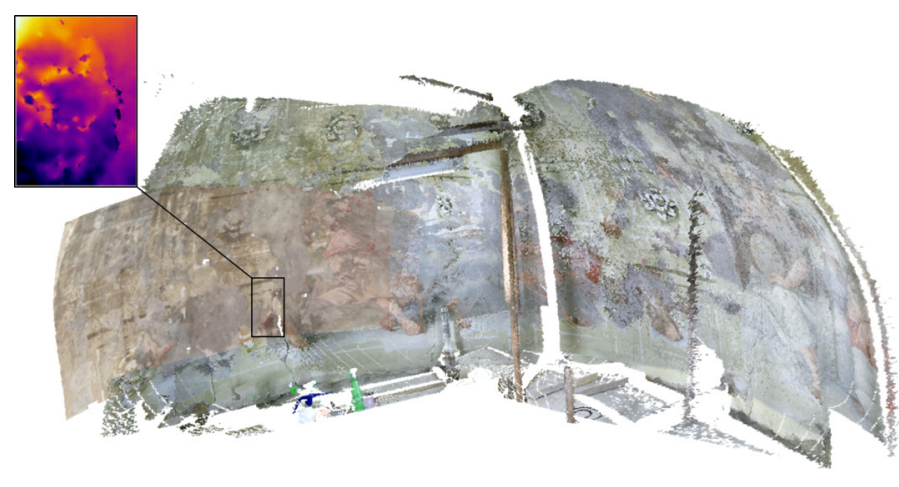

Fig. 5. on the left RGB-ITR scan (on the west sector of the dome) on the first tests with a new prototype, COBRAKIN. In the box a thermogram with a highlighted detachment area in white-yellow.

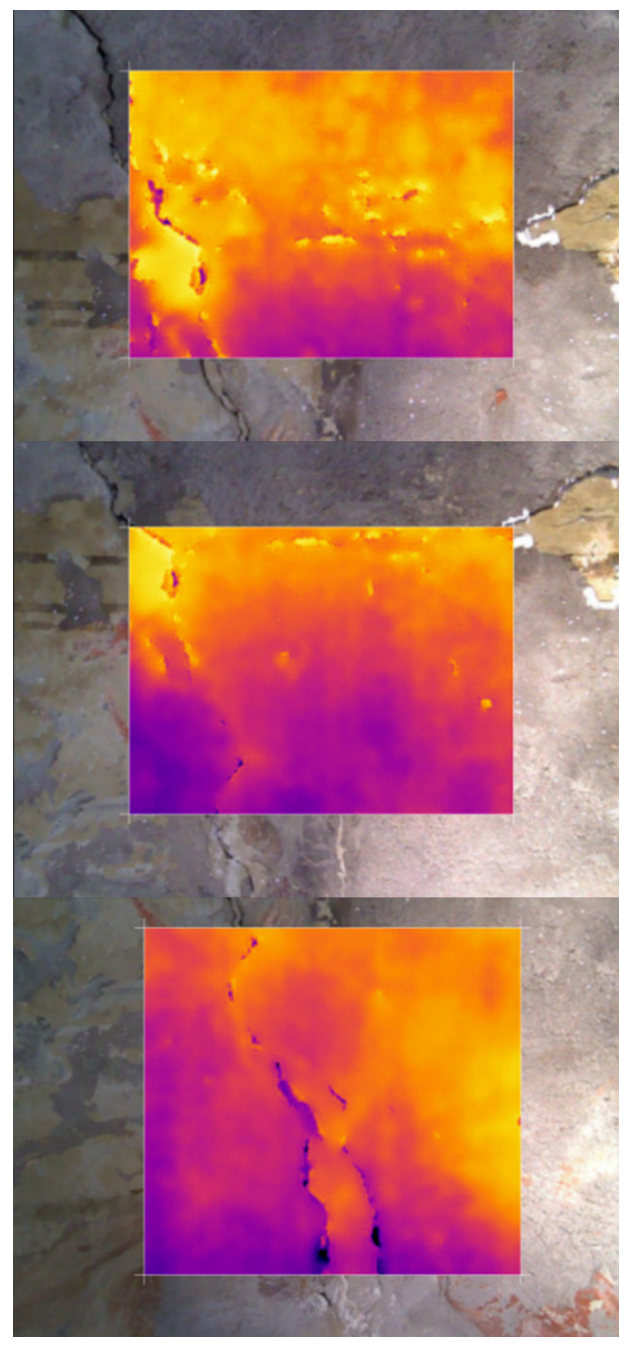

raphy revealed widespread detachments of the painted areas of the dome. They can be attributable to phenomena of crystallization of the soluble salts due to the presence of rainwater and/or the biological attack. The presence of overlapping layers of paint could triggered widespread detachments of the surface layers. External materials coming out from previous restorations can be the reason for the final degradation state. The images of the upper dome levels showed very critical conservation conditions due to rain water infiltration (Fig. 6).

\section{Laser cleaning}

The laser cleaning technique [14-16] was tested thanks to the collaboration with El.En. S.p.A. In particular, the system COMBO EOS 1000 was used. It is characterized by an emission at $1064 \mathrm{~nm}$ and two temporal regimes in one system: Short Free Running (SFR) mode, with a pulse duration ranging from 30 to 110 microseconds, and Long Q-switch (LQS) regime with 100ns. The energy ranges are 200-1400 $\mathrm{mJ}$ for the SFR mode and from $150 \mathrm{~mJ}$ to $450 \mathrm{~mJ}$ for the LQS mode.

$\leftarrow$ Fig. 6. Results of thermovision on the west sector, same area analysed by LIF and presented in Fig. 3 . 


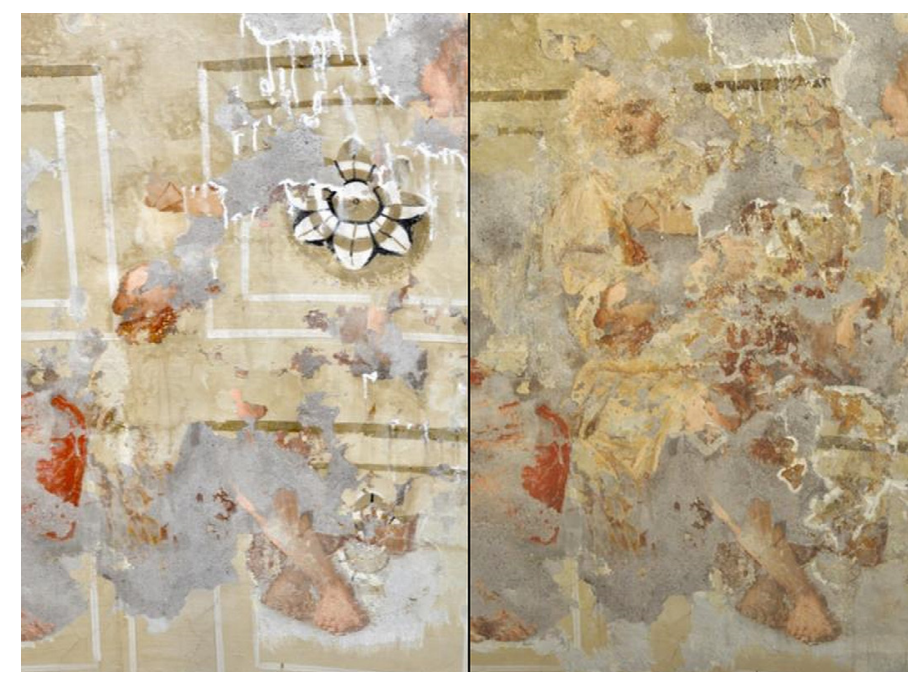

Fig. 7. Before (left) and after (right) the application of laser cleaning.
The selection of the operation parameters was particularly hard due to the high variability of impact points characteristics. In fact, the laser beam can impinge on areas with salts between fresco and the upper layers, on cohesive and tenacious layers, on fresco layer pulverized mixed with mortar, fresco layer damaged and patchy, on mortar, or on wide detachments. Moreover, chromatic selectivity was impossible because of the presence of polychromy in every different layer, which caused main difficulties. Nevertheless, after several tests the most suitable working conditions for every kind of problems were individuated. Both regimes were been used depending on the micro-area under study and for both of them the energy level was manually modified empirically to obtain the best result.

The successful outcomes of the laser cleaning are mainly due to a secondary spallation effect that allowed to remove micro-portions of the outer layers with high accuracy. An example of the obtained results is shown in Fig. 7. Here the same area of the dome before (left) and after (right) the laser cleaning is shown. As it is possible to notice on the left, originally a lacunar damaged motif is present and just some fragments of a human figure are visible (mainly feet at the bottom of the picture). The laser cleaning allowed discovering a whole cantor angel (clearly visible in the picture on the right). Thanks to this painstaking cleaning work several angel figures of good quality emerged.

Important results were obtained due to an accurate and slow work of restorers that cleared a large part of the surviving paintings from repainting with the use of the laser. As no other cleaning techniques could be successfully applied on the most part of the dome, the artwork was given up for lost up to the use of the laser cleaning. The frescoes in the dome of S. Costanzo proved to be a great surprise: there are just rare and sporadic documentary sources referring to it and no publications that remember the existence of this cycle. Further and deeper historic and artistic studies are being carried out by the SABAP RM MET.

After the removal of degradation and outer decoration layers, a laser cleaning 
tests were carried out on the pitting due to biological attack found on the original fresco. Moreover, in the areas previously cleaned by classical chemical manual methods, the EOS Combo removed also a residual thin layer of white patina. The remote colour measurements obtained by RGB-ITR on areas already treated with chemical approaches showed that the colours obtained after the laser cleaning are much more saturated than before the intervention, highlighting the ability of the laser cleaning to remove thin patinas, impossible to eliminate by means of other techniques.

The good performances of laser cleaning have now allowed other important interventions on the dome, first of all the consolidation of plaster and the refurbishment of the aesthetic appearance.

\section{Conclusions}

This work highlighted that the collaboration among different competences of people working on Cultural Heritage is necessary to achieve a correct and comprehensive restoration and conservation action. In fact, the collaboration between conservators, art historians and scientists allowed to verbalise open questions, to identify more suitable methodologies, to correctly read into the results, to reach complete answers. Different laser systems were used for diagnostics, documentation and cleaning of the dome of S. Costanzo church in Ronciglione (Italy). In particular, a LIF scanning system and Raman spectroscopy were applied to characterize the materials (both original and used during restoration actions) and to obtain information about techniques used for the different painting layers. Moreover, the ability of the LIF prototype to produce fluorescence images allowed to map bioattacks and preferential migration directions of consolidants used by restorers. The EOS Combo system by El.En. was used successfully for the effective cleaning of the surface, succeeding where classical mechanical and chemical cleaning methods had failed. The RGB-ITR prototype provided both the mapping of cracks and detachments in collaboration with thermography, and a 3D colour documentation for technical and media purposes before, during and after the cleaning of the dome.

\section{Acknowledgements}

The authors would like to thank dr Luisa Caporossi and dr Federica di Napoli Rampolla, from the Ministry of Cultural Heritage SABAP RM MET, dr Pietro Paolo Lateano, and Silvio Iacomi for making this work possible. This research was supported by the Latium Region under grant agreement lr13, n.1031.

\section{References}

[1] J.F. Asmus, C.G. Murphy, W.H. Munk, "Studies on the Interaction of Laser Radiation with Art Artifacts", in: Developments in Laser Technology II, San Diego, USA, August 27, 1973, Proceedings of SPIE 0041, 1974, DOI: $10.1117 / 12.953831$.

[2] A. Nevin, G. Spoto, D. Anglos, "Laser spectroscopies for elemental and molecular analysis in art and archaeology", Applied Physics A, 106 (2012) 339-361, DOI: 10.1007/s00339-011-6699-z.

[3] G. Ricci, L. Caneve, D. Pedron, Nadine Holesch, E. Zendri, "A multi-spectroscopic study for the characterization and definition of production techniques of German ceramic sherds", Microchemical Journal 126 (2016) 104-112, DOI: 10.1016/j.microc.2015.12.009.

[4] V. Spizzichino and R. Fantoni, "Laser induced breakdown spectroscopy in archeometry: a review of its application and future perspectives", Spectrochimica Acta B, 99 (2014), 201-209, DOI: 10.1016/j. sab.2014.07.003. 
[5] D. Bersani and P. P. Lottici, "Raman spectroscopy of minerals and mineral pigments in archaeometry" Journal of Raman Spectroscopy, 47 (2016) 499-530.

[6] J.L. Kinsey, "Laser Induced Fluorescence”, Annual Reviews Physical Chemistry 28 (1977) 349-372, DOI: 10.1146/annurev.pc.28.100177.002025.

[7] M. Guarneri, M. Ferri De Collibus, G. Fornetti, M. Francucci, M. Nuvoli, and R. Ricci, "Remote colorimetric and structural diagnosis by RGBITR color laser scanner prototype", Advances in Optical Technologies, 2012 (2012) 1-6, DOI: 10.1155/2012/512902.

[8] B. Park, R. Lu, Hyperspectral Imaging Technology in Food and Agriculture, Springer, New York 2015, DOI: 10.1007/978-1-4939-2836-1.

[9] V. Spizzichino, L. Caneve, R. Fantoni F. De Nicola, "Spectral database of Renaissance fresco pigments by LIBS, LIF and colorimetry", in: Proceedings of the $3^{\text {rd }}$ Balkan Symposium on Archaeometry, Bucharest, Romania, October 29-30, 2012, R. Rãdvan, S. Akyuz, M. Simileanu, V. Dragomir (Eds.) Integra natura et Omnia - INOE Bucharest 2013, pp. 9-16, http://certo. inoe.ro/watch/images/proceedings.pdf (accessed 16.06.2017).

[10] C. Lofrumento, M. Ricci, L. Bachechi, D. De Feo and E.M. Castellucci, "The first spectroscopic analysis of Ethiopian prehistoric rock painting", Journal of Raman Spectroscopy 43 (2012), 809-816, DOI: $10.1002 /$ jrs.3096.

[11] P. Vandenabeele, B. Wehling, L. Moens, H. Edwards, M. De Reu, G. Van Hooydonk, "Analysis with micro-Raman spectroscopy of natural organic binding media and varnishes used in art", Analytica Chimica Acta, 407 (2000) 261-274, DOI: 10.1016/ S0003-2670(99)00827-2.

[12] S.A.Centeno, "Identification of artistic materials in paintings and drawings by Raman spectroscopy: some challenges and future outlook", Journal of Raman Spectroscopy, 47 (2016) 9-15, DOI: 10.1002/ jrs.4767.

[13] D. Bikiaris, Sister Daniilia, S. Sotiropoulou, O. Katsimbiri, E. Pavlidou, A.P. Moutsatsou, Y. Chryssoulakis, "Ochre-differentiation through micro-Raman and micro-FTIR spectroscopies: application on wall paintings at Meteora and Mount Athos, Greece", Spectrochimica Acta Part A 56 (2000) 3-18, DOI: 10.1016/S1386-1425(99)00134-1.

[14] S. Siano, J. Agresti, I. Cacciari, D. Ciofini, M. Mascalchi, I. Osticioli, A.A. Mencaglia, "Laser cleaning in conservation of stone, metal, and painted artifacts: state of the art and new insights on the use of the Nd:YAG lasers", Applied Physics A 106 (2012) 419-446, DOI: 10.1007/s00339-011-6690-8.

[15] N.Arnold, “Theoretical description of dry laser cleaning" Applied Surface Science 208-209 (2003) 15-22, DOI: 10.1016/S0169-4332(02)01278-3.

[16] A. Sarzyński, K. Jach, and J. Marczak, “Comparison of Wet and Dry Laser Cleaning of Artworks", in: Proceedings of LACONA VI Lasers in the Conservation of Artworks, Vienna, Austria, Sept. 21-25, 2005 M.J. Nimmrichter, W. Kautek, M. Schreiner (Eds.), Proceedings in physics 116 (2007), DOI: 10.1007/978-3-540-72130-7_19. 


\title{
Archaeometric investigations of medieval stained glass panels from Grodziec in Poland
}

\author{
Dariusz Wilk $^{1 \star}$, Marta Kamińska ${ }^{2}$, Małgorzata Walczak $^{2}$, Ewa Bulska ${ }^{1}$ \\ 1 Faculty of Chemistry, Biological and Chemical Research Centre, University of Warsaw, Żwirki \\ i Wigury 101, 02-089 Warsaw, Poland \\ 2 Faculty of Conservation and Restoration of Works of Art, Jan Matejko Academy of Fine Arts in \\ Kraków, Pl. Matejki 13, 31-157 Kraków, Poland \\ * Corresponding author: dariusz.wilk@uj.edu.pl
}

\begin{abstract}
Two stained glass panels of Austrian origin from the $15^{\text {th }}$ century were formerly placed in Grodziec castle in Poland and nowadays they belong to the collection of the National Museum in Wrocław. The main aim of this work was to evaluate the chemical composition of glass from medieval and modern parts of the panels. Elemental composition of bulk glass and external layers of glass samples was determined with the use of SEM-EDX and LA-ICP-MS methods. Morphology of the deteriorated glass was investigated through SEM-BSE images of the cross-sections. Moreover, the longitudinal concentration profiles were determined by LA-ICP-MS measurements.

Results show that stained glass panels reveal characteristic elemental composition of wood ash glass produced from 1000 to $1400 \mathrm{AD}$. Almost equal proportions of potassium and calcium oxides indicate that high quality of beech wood was applied by manufacturers. Main and minor elements content was common for almost all investigated glass samples, which suggests that manufacturers follow strictly the assumed recipe during panels production. Differences in elemental composition detected for minor or trace elements were connected to colour additives. Stained glass samples from glazing exhibit composition of typical modern glass. Considerable differences between the composition of healthy bulk glass and the deteriorated surface of glass were detected though SEM-BSE images and LA-ICP-MS longitudinal concentration profiles. High concentrations of lead, copper and iron were noticed in external layers of glass samples, which can be explained by the presence of decorative paint layers and drawings.
\end{abstract}

Keywords: stained glass, wood ash glass, elemental composition, SEM-EDX, LA-ICP-MS

\section{Introduction}

Stained glass windows were one of the most important and precious features of medieval architecture and were used predominantly in churches, only rarely in private or public buildings. Technology and raw materials used in the manufacturing of glass have changed during ages. Glass is usually formed by melting a mixture of specific materials. It consists chiefly of three components: the network former, which in the case of silicate glasses is silicon dioxide $\mathrm{SiO}_{2}$, the alkaline ingredient working as a modifier, and the 
stabilizing agent. The melting temperature of pure silica, which is $1610^{\circ} \mathrm{C}$, makes the production of silicate glass complicated and expensive [1]. To obtain a glass easier to produce, it is necessary to add network modifiers and stabilizers. $\mathrm{SiO}_{2}$ was introduced in ancient glass generally as sea sand. According to Davison [2], network modifiers, called fluxants (usually alkali: $\mathrm{Na}, \mathrm{K}$ ), were added to the batch as natron or plants ashes in order to lower the melting temperature of the vitrifier. For glass becomes non-resistant to water after the addition of alkalis, it must be stabilized by the addition of alkaline earths (Mg, Ca). Seashells or other carbonic fragments occurring in marine sand were the sources of lime.

Three major glass types were used in the medieval period [3-5]. Soda ash glass was produced from about $1500 \mathrm{BC}$ to the Middle Ages in different parts of the world. The oldest soda ash glasses originated from ancient Mesopotamia and Egypt. Glass produced in central Europe and Venice between $11^{\text {th }}$ to $14^{\text {th }}$ centuries frequently reflects soda ash glass composition [4-6]. Typical soda ash glass contains sodium and calcium oxides in a weight proportion of almost 1.4:1 and relatively high amount of magnesium and potassium oxides due to use of quartz and the ash of halophytic plants for production [4-7]. Soda lime glass was produced from $900 \mathrm{BC}$ to the Middle Ages from quartz, trona and lime. Generally soda lime glass is characteristic for Roman glass and can be found also in post-Roman buildings and places. Low potassium and magnesium oxides concentration with high content of sodium and calcium oxides is typical of soda lime glasses $[4,5$, $8,9]$.

In the $8^{\text {th }}$ century, glass production was established on the basis of quartz and ash from beech trunks or from bulk beech trees. As a consequence of the use of such raw materials, elevated amounts of silicon, potassium and calcium oxides were found. In central Europe, during the Middle Ages, several subtypes of wood ash glasses were produced and they can be distinguished by the ratio of $\mathrm{CaO}$ vs. $\mathrm{K}_{2} \mathrm{O}$ [3]. The monk Theophilus Prestyber in his Diversarum Artium Schedula prescribed a wood ash glass recipe, where he suggested to mix and melt two parts ash of beech trunks with one part of quartz sand [3]. If producers follow that recipe, glass should contain no more than $50 \% \mathrm{SiO}_{2}$ and about $20 \% \mathrm{~K}_{2} \mathrm{O}$ [5]. Similar concentration of $\mathrm{CaO}$ and $\mathrm{K}_{2} \mathrm{O}$ is considered to be the evidence of the manufacture of glass with alkaline ashes obtained from good quality, purified wood with a low amount of bark. Medieval glass panels from English and French churches are characterized by higher concentrations of magnesium and phosphorus oxides in comparison to glass produced in Germany $[5,10]$. Wood ashes were used in the production of glass until the $19^{\text {th }}$ century. Therefore, discrimination of glass produced through the ages can be based on the ratio of specific elements [3]. Medieval glass technology utilized different materials and techniques for gaining specific colour of glass $[3,11]$.

Archaeometric research, defined as the application of scientific techniques and methodologies to archaeology and arts, provides important data for art historians, conservators and other professionals dealing with historical objects. Information about elemental composition of glass is necessary to determine the type of glass, the technology and provenance of the object. The determination of changes in the elemental composition of glass, which occurred due to deterioration processes, is vital for the proper evaluation of the condition of glass. Such information is 
also very important to establish appropriate conservation treatment. Many analytical methods can be applied for the investigations of glass samples. Scanning Electron Microscope coupled with an Energy Dispersive $\mathrm{X}$-Ray spectrometry (SEM-EDX) enables for measuring the content of main elements, but does not provide information on trace elements content, which can be essential for the provenance studies of historical glass. One of the methods allowing the identification of trace elements without sample preparation is Laser Ablation Inductively Coupled Plasma Mass Spectrometry (LA-ICP-MS).

This paper focuses on two stained glass panels from the so-called Grodziec Collection. A part of that collection consists of a group of fourteen panels, most likely of Austrian origin, depicting figures on architectural backgrounds, and is dated to $1420-1425$. There is no information on the time and person who brought the collection to Grodziec. Moreover, it is not clear whether the figurative panels embellished the windows of the castle, palace or the tower (Fig. 1) [12]. After the Second World War, eight panels were relocated to Kraków and are actually held by the Jagiellonian University Museum. The remaining six are held by the
National Museum in Wrocław where they were transported in 1966 [12]. Primarily the glass panels were determined as $19^{\text {th }}$-century copies. Later-on, the analyses performed during the conservation of the panels from the Jagiellonian University Museum in Kraków (2000-2004) and those from the National Museum in Wrocław (2013-2014) indicated medieval origins, modern composition of some of the glass panes and similarity of the panels. Further studies based on the analysis of historical documents and photos $[12,13]$, visual inspection of the glass panes, paints, leadlight glazing and physicochemical analyses performed by X-Ray Fluorescence (XRF), macro-XRF, micro-Raman spectroscopy, SEM-EDX, Optical Coherence Tomography [14-16] revealed more information about history, origin and degradation of the collection.

A detailed analysis of two medieval stained glass panels from the Grodziec collection was performed to determine the subtype of the medieval glass, confirm the provenance of the objects and indicate the accuracy of the production. Elemental composition of bulk glass and external layers of glass samples was determined using SEMEDX and LA-ICP-MS methods. The methods

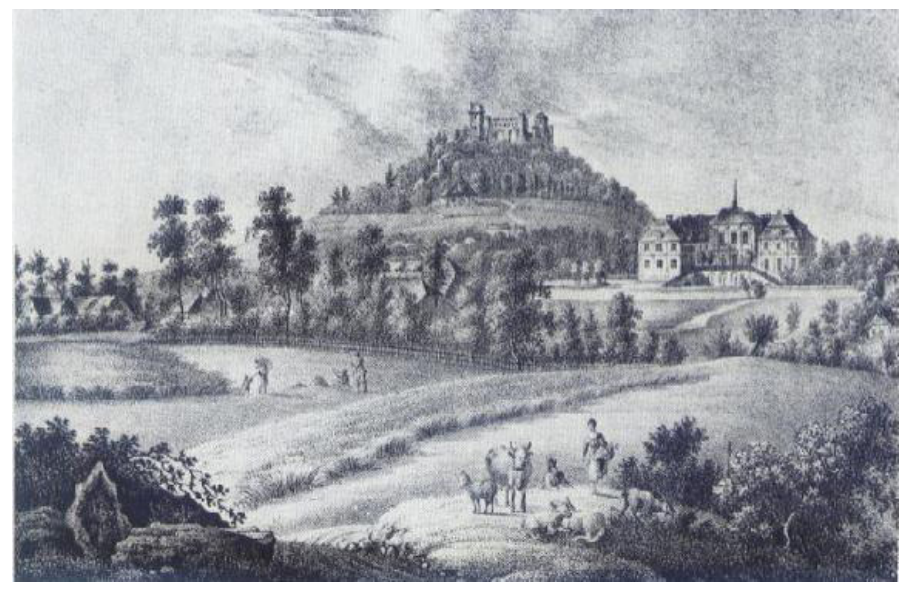

Fig 1. Historic buildings in Grodziec: castle on the hill and baroque palace (lithography from 1837). 
are capable to deliver information about the elemental composition and the state of the preservation of medieval glass due to chemical imaging (mapping). Additionally, LA-ICP-MS method allows for high spatial resolution measurements and for determination of trace elements. Morphology of the deteriorated glass was investigated through SEM-BSE (Backscattered Electrons) images of the cross-sections. LA-ICP-MS longitudinal concentration profiles were carried out to

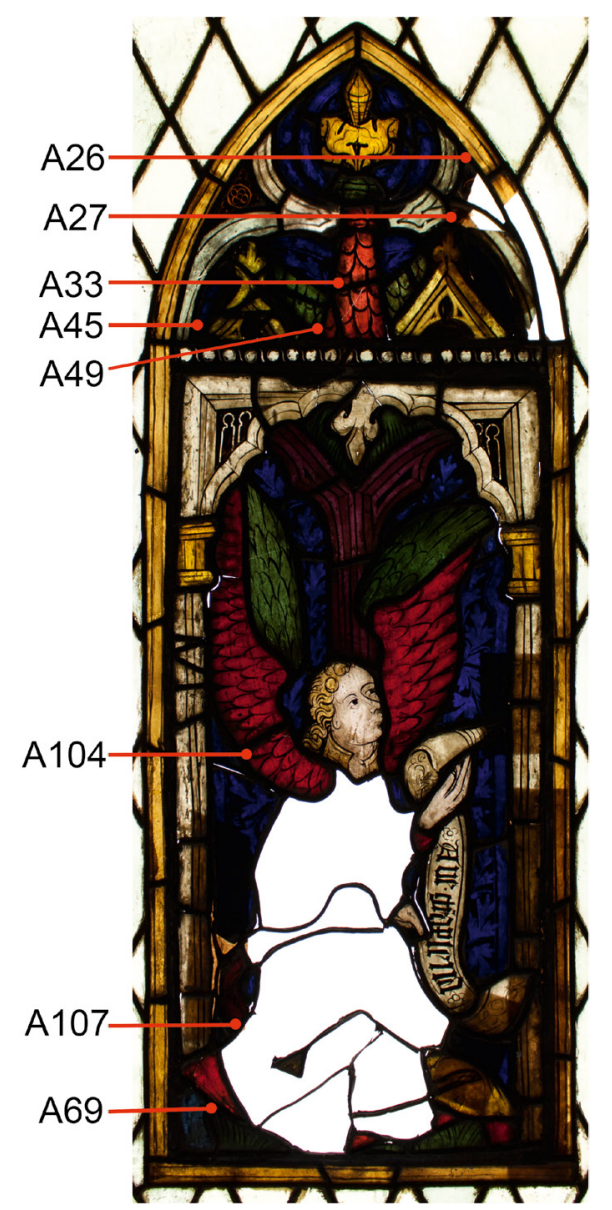

Fig. 2. Stained glass panel representing Annunciation Archangel Gabriel; Styria \& Carinthia, about 1430; samples collected from different glasses for the analysis are indicated by a number and arrows. reveal technology used to produce red glasses and corrosion phenomena.

\section{Materials and methods}

Two stained glass panels from Grodziec collection representing Annunciation Archangel Gabriel and St. Barbara (Fig. 2,3) were selected for the study. Nowadays, they belong to the collection of the National Museum in

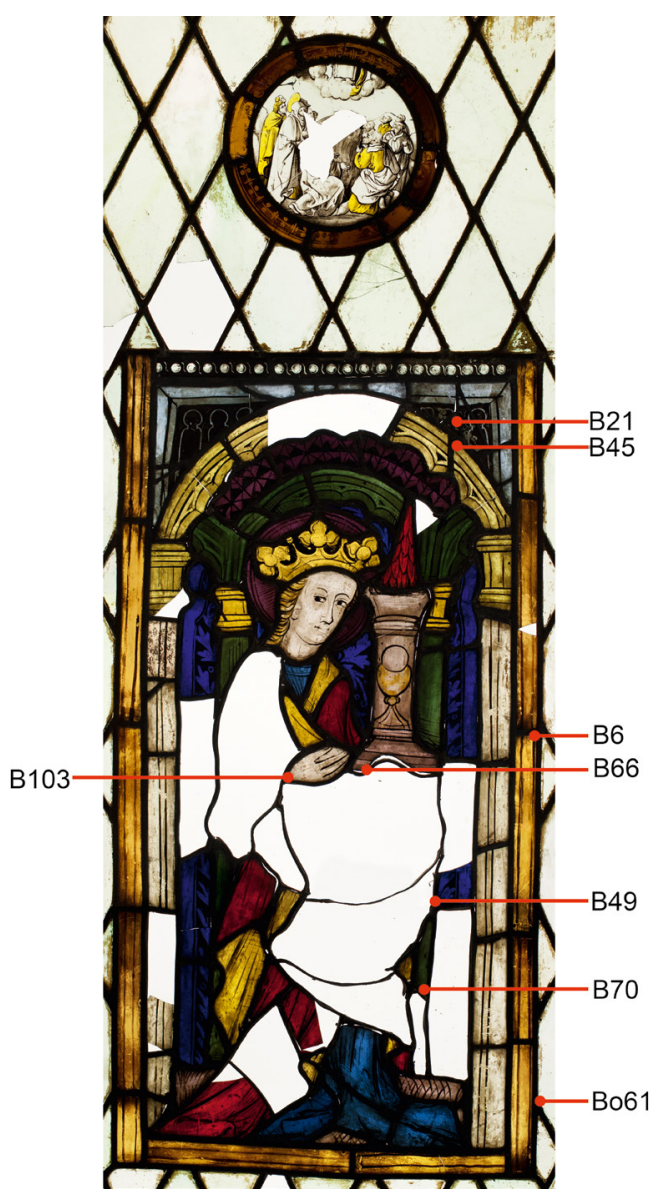

Fig. 3. Stained glass panel representing St. Barbara; Styria \& Carinthia, about 1430; samples collected from different glasses for the analysis are indicated by a number and arrows. 
Wrocław. Each panel has around $117 \mathrm{~cm}$ high and $43 \mathrm{~cm}$ width. According to historical data, those panels were manufactured about 1430 by stained glass workshop operated on the border between Styria and Carinthia in Austria [13].

Since many of glass pieces are broken, there was a possibility to prepare very small samples $\left(0,25 \mathrm{~cm}^{2}\right)$ for investigations. Samples differed with colour (yellow, red, blue, green) were selected from various parts of the panels and marked by a number. Three samples were selected from outside areas of the panels and glazing (A26, A27, Bo61). Location of the samples at the windows is presented in Figs. 2 and 3. Samples were immersed in epoxy resin and polished to receive thick sections.

Elemental composition was determined using laser ablation system (LSX-213 from Teledyne Cetac Technologies, USA) coupled with inductively coupled plasma mass spectrometer (NexION 300D from Perkin Elmer SCIEX, Canada). An LSX-213 system at $213 \mathrm{~nm}$ UV laser (Nd-YAG, solid state, Q-switched) with maximum energy up to 5 $\mathrm{mJ} /$ pulse and $5 \mathrm{~ns}$ pulse width was used for ablation. All experiments were performed using $\mathrm{Ar}$ as the carrier gas. Instrumental settings and data acquisition parameters are given in Table 1. Sample ablation was performed using different parameters. The spot diameter was adjusted to $100 \mu \mathrm{m}$ and pulse repetition rate adjusted to $10 \mathrm{~Hz}$ only for bulk analysis of the glass. The analyses of specific areas of the samples, i.e. colour or corroded layers, were carried out using smaller laser diameter $(25 \mu \mathrm{m})$ and higher pulse repetition rate $(20 \mathrm{~Hz})$. A number of experiments were performed to collect time resolved line profiles across different zones within a single sample. For these experiments the laser spot and scan rate were adjusted to $25 \mu \mathrm{m}$ and $1 \mu \mathrm{m} \cdot \mathrm{s}^{-1}$ respectively.

At least three replicate ablations at positions randomly selected were carried out on each sample. Transient signals were recorded and evaluated for elemental quantification.

Table 1. Instrumental settings and data acquisition parameters for LA-ICP-MS method

\begin{tabular}{|c|c|c|c|}
\hline \multicolumn{2}{|c|}{ ICP-MS characteristics and settings } & \multicolumn{2}{|c|}{ Laser ablation characteristics and settings } \\
\hline RF Power, $(\mathrm{W})$ & 1200 & Type of laser & Nd:YAG 213nm \\
\hline Neb. gas flow rate, $\left(\mathrm{L} \mathrm{min}^{-1}\right)$ & 0.94 & Pulse duration (ns) & 5 \\
\hline \multirow{2}{*}{ Carrier gas } & Ar & Energy (mJ) & 5.0 \\
\hline & & Ablation mode & spot, line \\
\hline \multicolumn{2}{|c|}{ ICP-MS data acquisition parameters } & Beam diameter $(\mu \mathrm{m})$ & $100 / 25$ \\
\hline Scanning mode & Peak hopping & Pulse repetition rate $(\mathrm{Hz})$ & $10 / 20$ \\
\hline Readings & 1 & Scan rate $\left(\mu \mathrm{m} \mathrm{s}^{-1}\right)$ & \\
\hline Replicates & 250 & (line mode only) & 1 \\
\hline Sweeps & 1 & & \\
\hline Dwell time, (ms) & 5 & & \\
\hline Pre-integration time, $(\mathrm{s})$ & 30 & & \\
\hline Integration time, $(\mathrm{s})$ & 60 & & \\
\hline Isotopes monitored & \multicolumn{3}{|c|}{$\begin{array}{l}{ }^{7} \mathrm{Li},{ }^{23} \mathrm{Na},{ }^{24} \mathrm{Mg},{ }^{27} \mathrm{Al},{ }^{29} \mathrm{Si},{ }^{39} \mathrm{~K},{ }^{43} \mathrm{Ca},{ }^{45} \mathrm{Sc},{ }^{49} \mathrm{Ti},{ }^{51} \mathrm{~V},{ }^{52} \mathrm{Cr},{ }^{55} \mathrm{Mn},{ }^{57} \mathrm{Fe},{ }^{59} \mathrm{Co}, \\
{ }^{60} \mathrm{Ni}{ }^{65} \mathrm{Cu},{ }^{66} \mathrm{Zn},{ }^{69} \mathrm{Ga},{ }^{85} \mathrm{Rb},{ }^{88} \mathrm{Sr},{ }^{89} \mathrm{Y},{ }^{90} \mathrm{Zr},{ }^{93} \mathrm{Nb},{ }^{118} \mathrm{Sn},{ }^{121} \mathrm{Sb},{ }^{137} \mathrm{Ba},{ }^{139} \mathrm{La}, \\
{ }^{140} \mathrm{Ce},{ }^{141} \mathrm{Pr},{ }^{142} \mathrm{Nd},{ }^{152} \mathrm{Sm},{ }^{153} \mathrm{Eu},{ }^{158} \mathrm{Gd},{ }^{159} \mathrm{~Tb},{ }^{164} \mathrm{Dy},{ }^{165} \mathrm{Ho},{ }^{166} \mathrm{Er},{ }^{169} \mathrm{Tm}, \\
{ }^{174} \mathrm{Yb},{ }^{175} \mathrm{Lu},{ }^{178} \mathrm{Hf},{ }^{181} \mathrm{Ta},{ }^{202} \mathrm{Hg},{ }^{207} \mathrm{~Pb},{ }^{208} \mathrm{~Pb},{ }^{232} \mathrm{Th},{ }^{238} \mathrm{U}\end{array}$} \\
\hline
\end{tabular}


NIST SRM 610 [17] and Corning D archeological glass [18] were used as the external standard for modern and medieval objects respectively. The results of elemental composition, for all samples, were recalculated to the content of the oxides using $\mathrm{SiO}_{2}$ as the internal standard. Sum normalization to 100 $\mathrm{wt} \%$ was applied based on the corresponding oxide concentrations [19]. The accuracy of the measurements was established by using Corning B archeological glass examined as an unknown sample.

The composition and morphology of glasses were examined by SEM-EDX (JEOL $5500 \mathrm{LV}$, Japan). The imaging was performed with $20 \mathrm{keV}$ electron beam. Prior to SEM imaging the glass sample was covered with thin carbon layer to avoid charging of the sample surface. The chemical composition of glass bulk and corrosion layers was determined by EDX (IXRF Systems, USA). Magnification from 200 to 900 times and live time of $50 \mathrm{~s}$ were adjusted during the analysis.

\section{Results and discussion}

\subsection{Elemental composition of modern and medieval samples}

Firstly, the bulk analysis of glass samples was carried out by using LA-ICP-MS and SEM-EDX methods to determine the concentration of major and minor elements. The analytical results obtained by the LA-ICP-MS are given in Table 2. Comparative data obtained for two selected red and green stained glasses from the analysis by using SEM-EDX and LA-ICP-MS methods are presented in Table 3.

As shown in Table 3, the results for the oxide content obtained with both techniques are generally in good agreement for most of the elements. However, some differences between the average of oxide content should be emphasized. Especially, differences for $\mathrm{CaO}, \mathrm{MgO}$ and $\mathrm{Al}_{2} \mathrm{O}_{3}$ contents are evident (about 20\% and 10\% relative error for red and green sample respectively). Similar errors were observed in the earlier complementary analysis of historical glass by SEM-EDX and LA-ICP-MS [20]. It can be explained by different sensitivities of those two techniques as well as due to different sampling zones used for signal acquisition [20,21]. It is well known that SEM-EDX gives information from deeper and larger volumes of the exposed matter. This is especially important when historical glasses are analyzed, as the concentration gradients between the surface and sub-surface regime of the glasses objects occurs due to weathering. Additionally, elemental fractionation during LA-ICP-MS analysis may play an important role during the analysis of transparent materials. For transparent glasses LA-ICP-MS analyses showed progressive volatility dependence with increasing transparency and measured concentrations of refractory elements being lower. On the other hand, measured concentrations of volatile elements being higher in comparison to certified concentrations of reference materials [22]. Thus, higher differences in results obtained from LA-ICP-MS and SEM-EDX analyses for red glass can be associated with higher transparency of red medieval glass in comparison to green glass. It is also worth reminding that phosphorus oxide was not detected in LA-ICP-MS due to the presence of a high background signal.

The accuracy of LA-ICP-MS measurements was established by using Corning $B$ archeological glass examined as an unknown sample. The composition of Corning Glass B measured by LA-ICP-MS in present study is 
Table 2. Composition of Grodziec stained glasses - concentrations of oxides in mass\% (average with standard deviation (SD) and range values in all measured samples by LA-ICP-MS)

\begin{tabular}{|c|c|c|c|c|c|c|}
\hline \multirow[t]{2}{*}{ wt. \% } & \multicolumn{3}{|c|}{$\begin{array}{l}\text { medieval samples } \\
\text { (13 samples) }\end{array}$} & \multicolumn{3}{|c|}{$\begin{array}{c}\text { modern samples } \\
\text { (3 samples: A26, A27, Bo61) }\end{array}$} \\
\hline & mean & SD & range & mean & SD & $\max$ \\
\hline $\mathrm{SiO}_{2}$ & 48.37 & 0.76 & $46.58 \div 49.09$ & 71.55 & 2.33 & $68.89 \div 73.25$ \\
\hline $\mathrm{Na}_{2} \mathrm{O}$ & 0.156 & 0.023 & $0.134 \div 0.212$ & 15.80 & 1.64 & $14.51 \div 17.65$ \\
\hline $\mathrm{MgO}$ & 3.46 & 0.09 & $3.22 \div 3.61$ & 0.240 & 0.068 & $0.163 \div 0.288$ \\
\hline $\mathrm{Al}_{2} \mathrm{O}_{3}$ & 1.00 & 0.12 & $0.82 \div 1.25$ & 0.428 & 0.010 & $0.422 \div 0.440$ \\
\hline $\mathrm{K}_{2} \mathrm{O}$ & 22.52 & 0.85 & $21.69 \div 25.24$ & 0.189 & 0.003 & $0.185 \div 0.190$ \\
\hline $\mathrm{CaO}$ & 22.35 & 0.60 & $20.11 \div 23.44$ & 11.33 & 0.174 & $11.21 \div 11.53$ \\
\hline $\mathrm{TiO}_{2}$ & 0.116 & 0.005 & $0.102 \div 0.126$ & 0.059 & 0.002 & $0.057 \div 0.060$ \\
\hline $\mathrm{MnO}$ & 0.768 & 0.082 & $0.635 \div 1.227$ & 0.374 & 0.594 & $0.031 \div 1.059$ \\
\hline $\mathrm{Fe}_{2} \mathrm{O}_{3}$ & 0.570 & 0.558 & $0.262 \div 2.128$ & & & \\
\hline $\mathrm{Li}_{2} \mathrm{O}$ & 0.0025 & 0.0007 & $0.0018 \div 0.0040$ & 0.0020 & 0.0005 & $0.0017 \div 0.0026$ \\
\hline $\mathrm{V}_{2} \mathrm{O}_{5}$ & 0.0010 & 0.0001 & $0.0008 \div 0.0012$ & 0.0011 & 0.0005 & $0.0009 \div 0.0017$ \\
\hline $\mathrm{CoO}$ & 0.0427 & 0.0694 & $0.0005 \div 0.1747$ & & & \\
\hline $\mathrm{NiO}$ & 0.0166 & 0.0207 & $0.0012 \div 0.0551$ & & & \\
\hline $\mathrm{CuO}$ & 0.0619 & 0.0662 & $0.0123 \div 0.1833$ & 0.0023 & - & \\
\hline $\mathrm{ZnO}$ & 0.0426 & 0.0036 & $0.0391 \div 0.0485$ & & & \\
\hline $\mathrm{Ga}_{2} \mathrm{O}_{3}$ & & & & 0.0008 & - & \\
\hline $\mathrm{Rb} 2 \mathrm{O}$ & 0.1181 & 0.0092 & $0.1019 \div 0.1469$ & & & $0.0003 \div 0.0004$ \\
\hline $\mathrm{SrO}$ & 0.0985 & 0.0098 & $0.0627 \div 0.1100$ & 0.0068 & 0.0003 & $0.0064 \div 0.0071$ \\
\hline $\mathrm{ZrO}_{2}$ & 0.0030 & 0.0004 & $0.0024 \div 0.0037$ & 0.0045 & 0.0006 & $0.0041 \div 0.0052$ \\
\hline $\mathrm{SnO}_{2}$ & 0.0045 & 0.0130 & $0.0000 \div 0.0416$ & 0.0008 & 0.0007 & $0.0004 \div 0.0016$ \\
\hline $\mathrm{Sb}_{2} \mathrm{O}_{5}$ & & & & 0.0009 & 0.0009 & $0,0003 \div 0,0015$ \\
\hline $\mathrm{BaO}$ & 0.3081 & 0.0443 & $0.1498 \div 0.3818$ & 0.0127 & 0.0078 & $0.0081 \div 0.0216$ \\
\hline $\mathrm{La}_{2} \mathrm{O}_{3}$ & & & & 0.0002 & - & \\
\hline $\mathrm{PbO}$ & 0.0021 & 0.0023 & $0.0008 \div 0.0084$ & 0.0148 & - & \\
\hline
\end{tabular}

compared to compositions published by Brill [23], Dussubieux et al. [24] and Wagner et al. [18] in Table 4 . The data clearly show that accuracy strongly differs for each element and depends on selected external standard for quantification. Relatively good accuracy is observed for trace elements and many major oxides, i.e. $\mathrm{SiO}_{2}, \mathrm{MgO}, \mathrm{CaO}, \mathrm{FeO}$ and $\mathrm{MnO}$, when Corning Glass D was used as external standard. Contrary, accuracy for $\mathrm{Al}_{2} \mathrm{O}_{3}, \mathrm{~K}_{2} \mathrm{O}$ and $\mathrm{Na}_{2} \mathrm{O}$ is low, which is expressed in high value of error. It should be underline that the difference in composition of Corning $\mathrm{B}$ and $\mathrm{D}$ glasses is pronounced for $\mathrm{K}_{2} \mathrm{O}$ and $\mathrm{Na}_{2} \mathrm{O}$.
Thus, lower accuracy for such oxides can be explained by use of non-matrix matched Corning Glass $\mathrm{D}$ for quantification of Corning Glass B. Because $\mathrm{K}_{2} \mathrm{O}$ and $\mathrm{Na}_{2} \mathrm{O}$ content in NIST 610 and Corning Glass B is much more similar, better accuracy for such oxides is observed (Table 4). The assessment of the accuracy indicates the importance of selection of matrix-matched reference materials for the analysis by LA-ICP-MS and allows to assume that results for analyzed samples are reliable due to good match of Corning Glass $\mathrm{D}$ and NIST610 to medieval and modern glasses respectively. 
Table 3. Element concentrations (average value \pm SD) for two selected samples obtained by SEM-EDX and LA-ICP-MS analyses

\begin{tabular}{lcccc}
\hline \multirow{2}{*}{$w t . \%$} & \multicolumn{2}{c}{ red glass (A104) } & \multicolumn{2}{c}{ green glass (A49) } \\
\cline { 2 - 5 } & SEM-EDX & LA-ICP-MS & SEM-EDX & LA-ICP-MS \\
\hline $\mathrm{SiO}_{2}$ & $50.30 \pm 0.60$ & $48.39 \pm 0.21$ & $51.23 \pm 0.38$ & $47.67 \pm 0.53$ \\
$\mathrm{Na}_{2} \mathrm{O}$ & & $0.135 \pm 0.001$ & & $0.208 \pm 0.008$ \\
$\mathrm{~K}_{2} \mathrm{O}$ & $20.00 \pm 0.27$ & $21.69 \pm 0.10$ & $19.63 \pm 0.05$ & $21.98 \pm 0.39$ \\
$\mathrm{CaO}$ & $19.73 \pm 0.25$ & $23.44 \pm 0.33$ & $20.16 \pm 0.09$ & $21.81 \pm 0.85$ \\
$\mathrm{MgO}$ & $2.99 \pm 0.13$ & $3.61 \pm 0.08$ & $3.22 \pm 0.26$ & $3.42 \pm 0.03$ \\
$\mathrm{Al}_{2} \mathrm{O}_{3}$ & $1.26 \pm 0.14$ & $1.01 \pm 0.02$ & $1.42 \pm 0.04$ & $1.24 \pm 0.05$ \\
$\mathrm{P}_{2} \mathrm{O}_{5}$ & $0.98 \pm 0.03$ & & $1.04 \pm 0.07$ & \\
$\mathrm{MnO}$ & $0.79 \pm 0.08$ & $0.74 \pm 0.03$ & & $0.736 \pm 0.005$ \\
$\mathrm{FeO}$ & 0.40 & $0.29 \pm 0.01$ & $2.06 \pm 0.03$ & $2.11 \pm 0.04$ \\
$\mathrm{TiO}_{2}$ & 0.34 & $0.121 \pm 0.002$ & & $0.123 \pm 0.004$ \\
\hline
\end{tabular}

The content of main elements allows to divide the glasses in two different groups, which means that the panels consist of glass parts originated from different centuries. Three samples taken from outside areas of the panels and from the glazing (denoted as A26, A27, Bo61; Fig. 2, 3) exhibit typical composition of soda lime glass. The high content of $\mathrm{SiO}_{2}, \mathrm{Na}_{2} \mathrm{O}$ and $\mathrm{CaO}$ and low content of other oxides reflecting the modern origins of the glasses. Thus, results confirm that some glasses in the panels and the leadlight glazing were added at the beginning of the $20^{\text {th }}$ century, most probably due to conservation works.

It is worth noticing that medieval and modern samples can be separated by main oxides content. Cluster analysis was employed for visualization of the data structure for analyzed glasses according to Zadora et al. [25]. $\mathrm{Na}, \mathrm{Mg}, \mathrm{Al}, \mathrm{Si}, \mathrm{K}$ and $\mathrm{Ca}$ content was used to describe objects. The distance between objects was calculated using Euclidean measure after autoscaling the data and Ward's algorithm was applied as the linkage method for clustering. The dendrogram presented on Fig. 4 clearly shows two different kinds of glass - medieval and modern. Especially, comparison of $\mathrm{SiO}_{2}, \mathrm{Na}_{2} \mathrm{O}, \mathrm{CaO}$ and $\mathrm{K}_{2} \mathrm{O}$ contents allows for distinction of medieval glasses (soda ash, soda lime and wood ash) and modern glasses with the greatest confidence. Modern glass (i.e. soda lime glass) contains the highest amount of $\mathrm{SiO}_{2}$ (above

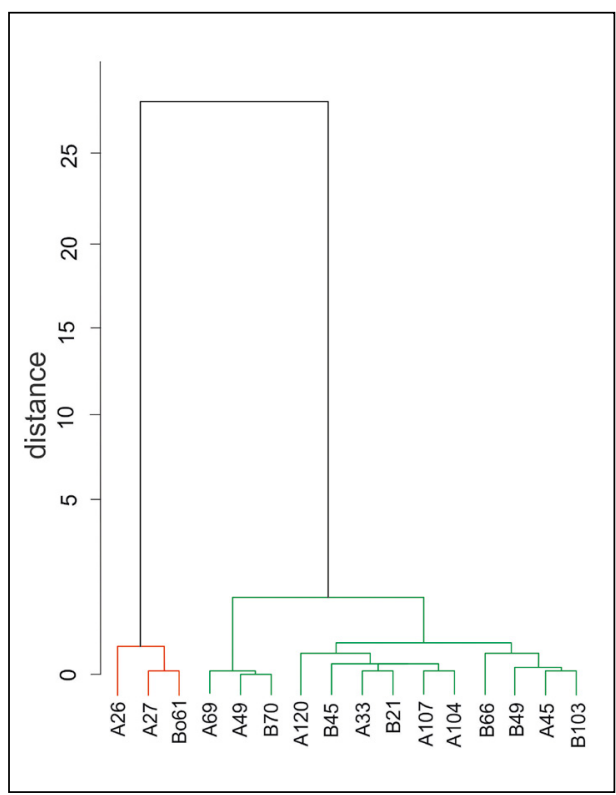

Fig. 4. The dendrogram for glass data including modern (A26, A27, Bo61) and medieval (other samples) glass. 
Table 4. Comparison between the composition of Corning Glass B measured by LA-ICP-MS in the present study with reproducibility (standard deviation) and accuracy (relative error based on mean value from published data) and composition published by Brill [23], Dussubieux et al. [24] and Wagner et al. [18]

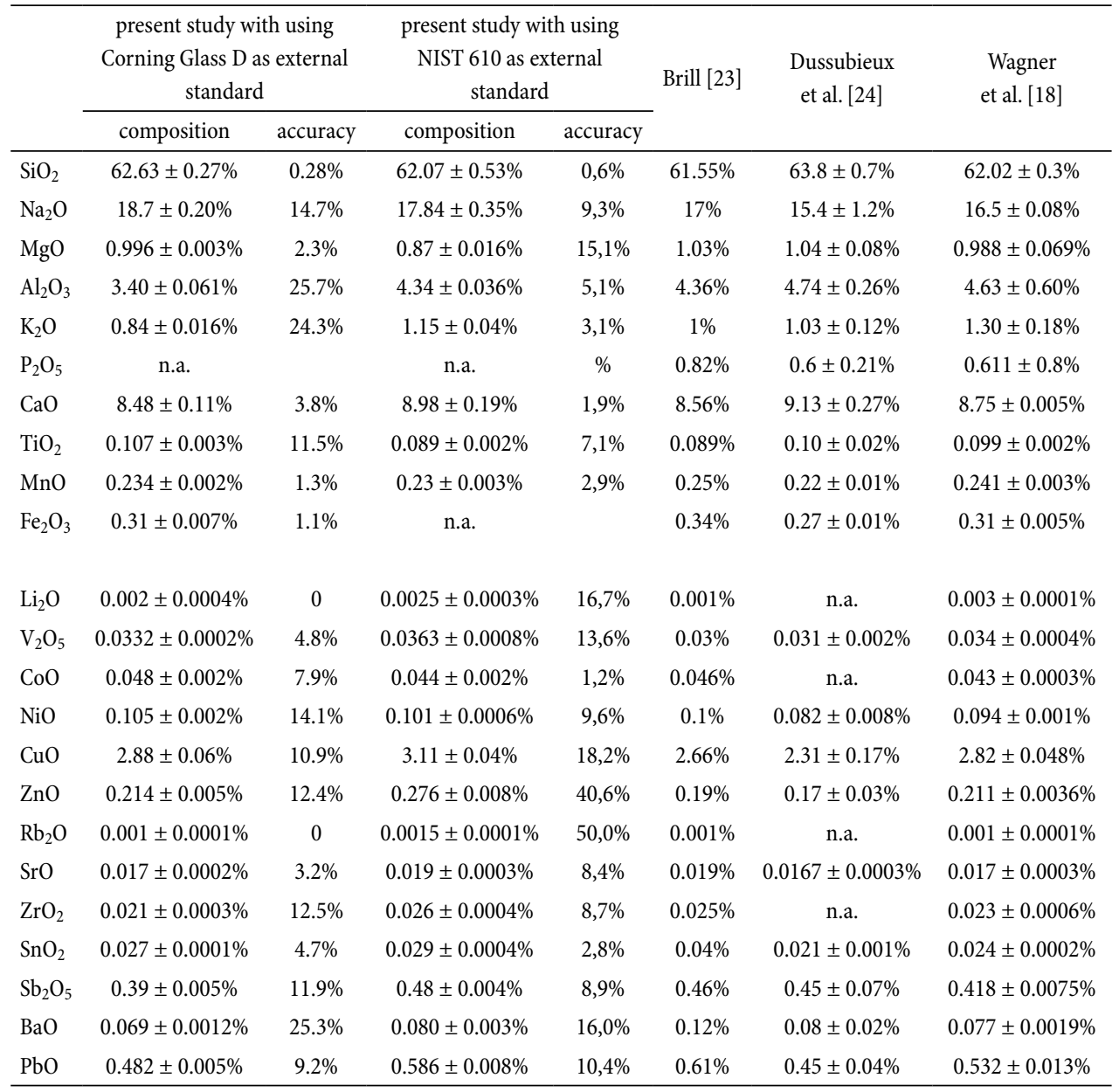

$70 \%)$ and very low amount of $\mathrm{K}_{2} \mathrm{O}$ in comparison to medieval glasses [3].

Big differences in elemental composition allows for classification glasses as medieval (i.e. wood ash glass) or modern. Because only 16 samples were analyzed, likelihood ratio models cannot be applied. Nevertheless, relatively simple Hotelling's $\mathrm{T}^{2}$ test was applied to check statistical confidence of classification based on elemental composition, i.e. multivariate data. Medieval sample A104 was randomly selected for calculations. The content of six major elements $(\mathrm{Na}, \mathrm{Mg}, \mathrm{Al}$, $\mathrm{Si}, \mathrm{K}$ and $\mathrm{Ca}$ ) was transformed by taking the logarithm to base 10. Composition of sample A104 was used as object A and composition of other medieval samples was acknowledged as object $\mathrm{B}$. The $p$-value calculated in $\mathrm{R}$ software (www.r-project.org) is 0.1124 , which suggests strongly that A104 is medieval glass. 
This means that the content of major elements is suitable for discrimination of wood ash and modern glass. On the other hand, the content of trace elements has a significant impact mainly for the discrimination of glasses manufactured in similar periods or provenance studies.

The great majority of analysed glasses have medieval origins. The oldest glass of the stained-glass panels from Grodziec is typical medieval wood ash glass. The amount of $\mathrm{SiO}_{2}$ is close to $50 \%$, while $\mathrm{CaO}$ and $\mathrm{K}_{2} \mathrm{O}$ content is around $40 \%$ (Table 2). The remaining oxides are present in an amount of around $10 \%$. The similar content of $\mathrm{CaO}$ and $\mathrm{K}_{2} \mathrm{O}$, that is $\mathrm{CaO} / \mathrm{K}_{2} \mathrm{O}$ ratio equals to 1 , is the evidence of the use of alkaline ashes obtained from good quality, purified wood with a low amount of bark [3]. That would also explain the relatively low phosphorus content in glass (Table 3 ). The results confirm that composition of the glasses is typical to $10^{\text {th }}-14^{\text {th }}$ century medieval glass and manufacturers had applied Theophilus recipe very strictly [3]. Low contents of $\mathrm{MgO}$ and $\mathrm{Na}_{2} \mathrm{O}$ (around $3.5 \%$ and $0.16 \%$ respectively) are consistent with the composition of stained glasses from the $12^{\text {th }}$ to $14^{\text {th }}$ centuries from Germanic countries. In agreement with literature data, these chemical differences give opportunity to distinguish Grodziec collection panels from French medieval glass [10]. Also $\mathrm{TiO}_{2}$ content ranging from 0.10 to $0.13 \%$ and $\mathrm{BaO}$ content ranging from 1498 to $3818 \mathrm{ppm}$ are characteristic of medieval wood ash glass [3].

An important observation can be made by considering differences in the concentration of minor and trace elements for all measured medieval samples (Fig. 5). There are no significant differences in the content of several oxides $\left(\mathrm{TiO}_{2}, \mathrm{~V}_{2} \mathrm{O}_{5}, \mathrm{MnO}, \mathrm{ZnO}, \mathrm{Rb}_{2} \mathrm{O}, \mathrm{SrO}\right.$, $\mathrm{BaO}$ ) related to raw materials, i.e. quartz sand and beech ash. Similar content of such oxides in all samples suggests the same source or the same recipe applied for the production of glass.

Contrary, significant differences in oxide concentrations are observed for colorants and related chemicals, i.e. $\mathrm{Fe}_{2} \mathrm{O}_{3}, \mathrm{CoO}, \mathrm{NiO}$ and $\mathrm{CuO}$. The green color of samples was evidently obtained with iron compounds (average concentration of $\mathrm{Fe}_{2} \mathrm{O}_{3}$ for three green samples is $2.11 \%$ ) and copper compounds (about $579 \mathrm{ppm} \mathrm{CuO}$ ). Blue glass was obtained by addition of $\mathrm{CoO}$ (ranging from $431 \mathrm{ppm}$ to $1747 \mathrm{ppm}$ ) and was characterized by a $\mathrm{Fe}_{2} \mathrm{O}_{3}$ content in the range between $0.4 \%$ and $0.7 \%$. The results are consistent with the composition of glass colorants used in the Middle Ages [1-3, 9, 20]. Nevertheless, yellow and red glasses are

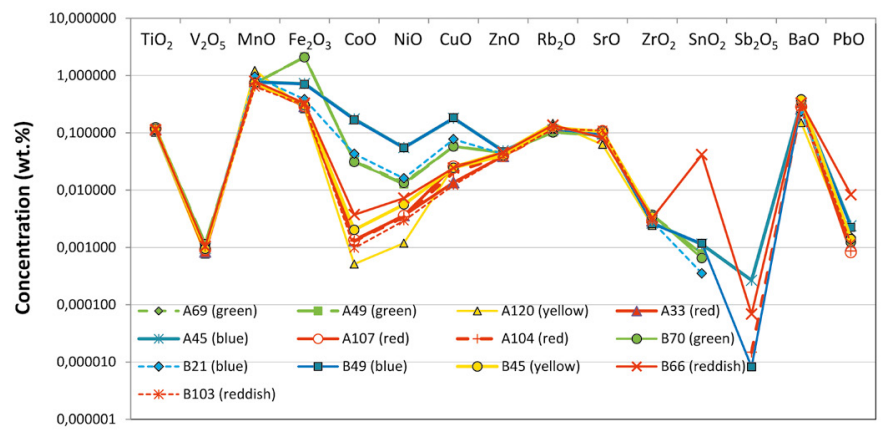

Fig. 5. The concentration of minor and trace elements in Grodziec medieval samples. 
not distinguishable from results obtained from bulk analyses.

It is worth noticing that green samples (A69, A49 and B70) from two stained glass panels exhibit almost the same composition. Similar observation can be stated for blue samples (A45 and B49) and red and yellow samples (A33, A104, A107, A120, B45, B66, B103). Exceptionally high content of $\mathrm{SnO}_{2}$ is observed in B66 sample, which can be explained by contamination during manufacture of glass or the panel. Such clear similarity of the samples confirms that stained glasses were produced at the same time from the same sources.

\subsection{Composition of medieval red samples}

Red colour of wood ash glasses was usually obtained by additions of about 0.1 to $0.5 \% \mathrm{Cu}$

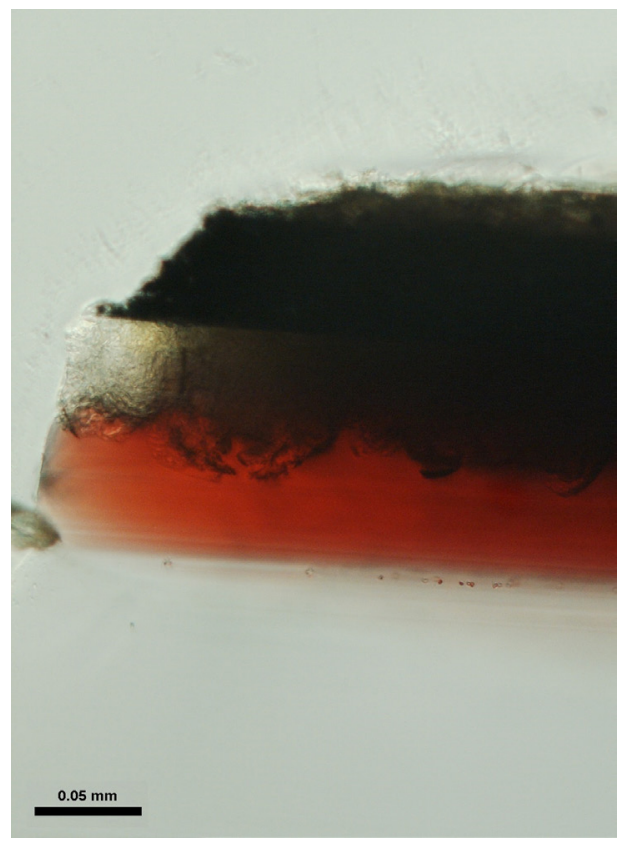

Fig. 6a. Microscopic view on cross-section of the red sample (A107) showing three separate layers (bar length: $0.05 \mathrm{~mm}$ ). to glass melts $[1,2]$. Results from bulk analysis of red samples indicate that the Grodziec glasses contain average $194 \mathrm{ppm}$ of $\mathrm{CuO}$. Thus copper content according to published literature should be expected to be present in a more distinctive amount [11]. Nevertheless, microscopy and SEM studies clarify composition and structure of red samples. Grodziec red glasses are mainly composed with three separate layers (Fig. 6a, 6b and 7). The biggest and innermost layer of the glass is colourless. As this layer was analysed during bulk analysis of red glasses by SEM-EDX and LA-ICP-MS, concentrations of oxides usually used as colorants were similar to slightly yellowish glasses (Fig. 5 and Table 5). Intensive red layer is located quite close to the surface of glass (Fig. 6a). The thin red layer is responsible for overall colour of the glass and contains higher amount of copper

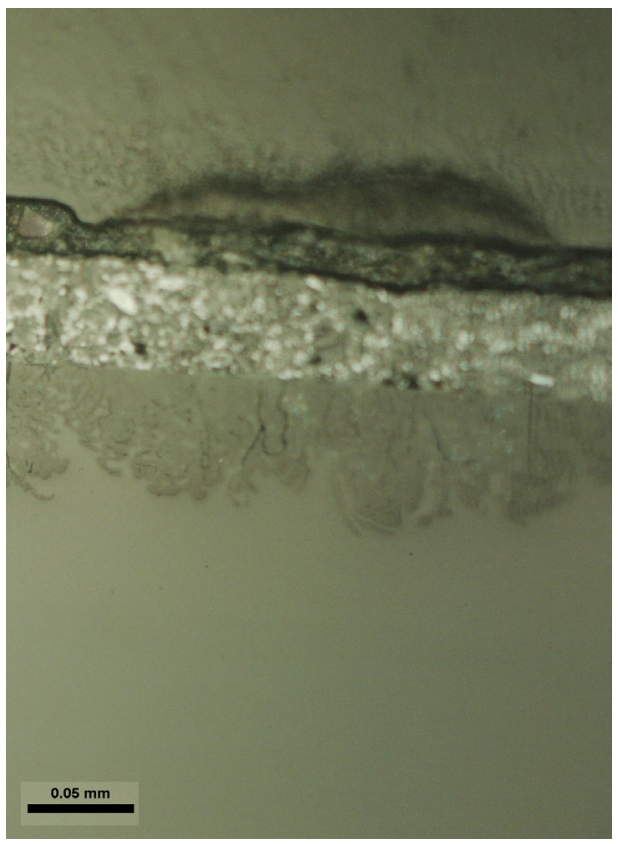

Fig. 6b. Microscopic view (in transparent light) on cross-section of the red sample (A107) showing three separate layers (bar length: $0.05 \mathrm{~mm}$ ). 
Table 5. Average concentrations of selected elements in different layers in the red glass (sample A107)

\begin{tabular}{llllll}
\hline wt.\%: & $\mathrm{CuO}$ & $\mathrm{Fe}_{2} \mathrm{O}_{3}$ & $\mathrm{PbO}$ & $\mathrm{CaO}$ & $\mathrm{SiO}_{2}$ \\
\hline outside colourless glass layer & 46 & 1.5 & 6.4 & 18.7 & 19.2 \\
red layer & $\mathbf{0 . 4 9}$ & 0.20 & 0.0407 & 20.9 & 51.7 \\
inside colourless glass layer & 0.026 & 0.29 & 0.0008 & 22.7 & 48.7 \\
\hline
\end{tabular}

(about $0.5 \% \mathrm{CuO}$ ). Thus the composition of the layer is agreed with the literature data $[3,4]$. Third additional colourless layer is overlaying the red. Such composition and structure of the glass is consistent with type B-3 red translucent glass, which was commonly applied from the $12^{\text {th }}$ century [11]. High amount of copper, iron and lead in the third layer (Table 5) can be explained with the presence of decorative paint layers and drawings [20].

The composition of the outside layers is affected also by corrosion processes manifested by microcracks, as can be seen on microscopic and BSE-SEM images (Fig. 6b, 7). This reflects in changes in the concentration of some elements in areas located near the surface of the glass. LA-ICP-MS longitudinal concentration profiles carried out from

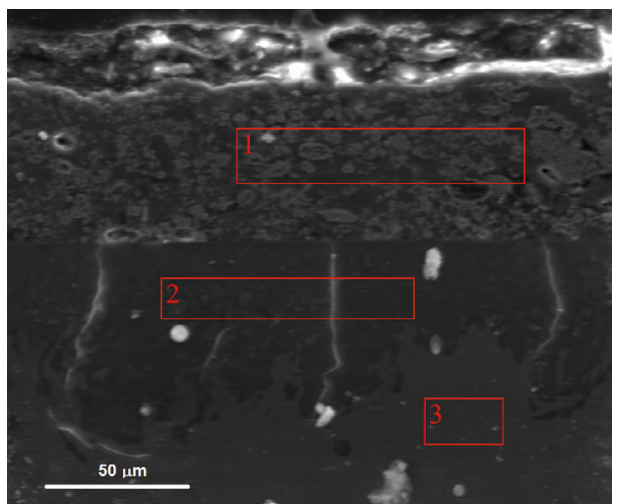

Fig. 7. BSE-SEM image showing different layers in the glass sample (A107): paint layer with the corrosion crust (1), outside colourless layer (2) and red layer (3). inside the glass to outside layers clearly show differences (Fig. $8 \mathrm{a}$ and $8 \mathrm{~b}$ ). $\mathrm{SiO}_{2}$ is enriched at the surface, while $\mathrm{CaO}$ and $\mathrm{K}_{2} \mathrm{O}$ shows decreasing concentration from the bulk to the surface of the glass (Fig. 8a).

Thus $\mathrm{Ca}$ and $\mathrm{K}$-ions are leached out of the glass, which is a typical result of glass

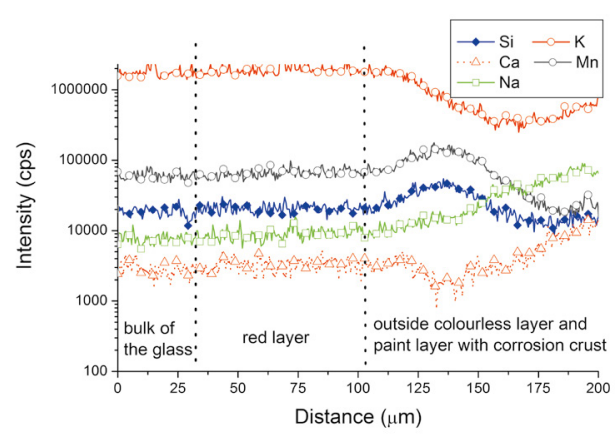

Fig. 8a. Longitudinal concentration profiles of alkali and manganese carried out from inside to outside layers in the red sample (A107).

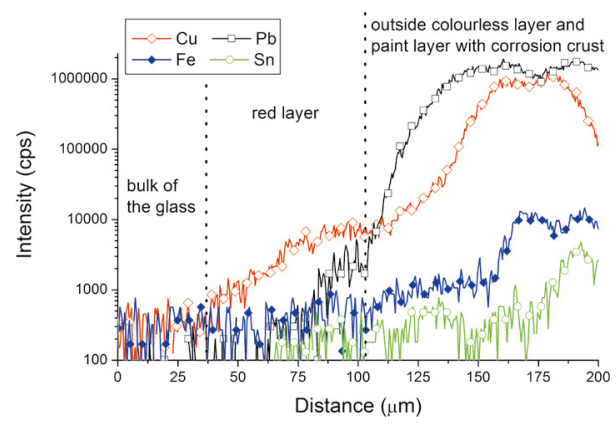

Fig. 8b. Longitudinal concentration profiles of elements related to paints carried out from inside to outside layers in the red sample (A107). 
deterioration caused by an ion exchange between $\mathrm{H}$-ions in water deposited on the glass surface. Enrichment of $\mathrm{SiO}_{2}$ at the surface is a result of increasing density of glass surface due to depletion of alkaline ions $[26,27]$. Nevertheless, $\mathrm{Na}_{2} \mathrm{O}$ enrichment at the surface (Fig. 8a) is not agreed with the well-known glass deterioration mechanism, because depletion of the $\mathrm{Na}_{2} \mathrm{O}$ as the most mobile alkaline is expected [27]. It can be explained only by low concentration of $\mathrm{Na}_{2} \mathrm{O}$ in wood ash glass and possible presence of sodium in paint layers. However, further studies are needed to clarify the result. The concentration of $\mathrm{MnO}$ in external surfaces is higher in comparison to the bulk, which can be explained by manganese browning [16]. The presence of high content of $\mathrm{Cu}, \mathrm{Pb}, \mathrm{Fe}$ and $\mathrm{Sn}$ in the outside colourless layers (Fig. $8 b$ ) is caused by the diffusion process from the paint layer

\section{Conclusions}

The applied analytical methods provided valuable information on the composition of Grodziec stained glasses, which was necessary to perform provenance studies of the investigated panels. Results confirm that stained glass panels reveal characteristic elemental composition of wood ash glass produced from 1000 to $1400 \mathrm{AD}$. Almost equal proportions of potassium and calcium oxides indicate that high quality of beech wood was applied by manufacturers. Main elements content is similar for almost all investigated glass samples, which means that manufacturers follow strictly the assumed recipe during panels production. Some glasses exhibit typical composition of modern glass, which were probably used during conservation treatments at $19^{\text {th }}$ or $20^{\text {th }}$ centuries.
LA-ICP-MS longitudinal concentration profiles together with microscopic and BSESEM images revealed specific areas in the glasses. The composition and structure of type B-3 red translucent glass was identified in the Grodziec red glasses. High concentrations of lead, copper and iron determined in external layers of glass samples can be connected with decorative paint layers and drawings. Considerable differences between the composition of healthy bulk glass and the deteriorated surface of glass were also detected and explained by dealkalinisation and manganese browning.

\section{Acknowledgements}

LA-ICP-MS measurements were supported by $\mathrm{Na}$ tional Science Centre of Poland from funds granted within post-doctoral internship based on decision no. DEC-2013/08/S/ST4/00560. SEM-EDX measurements were supported by the National Science Centre of Poland within the project no. 2012/05/E/ HS2/03867.

\section{References}

[1] G. Artioli, Scientific Methods and Cultural Heritage: an introduction to the application of materials science to archaeometry and conservation science, Oxford University Press, New York 2010.

[2] S. Davison, Conservation and Restoration of Glass, Butterworth Heinemann, Oxford 2003.

[3] K. Wedepohl, K. Simon, "The chemical composition of medieval wood ash glass from Central Europe", Chemie der Erde, 70 (2010) 89-97, DOI: 10.1016/j.chemer.2009.12.006.

[4] K. Wedepohl, K. Simon, A. Kronz, “The chemical composition including the Rare Earth Elements of the three major glass types of Europe and the Orient used in late antiquity and the Middle Ages", Chemie der Erde, 71 (2011) 289-296, DOI: 10.1016/j. chemer.2011.04.001.

[5] K. Wedepohl, K. Simon, A. Kronz, "Data on 61 chemical elements for the characterization of three 
major glass compositions in late antiquity and the middle ages", Archaeometry, 53 (2011) 81-102, DOI: 10.1111/j.1475-4754.2010.00536.x.

[6] Ž. Šmit, P. Pelicon, G. Vidmar, B. Zorko, M. Budnar, G. Demortier, B. Gratuze, S. Šturm, M. Nečemer, P. Kump, M. Kos, "Analysis of medieval glass by X-ray spectrometric methods", Nuclear Instruments and Methods in Physics Research B, 161-163 (2000) 718-723, DOI: 10.1016/S0168-583X(99)00947-7.

[7] Ž. Šmit, K. Janssens, E. Bulska, B. Wagner, M. Kos, I. Lazar, "Trace element fingerprinting of façonde-Venice glass", Nuclear Instruments and Methods in Physics Research B, 239 (2005) 94-99, DOI: 10.1016/j.nimb.2005.06.182.

[8] Ž. Šmit, T. Milavec, H. Fajfar, T. Rehren, J. Lankton, B. Gratuze, "Analysis of glass from the post-Roman settlement Tonovcov grad (Slovenia) by PIXE-PIGE and LA-ICP-MS", Nuclear Instruments and Methods in Physics Research B, 311 (2013) 53-59, DOI: 10.1016/j.nimb.2013.06.012.

[9] S. Conte, T. Chinni, R. Arletti, M. Vandini, "Butrint (Albania) between eastern and western Mediterranean glass production: EMPA and LAICP-MS of late antique and early medieval finds", Journal of Archaeological Science, 49 (2014) 6-20, DOI: 10.1016/j.jas.2014.04.014.

[10] B. Velde, "Glass Compositions over Several Millennia in the Western World", in: K. Janssens (Ed.), Modern Methods for Analysing Archaeological and Historical Glass, John Wiley \& Sons Ltd., Chichester 2013, pp. 67-78.

[11] J. Kunicki-Goldfinger, I. Freestone, I. McDonald, J. Hobot, H. Gilderdale-Scott, T. Ayers, “Technology, production and chronology of red window glass in the medieval period - rediscovery of a lost technology", Journal of Archaeological Science, 41 (2014) 89-105, DOI: 10.1016/j.jas.2013.07.029.

[12] E. Gajewska-Prorok, "Stained glass windows from Grodziec. Part I”, Opuscula Musealia, 22 (2014) 73-94, DOI: 10.4467/20843852.OM.14.004.3202.

[13] E. Gajewska-Prorok, "Stained glass windows from Grodziec. Part II”, Opuscula Musealia, 22 (2014) 95-116, DOI: 10.4467/20843852.OM.14.005.3203.

[14] M. Kamińska, P. Karaszkiewicz, "Old and modern methods of stained glass conservation and renovation, using as an example the stained glass depicting St Peter in the collection of the Collegium Maius of the Jagiellonian University in Kraków", Opuscula Musealia, 22 (2014) 123-136, DOI: 10.4467/20843852.OM.14.008.3206.

[15] M. Walczak, M. Kamińska, P. Karaszkiewicz, J. Szczerbiński, M. Szymoński, “The preliminary results on the investigation of historic stained glass panels from Grodziec collection, Poland", Proc. SPIE, 8790 (2013) 87901F, DOI: 10.1117/12.2021197.

[16] M.Walczak, M. Kamińska, J. Sobczyk, M. Płotek, D. Horzela, M. Sylwestrzak, P. Targowski, “The application of non-invasive analytical techniques in the investigation and documentation of medieval stained-glass windows from the Grodziec collection", in: H. Roemich, L. Fair (Eds.), Recent Advances in Glass and Ceramics Conservation, International Council of Museums - Committee for Conservation (ICOM-CC), Paris 2016, pp. 21-30.

[17] K. Jochum, U. Weis, B. Stoll, D. Kuzmin, Q. Yang, I. Raczek, D. Jacob, A. Stracke, K. Birbaum, D. Frick, D. Günther, J. Enzweiler, "Determination of Reference Values for NIST SRM 610-617 Glasses Following ISO Guidelines", Geostandards and Geoanalytical Research, 35 (2011) 397-429, DOI: 10.1111/j.175 1-908X.2011.00120.x.

[18] B. Wagner, A. Nowak, E. Bulska, K. Hametner, D. Günther, "Critical assessment of the elemental composition of Corning archeological reference glasses by LA-ICP-MS", Analytical and Bioanalytical Chemistry, 402 (2012) 1667-1677, DOI: 10.1007/ s00216-011-5597-8.

[19] Y. Liu, Z. Hu, S. Gao, D. Günther, J. Xu, C. Gao, H. Chen, "In situ analysis of major and trace elements of anhydrous minerals by LA-ICP-MS without applying an internal standard", Chemical Geology, 257 (2008) 34-43, DOI: 10.1016/j.chemgeo.2008.08.004.

[20] B. Wagner, A. Nowak, E. Bulska, J. Kunicki-Goldfinger, O. Schalm, K. Janssens, "Complementary analysis of historical glass by scanning electron microscopy with energy dispersive X-ray spectroscopy and laser ablation inductively coupled plasma mass spectrometry", Microchimica Acta, 162 (2008) 415-424, DOI: 10.1007/s00604-007-0835-7.

[21] J. Hormes, A. Roy, G.-L. Bovenkamp, K. Simon, C.-Y. Kim, N. Börste, S. Gai, "Medieval glass from the 
Cathedral in Paderborn: a comparative study using $\mathrm{X}$-ray absorption spectroscopy, X-ray fluorescence, and inductively coupled laser ablation mass spectrometry", Applied Physics A, 111 (2013) 91-97, DOI: 10.1007/s00339-012-7505-2.

[22] M. Gaboardi, M. Humayun, "Elemental fractionation during LA-ICP-MS analysis of silicate glasses: implications for matrix-independent standardization", Journal of Analytical Atomic Spectrometry, 24 (2009) 1188-1197, DOI: 10.1039/B900876D.

[23] E. Vicenzi, S. Eggins, A. Logan, R. Wysoczanski, "Microbeam Characterization of Corning Archeological Reference Glasses: New Additions to the Smithsonian Microbeam Standard Collection", Journal of Research of the National Institute of Standards and Technology, 107 (2002), 719-727, DOI: 10.6028/ jres.107.058.

[24] L. Dussubieux, P. Robertshaw, M. Glascock, "LAICP-MS analysis of African glass beads: Laboratory inter-comparison with an emphasis on the impact of corrosion on data interpretation", International Journal of Mass Spectrometry, 284 (2009) 152-161, DOI: 10.1016/j.ijms.2008.11.003.

[25] G. Zadora, A. Martyna, D. Ramos, C. Aitken, Statistical Analysis in Forensic Science. Evidential Value of Multivariate Physicochemical Data, Wiley, Chichester 2014.

[26] G. Van der Snickt, S. Legrand, J. Caen, F. Vanmeert, M. Alfeld, K. Janssens, "Chemical imaging of stained-glass windows by means of macro X-ray fluorescence (MA-XRF) scanning", Microchemical Journal, 124 (2016) 615-622, DOI: 10.1016/j.mi croc.2015.10.010.

[27] J. van Elteren, A. Izmer, M. Šala, E. Orsega, V. Šelih, S. Panighello, F. Vanhaecke, "3D laser ablation-ICP-mass spectrometry mapping for the study of surface layer phenomena - a case study for weathered glass", Journal of Analytical Atomic Spectrometry, 28 (2013) 994-1004, DOI: 10.1039/ C3JA30362D. 



\title{
Easel paintings on canvas and panel: application of Nd:YAG laser at $355 \mathrm{~nm}, 1064 \mathrm{~nm}$ and UV, IR and visible light for the development of new methodologies in conservation
}

\author{
Joakim Striber ${ }^{1 \star}$, Vanja Jovanović ${ }^{2}$, Maja Jovanović ${ }^{2}$ \\ 1 Alfa BK University, Palmira Toljatija 3, Belgrade 11000, Serbia \\ 2 Central Institute for Conservation, Terazije 26, Belgrade 11000, Serbia \\ * Corresponding author: striber65@yahoo.it
}

\begin{abstract}
Laser cleaning has been applied in the conservation of objects of cultural heritage since the early 1980s. Nowadays its technology is the object of many scientific studies. Laser can be applied on different objects of cultural heritage, while for objects with specific problems, in some cases, it is even indispensable. However, it has not yet become part of the standard procedure in the conservation studios. Despite the existence of an adequate legal framework and the scientific achievements in this field, the main conservation laboratories in Europe are still insufficiently equipped.

The general objective of this article is to join the efforts of other institutions in promoting the concept of modern approach to heritage conservation, which could be defined as a combination of art, science and technology. Therefore, it presents an equipment that can meet these requirements and is both available and economically acceptable. This equipment was applied in two case studies of the conservation of objects with specific problems.

The subject of research and conservation were two works of art: the painting Portrait of Jelena Milojevic with her daughters from 1922, the work of the Russian painter Valentin V. Volkov, and the Ukrainian icon Holy Mother of God from the $19^{\text {th }}$ century.

Portrait of Jelena Milojevic with her daughters: by observing the results of chemical cleaning tests under the UV light, it was concluded that due to the very high sensitivity of some pigments it was impossible to apply this methodology on such delicate surfaces. The laser cleaning, which had already proved to be an acceptable alternative for chemical cleaning of easel paintings, was the only solution in this case.

Icon Holy Mother of God: the icon was made with a technique of egg tempera in the $19^{\text {th }}$ century in Ukraine. A bronze coating was subsequently added on the aureoles, below which there was a gilding sheet. This sheet was poorly linked to the preparation layer so that any intervention, whether it was mechanical, chemical or laser cleaning, would have caused its removal together with the bronze coating. But when the surface layer was first irradiated at $1064 \mathrm{~nm}$, the added layer of coating was separated from the original layer, which allowed, subsequently, an easy mechanical removal of unwanted layers. Combination treatment of the aureoles using two techniques - laser cleaning and mechanical removal - is faster and more uniform than in the case of treatment by other techniques.
\end{abstract}

Keywords: easel paintings, laser treatment, mobile equipment, Nd:YAG laser, coating on gold leaf, combined treatment 


\section{Introduction}

To meet all the needs of modern conservation, it is necessary to define only the methods and research techniques whose application to objects of cultural heritage will not undermine the basic principles of conservation. These require that any intervention on an object must not compromise its integrity, in the broadest sense of the word: physical, aesthetic and historical [1].

Easel paintings represent the main challenge of laser cleaning - they are complex, multilayer systems, often very sensitive and difficult to clean. This kind of intervention is of high importance because it is the least reversible invasive intervention, as well as the most usual of all conservation treatments. Cleaning is important in maintaining the aesthetic aspect of easel paintings and prolonging their lifetime by removing potentially damaging environmental pollutants from the surface.

The application of laser technology to the paintings conservation field started in the early 1990s. Despite several works having been reported, including systematic investigations on laser interaction effects induced on pigments and binders, the laser approach was still far from conservation practice. Nevertheless, it was clear that the laser technology had great potential in the development of safer procedures for conservation because of its controllability and reproducibility.

In the case of thinning of varnish on the paintings, a method of laser cleaning with excimer laser was developed, where automatic monitoring of the cleaning process was applied [2]. This permitted an efficient, relatively fast, highly controlled and accurate removal of the varnish layer - only the desired thickness, with precision up to $1 \mathrm{~mm}$. However, such systems turned out to be very expensive, immobile and for the time being are not yet commercialised. For these reasons, in most cases where varnish thinning on the paintings is necessary and for which the laser would be optimal if not indispensable method of cleaning, the system is practically inaccessible.

On the other hand, at least $300 \mathrm{Nd}$ :YAG laser systems [3] are presently operative in conservation laboratories and restoration yards all over Europe and abroad. It is a proof that laser cleaning technologies moved from research laboratories to commercial production, and then to restoration work sites. Such a unique case of technological and methodological transfer in conservation of cultural assets was entirely determined by the scientific contribution provided by various research institutions. The latter can still play an important role in order to rigorously extend the exploitation of experimental results and address open cleaning problems.

In general, $\lambda=213 \mathrm{~nm}$ and $\lambda=355 \mathrm{~nm}$ are very promising wavelengths, giving new interesting results in the field of laser restoration [4-5]. In one part of this work, possibility and efficiency of thinning of varnish on the painting Portrait of Jelena Milojevic with her daughters will be examined using $355 \mathrm{~nm}$ wavelength. Laser cleaning proved to be an acceptable alternative for chemical methods of cleaning [6], but in this case it was the only possible solution. A mobile commercial pulse $\mathrm{Nd}$ :YAG laser with radiation at $355 \mathrm{~nm}$ was used instead of the excimer laser. Monitoring and control of the effects of cleaning was done before, during and after conservation treatment of the easel painting.

Another case of conservation of high risk was the removal of a thick bronze coating layer from a golden leaf, since any intervention of cleaning - mechanical, chemical 
or laser, could have caused its elimination together with the bronze coating.

Application of mechanical cleaning would have quite possibly resulted with a loss of the golden leaf. In case of chemical cleaning, it was very difficult to find a solution/product that would guarantee the safe removal of the liquid bronze layer from the layer of original gold. Solution for this problem was found in combining laser irradiation using Nd:YAG laser at $1064 \mathrm{~nm}$ in Q-switched regime and mechanical cleaning. This new technique opens the possibility for development of a new methodology that would provide safe, efficient and fast removal of non-original layers from layers of gilded surfaces or, in general, to separate one layer from another.

\section{Materials and methodologies}

\subsection{Portrait of Jelena Milojevic with her daughters}

The painting Portrait of Jelena Milojevic with her daughters from 1922 is a work of the Russian painter Valentin V. Volkov. After performing the tests of chemical cleaning and observing them under the UV light, it was concluded that due to the very high sensitivity of some pigments, the application of this methodology would be unsafe. The laser cleaning was therefore chosen as a safer alternative. Considering previous research results about absorption coefficients of varnish irradiation in VIS and NIR UV spectral region, an Nd:YAG laser was used for cleaning at $355 \mathrm{~nm}$, carefully controlling the process. The final decision about the method of thinning of varnish layer was taken after observing the efficiency and the effect of cleaning on the test area by a digital microscope. Visible and UV light were used for the control of laser cleaning.
In order to analyse the state of conservation and to obtain information about different painting layers, imaging techniques were applied.

\section{Multispectral analysis}

Using different parts of the light spectra (IR, VIS, and UV) to illuminate the surface of objects, mostly paintings, it is possible to obtain an image of various surface layers: the upmost surface layers can be observed with UV light, while going from UV toward IR region, deeper layers will be visible. This gives the possibility to study the underdrawings or to visualize the composition and condition of paintings. It can aid in the study of the applied artistic techniques, the analysis of colour and pigments and can help to reveal previous restorations.

\section{UV radiation in painting's analysis}

The objective of the UV-induced visible fluorescence photography is to localize prior restoration interventions on the painting. This technique can expose the old natural resin varnishes, as these often fluoresce under the UV light while newer varnishes do not. It is also possible to identify overpainting, repairs and floating signatures which usually appear as dark spots in contrast to the original fluorescent areas [7].

\section{Recording with an infrared camera}

Infrared reflectography (IRR) has become a classic image registration method in the technical examination of art [8]. This technique reveals the presence of preparatory drawings made of carbon black (infrared non-reflective material) and the artist's process of execution and changes in the composition (pentimenti) as well. Infrared spectroscopic imaging could also prove useful in document and forgery analysis, as well 
as in attributions of works of art [9]. In this case InGaAs camera was used in the spectral range from $900 \mathrm{~nm}$ to $1700 \mathrm{~nm}$, with filters for $1500 \mathrm{~nm}, 1600 \mathrm{~nm}$ and $1700 \mathrm{~nm}$, each of them having spectral width of $50 \mathrm{~nm}$.

\section{Laser cleaning}

Regarding laser cleaning of materials that have a very low thermal damage threshold, such as organic fibers, pigments or binders, a certain risk of adverse effects is present [10]. On the other hand, whenever there is a need to clean at micron level, which is often the case with paintings, selectivity, high precision and control of cleaning can be achieved in the process of photochemical ablation [11].

Studies have shown that the dominant mechanisam of laser cleaning using UV radiation is a photochemical process [12]. This means that the rise of temperature is very small. UV radiation is strongly absorbed by organic materials, which means that its penetration is only about a few microns [13]. Therefore, the UV radiation is very suitable for thinning of varnish on the paintings, which should remove only a thin layer of material, with high precision. In the case of Volkov's painting, various techniques have been used to create a new methodology for examination of all phases of the painting process, but also for the monitoring and control of laser cleaning. In this study, a Q-switched Nd:YAG laser Thunder art system for laser cleaning was used at $355 \mathrm{~nm}$.

\subsection{Holy Mother of God}

\section{Basic information on the object}

The subject of this icon, written in circle: "Sacred face of the Most Holy Mother of God, which is called the burning bush", is determined by prefiguration presented in corners, which is an Old Testament motive - prophet Moses's vision of the bush that burns, but does not burn out. Judging by the flesh tones and the use of characteristic blue background, this icon was painted in the early $19^{\text {th }}$ century.

\section{State of preservation (Fig. 1)}

The icon was painted using the egg tempera technique. The layer of paint was unstable in zones where cracks are grouped, and it was partially missing in zones of damage of the foundation layer. Colour coating is translucent and in some places covered with spots and stains.

Subsequently applied bronze coatings that were oxidized and darkened by ageing were visible on the aureoles, the ring with the inscription and the vestments of Virgin and Christ.

The gilding on the vestments of Christ and on the Archangel's icon lamp (in the

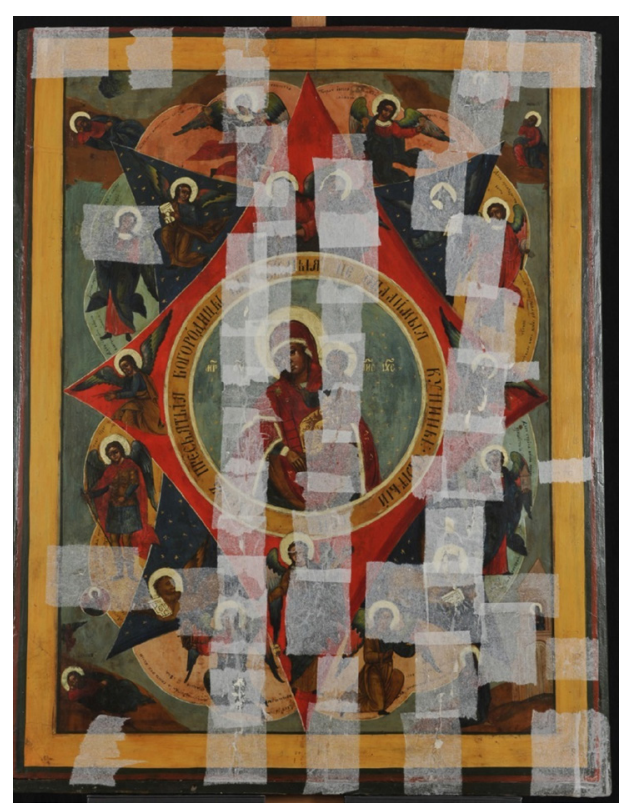

Fig. 1. State of preservation of the icon. 
central part, lower half) is made of gold leafs, which were thinned in some places due to the inadequate storage conditions.

The final varnish layer was of uneven thickness, probably applied at a later date.

\section{Optical microscopy -}

\section{application in the cultural heritage}

A microscopic analysis can give a new perspective on the origin and history of paintings by differentiating between natural ageing effects and changes deliberately instigated as part of the artist's technique. It is also used to study the conservation state of object's surface, and to determine the efficacy of conservation treatment on the surface. In this study, a ViTiny Pro10-3 Portable UV/ IR/White Light Digital Microscope was used with Ultra-violet, IR and White LED lighting and $10 \times$ to $200 \times$ magnification, $2 \mathrm{M}$ pixels Lens \& CMOS sensor.

\section{Chemical cleaning}

Solubility test was carried out by using Wolbers mixture of solvents, containing acetone.

\section{Laser irradiation}

During the laser ablation of material, measurements performed by the laser interferometry technique demonstrate that ablation induced high pressure on the surfaces of analyzed samples, which can provoke the appearance of delocalized defects formation [14].

In case of conservation of the Holy Mother of God, this effect, usually considered as a negative one, was used for safe and efficient cleaning. Laser radiation induced a phenomenon of layer delamination, where connections between the leaf of gold and the bronze coating became weak. When this was combined with subsequent mechanical cleaning, it allowed a quick and efficient removal of the bronze coating, with minor losses of the golden leaf.

\section{Results and discussion}

\subsection{Portrait of Jelena Milojevic with her daughters}

During the preview inspection of this painting in situ, a number of previous inadequate conservation interventions were noted in the form of darkened paint and retouch, as well as a very thick oxidised varnish layer, which could be clearly seen in the UV fluorescence images. This protective varnish had a yellowish tone - the result of aging and oxidation, and it was covered with a thin layer of surface dirt, which significantly harmed the aesthetic appearance of the work.

The main goal of the treatment, besides stabilization and revitalization of structural layers of the painting, was a selective removal of the thick layer of yellowed varnish and subsequent coatings, in order to reveal the original colors of the painting. The next goal was to apply adequate restoration treatments and bring back the original look and dimension of the painting, which would enable its overall perception.

The fact that the painting changed in the past, required a more detailed research and analysis of this work. Using modern methods such as multispectral analysis, it was possible to make the difference between the original and subsequent interventions on the painting - by the author and by the restorer. Also, selective and controlled removal of the restorer's intervention was successful.

Further tests, in which different parts of light spectra (IR, VIS, and UV) were used, revealed numerous pentimento, old retouching, micro-cracks in the varnish, as well as an 
advanced process of oxidation of the upper layer of the varnish.

\section{Multispectral Analysis}

IR reflectography discovered certain changes in the drawing, i.e. corrections that the artist made during the painting process working out the original idea and changing the composition of the painting. Darkened paint, retouch and a very thick oxidised varnish layer were clearly visible in the UV fluorescence images (Fig. 2 to 6 ).

IR 1600 - Besides visible initial drawing, the changes that the artist introduced during the process of painting are clearly visible on the IR recording: the girl's elbow is moved upwords while the dark stripes on the dress are covered with textile belt.

UV: UV recording reveals minor retouching and a thick layer of lacquer in the upper left zone (violet fluorescence).

\section{Microscopic analysis}

Instead of chemical cleaning, the laser cleaning was efficient: by thinning of the varnish layer craquelures, darkened paint and a thick oxidized varnish layer were eliminated. Following the application of a thin coat of new
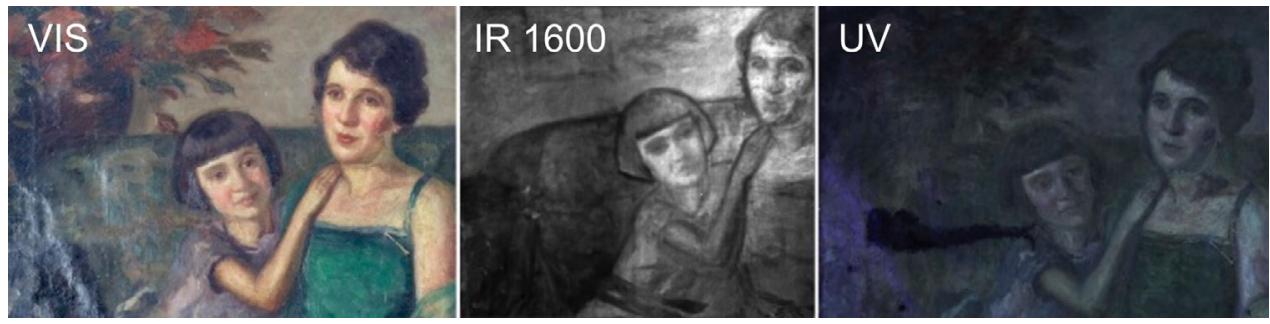

Fig. 2. VIS - The final appearance of the surface does not suggest through which phases/changes the painting went during and after the painting process; IR 1600 - Besides the visible initial drawings, the changes that the artist introduced during the painting process are clearly visible on the IR recording: the position of the girl's arm (elbow is moved upwards) and painting frame (upper left corner) is in further painter's procedure covered by the vase of flowers; UV - On the UV recording a large retouched area, which extends all the way to the girl's shoulders (dark zones on the left half of the photo) and several smaller retouched areas in the same zone are clearly visible.

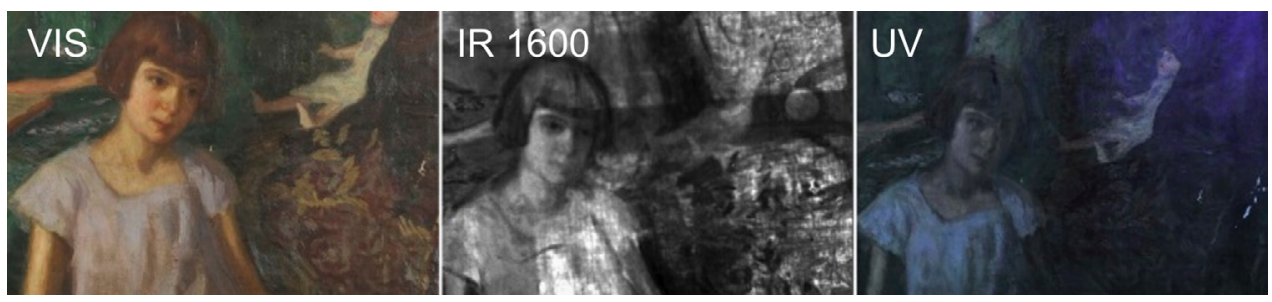

Fig. 3. VIS - At the final appearance on the surface of the painting, a thick layer of paint can be seen on the right half of the image and a circular shape appears on the brown drapery; IR 1600 - The IR recording reveals the original appearance of the divan and the drapery (or horizontal position and the track frame rails); UV - The UV recording reveals minor retouching on the hair of the little girl and a thick layer of lacquer in the upper right zone (violet fluorescence). 

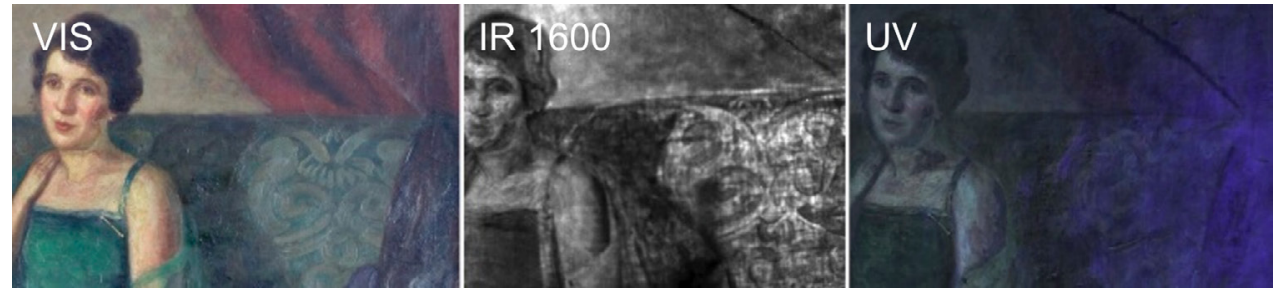

Fig. 4. VIS - At the final appearance of the painting's surface a thick layer of brown color can be seen, with which drapery over the divan was painted, as well as an inadequate retuch on a dark red curtain; IR 1600 - IR image reveals the original appearance of the right half of the image (the painter has added a dark red curtain, covered part of the divan with draperies and added a brown toy - a doll - in the final stages of painting); UV - UV image reveals the retouched positions on the skin of the woman as well as on the drapery in the right corner and a thick layer of varnish on the right half of the image (violet fluorescence).
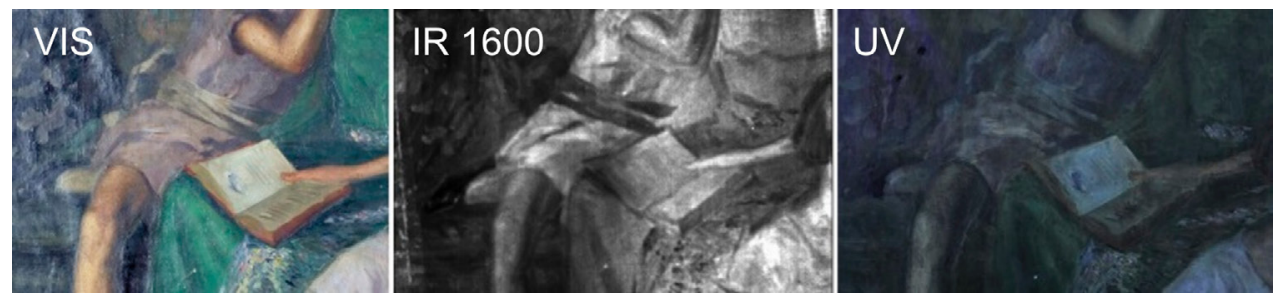

Fig. 5. VIS - The final appearance of the surface of the painting does not suggest through which phases/ changes the paintning has gone during the painting process.
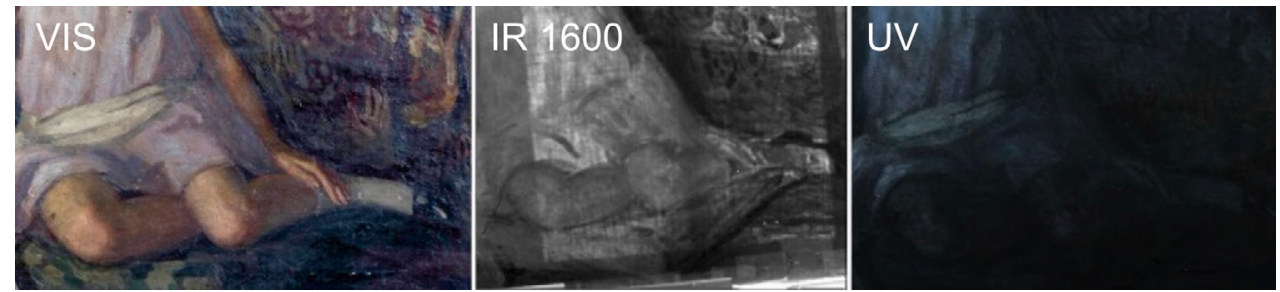

Fig. 6. VIS: The final appearance of the surface image does not suggest through which phases/changes the paintning has gone during the painting process; IR 1600 - The IC recording clearly shows that the girl's right leg is rounded (dark drawing) and also that the painter has put the textile belt after he has painted the dresses; UV - UV snapshot reveals minor retouch on the knees of a little girl.

varnish on the cleaned surface, satisfying aesthetic and visual integrity of paint was obtained.

Optical microscopy was used in order to identify surface variation during the laser cleaning. The microscopy recordings have shown that after laser cleaning, thickness of varnish cracks was reduced and, with the elimination of impurities, the visual unity of the surface was brought back (Figs. 7-9).

Summing the observation of analysis:

- Surface of varnish before cleaning - the uneven aspect of varnish, with very bright or 

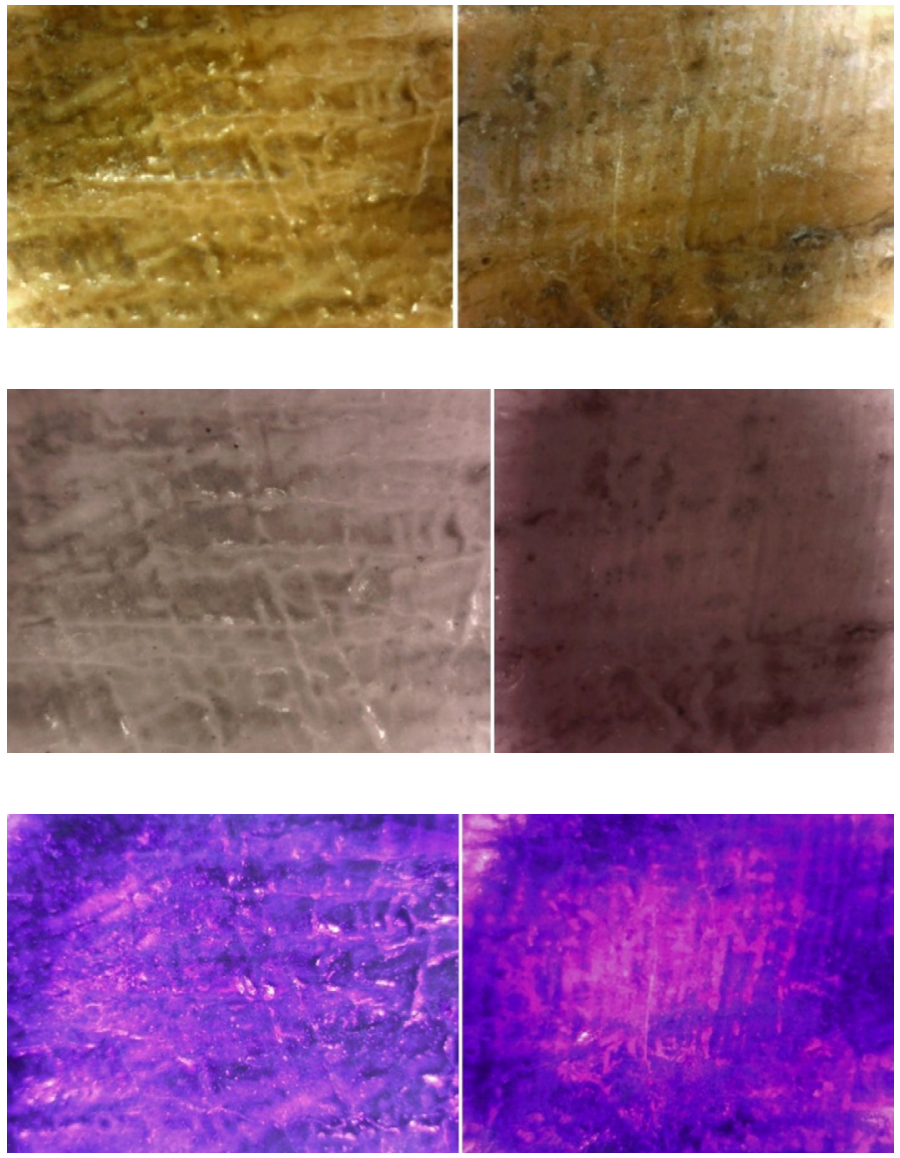

Fig. 7. Microscopy recordings of varnish using visible illumination, before and after cleaning.

Fig. 8. Microscopy recordings of varnish using infrared illumination, before and after cleaning.

Fig. 9. Microscopy recordings of varnish using ultraviolet illumination, before and after cleaning. completely matte surfaces is caused by the advanced stage of its aggregation.

- Surface of varnish after cleaning - after the thinning of varnish by laser, the aggregation of varnish was completely eliminated, and the surface is uniform in thickness and shine.

The process of varnish removal was accompanied by the emergence of light bleaching as a result of the creation of microcracks due to the break-off of the polymer chains in the layer. To eliminate this effect and obtain satisfying aesthetic and visual integrity of the paint, it was sufficient to apply a thin coat of new varnish on the cleaned surface.

\section{Cleaning of the painting: chemical and laser} cleaning

The final decision about the method of thinning of varnish layer was taken after observing the surface of varnish by a digital microscope which provided useful information about the layers and their mechanical structure. The process of laser cleaning of varnish was controlled by visible and UV light. Due to different effects of UV fluorescence on the varnish layer and on the paint layer, it was possible to control the presence of varnish, taking the paint layer as a reference. By combining these techniques, optimal results were achieved both from the 
conservation as well as from the aesthetic standpoint.

In order to remove the varnish, Wolbers dissolution test was performed, which is based on different mixtures of polar and nonpolar solvents. Results of this test have shown that by using different mixtures, the protective varnish was removed. Even more, by comparing (the parameter values of the three parameters $\left(f_{d}=\right.$ non polar dispersion forces, $\mathrm{f}_{\mathrm{p}}=$ polar dipole forces, $\mathrm{f}_{\mathrm{h}}=$ hydrogen bonds), it is possible to determine the nature of that varnish [15]. In the case of painting of Portrait of Jelena Milojevic with her daughters, the best result was obtained by the solution of cyclohexane and ethanol in ratio 40:60 (test Wolbers - CE6: Fig. 14), which indicates that a natural resin was used for preparation of the varnish.

This test has proved, however, that the paint layers which consisted of a pallet of natural, earthy pigments (brown, ochre, red, green) were very unstable, so it was impossible to continue cleaning with these solvents. Partial results were obtained using the concentrated mixture of isopropyl alcohol and distilled water, which was safe but only for bright areas of the picture.

The result pointed the fact that the chemical cleaning of the paint layer was unsatisfactory - most of the pigments being extremely unstable for this treatment, despite the fact that the protective varnish was removed. Because of the impossibility to selectively and partially remove the protective varnish due to difficult control of penetration of solvent into structural layers, this method proved to be inadmissible.

Very high control of laser cleaning, removing layers with thickness between 1 and $100 \mathrm{~mm}$ [16], was decisive to use it as a substitute method of cleaning. Using UV laser radiation at $355 \mathrm{~nm}$ with 4 pulses/s in Q-switched regime (pulse duration $\sim 10$ ns) made it possible to safely remove part of the varnish. Gradual increase in energy resulted in finding the optimal condition of laser cleaning: safely, efficiently and relatively rapidly removing desired thickness of varnish at $80 \mathrm{~mJ} / \mathrm{cm}^{2}$. Because of the very high absorption coefficient of varnish, UV radiation was totally absorbed by it and at the same time, the paint layer was protected. The process of laser cleaning of varnish was controlled by day light and UV light [17]. As mentioned above, thanks to a different effect of UV fluorescence on the varnish layer and on the paint layer, the presence of varnish was controlled using the paint layer as a reference. Treated surface had a lower fluorescence intensity of untreated surfaces and surfaces without varnish had no fluorescence. The cleaning process is stopped when the treated area satisfied the aesthetic aspect under ordinary light and UV fluorescence confirmed the continued presence of a layer of varnish on that area.

The process of varnish removal was accompanied by the emergence of light bleaching because of the creation of microcracks due to the break-off of the polymer chains in the layer. To eliminate this effect and obtain satisfying aesthetic and visual integrity of paint, it was sufficient to apply a thin coat of mineral spirit on the cleaned surface. The painting after conservation is shown on Fig. 10.

\subsection{Holy Mother of God}

\section{Chemical cleaning of the gilding}

(Figs. 11-14)

The liquid bronze coat, which was applied on the gilding in previous interventions, has oxidised during the ageing process and 

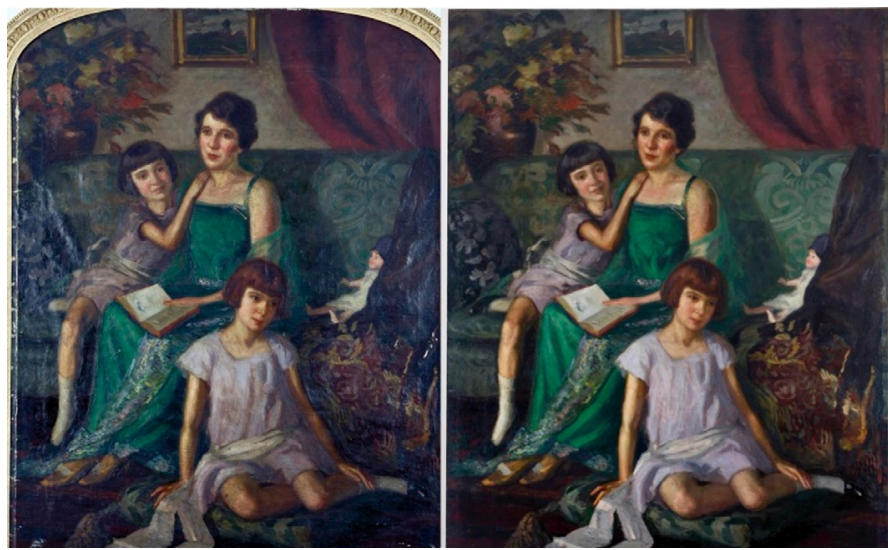

Fig. 10. The painting before and after the process of conservation.

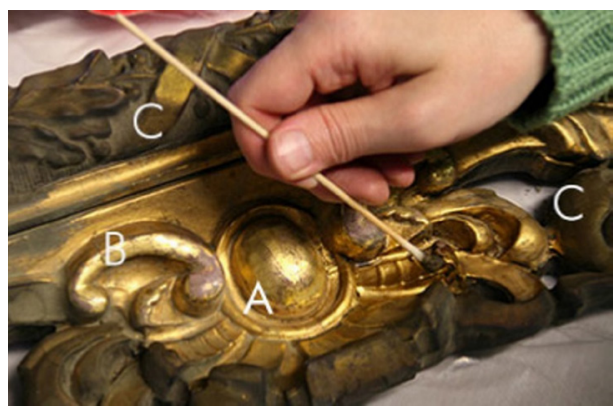

Fig. 11. Removing subsequently applied bronze coating A: original gilding; B: original gilding on oil mixtion; C: oxidised copper coating

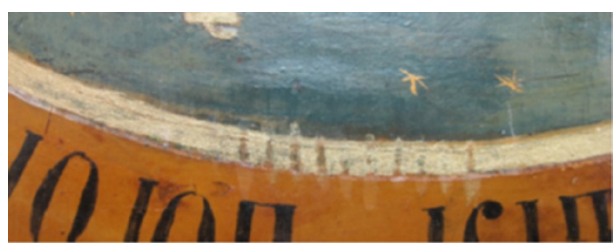

Fig. 12. Probe cleaning by Wolbers test.

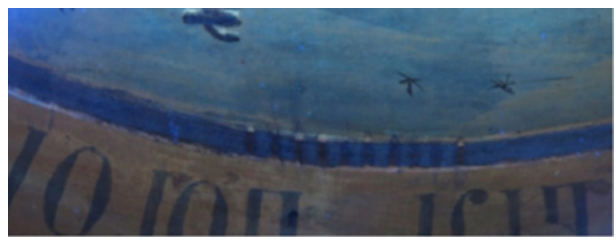

Fig. 13. Probe cleaning by Wolbers test under UV light.

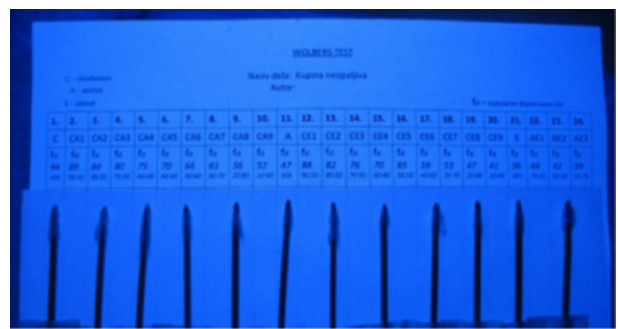

Fig. 14. Used swabs in Wolbers test: the best result was obtained by the solution of cyclohexane and ethanol in ratio 40:60 (test Wolbers - CE6), which indicates that a natural resin was used for preparation of the varnish.

harmed the aesthetic appearance of the icon. Chemical removal in such cases could be extremely risky, especially when the solubility parameters of the bronze coating and of the mixtion used in the original gilding coincide (e.g. when an oil based liquid bronze is directly applied on a gilding with oil mixtion).

Based on the preserved original gilding (the vestment of Christ) and the fact that real gold was used as an element in the painting of the Orthodox art, it was supposed that there is an original gilding underneath the bronze coating layer.

For the abovementioned reasons, it has been decided to remove this bronze coating. 
All solvent mixtures containing acetone removed the coating only partially, and the best results were achieved with pure acetone. However, the test results on the bronze coating have shown that the original gilding layer was not reached, and the research was continued.

\section{Laser irradiation}

In order to find an adequate solution, laser cleaning tests were done (Fig. 15) using infrared wavelength at $1064 \mathrm{~nm}$. Energy of radiation was increased gradually, from 150 to $650 \mathrm{~mJ} / \mathrm{cm}^{2}$, for 20 pulses per point. However, the coating had not been removed. On the other hand, with a further increase of energy there was a risk that by removing the bronze coating, the gold leaf would be removed, too.

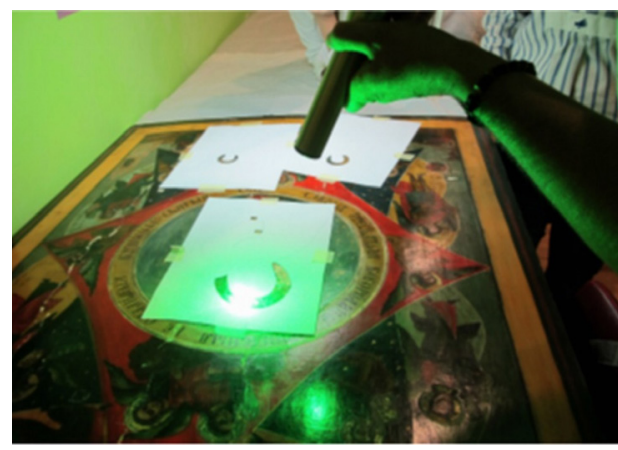

Fig. 15. Laser cleaning.

For this reason, energy of $200 \mathrm{~mJ} / \mathrm{cm}^{2}$ was used, increasing the number of pulses at 200 per point. But, the optimal result was not yet achieved.

However, in a new attempt of mechanical cleaning, it was noted that the bronze coating was easily removed from the surface which was previously irradiated with laser (Fig. 16). It was assumed that these are the surfaces where a delamination, i.e. weakening of linkage between the layers of coating and

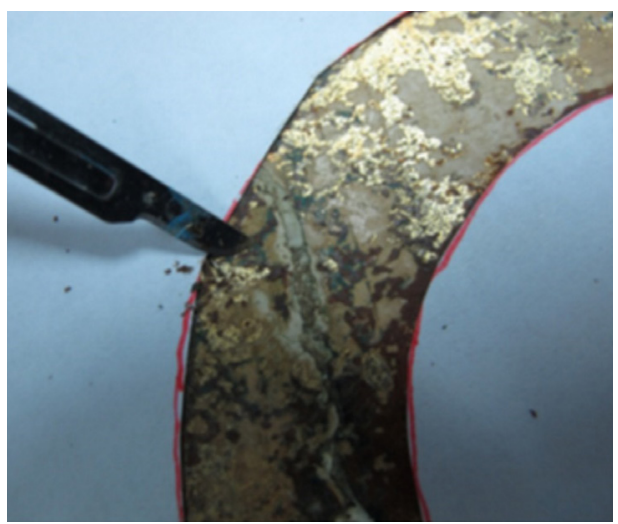

Fig. 16. Mechanical removal of coating after laser cleaning.

gold, occurred due to irradiation. Namely, the previous interferometric measurements have confirmed that ablation of material from the object's surface could induce great pressure on that surface, causing stress which induces the effect of delamination.

Laser irradiation was then applied on the remaining surface, with subsequent mechanical cleaning. Removal of bronze coating was fast and efficient, and the process of cleaning was safe for areas on which the gilding was solidly attached to the surface. Microscopic recordings confirm these facts (Fig. 17).

On the areas where the gilding was weakly attached to the surface, cleaning with scalpel could have led to the removal of gilding, so the answer was found in gel or, more precisely, jellified ethanol (Gellano was used for thickening) (Fig. 18). Tests of cleaning with this gel showed that following its application on the bronze coating a gradual, controlled softening was obtained and there was no penetration of solvent in layers underneath. Jellified ethanol was applied to the persistent coating and after a gradual softening the varnish was removed with a wet swab.

The icon after conservation is shown on Fig. 19. 

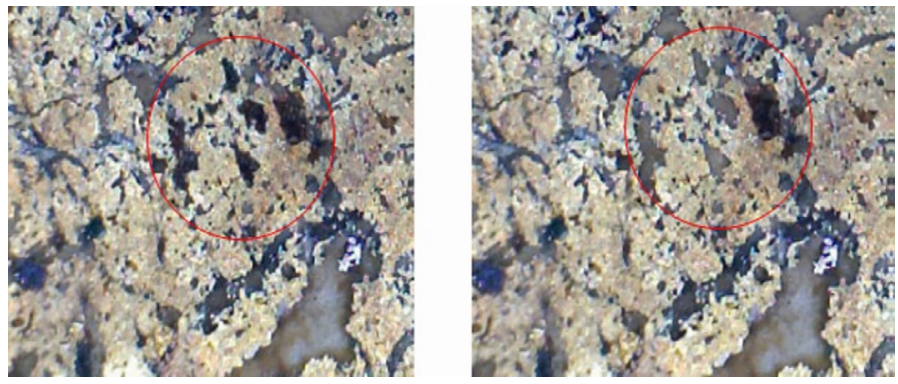

Fig. 17. Microscopy recording: before and after laser cleaning.

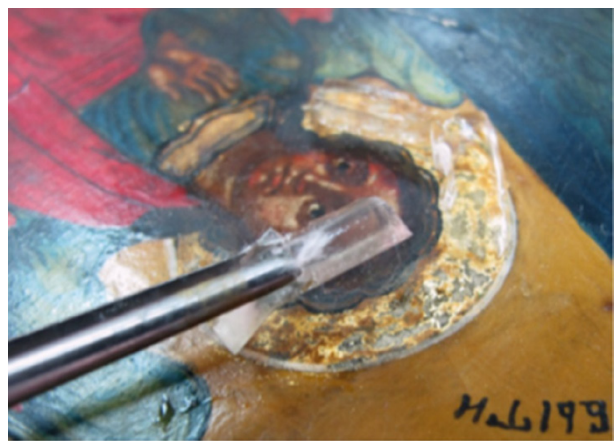

Fig. 18. Varnish removal with the jellified ethanol (Gellano).

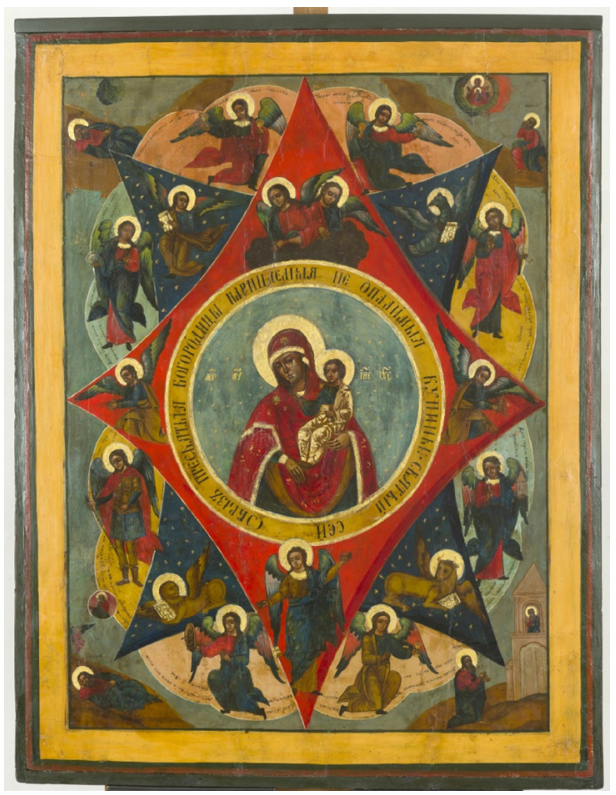

Fig. 19. Icon after the process of conservation.

\section{Conclusion}

\section{Portrait of Jelena Milojevic with her daughters}

Paintings are complex, multilayer systems, often very sensitive and difficult to clean. Laser cleaning/thinning of varnish proved to be an acceptable alternative. In the presented case, it was the only possible solution.

The results on the removal of degraded layer of varnish and deposits from the easel paintings suggest a potential practical usefulness of Nd:YAG laser's third harmonic (355 nm) in order to address such problems - it is possible to clean delicate substrates such as paintings or composite structures of polymeric layers (e.g. networks of polymerized resin or polymeric paints, respectively) when bearing in mind some fundamental criteria.

In certain demanding cases, in situ monitoring such as multispectral imaging or other diagnostic methods must be used for controlling the optimum cleaning depth.

\section{Holy Mother of God}

This new conservation treatment, combining the laser irradiation and the mechanical cleaning, brings the possibility of developing a new conservation methodology. The phenomenon of delamination of layers is used. 
However, in order to develop this new methodology, it is necessary to undertake a series of new experiments and examine the phenomenon of delamination in detail. There are several studies that have been carried out with the aim to study possible negative effects induced by laser irradiation on surfaces of artwork objects, for example separation between surfaces' layers. This new research goes in the other direction, in order to exploit this effect to improve and facilitate conservation in some specific cases.

\section{References}

[1] Cesare Brandi, Teoria del restauro, Enaudi, Torino 2000 pp 5-12.

[2] J.H. Scholten, J.M. Teule, V. Zafiropulos, R.M.A. Heeren; Controlled laser cleaning of painted artworks using accurate beam manipulation and on-line LIBS-detection, Journal of cultural heritage 1 (2000) S215-S220, DOI: 10.1016/S1296-2074(00)00142-4.

[3] S. Siano, J.Agresti, I. Cacciari, D. Ciofini, M. Mascalchi, I. Osticioli, A. A. Mencaglia "Laser cleaning in conservation of stone, metal and painted artifacts: state of the art and new insights on the use of the Nd:YAG lasers”, Appl. Phys. A 106 (2012) 419-446, DOI: 10.1007/s00339-011-6690-8.

[4] D. Ciofini, M. Oujja, M. Vega Cañamares, S. Siano, M. Castillejo, "Spectroscopic assessment of the UV laser removal of varnishes from painted surfaces", Microchemical Journal 124 (2016) 792-803, DOI: 10.1016/j.microc.2015.10.031.

[5] J. Hildenhagen, K. Dickma n; Nd:YAG laser with wavelengths from IR to UV $(\omega, 2 \omega, 3 \omega, 4 \omega)$ and corresponding applications in conservation of various artworks; Journal of Cultural Heritage, 4, Supplement 1, (2003) 174-178, DOI: 10.1016/S1296 -2074(02)01194-9.

[6] G. De Cesare, P. Iazurlo, P. Biocca, "La pulitura laser di vernici sintetiche su una tavolozza acrilico-vinilica: rimozione/resistenza e alterazioni", in Aplar 5 - Applicazione laser nel restauro, atti del convegno - Città del Vaticano, 18-19 settembre
2014 - an abstract available from: http://www.aplar. eu/pdfs/libro_abstracts_APLAR_5.pdf (accesed 8.07.2017).

[7] C.B. Carney Be Smart When Dealing With Art: Ultraviolet Light Inspection, 2014, http://www. gainsboroughproducts.com/uvl_inspection.html (accessed: 8.07.2017).

[8] S. Youn, Y. Kim, J. Lee, D. Har, "A study of infrared reflectography for underdrawing detection using a digital camera" in: M. Roccetti, Ed., Proceeding of the IASTED International Conference Internet and Multimedia Systems and Applications - March 17-19, 2008, Innsbruck, Austria ACTA Press Anaheim, CA, USA, 2008, pp. 128-134, http:// www.actapress.com/Abstract.aspx? paperId=32963 (accessed: 8.07.2018).

[9] M. Attas; E. Cloutis; C. Collins, D. Goltz, C. Majzels, J. R. Mansfield, H. H. Mantsch "Near-infrared spectroscopic imaging in art conservation: investigation of drawing constituents", Journal of Cultural Heritage 4 (2003) 127-136, DOI: 10.1016/ S1296-2074(03)00024-4.

[10] M. Chappé, J. Hildenhagen, K. Dickmann, M. Bredol, "Laser irradiation of medieval pigments at IR, VIS and UV wavelengths" Journal of Cultural Heritage 4 (2003) 264s-270s, DOI: 10.1016/S1296 -2074(02)01206-2.

[11] R. Salimbeni, "Laser techniques for conservation of artworks", Archeometriai Mühely (Archaeometry Workshop) 2006/1, p. 34-40, http://www. ace.hu/am/2006_1/AM-2006-1-RS.pdf (accessed: 25.06.2017).

[12] R. Teule, H. Scholten, O. F. van den Brink, R. M.A. Heeren, V. Zafiropulos, R. Hesterman, M. Castillejo, M. Martín, U. Ullenius, I. Larsson, F. Guerra-Librero, A. Silva, H. Gouveia, M. B. Albuquerque, "Controlled UV laser cleaning of painted artworks: a systematic effect study on egg tempera paint samples", Journal of Cultural Heritage 4 (2003) 209s-215s, DOI: $10.1016 /$ S1296-2074(02)01137-8.

[13] S. Georgiou, V. Zafiropulos, D. Anglos, C. Balas, V. Tornari, C. Fotakis, "Excimer laser restoration of painted artworks: procedures, mechanisms and effects", Applied Surface Science 127-129 (1998) 738-745, DOI: 10.1016/S0169-4332(97)00734-4. 
[14] J. Marczak, A. Koss. P. Targowski, M. Góra, M. Strzelec, A. Sarzyński, W. Skrzeczanowski, R. Ostrowski, A. Rycyk "Characterization of Laser Cleaning of Artworks”, Sensor (Basel) 8 (2008) 6507-654, DOI: 10.3390/s8106507.

[15] J.P. Teas, "Graphic analysis of resin solubility", Journal of Paint Technology, 40 (1968), 19-25.

[16] K. G. Watkins, Jong-Myoung Lee, C.L. Curran, "Underlying Mechanisms in Laser Techniques for Art Conservation: Two Improved Cleaning Methods", in: Laser Techniques and Systems in Art Conservation, Proc. SPIE 4402 (2001) 73, DOI: 10.1117/12.445647.

[17] M. Elias, N. Mas, P. Cotte, "Review of several optical non-destructive analyses of an easel painting. Complementarity and crosschecking of the results", Journal of Cultural Heritage 12 (2011) 335-345, DOI: 10.1016/j.culher.2011.05.006. 


\title{
White, yellow and green pigments on Polish artists' palettes in the period $1838-1938$
}

\author{
Mirosław Wachowiak $^{1 \star}$, Grzegorz Trykowski ${ }^{2}$, Iwona Żmuda-Trzebiatowska ${ }^{3}$ \\ 1 Department of Conservation and Restoration of Modern Art, Nicolaus Copernicus University, \\ Gagarina 11, 87-100 Toruń, Poland \\ 2 Instrumental Analysis Department, Faculty of Chemistry, Nicolaus Copernicus University, Gagarina \\ 11, 87-100 Toruń, Poland \\ 3 Photophysics Department, The Szewalski Institute, Polish Academy of Sciences, Fiszera 14, 80-231 \\ Gdańsk, Poland \\ * Corresponding author: miroszwach@wp.pl
}

\begin{abstract}
In the $19^{\text {th }}$ century, the evolution of white, yellow and other pigments was forced by numerous chemical discoveries. Dates of their inventions and patents, despite being well established in the literature, are not consistent with time of their implementation by painters. The survey of more than 300 hundred Polish artists' paintings from the period 1838-1938 confirmed this observation. The reported research allowed building chronological database of the first use, periods of intensive exploitation and of the decline of use or absence of specific pigments in the Polish artists' studio practice.

Portable XRF examination, Raman spectroscopy and SEM-EDX analysis enabled establishing dates of first applications of zinc white, chrome yellow, cadmium yellow, strontium yellow, zinc yellow, emerald green and viridian on paintings. Moreover, the research showed modifications of Naples yellow by zinc- or tin-based admixtures in the second half of the $19^{\text {th }}$ century as well as evolution of additives to basic lead white such as earth pigments, chalk, barites, zinc white or lithopone. The accuracy of dating and authentication of the artworks analysed was strengthened by examination of composition of their grounds. In some cases individual habits of artists of using special kinds of primings or characteristic pigments were revealed giving a support for authentication studies. The data gathered in this research proved to be a reliable basis for attribution and dating of paintings of uncertain origin.
\end{abstract}

Keywords: $19^{\text {th }}$ century pigments, Raman spectrometry, authentication, painting technology, non-invasive analyses, XRF portable spectrometry, J. Matejko, J. Pankiewicz

\section{Introduction}

As in the $19^{\text {th }}$ century numerous new pigments were introduced onto artists' palettes, their identification provides significant information on the artists' studio practice. Sufficient number of investigated paintings enables statistical conclusions based on pigments' identification, supporting dating authentication and in some cases, attribution of paintings. To a noticeable degree, it is possible also for the works from the first half on the $20^{\text {th }}$ century when subsequent synthetic pigments were introduced [1]. 
The preliminary data on non-organic pigments in a non-invasive way was collected by XRF examinations with portable instruments $[2,3]$. When sampling was possible, Raman spectroscopy, SEM-EDX and in some cases $\mathrm{XRD}$ were additionally used.

The number of objects examined exceeds three hundred items. Paintings originated from collections of the District Museum in Toruń, National Museum in Poznań, National Museum in Kraków and National Museum in Warsaw. There were also a few works from a private collection and the auction market; however, the core of the studied paintings were museum objects dated with certainty. The analysed period 1838-1938 starts with the first identified presence of a significant amount of barites and comes to an end a century later, time of preliminary, but still rare introduction of titanium white.

Whites were chosen as representative pigments because they were used on every painting and their chemical composition evolved significantly during $19^{\text {th }}$ and $20^{\text {th }}$ centuries. It is also easy to identify the pigments with the use of simple XRF examination. Moreover, white pigments are also present in grounds, which were investigated as well. The latter can give more systematic chronological data, especially in case of factory-made priming's offered on the market, as they were less susceptible to subjective artists' choices $[4,5]$. When both pigments and primings' fillers are identified, the chronological results are significantly more reliable than just pigments' examination considered alone [6].

The other group of pigments - yellows - is also present on nearly every painting and some significant continuous changes of chemical composition, taking place over the course of both centuries, are to be observed.
While investigating yellows it is also necessary to consider greens, to which they were commonly added.

The aim of the conducted research was to present a panorama of evolution of chemical composition and the use of white, yellow and green pigments from the $19^{\text {th }}$ to $20^{\text {th }}$ centuries, to display possible changes in time within certain kinds of pigments, trace some individual artists' habits and check to what extent the non-invasive portable XRF analysis is sufficient for such undertaking. The number of non-invasive measurements possible to be conducted, regardless of some limits of the technique, enables collecting statistical results impossible to gain with destructive analyses that need sampling. Importantly enough, the technique is convenient and relatively simple to use also on site. On the other hand, especially when building a chronological database, it is necessary to include methods like Raman spectroscopy. For stratigraphy recognition of the cross-section of sample BSE imaging and SEM-EDX examinations may be feasible.

\section{Materials and methods}

Further research was preceded by the observation of the UV excited fluorescence of the painting in order to avoid testing of non-original parts. The research methodology was to start with the identification of elemental composition of different areas of the paintings related to different colours with the portable XRF spectrometer. When sampling was possible, complementary Raman spectroscopy and SEM-EDX analysis was used for the identification of pigments ambiguous to detect, basing on the XRF examination only. 


\subsection{XRF}

The portable XRF spectrometer used in this study was the Genius 7000 from Skyray Instruments, equipped with the Ag lamp as a source of radiation, working at $40 \mathrm{kV} / 100$ $\mu \mathrm{A}$ conditions and a SDD detector. The instrument allows for detection of elements from magnesium to uranium. The time of a single measurement was $120 \mathrm{~s}$. The instrument was factory-optimised for pigments identification. The optimisation was based on the preceding analyses of historical-like model samples of paints of known composition.

In order to get data as reliable as possible, the measurements were repeated over a given area to avoid false identification from local contaminations. Since $\mathrm{X}$ rays penetrate the painting deeply enough to get fluorescence signal not only from the paint layer but from the priming as well, identification of pigments in the paint layer was preceded by the examination of priming itself. The latter was performed on tacking margins or from the back side of the paintings. In the case of pigments present in mixtures and/or in many layers the reliability of results was increased by collecting data along linear path over the area of the same hue but with gradually changed saturation and tonality. The reasonably stable ratio of intensities of lines of key elements indicated the use of factory-made paint only mixed with different amount of white to obtain desired saturation and tonality. As an example of such a procedure, the distinguishing between a viridian and a green paint made of chrome yellow mixed by artist with blues may be given. In the case of doubts additional examination with Raman spectroscopy [7] and SEM-EDX was used.

\subsection{Raman spectroscopy}

The Raman spectra were acquired by means of the InVia $\mu$-Raman spectrometer from Renishaw Group equipped with two lasers providing excitations at 785 and 514 $\mathrm{nm}$. The confocal microscope with objectives of magnification of 20,50 and $100 \times$ assured the spectral footprint of the sample surface area down to about $5 \times 5 \mu \mathrm{m}^{2}$. For dispersion of the backscattered signal, the grating of 1200 groves $/ \mathrm{mm}$ was used and detection was provided by a CCD array detector.

The spectrometer calibration was performed with the use of the polystyrene standard. Spectra were acquired over the range of $100-3200 \mathrm{~cm}^{-1}$ at resolution of $2 \mathrm{~cm}^{-1}$. Depending on the signal intensity up to 10 spectra of individual samples were accumulated and averaged. All spectra were processed and baseline corrected using the OriginLab $^{\odot}$ package. Pigments and paint components were identified basing on the reference spectra from digital Raman libraries [8-10]. It enabled not only the identification of pigments which were hardly distinguishable by XRF analysis, such as Scheele's green and emerald green or other chrome based pigments, but it also supported revealing the composition of mixtures of more than one colour [11].

\subsection{SEM-EDX}

Sample's cross-sections were observed with Scanning Electron Microscopy (SEM) technique using Scattered Electrons (SE) and Back SE imaging and examined with the Energy Dispersive X-ray (SEM-EDX) detector enabling elemental mapping. The instrument used was the SEM-LEO 1430VP microscope from Zeiss operated with BSE detector. The 
energy dispersive X-ray spectrometer (EDX) used in this research was Quantax 200 with XFlash 4010 from Bruker working at $28 \mathrm{kV}$ in variable pressure up to $50 \mathrm{~Pa}$.

\subsection{Objects}

Artists examined include the following names: Piotr Michałowski (1800-1855), Franciszek Kostrzewski (1826-1911), Józef Szementowski (1833-1876), Wojciech Gerson (1831-1901), Władysław Malecki (1836-1900), Jan Matejko (1838-1893), Witold Pruszkowski (1846-1896), Józef Chełmoński (1849-1914), Leon Wyczółkowski (1852-1936), Jacek Malczewski (1854-1929), Stanisław Lentz (1861-1920), Józef Pankiewicz (1866-1940), Stanisław Wyspiański (1869-1907), Józef Mehoffer (1869-1946), Wojciech Weiss (1875-1950), Fryderyk Pautsch (1877-1950), Władysław Jarocki (1879-1965), Stanisław Ignacy Witkiewicz (1885-1939), Vlastimil Hoffman (1881-1970) and Ludomir Ślendziński (1889-1980). Objects chosen were only of certain origin and dating mostly from museums, some also from private collection, considering representation of each year between the period 1838-1938. Number of examined objects exceeded 300 , enabled to draw some statistical conclusions. In the case of Jan Matejko and Józef Pankiewicz, when number of investigated paintings exceeded 60 , revealing the evolution of the palettes within certain periods of their artistic lives was possible $[12,13]$.

\section{Results}

\subsection{Whites}

Main white of the $19^{\text {th }}$ century is the lead white, pigment popular since antiquity. Yet as early as the end of the $18^{\text {th }}$ century, some alternatives were introduced - one of them was barium sulphate suggested for use as pigment in 1782. It could originate from the natural ores or could be synthesized and then was sold under the name blanc fixe. Its more significant production did not start before 1810-1820, yet already in 1825 Leuch mentioned the adulteration of lead white by admixtures of barite and Kulmans manufacture started its production in 1830 [14]. According to the presented research (Fig. 1), the earliest Polish paintings where admixtures of barium sulphate to the lead white were detected are dated 1838. Zinc white was found as admixture to the lead white in painting from the following year, even though it had been suggested as the lead white alternative as early as at the end of the $18^{\text {th }}$ century. The amount of zinc white admixed to the lead white raises with time especially from the year 1855 on, yet it is as late as 1883 when the pure zinc white with no addition of lead white is detected on Polish painting. In the sixties, a complex mixtures of lead, zinc and barium white are found on the paintings. In all the cases, significant shifts in time were observed in comparison to the dates of commercial introduction suggested by literature and the ones confirmed during research. The biggest "gap" for the pure zinc white exceeds half of the century.

The research (Fig. 1a) brought not only information on the real points in time of the first appearances of pigments significantly differing from the ones found in literature, but it also provided details on their abundance. Lead white is present throughout all the century. Barium sulphate identified since 1838 as admixture to lead white is slightly less common but invariably popular through the century. In the fifties, the zinc white is added to the lead white quite often but only 

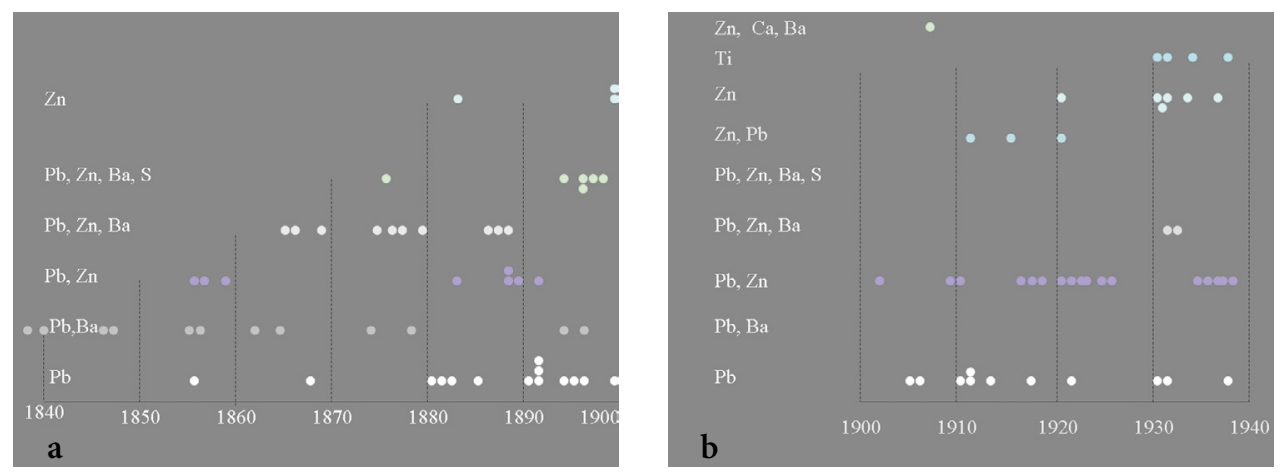

Fig. 1. Whites detected in paintings from a: $1838-1900$ period; b: $1901-1938$ period: lead white $(\mathrm{Pb})$, lead white with barium white $(\mathrm{Pb}, \mathrm{Ba})$, lead white with zinc white $(\mathrm{Pb}, \mathrm{Zn})$, zinc white with addition of lead white $(\mathrm{Zn}, \mathrm{Pb})$, pure zinc white $(\mathrm{Zn})$, titanium white mixtures $(\mathrm{Ti})$, mixture of lead and zinc and barium white $(\mathrm{Pb}$, $\mathrm{Zn}, \mathrm{Ba})$, zinc white admixed with chalk and barites ( $\mathrm{Zn}, \mathrm{Ca}, \mathrm{Ba})$, lithopone ( $\mathrm{Pb}, \mathrm{Zn}, \mathrm{Ba}, \mathrm{S})$, lead white admixed with chalk and barium white $(\mathrm{Pb}, \mathrm{Ca}, \mathrm{Ba})$.

for a short period, and seems to return again in this mixture some thirty years later. In the meantime, the three ingredient basing mixtures - lead, zinc and barium white - become popular between half of the sixties and half of the eighties, when it starts to decline to be used at all. As late as the beginning of the nineties, lithopone is added to the lead white.

As it is seen from Fig. 1b, titanium white was not used as pure pigment at least until the end of the Second World War. According to the written sources, the introduction of titanium white onto artists' palettes in a wider scale could take place in Europe about 1922 when so called Blumenfeld method was used for pigment production [15]. The present research revealed that in Poland it is present not earlier than in 1930, when titanium white is detected as minor admixtures to the zinc white in the Vilnius school compositions (with the zinc white as the main component and titanium white as relatively little admixture between 2-5\%) and in the case of other Polish painters even later - in the early thirties, as admixture to lead white and bar- ites. Another ingredient identified as added to the titanium white in a quite significant level - even in areas of pure white colour - is iron. It is possible, that the source - ilmenite - was not cleaned properly at the early stage of production [7]. In the case of Kazimierz Kwiatkowski, white paint being a mixture of zinc white and additions of titanium white is used on the same single painting (Portrait of Herta Dzikowska, from 1930) in parallel with the more traditional mixture of zinc white and lead white (Figs. 2-3).

In the painting of Ludomir Ślendziński from the next year, this zinc white (majority) and titanium white (minority) mixture is used as the only white. The example of painting of Edward Karniej from 1937 shows a mixture of zinc, lead white, chalk and titanium white. In the case of the Still life (1938, Silesian Museum in Katowice) by Józef Pankiewicz, white is the mixture that contains lead white, barites and titanium white. It is the first painting in artist's oeuvre in which the titanium white was detected. It seems that closer and more statistical recognition in the meaning of the number of paintings 


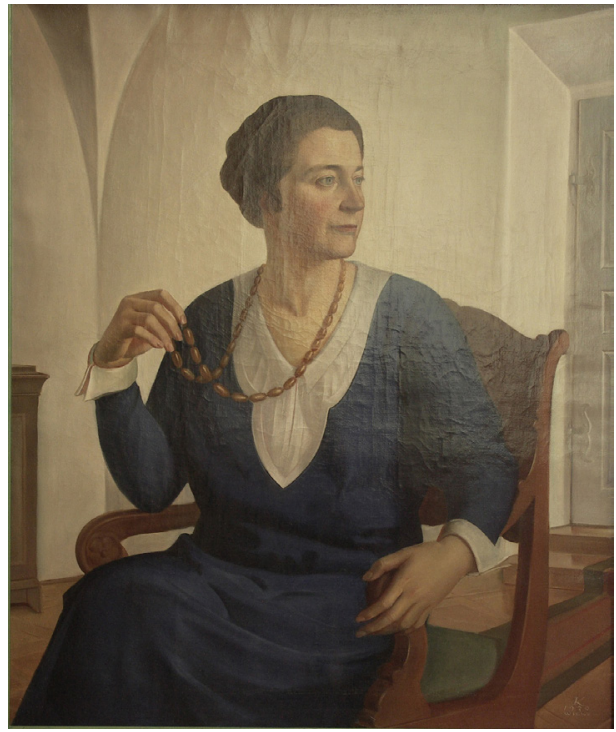

Fig. 2. Kazimierz Kwiatkowski, Portrait of Herta Dzikowska, 1930, District Museum in Torun. Both, traditional lead white and zinc white with titanium white admixed are used on the painting. Photo by M. Wachowiak. the Louis Nicholas Vauquelin researching crocoite, mineral found in Siberia [16]. The most important chromium containing yellow pigment - lead chromate - was identified on Polish paintings from the year 1836, nearly two decades after the first publication. Zinc chromate was detected on the painting from 1869 and for strontium chromate - on the one from 1874. As barium chromate was much more problematic in certain distinction from the barite or blanc fixe admixtures, no exact date was proposed for the moment of implementation by artists, yet it seems that in the 1880 s the pigment is already present on their palettes. Chromium yellows are common through all the second half of the $19^{\text {th }}$ century (Fig $4 a$ ).

Cadmium yellow was for the first time detected on the painting from 1861. Since its significance raises, in the seventies it is already common in use and in the eighties

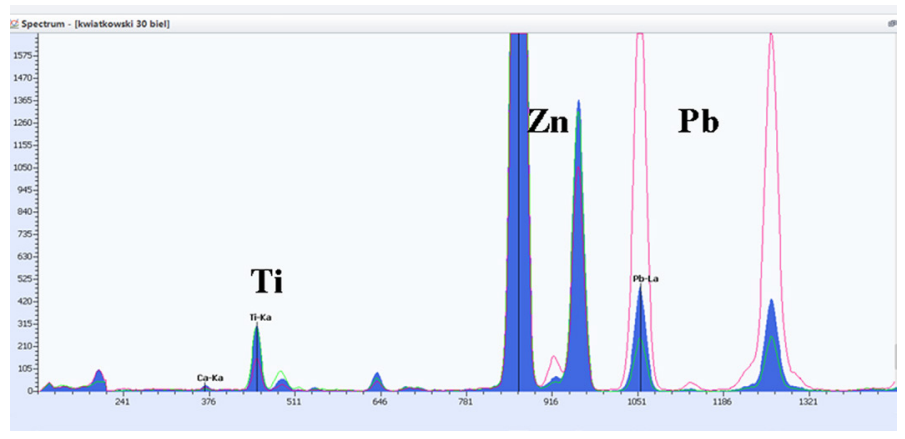

Fig. 3. XRF spectra of two whites: traditional lead white with zinc white (red line spectrum) and zinc white with admixture of titanium white (measured in two points: blue-filled spectrum and green line spectrum), detected on the painting by K. Kwiatkowski, Portrait of Herta Dzikowska, 1930.

examined can give further chronological, and maybe to some extent geographical, distinctions basing on ratios of the titanium and other whites included in complex white compositions of the 1930s and 1940s.

\subsection{Yellows}

A big group of yellows containing chromium were proposed as pigment in 1808-1809 by dominant. Cadmium yellow with significant addition of zinc is described in literature as popular one [17]. Basing only on non-invasive analyses, it is hard to say whether only metal zinc or its oxides or sulfides were added as well. Probably both cases took place. Interestingly enough, from the end of the $19^{\text {th }}$ century significant amount of potassium and aluminium appears as additional elements to cadmium (Figs. 5-6). 

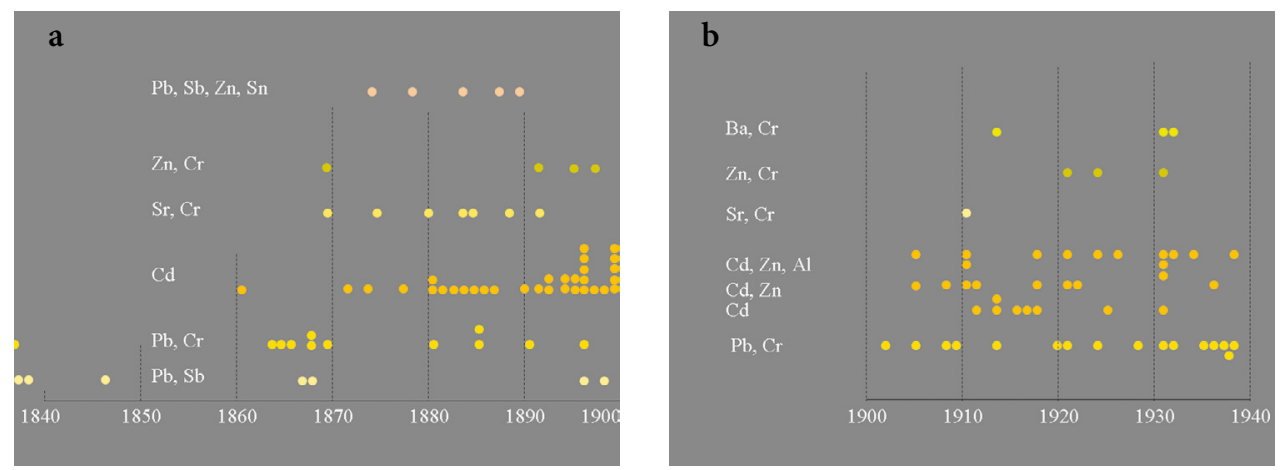

Fig. 4. Yellows detected in paintings from a: $1838-1900$ period; b: $1901-1938$ period: Naples yellow $(\mathrm{Pb}, \mathrm{Sb})$, modified Naples yellow ( $\mathrm{Pb}, \mathrm{Sb}, \mathrm{Zn}, \mathrm{Sn}$ ), chromium yellow ( $\mathrm{Pb}, \mathrm{Cr}$ ), zinc chromate yellow ( $\mathrm{Zn}, \mathrm{Cr}$ ), barium chromate yellow $(\mathrm{Ba}, \mathrm{Cr}$ ), strontium yellow $(\mathrm{Sr}, \mathrm{Cr})$, cadmium yellow $(\mathrm{Cd})$, cadmium yellow modified with $\mathrm{ZnO}$ or $\mathrm{ZnS}$ additions (Cd, $\mathrm{Zn})$, cadmium yellow containing zinc and aluminum based admixtures ( $\mathrm{Cd}, \mathrm{Zn}, \mathrm{Al})$.

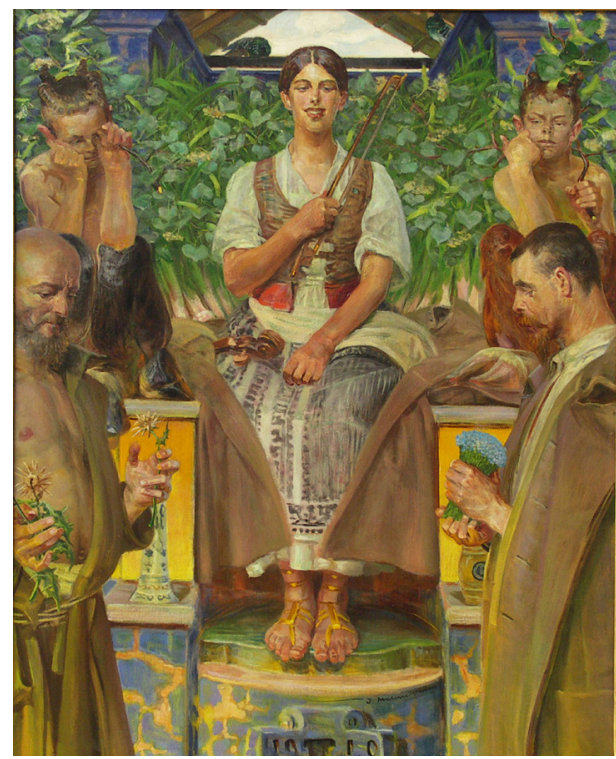

Fig. 5. Jacek Malczewski, For the Art and the Muse, 1910, District Museum in Toruń, Photo by M. Wachowiak.

However, there is a question concerning the source of the mentioned elements. On one painting, at least two kinds of cadmium yellow can be found, and these elements as well as amount of zinc in ratio to cadmium

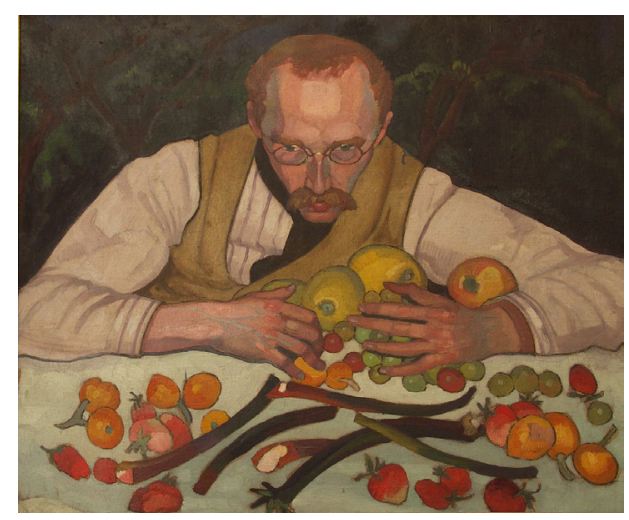

Fig. 6. Stanisław I. Witkiewicz, Portrait of dr Ignacy Wasserberg, about 1905-10, District Museum Toruń, Photo by M. Wachowiak.

added can support differentiation of cadmium yellows, as in the case of Malczewski's painting from 1910, where there is light lemon kind and intensive deep "egg-yolklike colour" cadmium yellow (Fig. 7a). In the latter pigment amount of potassium and aluminium is significant (Fig. 7b). Aluminium as admixture to the cadmium yellow appears at the end of the $19^{\text {th }}$ century and its ratio to the rest elements is growing with time. 

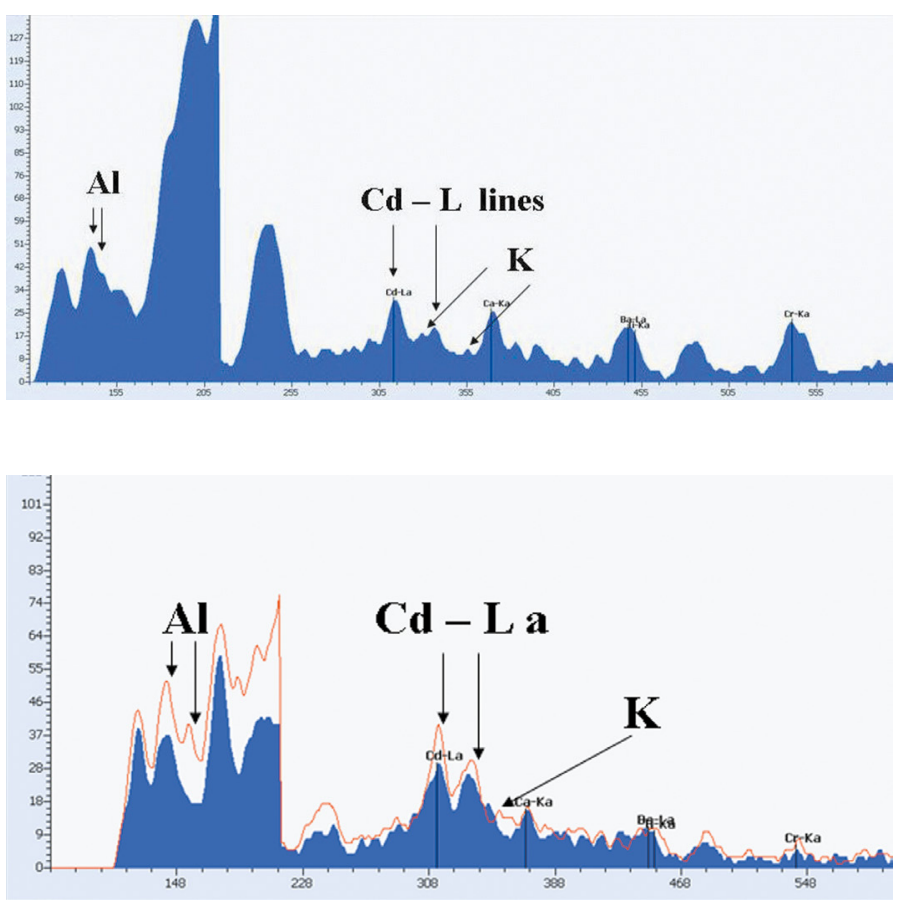

Fig. 7a. Fragment of XRF spectrum of cadmium yellow identified on Portrait of dr Ignacy Wasserberg by Stanisław I. Witkiewicz, containing additionally aluminium and potassium, and average amount of zinc as addition to the cadmium sulphide.

Fig. 7b. Fragment of XRF spectrum of cadmium yellow identified on painting For the Art and the Muse by Jacek Malczewski. Two kinds of cadmium yellows - light and dark - identified, one with majority of $\mathrm{Zn}$ and $\mathrm{Cd}$ (blue spectrum), the other with significant amounts of $\mathrm{Al}$ and $\mathrm{K}$, and minor amount of $\mathrm{Zn}$ (red line spectrum).
Whether this is alum - aluminium potassium sulphate or some other substance is still to be examined.

Another group of yellows are the factory-made mixtures of chromates and cadmium sulfides both of cadmium and chromium yellow and mixture of cadmium and strontium yellow, which are becoming common in the eighties of the $19^{\text {th }}$ century. Traditional Naples yellow, lead antimonite, is common in the first half of the $19^{\text {th }}$ century [18]. In the second half of the century, it is rather rare in traditional form, yet from the seventies on, there appear modified kinds containing zinc, tin and sometimes other compounds like aluminum, potassium, iron, copper and calcium in minor amounts [19]. According to different methods of production, they created various complex oxalate compounds in different ratios. In the case of Matejko, who was using them in extremely great amount, individual kinds are traceable on the painting when applied in pure impastos, basing on ratios of main elements and additionally recognized by characteristic ones represented in lesser amounts [20].

Seen as a whole, yellows are represented by chromium yellow and Naples yellow nearly throughout the whole $19^{\text {th }}$ century. In the sixties, there appear cadmium yellow which becomes popular in the seventies, and in the eighties its popularity is still raising. Zinc and strontium yellow occur much later than the date of their discovery. From the seventies on, modified Naples modern yellow is identified on paintings (Fig. 4a).

In the $20^{\text {th }}$ century, cadmium yellow is still common, there are some individual examples of the use of zinc yellow and in the case of cadmium yellow the kind containing aluminium is dominating (Fig. 7b). 


\subsection{Greens}

In the case of greens, there are a lot of difficulties in the accurate distinguishing of individual kinds as the main element chromium - is present in viridian, chromium oxide and greens from the group of variety of compositions based on mixtures of various yellows and blues.

For this reason, the XRF results are not conclusive. Further research was conducted using Raman spectroscopy to indentify exact greens (Figs. 8a, b).

Only in one case the chromium oxide green was identified with certainty. Yet it is possible that it was present on bigger number of paintings. Other chromium based green is viridian and comparable in frequency of occurrence - commercially available mixtures of yellow and blue greens. In their case, these with addition of cadmium yellow have appeared since the eighties of the $19^{\text {th }}$ century.

The copper based greens are less problematic in identification as most often it is the emerald green and the presence of arsenic that is distinctive. As the Scheeles green is

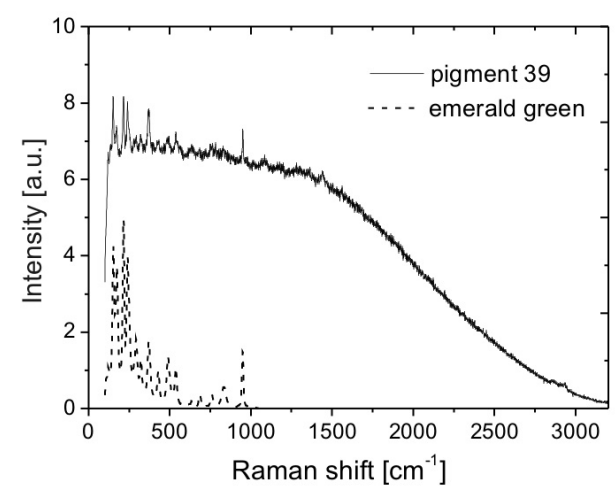

Fig. 8a. Raman spectrum of emerald green of Jan Matejko paint - paint tube sample from the collection of artist's materials from National Museum in Kraków. rather dull and declining in use in the second half of the century [21], there were hardly any examples from the first half of the $19^{\text {th }}$ century, so it could be concluded that most probable in all the cases was the emerald green, presence of which was confirmed with the Raman spectroscopy. Copper based green containing no arsenic is most probably the green verditer - synthetic version of malachite, suggested by comparisons of Raman spectra from available databases. Cobalt green is rare and was detected for the first time on the painting from 1883 .

Emerald green became popular in the seventies even though some early exceptional use was confirmed in 1845. Also in the case of viridian there was an exceptional isolated example of early use in 1839 and two later waves of popularity: first one in the early sixties and the second since the seventies, when it became to be common in use (Fig. 9a).

In the $20^{\text {th }}$ century, emerald green is less common. Although it continuously appears, it gives its place to viridian. There are hardly any copper based greens apart from copper/ arsenic emerald green and the cobalt green remains rare (Fig 9b).

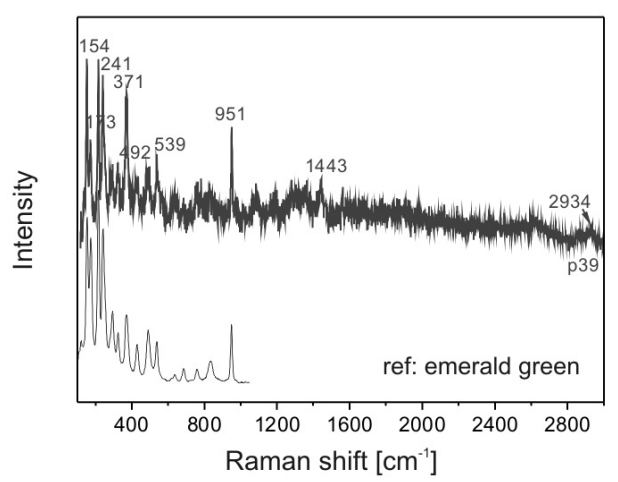

Fig. 8b. Raman spectrum of viridian from the J. Matejko paint - paint tube sample from the collection of artist's materials from National Museum in Kraków. 

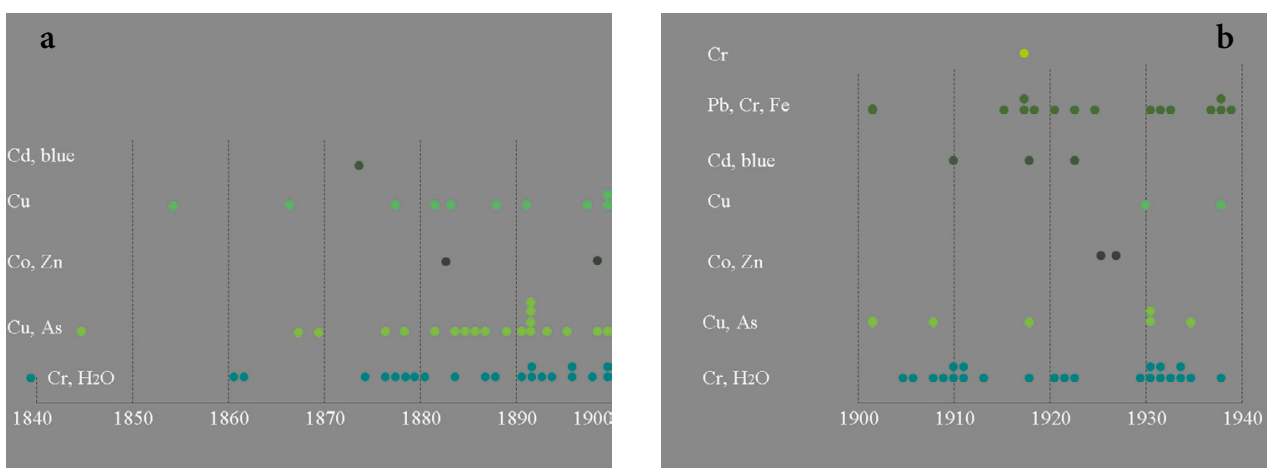

Fig. 9 Greens detected in paintings from a: $1838-1900$ period; b: $1901-1938$ period: viridian $\left(\mathrm{Cr}, \mathrm{H}_{2} \mathrm{O}\right)$, chromium oxide green $(\mathrm{Cr})$, copper based synthetic green (green verditer) $(\mathrm{Cu})$, emerald green $(\mathrm{Cu}, \mathrm{As})$, cobalt green $(\mathrm{Co}, \mathrm{Zn})$, composite green based on chrome yellow and Prussian blue $(\mathrm{Pb}, \mathrm{Cr}, \mathrm{Fe})$, composite yellow containing cadmium yellow and various kinds of blue (Cd, blue).

\subsection{Primings}

Whites being extenders in the grounds often occur in similar dates to the ones used in paint layers, yet in some cases they are introduced later. Until about half of the $19^{\text {th }}$ century most common are two-layered primings with iron based earth pigments in the bottom layers and lead white as the top one. Since approximately 1855 , when the significant additions of zinc white are identified in paints, they are also to be found in primings. Barites are found much later than in paints, as they have been detected in grounds since 1874. Lithopone is found in grounds in analogical time as in paints - in the late nineties. There are only two pure zinc white based grounds found till this moment in the $19^{\text {th }}$ century and these are artist self-applied primings by Podkowiński in 1894. Interestingly enough, after some time of diminished amounts of lead white detected only in complex mixtures, it returns as relatively pure individual main ingredient of primings in the nineties [22].

In the $20^{\text {th }}$ century, lead white is still quite common; however, more popular is its mix- ture with a significant amount of zinc white [23]. Another mixture that has appeared since about 1912 is the zinc white in majority with a slight addition of lead white. White fillers of the $20^{\text {th }}$ century primings when compared to pigments identified in paint layers show even greater popularity of zinc white with tiny admixture of lead. Pure zinc white grounds become popular similarly to the pigments in the thirties. No titanium is found in the priming in the period. Another issues are self-made and applied by artists' primings containing mostly chalk.

\subsection{Artists}

\section{Jan Matejko}

As individual painters' works have been investigated in a significant number, some further conclusions can be drawn. More than 60 paintings of Jan Matejko from the period $1852-1893$ have been analysed. From the very beginning, lead white and chromium yellow are present as well as cobalt blue. Soon after emerald green is introduced, other copper green broadens the palette in 1864. In 1869, four different pigments are implemented: 
cadmium yellow, zinc chromate yellow, viridian and zinc white - the latter exceptionally used as the lead white is the main white in all other years (Fig. 10). It seems that in 1869 is an extremely rich variety of pigments used. There are three kinds of chromate yellows neighboured with the cadmium yellow, emerald green and viridian, ultramarine

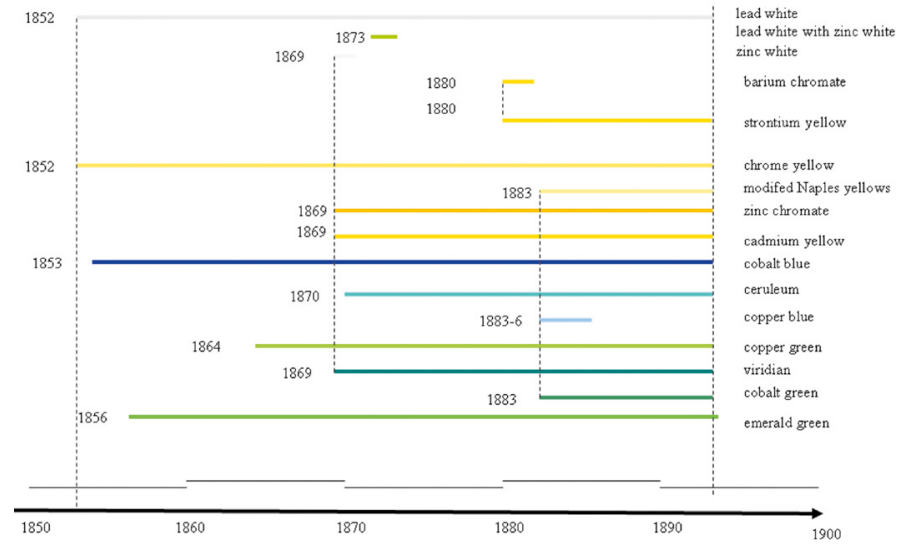

Fig. 10. Timelines of use of various pigment by Jan Matejko. Turning points of the same year of introduction of more than one pigment probably indicate moments of significant purchases of bigger number of painting materials. a big purchase of pigments was done. Next year, cerulean blue is found. Only for a short period, copper blue is used - just between the years 1883-1886. Additionally, in 1883, modified Naples yellow and cobalt green are introduced. Another result supportive in attribution, is the extremely wide use by Matejko of zinc and tin-modified modern Naples yellows, as well as of commercially available factory-prepared flash tone mixtures. Next characteristic detail of Matejko's technique is hardly any use of vermillion. A great variety of iron based reds is used instead of this red and the van Dyck brown in the underpainting.

\section{Józef Pankiewicz}

Pankiewicz is an artist who totally changed his style a few times, so it is really interesting to follow his stylistic changes and examine whether they were accompanied by the material ones. In the realistic period of his work, as in the painting The vegetable market (1888) from the National Museum in Poznań, there and cobalt blue as well as tiny amounts of cerulean and at least two different kinds of organic reds apart from the vermillion. Main white is the lead white; however, he could use zinc white as well.

In his impressionistic period 1890-1892, the palette is slightly narrowed, and the main white for the impastos rich landscape compositions is the lead white. There are less chromate yellows, cadmium yellow starts to dominate instead. Emerald green and viridian are widely used, accompanied by cobalt blue. There are hardly any black or earth iron based pigments. Priming is self-made, probably of gypsum.

The year 1983, in which Pankiewicz turns to symbolism, brings a lot of dark tones in his palette, which were absent in the impressionistic period. As the paintings are blurred and softly toned, more transparent zinc white surpasses lead white and there are hardly any blues on the palette.

Another turning into postimpressionistic period in 1907-1908 brings the rich color 
palette back. One of the new pigments introduced since 1911 is cobalt arsenate violet. Since 1911-1912 complex mixtures of chromate yellow containing greens have excluded emerald green and viridian. These greens return in the so-called Spanish period in the years 1915-1919, together with widely used cobalt arsenate violets, organic red precipitated on tin compounds, and since 1918 cadmium red has been implemented as well.

In the twenties of the $20^{\text {th }}$ century, after his return to Paris, Pankiewicz introduces cobalt greens apart from still used cadmium red. The late twenties and thirties is the mature period of the return of classical rules and a wide use of ochre's and umbers. In 1934, synthetic intensive rouge is found for the first time, and in 1938 Pankiewicz implements white admixed with titanium dioxide.

\section{Other artists}

In the seventies of the $19^{\text {th }}$ century, Józef Chełmoński used mainly a mixture of zinc white and lead white as a white colour and in the nineties - a mixture of zinc white, lead white and barium white, showing characteristic, distinguishable stable ratio of elements to each other. Additionally, these paintings from the nineties were executed on the same kind two layered priming containing lithopone.

Some rarely used pigments can be characteristic for individual painters as synthetic cobalt violets - arsenates and phosphates for Jacek Malczewski. Since 1894 he had been using cobalt arsenate in The Melancholy and a year later in The Circle (both from National Museum in Poznań). Other Polish artists started to use them about a decade later. Soon after, Malczewski introduced also manganese violet [24-26].

\section{Conclusions}

The study confirmed that written sources describing patents or dates of first examples of mass production are not consistent with the dates of real implementation onto paintings. In many cases a shift in time proves to be significant. Statistical overview enabled to draw a chronological panorama of the dates of the first appearance of exact pigments but also their raise in popularity of use and the decline or absence on some artists' palettes. There were also some examples of evolution of individual pigment as the case of cadmium yellow and especially of often complex compositions of whites. The latter, when identification is combined with the information on composition of primings, can be a supportive tool for dating, authentication and attribution (as in the case of Chełmoński works dated at the nineties of the $19^{\text {th }}$ century). Some individual characteristic choices can be traced as well. The core of the project was also the confirmation of reliability and accuracy of the portable XRF spectrometry when used for examination of numerous places of composition and related to the statistically significant number of investigated paintings of certain origin. Non-invasive recognition of paint layer pigment and primings fillers proved to be powerful, useful, effective, relatively simple, and cheap method which should be implemented into the museum's research kit and auction market practice in the preliminary examination of the painted works of art. The method is effective for dating in the periods of various introductions of new pigments. For that reason, the examination of the works from the $19^{\text {th }}$ and the first half of the $20^{\text {th }}$ century proves to be reasonable.

In many cases, it was the Raman spectroscopy resulting with conclusive identification 
and SEM-EDX to obtain the information on stratigraphy, important for proper interpretation of the XRF based results.

It is still a matter of question and a need for further research to situate Polish artists palettes' choice on a wider background of European art. From the sources and preserved paint tubes, it is known that most of painting material originated from abroad: Germany, Austria, France, England and Holland. When comparing to deeply analysed painters like impressionists or Van Gogh it seems that there was no significant delay in the use of newly introduced pigments, especially in the second half of the $19^{\text {th }}$ century.

Cerulean blue found in 1869 confirms a very early use of the pigment, as it is in the case of widely exploited cadmium yellows since the seventies of the $19^{\text {th }}$ century. More detailed comparisons and conclusions have to wait for further research on greater number both of western and central Europe artists' paintings from the $19^{\text {th }}$ and the first half of the $20^{\text {th }}$ century.

\section{Acknowledgements}

The authors would like to express their profound gratitude to the National Centre for Science, Poland, for approving founding for the project 2012/05/D/ HS2/03385, New Pigments of the $19^{\text {th }}$ century.

Special thanks to Anna Kroplewska from the District Museum in Torun for the possibility of the broadened research on the $20^{\text {th }}$ century paintings.

\section{References}

[1] M. Wachowiak, "New synthetic pigments of the $19^{\text {th }}$ and $20^{\text {th }}$ century", in: D. Markowski, S.Kamiński, M. Wachowiak (Eds.), Issues of Conservation and Restoration of Modern and Contemporary Art, Wydawnictwo Naukowe UMK, Toruń 2010, pp. 65-128.
[2] Ch.W. Mc Glinchey, "Handheld XRF for the examination of paintings: proper use and limitations", in: A.N. Shugar, J.L. Mass (Ed.), Handheld XRF for Art. Archaeology, Leuven University Press, 2013.

[3] M. Wachowiak, M. Sawczak, „Nieinwazyjna metoda identyfikacji pigmentów in situ - badania przenośnym spektroskopem XRF obrazów olejnych Józefa Pankiewicza", AUNC Zabytkoznawstwo i Konserwatorstwo, 39 (2010), pp. 15-37, http://apcz. umk.pl/czasopisma/index.php/AUNC_ZiK/article/ download/AUNC_ZiK.2010.007/2017 (accessed 10.07.2017)

[4] B. Haaf, „Industriell vorgrundierte Mallienen: Beitrage zur Entwicklungs-, Handels- und Materialgeschichte", Zeitschrift für Kunsttechnologie und Konservierung, 1/1987 Heft 2.

[5] M. Witlox, L. Carlyle, "A perfect ground is the very soul of the art': ground recipes for oil painting, 1600-1900", in: I. Verger (Ed.), ICOM-CC $14^{\text {th }}$ Triennial Meeting Preprints, London, James \&James, 2011, pp. 519-528.

[6] Ester S.B. Ferreira, R. Morrison, J. Boon, "Imaging chemical characterization of preparatory layers in fifteenth and sixteenth-century paintings", in: J.H. Townsend, T. Doherty, G. Heydenreich, J. Ridge (Eds.), Preparation for Painting. The artist's choice and its consequences, Archetype London 2008, pp. 52-58, ISBN: 9781904982326.

[7] F. Rosi, C. Miliani, I. Borgia, B. Brunetti and A. Sgamellotti, "Identification of nineteenth century blue and green pigments by in situ X-ray fluorescence and micro-Raman spectroscopy", Journal of Raman Spectroscopy, (2004) 35 610-615, DOI: 10.1002/ jrs. 1180 .

[8] Database of spectra of minerals, Department of Geosciences University of Arizona, http://rruff.info

[9] Infrared \& Raman Sers Group; http://www.irug. org

[10] Spectral Database for Organic Compounds, SDBS, National Institute of Advanced Industrial Science and Technology (AIST), Japan; http://sdbs. db.aist.go.jp

[11] M. Breitman, S. Ruiz-Moreno, A. Lopez-Gil, "Experimental problems in Raman spectroscopy applied to pigment identification in mixtures", 
Spectrochimica Acta A, (2007) 68 1114-1119, DOI: 10.1016/j.saa.2007.06.042.

[12] M. Wachowiak, A. Klisińska-Kopacz, "Issues of Jan Matejko painting technique", in: Interdisciplinary Research on the Works of Art, Justyna Olszewska-Świetlik, Joanna Arszyńska, Bożena Szmelter-Fausek (Eds.), Wydawnictwo Naukowe UMK, Toruń 2012, pp. 245-262.

[13] M. Wachowiak, Malarstwo olejne Józefa Pankiewicza - materiat i technika, $\mathrm{PhD}$ dissertation, Toruń 2008, manuscript.

[14] R.L. Feller, "Barium Sulfate - Natural and Synthetic", in: Robert L. Feller (Ed.), Artist's Pigments, A Handbook of Their History and Characteristics, Vol. 1, National Gallery of Art, Washington, Archetype Publications Ltd, London 2012, p. 49.

[15] M. Laver, Titanium dioxide whites, in: E. West Fitzgugh (Ed.), Artist's Pigments, A Handbook of Their History and Characteristics, Vol. 3, Archetype Publications Ltd, London 2012, p. 298.

[16] A.R. Burnstock, Ch. Jones, G. Cressey, "Characterisation of artists' chromium based yellow pigments", in: Zeitschrift für Kunsttechnologie und Konservierung, (2003) 17 , pp. 74-84.

[17] E. West Fitzgugh (Ed.), Artist's Pigments, A Handbook of Their History and Characteristics, Vol. 3, Archetype Publications Ltd, London 2012.

[18] J. Dik, F. Tichelaar, K. Goubitz, R. Peschar,

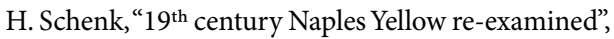
Zeitschrift für Kunsttechnologie und Konservierung, 16 (2002), 291-306.

[19] C. Sandalinas, S. Ruiz-Moreno, A. Lopez-Gil, Judith Miralles, "Experimental confirmation by Raman spectroscopy of a $\mathrm{Pb}$-Sn-Sb triple oxide yellow pigment in sixteenth-century Italian pottery", Journal of Raman Spectroscopy, 37 (2006), 1146-1153, DOI: 10.1002/jrs.1580.
[20] I. Żmuda-Trzebiatowska, M. Wachowiak, A. Klisińska-Kopacz, G. Trykowski, G. Śliwiński, Raman spectroscopic signatures of the yellow and ochre paints from artist palette of J. Matejko (1838-1893), Spectrochimica Acta, 136, (2015) 793-801, DOI: 10.1016/j.saa.2014.09.096.

[21] I. Fiedler, M.A. Bayard, "Emerald green and Scheele's green", in: Elisabeth West FitzHugh (Ed.), Artists' Pigments, A Handbook of their History and Characteristics, Vol. 3, Archetype London 1997, pp. 219-272.

[22] J. Salvant, M. Geldof, E. Ravaud, L. Megens, Ch. Walbert, M. Menu, Don. H. Johnson, "Investigation of the grounds of Tasset et L'hote commercially primed canvas used by Van Gogh in the period 1888 to 1890 , in: M. Vellekoop, M. Gedldof, E. Hendricks, L. Jansen, A. de Tagle (Ed.), Van Gogh's Studio Practice, Mercatorfonds, Brussels 2013, pp. 192-201.

[23] S. Morgan, J.H. Townsend, S.Hackney, R. Perry, "Canvas and its preparation in early twentieth century British paintings", in: J.H. Townsend, T. Doherty, G. Heydenreich, J. Ridge (Ed.), Preparation for Painting. The artist's choice and its consequences, Archetype Publications Ltd., London 2008, pp. 132-140.

[24] B. Fruhmann, M. Schreiner, M. Mantler, „Anorganische historische Pigmente einer Pigmentsammlung in Wien: Identifizierung zur Erstellung einer Datenbank", in: Zeitschrift für Kunsttechnologie und Konservierung, 17 (2003), 297-298.

[25] E.B.F. Pey, "The Hafkenscheid Collection", Maltechnik-Restauro, 93 (1987) 23-33; E.B.F. Pey, "The Organic Pigments of the Hafkenscheid Collection", Maltechnik-Restauro, 95 (1989), 146-150.

[26] E.L. Richter, H. Härlin, "A nineteenth century collection of pigments and painting materials", Studies in Conservation, 19 (1974), 76-82. 


\title{
Spectral imaging of Dutch gilt leather for improved conservation strategies
}

\author{
Roger M. Groves ${ }^{1 \star}$, Vassilis M. Papadakis ${ }^{1}$, Martine Posthuma de Boer ${ }^{1}$, \\ Tigran Mkhoyan ${ }^{1}$, Bianca van Velzen ${ }^{2}$, Kate Seymour ${ }^{2}$ \\ 1 Aerospace Non-Destructive Testing Laboratory (AeroNDT), Faculty of Aerospace Engineering, Delft \\ University of Technology, Kluyverweg 1, 2600 GB, Delft, The Netherlands \\ 2 Stichting Restauratie Atelier Limburg (SRAL), Avenue Ceramique 224, 6221 KX Maastricht, The \\ Netherlands \\ * Corresponding author: R.M.Groves@tudelft.nl
}

\begin{abstract}
Gilt leather was one of the most fashionable and costly types of wall-hangings in the Western world in the $16^{\text {th }}$ to $18^{\text {th }}$ centuries. It has a gold appearance which is the result of a silver leaf coated with an orange-brown varnish. It is a layered composite of organic and inorganic materials, including leather, animal glue, silver metal-leaf, varnish, and oil paint. Ageing processes are complex due to many interactions between these different materials, which makes conservation a real challenge. Furthermore, the common conservation treatments practiced in the past resulted in negative side-effects over-time such as gloss and colour change (darkening) and stiffening of the support. Hyperspectral imaging is a proven non-destructive method that has been used in conservation for more than two decades, mostly for the examination of paintings and manuscripts. With its use, it is possible to reveal features and their locations invisible to the naked eye, such as thin varnish layers, paint and overpaints or underdrawings.

In this study, a collection of gilt leather objects was studied at SRAL. Data were analysed using a software analysis platform (TIPP), developed at AeroNDT. The results show maps of quantitative pigment concentration and areas with possible chemical degradation or silver tarnishing.
\end{abstract}

Keywords: hyperspectral imaging, gilt leather, conservation, non-destructive testing, Dutch cultural heritage

\section{Introduction}

Gilt leather was one of the most fashionable and costly types of wall-hangings in the Western world from the $16^{\text {th }}$ to the $18^{\text {th }}$ centuries. The origins of this particular decorated leather lie in North-Africa (Ghadames, Libya). The craft spread through Spain towards the rest of Europe in the late Middle Ages. In the mid-1 $17^{\text {th }}$ century Dutch gilt leather had a similar fame to Delftware and Dutch paintings.

In The Netherlands, only 60 gilt leathers have survived in-situ in historic houses, castles, palaces, churches, town halls and other public or private institutions [1]. This is 
only a small percentage of what once existed. Archival research revealed that in Leiden, just on the Rapenburg alone, one of the main canals of the city, 60 rooms were decorated with gilt leather in the $17^{\text {th }}$ and $18^{\text {th }}$ centuries. None of these have survived. Due to the complex nature and sensitivity of the material, conservation of gilt leather is a challenge. Former craft-led conservation practices, focused solely on either the leather support or the decorative surface layers, negating the fragility of the separate materials used and the complexity of the layer build-up. As a consequence, a considerate amount of gilt leather ensembles and objects are in an alarming condition today.

Gilt leather is a layered composite of organic and inorganic materials. The aging of gilt leather is characterised by the specific degradations of each of the materials and the possible interactions between them. Typical chemical and physical damage and degradation processes have been studied for each of the materials - leather, animal glue, silver leaf, varnish, oil paint - and presented within the literature of the different conservation disciplines: leather, metals and paintings conservation. For example, much has been published on the complex ageing of leather [2, 3], as well as the degradation of oil paint [4]. However, there is scarce literature on the specific degradations of the composite materials of gilt leather or their interactions with each other. While the materials composing the aesthetic layers are often similar to oil paintings, the layer build-up of the laminate structure for gilt leather is opposed to traditional paintings practice as the varnish layer is found below the coloured paint. This leads to more complex problems in terms of cleaning these delicate surfaces with solvents. As a result, in the past many gilt leather ensembles were irrevocably damaged. The problem regarding the ageing and conservation of gilt leather has recently been brought to the attention in a white paper [5]. Some projects start taking into account possible interactions between the different materials and the resulting degradation products. Key to this new approach for the conservation of these precious beautiful ensembles is identifying the problem. This is where hyperspectral imaging can play a role, providing conservators with the appropriate diagnostic tools and resulting in data with which it will be possible to make suitable and ethical decisions for future treatment.

Hyperspectral Imaging (HSI) is an optical technique that holds the potential in characterising materials and degradation phenomena by their optical properties, or in determining and visualising mechanical properties and behaviour of materials, including defects. Within the domain of art history, archaeology and conservation, hyperspectral imaging has been used since the 1990s, mostly for the examination of paintings and manuscripts [6]. It has proved to be a successful tool for revealing things that are invisible to the naked eye. The near infrared spectrum (700-2500 nm) can be used for revealing underdrawings situated beneath opaque paint layers. To make faded writing in manuscripts visible, UV-reflected light and certain infrared bands are used. Retouchings can be made visible this way; moreover, their contrast reflectance pattern/ spectra is different under divergent light sources. Hyperspectral imaging can also be of use in the monitoring of conservation treatments. For example, monitoring the laser cleaning intervention on marble crusts $[7,8]$. This technique has also proved applicable for monitoring the partial cleaning of (yellowed aged) varnishes [9]. Another interesting application is pigment charac- 
terisation, which unfortunately remains complicated, as some pigments do not have very distinct spectral features, for instance yellow pigments [10]. The characterisation of pigment mixtures present in decorative paints is an even more demanding challenge; however, success is possible [6]. Deterioration analysis of painted surfaces using HSI focuses on the determination of the areas under risk based on the combined information deriving from both mechanical and chemical changes $[11,12]$.

Preliminary non-destructive analyses were performed with hyperspectral imaging (at AeroNDT) in order to get a better understanding of specific degradation phenomena taking place within the decorative layers. Understanding the data set provided during the preliminary study allowed researchers to progress to case studies. These were provided by SRAL. The case studies consisted of 5 sections of gilt leather ensembles currently undergoing treatment in the Maastricht conservation studios, of which the results of the panel from the Maastricht Town Hall will be presented here.

\section{Materials and methods}

The gilt leather sections were analysed using the modular 3D hyperspectral imaging scanner setup developed at AeroNDT, see Fig. 1. Particularly:

\subsection{Hyperspectral imaging}

The hyperspectral imaging system used was a line-scan spectral camera (IMSPECTOR V10E, Specim $\odot$ ) with a wavelength sensitivity range of 400-1000 $\mathrm{nm}$. The camera was attached to a 3D scanning system, as described in the following section. The

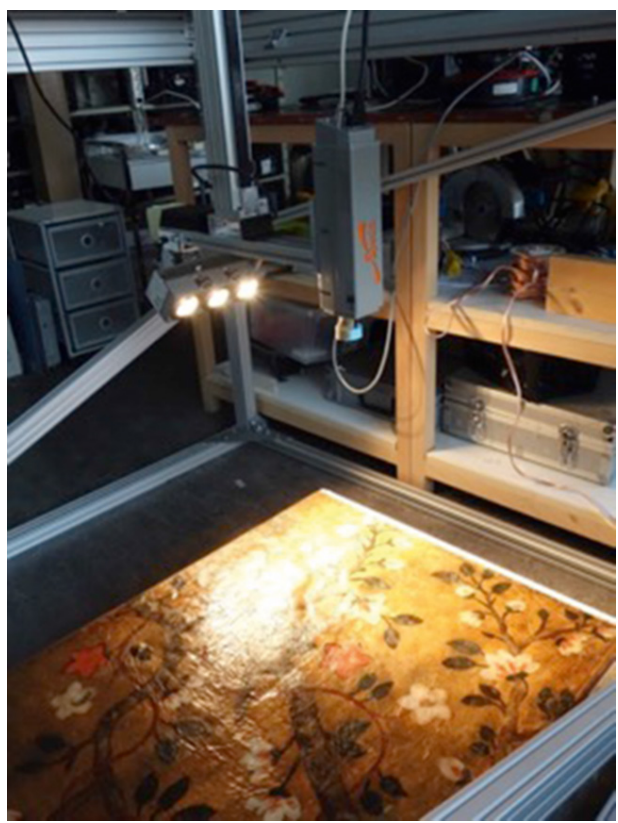

Fig. 1. The hyperspectral camera and light sources mounted on the modular 3D hyperspectral imaging scanner, developed at AeroNDT. Close-up of the setup in the conservation studio at SRAL. The gilt leather wall-hanging panel comes from the Town Hall in Maastricht.

objective lens of the camera was set to image a line of spatial information with a size of approximately $10 \mathrm{~cm}$. The frame rate of the camera was set to $3 \mathrm{fps}$ with a shutter time of $333 \mathrm{msec}$. The light sources were installed under a 45 degree angle with respect to the surface plane. The sample's surface temperature before measurements was measured and tests were performed related to the maximum temperature rise of the samples surface within 1 hour of illumination.

The calibration of the hyperspectral system was achieved by a white diffuse reflectance target made out of Spectralon (SRT-MS-050, Laser2000) and a dark reference image that was obtained with the shutter closed. Prior to the measurements, 
reference images were acquired from the white target, and with the camera's shutter closed. The white reference image contains much of the information needed for the calibration since it contains information related to the spectral variations of light, together with the differences deriving from the light source and optics. The image with the shutter closed contains the dark current background, which is subtracted from the signal image as it contains an offset. It should also be noted that the white and dark reference signals also contain a random noise component, so the calibration procedure slightly increases the overall signal noise.

\subsection{Scanning system}

The spectral camera is coupled to a $3 \mathrm{D}$ scanning system, which is a 3 axis Cartesian coordinate robot, capable of moving at maximum speed of $8 \mathrm{~cm} / \mathrm{s}$ in XY-axis and 5 $\mathrm{cm} / \mathrm{s}$ in Z-axis. The drive system and chassis are comprised of toothed-belt Linear Motion stages (MAXR23-S42-H42-C42, Schneider Electric) and are capable of delivering 0.05 $\mathrm{mm}$ repeatability. The chassis is motorised by means of 3 stepper motors/controller duo, the (QSH6018-86-28-310 and PD4-11360-SE-485, Trinamic Motion). The encoder allows micro-stepping and provides resolutions of up to 2.3 micron in the $\mathrm{X}$ - and Y-axes and 1.5 micron in the $\mathrm{Z}$-axis. The system is augmented with a Neugart planetary gear system of PLE 060 Series. Additionally, the $\mathrm{Z}$-axis is equipped with a mechanical brake and allows payload carrying capability of up to $12 \mathrm{~kg}$. The scanner assembly rests on a lightweight frame constructed with extruded aluminium beam members and perforated steel fortification elements to limit shearing. The scanner measures $1400 \mathrm{~mm}$ in $\mathrm{X}$ - and Y-, and $1200 \mathrm{~mm}$ in Z-axes. The active scanning volume, determined by the X-, Yand $\mathrm{Z}$-axis travel, is $785 \mathrm{~mm}, 895 \mathrm{~mm}$ and $500 \mathrm{~mm}$ respectively. Due to the additional elements (spectral camera and light source), the resulting bracket system was relatively large and limited either the X- or Y-axis travel by approximately $200 \mathrm{~mm}$. Finally, the entire setup is modular and can be dismantled and carried by two individuals.

In the current study, the modular 3D hyperspectral imaging scanner setup was initialised accordingly to the gilt leather sections. The main travel axis was limited to a speed of 0.0738 centimetres per second to match the camera acquisition speed. Hence, the larger pieces of guilt leather required several hours of scanning. Particularly large surfaces were scanned over the course of two days to prevent any damage that would be caused by the heat from the light source.

\subsection{Case Study: Chinoiserie gilt leather wall-hangings in the Mayor's office of the Town Hall of Maastricht}

Data presented in this paper, deriving from the hyperspectral images, were obtained from the $65 \mathrm{~cm}$ by $10 \mathrm{~cm}$ scanned section of gilt leather wall-hangings from the Town Hall in Maastricht. The section scanned was selected from the $178 \mathrm{~cm}$ by $71 \mathrm{~cm}$ panel removed from the interior setting (Fig. 2 and Fig. 3) for a pilot conservation and restoration project at SRAL in 2014-2016.

The panel is part of a larger ensemble that covers the four walls of the Mayor's office in the Town Hall. The Dutch architect Pieter Post (1608-1669) and his local assistant Cornelis Pessers designed and built the Town Hall in Maastricht between 1655 and 1662. Archival evidence dates the gilt leather wall-hangings to some seventy-five years later to the late 1730s. The Maas- 

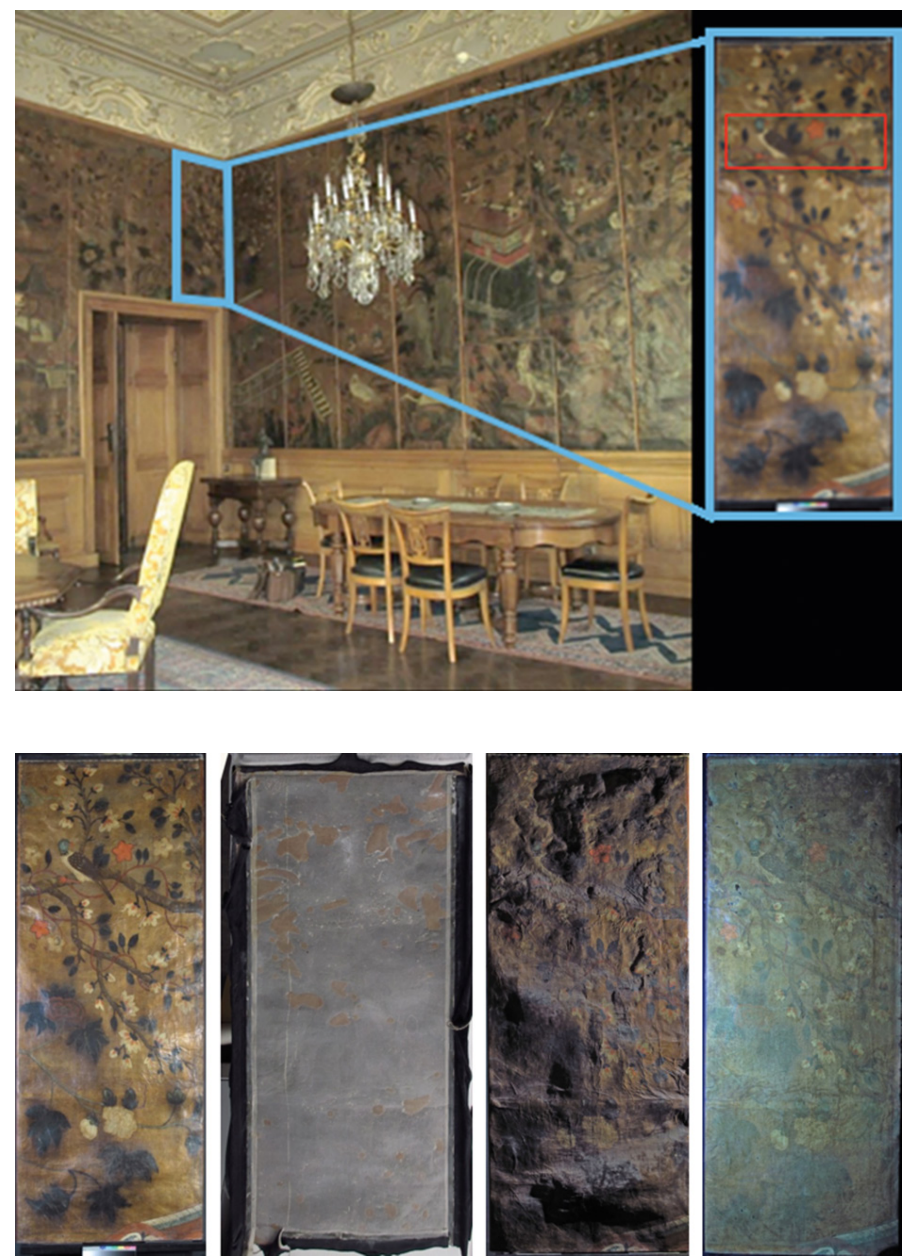

Fig. 3. The $18^{\text {th }}$ century English gilt leather panel $(178 \mathrm{~cm} \times 71 \mathrm{~cm})$ from the Town Hall in Maastricht. Left: front view in visible light; left centre: reverse view in visible light; right centre: front view in oblique/raking light; right: front view in ultraviolet light. @ SRAL
tricht-Brabant Mayor Godaert van Sleypen commissioned the ensemble in 1737 , via the cabinetmaker Van Thiel in The Hague, from an English manufacturer of gilt leather hangings [13]. The vertical panels depict a continuing, non-repeating oriental landscape painted in a "Chinoiserie" style. The pattern is unique and few other ensembles in this Chinoiserie style remain worldwide. Though manufactured in England, the Maastricht gilt leathers conform to Dutch production techniques, although the pattern is not embossed. As such, the ensemble can be described as a "flat type" of gilt leather. The layer build-up, seen in cross-sections (Fig. 4), consists of the (1) leather support, (2) silver leaf, (3) a "gold-coloured" varnish, and $(4,5)$ oil paint decorative layers.

The ensemble was treated at least three times in the past. The earliest documented treatment took place in 1861 by Mr. J. Krans, the second in 1927-1928 by Mr. Jan Mensing, and the latest around 1970 by Mr. H.A.B. van Soest. The current surface appearance is a result not only of original materials, but also of those left during past restoration cam- 


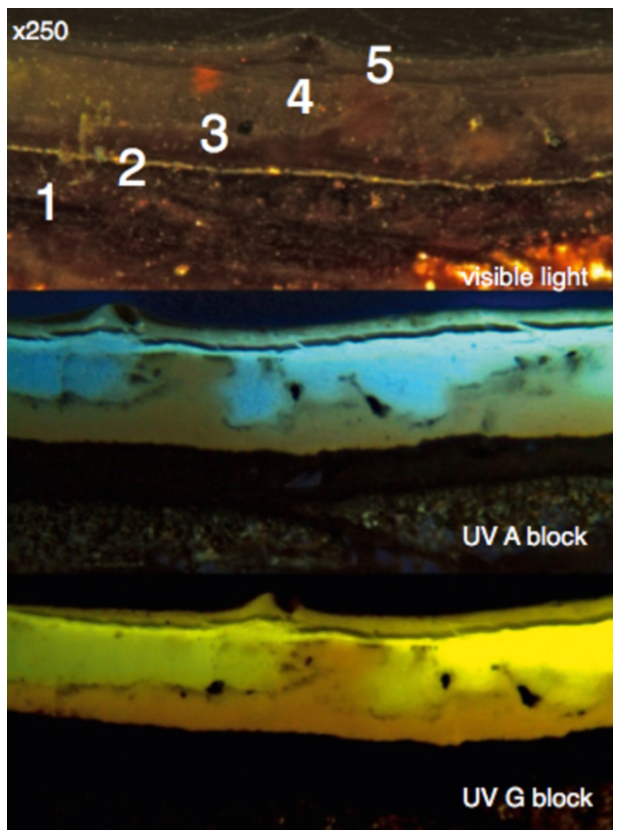

Fig. 4. Cross-section (MG4) removed from Maastricht Town Hall $18^{\text {th }}$ century English gilt leather showing representative layer build-up of manufacture process and materials. Magnification: 250×. Top: visible light; Middle: UV A block filter; Bottom: UV G block filter. The sample shows (1) leather support; (2) silver leaf; (3) a "gold-coloured" varnish; (5) non-original varnishes and dirt layers. The sample was removed from an area without decorative paint layers. Remnants of the original yellow coloured varnish layer can be seen migrating into more recently applied varnish layers. @ SRAL

paigns. Previous cleanings removed, partially or in some areas completely, the original orange-brown coloured varnish layer, and restorations added additional modern (coloured) varnish layers. Treatments carried out in the earlier documented restorations are not fully described. The report by Van Soest gives few details about the treatment of the pictorial layer, though it seems that his intension was to achieve a matte non-glossy surface and he retouched damages [14]. It is known that Van Soest consulted Mr.
H.S. Bloedhouwer, a restorer at the Rijksmuseum, Amsterdam. Bloedhouwer provides a piece of advice in Rapport betreffende restauratie goudleerbehang in het Raadhuis van de gemeente Maastricht ${ }^{*}$. He suggests that the vertical joints between the leather panels are re-stitched, the front is cleaned and paper/ glue backings on the reverse are removed. The leather support should be made more supple by massaging in a leather dressing ('...om een weinig leven in het leer terug te brengen...'), and the tears in the support should be mended by gluing leather patches and covering these with Tergal (polyester) fabric. It appears that Van Soest followed most of this advice. The stitched borders have been removed and replaced by new strips of leather, a Lycra fabric was adhered to the edges of the panels to provide flexible tensioning, old patches removed and replaced as suggested, a thin polyester gauze (Tergal) was glued using Mowilith (PVA) dispersion to the reverse of the original leather support, and finally the leather panels were stretched around double-sided aluminium honeycomb supports for additional strength.

Diagnosing the condition of the original surface-decorative materials, the extent of later restoration additions, and their effect is essential for determining conservation strategies and proved problematic due to the current surface appearance (Fig. 5). The surface was very dirty and dust had accumulated in deformations caused by a lack of tension across the support. "Flat-type" gilt leather

* The treatment proposal by H.S. Bloedhouwer was provided after a visit to the Mayor's Office at the Maastricht Town Hall on 21 November [1967]. Van Soest, H.A.B., Behandelingsvoorstel (Treatment proposal) 'Maatrichtse goudleer', C.L. dossier Maastricht, gemeentehuis: goudleer 67/3A 745.531.025, 1 page and photograph from the dossier $67 / 3$. 


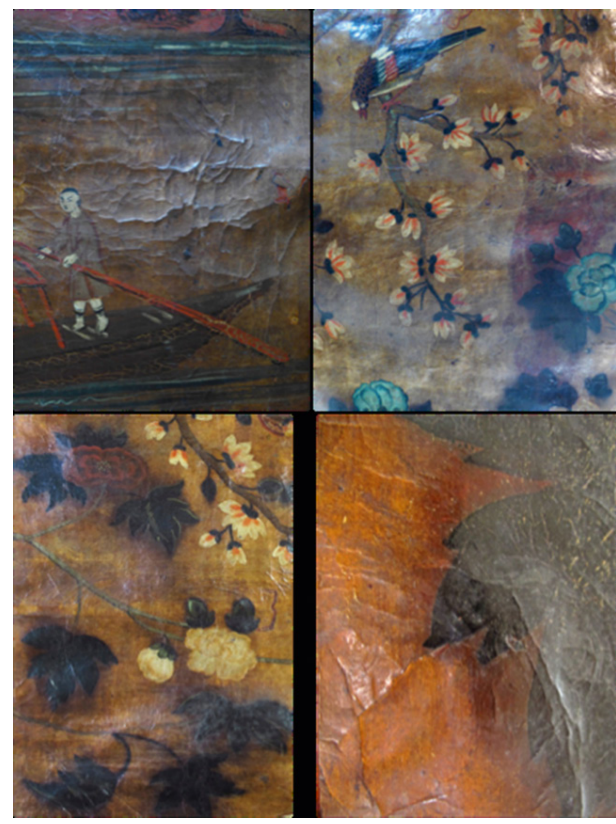

Fig. 5. Four images showing various surface deterioration and degradation effects. Top left: dusty surface and cracks in the leather support; top right: discoloured modern varnish layers muting original decorative scheme; bottom left: blackening of green leaves and dark surrounding halo; bottom right: detail of bottom left where the silver leaf has been tarnished. (C) SRAL

surfaces were often a contrast between matt (paint) and glossy areas (gilt background). This interplay of surface appearance is no longer apparent. The upper (later) varnish layers were applied indiscriminately over background and decorative areas. These had discoloured, changing the hue and tone of the original pictorial layers. The varnish layers have also become very glossy. The illusion of depth and the vivid colours of the composition are no longer apparent. Micro-fissures and cracks have propagated from the support through the varnish layers further disrupting the legibility of the image. Micro-chemical interactions between pigment, binding media, metal leaf and oil-resin varnish have caused the green leaves to become almost black in colour. Past cleaning solutions had dissolved paint, dispersing orpiment pigment particles from the green leaves over exposed areas of the silver leaf, exacerbating the tarnishing of this material. The visible appearance of the degradation results in a dark halo surrounding the leaves.

Visual observations using magnification and selective sampling of the surface aided the conservators in mapping original and non-original materials. Sampling confirmed the traditional layer build up and gave insight into pigment use. This was facilitated using traditional photographic documentation techniques employing lights of different wavelengths (ultraviolet, infra-red reflectography and $\mathrm{x}$-radiography). The traditional documentation techniques were supplemented by the results of the hyperspectral imaging carried out in conjunction with the AeroNDT team. Results aided the conservators in understanding the complex laminate structure and the (conservation) history of the object.

Hyperspectral imaging of the Maastricht gilt leather panel took place during a later phase in the conservation process. Initial surface cleaning had occurred and the aesthetical improvement (retouching) of the decorative image was completed. Optimally, HSI should take place during the initial diagnostic study and can be used throughout the treatment campaign to establish effective cleaning results. The removal of all remnants of non-original materials would provide a complete study of the original surface; however, in reality compromises are essential as often it is not ethically possible to remove all non-original material from delicate surfaces. Interpreting the images provided by the HSI would be 
difficult due to the complexity of materials on the surface. This challenge would allow the research team to establish protocol and parameters of the imaging system.

The presence of modern varnish layers on the Maastricht Town Hall gilt leather panel, applied in the 1970s/1980s, is clearly visible in the cross-sections (Fig. 4). The modern varnish was applied over the whole surface, including the pigmented layers, and had unknown additives (plasticisers or solvents) that caused the underlying original orangebrown varnish to migrate up into the newer varnish. The conservators had removed dust and dirt layers from the surface, and where safe had partially thinned the non-original modern varnish layers from non-decorative areas. It was not deemed safe to remove the varnish from the decorative layers due to the lack of interface between varnish applications. The conservators hoped that the hyperspectral scan would pin-point with greater accuracy areas presenting this problem. Then, a more accurate cleaning strategy could be implemented for the rest of the ensemble.

The second issue that the conservators desired was the clarification regarding the extent of tarnishing around the green leaves. Scanning electron microscopy linked with energy dispersive $\mathrm{x}$-ray spectroscopy (SEM-EDX) showed that the green paint is composed of a mixture of blue and yellow particles. The very fine blue particles could not be easily identified but contained the elements iron $(\mathrm{Fe})$ and aluminium $(\mathrm{Al})$; the yellow particles contained arsenic (As) and were therefore expected to be orpiment (arsenic sulphide $\mathrm{As}_{2} \mathrm{~S}_{3}$ ). Combinations of ultramarine (a pigment containing the elements iron and aluminium) or indigo and orpiment were common in the $18^{\text {th }}$ century to produce green coloured paint. The morphology of the pigment particles indicates a use of indigo (precipitated to an alum base) rather than ultramarine. An early use of Prussian blue is also possible as the manufacture of the pigment was widespread by 1730 (first publication in England in 1724). Cross-section samples removed from the area surrounding the leaves showed that particles of orpiment pigment had spread across the decorative borders. It is those, combined with atmospheric pollutants and solvents when applying the modern varnishes, which have probably caused the tarnishing. Conservators hoped to obtain an accurate map of the extent of tarnishing using the hyperspectral scanning system.

\subsection{Processing and analysis software}

Processing and the analysis of the gilt leather measurements were performed with an advanced image-processing platform (TIPP) that was developed at AeroNDT [15]. Initially, measurements are processed for calibration with the reference images in order to achieve repeatable and comparable data. This is simply achieved by subtraction of the dark reference and normalisation with the white reference. Data are processed through 32 bit floating point variables, which ensures that no data are lost from the calculations. Following calibration, the user has the option to process data to correct for the aspect ratio due to the acquisition properties, for dimension reduction, and for image stitching from multiple measurements. Advanced analysis algorithms are focused on principal component analysis (PCA) and on reference component analysis (RCA), which is based on non-negative least squares solutions for un-mixing spectral features of pixels. Viewing of data is achieved with a novel module that allows multi-dataset visualisation of spectral 
data and of the analysis results. Moreover, spectral reflection characteristic graphs can be visualised and exported from a single point of view after averaging with multiple different methods.

\section{Results}

\subsection{Surface temperature measurements}

The surface temperature of the scanned section, see Fig. 6, was initially measured with no illumination at 20.2 degrees Celsius $\left({ }^{\circ} \mathrm{C}\right)$. After one minute of exposure, the temperature rose to $22.3^{\circ} \mathrm{C}$, which was the most

\subsection{Gilt leather section measurements}

A $10 \mathrm{~cm}$ by $65 \mathrm{~cm}$ gilt leather section from the Maastricht Town Hall panel was examined and scanned. Measurements were performed across the whole sensitivity range of the sensor, starting at $400 \mathrm{~nm}$ and up to $1000 \mathrm{~nm}$ in the near infrared region. The total of spectral images acquired in this region were 196 bands, resulting to a tuning step of approximately $3 \mathrm{~nm}$. The dynamic range (bit depth) of the sensor was set to be 12 bit equivalent to 4096 grey levels.

Six different points of interest were selected as representative of the different

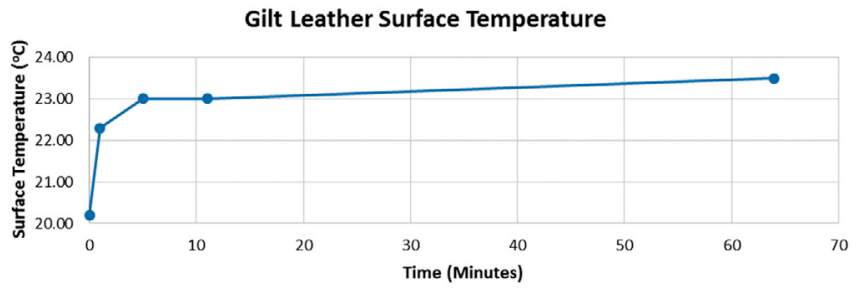

Fig. 6. Gilt leather surface temperature rise measurements. Temperature remains almost constant after 5 minutes of exposure in the systems light source.

significant raise in temperature observed. In the subsequent 5 minutes of illumination, the temperature reached $23.0^{\circ} \mathrm{C}$, where it remained almost constant for a full hour of measurements. The surface temperature was measured at $23.5^{\circ} \mathrm{C}$ after 65 minutes of exposure to the light source. The $3.3^{\circ} \mathrm{C}$ temperature increase was deemed minimal and not damaging to the gilt leather panel and its constituent materials. materials present on the surface of the gilt leather panel. These points were selected based on visual inspection and are shown in Fig. 7. The reference points were chosen where the decorative surface appeared to be most homogeneous and dense. As presented in the following Fig. 8, the reflection spectrums from each point were extracted Fig. 8 and recorded in TIPP database to be later used as reference points for the RCA.

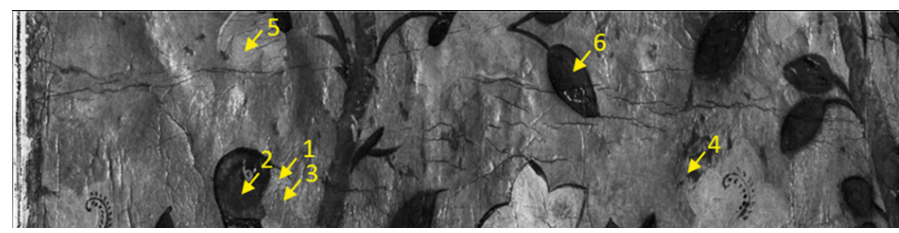

Fig. 7. Imaging at $700 \mathrm{~nm}$ of gilt leather section from the Maastricht Town Hall. The points of interest that were selected for spectral reflection characteristics extraction are displayed. 


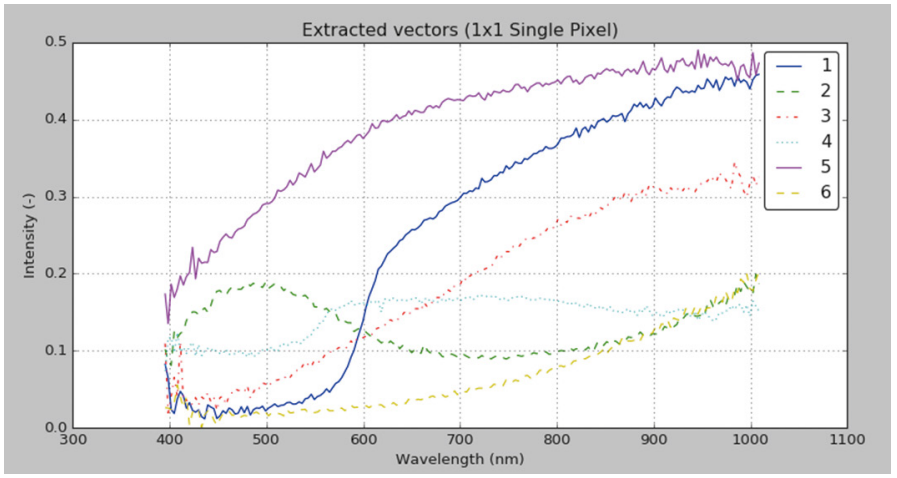

Fig. 8. The reflection spectral characteristics as displayed from the reference points selected in TIPP viewer. (1) red paint; (2) blue paint; (3) gold varnish; (4) white paint; (5) white-grey paint; and (6) green paint.
The reference points that were selected included: (1) red paint; (2) blue paint; (3) gold varnish; (4) white paint; (5) white paint with underlying corrosion; and (6) green paint. It is expected that the reference points selected contain for the paints more than one pigment and or a contribution from under-layers, such as the gold varnish and the silver leaf layers. Spectra obtained from similarly coloured surfaces may thus appear different. Ultimately, cross-section removed from the reference spectra points would aid in interpreting spectra and contrast false-colour images obtained at different wavelengths. Cross-section analysis and interpretation for the Maastricht Town Hall are reported elsewhere [16].

Following extraction of reference spectral characteristics, analysis was continued with RCA, which resulted in the mapping of the specific spectral characteristics in unique image maps. In Fig. 9, a colour image of the scanned area, shows the points where the 3 reference spectral characteristics were extracted from the blue, green and red coloured paint. After the RCA was completed, the scientists and conservators selected the 3 vectors of the main paints, as shown in Fig. 9, to create the false-colour image in Fig. 10, of the selected paint distribution. Each of those 3 image maps contain quantitative information related to the percentage of contribution of each reference.

\subsection{Interpretations}

Interpreting the resulting hyperspectral maps of the painted gilt leather panel from the Maastricht Town Hall proved challenging. The scans clearly show different spectral

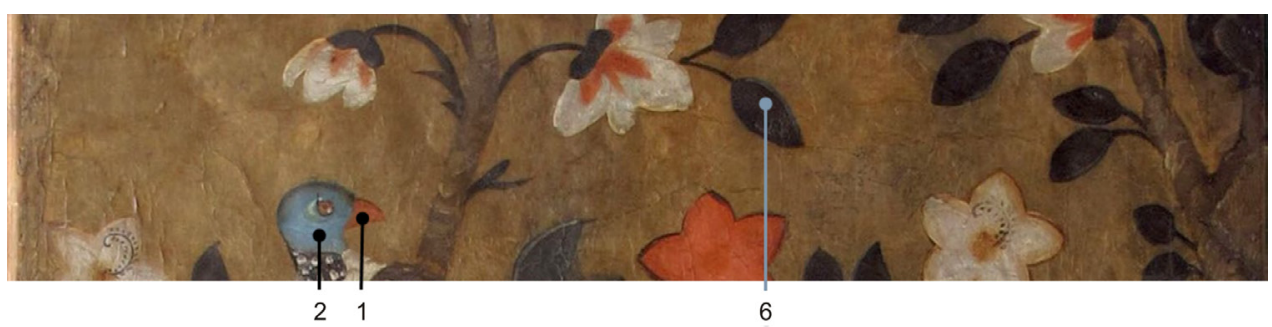

Fig. 9. Colour image from the Maastricht's gilt leather section. With numbers, the reference components that were used for further analysis are shown. 


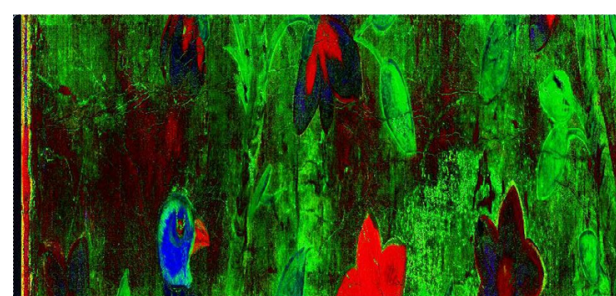

Fig. 10. The resulting false-colour image that was created by merging the reference component maps from the RCA. The false-colours represent: blue refers to the blue paint, green to the green paint, and red to the red paint.

responses to the different components used within the artwork (silver leaf, varnish, and paints). Studying the false-colour maps extracted at different wavelengths aided the differentiation of materials. Different paint mixtures in varying concentrations could be readily mapped, such as the blue colour of the bird's head and the red paint used for the flowers and the bird's beak. SEMEDX analyses of cross-sections confirmed a mixture of indigo (or Prussian blue) and lead white for the blue paint and a mixture of vermillion and minium for the red paint. The spectral response of a component in the paint of the white flowers (point 4, Fig. 7) is similar to the blue paint (point 2, Fig. 7), and is thus imaged in a similar shade of blue in the false-colour image (Fig. 10). The spectral response, at this wavelength represented in Fig. 10, of the blue paint of the bird's head is different from the paint applied around the outline of the head, which has similarities to the green paint mixture of the leaves. The spectral curves for the two white reference points (curve 4 and 5, Fig. 8) are dissimilar. This can be explained due to differences in layer build-up of the paints or a percentage difference in the concentration of pigment particles in these similarly appearing white flowers.
Prior SEM-EDX analyses of cross-sections pointed out that the green paint of the leaves is a mixture of orpiment and either indigo or Prussian blue. The false-colour maps show that, in the "golden" background, materials are present that have similar spectral characteristics as the green leaves. This could either correspond to orpiment particles that have been spread over the surface, or to silver corrosion, or both. HSI analyses on other gilt leather samples have shown that reference vectors taken from green paints tend to be difficult to differentiate from those taken from corrosion of the silver leaf. Both show a strong absorbance in the NIR. The visualisation of the extent of tarnishing of the silver leaves, due to the presence of arsenic sulphide (orpiment) thus remains ambiguous.

Imaging under higher optical magnification (3 cm field of view) was performed in selected areas where silver corrosion was visible under visual inspection. It was found that mapping of different corrosion levels is possible, and a broader spectral range (beyond 1000nm) could help in the differentiation between corrosion products of the silver leaf.

Subtle differences between similar materials were not as easily differentiated. The majority of the surface, mainly the gold coloured background, was composed of materials with similar chemical characteristics (organic natural resin varnishes) that, though applied at different times, gave similar readings throughout the spectral range used. It was not possible to delineate between areas where the upper varnish layers had been partially removed (coloured figurative areas) from those where the upper varnishes had not been removed (non-figurative areas). Nor was it possible to map where the original orange-brown varnish had migrated into the later applied varnish. 


\section{Conclusions}

In this study, the authors have shown that hyperspectral imaging can be a very useful tool for the diagnosis of gilt leather panels, as shown by the Maastricht Town Hall case study. The HSI of the surface did aid conservators in understanding the object more fully. Therefore, the technique can be a useful non-destructive tool in information gathering both prior to and during conservation treatments, and can be used in conjunction with micro-sampling to assist in understanding laminate build-up. A better understanding of the object and its constituent materials leads to a more informed decision-making process for determining conservation treatment strategies. In the case discussed, pigment characterisation was easily performed and image maps containing quantitative information regarding the pigment concentration was achieved. It was found that mapping of specific degradation phenomena, such as silver corrosion, requires high spatial resolution (approximately $30 \mathrm{um}$ ) and is still a challenge as material dynamics are complex. Specific pigment characterisation, deterioration analysis and mapping would become possible by the use of a database with reconstructions and reference samples, which is currently under development.

Further development on both hardware and software platforms will continue to enable the use of such technology in multiple fields of conservation without the limitation of the size of artwork. Furthermore, the scanner can be equipped with a wide variety of NDT specific measuring equipment, using the custom tailored brackets that will allow for multi-sensor measurements. One example is the combination of hyperspectral imaging with optical coherence tomography [17]. The TIPP platform is also progressing in such a direction to accept data from those sensors, and with advanced data-fusion techniques to enable analysis of the artwork from a single software tool.

\section{Acknowledgements}

The gilt leather project is funded by the Netherlands Organisation for Scientific Research (NWO) and is part of the Netherlands Institute for Conservation, Art and Science (NICAS).

\section{References}

[1] E.F. Koldeweij Goudleer in de Republiek der Zeven Verenigde Provinciën. Nationale ontwikkelingen en de Europese context. Leiden: Proefschrift ter verkrijging van de graad van Doctor aan de Rijksuniversiteit te Leiden 1998.

[2] R. Larsen ed. Environment Leather Project. Deterioration and Conservation of Vegetable Tanned Leather. Protection and conservation of European cultural heritage. Research Report Nr. 6. Copenhagen: 1996.

[3] R. Larsen "Experiments and observations in the study of environmental impact on historical vegetable tanned leathers". Thermochimica Acta, 365 (2000) 85-99, DOI: 10.1016/S0040-6031(00)00616-X.

[4] A. van Loon, P. Noble, A. Burnstock "Ageing and deterioration of traditional oil and tempera paints", in: Hill Stoner, J., R. Rushfield (Eds.) Conservation of easel paintings, Oxon 2012.

[5] M. Posthuma de Boer, E.F. Koldeweij, R.M. Groves, Gilt leather artefacts. White Paper on Material Characterization and Improved Conservation Strategies within NICAS, Delft 2016.

[6] H. Liang "Advances in multispectral and hyperspectral imaging for archaeology and art conservation", Applied Physics A, 106 (2012) 309-323, DOI: 10.1007/s00339-011-6689-1.

[7] V.M. Papadakis, A. Loukaiti, P. Pouli. "A spectral imaging methodology for determining on-line the optimum cleaning level of stonework", Journal of Cultural Heritage, 11 (2010) 325-328, 2010, DOI: 10.1016/j.culher.2009.10.007. 
[8] V.M. Papadakis, Y. Orphanos, S. Kogou, K. Melessanaki, P. Pouli, C. Fotakis, "IRIS: a novel spectral imaging system for the analysis of cultural heritage objects", Proc. SPIE 8084 80840W (2011), DOI: $10.1117 / 12.889510$.

[9] M. Kubic "Hyperspectral Imaging: A New Technique for the Non-Invasive Study of Artworks", in: Creagh, D., D. Bradley (Eds.), Physical Techniques in the Study of Art, Archaeology and Cultural Heritage, Elsevier 2007, pp. 200-259.

[10] S. Kogou, A. Lucian, S. Bellesia, L. Burgio, K. Bailey, C. Brooks, H. Liang,"A holistic multimodal approach to the non-invasive analysis of watercolour paintings", Applied Physics A, 121 (2015) 999-1014, DOI: 10.1007/s00339-015-9425-4.

[11] L.G. Montagud, C. Portalés, B.P. Carbonell, E.R. Gómez, A.G. Lucas, V. Tornari, V. Papadakis, R.M. Groves, B. Sirmacek, A. Bonazza, M. Föster, P. Aswendt, A. Borreman, J.D. Ward, A, Cardoso, L. Aguiar, F. Alves, P. Ropret, J.M. Luzón-Nogué, C. Dietz, "Deterioration estimation of paintings by means of combined 3D and hyperspectral data analysis", Proc. SPIE 8790 (2013) 879008, DOI: 10.1117/12.2020336.

[12] L.G. Montagud, C. Portalés, B.P. Carbonell, E.R. Gómez, A.G. Lucas, V. Tornari, V. Papadakis, R.M. Groves, B. Sirmacek, A. Bonazza, M. Föster, P. As- wendt, A. Borreman, J.D. Ward, A, Cardoso, L. Aguiar, F. Alves, P. Ropret, J.M. Luzón-Nogué, C. Dietz, "SYDDARTA: new methodology for digitization of deterioration estimation in paintings", Proc. SPIE 8790 (2013) 879011, DOI: 10.1117/12.2020333.

[13] E.F. Koldeweij, "Het goudleer-behang in de burgemeesterskamer in het stadhuis van Maastricht", Een seer magnifiek Stadthuys. Tien studies over de bouw en de inrichting van het stadhuis te Maastricht, Delft 1985, pp. 151-159.

[14] Van Soest, H.A.B., "Het goudleer en haar restauratie" (1969?1971?). C.L. dossier Den Haag, Gemeentemuseum: goudleer 70/2 745.531.025, 2-14 en $2-2$ p.13 en 14.

[15] TU Delft Image Processing Platform, http:// www.lr.tudelft.nl/TIPP (accessed 22.09.2016).

[16] B. van Velzen "Goudleer Maastricht Stadhuis vooronderzoek pilot: onderzoeksresultaten en behandelingsvoorstel" Internal report SRAL 2016.

[17] L.M. Dingemans, V.M. Papadakis, P. Liu, A.J.L. Adam, R.M. Groves. "Optical coherence tomography complemented by hyperspectral imaging for the study of protective wood coatings", Proc. SPIE. 9527, Optics for Arts, Architecture, and Archaeology V (2015), 952708, DOI: 10.1117/12.2184716. 

Author index 

Lasers in the Conservation of Artworks XI, Proceedings of LACONA XI, P. Targowski et al. (Eds.), NCU Press, Toruń 2017, DOI: 10.12775/3875-4.22

A

Andraud, Christine 63

Asmus, John F. 15, 115

\section{B}

Bartoli, Laura 253

Barucci, Marco 141

Basilissi, Giulia 127

Beier, Oliver 241

Binet, Laurent 63

Bonelli, Donatella 229

Botteon, Alessandra 229

Bourgon, Julie 63

Bulska, Ewa 263

\section{C}

Cagnini, Andrea 127

Caneve, Luisa 253

Cappuccini, Caterina 27

Carò, Federico 27

Cerea, Silvia 229

Cheung, Nai-Ho 205

Ciaffi, Massimiliano 253

Cicchi, Riccardo 141

Ciofini, Daniele 77

Conradi, Andrea 241

Corda, Ludovica 77
Czyż, Krzysztof 191

D

Daffara, Claudia 127

Dajnowski, Andrzej 217

Dajnowski, Bartosz A. 47, 217

Dal, Fovo Alice 141

Detalle, Vincent 205

E

Elford, Jessica P. 15, 115

$\mathbf{F}$

Falcon, Martinez Maria Fernanda 253

Fantoni, Roberta 253

Fontana, Raffaella 141

Francucci, Massimo 253

G

Gaburro, Nicola 127

Galeotti, Monica 127

Giuffrida, Chiara 253

Giuntini, Christine 77

Godet, Marie 63

Groves, Roger M. 307 
Grünler, Bernd 241

Guarneri, Massimiliano 253

H

Hartmann, Annett 241

\section{I}

Isella, Elisa 229

Iwanicka, Magdalena 105

J

Jovanović, Maja 279

Jovanović, Vanja 279

K

Kaczkowski, Rebecca A. 47

Kamińska, Marta 263

Krüger, Jörg 241

L

Leona, Marco 77

Leroy, Eric 63

Leung, Kelvin S. Y. 205

Londero, Pablo 27, 77

Lum, Judy T.S. 205

$\mathbf{E}$

Łukaszewicz, Jadwiga W. 105

M

Mancini, Francesca 229

Marchioro, Giacomo 127

Marinelli, Marco 179

Melessanaki, Kristalia 95

Mercatelli, Raffaella 141

Mkhoyan, Tigran 307

Monnier, Judith 63

Motto-Ros, Vincent 205

Musiela, Jędrzej 105
$\mathbf{P}$

Palucci, Antonio 253

Pampaloni, Enrico 141

Papadakis, Vassilis M. 307

Papanikolaou, Athanasia 95

Parfenov, Vadim A. 15, 115

Pasqualucci, Alessandra 179

Pawlak, Agnieszka 191

Pentzien, Simone 241

Persia, Franca 253

Pezzati, Luca 141

Philippidis, Aggelos 95

Posthuma, de Boer Martine 307

Pouli, Paraskevi 95

$\mathbf{R}$

Raffaelli, Marco 141

Rizzo, Adriana 27

Romani, Martina 179

Romeo, Alessandro 127

Roth, Constanze 241

Ruppen, Veronica 229

S

Saheb, Mandana 63

Sansonetti, Antonio 229

Sawicki, Marek 155

Schmidt, Birgit Angelika 241

Scirpa, Francesca 253

Seymour, Kate 307

Siozos, Panayiotis 95

Skrzeczanowski, Wojciech 191

Spizzichino, Valeria 253

Stoksik, Henryk 105

Striber, Joakim 279

Striova, Jana 141

Sylwestrzak, Marcin 105

Syta, Olga 155

T

Tatì, Angelo 253

Terranova, Gaetano 253

Trykowski, Grzegorz 293 
V

Velzen, Bianca van 307

Vergès-Belmin, Véronique 63

Verona-Rinati, Gianluca 179

Vicenzi, Edward P. 47

W

Wachowiak, Mirosław 293

Wagner, Barbara 155

Walczak, Małgorzata 263

Wilk, Dariusz 263
$\mathbf{Y}$

Yandrisevits, Marlene April 27

Yue, Cai Bruno 205

Z

Zanini, Alessandro 253

$\dot{\mathbf{Z}}$

Żmuda-Trzebiatowska, Iwona 293 
